Claudia Finger

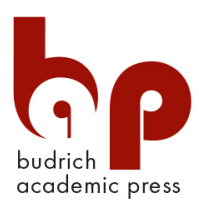

Soziale Herkunft und

die Umsetzung

von Studienaspirationen

Individuelle und institutionelle

Einflüsse in Phasen der

Selbst- und Fremdselektion 


\section{Claudia Finger Soziale Herkunft und die Umsetzung von Studienaspirationen}



Claudia Finger

\section{Soziale Herkunft \\ und die Umsetzung von Studienaspirationen}

Individuelle und institutionelle Einflüsse in Phasen der Selbst- und Fremdselektion

Budrich Academic Press

Opladen • Berlin • Toronto 2022 
Bibliografische Information der Deutschen Nationalbibliothek

Die Deutsche Nationalbibliothek verzeichnet diese Publikation in der Deutschen Nationalbibliografie; detaillierte bibliografische Daten sind im Internet über http://dnb.d-nb.de abrufbar.

\section{Die Publikation wurde durch den Leibniz-Publikationsfonds für Open-Access-Monografien und das Wissenschaftszentrum Berlin für Sozialforschung (WZB) gefördert.}

(C) 2022 Dieses Werk ist bei der Budrich Academic Press GmbH erschienen und steht unter der Creative Commons Lizenz Attribution 4.0 International

(CC BY 4.0): https://creativecommons.org/licenses/by/4.0/

Diese Lizenz erlaubt die Verbreitung, Speicherung, Vervielfältigung und Bearbeitung unter Angabe der UrheberInnen, Rechte, Änderungen und verwendeten Lizenz. www.budrich-academic-press.de

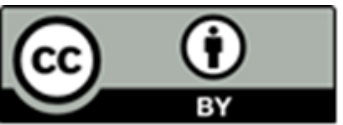

Dieses Buch steht im Open-Access-Bereich der Verlagsseite zum kostenlosen Download bereit (https://doi.org/10.3224/96665040).

Eine kostenpflichtige Druckversion kann über den Verlag bezogen werden. Die Seitenzahlen in der Druck- und Onlineversion sind identisch.

$\begin{array}{ll}\text { ISBN } & 978-3-96665-040-3 \\ \text { eISBN } & 978-3-96665-958-1 \\ \text { DOI } & 10.3224 / 96665040\end{array}$

Umschlaggestaltung: Bettina Lehfeldt, Kleinmachnow - www.lehfeldtgraphic.de Satz: Anja Borkam, Jena - kontakt@lektorat-borkam.de Druck: Books on Demand GmbH, Norderstedt Printed in Europe 


\section{Danksagung}

Das vorliegende Buch basiert auf einer überarbeiteten Fassung meiner Dissertation an der Freien Universität Berlin, die ich im Dezember 2017 verteidigte. Meine Dissertationszeit war eine intellektuelle wie auch emotionale Berg- und Talfahrt, die ohne die nötige Unterstützung beim Anschub, Bremsung und Gleiskorrekturen kaum zu bewältigen gewesen wäre.

Mein besonderer Dank gilt meiner Betreuerin und Erstgutachterin Heike Solga. Zunächst für die Möglichkeit am WZB in der Abteilung Ausbildung und Arbeitsmarkt und somit unter ausgezeichneten Bedingungen forschen und lernen zu können, die immer weit entfernt waren von den in anderen Kontexten oft als prekär beschriebenen Promotionsbedingungen. Zudem danke ich ihr für ihr Vertrauen und die vielen wichtigen Impulse und konstruktiv-kritischen Anregungen, mit denen sie meine Arbeit von Beginn an begleitet hat. Katrin Auspurg danke ich für das Verfassen des Zweitgutachtens sowie für die wertvolle methodische Beratung bei der Durchführung des Faktoriellen Surveys.

Die Dissertation entstand im Rahmen des von der Einstein Stiftung geförderten Projektes Berliner-Studienberechtigten-Panel (Förderkennzeichen A-2010-25). Die Erhebung von institutionellen Daten sowie fünf Befragungswellen war sehr intensiv und lehrreich und resultierte in Innovationsmöglichkeiten, von denen meine Dissertation stark profitieren konnte. Für das gemeinsame Meistern des Projektes danke ich meinen Kolleg/innen am WZB (Alessandra Rusconi, Martin Ehlert) und am DIW (Frauke Peter, Vaishali Zambre, Johanna Storck, Mathias Hübener, C. Katharina Spieß) sowie unseren studentischen Hilfskräften Christina Altmann, Cindy Fitzner, Judith Heinmüller, Fabio Krauthäuser und Torsten Hovorka. Insbesondere Cindy und Judith waren mir durch ihre absolute Zuverlässigkeit und akribischen Recherchen eine unverzichtbare Hilfe.

Ich danke all meinen AAM-Kolleg/innen für die immer anregende Atmosphäre und die vielen motivierenden Gespräche in den härteren (Tal-)Phasen. Zudem möchte ich mich bei den Teilnehmer/innen des Dokkolloquiums sowie insbesondere Jonna Blanck, Martina Dieckhoff, Jan Paul Heisig, Laura Menze und Alessandra Rusconi für das Lesen und Kommentieren einzelner Kapitel herzlich bedanken. Besonders hervorheben möchte ich die Unterstützung durch Alessandra Rusconi, mit der ich während der gesamten Projektzeit eng zusammengearbeitet habe und von der ich viel lernen durfte. Ich bin ihr sehr dankbar für ihr immer offenes Ohr und die viele Zeit, die sie im Laufe der Jahre aufbrauchte, um mit mir kleinere und größere Probleme zu diskutieren. Jan Paul Heisig danke ich für seine geduldige und immer erleuchtende Beratung in methodischen Fragen und Laura Menze für ihr beeindruckend genaues Lesen, ihre punktgenauen Kommentare, für ihre mentale Unterstützung und die 
Schokolade! Gunda Thielking gebührt großer Dank für das sorgfältige Korrekturlesen eines Großteils der Arbeit sowie die begleitenden sehr aufbauenden Worte.

Nicht zuletzt gilt mein Dank meinen Eltern, die meine Aspirationen geweckt und ihre Umsetzung immer unterstützt haben. Ich danke meinen Freunden, die im letzten Jahr des Schreibens geduldig auf mich gewartet haben. Und allen voran Simon: kurz für alles, da bereits eine grobe Annäherung den Rahmen dieser Arbeit sprengen würde.

Berlin, August 2021

Claudia Finger 


\section{Inhalt}

Abbildungsverzeichnis ................................................................... 11

Tabellenverzeichnis ............................................................... 13

Abkürzungsverzeichnis .............................................................. 15

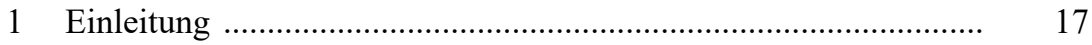

2 Institutioneller Kontext ............................................................ 27

2.1 Die Hochschulzugangsberechtigung: Voraussetzung

für den Übergang ins Studium .............................................. 28

2.1.1 Wege zur Hochschulzugangsberechtigung ............... 28

2.1.2 Pfade nach Erwerb der

Hochschulzugangsberechtigung ............................. 29

2.1.3 Soziale Herkunftsunterschiede bei und nach Erwerb der Hochschulzugangsberechtigung ........................ $\quad 30$

2.2 Die hochschulische Opportunitätsstruktur ............................... 33

2.2.1 Entwicklung und Struktur des deutschen Hochschulsystems .................................................. 33

2.2.2 Zulassung zum Hochschulstudium: Entwicklung und aktuelle Praxis ................................................. 41

2.2.3 Die Anwendung von Zulassungsbeschränkung und Eignungsfeststellung durch Hochschulen .......... $\quad 50$

2.2.4 Soziale Herkunftsunterschiede, Studienoptionen und Zulassungsregeln ............................................ 65

2.3 Berlin: ein spezieller Kontext ............................................. 67

2.4 Zusammenfassung ........................................................... $\quad 70$

3 Soziale Herkunftsunterschiede beim Übergang in die Hochschule: verbreitete Ansätze, Leerstellen und Erweiterungen ..................... $\quad 72$

3.1 Die Hauptstränge der deutschen „Übergangsforschung“ ....... 73

3.2 Leerstellen der „Übergangsforschung“ ................................ $\quad 75$

3.3 Alternative Ansätze: der Übergang als Phasenmodell ........... $\quad 78$

3.4 Zusammenfassung ............................................................. 84

4 Aspirationen und ihre Umsetzung: konzeptionelle und empirische Bestandsaufnahme ............................................................... 86

4.1 Aspirationen: Abgrenzung eines vielseitigen Begriffs ........... 86 
4.2 Herkunftsunterschiede bei der Umsetzung von

Studienaspirationen: empirische Befunde ............................ $\quad 89$

4.3 Die Phasen der Aspirationsumsetzung ................................. 98

4.3.1 Phase 1: die Übersetzung von Studienaspirationen in Studienintentionen .................................................. 100

4.3.2 Phase 2: Bewerbungsentscheidungen und Bewerbungsstrategien .................................... 102

4.3.3 Phase 3: Zulassung ................................................ 109

4.3.4 Phase 4: Studienaufnahme ...................................... 112

4.3.5 Soziale Ungleichheit durch Selbstoder Fremdselektion? .............................................. 112

4.4 Zusammenfassung .............................................................. 114

5 Soziale Herkunft, Studienaspirationen und ihre Umsetzung: theoretische Perspektiven

5.1 Aspirationen im Spiegel soziologischer

Ungleichheitstheorien

5.1.1 Der Regelfall: Aspirationen als Mediator von Herkunftsunterschieden

5.1.2 Die Ausnahme? Hohe Aspirationen trotz niedriger sozialer Herkunft

5.2 Die Umsetzung von Studienaspirationen: Zusammenspiel von individuellen Ressourcen und institutionellen

Strukturen

5.3 Primäre Herkunftseffekte und ihre Relevanz in Auswahlverfahren

5.4 Sekundäre Effekte: Bildungsentscheidungen innerhalb institutioneller Optionen und Barrieren

5.4.1 Entscheidungslogiken: unbewusst oder intentional?

5.4.2 Institutionelle Optionen: Wahlmöglichkeiten innerhalb und außerhalb des Hochschulsystems

5.4.3 Institutionelle Barrieren und deren Antizipation ....... 145

5.5 Zusammenfassung und Hypothesen ..................................... 153

6 Forschungsdesign ................................................................... 162

6.1 Übersetzung der Phasen in analytische Schritte .................... 162

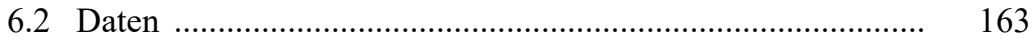

6.2.1 Best Up-Individualdaten ....................................... 164

6.2.2 Best Up-Rekrutierungsdaten .................................. 170

6.2.3 Mikrozensus .......................................................... 175 
6.3 Operationalisierung phasenübergreifender Variablen ........... 176

6.4 Definition des Ausgangssamples ........................................... 184

6.5 Methodisches Vorgehen ................................................... 188

6.5.1 Hierarchische Datenstruktur ................................... 188

6.5.2 Modelle für lineare und binäre abhängige Variablen ............................................................. 190

6.5.3 Anmerkungen zur Bedeutung von Signifikanz ......... 192

7 Phase 1: die Übersetzung von Studienaspiration in Studienintention ...................................................................... 194

7.1 Methodisches Vorgehen ........................................................ 194

7.1.1 Die Messung von Studienintentionen ...................... 195

7.1.2 Analyseschritte und Operationalisierungen .............. 197

7.1.3 Faktorieller Survey zur Modellierung institutioneller Einflussfaktoren ................................................. 199

7.1.4 Analysesample und Umgang mit fehlenden Werten ............................................................... 205

7.2 Ergebnisse I: Herkunftsunterschiede bei der Übersetzung und deren Erklärungen

7.2.1 Leistung, rationale Abwägung und soziale/kulturelle Ressourcen

7.2.2 Institutionelle Opportunitäten und Barrieren

214

7.3 Ergebnisse II: vertikal und horizontal differenzierte

Intentionen

228

7.4 Zusammenfassung ............................................................... 231

8 Phasen 2-4: die Umsetzung von Studienaspirationen .................... 236

8.1 Methodisches Vorgehen ....................................................... 236

8.1.1 Definition des Ausgangssamples .......................... 238

8.1.2 Analyseschritte und Operationalisierungen ............. 239

8.1.3 Auswertungsstrategie ............................................. 247

8.1.4 Umgang mit fehlenden Werten ............................... 250

8.2 Ergebnisse Phase 2: Herkunftsunterschiede im

Bewerbungsverhalten ..................................................... 251

8.2.1 Leistung, rationale Abwägung und soziale/kulturelle Ressourcen ......................................................... 253

8.2.2 Zugangshürden ..................................................... 259

8.2.3 Differenzierte Bewerbungen: Hochschultypen/

8.2.4 Bewerbungsstrategien ........................................... 276 
8.3 Ergebnisse Phase 3: Herkunftsunterschiede bei der Zulassung ................................................................ 281

8.4 Ergebnisse Phase 4: Herkunftsunterschiede bei der Studienaufnahme .................................................. 288

8.4.1 Studienaufnahme: konditional vs. unkonditional ...... 288

8.4.2 Differenzierte Übergänge: Hochschulorte, Hochschultypen und Studienfächer ........................ 292

8.5 Zusammenfassung .......................................................... 296

9 Zusammenfassung und Diskussion ............................................. 301

Literaturverzeichnis ................................................................... 317

Der Anhang steht online zum kostenfreien Download bereit und ist über die folgende DOI erreichbar: 10.3224/96665040A 


\section{Abbildungsverzeichnis}

Abb. 2.1: Anteil Erstsemester mit Bewerbung für ihr derzeitiges

Studienfach (in \%)

Abb. 2.2: Anteil Zulassungsbeschränkung/Eignungs-

feststellungsverfahren (in \%), WiSe 2014/15

Abb. 2.3: Durchschnittliche Auswahlgrenzen in der

Abiturbestenquote, WiSe 2014/15

Abb. 2.4: Durchschnittliche Auswahlgrenze in der Wartezeitquote, WiSe 2014/15

Abb. 2.5: Auswahlkriterien und -verfahren in zulassungsbeschränkten Studiengängen (in \%), WiSe 2014/15 ........ 61

Abb. 2.6: Verfahren zur Eignungsfeststellung (in \%), WiSe 2014/15

Abb. 4.1: Studienfachintention und -realisierung nach

Bildungsherkunft

Abb. 4.2: Modellhafte Darstellung der Phasen der

Aspirationsumsetzung

Abb. 6.1: Zeitstrahl zum Timing wichtiger Ereignisse im Best Up-Studienverlauf

Abb. 7.1: Beispielvignette

Abb. 7.2: Intentionswahrscheinlichkeit: Veränderung sozialer Herkunftsunterschiede über verschiedene Modellspezifikationen (90\%-CI)

Abb. 7.3: Vorhergesagte Intentionswahrscheinlichkeit (90\%-CI) nach Zugangshürden des angestrebten Faches und sozialer Herkunft

Abb. 7.4: Herkunftsunterschiede in der vorhergesagten Intentionswahrscheinlichkeit an unterschiedlichen Werten der Zugangshürden des angestrebten Faches (90\%-CI)

Abb. 7.5: Vorhergesagte Wahrscheinlichkeit der Übereinstimmung zwischen Studienfachaspiration und -intention (90\%-CI) nach Zugangshürden des angestrebten Faches und sozialer Herkunft

Abb. 7.6: Bewerbungsintention (Vignettenurteil), nach sozialer Herkunft

Abb. 8.1: Studienbewerbung nach sozialer Herkunft (Überlebenswahrscheinlichkeit) 
Abb. 8.2: Bewerbungswahrscheinlichkeit: Veränderung sozialer Herkunftsunterschiede über verschiedene Modellspezifikationen (Hazardratenmodelle)

Abb. 8.3: Vorhergesagte Bewerbungswahrscheinlichkeit (90\%-CI) nach Zugangshürden des angestrebten Faches und sozialer Herkunft (Hazardratenmodelle)

Abb. 8.4: Vorhergesagte Wahrscheinlichkeit der Übereinstimmung zwischen Studienfachaspiration und -bewerbung nach Zugangshürden des angestrebten Faches und sozialer Herkunft

Abb. 8.5: Übereinstimmung Fachaspiration und Bewerbung nach sozialer Herkunft (in \%)

Abb. 8.6: Bewerbungen in Berlin und Berlin/Brandenburg nach sozialer Herkunft

Abb. 8.7: Bewerbungen für verschiedene Hochschultypen nach sozialer Herkunft (in \%)

Abb. 8.8: Anzahl und Breite der Bewerbungen nach sozialer Herkunft (1. Bew.phase, in \%)

Abb. 8.9: Zulassungsverfahren und -chancen nach sozialer Herkunft (1. Bew.phase, in \%)

Abb. 8.10: Studienzulassung nach sozialer Herkunft (Überlebenswahrscheinlichkeit)

Abb. 8.11: Unkonditionale Studienaufnahme nach sozialer Herkunft (Überlebenswahrscheinlichkeit) 


\section{Tabellenverzeichnis}

Tab. 5.1: Hypothesen ……………………............................. 159

Tab. 6.1: $\quad$ Best Up-Erhebungsdesign ........................................... 166

Tab. 6.2: Zentrale Inhalte der Rekrutierungsrecherche .................. 172

Tab. 6.3: Recherchierte Studiengänge nach Region, Hochschultyp, Trägerschaft, Fächergruppe und Semester

Tab. 6.4: Durchschnittliche Zugangshürden pro Studienfächer und Semester

Tab. 6.5: Vergleich der Befragten mit Studienaspiration (im Analysesample) und der Befragten ohne Studienaspiration (nicht im Analysesample), Welle 2 .....

Tab. 7.1: Hypothesen Phase 1

Tab. 7.2: $\quad$ Operationalisierung und Verteilung der

Vignettendimensionen

Tab. 7.3: Verteilung der erklärenden Variablen nach sozialer Herkunft (Spaltenprozente) und Studienintention (Zeilenprozente)

Tab. 7.4: Übersetzungswahrscheinlichkeit von Studienaspirationen in -intentionen: multivariate Ergebnisse

Tab. 7.5: Durchschnittliche Zugangshürden des angestrebten Studienfaches: Deskriptive Information

Tab. 7.6: Intentionswahrscheinlichkeit: soziale Herkunft und Zugangsbarrieren des angestrebten Studienfaches

Tab. 7.7: Wahrscheinlichkeit der Übereinstimmung zwischen Studienfachaspiration und -intention: soziale Herkunft und Zugangsbarrieren des angestrebten Studienfaches ...

Tab. 7.8: Bewerbungsintention (FS): institutionelle Barrieren und Opportunitäten nach sozialer Herkunft

Tab. 7.9: Hochschultyp- und Fachintention: deskriptive Information nach sozialer Herkunft

Tab. 7.10: Soziale Herkunft und Hochschultyp-/Fachintention: multivariate Ergebnisse

Tab. 7.11: Unterschied zwischen Hochschultyp- und Fachaspirationen und -intentionen

Tab. 7.12: $\quad$ Ergebnisübersicht Phase 1 .............................................. 234

Tab. 8.1: $\quad$ Hypothesen Phase 2-4

Tab. 8.2: Operationalisierung Bewerbungsmuster und -strategien 
Tab. 8.3: Definition der risk sets, Perioden und hieraus resultierende Zensierungsgründe

Tab. 8.4: Bewerbungswahrscheinlichkeit in Abhängigkeit der sozialen Herkunft, Leistung, RC und Ressourcen (Hazardratenmodell)

Tab. 8.5: Bewerbungswahrscheinlichkeit: soziale Herkunft und Zugangsbarrieren des angestrebten Studienfaches (Hazardratenmodelle)

Tab. 8.6: Wahrscheinlichkeit der Übereinstimmung zwischen Studienfachaspiration und -bewerbung: soziale Herkunft und Zugangsbarrieren des angestrebten Studienfaches ...

Tab. 8.7: Entfernung der Bewerbungen nach sozialer Herkunft (in $\mathrm{km}$ )

Tab. 8.8: Soziale Herkunft und regionale Reichweite der Bewerbungen: multivariate Ergebnisse

Tab. 8.9: Soziale Herkunft und Hochschultypbewerbungen: multivariate Ergebnisse

Tab. 8.10: Studienfachbewerbungen: durchschnittliches Einkommen und Berufsprestige nach sozialer Herkunft .

Tab. 8.11: Soziale Herkunft und Studienfachbewerbung: multivariate Ergebnisse

Tab. 8.12: Unterschiede zwischen Hochschultyp- und Fachaspirationen und entsprechender erstgerankter Bewerbung

Tab. 8.13: Zulassungswahrscheinlichkeit und soziale Herkunft (Hazardratenmodell)

Tab. 8.14: Zulassungswahrscheinlichkeit und Bewerbungsstrategien (Hazardratenmodell)

Tab. 8.15: Konditionale Studienaufnahme nach sozialer Herkunft (Prozent/Fallzahl)

Tab. 8.16: Konditionale Studienwahrscheinlichkeit: multivariate Ergebnisse

Tab. 8.17: Unkonditionale Studienwahrscheinlichkeit: multivariate Ergebnisse (Hazardratenmodell)

Tab. 8.18: Studienort, Hochschultyp und Studienfach nach sozialer Herkunft

Tab. 8.19: Soziale Herkunft, Studienort, Hochschultyp, Studienfach: multivariate Ergebnisse

Tab. 8.20: Unterschiede zwischen Hochschultyp-/Fachaspirationen und dem entsprechenden Studium

Tab. 8.21: Ergebnisübersicht der Phasen 2-4 300 


\section{Abkürzungsverzeichnis}

$\begin{array}{ll}\text { ACT } & \text { American College Test } \\ \text { AdH } & \text { Auswahlverfahren der Hochschulen } \\ \text { AME } & \text { Average Marginal Effect } \\ \text { BAföG } & \text { Bundesausbildungsförderungsgesetz } \\ \text { Best Up } & \text { Berliner-Studienberechtigten-Panel } \\ \text { BVerfG } & \text { Bundesverfassungsgericht } \\ \text { CHE } & \text { Centrum für Hochschulentwicklung } \\ \text { CI } & \text { Confidence Intervall } \\ \text { DC } & \text { (Average) Discrete Change } \\ \text { DN } & \text { Durchschnittsnote } \\ \text { DoSV } & \text { Dialogorientiertes Serviceverfahren } \\ \text { DZHW } & \text { Deutsches Zentrum für Hochschul- } \\ & \text { und Wissenschaftsforschung } \\ \text { EMI } & \text { Effectively Maintained Inequality } \\ \text { FH } & \text { Fachhochschule } \\ \text { FML } & \text { Full Maximum Likelihood } \\ \text { FS } & \text { Faktorieller Survey } \\ \text { GLMM } & \text { Generalized Linear Mixed Models } \\ \text { HAM-Nat } & \text { Hamburger Naturwissenschaftstest } \\ \text { HoF } & \text { Institut für Hochschulforschung } \\ \text { HRG } & \text { Hochschulrahmengesetz } \\ \text { HRK } & \text { Hochschulrektorenkonferenz } \\ \text { ISCED } & \text { International Standard Classification of Education } \\ \text { ISCO } & \text { International Standard Classification of Occupations } \\ \text { ISEI } & \text { International Socio-Economic Index of Occupational Status } \\ \text { ISS } & \text { Integrierte Sekundarschule } \\ \text { KapVO } & \text { Kapazitätsverordnung } \\ \text { KldB } & \text { Klassifikation der Berufe } \\ \text { KMK } & \text { Kultusministerkonferenz } \\ \text { MCAR } & \text { Missing Completely at Random } \\ \text { MFS } & \text { Modell der Frameselektion } \\ \text { MINT } & \text { Mathematik, Informatik, Naturwissenschaft und Technik } \\ \text { ML } & \text { Maximum Likelihood } \\ \text { MPS } & \text { Magnitude-Prestigeskala } \\ \text { NC } & \text { Numerus Clausus } \\ \text { NEPS } & \text { Nationales Bildungspanel/National Educational Panel Study } \\ \text { OECD } & \text { Organisation for Economic Co-Operation and Development } \\ \text { OSZ } & \text { Oberstufenzentrum } \\ \text { PAPI } & \text { Paper and Pencil Interview } \\ & \end{array}$


PISA Programme for International Student Assessment

RC Rational Choice

RML Restricted Maximum Likelihood

RRA Relative Risk Aversion

SAT Scholastic Assessment Test

SfH

Stiftung für Hochschulzulassung

SOEP Sozio-oekonomisches Panel

SoSe Sommersemester

TMS

TOSCA

Test für medizinische Studiengänge

UCAS

Transformation des Sekundarschulsystems und akademische Karrieren

WiSe

Universities and Colleges Admissions Service

WZ

Wintersemester

ZVS

Wartezeit

Zentrale Vergabestelle für Studienplätze 


\section{Einleitung}

Breiter Konsens besteht darin, dass Bildung eine zentrale Bedeutung in modernen Gesellschaften zukommt, sowohl auf individueller als auch auf gesamtgesellschaftlicher Ebene (vgl. Mayer 2004). Für das Individuum ist Bildung mit vielen positiven Erträgen verbunden, die nicht allein auf Arbeitsmarktvorteile beschränkt sind, sondern ,sich in fast allen Lebensbereichen: Gesundheit, Lebenserwartung, Glück, Partnerschaften, soziale Integration, Partizipation, Mobilität, Gestaltungsmöglichkeiten im Lebensverlauf" zeigen (Allmendinger 2013). Gleichzeitig existieren deutliche soziale Disparitäten beim Bildungserwerb, die aufgrund der starken Verbindung zwischen Bildungszertifikaten und gesellschaftlichen Stellungen zur Reproduktion sozialer Ungleichheiten beitragen.

Es ist also nicht verwunderlich, dass sich der Themenkomplex Bildung und Chancen(un)gleichheit in der öffentlichen und wissenschaftlichen Debatte anhaltender Beliebtheit erfreut. In Deutschland erlangte das Thema mit den Veröffentlichungen der ersten PISA-Studie aus dem Jahr 2000 neue Brisanz, die Deutschland im internationalen Vergleich große sozialgruppenspezifische Unterschiede im Kompetenzerwerb bei insgesamt unterdurchschnittlichem Kompetenzniveau attestierte (vgl. Baumert et al. 2001). Doch nicht nur soziale Ungleichheit beim Kompetenzerwerb in der Schule, sondern auch die „Nadelöhre" (Becker 2009: 565) Hochschulzugangsberechtigung und Hochschulzugang sind ein immer wiederkehrender Bestandteil des Diskurses - wenn auch ein teilweise umstrittener, wie die Diskussion um Akademikerschwemme und Fachkräftemangel zeigt (für einen Überblick siehe Kreckel 2014).

Die Diskussion zum Thema soziale Ungleichheit beim Hochschulzugang wurde bereits in den 1960ern geführt. Zum einen wurde dabei die ökonomische Notwendigkeit der Mobilisierung von Begabungsreserven betont (vgl. Edding 1963, Picht 1964), zum anderen stand die normative Idee von „Bildung als Bürgerrecht" im Vordergrund (Dahrendorf 1965). Beide Argumentationslinien mündeten in der gleichen Forderung: eine stärkere Öffnung der Hochschulen auch für Kinder sozial benachteiligter Familien. Doch auch noch 50 Jahre später wird das deutsche Hochschulsystem mit zwei Problemen in Verbindung gebracht: „low university enrolment in combination with high inequality“ (Neugebauer und Schindler 2012: 20, OECD 2016). Ersteres mag aufgrund des deutlichen Aufholens Deutschlands, dessen Studienanfänger/innenquote sich dem OECD-Durchschnitt stetig annähert, und mit Blick auf das gut ausgestattete Berufsausbildungssystem weniger problematisch erscheinen (Helbig et al. 2015: 1). Doch den sozial ungleich verteilten Bildungs- und damit verbundenen Lebenschancen ist auch die Debatte um eine vermeintliche „Akademikerschwemme“ (Nida-Rümelin 2013) nicht gewachsen. 
Als eine zentrale Ursache für beide Phänomene - für geringe Studierendenraten und hohe soziale Ungleichheit beim Übergang ins Studium - wird in vielen bildungssoziologischen Beiträgen die Struktur des deutschen Schulsystems identifiziert, die Familien frühe und schwer zu korrigierende Bildungsentscheidungen abverlangt und somit dazu führt, dass viele sozial schwächere Schüler/innen die Hochschulzugangsberechtigung gar nicht erst erlangen (vgl. Becker 2009, Müller und Pollak 2008, Neugebauer et al. 2013, Powell und Solga 2011). Doch selbst innerhalb der bereits positiv selektierten Gruppe der Abiturient/innen finden sich noch beachtliche Herkunftsunterschiede beim Übergang in die Hochschule (ebd.). Während über $80 \%$ der Studienberechtigten aus Akademikerfamilien ein Studium aufnehmen, tun dies nur etwas mehr als $60 \%$ der Studienberechtigten, deren Eltern selbst keinen Hochschulabschluss haben (Autorengruppe Bildungsberichterstattung 2016). Dies wird wiederum der Attraktivität beruflicher Ausbildungswege als Alternative zum Studium zugeschrieben, die sozial schwächere Schüler/innen vom Hochschulstudium ,ablenken“, obwohl diese eigentlich formal hochschulzugangsberechtigt sind (Shavit und Müller 2000). Ein im Zuge der beachtlichen Expansion und Differenzierung der Sekundarstufe II oft bemühtes Argument für die anhaltende oder gar zunehmende soziale Ungleichheit beim konditionalen Übergang in die Hochschule ist eine mögliche (sozialgruppenspezifische) Differenzierung der Motive, die Jugendliche zur Hochschulzugangsberechtigung führen (vgl. Schindler und Lörz 2011). Demnach streben viele Jugendliche heute ein Abitur nicht in erster Linie an, um zu studieren, sondern um sich einen Wettbewerbsvorteil im Kampf um beliebte Ausbildungsplätze zu verschaffen (vgl. Jacob und Solga 2015, Mayer et al. 2007, Schindler 2012).

Doch wie verhält es sich mit den Schüler/innen, die sich trotz attraktiver beruflicher Alternativen ein Hochschulstudium wünschen? Nehmen selbst in dieser Gruppe nicht alle ein Studium auf, und können selbst hier noch soziale Herkunftsunterschiede beim Übergang ins Studium beobachtet werden? Mit welchen individuellen und institutionellen Barrieren sind junge Menschen konfrontiert, die trotz Berechtigung und Aspiration kein Hochschulstudium aufnehmen? Das Ziel dieser Arbeit ist es, diese grundlegenden Fragen zu beantworten. Der Fokus auf Studienberechtigte mit Studienaspiration kann über die bereits bekannten institutionellen Faktoren (frühes Tracking, Differenzierung der gymnasialen Oberstufe und Attraktivität beruflicher Ausbildungsalternativen) hinaus zu einem umfassenderen Verständnis existierender und potentiell ungleichheitsgenerierender Barrieren beitragen.

Die Untersuchung dieser Gruppe ist jedoch auch über ihre wissenschaftliche Relevanz hinaus bedeutsam. Aus einer humankapitaltheoretisch-funktionalistischen Perspektive (die etwa die OECD einnimmt) erfordert die schnell voranschreitende Technologisierung, globaler Wettbewerbsdruck und der Wandel Deutschlands hin zu einer Wissensgesellschaft vor allem im Kontext des demographischen Wandels einen höheren Akademiker/innenanteil in der 
Bevölkerung (vgl. Europäische Kommission 2010, OECD 2008, für eine kritische Diskussion auch der Konterargumente siehe Kreckel 2014). In der in dieser Arbeit betrachteten Gruppe von Studienberechtigten mit Studienaspiration liegt ein besonders hohes Mobilisierungspotential, sodass die Frage, was diese hoch motivierte Gruppe vom Studium abhält, sowohl politische als auch wirtschaftliche Relevanz besitzt. Doch es ist auch unter normativen Gesichtspunkten zu diskutieren, warum junge Menschen - trotz formaler Berechtigung - ihren Bildungswünschen nicht nachkommen (können), insbesondere wenn dabei bestimmte soziale Gruppen stärker benachteiligt sind.

Für den US-amerikanischen Kontext konnte wiederholt gezeigt werden, dass nicht alle Schüler/innen ihre hohen Bildungsaspirationen umsetzen können und dass es vor allem Schüler/innen aus sozial schwächeren Familien sind, die trotz Studienaspiration letztlich nicht ins Studium übergehen (vgl. Hanson 1994, Roderick et al. 2011, Klasik 2012). Diese Befunde können nur sehr eingeschränkt auf Deutschland übertragen werden, da die Gruppe der high schoolAbsolvent/innen aufgrund der relativ geringen Stratifizierung des US-amerikanischen Schulsystems viel heterogener ist als die Gruppe deutscher Abiturient/innen und ein Studium in den USA häufig mit hohen Kosten und $\mathrm{Zu}-$ gangshürden einhergeht (vgl. Buchmann et al. 2010, Page und Scott-Clayton 2016). Deutsche Studienberechtigte sind hingegen stärker positiv selektiert, und die Studienaufnahme wurde lange Zeit als ,,der relativ undramatische Moment der Allokation“ (Teichler 2005: 139) beschrieben.

Womöglich sind dies Gründe dafür, dass es für den deutschen Kontext bisher kaum empirisch gesicherte Antworten auf die Frage nach sozialer Ungleichheit bei der Umsetzung von Studienaspirationen gibt. Doch selbst wenn das „Nadelöhr“ Hochschulzugangsberechtigung (Becker 2009: 565) bezwungen ist und Studienaspirant/innen somit nur noch ,einen kurzen Schritt“ vom Hochschulsystem entfernt sind, kann auch im deutschen Kontext der Übergang steinig sein. Im internationalen Vergleich kann das deutsche binäre Hochschulsystem zwar immer noch als wenig stratifiziert bezeichnet werden (vgl. Arum et al. 2007). Lenkt man den Blick aber auf die innerdeutschen Entwicklungen, kann eine zunehmende Wettbewerbsorientierung, Deregulierung und Differenzierung attestiert werden, die sich zum Beispiel in der Exzellenzinitiative (vgl. Banscherus et al. 2015), in der stetig wachsenden Zahl inhaltlich differenzierter Studienangebote (vgl. Hachmeister und Grevers 2019, Schröder 2015) oder in der Autonomiesteigerung der Hochschulen bei der Zulassung zum Studium widerspiegelt (vgl. Bode 2015, Täger 2010). Durch die Expansion sekundärer Bildung verschiebt sich die Allokationsfunktion des Bildungssystems zunehmend auf die Hochschulen, sodass die schulische Hochschulzugangsberechtigung - das Abitur - ihre Stellung als alleiniges Berechtigungszertifikat sukzessive einbüßt (vgl. Wolter 2008). All das macht den Übergang voraussetzungsvoller und birgt das Potential, soziale Reproduktion zu verstärken. 
Forschung zum Thema Hochschulpartizipation befindet sich insbesondere seit den letzten zwei Dekaden im Aufwind, was nicht lediglich eine durch neue Datenquellen hervorgerufene ,wissenschaftliche Modeerscheinung“ darstellt, sondern ,einen Forschungsbedarf [reflektiert], der durch politisch formulierte Zielsetzungen genährt wurde" (Schindler 2014: 19). Der Befund sozialer Ungleichheit beim Übergang in die Hochschule ist hier eindeutig und robust gegenüber der Verwendung unterschiedlicher Datenquellen und Methoden (vgl. Becker und Hecken 2007, 2008, 2009a/b, Blossfeld et al. 2015, Hillmert und Jacob 2003, 2010, Lörz 2012, Lörz und Schindler 2011, Maaz 2006, Mayer et al. 2007, Müller und Pollak 2008, Neugebauer et al. 2013, Obermeier und Schneider 2015, Schimpl-Neimanns 2000, Schindler 2012, 2014, Schindler und Reimer 2010, Watermann und Maaz 2006, 2010).

Zudem wird die Frage nach differenzierten Entscheidungen (und Zugangschancen) innerhalb des Hochschulsystems und dabei entstehender sozialer Ungleichheit im Zuge von Expansions- und Differenzierungsprozessen wichtiger. So konnte für den deutschen Kontext soziale Ungleichheit beim Übergang in mehr oder weniger prestigereiche Fächer und in anwendungsorientierte Fachhochschulen und forschungsorientierte Universitäten nachgewiesen werden (vgl. Becker et al. 2010, Lörz 2012, Reimer und Pollak 2010, Trautwein et al. 2006).

Theoretisch orientiert sich der ,Mainstream der deutschen Bildungssoziologie" (Solga und Becker 2012: 24, Hervorhebung im Original) an mikrotheoretisch fundierten Entscheidungsmodellen: Demnach evaluieren Schüler/innen (und deren Familien) die ihnen zur Verfügung stehenden Optionen und treffen auf Grundlage dieses Abwägungsprozesses aus ihrem Blickwinkel rationale (und daher sozialgruppenspezifische) Bildungsentscheidungen. Aus dieser Perspektive werden alle beobachteten Nicht-Übergänge als (rationale) Entscheidungen gegen ein Studium interpretiert. Der potentielle Einfluss von Fremdselektionsprozessen wird - auch in aktuelleren Studien - selten in die Diskussion einbezogen.

Empirisch stehen quantitative Studien im Vordergrund, die zumeist Studienintentionen oder bereits realisierte Übergänge betrachten. Diesem Vorgehen liegt die (teils implizite) Annahme zugrunde, dass beide Konstrukte sich gegenseitig gut abbilden, dass also Übergänge Präferenzen und Intentionen Übergänge gut approximieren, da institutionelle Hürden - ist die Studienberechtigung erst einmal erreicht - als vergleichsweise gering angesehen werden, etwa aufgrund geringer direkter Kosten, einem vergleichsweise dichten Hochschulnetz (vgl. Spieß und Wrohlich 2010) oder als gering eingeschätzter Zugangsbarrieren. Aufgrund der Existenz zulassungsfreier Studiengänge kann theoretisch jede/r Studienberechtigte ein Studium aufnehmen (vgl. etwa Schindler 2012). Gestützt wird diese Annahme von regelmäßigen Veröffentlichungen, die zeigen, dass in den vergangenen Jahren etwa $60 \%$ des grundständigen Studienangebots an deutschen Hochschulen ,zulassungsfrei“ war (Gehlke et al. 
2019a). Jedoch kann eine deutliche fachliche und regionale Varianz bezüglich existierender Zugangsbarrieren beobachtet werden (ebd.), sodass die Umsetzungschancen von Studienaspirationen auch von der Art und Stärke inhaltlicher und örtlicher Präferenzen abhängen sollten. Zudem wurde die Rolle von Hochschulen als Gatekeeper in den letzten Jahrzehnten, insbesondere seit der Novelle des Hochschulrahmengesetzes aus dem Jahre 2004, stärker institutionalisiert. Seitdem genießen deutsche Hochschulen größere Autonomie bei der Studienplatzvergabe und der Auswahl, geeigneter" Bewerber/innen (vgl. Bode 2015, Täger 2010, Zimmerhofer und Trost 2008).

Welche (direkte und indirekte) Rolle Zulassungshürden für soziale Ungleichheit beim Übergang ins Studium spielen, ist bisher für den deutschen Kontext allerdings weitestgehend unklar. Dabei sind Übergänge ins Studium nicht allein das Resultat individueller Bildungsentscheidungen, sondern vielmehr „das Ergebnis des Zusammenwirkens eigener Wahlentscheidungen (,Selbstselektion') und Auswahlentscheidungen anderer (,Fremdselektion')“ (Hillmert 2005: 177). Während Intentionen somit vor allem die individuelle (jedoch möglicherweise bereits durch antizipierte Hürden angepasste) Entscheidung von Studienberechtigten widerspiegeln, die nicht in jedem Fall mit institutionellen Entscheidungen übereinstimmen muss (vgl. Jacob et al. 2013), birgt die Untersuchung realisierter Übergänge das Problem, dass individuelle und institutionelle Entscheidungsprozesse (Selbstselektion und Fremdselektion) konfundiert sind (Boliver 2013).

Daher wähle ich in der vorliegenden Arbeit einen anderen konzeptionellen und empirischen Ansatz, mit dessen Hilfe eine differenzierte Betrachtung der Umsetzung von Studienaspirationen ermöglicht wird. Grundlegend ist dabei, den Übergang in die Hochschule nicht als singulären Schritt zu betrachten, sondern aus verschiedenen, miteinander verbundenen Phasen. Weitestgehend unstrittig ist, dass die Studienaufnahme das Ergebnis kumulativer Entscheidungen ist (vgl. Hillmert und Jacob 2010, Mare 1980). Welche Phasen hierbei als relevant erachtet werden, hängt vom jeweiligen Forschungsinteresse und dem damit verbundenen Aggregationsniveau ab.

Aus einer Lebensverlaufsperspektive fragen etwa Hillmert und Jacob (2010: 59) in einer Studie zu sozialer Ungleichheit auf dem akademischen Track: "Where on the long way to the university degree does Germany 'lose' its potential academics and in particular its lower class children?" Sie beziehen dabei die gesamte Bildungslaufbahn von Individuen ein und untersuchen, in welchen mehr oder weniger stark institutionalisierten Phasen und an welchen Schwellen soziale Selektivität besonders ausgeprägt ist - verbleiben dabei aber aufgrund des Umfangs ihrer Fragestellung empirisch auf einem eher hohen Abstraktionsniveau. Die hieraus gewonnenen Erkenntnisse sind ohne Frage wertvoll - doch sollten sie durch differenziertere, stärker erklärende Ansätze ergänzt werden. Denn der Übergang in die Hochschule ist auch dann kein singulärer Prozess, wenn man hochschulzugangsberechtigte Studienaspirant/in- 
nen betrachtet, die in abstrakteren Modellen „nur noch eine Stufe“ vom Studium entfernt sind. Vielmehr muss auch diese bereits stark selektierte Gruppe verschiedene Hürden nehmen.

Um diese Überlegungen theoretisch und empirisch einzubetten, orientiere ich mich an in der US-amerikanischen Forschung verbreiteten Phasenmodellen (oder auch college choice-Modelle) (vgl. Avery und Kane 2004, Hossler und Gallagher 1987, Jackson 1982, Klasik 2012, Litten 1982, Roderick et al. 2011). Diese gehen davon aus, dass Schüler/innen verschiedene, miteinander verbundene Phasen durchlaufen müssen, in denen institutionelle Faktoren mit individuellen Merkmalen interagieren und sowohl individuelle als auch institutionelle Akteure folgenreiche (ungleichheitsgenerierende oder ungleichheitsreduzierende) Entscheidungen treffen. Die Anzahl und Abgrenzung der Phasen variiert zwischen den verschiedenen Studien. In der vorliegenden Arbeit stehen vier Phasen der Aspirationsumsetzung im Vordergrund: 1) Die Übersetzung von (idealistischen) Aspirationen in (realistische) Intentionen sowie die $\mathrm{Um}$ setzung von Aspirationen, die sich in 2) Bewerbungen, 3) Zulassungen und 4) die finale Studienaufnahme unterteilt.

Durch diese detaillierte Betrachtung soll ein tieferes und umfassenderes Verständnis sozial ungleicher Übergänge in die Hochschule generiert werden, das das von breiteren Studien gezeichnete Bild ergänzen soll. Dabei stehen folgende forschungsleitenden Fragestellungen im Mittelpunkt:

- In welchen Phasen auf dem ,kurzen Weg“ vom letzten Schuljahr bis zum Übergang in die Hochschule entsteht soziale Ungleichheit innerhalb der hoch selektiven Gruppe von Studienberechtigten mit Studienaspiration, und wie kann diese erklärt werden?

- Inwiefern sind dabei nicht nur individuelle Entscheidungsprozesse (Selbstselektion), sondern auch institutionelle Entscheidungen (Fremdselektion) und deren Zusammenspiel von Bedeutung?

- Mit welchen (weiteren) institutionellen Barrieren sind Studienaspirant/innen konfrontiert, und wie wirken diese auf deren Entscheidungen zurück?

Dabei sollen nicht nur soziale Herkunftsunterschiede bei der Umsetzung von Studienaspirationen an sich untersucht werden, sondern auch soziale Ungleichheit innerhalb des Hochschulsystems, bezüglich der Wahl differenzierter Studienoptionen (Fach und Hochschultyp).

Um diesen Themenkomplex untersuchen zu können, benötige ich Längsschnittdaten, die möglichst detaillierte Informationen $\mathrm{zu}$ den verschiedenen Phasen beinhalten. Zwar stehen mit dem Studienberechtigtenpanel des Deutschen Zentrums für Hochschul- und Wissenschaftsforschung und seit einiger Zeit auch den umfangreichen Befragungen des Nationalen Bildungspanels für Gesamtdeutschland repräsentative Längsschnittdaten zur Verfügung. Doch erlauben die in diesen Studien erhobenen Konstrukte nur eine sehr oberflächliche Betrachtung der aufgezeigten Phasen des Übergangsprozesses. Hieran anknüp- 
fend ist ein Grund dafür, dass in der quantitativen Forschung in Deutschland (fast ausschließlich) Bildungsintentionen und realisierte Übergänge im Mittelpunkt stehen, sicherlich auch in der fehlenden Datengrundlage zu suchen: Zwar werden sowohl Studienintentionen als auch letztliche Übergänge je nach Befragungszeitpunkt standardmäßig erfasst, doch stehen für die Analyse des Bewerbungsverhaltens von Abiturient/innen kaum quantitative Daten zur Verfügung. Auch gibt es bisher keine Daten zu Zulassungsentscheidungen der Hochschulen, weshalb bisher empirisch nicht klar zwischen Selbstselektion (keine Bewerbung) und Fremdselektion (keine Zusage trotz Bewerbung) differenziert werden kann.

Detaillierte Informationen zu den verschiedenen Phasen stehen mit dem Berliner-Studienberechtigten-Panel (Best Up) zur Verfügung (vgl. Ehlert et al. $2017 \mathrm{a} / \mathrm{b}$ ), das neben fünf Befragungswellen, in denen Schüler/innen vom Ende des vorletzten Schuljahres bis circa 1,5 Jahre nach Erhalt der Studienberechtigung verfolgt wurden, auch einen Faktoriellen Survey zum Einfluss institutioneller Merkmale auf Bewerbungsintentionen und umfangreiche institutionelle Daten zu Zulassungsbeschränkungen und -kriterien enthält.

Wie der Name vermuten lässt, handelt es sich bei Best Up um eine Berliner Studie. Berlin stellt einen spezifischen Kontext dar, zum Beispiel durch ein großes und vielfältiges Angebot an nahegelegenen Studienoptionen. Auf der anderen Seite sind die Berliner Hochschulen stark nachgefragt, was den $\mathrm{Zu}-$ gang zum Studium schwieriger gestalten kann als in anderen, weniger nachgefragten Regionen Deutschlands. Auch wenn durch diesen regionalen Fokus keine generalisierenden Aussagen für deutsche Hochschulzugangsberechtigte getroffen werden können, birgt die Untersuchung eines bestimmten Kontextes auch Vorteile, da der Einfluss von divergierenden institutionellen und strukturellen Besonderheiten zwischen und innerhalb der Bundesländer (vgl. Helbig und Nikolai 2015) nicht modelliert (wenn auch abschließend reflektiert) werden muss. Trotz der Fülle der Best Up-Daten muss zudem einschränkend betont werden, dass die Samplegröße zum Teil an die Grenzen des analytisch Machbaren stößt. Daher ist der eigentliche Beitrag der Arbeit theoretischer und konzeptionell empirischer Natur; die Auswertungen und Ergebnisse haben aus genannten Gründen - eher explorativen Charakter.

\section{Aufbau der Arbeit}

Mit dieser Arbeit verfolge ich das Ziel, durch eine detaillierte und differenzierte Betrachtung des Übergangsprozesses in die Hochschule einen Beitrag zur soziologischen Bildungs- und Ungleichheitsforschung zu leisten. Der Fokus auf Studienberechtigte mit Studienaspiration, auf junge Menschen also, die nicht bereits im Vorfeld durch frühere Bildungsentscheidungen oder berufsbildende Alternativen vom Hochschulstudium ,,abgelenkt" wurden, eröffnet den Blick auf weitere institutionelle Barrieren, die im Zusammenspiel mit indivi- 
duellen Ressourcen Bildungsübergänge beeinflussen können, deren direkter und indirekter Einfluss jedoch bisher - zumindest für den deutschen Kontext vergleichsweise wenig erforscht ist.

Kapitel 2 gibt einen Überblick über den institutionellen Kontext, innerhalb dessen Studienaspirationen und Studienchancen von Schüler/innen durch formale Vorgaben und vorhandene Bildungsoptionen strukturiert werden. Dabei steht zunächst die Hochschulzugangsberechtigung als zentrale Voraussetzung der Studienaufnahme im Mittelpunkt. Ist diese erreicht, stehen Studienberechtigte vor einer zunehmend komplexen (hochschulischen) Opportunitätsstruktur, deren Entwicklung und aktuelle Ausgestaltung in den weiteren Ausführungen beschrieben wird. Der Fokus liegt dabei auf Zulassungsregelungen und deren Anwendung durch die Hochschulen. Da die Datenlage hierzu eingeschränkt ist, werden bereits in Kapitel 2 zentrale Befunde aus den im Best UpProjekt gesammelten institutionellen Daten zu Auswahlverfahren an deutschen Hochschulen (,Rekrutierungsdaten“) präsentiert, die zeigen, dass der Hochschulzugang weniger offen ist als häufig angenommen. Zuletzt werden institutionelle Besonderheiten Berlins als spezifischer Kontext der vorliegenden Arbeit vorgestellt.

In Kapitel 3 werden verbreitete theoretische und empirische Ansätze der (deutschen) Forschung zu sozialer Ungleichheit beim Übergang in die Hochschule vorgestellt. Dabei wird diskutiert, warum die verbreitete Untersuchung von Studienintentionen oder bereits realisierten Übergängen in Kombination mit den ebenfalls verbreiteten mikrotheoretisch orientierten Entscheidungsmodellen ergänzt werden sollte durch einen differenzierteren Blick, der nicht nur individuelle, sondern auch institutionelle Entscheidungen (sowie deren Interaktion) einbezieht. Ein solcher Blick kann mithilfe von Phasenmodellen erreicht werden, die als Vorbereitung auf die theoretischen Überlegungen und empirischen Untersuchungen vorgestellt werden.

Im Anschluss hieran dient Kapitel 4 der konzeptionellen und empirischen Bestandsaufnahme zu sozialer Ungleichheit in den Phasen der Aspirationsumsetzung. Zunächst soll das sehr vielseitig genutzte Konzept Aspirationen ein- und abgegrenzt und somit eine Definitionsgrundlage geschaffen werden, der die nachstehenden Kapitel folgen. Bildungsaspirationen als zentraler Ausgangspunkt der vorliegenden Arbeit werden - in Abgrenzung zu dem ebenfalls häufig genutzten Konzept der Intentionen - als Bildungswünsche definiert, denen ein idealistisches Element innewohnt und die weniger stark von wahrgenommenen individuellen und institutionellen Barrieren beeinflusst sind. Im Anschluss daran werden empirische Befunde zur Umsetzung von Studienaspirationen und dabei auftretender sozialer Ungleichheit präsentiert. Zuletzt wird die Umsetzung von Studienaspirationen modellhaft in die vier oben bereits genannten Phasen unterteilt und der Forschungsstand zu sozialer Ungleichheit in diesen Phasen aufbereitet. Abschließend wird diskutiert, ob soziale Ungleich- 
heit bei der Umsetzung von Studienaspirationen eher in den Phasen der Selbstselektion oder der Fremdselektion zustande kommt.

In Kapitel 5 soll ein theoretischer Rahmen für die Fragestellung dieser Arbeit entwickelt werden. Dafür wird vor allem auf Bourdieus Reproduktionstheorie, das sozialpsychologisch orientierte status attainment-Modell sowie soziologische Varianten des rational choice-Ansatzes zurückgegriffen. Zunächst wird der Stellenwert von Aspirationen in diesen Ansätzen betrachtet, um ein theoretisches Verständnis dafür zu entwickeln, unter welchen Bedingungen Studienaspirationen auch bei sozial schwächeren Schüler/innen erwartet werden können.

Um sich der Frage sozialgruppenspezifischer Umsetzungschancen von Studienaspirationen theoretisch anzunähern und mögliche ungleichheitsgenerierende Mechanismen abzuleiten, wird auf die in der Bildungssoziologie zentrale Unterscheidung zwischen primären und sekundären Effekten (Boudon 1974) zurückgegriffen. Der theoretischen Annahme folgend, dass sozialgruppenspezifische Umsetzungschancen durch das Zusammenspiel individueller Ressourcen und institutioneller Opportunitäten und Barrieren entstehen können, wird dabei die Wechselwirkung zwischen a) Leistungsunterschieden (primären Effekten) und institutionellen Auswahlkriterien sowie zwischen b) (mehr oder weniger rational reflektierten) Bildungsentscheidungen (sekundären Effekten) und der (hochschulischen) Opportunitätsstruktur genauer betrachtet. Letztere ist gekennzeichnet durch die Vorgabe institutioneller Wahloptionen, die auf Studienberechtigte unterschiedlicher sozialer Herkunft unterschiedlich attraktiv wirken können sowie durch institutionelle Barrieren zum Beispiel in Form von entstehenden Kosten oder Zugangshürden, die (mehr oder weniger vollständig und korrekt) antizipiert werden und Bildungsentscheidungen sozialgruppenspezifisch beeinflussen können. Auf Grundlage dieser Überlegungen und mit Rückgriff auf die in Kapitel 4 ausführlich vorgestellten Phasen werden letztlich Hypothesen zu sozialer Ungleichheit bei der Umsetzung von Studienaspirationen abgeleitet.

Bevor diese empirisch geprüft werden, wird in Kapitel 6 das Forschungsdesign der Arbeit vorgestellt: die verwendeten Daten (Best Up-Individual- und Rekrutierungsdaten sowie der Mikrozensus zur Messung des Einkommens und Berufsprestiges, zu dem unterschiedliche Studienfächer führen), die Operationalisierung der theoretischen (phasenübergreifenden) Konstrukte, grundlegende Sampledefinitionen sowie die zur Anwendung kommenden Analysemethoden.

Im ersten empirischen Teil der Arbeit steht in Kapitel 7 die erste Phase im Fokus, in der Schüler/innen mit Studienaspiration diese in eine ebenso hohe Intention übersetzen oder aufgrund antizipierter, individueller und/oder institutioneller Hürden hiervon abweichen. Zunächst wird den Fragen nachgegangen, ob es Herkunftsunterschiede bei der Übersetzung von Studienaspirationen in Studienintentionen gibt und wie diese erklärt werden können. Als mögliche 
Erklärungen werden dabei Unterschiede in den Schulleistungen und in den Bildungsentscheidungen untersucht, die sowohl aufgrund sozialgruppenspezifischer rationaler Abwägungsprozesse als auch durch Erwartungshaltungen und Unterstützung aus dem sozialen Umfeld zustande kommen können. Zudem wird untersucht, inwiefern antizipierte institutionelle Barrieren und Opportunitäten sozialgruppenspezifisch auf die Übersetzungswahrscheinlichkeit wirken. Hierfür werden zum einen die Best Up-Rekrutierungsdaten genutzt, um Zugangshürden zu operationalisieren. Zum anderen kann der (sozialgruppenspezifische) Einfluss weiterer institutioneller Merkmale (z.B. der Reputation von Hochschulen oder geographischer Distanz) mithilfe eines quasi-experimentellen Faktoriellen Surveys isoliert erfasst werden.

Sofern eine Studienintention besteht, soll zuletzt untersucht werden, inwiefern sich Schüler/innen unterschiedlicher sozialer Herkunft bezüglich ihrer Intention für verschiedene Hochschultypen und Fächer unterscheiden. So soll ein Beitrag zur Frage sozialer Ungleichheit innerhalb des Hochschulsystems geleistet werden.

Im zweiten empirischen Teil steht in Kapitel 8 schließlich die Umsetzung von Studienaspirationen im Vordergrund, die die Phasen 2 (Bewerbung), 3 (Zulassung) und 4 (Studienaufnahme) umfasst und mithilfe ereignisdatenanalytischer Methoden untersucht wird. Zunächst wird hier - inhaltlich weitestgehend parallel zu den Analysen in Kapitel 7 - analysiert, inwiefern sich Studienberechtigte unterschiedlicher sozialer Herkunft darin unterscheiden, ob und wofür sie sich bewerben. Weiterhin sollen mögliche Bewerbungsstrategien für den deutschen Kontext erstmalig explorativ dargestellt werden. Im Anschluss daran liegt der Fokus auf der fremdselektiven Phase und der Frage, ob die soziale Herkunft von Bewerber/innen mit deren Zulassungswahrscheinlichkeit zusammenhängt. Zuletzt soll die Studienaufnahme und damit die letzte Phase im Übergangsprozess betrachtet werden. Zunächst wird untersucht, ob Studienberechtigte mit einer oder mehreren Zulassungen ein Studium aufnehmen (konditionale Analyse). Der Übergang soll aber - wie in vielen anderen Studien - auch unkonditional, d.h. hier für alle Befragten mit Hochschulzugangsberechtigung und Studienaspiration betrachtet werden, um das Ausmaß sozialer Ungleichheit bei der Umsetzung von Studienaspirationen abbilden zu können.

Abgerundet wird die Arbeit mit Kapitel 9, in dem die zentralen theoretischen Überlegungen und empirischen Befunde zusammengefasst und reflektiert werden. Neben einer Darstellung der Grenzen der empirischen Analysen und hieran anknüpfender Ansatzpunkte für weiterführende Forschung werden hier abschließend gesellschaftliche Implikationen diskutiert. 


\section{Institutioneller Kontext}

Dieses Kapitel verfolgt das Ziel, einen Überblick über den institutionellen Kontext zu geben, innerhalb dessen Schüler/innen die Studienberechtigung erhalten, Aspirationen ausbilden und letztlich als Resultat des Zusammenspiels zwischen den eigenen und institutionellen Entscheidungen ins Studium - und hier: in bestimmte inhaltlich und institutionell differenzierte Studienoptionen übergehen oder vom Hochschulpfad abgelenkt werden. Dabei wird auf Grundlage existierender Befunde auch aufgezeigt, an welchen Schwellen soziale Herkunftsunterschiede entstehen.

Im ersten Teil (2.1) stehen zum einen die Wege zur schulischen Hochschulzugangsberechtigung als zentrale Voraussetzung der Studienaufnahme im Mittelpunkt. Zum anderen werden verschiedene (Bildungs-)Optionen nach Erhalt der Hochschulzugangsberechtigung vorgestellt. Hier sind vor allem berufliche Ausbildungswege zentral, die von vielen Schüler/innen - trotz Studienberechtigung - eingeschlagen werden. Um die empirischen Ergebnisse der vorliegenden Arbeit besser einordnen zu können, beziehen sich die ausgewiesenen Anteilswerte hier in der Regel auf das Jahr 2014, in dem die Teilnehmer/innen der Best Up-Studie ihre Hochschulzugangsberechtigung erhielten.

Die hochschulische Opportunitätsstruktur, vor der diejenigen stehen, die sich für ein Studium entscheiden, wird in Abschnitt 2.2 vorgestellt. Dabei steht zunächst die institutionelle Struktur des deutschen Hochschulsystems im Vordergrund, die vorgibt, welche (hochschulischen) Optionen Studienberechtigte haben. Daneben wird die institutionelle Opportunitätsstruktur jedoch auch durch Zulassungsregelungen geprägt. Als zentraler Bestandteil der vorliegenden Arbeit werden diese und deren tatsächliche Anwendung durch die Hochschulen detailliert beschrieben. Auch wenn Entwicklungen über die Zeit in dieser Arbeit nicht empirisch untersucht werden, ist es dennoch wichtig, einige zentrale institutionelle Entwicklungen im deutschen Hochschulsystem zu thematisiert. Denn diese verdeutlichen, dass Studienberechtigte vor einer zunehmend komplexen hochschulischen Opportunitätsstruktur stehen, deren stärkerer Einbezug in Entscheidungsmodelle für den deutschen Fall überfällig erscheint.

Auch wenn institutionelle Rahmenbedingungen oft für Gesamtdeutschland zutreffen, gibt es aufgrund der Kulturhoheit der Länder und Föderalismusreformen regionale Besonderheien. Diese werden in Abschnitt 2.3 für Berlin als spezifischen Kontext der vorliegenden Arbeit dargestellt. 


\subsection{Die Hochschulzugangsberechtigung: Voraussetzung für den Übergang ins Studium}

\subsubsection{Wege zur Hochschulzugangsberechtigung}

Um Zugang zu deutschen Hochschulen zu erhalten, müssen Bewerber/innen ihre Hochschulreife nachweisen, meist in Form der Hochschulzugangsberechtigung (synonym: Studienberechtigung). Diese kann in unterschiedlichen Formen vorliegen und auf unterschiedlichen (meist schulischen) Wegen erlangt werden, die zwischen den Bundesländern variieren (vgl. Helbig und Nikolai 2015).

In Deutschland folgt die enge Verknüpfung von Schul- und Hochschulsystem einer langen Tradition: Seit dem frühen 19. Jahrhundert ist der Hochschulzugang hier an die schulische Maturitätsprüfung geknüpft, und die Vorbereitung auf das Studium wurde zur Hauptaufgabe der Gymnasien (vgl. Wissenschaftsrat 2004: 66). Die Hochschulzugangsberechtigung stellt nach wie vor die zentrale formale Voraussetzung für die Aufnahme eines Studiums dar. Ihr Nachweis erfolgt laut Gesetzgeber ,grundsätzlich durch den erfolgreichen Abschluß einer auf das Studium vorbereitenden Schulbildung" ( $\$ 7$ Abs. 2 Hochschulrahmengesetz (HRG)).

Der Königsweg ins Studium verläuft daher nach wie vor über Schulformen mit gymnasialer Oberstufe, an denen die Hochschulreife nach insgesamt zwölf oder 13 Schuljahren in Form des Abiturs zertifiziert wird (erster Bildungsweg). Daneben existiert die Möglichkeit, Schulabschlüsse auf dem zweiten Bildungsweg, etwa an einem Kolleg oder Abendgymnasium, nachzuholen. Über den sogenannten dritten Bildungsweg kann ein Studium unter bestimmten, landesrechtlich geregelten Bedingungen auch ohne schulische Studienberechtigung aufgenommen werden. 2014 betrug der Anteil an Studienanfänger/innen des zweiten und dritten Bildungsweges 4,1 bzw. 3,5\% (Autorengruppe Bildungsberichterstattung 2016: 299).

Neben der Unterscheidung zwischen erstem, zweitem und drittem Bildungsweg können verschiedene Arten der Hochschulzugangsberechtigung erworben werden: die allgemeine Hochschulreife, die Fachhochschulreife sowie die fachgebundene Hochschulreife. Nur die allgemeine Hochschulreife - das Abitur - berechtigt generell zur Aufnahme eines Studiums an allen Hochschularten und in allen Fächern (zu faktischen Einschränkungen siehe Abschnitt 2.2.2). ${ }^{1}$ Aufgrund des empirischen und theoretischen Fokus der vorliegenden

1 Die Fachhochschulreife berechtigt in der Regel zum Studium an einer Fachhochschule. Die fachgebundene Hochschulreife berechtigt uneingeschränkt für ein Studium an Fachhochschulen sowie für ein Studium an Universitäten in den jeweiligen Fächern. 
Arbeit liegt im Folgenden der Schwerpunkt auf dem vorherrschenden ersten Bildungsweg sowie der allgemeinen und Fachhochschulreife.

Zentral für den Erhalt der Hochschulzugangsberechtigung ist die Wahl der Schulform nach der Grundschule. Im stratifizierten deutschen Schulsystem (Allmendinger 1989) findet diese in den meisten Bundesländern bereits nach der vierten Klasse statt (nur in Berlin und Brandenburg regulär nach der sechsten Klasse). Traditionell führte diese Entscheidung zur Verteilung auf Hauptschulen (Hauptschulabschluss), Realschulen (mittlerer Schulabschluss) und Gymnasien (Hochschulreife). Hauptschulen und zu einem geringeren Teil auch Realschulen verlieren jedoch zunehmend an Relevanz. Dafür werden Schüler/innen nach der Grundschule verstärkt an kombinierten Schularten (z.B. Integrierten Sekundarschulen), an denen unterschiedliche Abschlüsse erworben werden können, beschult. Zu nennen ist außerdem die Etablierung und zunehmende Relevanz beruflicher Gymnasialformen in allen Bundesländern (vgl. Schindler 2014). ${ }^{2}$

Seit Mitte des 20. Jahrhunderts kam es zu einer starken Expansion der schulischen Möglichkeiten, die zur Hochschulreife führen, sowie des Anteils an hochschulzugangsberechtigten Schüler/innen, sodass sich das Abitur als vormals ,exklusives Bildungszertifikat zu einem Massenzertifikat gewandelt hat" (Schindler 2012: 11). Während in den 1960ern noch circa 10\% der (westdeutschen) Schulabsolvent/innen bzw. Schulabgänger/innen die Schule mit einer Hochschulzugangsberechtigung verließen, stieg dieser Anteil sukzessive an und lag 2014 bereits bei 53\% (Abitur: 41\%, Fachabitur: 12\%) (Autorengruppe Bildungsberichterstattung 2016: 96, 273, Schindler 2012: 3).

\subsubsection{Pfade nach Erwerb der Hochschulzugangsberechtigung}

Mehr als die Hälfte der Schüler/innen verlassen aktuell das deutsche Schulsystem mit einer Hochschulzugangsberechtigung. Doch bei weitem nicht alle Studienberechtigten nehmen ein Studium auf. Lange Zeit galt der Besuch eines Gymnasiums als Vorbereitung auf das folgende Studium (vgl. Kreckel 2015, Wissenschaftsrat 2004, Wolter 2008). Dies ist heute weitaus weniger selbstverständlich, denn mit der Expansion der Wege zur Hochschulzugangsberechtigung sowie der Anzahl der Studienberechtigten kam es auch zu einer Differenzierung der Motive (vgl. Schindler und Lörz 2012) sowie zu einer teilweisen Verschiebung der Selektion vom Schul- auf das Hochschulsystem (siehe Abschnitt 2.2.2).

2 Im Schuljahr 2014/15 besuchten 29\% der Schüler/innen der 5./7. Klasse eine kombinierte Schulart, 2004/05 waren es noch 14\% (Autorengruppe Bildungsberichterstattung 2016: 73, 259). An berufliche Gymnasien erwirbt mittlerweile circa ein Drittel aller (Fach-)Abiturient/innen die Hochschulzugangsberechtigung (ebd.: 273). 
Ein beachtlicher Anteil der Studienberechtigten, die letztlich ein Studium aufnehmen, beginnt dieses nicht direkt im Anschluss an die Schule: Nur 45\% der 2014er (Fach-)Abiturient/innen nahmen das Studium im Jahr des Erhalts der Studienberechtigung auf; 23\% ein Jahr später. In den Folgejahren liegt die Übergangsquote nur noch im mittleren und unteren einstelligem Bereich (Autorengruppe Bildungsberichterstattung 2020: F2-4web). Dieses Muster verdeutlicht, dass gap year Tätigkeiten (z.B. Freiwilliges Soziales Jahr, Work and Travel, Praktika oder Jobben) durchaus bedeutungsvoll sind.

Bei der berichteten Übergangsquote von etwa $80 \%$ ist zu berücksichtigen, dass die Gruppe der Hochschulzugangsberechtigten aufgrund der beschriebenen Struktur des stratifizierten deutschen Schulsystems bereits sehr selektiv ist. Ein Grund für die unter diesen Bedingungen relativ geringe Übergangsquote wird von vielen Autor/innen in der Attraktivität des Berufsbildungssystems, insbesondere der dualen Ausbildung gesehen (vgl. Müller und Pollak 2008, Powell und Solga 2011, Shavit und Müller 2000). Demnach erlangen viele Schüler/innen die Studienberechtigung nicht mit dem vorrangigen Ziel zu studieren, sondern um ihre Chancen auf attraktive Ausbildungsplätze etwa im Finanzbereich zu erhöhen (vgl. Jacob und Solga 2015, Protsch und Solga 2016, Quast et al. 2014). Laut Daten des Deutschen Zentrums für Hochschulund Wissenschaftsforschung (DZHW) ${ }^{3}$ befanden sich 17\% der 2010er Studienberechtigtenkohorte ein halbes Jahr nach Verlassen der Schule in einer Ausbildung (Schneider und Franke 2014: 121).

\subsubsection{Soziale Herkunftsunterschiede bei und nach Erwerb der Hochschulzugangsberechtigung}

Die gerade beschriebenen institutionellen Aspekte des deutschen Bildungssystems führen nicht nur zu vergleichsweise geringen Studierendenquoten (OECD 2016), sondern auch zu deutlichen sozialen Selektionsprozessen beim Übergang ins Studium. Auf Grundlage der Lebensverlaufsstudie (Geburtskohorte 1964) zeigen etwa Hillmert und Jacob (2010: 72), dass 44\% der Kinder

3 Vor 2013 war das DZHW als Teil des Hochschul-Informations-Systems (HIS) bekannt und Publikationen und Datenquellen entsprechend institutionell dem „HIS““ zugeordnet. 2013 wurde das DZHW durch Ausgliederung des HIS-Instituts für Hochschulforschung aus der Hochschul-Informations-System $\mathrm{GmbH}$ gegründet. Das HIS/DZHW ist im Hochschulbereich ein zentraler Datenproduzent und führt entsprechend zahlreiche Zeitreihen- und Panelerhebungen durch (z.B. Studienberechtigtenpanel, Absolvent/innenpanel, Sozialerhebung, Studienanfänger/innenbefragung). Im Folgenden werden häufig auf diesen Erhebungen beruhende Ergebnisse zitiert. Um Verständlichkeit und Lesbarkeit zu erhöhen, verweise ich dabei ausschließlich auf den aktuellen Institutsnamen DZHW, auch wenn es sich um ältere Daten/Veröffentlichungen handelt. 
aus höher gebildeten Familien (Eltern mit Abitur) ein Studium aufnahmen, aber nur $10 \%$ der Kinder aus Elternhäusern ohne Abitur. Für die aktuellere Geburtskohorte von 1985 berichten Neugebauer und Schindler (2012: 20), dass Kinder aus der unteren Herkunftsklasse zwar $45 \%$ der Grundschulabsolvent/innen ausmachten, aber nur 15\% der Studierenden, die zwischen 2003 und 2008 ein Studium aufnahmen. Für die obere Herkunftsklasse lagen diese Werte bei 27\% (Grundschule) und 51\% (Studium).

Wiederholt konnte gezeigt werden, dass soziale Herkunftsunterschiede sowohl auf dem Weg zur Hochschulzugangsberechtigung entstehen als auch nach deren Erhalt (vgl. Becker 2009, Hillmert und Jacob 2010, Neugebauer und Schindler 2012). Auf institutioneller Ebene wurden dabei drei zentrale Gründe identifiziert, die in den vorangegangenen Abschnitten bereits ohne Fokus auf herkunftsspezifische Ungleichheiten angesprochen wurden: die Stratifizierung und die Differenzierung des Schulsystems sowie die Attraktivität beruflicher Bildungsalternativen.

Zahlreiche Befunde aus Deutschland zeigen, dass an der ersten Schwelle im deutschen Schulsystem - dem Übergang nach der Grundschule - die meisten Kinder aus sozial benachteiligten Familien ,verloren gehen“ (Hillmert und Jacob 2010: 59). Dieser Verlust macht entsprechend den größten Anteil der beim Übergang in die Hochschule beobachteten Ungleichheit aus (vgl. Becker 2009, Hillmert und Jacob 2010, Neugebauer et al. 2013, Neugebauer und Schindler 2012).

Die gerade beschriebene Selektionswirkung des stratifizierten deutschen Schulsystems führt dazu, dass es sich bei Hochschulzugangsberechtigten - besonders bei denjenigen aus sozial schwächeren Familien - bezüglich Leistung, Motivation und Aspiration um eine insgesamt sehr positiv selektierte Gruppe handelt (Mare 1980). Daher liegt zunächst die Annahme nahe, dass soziale Ungleichheit beim Übergang in die Hochschulen vergleichsweise gering ist, sofern man nur die Gruppe der Hochschulzugangsberechtigten betrachtet. Doch auch bei diesem konditionalen Übergang zeigen sich deutliche Herkunftsunterschiede: $82 \%$ der (Fach-)Abiturient/innen (Studienberechtigtenjahrgang 2012) mit mindestens einem Elternteil mit Universitätsabschluss nehmen ein Studium auf. Mit einer Differenz von 12 Prozentpunkten folgen Studienberechtigte mit mindestens einem Elternteil mit Fachhochschulabschluss (70\%). Studienberechtigte ohne akademisch gebildete Eltern gehen zu 65\% (Meisterabschluss) und 61\% (Lehre oder kein beruflicher Abschluss) ins Studium über (Autorengruppe Bildungsberichterstattung 2016: Abb. F2-5web). Für den Studienberechtigtenjahrgang 2015 zeigt der Bildungsbericht (weniger differenziert) etwas geringere Herkunftsunterschiede (Nichtakademikerkinder: $76 \%$, Akademikerkinder $86 \%$, vgl. Autorengruppe Bildungsberichterstattung 2020: 186).

Diese sozialgruppenspezifischen Unterschiede erscheinen in Anbetracht der vergleichsweise geringen Studienkosten und bedarfsabhängigen finanziel- 
len Fördermöglichkeiten in Deutschland erstaunlich (vgl. Abschnitt 2.2.1). Im deutschen Kontext werden sozialgruppenspezifische Übergangsquoten daher weniger auf die Finanzierbarkeit eines Hochschulstudiums zurückgeführt als vielmehr auf die Attraktivität des deutschen Berufsbildungssystems sowie auf Expansionsprozesse im Sekundarschulbereich (vgl. Becker und Hecken 2008, Müller und Pollak 2008, Powell und Solga 2011, Schindler 2014, Shavit und Müller 2000). Die Attraktivität des Berufsbildungssystems führt nicht nur wie in Abschnitt 2.1.2 beschrieben - zu einer relativ geringen Übergangsquote, sondern trägt auch zu sozial selektiven Übergängen bei (vgl. Becker und Hecken 2008, Jacob und Hillmert 2003, Mayer et al. 2007, Müller und Pollak 2008, Powell und Solga 2011). Denn das deutsche Berufsausbildungssystem dient vor allem sozial schwächeren Schüler/innen einerseits als „Sicherheitsnetz", da es erstens vergleichsweise sichere Arbeitsmarktchancen und gute berufliche Stellungen bietet und zweitens geringere Kosten und Investitionsrisiken birgt. Andererseits sind es eben diese, insbesondere für sozial schwächere Familien attraktiven Merkmale, die von Hochschulbildung und somit dem Erreichen der damit verbundenen positiven Erträge ,ablenken“ (vgl. Shavit und Müller 2000).

Ein zweiter Grund für die ausgeprägte soziale Selektivität beim konditionalen Übergang in die Hochschule wird in der in Abschnitt 2.1.1 beschriebenen Expansion und Differenzierung der Wege zur Hochschulzugangsberechtigung und der daraus folgenden Heterogenisierung der Schüler/innenschaft gesehen. Die Expansion der gymnasialen Oberstufe wurde in Deutschland vor allem durch neu entstandene beruflich orientierte Schulen und die Einführung des Fachabiturs getragen, die wiederum vor allem von sozial schwächeren Schüler/innen genutzt werden und somit zwar den Weg zum (Fach-)Abitur auch für diese Gruppe erleichtern, aber auch zur Etablierung einer „,neue[n] Ungleichheitsdimension innerhalb der Sekundarstufe II" führten (Schindler 2012: 16). Daraus resultiert eine Verschiebung der Selektionsprozesse von früheren auf spätere Schwellen im Bildungssystem (vgl. Becker 2006, Blossfeld et al. 2015, Blossfeld und Shavit 1993, Breen et al. 2009, Lörz und Schindler 2011, Mayer et al. 2007, Müller und Pollak 2008, Schindler 2012, Schindler und Lörz 2012). In Abschnitt 2.1.2 wurde argumentiert, dass heute viele Schüler/innen das (Fach-)Abitur nicht aufgrund eines Studienwunsches absolvieren, sondern um sich einen kompetitiven Vorteil im Kampf um attraktive Ausbildungsplätze $\mathrm{zu}$ verschaffen. Diesbezüglich zeigen Schindler und Lörz (2012: 656), dass die tendenziell zunehmende soziale Ungleichheit beim konditionalen Übergang in die Hochschule auf eine Motivverschiebung unter sozial schwächeren Studienberechtigten zurückzuführen ist: ,the percentage of [working class] students that are not interested in academic work but want to enter paid employment as soon as possible increased" (siehe auch Schindler und Reimer 2010). 
Zusammenfassend führt der Königsweg ins Studium über die schulische Hochschulzugangsberechtigung, die die zentrale und formalrechtlich einzige Voraussetzung für die Studienaufnahme darstellt. In Deutschland nimmt das Schulsystem hier traditionell eine starke Selektionsfunktion wahr. Zwar expandierten die Wege zur Hochschulzugangsberechtigung, sodass mittlerweile mehr als 50\% der Schulabgänger/innen das (Fach-)Abitur erreichen. Andersherum bedeutet dies aber auch, dass fast die Hälfte der Schulabgänger/innen nicht formal studienberechtigt sind, wenn sie das Schulsystem verlassen. Schüler/innen aus sozial schwächeren Familien sind hierbei deutlich überrepräsentiert. Trotz dieser Vorselektion nehmen nur etwa $80 \%$ der Studienberechtigten ein Studium auf. Wiederum stammen diese überdurchschnittlich häufig aus sozial privilegierten Familien. Einer der Hauptgründe für geringe Übergangsquoten und deren soziale Selektivität wird in der Attraktivität des Berufsbildungssystems gesehen. Weitere Gründe können aber auch vom Hochschulsystem ausgehen, das im Folgenden im Fokus stehen soll.

\subsection{Die hochschulische Opportunitätsstruktur}

Ist die zentrale Hürde - der Erhalt der Hochschulzugangsberechtigung - überwunden, stehen diejenigen Studienberechtigten, die ein Studium und nicht etwa eine Berufsausbildung anstreben, vor einer Vielzahl an Optionen und vor weiteren Hürden, die es zu überwinden gilt. Im Folgenden soll diese institutionelle Opportunitätsstruktur beschrieben werden. Dabei stehen erstens zentrale Entwicklungen und die aktuelle Ausgestaltung des deutschen Hochschulsystems im Mittelpunkt (2.2.1). Zweitens sollen formalrechtliche und faktisch zur Anwendung kommende Zulassungsbedingungen eingehend beschrieben werden (2.2.2 und 2.2.3), die neben dem Nachweis der „Hochschulreife“ zunehmend an Relevanz gewinnen und potentiell eine weitere zu überwindende Hürde auf dem Weg ins Studium darstellen.

\subsubsection{Entwicklung und Struktur des deutschen Hochschulsystems}

Quantitative Entwicklung: die Expansion der Hochschulbildung

Seit etwa Mitte des 20. Jahrhunderts kam es weltweit zu einer massiven Expansion der Hochschulbildung (vgl. Schofer und Meyer 2005). Auch das deutsche Hochschulsystem ist Teil dieser Entwicklung und nähert sich trotz des lange diagnostizierten Nachholbedarfs (vgl. Powell und Solga 2011) der durchschnittlichen OECD Studienanfängerquote - circa 60\% im Jahr 2014 - 
stetig an (OECD 2016: 316). Diese stieg in Deutschland - von einigen Schwankungen abgesehen - von circa 5\% in den 1950ern kontinuierlich auf circa 30\% zum Jahrtausendwechsel, um seit Mitte der 2000er nochmals ,eruptiv" (Wolter 2014: 23) anzusteigen und 2011 erstmals die 50\%-Marke zu überschreiten (Autorengruppe Bildungsberichterstattung 2012: 126). ${ }^{4}$ Zudem nahmen im Jahr 2011 erstmals etwa genauso viele Personen ein Studium auf wie eine duale Ausbildung, was der spezifisch deutschen Debatte um Fachkräftemangel, Überqualifizierung und „Akademikerschwemme“ neuen Auftrieb verlieh (vgl. Kreckel 2014).

Institutionell spiegelt sich die Expansion sowohl im Ausbau existierender Hochschulen als auch in Neugründungen wider. Mitte der 1990er Jahre betrug die Anzahl (staatlich anerkannter) deutscher Hochschulen etwa 300; im Studienjahr 2014/15 lag dieser Wert bei 400 Hochschulen, an denen fast 10.000 grundständige Studiengänge angeboten werden. Die meisten Neugründungen gehen auf (private) Fachhochschulen zurück (Autorengruppe Bildungsberichterstattung 2016: 124); auf Studierendenebene wird die Expansion allerdings in großem Maße von den staatlichen Hochschulen getragen (vgl. Darraz et al. 2009). Im Vergleich zu Ländern mit größerer geographischer Ausdehnung und Disparitäten (vgl. für Australien: James 2001, USA: Turley 2009, Finnland: Suhonen 2014) kann das deutsche Hochschulnetz als engmaschig bezeichnet werden. Spieß und Wrohlich (2010: 474) etwa berechnen für studienberechtigte Teilnehmer/innen des Sozio-ökonomischen Panels (SOEP) eine durchschnittliche Distanz vom Heimatort zur nächsten Hochschule von etwa $21 \mathrm{~km}$ und eine Maximaldistanz von $86 \mathrm{~km}$ (siehe auch Helbig et al. 2017).

Mit der beschriebenen Expansion ist Hochschulbildung kein exklusives Gut mehr, an dem nur ein kleiner (elitärer) Teil der Bevölkerung teilhaben und entsprechend von den positiven Erträgen profitieren kann (vgl. Kreckel 2015).

4 Dies mag in Anbetracht der oben zitierten Studienberechtigtenquoten von etwas mehr als 50\% und Übergangsraten von etwa $80 \%$ erstaunen. Daher scheint hier eine technische Erläuterung angebracht: Die Studienanfänger/innenquote wird jährlich vom Statistischen Bundesamt veröffentlicht und gibt den Anteil von Studienanfänger/innen an der altersgleichen Bevölkerung an. Für jeden Altersjahrgang wird dieser Anteil berechnet und anschließend aufsummiert, sodass alle Studienanfänger/innen unabhängig von ihrem Alter in die Berechnung eingehen. Dieses sogenannte Quotensummenverfahren entspricht dem OECD Standard (Statistisches Bundesamt 2016a: 12). Der starke Anstieg des „Rohwertes“ in den letzten Jahren ist zum Teil auf die Abschaffung der Wehrpflicht sowie auf die doppelten Abiturjahrgänge zurückzuführen. Zudem ist in der Studienanfänger/-innenquote auch die steigende Zahl an Studienanfänger/innen mit ausländischer Hochschulzugangsberechtigung (sogenannte Bildungsausländer/innen) enthalten. Der um die doppelten Abiturjahrgänge und um Bildungsausländer/innen bereinigte Wert lag 2011 bei 44,7\% (ohne Korrektur: 55,6\%). 2014 stiegen die entsprechenden Werte nochmals leicht an auf 47,9\% (ohne Korrektur: 58,3\%) (Autorengruppe Bildungsberichterstattung 2016: Tab. F2-2A). 
Wie jedoch in Abschnitt 2.1.3 gezeigt wurde, konnte diese Entwicklung allenfalls teilweise zur Reduktion sozialer Ungleichheit beim Zugang zu Hochschulbildung beitragen.

Institutionelle Ausgestaltung: Typen und Trägerschaft

In vielen Ländern wurde die expandierende Nachfrage nach Hochschulbildung, speziell durch Bildungsaufsteiger/-innen, zum Teil durch die Gründung neuer, weniger prestigereicher und oft kostengünstigerer Institutionen innerhalb des Hochschulsystems gedeckt (z.B. UK: Polytechnics, USA: Community Colleges, Frankreich: Instituts Universitaires de Technologie, vgl. Teichler 2005: 127). In Deutschland manifestierte sich dieser Trend in der Gründung der ersten Fachhochschulen zu Beginn der 1970er (vgl. Banscherus et al. 2015, Mayer et al. 2007, Teichler 2005). Das deutsche Hochschulsystem gliedert sich seitdem in zwei zentrale institutionelle Typen - Universitäten und Fachhochschulen $(\mathrm{FH})$ - und wird deshalb im internationalen Vergleich als binär (vs. einheitlich oder diversifiziert) charakterisiert (vgl. Arum et al. 2007).

Im Vergleich zu Universitäten fokussieren FHs primär auf den Bereich Lehre und sind dabei anwendungsorientierter ausgerichtet. Weitere zentrale Unterschiede bestehen bezüglich des angebotenen Fächerspektrums sowie des Promotions- und Habilitationsrechts, das nach wie vor weitestgehend uneingeschränkt den Universitäten obliegt. ${ }^{5}$ Bezüglich verschiedener Ertragsdimensionen (Einkommen, Arbeitslosigkeitsrisiko, inhaltlicher und Statusadäquanz) unterscheiden sich Universitätsabsolvent/innen der gleichen Fachgruppe im Durchschnitt jedoch kaum von Fachhochschulabsolvent/innen (vgl. Autorengruppe Bildungsberichterstattung 2016, Müller et al. 2002, Spangenberg et al. 2012).

Die vor fast 50 Jahren etablierte Zweiteilung des deutschen Hochschulsystems existiert nach wie vor, was sich zum Beispiel im „Typenzwang“, also der erzwungenen Zuordnung bei Neugründung, manifestiert (Wissenschaftsrat 2010). Nichtsdestotrotz kam es im Laufe der Zeit zur Annäherung zwischen beiden Sektoren, etwa durch den Bedeutungsgewinn der Forschung an FHs, durch die Etablierung forschungs- und anwendungsorientierter Studiengänge an beiden Hochschultypen und nicht zuletzt durch die Gleichstellung der im Zuge des Bologna Prozesses neu eingeführten Bachelor- und Master-Ab-

5 Dieses stark umkämpfte Exklusivrecht wird nach und nach aufgeweicht. Seit den 1990ern können Fachhochschulabsolvent/innen ohne zusätzlichen Universitätsabschluss promovieren - allerdings zunächst nur an Universitäten. Die ,kooperative Promotion“" ist mittlerweile in allen Bundesländern möglich. In Hessen besteht seit 2016 erstmalig die Möglichkeit, an Fachhochschulen zu promovieren, sofern diese „eine ausreichende Forschungsstärke besitzen“ (Keller 2016: 2). 
schlüsse,${ }^{6}$ die für die FHs einen großen Schritt ,im Hinblick auf eine Statusangleichung zu den Universitäten“" (Teichler 2005: 203) bedeutete. Trotz dieser „Entdifferenzierungstendenzen“ (Wissenschaftsrat 2010: 22) gehen manche Beobachter (vgl. Hillmert und Jacob 2010, Reimer und Pollak 2010) nach wie vor von Reputationsunterschieden aus, schon aufgrund des meist fehlenden Promotionsrechts sowie des begrenzten Fächerangebots an FHs (gerade Medizin und Jura - zwei Fächer, die zu besonders prestigereichen Berufen führen können nur an Universitäten studiert werden).

In Deutschland hat sich seit den 1970ern mit der Berufsakademie ein weiterer Hochschultyp herausgebildet, der durch die Etablierung dualer Studiengänge der traditionell starken Trennung zwischen beruflicher und Hochschulbildung - dem „Bildungs-Schisma“ (Baethge 2006) - eine hybride Ausbildungsform entgegenstellt (vgl. Graf 2013). Laut Bildungsbericht 2016 waren im Studienjahr 2014 circa 5\% der Studienanfänger/innen in dualen Studiengängen eingeschrieben (Autorengruppe Bildungsberichterstattung 2016: F110web).

Neben dem Typ können Hochschulen anhand ihrer Trägerschaft unterschieden werden, die größtenteils staatlich ist (vgl. Darraz et al. 2009). Daneben existieren staatlich anerkannte Hochschulen in kirchlicher und privater Trägerschaft. In den 1990ern setzte eine Gründungswelle vor allem privater (Fach-)Hochschulen mit spezifisch fachlichem Profil (meist im Bereich Wirtschaft und Recht) ein, deren Zahl sich von 25 im WiSe 1995/96 auf 117 im WiSe 2014/15 fast verfünffachte. Der Studienanfänger/innenanteil stieg zwar entsprechend an, liegt aber immer noch bei unter 8\% (Autorengruppe Bildungsberichterstattung 2016: 124). Ein Grund hierfür ist, dass private Hochschulen nicht an die Kapazitätsverordnung (siehe Abschnitt 2.2.2) gebunden sind und so ihren exklusiven (wenn auch meist nicht elitären) Status durch Einschränkung der Studierendenzahl aufrechterhalten können. Zudem können private Hochschulen uneingeschränkt Studiengebühren erheben, was staatlichen Hochschulen lange Zeit verwehrt blieb. Nach den Klagen von fünf Bundesländern wurde das Studiengebührenverbot zwar gekippt und daraufhin ab 2005 Gebühren (bis zu 500€ pro Semester) in sieben Bundesländern auch an staatlichen Einrichtungen eingeführt. Diese wurden allerdings in den Folgejahren nach Kritik und Regierungswechseln im gesamten Bundesgebiet wieder abgeschafft (vgl. Kroth 2016: 16).

Die Differenzierung zwischen staatlichen und privaten Hochschulen in Deutschland entspricht nicht der etwa in den USA etablierten Unterteilung in Breiten- und Elitebildung (vgl. Darraz et al. 2009), auch wenn einige private Hochschulen versuchen, sich in ihrem Bereich als Eliteeinrichtungen zu positionieren (vgl. Deppe et al. 2015).

6 Unabhängig vom Hochschultyp werden Bachelor- und Masterabschlüsse den ISCED-2011 Stufen 6 bzw. 7 zugeordnet. 


\section{Studienkosten}

Wie gerade bereits angedeutet, sind die direkten Studienkosten - mit Ausnahme des Studiums an einigen privaten Hochschulen - in Deutschland im Vergleich zu vielen anderen Ländern (vgl. Europäische Kommission 2016, Page und Scott-Clayton 2016) einheitlich gering. Seit der Abschaffung von Studiengebühren an staatlichen Hochschulen in allen Bundesländern belaufen sich die Kosten, die direkt an die Hochschulen abgeführt werden müssen, meist auf weniger als $600 €$ pro Jahr (Verwaltungsgebühren, Sozialbeitrag, inklusive Semesterticket für öffentlichen Nahverkehr). Gleichzeitig besteht auf Grundlage des Bundesausbildungsförderungsgesetzes (BAföG) die Möglichkeit einer bedarfsabhängigen finanziellen Studienförderung. ${ }^{7}$

Gleichwohl werden Finanzierungsschwierigkeiten von etwa 30\% der Schüler/innen im letzten Schuljahr als eine Schwierigkeit bei der Wahl des nachschulischen Werdeganges genannt (Schneider und Franke 2014: 34, DZHW Studienberechtigte 2012). Gründe hierfür können neben der Fehleinschätzung direkter Kosten und Finanzierungsmöglichkeiten in der Antizipation finanzieller (und auch sozialer) Kosten in Form von Umzugs-, Wohn- und Reisekosten liegen, die durch etwaige Mobilitätszwänge entstehen.

Zudem fallen mit einem Studium Opportunitätskosten an, da sich der Arbeitsmarkteinstieg verschiebt. Zwei Entwicklungen des deutschen Hochschulsystems wirken dem allerdings entgegen: Zum einen wurde die Studiendauer bis zum ersten berufsqualifizierenden Abschluss im Zuge der Bologna-Reform in den meisten Studiengängen auf drei Jahre verkürzt (wichtige Ausnahmen sind die Staatsexamensstudiengänge im Bereich Medizin und Jura). Nichtsdestotrotz können Studienberechtigte einen Ausbildungsabschluss oft bereits nach zwei Jahren erreichen, währenddessen sie zudem in der Regel eine Ausbildungsvergütung erhalten. Zum anderen bieten die oben genannten dualen Studiengänge die Möglichkeit, ein Studium mit den Vorzügen einer Ausbildung (Vergütung, bereits erfolgte Integration in den Arbeitsmarkt) zu kombinieren. Trotz stetigen Wachstums ist diese Option bisher jedoch rein quantitativ wenig relevant.

7 Die durchschnittliche Förderhöhe betrug im Jahr 2020 514€, der Höchstsatz 861€, vgl. https://www.bafög.de/de/904.php sowie https://de.statista.com/statistik/daten/ studie/220/umfrage/monatlicher-bafoeg-foerderbetrag-pro-student/[letzter Zugriff 05.08.2021]). Laut Sozialerhebung wurde 2016 etwa ein Fünftel der Studierenden durch BAföG gefördert (Middendorff et al. 2017: 14). Zurückgezahlt werden muss höchstens die Hälfte des erhaltenen Gesamtbetrags bis zu einer Höchstsumme von 10.010€. Die Rückzahlung beginnt regulär fünf Jahre nach Ende der Förderungshöchstdauer und kann bei zu geringem Einkommen aufgeschoben bzw. ausgesetzt werden. https://www.bafoeg-rechner.de/FAQ/rueckzahlung.php [letzter Zugriff: 16.04.2017] 
Neue Entwicklungen: Deregulierung, Wettbewerbsorientierung und Differenzierung

In den 1980ern setzten erste Diskussionen über die Notwendigkeit eines größeren Wettbewerbs zwischen den Hochschulen und damit verbundenen Deregulierungsmaßnahmen ein. Diese Debatte wurde in den 1990ern verstärkt und führte zu ersten, durchaus scharf kritisierten Reformen mit dem Ziel, durch die schrittweise Einführung von Managementstrukturen (,New Public Management ${ }^{*}$ ) die Selbststeuerungsfähigkeit der Hochschulen zu stärken. ${ }^{8}$ Hierzu gehörten etwa die Umstellung auf Outputorientierung bei Finanzierungsfragen, die Festlegung und Evaluation von Zielvereinbarungen und Leistungsindikatoren sowie die Stärkung der hochschulischen Autonomie bei der Studierendenauswahl (vgl. Kamm und Köller 2010, Krüger et al. 2012, Pritchard 2006). Maßnahmen dieser Art sollten dazu beitragen, den Wettbewerb in einem expandierenden Hochschulsystem zu stärken.

Sowohl Expansion als auch Wettbewerb sind unweigerlich mit Differenzierung verknüpft (vgl. Arum et al. 2007, Banscherus et al. 2015, Teichler 2005). So war auch die Einführung der Fachhochschulen vor allem eine Reaktion auf die erste Expansionswelle in den 1960ern (vgl. Banscherus et al. 2015). Innerhalb eines Hochschultyps war allerdings die Vorstellung verbreitet, dass sich verschiedene Universitäten und Fachhochschulen in Qualität und Reputation kaum voneinander unterscheiden (sollen). Diese „Gleichheitsfiktion“ (Kreckel 2015: 406) sollte sowohl durch zunehmende horizontale (stärkere Profilbildung mithilfe der oben genannten Maßnahmen) als auch vertikale Differenzierung ersetzt werden. Letztere wurde insbesondere durch die Exzellenzinitiative bzw. Exzellenzstrategie gefördert, ${ }^{9}$ die aufgrund der Überzeugung ins Leben gerufen wurde, dass das deutsche Hochschulsystem ,,Leuchttürme' und ,Elite' statt ,Gießkanne“ und ,Egalität"“ (Barlösius 2008: 151) brauche, um im ,weltweite[n] ,battle of excellence“" (Banscherus et al. 2015: 25, vgl. auch Deppe et al. 2015) bestehen zu können. Seit 2005/2006 wurden in diesem Rahmen in drei Runden Exzellenzcluster, Graduiertenschulen und Zukunftskonzepte gefördert. Mit der Exzellenzstrategie soll es zu einer dauerhaften Förderung von ausgewählten Exzellenzuniversitäten und -verbünden kommen (vgl. DFG 2019). Inwiefern dies zu einer langfristigen Institutionali-

8 Tatsächlich kam es zu einer regelrechten Reformwelle mit vier Novellen des Hochschulrahmengesetzes in nur sechs Jahren (zwischen 1998 und 2004) (vgl. Pritchard 2006).

9 Neben der Exzellenzinitiative könnten auch Rankings zur vertikalen Differenzierung beitragen. In den großen internationalen Rankings (Shanghai Ranking, Times Higher Education World University Ranking), die deutlich von US-amerikanischen Hochschulen dominiert werden, schaffen es einige deutsche Hochschulen vereinzelt unter die Top 50. Innerhalb Deutschlands deckt das „CHE-Ranking“ einen Teil des Studienangebots ab (vgl. Weiss et al. 2015, Winkler 2014). 
sierung vertikaler Hierarchien innerhalb des Universitätssektors führt, bleibt abzuwarten.

Seit etwa zwei Jahrzehnten befindet sich das deutsche Hochschulsystem also in einer „Umbruchs- und Deregulierungsphase“, die zu größerer institutioneller Autonomie, Differenzierung und somit auch Komplexität führt. Aus diesen ersten Schritten lassen sich aber noch keine ,sedimentierten stratifikatorischen Unterscheidungen“ (Bloch et al. 2014: 245) ableiten. Im Vergleich zu stärker stratifizierten Hochschulsystemen ist das deutsche Hochschulsystem trotz der beschriebenen Tendenzen als homogen zu bezeichnen: „Still far off on the horizon is an American-style higher education market with highly differentiated institutions, all costing different amounts and offering different incentive schemes and rewards" (Pritchard 2006: 111).

Neben den beschriebenen vertikalen Differenzierungstendenzen spielt in Deutschland (und vielen anderen europäischen Ländern) eine horizontale Differenzierungsdimension eine wichtige Rolle: die fachliche Differenzierung. Anders als etwa an anglo-amerikanischen Hochschulen, an denen die fachliche Spezialisierung oft erst im Laufe des Studiums stattfindet und somit zunächst die Entscheidung zwischen hierarchisch gegliederten Hochschulen im Vordergrund steht (vgl. Haase 2008), wird die Entscheidung für ein bestimmtes Fach und somit in einigen Fällen auch für die damit einhergehende berufliche Ausrichtung (vgl. Guggenberger 1991, Teichler 2005) im deutschen Kontext formal zeitgleich zur Studienentscheidung getroffen. ${ }^{10}$ Auch aufgrund der bisher (noch) geringen vertikalen Stratifizierung sind Studien- und spätere Arbeitsmarktchancen in Deutschland vor allem fachlich strukturiert. Die Zugangschancen zum Studium etwa variieren deutlich zwischen den Studienfächern (siehe hierzu Abschnitt 2.2.3). Auch unterscheiden sich, wie weiter oben bereits erwähnt, die Einkommenserträge kaum zwischen Universitäts- und Fachhochschulabsolvent/innen des gleichen Fachs, während zwischen den Fächern eklatante Unterschiede bestehen (vgl. Autorengruppe Bildungsberichterstattung 2016: F4-16web, siehe auch Abschnitt 6.2.3). Die horizontale Differenzierungsdimension Studienfach bekommt durch diese Verknüpfung mit $\mathrm{Zu}-$ gangs- und Arbeitsmarktchancen eine klar hierarchische und in ihrer Konsequenz möglicherweise ungleichheitsgenerierende Komponente. Dieser Befund

10 Dies ist darauf zurückzuführen, dass sich Abiturient/innen nicht allein für ein Studium an einer oder mehreren Hochschule bewerben, sondern für einen oder mehrere spezifische, fachlich ausgerichtete Studiengänge. Aus dieser Parallelität kann die tatsächliche Entscheidungssequenz allerdings noch nicht abgeleitet werden. Für manche Schüler/innen mag die Entscheidung für oder gegen ein Studium vorgelagert sein und die spezifische Fachwahl (wie auch Hochschul- und Ortswahl) innerhalb der gegebenen Zugangshürden getroffen werden. Für andere mag eine spezifische fachliche oder lokale Ausrichtung Vorrang haben, sodass bei geringen Zugangschancen in diesem Bereich eher vom Studium abgesehen wird (für eine ausführlichere Diskussion siehe Kapitel 5). 
ist prinzipiell nicht neu. Neu ist allerdings, dass es im Zuge der Bologna-Reform und dem stärkeren Fokus auf Wettbewerb und Profilbildung zu einer „radikalen Ausdifferenzierung des Studienangebots“ (Schröder 2015: 98) kam. Studieninteressierte können laut dem Hochschulkompass der Hochschulrektorenkonferenz (HRK, Stand 2021) heute zwischen mehr als 10.000 grundständigen Studiengängen an staatlich anerkannten Hochschulen wählen. ${ }^{11}$ In Anbetracht dieser ,unübersichtlichen Vielfalt" (Autorengruppe Bildungsberichterstattung 2016: 125) warnt der Wissenschaftsrat (2010: 68) als zentrales wissenschaftspolitisches Beratungsgremium in Deutschland vor zu starken Spezialisierungen im Bachelorbereich und fordert eine ,klare Differenzierungsgrenze“. Die „nur schwer überschaubare Zahl der Möglichkeiten“ stellt auch viele Studienberechtigte vor Herausforderungen: sie wird von $43 \%$ der Schüler/innen im letzten Schuljahr als Problem „,bei der Wahl des nachschulischen Werdegangs" genannt und stellt damit die am häufigsten genannte Schwierigkeit dar (Schneider und Franke 2014: 34).

Abschnitt 2.2.1 diente vorrangig dazu, wichtige Entwicklungen, die institutionelle Struktur und zentrale Differenzierungslinien des deutschen Hochschulsystems zu beschreiben, um ein grundlegendes Verständnis für die (hochschulische) Opportunitätsstruktur, mit der Studienberechtigte konfrontiert sind, zu schaffen. Zusammenfassend führten die Hochschulexpansion und die geführte Debatte um die Wettbewerbsfähigkeit des deutschen Hochschulsystems zu Reformen und Ausdifferenzierungen auf verschiedenen Ebenen. Dies hat zur Folge, dass die nachschulische Entscheidung, vor der Studienberechtigte stehen, im innerdeutschen, zeitlichen Vergleich zunehmend komplex geworden ist. Im Vergleich zu vielen anderen Hochschulsystemen kann das deutsche aber immer noch als relativ homogen bezeichnet werden. Trotz des Gründungsbooms privater Hochschulen, vertikaler Hierarchisierungstendenzen durch die Exzellenzinitiative und der Aufweichung der starken Trennung von Hochschul- und Berufsbildungssystem durch duale Studiengänge verlaufen die zentralen Differenzierungslinien nach wie vor zwischen Universitäten und Fachhochschulen auf der vertikalen und zwischen Studienfächern auf der horizontalen Achse.

Die hochschulische Opportunitätsstruktur ist jedoch nicht nur durch Kosten und vorhandene Studienoptionen bestimmt, sondern auch durch Zulassungsregelungen, die im Folgenden beschrieben werden.

11 Hierbei handelt es sich nicht in allen Fällen um eigene fachliche Spezialisierungsbereiche, sondern um einzelne Programm-Hochschulkombinationen. Laut einer inhaltsanalytischen Kategorisierung durch Schröder (2015) konnten 2011 etwa 3000 inhaltlich unterscheidbare interdisziplinäre oder spezialisierte ,teildisziplinäre“ Studiengänge identifiziert werden. 1970 betrug diese Zahl noch 70 und im Jahre 1999 belief sie sich auf 180 (ebd.). 


\subsubsection{Zulassung zum Hochschulstudium: Entwicklung und aktuelle Praxis}

Im internationalen Vergleich kann grundsätzlich zwischen zwei Hochschulzugangsmodellen unterschieden werden: einem Berechtigungsmodell (ein definierter Schulabschluss berechtigt zur Studienaufnahme) und einem Prüfungsmodell (mit zusätzlichen Zugangsprüfungen) (vgl. Banscherus 2010: 51). Aufgrund der Stellung des (Fach-)Abitur als zentrale formale Hochschulzugangsvoraussetzung wird Deutschland dem Berechtigungsmodell zugeordnet, das darauf fußt, dass ein Großteil der Selektion bereits im Schulsystem stattfindet. In Deutschland war (und ist) dies traditionell der Fall, und der Hochschulzugang wurde entsprechend als ,undramatische Übergangsstation für die Bildungselite" (Teichler 1984: 11) beschrieben. Diese Offenheit des Hochschulsystems für formal Hochschulzugangsberechtigte war funktional, ,[s]olange die soziale und fachliche Filterwirkung des dreigliedrigen Schulsystems dafür sorgte, dass die Abiturientenzahl begrenzt blieb“ (Kreckel 2015: 408). Zwar stellt das Abitur nach wie vor einen „Flaschenhals“ dar (Mayer et al. 2007: 246), doch weitet sich dieser, wie oben beschrieben, zusehends: Eine immer größere Zahl Studienberechtigter strebt an die Hochschulen, die als Reaktion hierauf vermehrt selbst filtern. Entsprechend deuten jüngere Entwicklungen darauf hin, dass das schulische Zertifikat in der Regel zwar nach wie vor notwendige, in vielen Fällen aber nicht mehr hinreichende Bedingung für die $\mathrm{Zu}$ lassung zum Studium ist. Diese Entwicklungen und der aktuelle Status quo sollen im Folgenden dargestellt werden.

Von der Hochschulzugangsberechtigung zum Auswahlrecht der Hochschulen: neuere Debatten

Traditionell werden in Deutschland Gymnasium und Universität als ,zwei konsekutive Stufen einer einheitlichen, durchgehenden Bildungsbiographie gedacht" (Wolter 2008: 19). Hierzu zählt auch die Vorstellung, dass mit dem Bestehen des Abiturs die allgemeine Hochschulreife und somit auch allgemeine Studierfähigkeit attestiert wird, unabhängig vom angestrebten Fach (vgl. Tarazona 2006). Tatsächlich konnten bis etwa Mitte der 1960er Jahre nahezu alle Studienberechtigten davon ausgehen, ,das Studium [ihrer] Wahl, meistens auch am [...] präferierten Ort, aufnehmen zu können“ (Bode 2015: 8).

Dieses ,alte Modell“ der engen Kopplung von Abitur und Hochschulzugang (Wolter 2008: 18) wurde und wird jedoch von zentralen hochschulpolitischen Akteuren in Anbetracht neuerer Entwicklungen, wie der Nachfrageexpansion nach hochschulischer Bildung, der Heterogenisierung der Studienberechtigten sowie der zunehmenden Ausdifferenzierung des Studienangebots, als nicht mehr angemessen kritisiert (vgl. Heine et al. 2008, HRK 2004, Lewin 2004, Schuler und Hell 2008, Wissenschaftsrat 2004). Im Einklang mit den im 
vorangegangenen Abschnitt diskutierten Deregulierungs-, Wettbewerbs- und Profilbildungstendenzen wurde daher in der Diskussion um den Hochschulzugang ein „Paradigmenwechsel von Studierfähigkeit zu Passfähigkeit“ vollzogen (Lewin und Lischka 2004: 82). ${ }^{12}$ Dieser spiegelt sich auch in den bundesund landespolitischen Neuerungen des Zulassungsrechts der letzten Jahrzehnte wider (siehe unten), die die aktive Rolle der Hochschulen bei der Bewerber/innenauswahl stärkten. Befürworter/innen verknüpfen diese Entwicklung mit verschiedenen positiven Auswirkungen auf individueller, organisationaler und gesellschaftlicher Ebene: Eine optimierte Auswahl von Studierenden steigere etwa deren Wohlbefinden und Leistung und führe zu besseren Noten, kürzeren Studienzeiten und geringeren Abbruchquoten. Hochschulen könnten ihr spezifisches Profil besser ausbilden und somit konkurrenzfähiger werden, was letztlich die Wettbewerbsfähigkeit des gesamten deutschen Hochschulsystems steigern könne. Letztlich gebe es auch einen unmittelbar wirtschaftlichen Nutzen durch geringere BAföG-Leistungen, längere Lebensarbeitszeit und höhere Produktivität durch die bessere Nutzung geistiger Potentiale (vgl. Haug 2006: 97, Schuler und Hell 2008: 11, Wissenschaftsrat 2004). Kritiker hingegen warnen vor einer „Zersplitterung der Zulassungskriterien“ (Hauck-Scholz 2010: 89), vor „,verwirrender Vielfalt“ (Selbmann 2012: 1373) oder einem „Zulassungschaos“, das „Semester für Semester tausende junge Menschen ihrer $\mathrm{Zu}$ kunftschancen" beraube (Keller 2009: 30).

Tatsächlich haben sich die Zugangswege und -modalitäten in den letzten Dekaden vervielfältigt. Neben bundesweit oder örtlich zulassungsbeschränkten Studiengängen (mit variierenden, bundes- und/oder landesrechtlich festgelegten Kapazitäten, Quoten und Verfahren) gibt es zulassungsfreie Studiengänge. Letzteres bedeutet dabei ,nicht zwangsläufig, wie es der Terminus nahelegt, dass jene Studiengänge allen Studieninteressierten, die die allgemeinen Studienvoraussetzungen erfüllen, offen steht und diese sich unmittelbar immatrikulieren können“ (Winter et al. 2012: 132). Denn losgelöst von der Zulassungsbeschränkung wenden einige Universitäten für bestimmte Studiengänge Eignungsfeststellungsverfahren an, um ,nicht geeignete“ Bewerber/innen zu identifizieren und von vorn herein aus dem Pool ihrer potentiell Studierenden auszuschließen. Dies hat zur Folge, dass zum Teil auch in zulassungsfreien Studiengängen „Zulassungsvoraussetzungen erbracht, mithin Zulassungshürden überwunden werden müssen“ (ebd.: 143f.).

Das zugrunde liegende Zulassungsrecht ist für Laien nur schwer überschaubar: „Die Rechtsquellen stehen in einem komplizierten Hierarchiegefüge“ - so ein Hochschulrechtsexperte - ,nur wenige Rechtsgebiete sind derart stark durch das Nebeneinander von verfassungsrechtlichen, einfachgesetz-

12 Mit Passfähigkeit soll das Ziel einer ,,möglichst hohe[n] Übereinstimmung individueller Kompetenzen der StudienanfängerInnen mit den grundlegenden und spezifischen Anforderungen eines Studiums“ (Lewin und Lischka 2004: 87) erreicht werden. 
lichen, verordnungs- und satzungsrechtlichen Normen geprägt" (Bode 2013: 372). Weitere Vielfalt kommt durch die oft komplexen Zulassungsverfahren privater Hochschulen hinzu, denen vom Gesetzgeber hier kaum Grenzen gesetzt werden (vgl. Zimmerhofer und Trost 2008: 37).

Im Folgenden beschreibe ich die rechtlichen Grundlagen und Verfahrensweisen für die vier im vorherigen Absatz genannten Zulassungswege. Insbesondere mit Blick auf die bundesweit zulassungsbeschränkten Studiengänge soll auch die rechtliche Entwicklung kurz dargestellt werden, da Entscheidungen, insbesondere aus den 1970ern, noch heute maßgeblich sind und auch für landesgesetzlich geregelte Zulassungsformen oft als Orientierung dienen. ${ }^{13}$

Bundesweit zulassungsbeschränkte Studiengänge

\section{Rechtliche Entwicklung}

Bis in die 1960er hinein war die Hochschulautonomie bezüglich der Studienplatzvergabe hoch; es oblag weitestgehend den Hochschulen, Auswahlkriterien festzulegen. Gleichzeitig waren die Nachfrage nach Studienplätzen noch überschaubar und Studienberechtigte in ihrer Wahlfreiheit kaum eingeschränkt, sodass hier zunächst kein dringender Regulierungsbedarf bestand (vgl. Bode 2013, 2015). Dies änderte sich mit steigender Nachfrage und einem „mehrjährigen ,Wildwuchs““ (Zimmerhofer und Trost 2008: 33) der angewandten Zulassungsmodalitäten, die es für viele Studienberechtigte immer schwerer machten, einen Studienplatz zu bekommen, vor allem in den stark nachgefragten (medizinischen) Studienfächern.

In diesem Kontext ist das erste, bis heute grundlegende Numerus claususUrteil des Bundesverfassungsgerichts (BVerfG) von $1972 \mathrm{zu}$ verorten, mit dem ein „Perspektivwechsel von einem Recht der Hochschule zum Grundrecht der Bewerber" (Bode 2013: 9) vollzogen wurde. Aus der grundgesetzlich verankerten Wahlfreiheit des Berufes und der Ausbildungsstätte (Art. 12, Abs. 1 Grundgesetz) wurde der prinzipielle Anspruch jedes/jeder Hochschulzugangsberechtigten abgeleitet, für das „Fach seiner Wahl am Ort seiner Wahl“ (Zimmerhofer und Trost 2008: 33) zugelassen zu werden. Eine Verweigerung der Zulassung, so die Argumentation des BVerfG, gehe daher mit einer Grundrechtsverletzung einher und bedürfe strikter rechtlicher Regelung. Ein absoluter Numerus clausus (NC) (d.h. auf das gesamte Bundesgebiet zutreffende Zulassungsbeschränkungen in einem Fach) sei nur verfassungsmäßig, sofern er

13 Für Eignungsfeststellungsverfahren, örtlich zulassungsbeschränkte und zulassungsfreie Studiengänge ist das Landesrecht maßgeblich und eine umfassende Darstellung der Entwicklung und des Status quo daher im Rahmen der vorliegenden Arbeit nicht möglich. Zudem beschränke ich mich auf die Entwicklung in den alten Bundesländern, da diese für spätere gesamtdeutsche Regelungen ausschlaggebend war. 
„in den Grenzen des unbedingt Erforderlichen unter erschöpfender Nutzung der vorhandenen, mit öffentlichen Mitteln geschaffenen Ausbildungskapazitäten angeordnet wird" und „Auswahl und Verteilung nach sachgerechten Kriterien mit einer Chance für jeden an sich hochschulreifen Bewerber" erfolgten. ${ }^{14}$

Dieses Urteil bildete die rechtliche Grundlage für den ersten Staatsvertrag über die Vergabe von Studienplätzen, auf dessen Grundlage die Zentrale Vergabestelle für Studienplätze (ZVS) und die Kapazitätsverordnung (KapVO) ins Leben gerufen wurden. Mit Letzterer wurde eine bundeseinheitliche Verfahrensgrundlage für die Ermittlung zur Verfügung stehender Ausbildungskapazitäten festgelegt, mit der weitestgehend einheitliche Studienund Prüfungsbedingungen geschaffen werden sollten, um mit der Verteilung von Studienplätzen nicht unzulässigerweise Lebenschancen zu beeinflussen (vgl. Weber 2010: 78, Zimmermann 2010). ${ }^{15}$ Die ZVS wurde mit der Studienplatzvergabe von Studiengängen betraut, für die an (fast) allen staatlichen Hochschulen Zulassungsbeschränkungen bestanden und für die mit einer die Studienplatzkapazitäten übersteigenden Bewerber/innenzahl zu rechnen war. Bis zum WiSe 2009/2010 wurden die Plätze für die drei medizinischen Fächer, Pharmazie, Psychologie und Biologie kontinuierlich zentral vergeben; heute gilt dies nur noch für die vier Erstgenannten.

Die ZVS vergab zunächst $40 \%$ der Studienplätze nach dem Anciennitätsprinzip (Wartezeit (WZ)) sowie 60\% nach dem Leistungsprinzip (Durchschnittsnote (DN) des Abiturzeugnisses). Mit weiterhin steigender Nachfrage, vor allem in den medizinischen Fächern, wurden die Auswahlgrenzen (d.h., die Höhe der benötigten Abiturnote und die Länge der Wartezeit) unzumutbar. 1977 kam es daraufhin zum zweiten Numerus clausus-Urteil des BVerfG (vgl. Zimmerhofer und Trost 2008). Da jede/jeder Hochschulzugangsberechtigte eine Chance auf Zulassung haben müsse, sei das Vergabeverfahren in Fächern mit starkem Bewerber/innenüberhang ,,beschleunigt durch ein anderes Auswahlverfahren zu ersetzen“. ${ }^{16}$ Infolgedessen wurde der Test für medizinische Studiengänge (TMS) entwickelt, der von Mitte der 1980er bis Mitte der 1990er

14 Der Urteiltext steht unter folgendem Link zur Verfügung: https://www.hrk.de/file $\mathrm{admin} / \mathrm{redaktion} / \mathrm{hrk} / 02-D o k u m e n t e / 02-03-S t u d i u m / 02-03-04-H o c h s c h u l z u l a s-$ sung/bverfg_nc-urteil_18071972.pdf [letzter Zugriff: 29.07.2021]

15 Der Berechnung verfügbarer Kapazitäten liegen definierte Curricularnormwerte zugrunde, die den Betreuungsumfang bis zum Abschluss eines Studienfaches umfassen, der mit dem jeweils vorhandenen Lehrdeputat der zur Verfügung stehenden Lehreinheiten in Beziehung gesetzt wird (vgl. Zimmermann 2010). Zunächst galt die bundeseinheitliche Regelung nicht nur für die bundesweit, sondern auch örtlich zulassungsbeschränkten Studiengänge. Seit der Föderalismusreform von 2006 wird die Kapazitätsfestlegung für Letztere jedoch landesrechtlich geregelt (vgl. Winter et al. 2012).

$16 \mathrm{http} / /$ datenbank.flsp.de/flsp/lpext.dll/Infobase8/h/hochschulzulassung/328nr23? $\mathrm{fn}=$ document-frame.htm\&f=templates\&2.0\# [letzter Zugriff: 29.07.2021] 
fester Bestandteil des Zulassungsverfahrens zu einem Teil der medizinischen Studiengängen war (ebd.).

Im Zuge der bereits diskutierten Debatte um Wettbewerbsfähigkeit und Profilbildung wurde in den 1990ern die Forderung lauter, das Auswahlrecht der Hochschulen zu stärken. Die 4. und 5. Novelle des HRG (1998 und 2002) entsprachen bereits diesen Forderungen, indem den Hochschulen die Möglichkeit eingeräumt wurde, bei der Vergabe bundesweit zulassungsbeschränkter Studienplätze Bewerber/innen für 20 bzw. 24\% der verfügbaren Kapazitäten mittels eigener Kriterien auszuwählen (vgl. Täger 2010: 37). In seinen „Empfehlungen zur Reform des Hochschulzugangs" bekräftigte der Wissenschaftsrat (2004: 33) die Funktion des Hochschulzugangs als „Mittel zur Förderung von Profilbildung und Wettbewerb der Hochschulen" und sprach sich explizit für eine weitere Stärkung des Auswahlrechts der Hochschulen aus. Die 7. Novelle des HRG von 2004 vollzog mit der Einführung einer 60\% „AdH-Quote“ (Auswahlverfahren der Hochschulen) daraufhin die endgültige Trendwende hin zu mehr Hochschulautonomie (erstmalige Anwendung WiSe 2005/06). Die zulassungsbezogenen Inhalte des HRG finden sich auch im Staatsvertrag von 2008 (seit 2010 in Kraft), durch den die Stiftung für Hochschulzulassung (SfH) die ZVS bei der Vergabe bundesweit zulassungsbeschränkter Studiengänge ablöste.

2017 befasste sich das BVerfG erneut mit der Zulassung für die bundesweit zulassungsbeschränkten Studiengänge und erklärte das geltende Verfahren als teilweise verfassungswidrig. ${ }^{17}$ Daraufhin wurde das $\mathrm{HRG}^{18}$ überarbeitet und ein neuer Staasvertrag ${ }^{19}$ verabschiedet, der 2019 in Kraft trat.

Da sich der empirische Teil der vorliegenden Arbeit auf Schüler/innen bezieht, die ihre Hochschulzugangsberechtigung im Jahr 2014 erhielten, sind jedoch die zuvor geltenden Regelungen entscheidend. Diese sollen im Folgenden kurz vorgestellt werden. Auf aktuellere Änderungen wird an gegebener Stelle in Kürze eingegangen (für einen ausführlicheren Überblick vgl. Konegen-Grenier 2018).

17 https://www.bundesverfassungsgericht.de/entscheidungen/ls20171219_1bv10003 14.html [letzter Zugriff: 29.07.2021]

18 Aktuelle Fassung: https://www.gesetze-im-internet.de/hrg/HRG.pdf [letzter Zugriff: 29.07.2021]

19 https://www.hochschulstart.de/fileadmin/downloads/gesetze/StV_2019.pdf [letzter Zugriff: 29.07.2021] 


\section{Vergabeverfahren (Pharmazie, Human-, Zahn- und Tiermedizin) bis 2019}

Nach Abzug einer Vorabquote von bis zu $20 \%{ }^{20}$ wurden die verbleibenden Studienplätze der zentral zu vergebenden Studienfächer nach folgenden Quoten verteilt (vgl. Bode 2015: 86 ff.):

- 20\% Abiturbestenquote: nach dem Grad der Qualifikation (DN des Abiturzeugnisses).

- $\quad 20 \%$ Wartezeitquote: nach der Anzahl verstrichener Halbjahre seit Erhalt der Hochschulzugangsberechtigung. Studienzeiten an deutschen Hochschulen („Parkstudium“) zählen nicht.

- $60 \%$ AdH-Quote: anhand folgender Kriterien oder deren Kombination: DN, gewichtete Einzelnoten, Ergebnis eines fachspezifischen Studierfähigkeitstests oder Auswahlgesprächs, Art der Berufsausbildung oder Berufstätigkeit. In den Hochschulzulassungsgesetzen der Länder können die genauen Kriterien konkretisiert und ergänzt werden; deren Auswahl und Anwendung obliegt den Hochschulen. Der DN soll ein maßgebliches Gewicht zukommen; meist bedeutet dies das relativ stärkste Gewicht.

Hochschulzugangsberechtigte, die eines der vier bundesweit zulassungsbeschränkten Fächer studieren wollten, mussten sich direkt bei der SfH bewerben und pro Quote bis zu sechs Hochschulen angeben und ranken. Das zentrale Vergabeverfahren ist komplex, erfordert ein ausreichendes Maß an Informiertheit auf Seiten der Bewerber/innen und fördert strategisches Bewerbungsverhalten (vgl. Braun et al. 2007).

Die bundesweit zulassungsbeschränkten Studiengänge sind naturgemäß sehr selektiv. Im Fach Medizin kamen im WiSe 2014/15 43.000 Bewerber/innen auf 9.000 Studienplätze, sodass die durchschnittliche Zulassungschance nur etwa 20\% betrug (Bode 2015: 24). Die Zulassung über die Abiturbestenquote erforderte in der Regel Spitzennoten, die je Bundesland des Erwerbs der Studienberechtigung zwischen 1,0 und 1,3 lagen. ${ }^{21}$ Die Auswahlgrenze in der Wartezeitquote lag regelmäßig bei zwölf Semestern oder mehr, also im Bereich der Regelstudienzeit des Medizinstudiums. Die Zumutbarkeit solcher Wartezeiten sowie deren ursprünglich angedachtes chancenausgleichendes Potential wird in Anbetracht dieser Werte bezweifelt (vgl. Bode 2013, 2015) und war ein Grund für die Verfassungsklage im Jahr 2017. Eine Hoffnung, die mit den in der AdH-Quote zur Anwendung kommenden Kriterien verknüpft wird,

20 Die Vorabquote umfasst folgende Personengruppen: Härtefälle, Personen mit Beruf im öffentlichen Bedarf, ausländische Staatsangehörige und Staatenlose, Studiengangswechsler, Personen im Zweitstudium und mit abgeschlossener Berufsausbildung.

21 Solange die Abiturnoten zwischen den Bundesländern eingeschränkt vergleichbar sind, werden Landesquoten zugrunde gelegt, wodurch nur Bewerber/innen, die ihre Hochschulzugangsberechtigung im selben Bundesland erworben haben, miteinander konkurrieren. 
ist, dass sich so eine besondere Motivation oder Eignung von Bewerber/innen identifizieren lässt, die in der hoch aggregierten DN nicht zum Ausdruck kommt (vgl. Lewin und Lischka 2004, Wissenschaftsrat 2004). ${ }^{22}$ Tatsächlich erfolgte durch den Nachweis weiterer Kriterien eine „Bonierung“ oder Relativierung der Note. Im WiSe 2014/15 wurden an zehn von 35 Hochschulen Bewerber/innen mit einer Abiturnote von 2,0 oder schlechter über die AdH-Quote zum Medizinstudium zugelassen (Bode 2015: 108). Es kam somit teilweise zu einem Austausch zwischen Bewerber/innen mit besserem und schlechterem Abiturdurchschnitt (vgl. Gentsch und Gold 2008, Reuter und Spoun 2008, Simmenroth-Nayda et al. 2014, Trost et al. 1998).

Mit der oben angesprochenen Änderung des Staatsvertrages, werden die Studienplätze für die bundesweit zulassungsbeschränkten Studienplätze seit dem SoSe 2020 nach folgenden Hauptquoten vergeben: Abiturbestenquote $(30 \%)$, Eignungsquote (10\%), AdH-Quote (60\%); die Wartezeitquote entfällt. Sowohl durch die Einführung der Eignungsquote als auch Änderungen der AdH-Quote erhalten schulnotenunabhängige Kriterien eine größere Relevanz. Insbesondere Studierfähigkeitstests sind seitdem im Aufwind, wie dem Auswahlkriterienkatalog entnommen werden kann. ${ }^{23}$

\section{Örtlich zulassungsbeschränkte Studiengänge}

Im Vergleich zu den gerade vorgestellten bundesweit zulassungsbeschränkten Studiengängen sind die Zulassungsmodalitäten für Studiengänge mit örtlichem NC (noch) stärker fragmentiert. Das vom BVerfG formulierte Gebot, bestehende Ausbildungskapazitäten voll auszunutzen, trifft prinzipiell auf alle Studiengänge an staatlichen Hochschulen zu. Eine einheitliche Kapazitätsermittlung sahen Kritiker/innen jedoch als Behinderung von Wettbewerb und Profilbildung, die ,faktisch an Bedeutung und grundsätzlich an Berechtigung verloren habe" (Wissenschaftsrat 2004: 54). Seit der Föderalismusreform 2006 kam es hier zu einer Liberalisierung. Regelungen für die Kapazitätsermittlung örtlich zulassungsbeschränkter Studiengänge liegen seitdem bei den Bundesländern (vgl. Weber 2010). Will eine Hochschule die Zulassung für einen Studiengang beschränken, muss dies in der Regel jährlich beim Landesministerium

22 Tatsächlich sind sich verschiedene Evaluationsstudien einig, dass die durchschnittliche Abiturnote der beste Einzelprädiktor für die Vorhersage des Studienerfolgs ist und daher berechtigterweise einen zentralen Stellenwert bei der Vergabe von Studienplätzen innehat. Ergänzend tragen auch Eignungstests zur Varianzaufklärung bei. Eher qualitativen Verfahren, wie Auswahlgesprächen oder Essays, wird hingegen eine eher geringe prognostische Validität zugesprochen (vgl. Hell et al. 2008, Nickolaus und Abele 2008, Tarazona 2006).

23 Vgl. https://www.hochschulstart.de/fileadmin/media/epaper/hilfe21-22/zeq_ws 21-22.pdf [letzter Zugriff: 06.08.2021] 
beantragt und eingeschränkte Kapazitäten nachgewiesen werden (vgl. Banscherus 2010).

Wurden Zulassungszahlen festgelegt, gilt das Hochschulzulassungsgesetz des jeweiligen Bundeslandes, das - oft in Anlehnung an den Staatsvertrag Quoten und erlaubte Auswahlverfahren innerhalb der AdH-Quote vorgibt. Baden-Württemberg galt hier lange Zeit als Vorreiter der Liberalisierung und räumte bereits 2003 seinen Hochschulen das Recht ein, 90\% ihrer Studierenden selbst auszuwählen. Mittlerweile folgen viele Bundesländer dieser Entwicklung (z.B. Hamburg, Niedersachsen). Die niedrigste zur Anwendung kommende AdH-Quote beträgt $60 \%$ (z.B. in Berlin oder Sachsen) [Stand 2021].

Neben der zentralen Vergabe der Studienplätze bundesweit zulassungsbeschränkter Studiengänge soll die SfH Hochschulen durch das sogenannte Dialogorientierte Serviceverfahren (DoSV) dabei unterstützen, die Vergabe örtlich zulassungsbeschränkter Studiengänge zu koordinieren, vorrangig um dem Problem von Mehrfachzulassung, unbesetzter Studienplätze und überbuchter Studiengänge entgegenzuwirken und die Planungssicherheit von Hochschulen und Bewerber/innen zu erhöhen (vgl. Bode 2013, Hüber und Kübler 2011). Trotz der genannten Vorzüge scheinen die Hochschulen bezüglich ihrer Teilnahmebereitschaft am DoSV eher zögerlich, was die Wirksamkeit des Verfahrens nach wie vor einschränkt: Im WiSe 2016/17 beteiligten sich insgesamt 103 Hochschulen mit 748 Studiengängen (circa 17\% des zulassungsbeschränkten grundständigen Studienangebots) (vgl. Preuß 2017). ${ }^{24}$ Für Studienbewerber/innen bedeutet das Nebeneinander von örtlich zulassungsbeschränkten Studiengängen, die das DoSV nutzen oder darauf verzichten, eine weitere Komplexitätssteigerung.

\section{Zulassungsfreie Studiengänge}

Für zulassungsfreie Studiengänge gibt es keine festgelegten Kapazitätsgrenzen, sodass sich zumindest theoretisch alle Studienberechtigten (mit allgemeiner Hochschulreife) für den Studiengang ihrer Wahl immatrikulieren können. ${ }^{25}$ Wie bereits erwähnt ist der Nachweis der Hochschulzugsberechtigung jedoch oft nicht die einzige Voraussetzung für das Studium eines zulassungsfreien Studiengangs. Dies ist immer dann der Fall, wenn Eignungsfeststellungsverfahren zur Anwendung kommen, die im Folgenden diskutiert werden.

24 Zum WiSe 2020/21 stiegen die Zahlen auf 143 Hochschulen und 1724 Studiengänge (https://www.hochschulstart.de/startseite/statistik [letzter Zugriff: 28.07. 2021])

25 Daraus kann nicht geschlossen werden, dass keine Selektion stattfindet. Diese ist jedoch vom „Tor“ ins Studium verschoben und kann durch Pflichtprüfungen im Nachhinein stattfinden. 
Eignungsfeststellungsverfahren

Unabhängig von der Frage nach (relativer) Zulassungsbeschränkung und Kapazitätsermittlung können (absolute) Eignungsfeststellungsverfahren als weiteres Mittel der Bewerber/innenauswahl durch die Hochschulen angesehen werden (vgl. Haug 2006: 96). Diskutiert wird dieses Instrument meist im $\mathrm{Zu}-$ sammenhang mit zulassungsfreien Studiengängen (vgl. Winter et al. 2012, Wissenschaftsrat 2004). Verlangt werden Eignungsnachweise jedoch zum Teil auch für die Zulassung zu örtlich zulassungsbeschränkten Studiengängen (z.B. Sprachnachweise bei sprachwissenschaftlichen Studiengängen).

Die grundlegende Idee von Zulassungsbeschränkungen ist, dass aufgrund von Kapazitätsbeschränkungen nur eine bestimmte Anzahl hochschulzugangsberechtiger Bewerber/innen für bestimmte Studiengänge zugelassen werden kann, um angemessene Studien- und Prüfungsbedingungen zu gewährleisten. Die Höchstzahl wird daher festgesetzt und alle prinzipiell hochschulzugangsberechtigten Bewerber/innen entsprechend der zur Anwendung kommenden Kriterien (und innerhalb der definierten Quoten) gerankt und ausgewählt. Eignungsfeststellungsverfahren basieren hingegen auf der Annahme, dass bestimmte Studiengänge eine spezifische Eignung voraussetzen, die nicht allein durch die Vorlage des (Fach-)Abiturzeugnisses feststellbar ist. Die damit verbundene Frage ist dann nicht mehr: Bis zu welcher Auswahlgrenze werden berechtigte Bewerber/innen ausgewählt? Sondern: Wer ist überhaupt zugangsberechtigt? Für Studiengänge im Bereich Kunst oder Sport ist die Eignungsfeststellung durch Vorspielen, Arbeitsproben, Sportprüfungen und ärztliche Atteste seit Langem fester (und rechtlich geregelter) Bestandteil der Studienzulassung (vgl. Wolter 2001).

Über diese speziellen Fächer hinaus ist die Frage nach Angemessenheit und Zulässigkeit von Eignungsfeststellungsverfahren bildungspolitisch und -rechtlich kaum geklärt (vgl. Bode 2015: 30, Wolter 2008: 32). Die Debatte kreist dabei vordergründig ,um die Frage der allgemeinen Studierfähigkeit, die mit dem Besuch der gymnasialen Oberstufe erlangt werden soll, und die Frage, inwiefern das Abitur als einheitlicher, vergleichbarer Indikator ein Anhaltspunkt für die Eignung zum Studium aller Studienfächer sein kann“ (Täger 2010: 51, Hervorhebung: CF). In Anbetracht der Heterogenisierung der Hochschulzugangsberechtigten und Diversifizierung des Studienangebots wird „diese Funktion des Abiturs [...] jedoch von einigen Vertretern aus Hochschule und Wirtschaft mit Skepsis betrachtet" (Tarazona 2006: 69). So spricht sich der Wissenschaftsrat (2004: 56f.) in seinen Empfehlungen zur Reform des Hochschulzugangs explizit dafür aus, dass Hochschulen auch in zulassungsfreien Studiengängen die Möglichkeit erhalten sollten, ,durch Eignungsfeststellungsverfahren bereits vor Studienbeginn vertiefte Kenntnisse über Motivation, Qualifikationsprofil und -niveau von Studierwilligen zu gewinnen“. Diese Kenntnisse sollten zum einen als „Grundlage für eine vertiefte Studien- 
beratung" genutzt werden und somit eine informierte Studienwahl seitens der Bewerber/innen ermöglichen. Zum anderen sollten Hochschulen aber auch die Möglichkeit haben, Eignungsfeststellungsverfahren ,in Fällen, in denen dies sinnvoll ist“, als Selektionskriterium zu nutzen (für noch explizitere Forderungen siehe Frankenberg 2008).

Über die gesondert geregelten Zulassungsvoraussetzungen im Bereich Sport und Kunst hinaus gewähren fast alle Landeshochschulgesetze die Möglichkeit, Eignungsfeststellungsverfahren für die Zulassung zu Studiengängen mit „besonderen qualitativen Anforderungen“ (Bayrisches Hochschulgesetz, Abschnitt III, Art. 44) anzuwenden, oft nach Antrag und Genehmigung und „,jenseits kapazitärer Notwendigkeiten“ (Winter et al. 2012: 143). Wie stark die Anwendung von Eignungsfeststellungsverfahren reguliert ist, d.h. ob sie nur für bestimmte Fächer gestattet ist (z.B. Brandenburg: neben Kunst und Sport nur Sprachwissenschaften) oder ob erlaubte Kriterien festgelegt werden (z.B. Bayern oder Thüringen), unterscheidet sich beträchtlich zwischen den Bundesländern.

$\mathrm{Ob}$ eine flächendeckende Einführung von Eignungsfeststellungsverfahren mit der grundgesetzlich verankerten Freiheit der Berufswahl und Wahl der Ausbildungsstätte vereinbar ist, ist fraglich (Bode 2015: 29), da dies eine ,,vollständige Verriegelung des Hochschulzugangs, unabhängig von Kapazitätsfragen“ (Wolter 2008: 32) bedeuten würde. Fest steht, dass das Abitur als „Nachweis für Berechtigung und Befähigung" (Täger 2010: 53, Hervorhebung im Original) die zuletzt genannte Funktion einbüßen würde und dies zur Zeit bereits in Teilen tut.

Mit der beschriebenen Entwicklung des Zulassungsrechts wird die Stellung von Hochschulen als Gatekeeper zunehmend institutionalisiert. Zulassungsbeschränkungen sind zwar nur unter Nutzung der zur Verfügung stehenden Kapazitäten zulässig, sodass zumindest den staatlichen Hochschulen hier bezüglich ihres Selbstauswahlrechts Grenzen gesetzt sind (vgl. Kreckel 2015). Jedoch können die Hochschulen durch die Möglichkeit der Eignungsfeststellung auch ohne Kapazitätsbeschränkung Einfluss auf die Bewerber/innenauswahl nehmen.

\subsubsection{Die Anwendung von Zulassungsbeschränkung und Eignungsfeststellung durch Hochschulen}

Im Folgenden soll beschrieben werden, inwiefern Hochschulen von den Möglichkeiten der Auswahl und Eignungsfeststellung Gebrauch machen. Hierfür werden zunächst verfügbare Publikationen und Datenquellen herangezogen, insbesondere der HRK und des DZHW. Wie ich zeigen werde, weisen diese Publikationen einige Lücken auf, die durch die Beschreibung der im Rahmen des Best Up-Projektes gesammelten Daten zu Auswahlverfahren an deutschen 
Hochschulen (im Folgenden: „Rekrutierungsdaten“) (für detaillierte Informationen siehe Ehlert et al. 2017b, Finger et al. 2018 und Abschnitt 6.2.2) ergänzt werden sollen. Diese Daten stehen in leicht angepasster Form zum Download zur Verfügung: https://doi.org/10.7802/2292 (Finger 2021).

\section{Informationen aus existierenden Datenquellen (HRK, DZHW)}

Die HRK pflegt mit dem Hochschulkompass eine Datenbank, die laut eigener Angabe das gesamte Studienangebot an deutschen, staatlich anerkannten Hochschulen umfasst und hierzu Informationen für Studieninteressierte bereitstellt. Die nötigen Angaben werden von den Hochschulen selbst eingepflegt. In der seit 2007 jährlich erscheinenden Veröffentlichung „Statistische Daten zu Studienangeboten an Hochschulen in Deutschland“" werden diese Informationen zusammenfassend dargestellt (siehe Anhangstabelle A2.1). Hier zeigt sich, dass der Anteil örtlich zulassungsbeschränkter Studiengänge bis zum WiSe 2013/14 bei circa 50\% lag; in den Folgejahren kann eine abnehmende Tendenz beobachtet werden (WiSe 2016/17: 44\%, WiSe 2020/21: 40\% ${ }^{26}$ ). Bundesweit zulassungsbeschränkte Studiengänge machen mit etwa 1-2\% nur einen geringen Anteil am gesamtdeutschen Studienangebot aus. Zudem kann eine starke Variation nach Bundesländern beobachtet werden. Studiengänge an Hochschulen der neuen Bundesländer sind im Durchschnitt seltener zulassungsbeschränkt (geringster Anteil: Thüringen mit 10 bis 25\%). Aber auch Bayern hat einen vergleichsweise geringen Anteil örtlicher Zulassungsbeschränkungen (28 bis 34\%). Vor allem in den Stadtstaaten unterliegt das Studienangebot - bis auf einige Schwankungen - überdurchschnittlichen Zulassungsbeschränkungen mit einem Höchstwert von 84,3\% in Hamburg im WiSe 2013/14. In Berlin sank der Anteil örtlich zulassungsbeschränkter Studiengänge: von über $80 \%$ im WiSe 2007/08 auf 50\% 2016/17, stieg danach aber wieder an auf 69\% im WiSe 2020/21. Der „Numerus Clausus-Check“ des Cen-

26 Die veröffentlichten Werte ab dem WiSe 2017/18 sind nur noch bedingt vergleichbar mit den Vorjahreswerten, da die Kategorisierung der Zulassungsmodi im Hochschulkompass geändert wurden: „Der Zulassungsmodus ,örtliche Zulassungsbeschränkung mit NC' wurde enger gefasst und bezieht sich ausschließlich auf Studiengänge der Hochschulen mit örtlicher Zulassungsbeschränkung, die dem Kapazitätsausschöpfungsgebot unterliegen. Der neu eingeführte Zulassungsmodus ,Auswahlverfahren/Eignungsprüfung' bezieht sich auf zulassungsbeschränkte Studiengänge, für die der Numerus Clausus (NC) weder örtlich noch bundesweit verbindlich ist" (https://www.hrk.de/fileadmin/redaktion/hrk/02-Dokumente/0203-Studium/02-03-01-Studium-Studienreform/HRK_Statistik_BA_MA_UEbrige

WiSe_2020_21_finale.pdf [letzter Zugriff: 02.08.2021]). Der im Text genannte Wert für $202 \overline{1}$ von $40 \%$ ergibt sich aus der Summe örtlich zulassungsbeschränkter Studiengänge (33,3\%) und solchen mit Eignungsprüfung (6,6\%). Anhangstabelle A2.1 schließt aus Vergleichsgründen nur Werte bis 2016/17 ein. 
trums für Hochschulentwicklung (CHE) beruht auf denselben Daten, differenziert aber zusätzlich nach groben Fächergruppen. Im WiSe 2016/17 lag die Varianz hier bei 20 Prozentpunkten (38\% Sprach- und Kulturwissenschaften, $58 \%$ Rechts-, Wirtschafts- und Sozialwissenschaften) (Hachmeister et al. 2016: 23, siehe Gehlke et al. 2019a für sehr ähnliche Unterschiede im WiSe 2019/20).

Noch 2010 stellte Banscherus (2010: 43f.) fest, dass „die Einschränkung des individuellen Rechts zur Aufnahme eines Studiums vom Ausnahme- zum Regelfall geworden ist". Mit Blick auf den abnehmenden Anteil zulassungsbeschränkter Studiengänge kann diese Aussage zunächst relativiert werden. Allerdings umfassten die Daten der HRK zumindest bis zum Wintersemester 2017/18 keine Angaben zu Eignungsfeststellungsverfahren, ${ }^{27}$ sodass auf deren Grundlage nur ein Teil der „Einschränkung des individuellen Rechts zur Aufnahme eines Studiums“" erfasst werden konnte. Zudem liegt mit der Angabe zu Zulassungsbeschränkungen noch keine Auskunft darüber vor, welche Verfahren konkret angewendet werden. Hierzu gibt eine mittlerweile ältere Studie des DZHW aus dem Jahre 2005 Auskunft (Heine et al. 2006). Es handelt sich hierbei um eine Bestandsaufnahme der zur Anwendung kommenden Auswahl- und Eignungsfeststellungsverfahren für die Zulassung zu 7.989 grundständigen Studiengängen an insgesamt 287 deutschen Hochschulen. Nicht klar differenziert wird allerdings, welche der Verfahren zur Erstellung der Rangliste der Bewerber/innen und welche zur Prüfung der Eignung genutzt wurden.

Im Studienjahr 2005 wendeten 3.888, also fast 50\% der Studiengänge Auswahl- oder Eignungsfeststellungsverfahren an; weitere 325 hatten dies fest geplant. $\mathrm{Zu}$ beobachten waren deutliche Unterschiede zwischen den Studienfächern mit überdurchschnittlich häufigem Rückgriff auf Auswahlverfahren im Bereich Medizin (88\%), Kunst (77\%), Sport (71\%) und Rechts-/Wirtschafts-/ Sozialwissenschaften (69\%). In den Bereichen Ingenieurswissenschaften und Sprach-/Kulturwissenschaften machten hingegen nur 39 bzw. 37\% der Studiengänge von solchen Verfahren Gebrauch. Dies spiegelt laut Heine et al. (2006: 36) sowohl das Angebots-Nachfrage-Verhältnis als auch eine spezifische Auswahltradition in Fächern wider, die besondere „Begabungen“ voraussetzen (etwa Kunst, Sport). Insgesamt identifiziert die Studie 26 Kriterien, die zur Anwendung kamen; davon jedoch nur sechs bei mindestens 5\% der Studiengänge: Abiturnote (82\%), Wartezeit (37\%), Nachweis Berufserfahrung (21\%), praktische Eignungsprüfung (19\%), Auswahlgespräch (8\%) und Motivationsschreiben (5\%). Zudem wurden 203 Kombinationen von Einzelkriterien identifiziert.

27 Zuvor enthielt die Kategorie „zulassungsfrei“ den Hinweis auf möglicherweise stattfindende „Anmelde- oder Auswahlverfahren“. Die in dieser Arbeit betrachteten Berliner Studienberechtigten (Abschlussjahrgang 2014) konnten von der aussagekräftigeren Kategorisierung entsprechend noch keinen Gebrauch machen. 
Einen etwas aktuelleren Überblick, allerdings aus der Perspektive von Studierenden, liefern zudem die Studienanfänger/innenbefragungen des DZHW. Es handelt sich hierbei um eine repräsentative Zeitreihenbefragung von Studierenden im ersten Semester an deutschen Universitäten und Fachhochschulen, die bis zum WiSe 2011/12 im 2-3 Jahresturnus durchgeführt wurde; zuletzt nahmen 7.638 Studierende an 60 Hochschulen teil (vgl. Scheller et al. 2013).

Abb. 2.1: Anteil Erstsemester mit Bewerbung für ihr derzeitiges Studienfach (in \%)

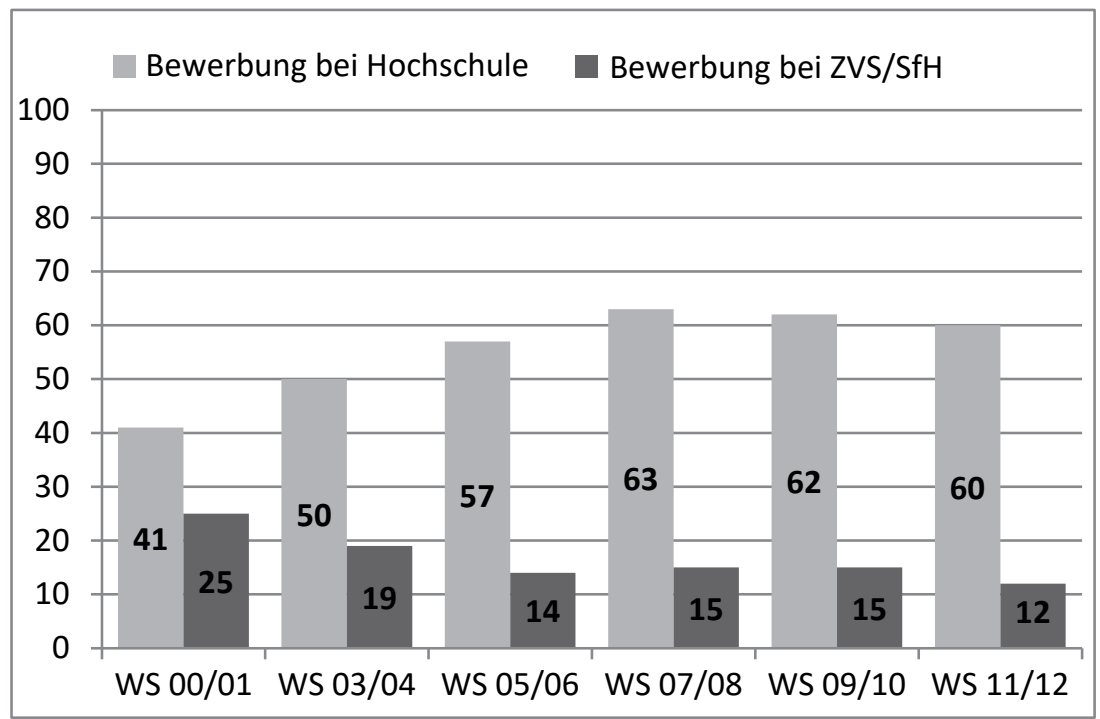

Quelle: DZHW Studienanfänger/innenbefragung; Scheller et al. (2013: 68); eigene Darstellung

Die Befragten gaben an, ob sie sich bei der ZVS/SfH oder direkt an den Hochschulen für ihr jetziges Studienfach bewerben mussten. Abbildung 2.1 gibt einen Überblick über die Ergebnisse: Der Anteil an Erstsemestern, die sich direkt bei den Hochschulen bewerben mussten, stieg zunächst bis zum WiSe 2007/08 auf $63 \%$ an und ist seitdem leicht rückläufig. Der Anteil an Befragten, die sich über die ZVS bewarben, nahm deutlich ab und lag im WiSe 2011/12 noch bei $12 \%{ }^{28}$ Die Fächervarianz ist dabei beträchtlich: Der Anteil an Studienanfän-

28 Dies liegt zum einen daran, dass Biologie und Psychologie nur bis 2008/09 bzw. 2009/2010 zentral vergeben wurden. Zudem nutzten zwischenzeitlich einzelne Bundesländer die ZVS auch für die Unterstützung bei der Vergabe örtlich zulassungsbeschränkter Studiengänge. Die in Abbildung 2.1 gezeigten Anteile beziehen sich damit nicht ausschließlich auf bundesweit zulassungsbeschränkte Studiengänge. 
ger/innen im WiSe 2011/12, die sich für ihr aktuelles Studienfach bewerben mussten (beide Bewerbungswege), liegt bei über 90\% in den Fachbereichen Medizin, Wirtschafts- und Sozialwissenschaften und Kunst, jedoch bei unter $60 \%$ in den natur- und ingenieurswissenschaftlichen Studiengängen (Scheller et al. 2013: 69).

Zudem wurde erhoben, ob die Studienanfänger/innen an spezifischen Auswahlverfahren (Bewerbungsschreiben, Auswahlgespräch, Fremdsprachentest, praktische Eignungsprüfung, Mappe etc.) teilnehmen mussten. Der Anteil an Befragten, die dies bejahten, stieg von 7\% im WiSe 2003/2004 auf 14\% im WiSe 2008/09 und sank danach wieder etwas auf 10\% im WiSe 2011/12.

Eine Veröffentlichung vom Institut für Hochschulforschung (HoF) setzt sich explizit mit Eignungsfeststellungsverfahren zulassungsfreier Studiengänge auseinander (Winter et al. 2012). Die Ergebnisse beziehen sich auf 20 Hochschulen in vier Bundesländern (Hessen, Niedersachsen, Rheinland-Pfalz und Sachsen-Anhalt). Von den 450 hier im WiSe 2011/12 angesiedelten zulassungsfreien Studiengängen (knapp 50\% aller Studiengänge) wendete fast die Hälfte Eignungsfeststellungsverfahren an. ,Tatsächlich ,hürdenfrei‘ ist der Zugang zur ersten Studienphase demnach [...] lediglich in einem Viertel $(26 \%)$ aller grundständigen Studienangebote“ (ebd.: 132). Am häufigsten wird dabei der Nachweis von Sprachkenntnissen gefordert (63\%), gefolgt von Testverfahren (27\%) und Praktika oder Berufserfahrung (19\%). Die Abiturnote, Fachnoten und Auswahlgespräche kommen mit jeweils etwa 3\% hingegen seltener zum Einsatz. Die Autor/innen beobachten weiterhin, dass teilweise nicht alle Studienberechtigten am Eignungsfeststellungsverfahren teilnehmen dürfen, sondern zum Beispiel nur solche mit einer bestimmten Abiturnote. Damit ist „bereits die Teilnahme am Auswahltest ein Ereignis [...], das selektiven Maßstäben folgt und daher mit Beschränkungen versehen ist." (ebd.: 144)

Diese Quellen geben einen Eindruck zu den Zulassungsbedingungen der letzten Jahre, weisen jedoch einige Leerstellen auf, die eine umfassende Beurteilung der Zulassungsbedingungen, mit denen Studienberechtigte konfrontiert sind, erschweren. Zum einen stellt sich die Frage nach aktuelleren Informationen zu konkreten Auswahlverfahren. Zum anderen liegen keine Informationen zu den Auswahlgrenzen bei zulassungsbeschränkten Studiengängen vor, die Hinweise zur Selektivität der Studiengänge liefern können und eine wichtige wenn auch nicht perfekte - Orientierungshilfe für Studienberechtigte darstellen. Die im Zuge des Berliner-Studienberechtigten-Panels (Best Up) erhobenen Rekrutierungsdaten werden daher im Folgenden ergänzend herangezogen. Diese enthalten sehr detaillierte Informationen zu Zulassungsbeschränkungen, Auswahl- und Eignungsfeststellungsverfahren und enthalten auch erstmalig genaue Angaben zu Auswahlgrenzen und gehen damit über den Informationsgehalt existierender Datenquellen hinaus. 


\section{Informationen aus den Best Up-Rekrutierungsdaten}

Bei den Rekrutierungsdaten handelt es sich um institutionelle Daten zu Zulassungsbeschränkungen, Auswahlgrenzen sowie Auswahl- und Eignungsfeststellungsverfahren für 391 (WiSe 2013/14), 444 (WiSe 2014/15) und 171 (WiSe 2015/16) Studiengänge (vgl. Ehlert et al. 2017b, Finger et al. 2016). Die Auswahl der Studiengänge basiert auf den Angaben der Best Up-Befragten. Es ist daher zu beachten, dass es sich nicht um einen repräsentativen Ausschnitt der deutschen oder Berliner Studiengänge handelt.$^{29}$ Detaillierte Informationen finden sich in Kapitel 6.2.2. An dieser Stelle sollen die recherchierten Informationen beschrieben werden, da diese einen detaillierteren und aktuelleren Einblick in die Zulassungsbedingungen ausgewählter Studiengänge gewähren. Dies geschieht hier exemplarisch für das WiSe 2014/15.

Abbildung 2.2 zeigt den Anteil an recherchierten Studiengängen, die Eignungsfeststellungsverfahren, Zulassungsbeschränkungen oder mindestens eines von beiden im WiSe 2014/15 anwendeten, differenziert nach Standort, Hochschultyp und grober Fächergruppe. Insgesamt wenden knapp 30\% der Studiengänge ein Eignungsfeststellungsverfahren an, und insgesamt sind etwas mehr als $70 \%$ zulassungsbeschränkt. ${ }^{30}$ In den oben zitierten Analysen von Winter et al. (2012) konnten sich Studienberechtigte ohne weiteren Nachweis nur in ein Viertel aller betrachteten Studiengänge einschreiben. In den im Rahmen des Best Up-Projektes recherchierten Daten liegt dieser Anteil sogar nur bei $15 \%$. Während Studiengänge an Universitäten häufig zulassungsbeschränkt sind, greifen FHs eher auf Eignungsfeststellungsverfahren zurück.

29 Ein Vergleich mit den im Hochschulkompass gelisteten Studiengängen [Stand April 2017] zeigt, dass Studiengänge an Berliner Hochschulen, an Universitäten und an privaten Hochschulen, in den Rekrutierungsdaten überrepräsentiert sind. Innerhalb Berlins kommen Studiengänge an Universitäten im Vergleich zu Fachhochschulen deutlich häufiger vor. Mit besonderer Vorsicht sind die Angaben zu Studiengängen außerhalb Berlins zu interpretieren, da diese weniger als 5\% des entsprechenden Studienangebots widerspiegeln.

30 Der Anteil an zulassungsbeschränkten Studiengängen ist in den Rekrutierungsdaten deutlich höher als in den oben berichteten HRK-Daten. Ein Grund hierfür ist sicherlich die Auswahl der Studiengänge, die sich an den Präferenzen der Befragten orientierte (vgl. Abschnitt 6.2.2). Daher sind in den Rekrutierungsdaten begehrte Studiengänge vermutlich überrepräsentiert, die möglicherweise gerade aufgrund einer höheren Nachfrage häufiger zulassungsbeschränkt sind. 


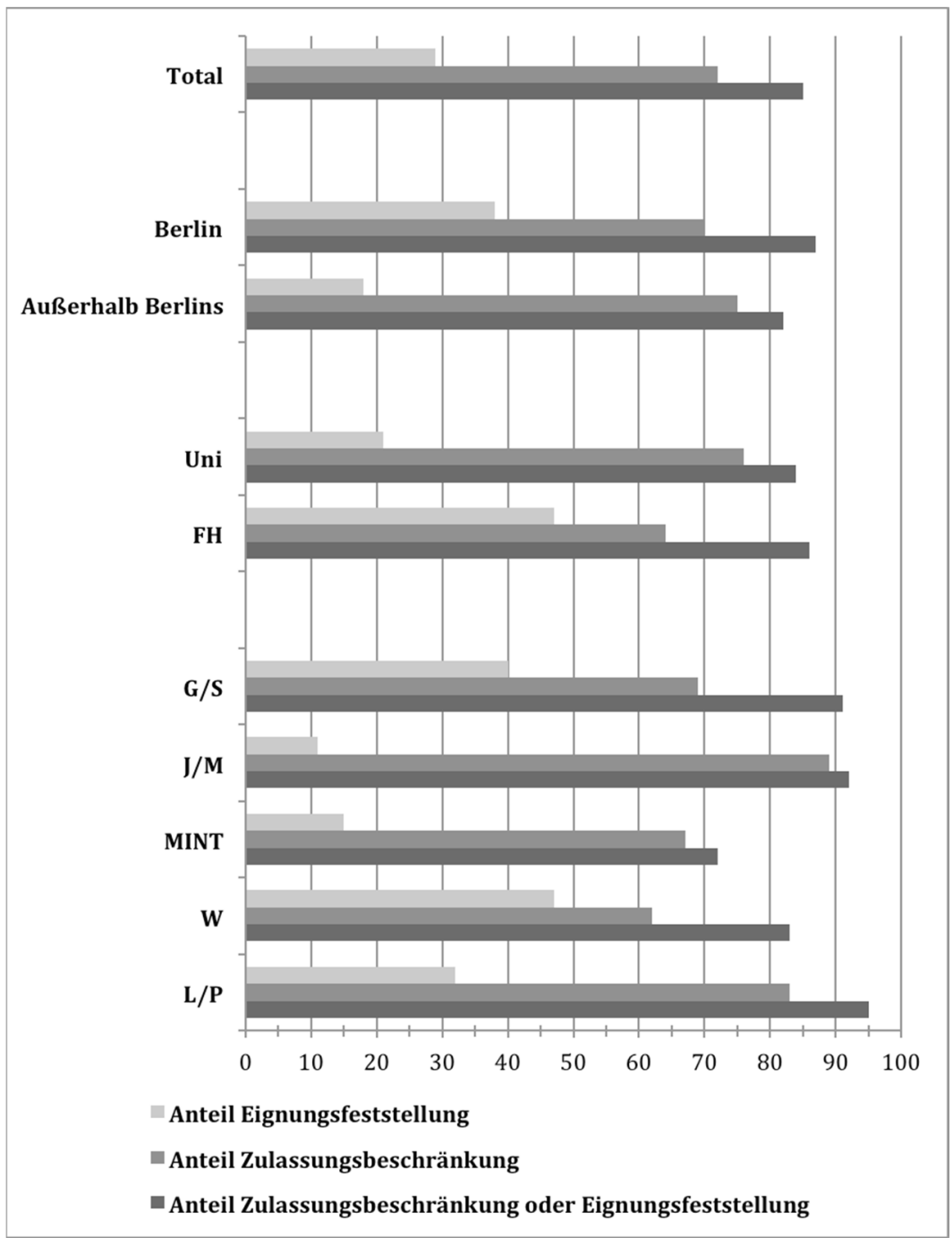

Quelle: Best Up-Rekrutierungsdaten, eigene Berechnungen

Fallzahlen: Total: 444; Berlin: 236, Außerhalb Berlins: 208; Uni (inklusive Kunst- und Musikhochschulen): 315; FH: 129; Geistes- und Sozialwissenschaften, Kunst (G/S): 126; Jura und Medizin (J/M): 53; Mathematik, Natur- und Ingenieurswissenschaften (MINT): 132; Wirtschaftswissenschaften (W): 58 ; Lehramt, Pädagogik und Erziehungswissenschaften (L/P): 60. Kategorie „Sonstige“ (N: 15) nicht ausgewiesen. 
Ein deutlicher Unterschied existiert zudem bezüglich der Fächergruppe. Eignungsfeststellungsverfahren kommen in den Rekrutierungsdaten relativ häufig in den Geistes-/Sozialwissenschaften, den Wirtschaftswissenschaften sowie Pädagogik- und Lehramtsstudiengängen vor. Letztere sind zudem ähnlich häufig zulassungsbeschränkt wie die Fächergruppen Medizin/Jura. Bemerkenswert ist, dass man sich in nur 5\% der recherchierten Lehramtsstudiengänge und weniger als 10\% der Studiengänge der Geistes- und Sozialwissenschaften „einfach einschreiben" kann.

Auch wenn nochmals betont werden muss, dass diese Daten nicht repräsentativ sind, liefern sie deutliche Hinweise darauf, dass eine alleinige Betrachtung von Zulassungsbeschränkung über weitere Zulassungshürden hinwegtäuschen kann, und stützen somit die Ergebnisse von Winter et al. (2012).

Wie bereits diskutiert, wird hieraus nicht ersichtlich, wie selektiv zulassungsbeschränkte Studiengänge sind. Eine Zulassungsbeschränkung an sich bedeutet noch nicht, dass ,schlechtere“ Abiturient/innen eine geringe Zulassungschance haben und stellt daher nur ein sehr grobes Maß für Zulassungshürden dar. Abbildung 2.3 zeigt die durchschnittliche Auswahlgrenze in der Abiturbestenquote, d.h. die Durchschnittsnote des/der zuletzt zugelassenen Bewerber/in.

Wenn die Nachfrage nach einem Studiengang dessen Angebot an Plätzen nicht übersteigt, werden trotz Zulassungsbeschränkung alle Bewerber/innen zugelassen. Dies war im WiSe 2014/15 bei etwa 20\% der recherchierten Studiengänge der Fall. In diesem Fall wird keine Auswahlgrenze ausgewiesen. Für diese Studiengänge wurde die nötige Abiturnote auf 4,0 (=bestanden) gesetzt. Im Durchschnitt lag die Auswahlgrenze im WiSe 2014/15 bei einem Notendurchschnitt von 1,9. Zum Vergleich erzielen Abiturient/innen im Mittel eine Abiturnote von 2,2 (Schneider und Franke 2014: 55). Unterschiede nach Standort und Hochschultyp sind gering. Deutliche Unterschiede lassen sich allerdings erneut bezüglich der Fachgruppe beobachten: Studiengänge im Bereich Medizin/Jura sind mit einer durchschnittlichen Auswahlgrenze in der Abiturbestenquote von 1,3 wenig überraschend mit Abstand am selektivsten. Die recherchierten Lehramtsstudiengänge sind zwar häufig zulassungsbeschränkt, dafür aber weniger leistungsselektiv. Deutlich wird auch, dass der Umstand, dass einige Studiengänge trotz Beschränkung aufgrund einer zu geringen Nachfrage (sowie Verteilungsproblemen aufgrund von Mehrfachzulassungen) alle Bewerber/innen zulassen müssen, Zugangsbarrieren insgesamt reduziert. Dies ist wiederum für die Fächergruppen Medizin/Jura am seltensten der Fall. 


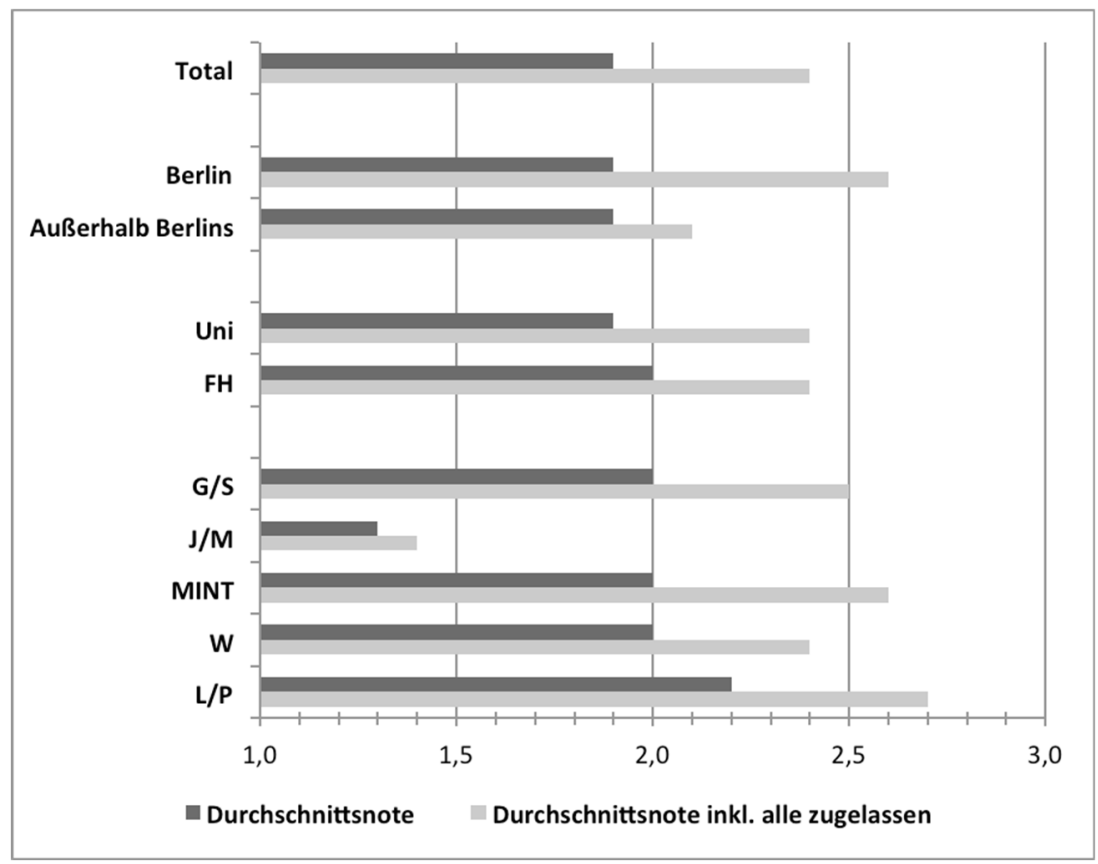

Quelle: Best Up-Rekrutierungsdaten, eigene Berechnungen.

Fallzahlen: Total: 444; Berlin: 236, Außerhalb Berlins: 208; Uni (inklusive Kunst- und Musikhochschulen): 315; FH: 129; Geistes- und Sozialwissenschaften, Kunst (G/S): 126; Jura und Medizin (J/M): 53; Mathematik, Natur- und Ingenieurswissenschaften (MINT): 132; Wirtschaftswissenschaften (W): 58 ; Lehramt, Pädagogik und Erziehungswissenschaften (L/P): 60. Kategorie "Sonstige“ (N: 15) nicht ausgewiesen.

Angaben basieren auf grundständigen, zulassungsbeschränkten Studiengängen mit Abiturbestenquote. Werden alle Bewerber/innen zugelassen, wird keine Auswahlgrenze ausgewiesen. Solchen Studiengängen wurde der Wert 4,0 (bestanden) zugewiesen (graue Balken). Dadurch erhöhen sich die Fallzahl sowie die durchschnittliche DN. Zudem war für einige Studiengänge keine Auswahlgrenze recherchierbar (N: 37). Diese wurden hier nicht berücksichtigt.

Total: 195 (inkl. alle zugelassen: 255); Berlin: 91 (137), Außerhalb Berlins: 104 (118); Uni (inklusive Kunst- und Musikhochschulen): 160 (212); FH: 35 (43); Geistes- und Sozialwissenschaften, Kunst (G/S): 54 (74); Jura und Medizin (J/M): 41 (42); Mathematik, Natur- und Ingenieurswissenschaften (MINT): 43 (64); Wirtschaftswissenschaften (W): 17 (21); Lehramt, Pädagogik und Erziehungswissenschaften (L/P): 31 (43). Kategorie „Sonstige“ (N: 9/11) nicht ausgewiesen.

Ein weiteres Kriterium für die Selektivität von Studiengängen ist die Auswahlgrenze in der Wartezeitquote, die angibt, wie viele Halbjahre der Erhalt der Studienberechtigung des/der zuletzt zugelassenen Bewerber/in zurückliegt. Wurden alle zugelassen, wurde die Wartezeit hier auf 0 gesetzt. Wie Abbildung 2.4 zeigt, lag die Auswahlgrenze im Durchschnitt bei 7 Halbjahren. Deut- 
lich höher bei Fachhochschulen (10 Halbjahre) $)^{31}$ und in den Fachgruppen Jura/Medizin (11 Halbjahre).

Abb. 2.4: durchschnittliche Auswahlgrenze in der Wartezeitquote, WiSe 2014/15

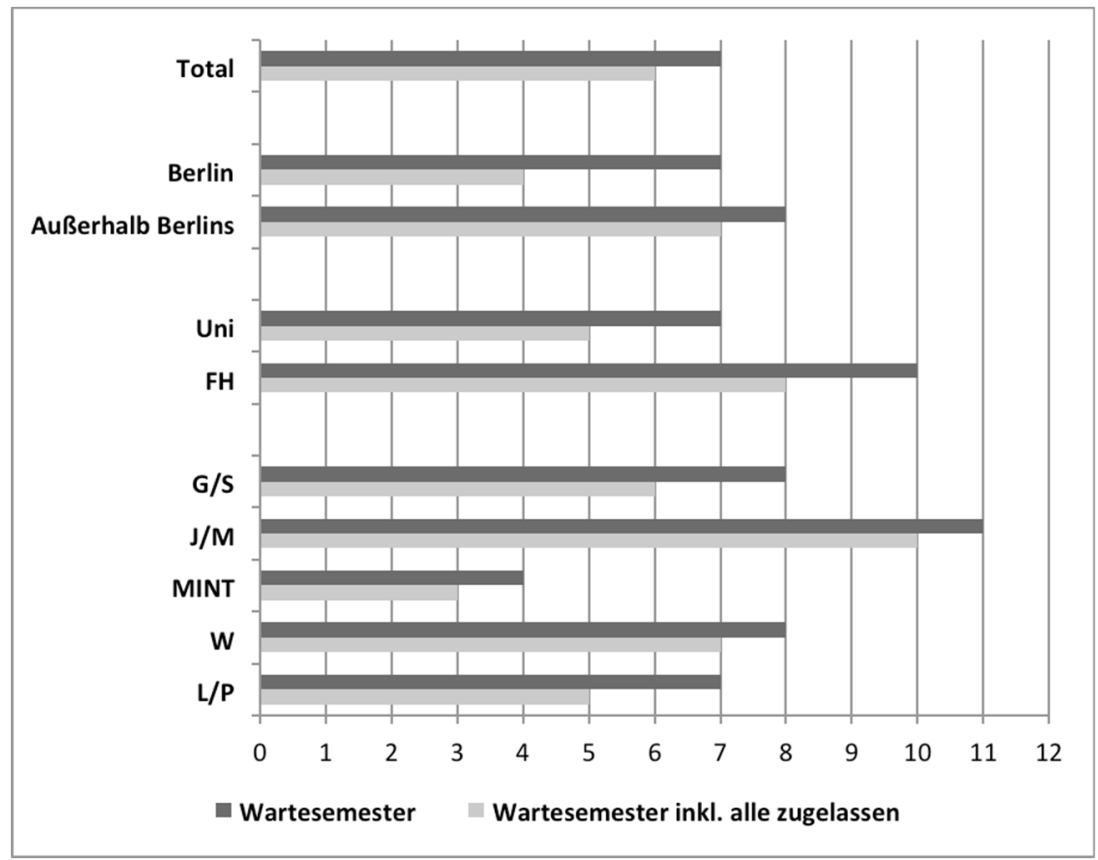

Quelle: Best Up-Rekrutierungsdaten, eigene Berechnungen

Angaben basieren auf grundständigen, zulassungsbeschränkten Studiengängen mit Wartezeitquote. Werden alle Bewerber/innen zugelassen, wird keine Auswahlgrenze ausgewiesen. Solchen Studiengängen wurde der Wert 0 zugewiesen (graue Balken). Dadurch erhöht sich die Fallzahl, und die durchschnittliche WZ nimmt ab. Zudem war für einige Studiengänge keine WZ-Grenze recherchierbar (N:50). Diese wurden hier nicht berücksichtigt.

Total: 203 (inkl. alle zugelassen: 263); Berlin: 91 (137), Außerhalb Berlins: 112 (126); Uni (inklusive Kunst- und Musikhochschulen): 166 (218); FH: 37 (45); Geistes- und Sozialwissenschaften, Kunst (G/S): 55 (75); Jura und Medizin (J/M): 42 (43); Mathematik, Natur- und Ingenieurswissenschaften (MINT): 48 (69); Wirtschaftswissenschaften (W): 17 (21); Lehramt, Pädagogik und Erziehungswissenschaften (L/P): 32 (44). Kategorie „Sonstige“ (N: 9/11) nicht ausgewiesen.

31 Dies könnte daran liegen, dass Fachhochschulen möglicherweise besonders attraktiv für bereits beruflich gebildete Studienberechtigte sind, die nach dem (Fach)Abitur eine Ausbildung absolvieren und im Anschluss in einem verwandten Bereich studieren. Die während der Ausbildung gesammelten Wartesemester treiben dann die Auswahlgrenze nach oben. 
Abbildung 2.5 gibt einen Überblick über die zur Anwendung kommenden Verfahren und Kriterien für die Bewerber/innenauswahl in den recherchierten zulassungsbeschränkten Studiengängen. Berücksichtigt wurden dabei alle Hauptquoten. Wie vom Gesetzgeber gewollt, greifen fast alle Studiengänge auf die Abiturnote zurück. Fast genauso häufig wird die Wartezeit als Kriterium genutzt. Diese hohen Anteile kommen auch dadurch zustande, dass sowohl Abiturnote als auch Wartezeit aufgrund der Quotenregelung in den meisten Bundesländern „,eigenständige“ Kriterien sind (Bode 2013: 366).

Alle weiteren Kriterien können als ,uneigenständige Kriterien“ verstanden werden, mit deren Hilfe die Bewerber/innenrangliste in der AdH-Quote erstellt wird (auch hier kommt die Abiturnote häufig zur Anwendung). Das am häufigsten zum Einsatz kommende ,uneigenständige Kriterium“ ist Berufserfahrung (50\%) gefolgt von Fachnoten (25\%). Weitere Kriterien und Verfahren wie Fremdsprachenkenntnisse, Tests oder Auswahlgespräche werden nur von einem geringen Anteil der Studiengänge verwendet. Auch hier spiegelt sich die bereits festgestellte Tendenz hin zu eher standardisierten Verfahren wider.

Wie in Abbildung 2.2 gezeigt, wenden etwa 30\% der recherchierten Studiengänge Eignungsfeststellungsverfahren an. Abbildung 2.6 kann entnommen werden, um welche Verfahren oder Kriterien es sich hierbei handelt. Insgesamt werden Fremdsprachenkenntnisse am häufigsten vorausgesetzt (46\%). Zudem müssen Bewerber/innen häufig an Tests teilnehmen, um ihre Eignung unter Beweis zu stellen (43\%). Berufserfahrung ist in 24\% und ein Motivationsschreiben, eine Mappe mit Arbeitsproben oder Ähnliches in $22 \%$ der Studiengänge Bestandteil der Eignungsfeststellung. Auswahlgespräche finden nur in $10 \%$ der recherchierten Fälle statt. An Universitäten und in den Fachbereichen Geistes- und Sozialwissenschaften, Kunst, Wirtschaftswissenschaften und Lehramt/Pädagogik scheint der Nachweis von Fremdsprachenkenntnissen häufig als notwendig angesehen zu werden. Berufserfahrungen (meist in Form eines mehrwöchigen Praktikums vor Studienbeginn) scheinen hingegen besonders relevant für ein Studium in der Fächergruppe MINT und an FHs. 
Abb. 2.5: Auswahlkriterien und -verfahren in zulassungsbeschränkten Studiengängen (in \%), WiSe 2014/15

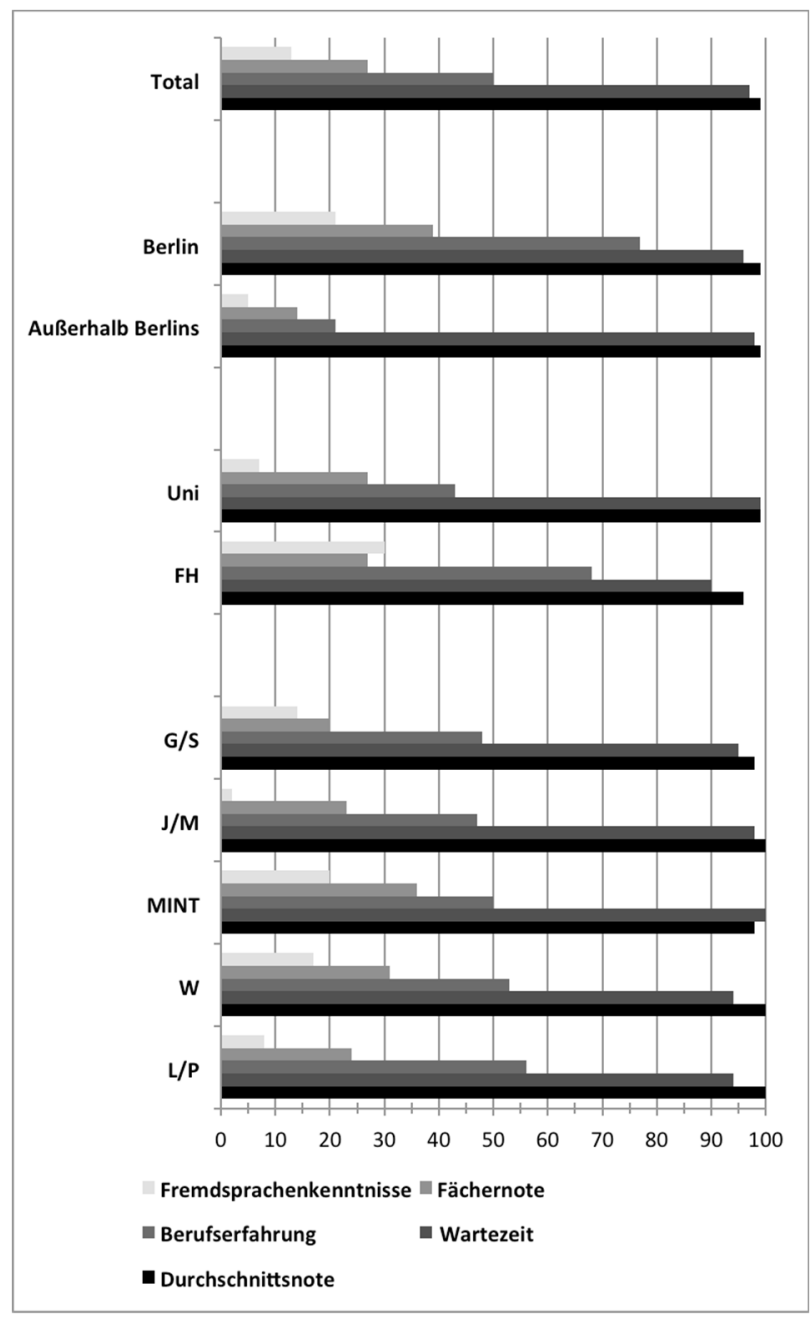

Quelle: Best Up-Rekrutierungsdaten (zulassungsbeschränkte Studiengänge), eigene Berechnungen Fallzahlen: Total: 320; Berlin: 165, Außerhalb Berlins: 155; Uni (inklusive Kunst- und Musikhochschulen): 238; FH: 82; Geistes- und Sozialwissenschaften, Kunst (G/S): 87; Jura und Medizin (J/M): 47; Mathematik, Natur- und Ingenieurswissenschaften (MINT): 88; Wirtschaftswissenschaften (W): 36; Lehramt, Pädagogik und Erziehungswissenschaften (L/P): 50. Kategorie „Sonstige“ (N: 12) nicht ausgewiesen.

Folgende Auswahlkategorie nicht abgetragen: Studienvorbereitender Kurs (N: 42/13\%), Test (N: $21 / 7 \%)$, Auswahlgespräch (N: 8/3\%), Sonstige (N: 13/4\%) 
Abb. 2.6: Verfahren zur Eignungsfeststellung (in \%), WiSe 2014/15

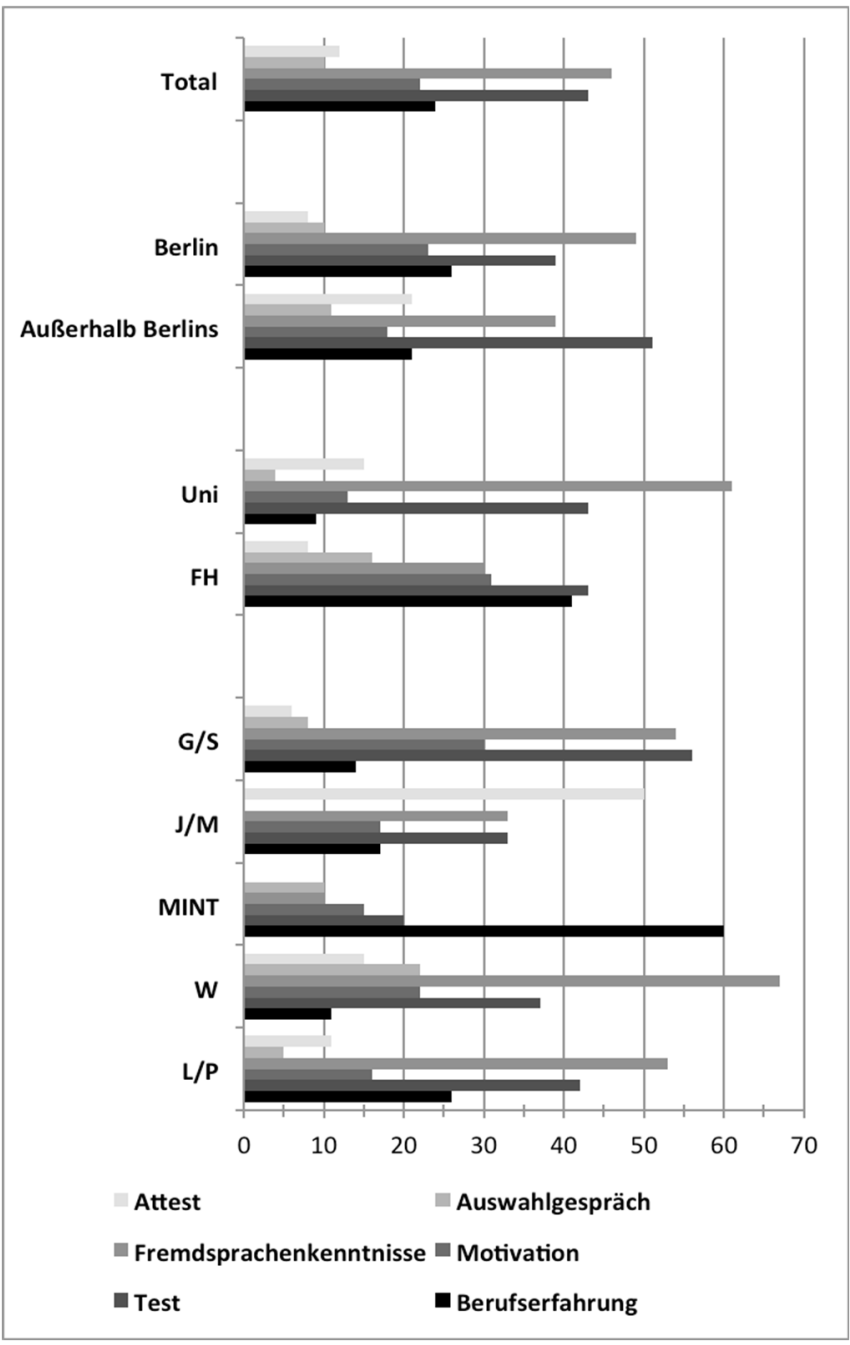

Quelle: Best Up-Rekrutierungsdaten (Studiengänge mit Eignungsfeststellungsverfahren), eigene Berechnungen.

Attest umfasst: ärztliches Attest, polizeiliches Führungszeugnis. Motivation umfasst: sämtliche Unterlagen, die eingereicht werden müssen, um Motivation und Eignung zu demonstrieren (z.B. Essay, Motivationsschreiben, Arbeitsproben, Mappen etc.).

Fallzahlen: Total: 128; Berlin: 90, Außerhalb Berlins: 38; Uni (inklusive Kunst- und Musikhochschulen): 67; FH: 61; Geistes- und Sozialwissenschaften, Kunst (G/S): 50; Jura und Medizin (J/M): 6; Mathematik, Natur- und Ingenieurswissenschaften (MINT): 20; Wirtschaftswissenschaften (W): 27; Lehramt, Pädagogik und Erziehungswissenschaften (L/P): 19. Kategorie „Sonstige“ (N: 6) nicht ausgewiesen. 
Welche Informationen stehen Studienberechtigten zur Verfügung?

Die hier berichteten Informationen stehen Studienberechtigten natürlich nicht vollständig zur Verfügung. Gleichwohl können diese in Anbetracht der Komplexität des Studienangebots und des Zulassungssystems zentral für einen erfolgreichen Übergang ins Studium sein.

Auswertungen des DZHW Studienberechtigtenpanels zeigen, dass das Internet die wichtigste Informationsquelle für Studienberechtigte ist, die jedoch im Zeitverlauf seltener als ,hilfreich" angesehen wird (vgl. Franke und Schneider 2015, Heine et al. 2010), was darauf hindeutet, dass mit steigendem Informationsangebot die Orientierung zunehmend schwer fällt. Eine relevante Anlaufstelle ist hier sicherlich der bereits vorgestellte Hochschulkompass der HRK, der neben der Übersicht zum Studienangebot auch erste Hinweise zu den Zulassungswegen bereithält. Für genauere Informationen wird allerdings entsprechend der Dezentralität der Zulassung für einen Großteil der etwa 10.000 Studiengänge - auf die einzelnen, etwa 400 Hochschulen verwiesen. Die Hochschulen veröffentlichen in der Regel die Zulassungsmodi, Verfahren und Auswahlgrenzen auf ihren Websites. Einige Hochschulen - insbesondere große Universitäten - haben hierfür eine zentrale Seite, auf der alle nötigen Informationen gebündelt werden. Wie die Rekrutierungsrecherche zeigte, sind jedoch nicht immer alle Angaben verfügbar und einfach auffindbar (für Details siehe Abschnitt 6.2.2). ${ }^{32}$

Daneben gibt es eine hohe Anzahl (teils kommerzieller) Internetseiten und Publikationen, die Informationen zu Studienangebot und Zulassung zusammentragen und Studieninteressierten ein Forum zum Austausch bieten. Die Qualität der angebotenen Informationen kann dabei vermutlich oft nur unzureichend eingeschätzt werden (vgl. Morgan 2010). Dabei scheinen selbst seriöse Quellen Informationen weiterzutragen, die, wenn nicht unkorrekt, so zumindest unvollständig sind. So wird etwa die durchaus verbreitete Anwendung von Eignungsfeststellungsverfahren kaum öffentlich thematisiert und mit Verweis auf zulassungsfreie Studiengänge eine Offenheit des deutschen Hochschulsystems suggeriert, die in dieser Form nicht zutrifft. Das CHE bietet mittlerweile erweiterte Informationen zum Thema Hochschulzugang, die mit existierenden Mythen zum NC aufräumen und auch das Thema Eignungsfeststellung thematisieren (vgl. Hachmeister et al. 2020). ${ }^{33}$

32 Von der Dezentralität ausgenommen sind die bundesweit zulassungsbeschränkten Studiengänge. Die SfH bündelt auf ihrer Internetseite (https:/www.hochschulstart.de) vielfältige Informationen zum Bewerbungs- und Zulassungsprozess.

33 Stellenweise würde ich die bereitgestellten Informationen dennoch als problematisch bewerten, etwa, wenn Eignungsfeststellungsverfahren bestimmten Studienfächern (Sport, Kunst) und darüber hinaus ,,so genannte[n] ,Elitestudiengänge[n]““ zugewiesen werden (Gehlke et al. 2019b: 6) und den Bewerbungstipps für Studienberechtigte ohne ,Spitzenabitur“ zu entnehmen ist: „Wie oben beschrieben ist we- 
Basierend auf den gesammelten Erfahrungen im Zuge der Rekrutierungsrecherche und darüber hinaus kann davon ausgegangen werden, dass es aufgrund der Komplexität und Fragmentierung der Verfahren und Informationen für Studienberechtigte insgesamt schwer sein dürfte, sich einen umfassenden Überblick über Zulassungsbeschränkungen und Auswahlverfahren und somit ihre tatsächlichen Zulassungschancen zu verschaffen.

Zusammenfassend konnte gezeigt werden, dass das (Fach-)Abitur die Türen nur noch für eine Minderheit der Studiengänge an deutschen Hochschulen automatisch öffnet. Zwar bekräftigte das BVerfG in seinen Numerus claususUrteilen, dass Hochschulzugangsberechtigten die freie Wahl des Berufes und der Ausbildungsstätte verfassungsrechtlich zusteht und nur unter streng geprüften Bedingungen eingeschränkt werden darf. Diese Einschränkungen betreffen seit einigen Jahren weniger als die Hälfte aller Studiengänge an deutschen Hochschulen, die entweder örtlich oder bundesweit zulassungsbeschränkt sind. Doch bildet dies nur einen Teil der „Zulassungsrealität" ab. Von der rein an kapazitätsrechtliche Fragen gebundenen Unterscheidung zwischen zulassungsbeschränkt und zulassungsfrei losgelöst sind - ,jenseits semantischer Plausibilitäten“" (Winter 2012: 143) - Aspekte der Eignungsfeststellung, die ebenso wie Zulassungsbeschränkungen die Wahlfreiheit von Studienberechtigten begrenzen.

Während die freie Wahl für Hochschulzugangsberechtigte also zunehmend eingeschränkt wird, wurden die Hochschulen in ihrem Auswahlrecht gestärkt. Die insbesondere seit 2004 stattgefundenen Entwicklungen können daher als ,eine modifizierte Rückkehr zur traditionellen, vor den 1960er Jahren herrschenden Vergabe- und Zulassungspraxis von Studienplätzen durch die Hochschulen selbst verstanden werden“" (Täger 2010: 38). Kritiker sehen sogar die Restauration des verfassungswidrigen Zustandes, der zum ersten Numerus clausus-Urteil führte (vgl. Keller 2009: 30).

Insgesamt entwickelt sich das deutsche System weg vom Berechtigungsmodell. Dem Prüfungsmodell, in dem wie etwa in Japan, Spanien (vgl. Haase 2008) oder zu großen Teilen auch in den USA (vgl. Buchmann et al. 2010) eine weitere Prüfung Voraussetzung für die Zulassung zum Studium ist, kann es jedoch ebenfalls nicht zugeordnet werden. Vielmehr entwickelt sich der Hochschulzugang in Deutschland zu einem „Berechtigungsmodell Plus“, in dem neben der zentralen Voraussetzung Hochschulzugangsberechtigung zusätzliche Kriterien Zulassungschancen entweder in Form von Eignungsfeststellungsverfahren determinieren oder in Form von Auswahlverfahren zumindest verbessern können. Zur Anwendung kommen dabei vorwiegend bereits vor der Bewerbung erworbene, leicht nachzuprüfende und standardisiert er-

niger als die Hälfte der Studienplätze überhaupt zulassungsbeschränkt. Es stehen also rechnerisch tausende Studiengänge offen, in die man sich mit seiner (Fach)Abiturnote sofort einschreiben kann, unabhängig davon, wie gut diese ist." (ebd.:

10) 
fassbare Kriterien (z.B. Berufserfahrung oder Sprachkenntnisse). Weitere Verfahren - wie Tests oder Auswahlgespräche - werden zwar auch genutzt, jedoch nur in der Minderheit der Fälle. Diese sind vor allem in bestimmten, prüfungserprobten Fachrichtungen wie Kunst und Sport, an privaten Hochschulen oder an diesbezüglich besonders ambitionierten staatlichen Hochschulen zu finden. Seit Inkrafttreten der Zulassungsreform für bundesweit zulassungsbeschränkte Studiengänge werden Tests auch hier flächendeckend eingesetzt.

Fest steht, dass Studienberechtigte heute nicht nur aufgrund institutioneller Differenzierungsprozesse vor komplexeren Wahlentscheidungen stehen, sondern dass auch der Zugang zu bestehenden Studienoptionen im deutschen Kontext komplexer und voraussetzungsvoller geworden ist. Inwiefern diese hochschulische Opportunitätsstruktur zu sozialen Selektionsprozessen beiträgt, soll im folgenden Abschnitt diskutiert werden.

\subsubsection{Soziale Herkunftsunterschiede, Studienoptionen und Zulassungsregeln}

In Abschnitt 2.1.3 wurde argumentiert, dass es im Zuge der Expansion und Differenzierung des Schulsystems zu einer Verschiebung der sozialen Selektionsprozesse von früheren auf spätere Schwellen im Bildungssystem kam. Expansions- und Differenzierungsprozesse fanden aber ebenso im Hochschulsystem statt (2.2.1), sodass selbst für die bereits stark vorselektierte Gruppe der Studienberechtigten, die ein Hochschulstudium aufnehmen, Herkunftsdifferenzen bezüglich der oben eingeführten vertikalen und horizontalen Differenzierungslinien beobachtet werden können.

Wie in Abschnitt 2.2.1 beschrieben, ist im binären Hochschulsystem trotz beobachtbarer Tendenzen hin zu einer reputationsbasierten Differenzierung - die Unterscheidung zwischen Universitäten und Fachhochschulen zentral. Der empirische Befund sozialer Ungleichheit bei der Hochschultypenwahl ist dabei weitestgehend unstrittig. ${ }^{34}$ Fachhochschulen entfalten eine ähnlich „ablenkende“ Wirkung wie berufliche Ausbildungsalternativen und werden überdurchschnittlich häufig von sozial schwächeren Studienberechtigten besucht. Damit ist zwar der Übergang ins Universitätsstudium, nicht aber an Fachhochschulen sozial selektiv (vgl. Blossfeld et al. 2015, Duru-Bellat et al. 2008, Maaz 2006, Müller und Pollak 2008, Reimer und Pollak 2010). Weiter-

34 Zum Thema soziale Ungleichheit beim Übergang in ,prestigereiche“ Studienoptionen existiert bislang kaum Forschung für den deutschen Kontext. Erste Studien weisen auf Grundlage von Studierendenbefragungen auf einen tendenziell positiven Zusammenhang zwischen sozialer Herkunft und dem Studium eines hochgerankten Studiengangs oder an einer „Exzellenzuniversität“ hin, der jedoch zum Teil schon vor Einführung des Exzellenzlabels bestand (Lörz und Quast 2011, Wagner 2018, Weiss et al. 2015, Winkler 2014, 2019). 
hin konnte gezeigt werden, dass Schüler/innen aus sozial schwächeren Familien duale Studienangebote überdurchschnittlich häufig anstreben und studieren (Schindler und Reimer 2010, Maaz 2006, Trautwein et al. 2006).

Auch für die horizontale Dimension - das Studium eines bestimmten Faches - wurden Herkunftsdifferenzen nachgewiesen. Hier zeigt sich insbesondere, dass die prestigereichen Fächer Medizin und Jura hochgradig sozial selektiv sind (vgl. Becker et al. 2010, Duru-Bellat et al. 2008, Lörz 2012, Reimer und Pollak 2010).

Weiterhin wurde in den vorherigen Abschnitten gezeigt, dass das deutsche Hochschulsystem und somit auch der Übergang ins Studium deutlich an Komplexität gewonnen haben. Dies betrifft nicht nur das stetig wachsende Angebot an Hochschulen und an inhaltlich mehr oder weniger eindeutig abgrenzbaren Studiengängen, sondern insbesondere auch das Zulassungssystem, dessen potentiell ungleichheitsgenerierende Konsequenzen in der deutschen hochschulpolitischen Debatte jedoch kaum diskutiert werden. Auch empirische Befunde hierzu fehlen.

Geht es um Konsequenzen der Studierendenzulassung, liegt der Fokus der deutschen Debatte wie beschrieben klar auf Wettbewerb, Leistungssteigerung und Profilbildung. Der Wissenschaftsrat (2004: 48) geht in seinen Empfehlungen zur Reform des Hochschulzugangs an nur einer Stelle explizit auf mögliche ungleichheitsgenerierende Folgen diversifizierter Zulassungsverfahren ein: „Befürchtet wird, dass flächendeckende Hochschulauswahlverfahren, insbesondere Auswahlgespräche, die soziale Selektivität des Bildungssystems erhöhen“. Eingeräumt und auch kritisiert wird zudem die zunehmende Komplexität und Unübersichtlichkeit der Zulassungsregelungen (vgl. Keller 2009, Selbmann 2012, Wolter 2008), deren Transparenz durch begleitende Informationen seitens der Hochschule als wünschenswert angesehen wird (vgl. Wissenschaftsrat 2004).

Insgesamt zeigt sich also, dass sich soziale Ungleichheit - trotz der vielen vorgelagerten Selektionsstufen - auch innerhalb des Hochschulsystems fortsetzt. Welche Rolle Zugangshürden dabei spielen, ist bisher für den deutschen Kontext weitestgehend unbekannt. Bevor ich mich im weiteren Verlauf intensiver mit Fragen der sozialen Ungleichheit beim Übergang ins Studium - oder genauer: bei der Realisierung von Studienaspirationen - auseinandersetze, möchte ich zunächst beschreiben, inwiefern die beschriebenen institutionellen Rahmenbedingungen auf Berlin als Kontext des empirischen Teils der vorliegenden Arbeit zutreffen. 


\subsection{Berlin: ein spezieller Kontext}

Viele der bisher diskutierten generellen Strukturen und Regelungen treffen auf den gesamtdeutschen Kontext zu. Jedoch wurde in den vorangegangenen Abschnitten immer wieder angedeutet, dass auch beträchtliche Unterschiede zwischen den Bundesländern bestehen (vgl. Helbig und Nikolai 2015), sowohl bezüglich der rechtlichen Regelungen als auch der institutionellen Ausgestaltung und Abdeckung. Zurückzuführen ist dies auf die Kulturhoheit der Länder, also deren primäre Gesetzgebungszuständigkeit u.a. im Schul- und Hochschulbereich (vgl. Pritchard 2006), die durch die Föderalismusreform von 2006 insbesondere im Bereich des Hochschulrechts gestärkt wurde (vgl. Täger 2010). Zudem variiert auch das Angebot an Hochschulen und Studiengängen zwischen den und auch innerhalb der Bundesländer und ist in den Stadtstaaten - und hier wiederum speziell in der deutschen Hauptstadt - besonders vielfältig und engmaschig.

Dem empirischen Teil der vorliegenden Arbeit liegen Daten zu Berliner Studienberechtigten zugrunde (Best Up). Im Folgenden soll daher der Berliner Kontext detaillierter dargestellt werden, um den Kontext der Studie und daraus resultierende Besonderheiten besser einordnen zu können.

Der Weg zur Hochschulzugangsberechtigung

Die allgemeine Hochschulzugangsberechtigung kann in Berlin auf dem ersten Bildungsweg an verschiedenen allgemeinbildenden und beruflichen Schulformen erworben werden. Neben den Gymnasien ist dies seit dem Schuljahr 2010/11 an Integrierten Sekundarschulen möglich, die durch die Zusammenführung von Haupt-, Real- und Gesamtschulen entstanden (vgl. Helbig und Nikolai 2017). ${ }^{35}$ Zudem existiert mit den Oberstufenzentren (OSZ) eine Schulform mit mehreren Bildungsgängen innerhalb eines Berufsfeldes (z.B. Gesundheit, Energietechnik oder Handel). Einer dieser Bildungsgänge ist das berufliche Gymnasium, das nach Abschluss der 10. Klasse an einer anderen Schulform besucht werden kann (SenBJF 2014). Die größte Relevanz für die Zertifizierung der Hochschulreife besitzt aber nach wie vor das Gymnasium (Autorengruppe Bildungsberichterstattung 2016: 80). Das Abitur wird an Gymnasien seit dem Schuljahr 2005/06 nach zwölf Schuljahren erlangt, an beruflichen Gymnasien und ISS nach 13 Jahren.

35 Seit dem Schuljahr 2008/09 wurden zudem Gesamtschulen als Modellversuch eingeführt, an denen Schüler/innen durchgängig von der ersten Klasse bis zum Abitur beschult werden können. 
Berliner Studienberechtigte und ihre Bildungswege

Die Berliner (Fach-)Abiturient/innenquote lag im Jahr 2014 über dem deutschen Durchschnitt von 53\%: 57\% der Schüler/innen verließen das allgemeinbildende und berufliche Schulsystem mit einer Hochschulzugangsberechtigung (48\% Abitur, 9\% Fachabitur) (Autorengruppe Bildungsberichterstattung 2016: D7-web5). 81,5\% hiervon nahmen in den Folgejahren ein Studium auf (bundesweiter Durchschnitt: 80\%) (Autorengruppe Bildungsberichterstattung 2020: F2-3web).

Die gleichzeitig hohe Abiturient/innen- und Übergangsquote (bei gleichzeitig hoher Nachfrage nach Studienplätzen in Berlin) könnte eine Erklärung dafür sein, dass Berliner Hochschulzugangsberechtigte zu Studienbeginn leicht überdurchschnittlich regional mobil sind: $41 \%$ derjenigen Berliner Studienberechtigten, die $2012 \mathrm{ihr}$ Studium aufnahmen, taten dies außerhalb Berlins und insgesamt 24\% wanderten über das Nachbarland Brandenburg hinaus (bundesweiter Durchschnitt: 34 und 14\%). Gleichzeitig war der Anteil an Studienanfänger/innen in Berlin, die ihre Hochschulzugangsberechtigung in einem anderen Bundesland erhalten hatten, mit $65 \%$ überdurchschnittlich hoch (bundesweiter Durchschnitt: 45\%) (KMK 2014: 25, 32) ${ }^{36}$ Berliner Abiturient/innen sind entsprechend mit einem tendenziell ungünstigen Wettbewerbsverhältnis konfrontiert: Ein hoher Anteil erlangt das Abitur, und gleichzeitig drängen überdurchschnittlich viele Nicht-Berliner/innen in die Hauptstadt. Jedoch ist nicht nur die Nachfrage nach Hochschulbildung groß, sondern auch das Angebot, wie im Folgenden gezeigt wird.

\section{Berliner Hochschullandschaft}

Die Berliner Hochschullandschaft ist mit insgesamt 38 Hochschulen und über 600 grundständigen Studiengängen durch ein diverses und dichtes Angebot gekennzeichnet [Stand 2021]; in keiner anderen Stadt (oder Ballungsgebiet) sind ähnlich viele Hochschulen angesiedelt. ${ }^{37}$ Die 38 Hochschulen unterteilen sich in zehn Universitäten (sieben davon privat), fünf Kunst- und Musikhochschulen sowie 23 Fachhochschulen. Mit insgesamt 25 privaten Hochschulen floriert insbesondere dieser Markt in der deutschen Hauptstadt. Zudem kann Berlin mit der Freien und der Humboldt Universität zwei „Exzellenzuniversitäten“ vorweisen, deren Zukunftskonzepte in den Runden der Exzellenzinitiative ausgewählt wurden; zudem wurde die Berlin University Alliance als Teil

36 Interessanterweise führt Brandenburg mit 75\% hier die Statistik an, was an den vielen Zuwanderungen aus Berlin in das brandenburgische Hochschulsystem liegt.

37 Hamburg: 18, München: 14, Bremen: 7, Köln: 9 (zusammen mit Bonn, Düsseldorf, Dortmund, Essen: 24). https://www.hochschulkompass.de/hochschulen/die-hochschulsuche.html [letzter Zugriff: 05.08.2021] 
der Exzellenzstrategie ausgewählt. Für Berliner Hochschulzugangsberechtigte attraktiv scheinen laut der oben beschriebenen Wanderungsbewegungen zusätzlich die 18 Hochschulen im Land Brandenburg (vier davon in Potsdam), die in der Regel in Pendelnähe sind; durch das oft verfügbare Semesterticket fallen zudem keine zusätzlichen Fahrtkosten an.

Auf Studiengangsebene werden 197 der insgesamt 647 grundständigen Studiengänge an Universitäten angeboten, 52 an Kunst- und Musikhochschulen sowie 378 an Fachhochschulen. 275 können an Hochschulen in privater Trägerschaft studiert werden. ${ }^{38}$ Im Land Brandenburg werden zusätzlich 232 grundständige Studiengänge angeboten.

Durch die Vielzahl an Hochschulen und Studierenden - im WiSe 2015/16 wurden mehr als 175.000 Rückmeldungen registriert (Amt für Statistik BerlinBrandenburg 2016) - kann von einer hohen Präsenz von Hochschulbildung in der Hauptstadt ausgegangen werden, die Berliner Schüler/innen vielfältige Informationsmöglichkeiten eröffnet und zu Nachbarschafteffekten führen kann, die wiederum Studienaspirationen und -entscheidungen positiv beeinflussen können (Spieß und Wrohlich 2010: 472). ${ }^{39}$

Der Anteil an zulassungsbeschränkten Studiengängen war in Berlin in den letzten zehn Jahren stark rückläufig: Im WiSe 2007/08 lag dieser noch bei $82 \%$, im WiSe $2012 / 13$ bei $67 \%$, und im im WiSe $2016 / 17$ nur noch bei $50 \%$, stieg aber danach wieder auf 69\% im WiSe 2020/21 (vgl. Anhangstabelle A2.1, Abschnitt 2.2.3). Trotz dieses starken Rückgangs waren in Berlin dennoch in jedem Jahr überdurchschnittlich viele Studiengänge zulassungsbeschränkt (der bundesweite Durchschnitt variierte im gleichen Zeitraum zwischen 52 und 41\%). Zudem sind diese Werte - wie in Abschnitt 2.2.3 beschrieben - mit Vorsicht zu bewerten, da sie zumindest bis zum WiSe 2017/18 nur eingeschränkt Angaben zu Eignungsfeststellungsverfahren beinhalten.

Wie oben beschrieben, legen die Länder für staatliche Hochschulen in ihren Hochschulzulassungsgesetzen fest, nach welchen Quoten die Plätze für örtlich zulassungsbeschränkte Studiengänge vergeben werden und nach welchen Kriterien in der AdH-Quote ausgewählt werden darf. Das Land Berlin folgt hier dem (vor 2019 geltenden) HRG und schließt sich damit den in Abschnitt 2.2.2 beschriebenen Regelungen für bundesweit zulassungsbeschränkte Studiengänge sowohl bezüglich der Quoten (Abiturbeste: 20, Wartezeit: 20, AdH:

38 Sämtliche Angaben zu Hochschulen und Studiengängen sind dem Hochschulkompass entnommen. Letzter Abruf: 05.08.2021. Insgesamt scheint es eine relativ starke Fluktuation bezüglich der Anzahl der Studiengänge zu geben (Anfang März 2017 waren zum Beispiel nur 515 Berliner Studiengänge gelistet), die insbesondere durch einen starken Anstieg privater Studienangebote zustande kommt.

39 Auch wenn Berlin absolut gesehen deutschlandweit die Stadt mit den meisten Hochschulen ist, sollte dieses Argument aber stärker noch auf kleinere Universitätsstädte zutreffen. So machen Studierende etwa 5\% der Berliner Bevölkerung aus. In Heidelberg sind es etwa $20 \%$ und in Tübingen sogar etwa $30 \%$. 
60) als auch der erlaubten AdH-Kriterien an (Berliner Hochschulzulassungsgesetz). Bezüglich der Anwendung von Eignungsfeststellungsverfahren zeigt sich Berlin besonders liberal: „Die Hochschulen regeln in der Zugangssatzung, in welchen Studiengängen über die Hochschulzugangsberechtigung hinaus zusätzliche Eignungs- und Qualifikationsvoraussetzungen gefordert werden und wie diese nachzuweisen sind." ( $\$ 10$ Abs. 5 Berliner Hochschulgesetz) ${ }^{40}$ Wie die Deskription der Rekrutierungsdaten in Abschnitt 2.2.3 zeigte, scheinen die Berliner Hochschulen von dieser Möglichkeit regen Gebrauch zu machen

Zusammenfassend ist der Berliner Kontext gekennzeichnet durch ein groBes und vielfältiges Angebot an nahegelegenen Studienoptionen, vielfältigen, relativ leicht erreichbaren Informationsmöglichkeiten und zwei Exzellenzuniversitäten. Der Anteil an zulassungsbeschränkten Studiengängen sank zunächst auf etwa 50\% im WiSe 2015/16, um danach wieder anzusteigen. Wie in Abschnitt 2.2.3 argumentiert, sollte dies jedoch nicht über zusätzliche Hürden in Form von Eignungsfeststellungsverfahren hinwegtäuschen, deren Nutzung im Berliner Hochschulgesetz kaum reglementiert wird. Weiterhin führt das Berliner Schulsystem überdurchschnittlich viele junge Menschen zur Hochschulzugangsberechtigung, die überdurchschnittlich häufig ein Studium aufnehmen. Gleichzeitig ist die externe Nachfrage nach Berliner Studienplätzen hoch, was zwangsläufig zu Wanderungsbewegungen vor allem auch ins Brandenburger Umland führt.

Im Berliner Kontext treffen also unterschiedliche Faktoren aufeinander: Zwar ist sowohl die interne als auch externe Nachfrage nach Hochschulbildung in der Hauptstadt hoch, doch bietet diese gleichzeitig ein großes und vielfältiges Angebot. Zwar führt diese Vielfalt zu einer hohen, nur schwer überschaubaren Komplexität, doch sind viele Hochschulen und somit auch Informationsmöglichkeiten für Berliner Schüler/innen einfach erreichbar. Die Diskussion, wie diese Faktoren Studienchancen und Herkunftsunterschiede rahmen, wird später wieder aufgenommen

\subsection{Zusammenfassung}

Kapitel 2 diente dazu, den institutionellen Kontext, in den die folgenden theoretischen Überlegungen und empirischen Untersuchungen eingebettet sind, vorzustellen. Wie zu Beginn dargestellt, ist die schulische Hochschulzugangsberechtigung - das (Fach-)Abitur - in Deutschland die zentrale und formalrechtlich einzige Bedingung für die Aufnahme eines Studiums. Aufgrund der starken Stratifizierung des Schulsystems erlangt dieses Berechtigungszertifi-

40 Die Gesetzestexte wurden 2017 recherchiert. Die Aussagen sind auch 2021 noch aktuell. 
kat - trotz deutlicher Expansionsprozesse im Bereich sekundärer Bildung nur etwa gut die Hälfte eines Geburtsjahrgangs. Trotz dieser starken Selektionswirkung nehmen nur etwa $80 \%$ der Studienberechtigten ein Studium auf. Ein zentraler Grund hierfür ist die Attraktivität des Berufsbildungssystems, die dazu führt, dass ein Teil der Schüler/innen das Abitur vordergründig erlangen, um die eigenen Chancen auf einen attraktiven Ausbildungsplatz zu erhöhen. Diejenigen mit Studienwunsch - also Schüler/innen mit Studienaspiration, die im Fokus dieser Arbeit stehen - stehen vor der Wahl zwischen unterschiedlichen Studienoptionen und sind dabei mit verschiedenen Hürden konfrontiert.

Insgesamt ist das deutsche Hochschulsystem durch geringe direkte Studienkosten und eine relativ dichte regionale Abdeckung an Hochschulinstitutionen gekennzeichnet. Seit den 1970er Jahren hat sich die binäre Trennung zwischen Fachhochschulen und Universitäten zur zentralen Differenzierungsdimension entwickelt; ein Elitesektor hat keine lange Tradition und private Hochschulen spielen eine untergeordnete Rolle. Im Vergleich zu vielen anderen Ländern wurde das deutsche Hochschulsystem entsprechend als homogen charakterisiert.

Fokussiert man jedoch auf die innerdeutsche Entwicklung, zeigt sich ein deutlicher Trend in Richtung Deregulierung, Differenzierung und Komplexitätssteigerung, der sich zum Beispiel in Form der Exzellenzinitiative, der Etablierung hybrider Studienformen und der inhaltlichen Vervielfältigung des Studienangebots zeigt. Dieser Trend betrifft auch das Zulassungssystem: Das (Fach-)Abitur stellt zwar nach wie vor (in aller Regel) eine notwendige, in vielen Fällen aber keine hinreichende Bedingung für eine Studienaufnahme mehr dar. Das Wahlrecht von Studienberechtigten ist durch Zulassungsbeschränkungen und Eignungsfeststellungsverfahren häufig eingeschränkt, und der Weg in die Hochschule wird damit voraussetzungsvoller und komplexer.

Innerhalb dieser institutionellen Struktur entsteht soziale Ungleichheit sowohl auf dem Weg ins Studium als auch innerhalb des Hochschulsystems: Sozial schwächere Schüler/innen erlangen erstens seltener die Hochschulzugangsberechtigung und gehen zweitens auch nach deren Erhalt seltener ins Studium über. Sofern sie nicht von attraktiven beruflichen Optionen abgelenkt werden und ein Studium aufnehmen, tun sie dies drittens seltener an Universitäten und in prestigereichen Studienfächern als ihre sozial privilegierten Peers, sodass sich soziale Ungleichheit - trotz der vielen vorgelagerten Selektionsstufen - auch innerhalb des Hochschulsystems fortsetzt. Welche Rolle Zugangshürden dabei spielen, ist bisher für den deutschen Kontext weitestgehend unbekannt.

In den folgenden Kapiteln wird auf die hier ausführlich beschriebene institutionelle Opportunitätsstruktur und deren potentiell ermöglichende und einschränkende Wirkung wiederholt sowohl theoretisch als auch empirisch zurückgegriffen. 


\section{Soziale Herkunftsunterschiede beim Übergang in die Hochschule: verbreitete Ansätze, Leerstellen und Erweiterungen}

In Kapitel 2 wurde der institutionelle Kontext in Deutschland vorgestellt, der Studienaspirationen und Studienchancen von Schüler/innen durch formale Vorgaben und vorhandene Bildungsoptionen strukturiert. Zudem wurden empirische Befunde zitiert, die verdeutlichen, dass sowohl der Übergang ins Studium als auch in unterschiedliche vertikal und horizontal stratifizierte Studienoptionen in Deutschland sozial selektiv ist.

In Kapitel 3 werden verbreitete theoretische und insbesondere empirische Ansätze, die zur Untersuchung sozialer Ungleichheit beim Übergang ins Hochschulstudium genutzt werden, vorgestellt, kritisch diskutiert und hieran anknüpfend die konzeptionellen, theoretischen und empirische Erweiterungen vorbereitet, die zentraler Bestandteil der nachfolgenden Kapitel sind. Abschnitt 3.1 gibt - mit Fokus auf die deutsche Forschung - einen Überblick über die zentralen Ansätze. ${ }^{41}$ Hieraus ergeben sich konzeptionelle und empirische Leerstellen (3.2), aus denen sich der Bedarf einer detaillierten Analyse des Übergangsprozesses ableitet, mit deren Hilfe das Zustandekommen von sozialer Ungleichheit umfassender verstanden werden kann. Zentral erscheint hierbei, den Übergang ins Hochschulstudium nicht als singuläres Ereignis zu verstehen und zu modellieren, sondern als aus verschiedenen, miteinander verbundenen Phasen bestehend, in denen sozialgruppenspezifische Selbst- und Fremdselektionsprozesse stattfinden. In Deutschland werden zwar der Besuch eines Gymnasiums oder einer ähnlichen Schulform und das Erreichen der Hochschulzugangsberechtigung als wichtige Schritte auf dem Weg in die Hochschule diskutiert, jedoch sind die Phasen nach Erhalt der Studienberechtigung und hierin stattfindende soziale Selektionsprozesse bisher weitestgehend unerforscht. Anders in der US-amerikanischen Übergangsforschung: Hier dienen verschiedene Phasenmodelle häufig als Grundlage empirischer Studien (vgl. Avery und Kane 2004, Hossler und Gallagher 1987, Jackson 1982, Klasik 2012, Litten 1982, Roderick et al. 2011). ${ }^{42}$ Diese Modelle, die als wichtige Orientierung für die vorliegende Arbeit dienen, werden in Abschnitt 3.3 vorgestellt.

41 Diese und weitere Ansätze und Befunde werden an gegebener Stelle, insbesondere in Kapitel 5, weiter ausgeführt und genutzt, um den empirischen Teil der vorliegenden Arbeit theoretisch anzureichern.

42 Der Ansatz, den Übergang als aus verschiedenen Phasen bestehend zu begreifen, findet auch in anderen Ländern Anwendung (Schweden: Hällsten 2010, UK: Anders 2012, Boliver 2013, Kanada: Drewes und Michael 2006). 


\subsection{Die Hauptstränge der deutschen „Übergangsforschung“"}

Soziale Ungleichheit beim Übergang in die Hochschule ist ein Thema, zu dem bereits umfangreiche Forschung vorliegt. Diese reicht von international vergleichenden Studien, die den Einfluss variierender institutioneller Merkmale betrachten (vgl. Hillmert und Jacob 2003, Jackson und Jonsson 2013, Müller und Karle 1993, Pfeffer 2008, Shavit und Müller 2000), über historische Vergleiche, die die Wirkung institutionellen Wandels (insbesondere der Bildungsexpansion und institutioneller Differenzierung) untersuchen (vgl. Blossfeld et al. 2015, Blossfeld und Shavit 1993, Breen et al. 2009, Lörz und Schindler 2011, Mayer et al. 2007, Müller und Haun 1994, Müller und Pollak 2008, Reimer und Pollak 2010, Schimpl-Neimanns 2000, Schindler 2012, 2014), bis hin zu Studien, die Mechanismen auf der Mikroebene testen und den individuellen Entscheidungsprozess in den Blick nehmen (vgl. Becker und Hecken 2007, 2008, 2009a/b, Lörz 2012, Maaz 2006, Obermeier und Schneider 2015, Schindler und Reimer 2010, Watermann und Maaz 2006, 2010). Noch 2006 resümierte Kai Maaz, dass im deutschen Kontext „für den Hochschulzugang [...] vergleichsweise wenige Untersuchungen vor[liegen], die über eine beschreibende Darstellung hinausgehen" (Maaz 2006: 231). Seitdem befindet sich dieser Forschungsbereich aber auch in Deutschland im Aufwind, was sich in einer zunehmenden Zahl wissenschaftlicher Publikationen widerspiegelt, deren zentrale Befunde zum Teil bereits in Kapitel 2 vorgestellt wurden.

Wie in Kapitel 2 beschrieben, wurden für den deutschen Kontext auf institutioneller Ebene das stratifizierte Schulsystem, Expansionsprozesse im Sekundarschulbereich sowie die Attraktivität beruflicher Bildungswege als zentrale Erklärungsfaktoren für soziale Ungleichheit beim Übergang in die Hochschule identifiziert. Ein Großteil der neueren (im vorangegangenen Absatz größtenteils bereits zitierten) deutschen Forschung zu sozialer Ungleichheit beim Übergang ins Studium interpretiert diese Opportunitätsstruktur als Rahmen, innerhalb dessen Schüler/innen (und ihre Familien) Entscheidungen treffen. Theoretisch knüpfen viele dieser Studien an dem ,methodologischen Individualismus verpflichtete handlungs- und entscheidungstheoretische Ansätze aus der Familie der Rational-Choice-Theorien“ (Solga und Becker 2012: 19) an, die mittlerweile zum „Mainstream der deutschen Bildungssoziologie“ (ebd.: 24, Hervorhebung im Original) geworden sind. ${ }^{43}$ Viele der gestellten

43 Zwar werden konflikttheoretische Ansätze insbesondere mit Bezug auf Bourdieu (1973, 1982, Bourdieu und Passeron 1971) als ergänzende theoretische Einbettung oder zur Plausibilisierung von Ergebnissen herangezogen, doch werden sie - sicherlich auch aufgrund der Schwierigkeit, die zentralen Konstrukte und Relationen zu operationalisieren (vgl. Sullivan 2002) - in quantitativen Studien kaum empirisch geprüft (vgl. Solga und Becker 2012: 22). 
Fragen drehen sich also um die Aufdeckung ungleichheitsgenerierender Mechanismen auf der (institutionell gerahmten) Individualebene - um Gründe für sozialgruppenspezifische Bildungsentscheidungen individueller, rational handelnder Akteure. Soziale Ungleichheit in den Bildungsentscheidungen entsteht demnach durch sozialgruppenspezifische Kosten-Nutzen-Abwägungen. Eine wachsende Zahl an Studien widmet sich der empirischen Überprüfung dieser Mechanismen im deutschen Kontext (vgl. Becker und Hecken 2007, 2009a, Lörz 2012, Maaz 2006, Schindler und Reimer 2010).

Empirisch greifen diese Studien auf quantitative Methoden zurück, wobei als Datengrundlage neben anderen Quellen wie dem SOEP (vgl. Obermeier und Schneider 2010, Spieß und Wrohlich 2010) oder der Lebensverlaufsstudie (vgl. Blossfeld et al. 2015, Hillmert und Jacob 2010, Müller und Pollak 2008) insbesondere Studienberechtigtenbefragungen zum Einsatz kommen. $\mathrm{Zu}$ nennen sind hierbei vor allem die querschnittliche Sächsische Abiturient/innenbefragung (vgl. Becker und Hecken 2007, 2008, 2009 a/b, Becker et al. 2010) sowie die Panelbefragungen baden-württembergischer (TOSCA, vgl. Maaz 2006, Trautwein et al. 2006, Watermann und Maaz 2006) sowie gesamtdeutscher Studienberechtigter (DZHW Studienberechtigtenpanel, vgl. Lörz 2012, Lörz und Schindler 2011, Neugebauer et al. 2013, Reimer und Pollak 2010, Schindler und Reimer 2010). Untersucht werden dabei meist soziale Herkunftsunterschiede bezüglich tatsächlich realisierter Übergänge (vgl. Hillmert und Jacob 2010, Reimer und Pollak 2010, Schindler und Lörz 2012, Schindler und Reimer 2010, Neugebauer et al. 2013), geäußerte Studienintentionen (vgl. Becker und Hecken 2007, 2008, 2009a/b, Obermeier und Schneider 2015, Schnabel und Gruehn 2000, Waterman und Maaz 2006) oder eine Mischung aus beidem (vgl. Helbig et al. 2015, Lörz 2012, Lörz et al. 2015 für den Übergang ins Masterstudium). ${ }^{44}$

44 Neben Übergängen und Intentionen betrachten verschiedene Studien meist anhand repräsentativer Bevölkerungsbefragungen die Verteilung von Bildungszertifikaten (vgl. Blossfeld et al. 2015, Blossfeld und Shavit 1993, Mayer et al. 2007, Müller und Pollak 2008, Müller und Karle 1993, Breen et al. 2009). Der Fokus dieses Ansatzes liegt damit stärker auf Ergebnisungleichheit als auf Chancenungleichheit bezüglich des Zugangs zu Hochschulbildung. Einschränkend wird diskutiert, dass er (meist ideal-typische) Übergangssequenzen nur indirekt rekonstruieren kann (Hillmert und Jacob 2010: 61), sodass Übergangsraten zum Beispiel unterschätzt werden, da Studienabbrecher/innen nicht in das Maß für Hochschulabsolvent/innen einfließen. 


\subsection{Leerstellen der „Übergangsforschung“}

Diese umfangreiche Forschung liefert wertvolle Erkenntnisse zu Ausmaß, Entwicklung und Erklärungen sozialer Ungleichheit beim Übergang in die Hochschule. Die hierbei verbreiteten (empirischen) Ansätze weisen aber zum Teil Leerstellen auf. Eine vor allem konzeptionell-empirische Leerstelle der deutschen Übergangsforschung soll im Folgenden kritisch diskutiert und eingeordnet werden. Die meisten Studien, die sich mit sozialen Herkunftsunterschieden beim Übergang ins Studium beschäftigen, betrachten - wie im vorangegangenen Abschnitt beschrieben - (1) tatsächlich realisierte Übergänge oder (2) geäußerte Studienintentionen. Beide Ansätze können nur Teilaspekte des Übergangs ins Studium und hierbei auftretender sozialer Ungleichheit erfassen (vgl. Becker 2000, Jacob et al. 2013, Watermann und Maaz 2010).

Beobachtete Übergänge = individuelle Entscheidungen?

Ein Vorteil des ersten Ansatzes ist sicherlich, dass durch die Untersuchung realisierter Übergänge das letztliche Ausmaß sozialer Ungleichheit beim $\mathrm{Zu}-$ gang zum Studium erfasst werden kann. Dieser Ansatz kann als ,,revealed outcome approach" (Sen 1986) bezeichnet werden, der von der voraussetzungsvollen Annahme ausgeht, dass allein vom Outcome (dem Übergang ins Studium) auf individuelle Präferenzen und Entscheidungen rational handelnder Akteure geschlossen werden kann. Übergänge ins Studium sind allerdings nicht allein das Resultat individueller Bildungsentscheidungen, sondern vielmehr „das Ergebnis des Zusammenwirkens eigener Wahlentscheidungen (,Selbstselektion') und Auswahlentscheidungen anderer (,Fremdselektion')“ (Hillmert 2005: 177). Die Untersuchung realisierter Übergänge birgt daher das Problem, dass individuelle und institutionelle Entscheidungsprozesse (Selbstselektion und Fremdselektion) konfundiert sind (Boliver 2013).

Der Großteil der Studien, die realisierte Übergänge betrachten, greift - wie im vorangegangenen Abschnitt beschrieben - auf mikrotheoretische Entscheidungstheorien zurück. Aus dieser Perspektive haben sich Schüler/innen, die nicht ins Studium übergehen, auf Grundlage rationaler Überlegungen gegen ein Studium entschieden. Diese Interpretation betrifft auch diejenigen, die selbst zwar die Wahl für ein Studium getroffen haben (und die entsprechende Schritte ergreifen, etwa in Form einer Bewerbung), aber denen der Zugang zumindest zum präferierten Studium - verwehrt wird. Studien aus stratifizierteren Hochschulsystemen reflektieren und untersuchen diesen Sachverhalt häufiger, oft mit Blick auf den Zugang zu prestigereichen Hochschulen oder Hochschultypen (vgl. An 2010, Anders 2012, Boliver 2013, Klasik 2012, Roderick et al. 2011). In einer der wenigen deutschen (quantitativen) Studien, die sich explizit mit dem Verhältnis individueller und institutioneller Bildungsent- 
scheidungen beschäftigt, kritisiert Rolf Becker (2000) die eingeschränkte Sicht auf institutionelle Einflüsse und die damit verbundenen Fehlschlüsse bezüglich sozialer Ungleichheit:

„Der Stellenwert des Bildungssystems [...] beschränkt sich bei den angeführten Rational Choice-Modellen hauptsächlich auf dessen Einfluss auf die elterliche Bildungsentscheidung [...]. Bei der Aggregation vieler einzelner elterlicher Bildungsentscheidungen muss jedoch die hochgradig institutionalisierte Selektionsleistung des Bildungssystems systematisch eingeschlossen werden [...]. So können über die Selektion seitens des Bildungssystems die elterlichen Bildungsentscheidungen dahin gehend modifiziert werden, dass die sozial ungleiche Verteilung der Bildungsentscheidungen nicht mehr mit der sozialen Ungleichheit von tatsächlich vollzogenen Bildungsübergängen übereinstimmt.“ (ebd.: 456, Hervorhebung: CF)

Wie das Zitat andeutet, geht es in Beckers Studie um die elterliche Entscheidung für den weiterführenden Schultyp nach der Grundschule, die je nach Bundesland durch institutionelle Vorgaben, etwa in Form bindender Gymnasialempfehlungen, eingeschränkt sein kann. In seiner Forschung zu Studienentscheidungen sächsischer Abiturient/innen räumt Becker solchen Reflexionen allerdings weniger Raum ein.

Generell wird institutionellen Auswahlprozessen und deren Beitrag zu sozialer Ungleichheit beim Übergang in das deutsche Hochschulsystem bisher zumindest empirisch wenig Bedeutung beigemessen. ${ }^{45}$ Im Kontext des deutschen Hochschulsystems mag diese Leerstelle kaum verwunderlich erscheinen, da die Gatekeepingfunktion von Hochschulen lange Zeit eingeschränkt war und im Vergleich zu einigen anderen Hochschulsystemen immer noch ist (vgl. Kapitel 2). So wird argumentiert, dass der Einfluss von Zulassungshürden für die individuelle Entscheidung für oder gegen ein Studium in Anbetracht der Existenz zulassungsfreier Studiengänge als nachrangig betrachtet werden kann (vgl. Schindler 2012). ${ }^{46}$ Doch wie in Kapitel 2 gezeigt wurde, ist dies zumindest in Teilen ein Fehlschluss. Daher ist es überfällig, neben den verschiedenen Stufen der individuellen auch der institutionellen Selektion und deren Zusammenspiel stärkere Aufmerksamkeit zu schenken (vgl. Helsper und Krüger 2015), um soziale Ungleichheit beim Übergang in die Hochschule umfassender verstehen zu können.

45 Ein konzeptioneller und theoretischer Überblick findet sich in Helsper und Krüger (2015), Sackmann (2015) und Täger (2010).

46 Gleiches trifft im Übrigen auch auf das US-amerikanische Hochschulsystem zu, in dem nur etwa 80\% der four-year colleges eine Bewerbung erfordern (An 2010: 311) und viele community colleges freien Zugang gewähren. Gerade letztere sind aber häufig kein Sprungbrett für die Aufnahme eines Studiums an einem four-year college, sondern kanalisieren insbesondere sozial schwächere Individuen in weniger prestigereiche soziale und berufliche Positionen (vgl. Brint und Karabel 1989). 
Beobachtete Intention $=$ Übergang?

Der zweite weit verbreitete Ansatz versteht Studienintentionen oder -pläne als Approximationen individueller Entscheidungen (vgl. Obermeier und Schneider 2015: 111). Die Studienintention wird dabei als individuelle „Entscheidung, im Anschluss an den Schulabschluss an einer Universität oder an einer Fachhochschule zu studieren" (Becker und Hecken 2007: 106, Hervorhebung: $\mathrm{CF}$ ) interpretiert und als guter Indikator für tatsächliches Verhalten angesehen. Auf diesem Argument aufbauend werden mitunter abhängige Variablen gebildet, die Studienintentionen und realisierte Übergänge gemeinsam erfassen (vgl. Helbig et al. 2015, 2017, Lörz 2012). ${ }^{47}$

Unstrittig bleibt jedoch, ,dass nicht in allen Fällen Bildungsintention und Realisierung übereinstimmen" (Jacob et al. 2013: 308). Setzt man Intentionen mit realisierten Übergängen gleich, können entsprechend Verzerrungen aufgrund späterer Umentscheidungsprozesse und unzureichend antizipierter $\mathrm{Zu}$ gangshürden entstehen (vgl. Azjen 1991). Träfe Ersteres nicht zu, wären also geäußerte Intentionen stabil und spiegelten individuelle Entscheidungen perfekt wider, sollten Intentionen in Hochschulsystemen mit uneingeschränktem Zugang tatsächlich realisierte Übergänge gut approximieren. In Hochschulsystemen, in denen institutionelle Zulassungsbeschränkungen flächendeckend zum Einsatz kommen, oder im Falle individueller Präferenzen für selektive Fächer oder Hochschulen sind individuelle Bildungsentscheidungen jedoch lediglich ,eine notwendige Voraussetzung, aber keine hinreichende Garantie für ihre Realisierung" (Becker 2000: 458). Mit Intentionen kann somit ein Teil des Übergangsprozesses - Fremdselektion - über antizipatorische Prozesse nur indirekt und je nach Informationsverfügbarkeit und Informiertheit mehr oder weniger umfassend und korrekt erfasst werden.

Im deutschen Kontext kann von einer guten Approximation ausgegangen werden, wenn (1) Studienberechtigte entweder so gute Noten vorweisen können, dass sie Zugang zu (fast) allen Studiengängen haben, wenn (2) sie eine Studienrichtung anstreben, deren Selektivität in der Regel unter dem eigenen Leistungsniveau liegt oder die weitestgehend zulassungsfrei ist und in der Eignungsfeststellungsverfahren kaum zum Einsatz kommen, oder wenn (3) sie keine klare fachliche (oder örtliche) Präferenz haben und somit uneingeschränkt zwischen den Studiengängen ohne (oder mit ausreichend niedrigen) Zugangsbarrieren wählen können. In allen anderen Fällen kann nicht ohne

47 Ein Grund für dieses Vorgehen ist häufig auch in der Rechtszensierung der Daten zu suchen. Wird zum Beispiel - wie in den zitierten Studien - die zweite Welle des DZHW Studienberechtigtenpanels genutzt (ein halbes Jahr nach Erhalt der Hochschulzugangsberechtigung), können nur direkte Übergänge ins Studium beobachtet werden, was zu einer deutlichen Unterschätzung der Übergangswahrscheinlichkeiten führen würde. 
Weiteres von einer Intention auf den tatsächlichen Übergang geschlossen werden.

Zusammenfassend erlaubt der Fokus auf Intentionen und damit eine (frühe) Phase der Selbstselektion keine endgültige Aussage über deren Realisierung, da institutionelle Selektionsprozesse und andere Hürden nicht (oder durch Antizipation allenfalls indirekt) erfasst werden können. Das tatsächliche Ausmaß an sozialer Ungleichheit beim Übergang ins Studium kann daher lediglich approximiert werden. Anders verhält es sich mit Studien zu realisierten Übergängen: Diese schließen faktisch sowohl Phasen der Selbst- als auch Fremdselektion mit ein. Sie können allerdings keine Aussage darüber treffen, in welchen Phasen Studienberechtigte (aus sozial schwächeren Familien) den Hochschulpfad (vorerst) verlassen.

Die dargestellten Leerstellen beider hier diskutierten empirischen Ansätze verdeutlichen den Mehrwert einer differenzierten Analyse des Übergangsprozesses. Faktisch bedeutet dies einerseits, nicht bei Studienintention stehen zu bleiben, und andererseits, nicht erst beim bereits vollzogenen Übergang zu starten, sondern auch „das Dazwischen“ zu untersuchen. Entsprechend fordert etwa An (2010: 312), ,[c]ontinued efforts that unpack the 'black box' in the transition from high school to college $[\ldots]$ in order to better understanding the source of social inequalities in college destinations." Modelle, die den Übergang ins Studium als aus verschiedenen Phasen bestehend verstehen, nähern sich dieser Forderung an und sollen im Folgenden vorgestellt werden.

\subsection{Alternative Ansätze: der Übergang als Phasenmodell}

Weitestgehend unstrittig - auch in der mikrotheoretisch orientierten Forschung - ist, dass der Übergang in die Hochschule kein losgelöster singulärer Schritt ist, sondern das Ergebnis kumulativer Entscheidungen. Welche Phasen hierbei als relevant erachtet werden, hängt vom jeweiligen Forschungsinteresse und dem damit verbundenen Aggregationsniveau ab.

Der lange Weg zur Hochschule: sequentielle Entscheidungsmodelle

Großer Beliebtheit erfreut sich etwa das Selektionsmodell von Mare (1980), das sowohl international als auch in Deutschland den Standardrahmen für die Untersuchung von Bildungsungleichheit darstellt (vgl. Breen und Jonsson 2005: 235, Schindler und Lörz 2012: 648). Der Übergang in die Hochschule wird dabei als Ergebnis sequentieller Bildungsentscheidungen interpretiert. An jeder Schwelle im Bildungssystem treffen Schüler/innen und deren Familien 
eine Entscheidung über Verbleib oder Ausscheiden. ${ }^{48}$ Schüler/innen aus sozial schwächeren Familien scheiden dabei überdurchschnittlich häufig aus, vor allem an den frühen Schwellen. Dass dies gerade auch im stratifizierten deutschen Schulsystem der Fall ist, wurde bereits in Abschnitt 2.1.3 beschrieben. Aus dieser Perspektive definieren etwa Lörz und Schindler (2012: 460) den Hochschulabschluss als

„das Ergebnis der Entscheidung, einen zur Hochschulreife führenden Bildungsweg einzuschlagen (1. Übergang), auf diesem Weg die Hochschulzugangsberechtigung zu erwerben (1. Abschluss), im Anschluss daran ein Studium aufzunehmen (2. Übergang) und dieses erfolgreich mit einem Hochschulabschluss zu beenden ( 2 . Abschluss).“

Auch Hillmert und Jacob (2010: 59) fragen ,where on the long way to the university degree does Germany ,lose“ its potential academics?" Dabei betrachten sie den gesamten Bildungsverlauf von der Grundschule bis zum Hochschulabschluss und schließen nicht nur den Standardweg (Grundschule Gymnasium - Abitur - Hochschule) einer akademischen Bildungslaufbahn ein, sondern auch Trackmobilität im Sekundarschulbereich, nachgeholte Bildungsabschlüsse und Studienabbrüche. ${ }^{49}$

Durch die Betrachtung der gesamten Bildungslaufbahn bleiben solche Studien zwangsläufig auf einem relativ hohen Abstraktionsniveau. In Anlehnung an Hillmert und Jacob (2010) bleibt demnach die Frage, wo und warum auf dem kurzen Weg vom letzten Schuljahr (und dem Erhalt der Hochschulzugangsberechtigung) bis zum Übergang in die Hochschule potentielle Studierende (aus sozial schwächeren Familien), ,verloren gehen“ (ebd.: 59). Um dies zu beantworten, ist es nötig, potentielle Stolpersteine auf diesem „,kurzen Weg“" näher zu betrachten.

Der kurze Weg zur Hochschule: college choice- und „Meilenstein“-Modelle

In der deutschsprachigen Hochschulforschung finden sich einige, meist recht breite Konzeptualisierungen des „Hochschulzugangs“, die dessen prozesshaf-

48 Eine wichtige Kritik an diesem Ansatz ist, dass er sich ausschließlich auf binäre Entscheidungen bezieht (Übergang in die nächste Stufe oder Ausscheiden), die die Entscheidungsoptionen in vielen Bildungssystemen mit parallelen Optionen - wie auch dem deutschen - nicht adäquat widerspiegeln (Hillmert und Jacob 2010: 61). Daher greifen zum Beispiel Breen und Jonsson (2000) oder Schimpl-Neimanns (2000) auf multinomiale Logitmodelle zurück, die multiple, parallele Entscheidungsoptionen erfassen können.

49 Zusammenfassend berichten sie, dass nicht nur der oft beschriebene Königsweg zum Hochschulabschluss sozial selektiv ist, sondern auch Zwischenphasen und institutionelle Korrekturmöglichkeiten: ,virtually all transitions along the academic track are selective in favor of children from higher educational backgrounds" (Hillmert und Jacob 2010: 70). 
ten Charakter unterstreichen. Kellermann (1984: 245) zum Beispiel versteht diesen als ,komplexen Prozeß [...], der sich zeitlich wenigstens von den Abschlußklassen der höheren Schulen bis hin zur gelungenen Aufnahme beziehungsweise erfolgreichen oder erfolglosen Beendigung des Studiums erstreckt". Lischka (2001: 30) kritisiert dieses breite Begriffsverständnis und definiert Hochschulzugang ,sowohl als Prozess als auch Ergebnis der Aufnahme des Studiums an Hochschulen“, wobei sie vier „Prozessphasen“ identifiziert: „,den Prozess der Information und Beratung, den Prozess des Prüfens, den Prozess der Entscheidung, den Prozess des Studienbeginns“ (ebd.). Mit dem Ziel, den Hochschulzugang in seiner Komplexität darstellen zu können, nennen beide Autor/innen eine Vielzahl an Determinanten auf verschiedenen Ebenen. Dabei bleiben sie bezüglich der genauen Definition der Phasen, ihrer Ausgestaltung, relevanten Ursachen und Wirkungen allerdings auf einem sehr abstrakten Niveau, sodass die Ausführungen für eine detaillierte Analyse der einzelnen Phasen allenfalls eine grobe Orientierung bieten können.

Was genau geschieht im Zeitraum vom letzten Schuljahr vor Erhalt der Hochschulzugangsberechtigung bis zum realisierten Übergang ins Studium? Um der Antwort auf diese Frage näher zu kommen, orientiere ich mich in der vorliegenden Arbeit an der grundlegenden Idee von vornehmlich in den USA entwickelten und angewendeten Phasenmodellen, die sich spezifisch mit diesem Zeitraum befassen. Es handelt sich dabei zum einen um sogenannte college choice-Modelle und zum anderen um Modelle, die im Folgenden als Meilenstein-Modelle ${ }^{50}$ bezeichnet werden.

\section{College Choice-Modelle}

Vor dem Hintergrund eines prognostizierten Nachfragerückgangs nach Hochschulbildung (Paulsen 1990) entwickelten in den USA der 1980er Jahre verschiedene Autor/innen sogenannte college choice-Modelle (vgl. Chapman 1986, Hanson und Litten 1982, Hossler und Gallagher 1987, Hossler et al. 1999, Jackson 1982, Litten 1982). Der Marketinghintergrund dieser vornehmlich deskriptiven Publikationen, in denen potentiell Studierende als Kund/innen agieren und sich für die Hochschulen mit den für sie attraktivsten Markenmerkmalen entscheiden, ist dabei mehr (vgl. Litten 1982, Paulsen 1990) oder weniger (vgl. Jackson 1982) zentral. Es wäre allerdings verfehlt, diese Arbeiten auf ein reines Beratungsangebot für konkurrierende Hochschulen zu reduzieren, denn die entwickelten Modelle erfüllen vor allem auch eine theoreti-

50 Der Terminus „Meilenstein-Modelle“ ist im Gegensatz zu college choice-Modellen kein in der Literatur etablierter Begriff. Ich nutze diesen hier, da er den Kern der Modelle gut beschreibt, die sich damit beschäftigen, welche Phasen oder Schritte Schüler/innen vor der Studienaufnahme durchlaufen müssen oder sollten. Tatsächlich wird der Begriff ,,milestones“ in einigen der entsprechenden Studien verwendet, um die Relevanz dieser Phasen zu verdeutlichen. 
sche und analytische Ordnungsfunktion, die vielen ökonomischen und soziologischen Forschungsarbeiten als Grundlage dient (vgl. DesJardins et al. 2006, Drewes und Michael 2006, Hurtado et al. 1997, Niu und Tienda 2008, Turley 2009). College choice-Modelle lenken dabei den Fokus zum einen auf den prozessualen, aus verschiedenen Phasen bestehenden Charakter des Übergangs in die Hochschule und zum anderen auf die Relevanz institutioneller Merkmale (in Interaktion mit individuellen Merkmalen) für die Studienwahl. Damit leisten sie einen wichtigen Beitrag zum besseren Verständnis des Übergangs in die Hochschule.

Die verschiedenen Modelle integrieren ökonomische (utility maximization) und soziologische (status attainment) Ansätze und teilen dabei einen Phasenansatz: Je nach Differenzierungsgrad werden zwischen drei und sieben Phasen unterschieden, die ungefähr den gleichen Zeitraum umfassen (einige Jahre vor Schulabschluss bis zur finalen Hochschulimmatrikulation) und sich vor allem in den genutzten Labels und der genauen Abgrenzung unterscheiden. ${ }^{51}$

Zur Einbettung der ersten Phase - der Prädispositionsphase - wird zumeist auf soziologische und sozialpsychologische Erkenntnisse aus der status attainment-Forschung (vgl. Sewell et al. 2004) zurückgegriffen. In dieser Phase wird vor allem der Einfluss der individuellen Schulleistung, des familiären Hintergrunds, des sozialen Netzwerkes und des Schulkontextes auf die Entwicklung von Studienaspirationen oder -intentionen hervorgehoben (zur Abgrenzung der Konzepte Aspiration und Intention siehe Abschnitt 4.1).

Erst nachdem Schüler/innen Studienaspirationen bzw. -intentionen entwickelt haben, treten sie in die zweite Phase des college choice-Prozesses - die Suchphase - ein und wägen zwischen verschiedenen Hochschulen ab, sodass hochschulbezogene, institutionelle Charakteristika an Einfluss gewinnen. In dieser Such- und Exklusionsphase kommt es zum einen zu einer vermehrten Interaktion zwischen aktiv nach Informationen suchenden Schüler/innen und werbenden Hochschulen (vgl. Hossler und Gallagher 1987). Wie umfangreich und gekonnt diese Informationssuche ist, variiert dabei mit individuellen Merkmalen wie der sozialen Herkunft (vgl. Hossler et al. 1989, Litten 1982, Morgan 2010). Zum anderen legen Schüler/innen Exklusionskriterien fest (z.B. maximale Distanz oder maximale Kosten), anhand derer sie die Anzahl in Frage kommender Institutionen einschränken und somit ihr choice set bilden. Dabei interagieren individuelle (z.B. Schulleistung, Ressourcenausstattung) und institutionelle Merkmale (z.B. Distanz, Selektivität, Kosten) und führen zu unterschiedlicher Antizipation von Barrieren, die wiederum zu unterschiedlichen Exklusionskriterien führen (Hossler et al. 1989: 244). Dies führt dazu, dass die Studienoptionen sozial benachteiligter Schüler/innen ein-

51 So differenziert das Drei-Phasen-Modell von Hossler und Gallagher (1987) zwischen predisposition, search und choice, während Jackson (1982) zwischen preference, exclusion und evaluation unterscheidet. 
geschränkter sind (vgl. auch Hällsten 2010, Vergolini und Zanini 2015). Die zweite Phase mündet in Bewerbungen für in Frage kommende Institutionen.

In der letzten Phase - der Entscheidungsphase - treffen die Bewerber/innen ihre finale Entscheidung. Dabei wägen sie anhand individuell variierender Kriterien zwischen Institutionen ab, für die sie zugelassen wurden (vgl. Chapman 1986). Insbesondere Litten (1982) und Hanson und Litten (1982: 82) räumen auch den aktiven Part, den Hochschulen im Übergangsprozess einnehmen, ein und integrieren dies in ihr Modell: ,the college-selection process involves both students as information seekers and applicants and institutions that provide information and admit applicants." Der Fokus dieser und anderer college choice-Modelle liegt aber eindeutig auf individuellen Entscheidungen rationaler (Kosten und Nutzen abwägender) Akteure, während institutionelle Entscheidungen oft nur indirekt angesprochen werden, vor allem als Kriterium zur Exklusion bestimmter Hochschulen aufgrund antizipierter Zulassungshürden oder als letzter „Unsicherheitsfaktor“ (vgl. Chapman 1986, siehe auch Hossler et al. 1989).

Soziale Herkunft ist in den college choice-Modellen in der Regel auf zweifache Weise relevant. Zum einen indem sie in der ersten Phase auf die sozial eingebettete Entstehung von Aspirationen wirkt. Zum anderen können die festgelegten Exklusionskriterien mit der sozialen Herkunft (und anderen Merkmalen wie Schulleistung) variieren (vgl. Jackson 1982), was wiederum zu sozialgruppenspezifischen Bewerbungen und letztlichen Entscheidungen führen kann (vgl. Hoxby und Avery 2013, Griffith und Rothstein 2009, Turley et al. 2007). Insgesamt hat die Frage nach dem Einfluss, den die soziale Herkunft von Schüler/innen in den verschiedenen Phasen spielt, in den college choiceModellen jedoch nur eine untergeordnete Bedeutung.

Meilenstein-Modelle

Teilweise anknüpfend an die gerade vorgestellten college choice-Modelle stellen verschiedene Autor/innen Meilenstein-Modelle auf und testen diese empirisch (vgl. Avery und Kane 2004, Berkner und Chavez 1997, Cabrera und La Nasa 2001, Horn 1997, Klasik 2012, Roderick et al. 2011). Auch diese Modelle umfassen in etwa den Zeitraum der letzten Schuljahre bis hin zur Studienaufnahme und beinhalten jeweils unterschiedliche Meilensteine, die Schüler/innen bewältigen müssen, um eine realistische Chance auf ein Hochschulstudium - zumeist an einem four-year college - zu haben.

Cabrera und La Nasa (2001) zum Beispiel beschränken sich auf drei Schritte: die richtige Kurswahl in der Schule, das Erlangen des Schulabschlusses und das Versenden von Bewerbungen. Horn (1997) betrachtet hingegen 
fünf Meilensteine und Klasik (2012) sogar neun. ${ }^{52}$ Auf Grundlage der Daten der Education Longitudinal Study: 2002 zeigt Klasik (2012), dass der Anteil an verbleibenden Schüler/innen mit fast jedem Meilenstein auf dem Weg zur Hochschule abnimmt: Während knapp über $90 \%$ der Zehntklässler/innen eine Studienintention äußern, studieren vier Jahr später etwas weniger als $50 \%$ an einem four-year college. Schüler/innen aus einkommensschwachen Familien erreichen jeden der neun Meilensteine signifikant seltener als ihre einkommensstarken Peers, ${ }^{53}$ wobei die Stärke der Herkunftsdifferenz je nach Meilenstein variiert: Während diese bezüglich des ersten Schrittes (Studienintention in Klasse 10) nur 8 Prozentpunkte beträgt, liegt sie bei einigen späteren Schritten deutlich höher (Bewerbung: 25 Prozentpunkte, Zulassung: 32, Immatrikulation: 38). Selbst die Bewerbung für finanzielle Unterstützung wird seltener von Schüler/innen in der niedrigsten Einkommensklasse getätigt (9 Prozentpunkte).

Während für den US-amerikanischen Kontext diese kleinteilige Differenzierung notwendiger Schritte durchaus angemessen erscheint, stellen bestimmte Phasen - zum Beispiel das Absolvieren von Tests oder die Inanspruchnahme von Beratungsangeboten - im deutschen Kontext (noch?) keinen standardmäßigen Meilenstein auf dem Weg in die Hochschule dar. Hierfür bietet die Differenzierung von Roderick et al. (2011) eine angemessenere Schablone. Die Autorinnen beschränken ihr Sample bestehend aus Chicago high school seniors auf diejenigen mit einer Studienaspiration und analysieren, inwiefern diese (1) auch eine Studienintention äußern (72\%), (2) sich für fouryear colleges bewerben (59\%), (3) zugelassen werden (51\%) und (4) sich final immatrikulieren (41\%). Dabei sind vor allem Leistungsindikatoren sehr prädiktiv für das Erreichen der einzelnen Meilensteine. Die im Sample enthaltenen Schüler/innen besuchen vorwiegend Schulen, in denen (ökonomisch) benachteiligte und minority Schüler/innen deutlich überrepräsentiert sind. Darüber hinaus steht deren soziale Herkunft nicht im Fokus der Studie.

Die Studie von Roderick et al. (2011) ist konzeptionell hochgradig relevant für die vorliegende Arbeit. Dies ergibt sich zum einen schon daraus, dass die Autorinnen ihr Sample auf Schüler/innen mit Studienaspirationen beschränken und untersuchen, inwiefern diese realisiert werden können. Damit kommen sie der zentralen Forschungsfrage dieser Arbeit sehr nahe. Die Relevanz ergibt sich jedoch nicht nur aus der Sampleeinschränkung, sondern

52 1) Studienintention in Klasse 10, 2) Studienintention in Klasse 12, 3) Teilnahme an Eignungstests, 4) Erbringung ausreichender Schulleistung, 5) Aufsuchen eines college counselor 6) Versenden von Bewerbungen 7) Zulassungen durch Hochschulen 8) Bewerbungen für finanzielle Unterstützung und 9) finale Immatrikulation.

53 Klasik (2012) differenziert fünf Einkommenskategorien. Die zitierten Ergebnisse beziehen sich jeweils auf Differenzen zwischen den Extremwerten (Haushaltseinkommen bis 25.000 Dollar vs. mehr als 75.000 Dollar). 
ebenso aus den identifizierten Phasen oder Meilensteinen, die auch in der vorliegenden Arbeit eine zentrale Rolle spielen.

Sie dient daher als wichtiger Ausgangspunkt, der jedoch konzeptionell erweitert werden soll. So stehen im Folgenden im Gegensatz zu Roderick et al. (2011) soziale Herkunftsunterschiede in den verschiedenen Phasen der Realisierung von Studienaspirationen und zugrunde liegende Mechanismen im Fokus. In Anlehnung an die vorgestellten college choice-Modelle werden zudem institutionelle Merkmale von Hochschulen und deren Interaktion mit der sozialen Herkunft der Schüler/innen stärker in den Mittelpunkt rücken.

\subsection{Zusammenfassung}

In diesem Kapitel standen zunächst im deutschen Kontext verbreitete Ansätze zur Untersuchung sozialer Selektivität beim Übergang ins Studium im Mittelpunkt. Innerhalb des institutionellen Rahmens treffen dem Hauptstrang der deutschen „Übergangsforschung“ zufolge Individuen rationale - und sozialgruppenspezifische - Entscheidungen für oder gegen ein Studium und für oder gegen bestimmte Studienoptionen. In den folgenden Kapiteln werden diese und weitere Ansätze und Befunde (erneut) aufgegriffen, stärker theoretisch eingebettet und diskutiert, inwiefern sie auf den Gegenstand der vorliegenden Arbeit - die Realisierung von Studienaspirationen - übertragen werden können.

Empirisch stehen in der Forschung zum deutschen Kontext quantitative Studien im Vordergrund, die zumeist Studienintentionen oder bereits realisierte Übergänge betrachten. Diesem Vorgehen liegt die (teils implizite) Annahme zugrunde, dass aufgrund der als gering angesehenen Selektionsleistung des Hochschulsystems Intentionen realisierte Übergänge gut approximieren und vice versa. Eine möglicherweise einschränkende Wirkung durch institutionelle Selektionsprozesse wird bestenfalls diskutiert (vgl. Becker et al. 2010, Lörz 2012, Watermann und Maaz 2006). Wie in Kapitel 2 gezeigt wurde, ist die Gatekeepingfunktion der Hochschulen zwar innerhalb des rechtlichen Rahmens eingeschränkt, doch findet mittlerweile für eine Mehrheit der Studiengänge eine Auswahl oder über die Hochschulzugangsberechtigung hinausgehende Eignungsfeststellung statt. Daraus folgt auch, dass einige der bereits stark vorselektierten hochschulzugangsberechtigten Studienaspirant/innen ihren Studienwunsch möglicherweise nicht umsetzen können.

Inwiefern können Schüler/innen ihre Studienaspirationen umsetzen und gibt es hierbei soziale Herkunftsunterschiede? Um sich dieser Frage anzunähern, bleibt die vorliegende Arbeit nicht bei Intentionen stehen und setzt nicht erst bei realisierten Übergängen an, sondern betrachtet die dazwischenliegenden Phasen. Ich fokussiere dabei auf Schüler/innen mit dem Wunsch zu stu- 
dieren und schließe diejenigen aus den folgenden Betrachtungen aus, die ein Studium gar nicht erst anstreben und für die sich die Frage nach Übergangschancen daher nur sehr eingeschränkt stellt. Erstere verfolge ich auf dem ,kurzen Weg in die Hochschule“. Hierbei dienen die vorgestellten college choiceund Meilenstein-Modelle (insbesondere die Studie von Roderick et al. (2011)), die den Übergang ins Studium in miteinander verbundene Phasen unterteilen, als Ausgangspunkt und Orientierung. 


\section{Aspirationen und ihre Umsetzung: konzeptionelle und empirische Bestandsaufnahme}

Im vorangegangenen Kapitel lag der Fokus auf zentralen Ansätzen der (deutschen) Forschung zu sozialer Ungleichheit beim Übergang ins Studium. In den folgenden Kapiteln wird der Fokus konzeptionell, theoretisch und empirisch stärker zum Forschungsgegenstand der vorliegenden Arbeit gelenkt: der Frage nach sozialer Ungleichheit in den Phasen der Umsetzung von Studienaspirationen.

In Kapitel 4 soll hierfür zunächst eine konzeptionelle Grundlage gelegt, der Forschungsstand aufbereitet und existierende Forschungslücken identifiziert werden. Konkreter werden dabei folgende Ziele verfolgt: Zunächst soll das sehr vielseitig genutzte Konzept Aspirationen ein- und abgegrenzt und somit eine Definitionsgrundlage geschaffen werden, der die nachstehenden Kapitel folgen (4.1). Im Anschluss daran werden empirische Befunde zur Umsetzung von Studienaspirationen und dabei auftretender sozialer Ungleichheit präsentiert (4.2). Diese stammen vornehmlich aus den USA, wo diesem Thema im Zuge der Ausbreitung des ,college-for-all“" Mythos (vgl. Rosenbaum 2001) größere Beachtung geschenkt wird. Erste Befunde liegen allerdings auch für Deutschland vor. Zuletzt wird - wie in Kapitel 3 angekündigt - die Umsetzung von Studienaspirationen modellhaft in vier zentrale Phasen unterteilt, die im empirischen Teil der vorliegenden Arbeit aufgegriffen werden: (1) die Übersetzung von Studienaspirationen in realistischere Intentionen, (2) Bewerbungsentscheidungen und Bewerbungsmuster, (3) institutionelle Selektionsprozesse und Zulassungschancen und (4) die Entscheidung, ein Studium tatsächlich aufzunehmen. Empirische Ansätze und Befunde zu den vier Phasen werden in Abschnitt 4.3 vorgestellt und abschließend diskutiert, ob soziale Ungleichheit bei der Umsetzung von Studienaspirationen eher in den Phasen der Selbstselektion oder der Fremdselektion zustande kommt.

\subsection{Aspirationen: Abgrenzung eines vielseitigen Begriffs}

Aspirationen sind wichtiger Bestandteil vieler soziologischer Theorien, die sich mit Bildungsentscheidungen und Bildungsungleichheiten beschäftigen: Hierzu gehören so unterschiedliche Ansätze wie das status attainment-Modell, Bourdieus Reproduktionstheorie oder der rational choice-Ansatz (RC) (siehe Abschnitt 5.1). Somit ist es nicht verwunderlich, dass Aspirationen und verwandte Begriffe zu Schlagwörtern der soziologischen Bildungsforschung ge- 
worden sind, die in vielen Veröffentlichungen auf unterschiedliche Art und Weise in Verbindung mit Bildungsverläufen und Bildungsungleichheit gebracht werden. Doch was genau unter dem Begriff zu verstehen ist, wird häufig nicht eindeutig definiert. Entsprechend existiert eine ,terminological confusion“ (Haller 1968: 485, vgl. auch Kerckhoff 1976, Morgan 2007, Stocké et al. 2011, Trebbels 2015, Vaisey 2010) bestehend aus Begriffen wie Erwartungen, Plänen, Zielen, Wünschen, Normen, Ambitionen, Motivationen, Präferenzen, Neigungen, Absichten und Intentionen, die zum Teil synonym verwendet werden, zum Teil aber auch Unterschiedliches bedeuten. Die zentrale Differenzierungslinie läuft dabei zwischen idealistischen Bildungswünschen (hier: Aspirationen) auf der einen und realistischen Bildungsintentionen auf der anderen Seite.

Neben dem unbestreitbaren Vorteil terminologischer und empirischer Präzision, ,[to] avoid conflating what people want to happen with what they predict will happen" (Vaisey 2010: 84, Hervorhebung im Original) ist es auch aus einer theoretischen Perspektive wichtig, zwischen verschiedenen Bedeutungen von „Aspirationen“ zu differenzieren, da Ursachen und Wirkungen, wenn nicht in ihrer Richtung, so zumindest in ihrer Stärke zwischen verschiedenen Konzepten variieren können. Entsprechend forderte Haller (1968: 484) bereits früh, zwischen realistischen und idealistischen Aspirationen zu unterscheiden: „the former being what the person thought he might really be able to attain, and the latter what he hoped to attain if everything went well". In der englischsprachigen Literatur ist auch die Trennung zwischen expectations und aspirations verbreitet ${ }^{54}$ während in deutschsprachigen Texten oft auf die Begriffe „Absichten“ (z.B. Helbig et al. 2015, Lörz 2012) oder „Intentionen“ zurückgegriffen wird, um konkretere Bildungspläne im Sinne von realistischen Aspirationen zu benennen (vgl. Maaz 2006, Obermeier und Schneider 2015, Watermann und Maaz 2006, Weiss und Steininger 2013). Im Weiteren folge ich dieser terminologischen Trennung: Aspiration - Intention.

In der Literatur werden Aspirationen oft als „,desired outcomes“ (Hauser und Anderson 1991: 271) definiert, als „Hoffnungen“ und „Träume“ (Katthab 2015: 732) oder ,innere Selbstverpflichtungen“ (Stocké 2013: 269), die sozialisationsbasierte Werte widerspiegeln und eine wichtige Motivationsquelle darstellen (Stocké et. al 2011: 107). Intentionen hingegen bezeichnen „realistic appraisals" (Morgan 2007: 1529), beinhalten "real-life perceptions of what is possible" (Lloyed et al. 2008: 3) und besitzen daher in der Regel eine größere Handlungsrelevanz (Haller 1968, Vaisey 2010). Entsprechend entstehen sie durch eine bewusste Auseinandersetzung mit wahrgenommenen Barrieren und werden auch als ,antizipierte“ (Becker 2010: 6), ,intendierte“ (Stocké 2005b:

54 Der Begriff Erwartungen ist allerdings nicht trennscharf, da er sowohl für realistische Aspirationen von Schüler/innen als auch für Erwartungen aus dem sozialen Umfeld, die an die individuellen Schüler/innen gestellt werden, genutzt wird (vgl. Williams 1972). 
2) oder „Vorweggenommene Bildungsentscheidungen“ (Kurz und Paulus 2008: 5490) interpretiert. Bildungsintentionen und letztliche Entscheidungen werden daher oft mithilfe der gleichen Faktoren - häufig Komponenten der rationalen Wahl - modelliert (vgl. Becker 2010, Becker und Hecken 2007, Lörz 2012, Stocké 2005b). Der Fokus verlagert sich vom durch die soziale Umwelt geprägten Individuum auf rational abwägende Akteure (vgl. Jencks et al. 1983, Morgan 1998), die ihre Aspirationen im Lichte zur Verfügung stehender Informationen, wahrgenommener Barrieren und der eigenen Ressourcenausstattung reflektieren und entsprechend in Intentionen übersetzen und weiterverfolgen oder verwerfen. ${ }^{55}$

Aspirationen sind keine konstanten Größen, sondern werden mit herannahender Entscheidung zum Teil korrigiert und sind somit je nach Messzeitpunkt noch mehr oder weniger ,,idealistisch“. Andersherum können auch Intentionen - je nach Messzeitpunkt (und Messung) - mehr oder weniger stark durch idealistische Wünsche geprägt und mehr oder weniger stark an wahrgenommene, ,realistische“ Gegebenheiten angepasst sein (vgl. Alexander und Cook 1979, Goyette 2008: 477). Eine klare Trennung zwischen beiden Konzepten als ,polar points on an [intention]-aspiration continuum“" (Stephenson 1957: 205 ) ist daher zwar theoretisch sinnvoll, aber empirisch herausfordernd.

Daher werden beide Konzepte auch empirisch - und entsprechend der häufig fehlenden konzeptionellen Differenzierung - oft nicht voneinander getrennt, sondern die Frage nach „Bildungsplänen“ unabhängig vom zugrundeliegenden Konzept und der theoretischen Einbettung genutzt (vgl. Morgan 2007: 1529). Sofern eine getrennte Messung und Auswertung erfolgt (vgl. Abschnitt 7.1.1), zeigt sich erwartungsgemäß, dass beide zwar stark miteinander korrelieren, das Aspirationsniveau aber im Durchschnitt meist höher ist als das Intentionsniveau - vor allem bei benachteiligten Gruppen (vgl. Haller 1968, Hanson 1994, Katthab 2015, Stephenson 1957, Vaisey 2010). Dies stützt die theoretische Grundannahme, dass Aspirationen stärker von Wünschen und Idealvorstellungen geprägt sind als von realistischen (und rationalen) Abwägungen.

Dies bedeutet jedoch nicht zwangsläufig, dass Aspirationen nicht im Zusammenhang mit sozialer Herkunft stehen (für eine theoretische Diskussion

55 Birgit Becker (2010: 16) präsentiert ein entsprechendes theoretisches Modell, das die unterschiedlichen Entstehungs- und Wirkungslogiken von Aspirationen und Intentionen enthält. Demnach beeinflussen (sozialgruppenspezifische) Einstellungen, Normen und Werte in einem ersten Schritt Aspirationen. Intentionen werden durch Aspirationen geprägt sowie zusätzlich durch Schulleistung, soziale und kulturelle Ressourcen sowie die Wahrnehmung externer Barrieren beeinflusst, die in den rationalen Entscheidungsprozess mit einbezogen werden. Wie wahrscheinlich die Realisierung von Intentionen ist - d.h. wie realistisch sie wirklich sind -, hängt hier entscheidend davon ab, wie gut Barrieren mithilfe der richtigen Systemkenntnis antizipiert werden können (siehe hierzu auch Azjen 1991). 
hierzu siehe Abschnitt 5.1). Zum Beispiel ist zwar im US-amerikanischen Kontext der Anteil an Schüler/innen mit Studienaspirationen in allen Herkunftsgruppen hoch, doch besteht auch ein deutlicher Unterschied von 20 Prozentpunkten zwischen als arm klassifizierten Jugendlichen und solchen aus einkommensstärkeren Familien (70 vs. 90\%) (Vaisey 2010: 87). Für Deutschland gibt es bisher keine Studie, die die Anteile an (Fach-)Abiturient/innen mit Studienaspiration getrennt nach sozialer Herkunft berichtet. ${ }^{56} \mathrm{Im}$ Gegensatz zum US-amerikanischen contest mobility regime, das das Ziel verfolgt, möglichst viele Kandidat/innen möglichst lange „im Rennen“ zu halten (Turner 1960: 855, Kerckhoff 1977), ist das deutsche sponsored mobility regime - wie in Kapitel 2 beschrieben - durch frühe (sozial selektive) Sortierung der Schüler/innen und zahlreiche Feedback-Mechanismen gekennzeichnet, sodass Aspirationen hier früher ,realistisch“ werden (vgl. Buchmann und Park 2009). Innerhalb der bereits positiv, auch auf Grundlage familiärer Bildungsaspirationen selektierten Gruppe deutscher (Fach-)Abiturient/innen könnte daher zum einen davon ausgegangen werden, dass Studienaspirationen kaum mit der sozialen Herkunft variieren. Zum anderen stehen deutschen Studienberechtigten im Gegensatz zu US-amerikanischen high school-Absolvent/innen die beschriebenen attraktiven und daher ,ablenkenden“ (Shavit und Müller 2000) Ausbildungsoptionen zur Verfügung, sodass selbst für diese Gruppe ein $\mathrm{Zu}-$ sammenhang zwischen sozialer Herkunft und Aspiration vermutet werden kann.

Auch wenn Aspirationen eine wichtige Motivationsquelle für individuelles Handeln darstellen (vgl. Haller 1968), bedeutet dies nicht automatisch, dass sie in jedem Fall umgesetzt werden (können). Im Folgenden sollen empirische Studien vorgestellt werden, die sich mit eben diesem Thema befassen.

\subsection{Herkunftsunterschiede bei der Umsetzung von Studienaspirationen: empirische Befunde}

Während es umfassende Forschung zum Übergang ins Studium und dessen sozialer Selektivität gibt (vgl. Kapitel 3), beschäftigen sich deutlich weniger

56 Auf Grundlage des DZHW Studienberechtigtenpanels berichten Schneider und Franke (2014: 131ff.) beispielhaft für den Abiturjahrgang 2012, dass ein halbes Jahr vor dem voraussichtlichen Erhalt des (Fach-)Abiturs 63\% der Schüler/innen aus Akademikerfamilien, aber nur 49\% der Schüler/innen aus Nicht-Akademikerfamilien sicher/wahrscheinlich beabsichtigen, ein Studium aufzunehmen. Inwiefern diese Ergebnisse auch auf Aspirationen im Sinne eines Studienwunsches zutreffen, kann hieraus nicht abgeleitet werden. Dies wird auf Grundlage der in der vorliegenden Arbeit genutzten Daten in Abschnitt 6.4 näher betrachtet. 
Studien mit der Umsetzung von Studienaspirationen. Zudem bleibt trotz der zunehmenden Anerkennung der Wichtigkeit einer theoretischen und analytischen Trennung zwischen Aspirationen und Intentionen in vielen Studien unklar, welches Verständnis dem jeweiligen ,Aspirationskonzept“" zugrunde liegt (vgl. Stocké et al. 2011: 107). Empirisch dienen als Ausgangspunkt für die Analysen zu Umsetzungschancen oft Surveyfragen, die konzeptionell eher Intentionen abbilden (vgl. Morgan 2007). Im folgenden Literaturüberblick werden daher nicht ausschließlich Studien eingeschlossen, die eindeutig Aspirationen und deren Umsetzungschancen messen, sondern auch solche, in denen zumindest empirisch eher Intentionen erfasst werden. Daraus resultierende Besonderheiten und Konsequenzen werden an den gegebenen Stellen reflektiert. Nachfolgend werden zunächst Studien betrachtet, die sich generell mit der Umsetzung von Studienaspirationen (bzw. häufig: Studienintentionen) beschäftigen. Im Anschluss daran werden einige Befunde zur Umsetzung von Studienfachaspirationen berichtet, die im deutschen Kontext aufgrund der starken fachlichen Variation in den Studienchancen (vgl. Abschnitt 2.2.3) (sozialgruppenspezifisch) eingeschränkt sein könnte.

\section{Die Umsetzung von Studienaspirationen}

Bevor mit Fokus auf Studien zum deutschen Kontext einzelne Befunde eingehender diskutiert werden, sollen zunächst einige generelle Anmerkungen zum Forschungsstand geliefert werden: Erstens zeigt sich, wie bereits angedeutet, dass Studienintentionen und deren Umsetzung häufiger empirisch untersucht werden als Aspirationen (vgl. Alexander und Cook 1979, Avery und Kane 2004, Ehlert et al. 2017a, Hossler et al. 1999, Grodsky und Riegele-Crumb 2010, Spangenberg und Quast 2016; zu den Außnahmen gehören: Lloyed et al. 2008, Roderick et al. 2011). Zweitens betreffen Unterschiede in den Operationalisierungen nicht nur das Aspirations- oder Intentionsmaß, sondern auch die Messung der erfolgreichen „Umsetzung“, die teils über Bewerbungen (vgl. Ehlert et al. 2017a, Grodsky und Riegele-Crumb 2010, Lloyed et al. 2008), teils über die finale Immatrikulation erfasst wird (vgl. Avery und Kane 2004, Hossler et al. 1999, Schneider und Saw 2016, Spangenberg und Quast 2016, Weiss und Steininger 2013). Drittens beschäftigen sich einige Studien zwar sowohl mit Aspirationen/Intentionen als auch Bewerbungen/Übergängen, jedoch kann die Umsetzung vorhandener Aspirationen nur indirekt abgeleitet werden (vgl. Klasik 2012, Lloyed et al. 2008, Maaz 2006, Schneider und Franke 2014). Dies ist dann der Fall, wenn die aggregierten Anteile an Schüler/innen mit Studienaspiration/-intention sowie mit Bewerbungen/Übergängen separat ausgewiesen werden. Viertens wird deutlich, dass dieser Forschungsbereich die stärkste „Tradition“ in den USA hat, wo die Gruppe der Hochschulzugangsberechtigten aufgrund der im Vergleich zu Deutschland weitaus geringeren Stratifizierung des Schulsystems groß und heterogen ist 
und gleichzeitig hohe Bildungsaspirationen weit verbreitet sind (vgl. Domina et al. 2011, Goyette 2008, Rosenbaum 2001).

Für diesen Kontext zeigen verschiedene Studien basierend auf unterschiedlichen Datenquellen, dass ein beträchtlicher und stark variierender Anteil an Schüler/innen - zwischen etwa 20\% (Hossler et al. 1999) und 60\% (Roderick et al. 2011) - ihre Bildungsaspiration bzw. -intentionen nicht umsetzen (können). Diese großen Abweichungen können verschiedene Ursachen haben, die zum Teil im vorangegangenen Absatz bereits angesprochen wurden. ${ }^{57}$

Die einzige mir bekannte Studie, die bei Aspirationen ansetzt und die finale Umsetzung im Sinne einer Studienaufnahme betrachtet, ist die bereits in Abschnitt 3.3 vorgestellte Arbeit von Roderick et al. (2011). Hier zeigt sich, dass nur $41 \%$ derjenigen Chicago high school seniors mit einer hohen Aspiration (für einen four-year college Abschluss) im Anschluss an ihren Schulabschluss ein Studium aufnehmen (ebd.: 185). Eine Ursache für die im Vergleich zu anderen Studien sehr geringe Umsetzungswahrscheinlichkeit kann darin begründet liegen, dass mit idealistischen Aspirationen und deren Umsetzung durch eine Studienaufnahme zwei ,weit"voneinander entfernte Phasen untersucht wurden. ${ }^{58}$ Ein weiterer Grund ist in der Datengrundlage zu suchen: Die im Chicago Sample enthaltenen Schüler/innen besuchen vorwiegend Schulen, in denen (ökonomisch) benachteiligte und minority Schüler/innen deutlich überrepräsentiert sind (ebd.: 183). Dies liefert erste Hinweise darauf, dass eine Diskrepanz zwischen Aspirationen und deren Umsetzung insbesondere auf sozial benachteiligte Schüler/innen zutreffen könnte. Bestätigung hierfür findet sich in einer Reihe von anderen Studien (etwa Grodsky und Riegele-Crumb 2010, Hanson 1994, Klasik 2012, McCarron und Inklas 2006).

Für Deutschland gibt es meines Wissens nach kaum empirische Evidenz zur Umsetzung von Studienaspirationen. Auf Grundlage von Längsschnittdaten berichten einige Autor/innen getrennt die Anteile an Schüler/innen mit Studienintention und tatsächlich erfolgter Studienaufnahme. Anhand der TOSCA Daten nennt etwa Maaz (2006: 212) für baden-württembergische Abiturient/innen am Ende ihres letzten Schuljahres eine Intentionsquote von 75\%.

57 Erstens greifen die Studien auf sehr unterschiedliche Daten zurück, die sich auf verschiedene Kohorten beziehen (z.B. Alexander und Cook 1979: 1968er high school seniors; Schneider und Saw 2016: 2012 high school seniors). Zweitens liegen den Studien die beschriebenen unterschiedlichen Operationalisierungen bezüglich des Aspirationsmaßes und der Umsetzung zugrunde. Drittens wurden sowohl Aspirationen/Intentionen als auch deren Umsetzung zu unterschiedlichen Zeitpunkten individueller Bildungsverläufe gemessen (Aspirationen: Klasse 9 bis 12; Umsetzung: während des letzten Schuljahres (Bewerbung) oder direkt im Anschluss an den Schulabschluss bzw. in den Folgejahren (Übergang)).

58 Tatsächlich vergrößert sich der Anteil an „Umsetzungen“ auf 57\%, wenn Befragte mit Studienintention als Ausgangspunkt genommen werden, oder auf 59\%, wenn Bewerbungen als Umsetzungsmaß dienen. 
Zwei Jahre später studieren 65\% aller Befragten. Schneider und Franke (2014: $131,135)$ berichten auf Grundlage des DZHW Studienberechtigtenpanels (Abiturient/innenjahrgang 2012) den Anteil an Schüler/innen mit eventueller bis sicherer Studienintention im letzten Schuljahr (79\%) und den Anteil an Schüler/innen mit realisierten Übergängen direkt nach dem Abitur sowie geäußerten Plänen einer späteren Studienaufnahme (insgesamt 74\%). Bei Befragten aus akademisch gebildeten Elternhäusern liegen sowohl die Anteile für die Intention als auch für die (geplante) Studienaufnahme mit $83 \%$ und $80 \%$ höher als bei ihren Peers aus nicht-akademischen Elternhäusern $(72 \%$ und $66 \%$ ). Herkunftsunterschiede bezüglich der indirekt approximierten Realisierungschancen (Akademikerkinder: 87\%, Nichtakademikerkinder: 83\%) sind demnach mit circa 4 Prozentpunkten nicht substantiell und weisen darauf hin, dass soziale Ungleichheit vor allem bei der Herausbildung von Studienintentionen entsteht. Anhand dieser Aggregatwerte ist es - wie weiter oben bereits angedeutet - allerdings nur bedingt möglich, von der Studienintention auf deren Umsetzungswahrscheinlichkeit zu schließen, da es zu Verschiebungen zwischen den Gruppen kommen kann, wenn Abiturient/innen ohne Studienintention doch ein Studium aufnehmen. ${ }^{59}$ Zudem bleibt offen, wer die Pläne für eine spätere Studienaufnahme tatsächlich umsetzt und ob hierbei soziale Herkunftsunterschiede beobachtet werden können. Die folgenden Studien geben hierüber zum Teil Aufschluss.

Neben diesen deskriptiven Gegenüberstellungen von Studienberechtigten mit Studienintention und realisiertem bzw. geplantem Übergang gibt es für den deutschen Kontext einige wenige weitere Studien, die auch multivariat untersuchen, inwiefern Schüler/innen mit Studienaspiration/-intention ein Studium aufnehmen (vgl. Helbig et al. 2015, Spangenberg und Quast 2016, Weiss und Steininger 2013). ${ }^{60}$

Weiss und Steininger (2013) nutzen hierfür die 1999er Kohorte des DZHW Studienberechtigtenpanels. Sie untersuchen die Übergänge ins Studium von vier Gruppen: Studienberechtigten mit einer „sofortigen“ Studien-

59 Tatsächlich kommt es im nachschulischen Verlauf häufig zu einer „Umorientierung zugunsten einer akademischen Qualifizierung“ (Spangenberg und Quast 2016: 19, siehe auch Spangenberg et al. 2011). Von den 2010er Studienberechtigten ohne Studienintention nahmen 36\% fünf Jahre nach Erhalt der Studienberechtigung doch ein Studium auf, häufig als Teil einer Doppelqualifizierung. Diese Umorientierung fand überdurchschnittlich häufig bei Akademikerkindern statt: „Akademikerkinder realisieren somit ihre Studienabsicht nicht nur öfter, sondern revidieren eine ursprüngliche Entscheidung gegen ein Hochschulstudium häufiger als Studienberechtigte ohne einen solchen Bildungshintergrund" (Spangenberg et al. 2011: 81, siehe auch Helbig et al. 2015: 26, Weiss und Steininger 2013).

60 Zudem untersuchen Ehlert et al. (2017a) die Bewerbungswahrscheinlichkeit von Schüler/innen mit Studienintention. Diese Studie wird unter 4.3.2 eingehender diskutiert. 
aspiration (Umsetzung insgesamt: 86\%), einer Studienaspiration für die Zeit nach einer Ausbildung (46\%) oder anderen Aktivität (90\%) und Unentschlossenen $(61 \%)$. Herkunftsdifferenzen können hier vor allem bezüglich verzögerter (nicht aber direkter) Übergänge beobachtet werden: Nichtakademikerkinder, die sich bei Erhalt der Hochschulzugangsberechtigung wünschten, das Studium zu einem späteren Zeitpunkt aufzunehmen, setzten diesen Wunsch bis dreieinhalb Jahre später seltener um als Akademikerkinder. Besonders stark ausgeprägt sind die Herkunftsdifferenzen zudem in der Gruppe derjenigen, die sich bezüglich ihres Studienwunsches nicht sicher waren. ${ }^{61}$ Multivariat können diese Herkunftsdifferenzen zum Teil durch die Abiturnote erklärt werden, nicht aber durch das Hinzufügen von Indikatoren der rationalen Wahl. Insgesamt sind die Ergebnisse von Weiss und Steininger aber mit Vorsicht zu interpretieren. In den frühen DZHW Erhebungen startete die Befragung erst ein halbes Jahr nach Erhalt des Abiturs und nicht wie für spätere Erhebungskohorten bereits während des letzten Schuljahres. Somit wurde die Aspirationsfrage retrospektiv erfasst ${ }^{62}$ was zu Erinnerungsfehlern und Rationalisierungsprozessen führen kann und somit zu einer zu häufigen Nennung von Aspirationen, die im Einklang mit dem tatsächlichen Verhalten stehen (vgl. Ayan und Isikal 2004, Sudman et al. 1996, für eine Diskussion dieses Problems siehe Weiss und Steiniger 2013: 194f.). Entsprechend ist eine Diskrepanz von insgesamt 14 Prozentpunkten zwischen sofortiger Studienaspiration und deren sofortiger Umsetzung beachtlicher, als es auf den ersten Blick scheinen mag. Und auch die fehlende Herkunftsdifferenz könnte zum Teil hierauf zurückzuführen sein.

Spangenberg und Quast (2016) nutzen die erste und dritte Welle einer späteren Studienberechtigtenkohorte (Jahrgang 2010) und umgehen so dieses Problem. Deskriptiv zeigt sich hier zunächst, dass 93\% derjenigen mit sicherer Studienintention ein halbes Jahr vor Erhalt der Hochschulzugangsberechtigung (insgesamt $70 \%$ der Befragten) fünf Jahre später ein Studium aufgenommen hatten. In multivariaten Modellen zeigt sich, dass die Umsetzungswahr-

61 Es wurden drei Herkunftsgruppen betrachtet: Studienberechtigte ohne, mit einem oder zwei akademisch gebildeten Eltern. Die Herkunftsunterschiede bezüglich der Realisierung eines Studienwunsches nach einer Ausbildung betragen: 12 und 25 Prozentpunkte (Ein- und Doppelakademikerkinder vs. Nichtakademikerkinder), bezüglich der Realisierung eines Studienwunsches nach einer anderen Aktivität: 7 und 16 Prozentpunkte, in der Gruppe der „Unentschiedenen“: 18 und 40 Prozentpunkte (Weiss und Steininger 2013: 196)

62 Im Text nutzen Weiss und Steininger (2013) sowohl die Begriffe ,aspirations“ als auch ,intentions“ und ,plans“. Sie lassen allerdings an mehreren Stellen zunächst vermuten, dass es sich bei der Messung eher um ein Intentions- als ein Aspirationsmaß handelt. So lautet ihr Titel: "Educational family background and the realization of educational career intentions“. Tatsächlich bezieht sich die Originalfrage im Wortlaut jedoch eher auf Bildungswünsche: „Hatten Sie bis zum Abgang von der Schule, an der Sie die Hochschulreife erworben haben, den Wunsch zu studieren?" 
scheinlichkeit einer sicheren Studienintention signifikant positiv mit dem Bildungshintergrund der Eltern (akademisch vs. nicht-akademisch), der Schulleistung und positiven Ertragserwartungen zusammenhängt (nicht aber mit der subjektiven Erfolgsaussicht sowie Kostenaspekten). Die Effektstärke für die Bildungsherkunft ist mit 2 bis 4 Prozentpunkten (je nach Modellspezifikation) nicht sehr substantiell. Anders die Schulleistung: Eine um einen Notenpunkt bessere Abiturnote erhöht die Realisierungswahrscheinlichkeit um circa 6 Prozentpunkte (für ähnliche Ergebnisse vgl. Helbig et al. 2015).

\section{Die Umsetzung von Studienfachaspirationen}

In Kapitel 2 wurde darauf verwiesen, dass sich Zugangshürden in Deutschland stark zwischen Studienfächern unterscheiden. Gleichzeitig ist das ,fachliche Interesse" ein vordergründiges Studienwahlmotiv, das von 90\% der Erstsemester genannt wird (Scheller et al. 2013). Neben der Frage, ob Studienaspirationen umgesetzt werden können, stellt sich daher im deutschen Kontext zudem die Frage, inwiefern dies für Fachaspirationen gilt, und wenn nicht: ob damit eher eine Umentscheidung (kein Studium) oder eine Umorientierung (anderes Studienfach) einhergeht. Die wenigen empirischen Befunde zu diesen Fragen sind rein deskriptiver Natur.

Die vom DZHW durchgeführte repräsentative Befragung von Studienanfänger/innen liefert hier erste Erkenntnisse mithilfe der Frage, ob sich die Studienwahl der Befragten mit ihren Wünschen und Zielen deckt (Scheller et al. 2013: 64-66). Der Anteil an Studierenden im ersten Semester, die diese Frage bejahten, lag 2011/12 bei $89 \%$. Nur $9 \%$ gaben an, dass sie lieber ein anderes Fach studiert hätten; 2\% hätten lieber eine Berufsausbildung begonnen. Zwischen den Studierenden unterschiedlicher Fächer liegt die Anteile zwischen 93\% (Medizin) und 84\% (Kunst). ${ }^{63}$ Die Daten beschränken sich auf Studierende, sodass keine Aussagen über Studienberechtigte getroffen werden können, die sich mehr oder weniger freiwillig gegen ein Studium entschieden haben. Die Annahme liegt nahe, dass es sich hierbei überdurchschnittlich häufig um Studienberechtigte handelt, die ihre Studienwünsche nicht umsetzen konnten.

Heine et al. (2007: 60ff.) liefern anhand des Studienberechtigtenpanels von 2002 erste Befunde zu der Frage, inwiefern ein halbes Jahr nach Erhalt der Hochschulzugangsberechtigung geäußerte Studienfachintentionen bis zu drei Jahre später umgesetzt wurden. Wie bei Weiss und Steiniger (2013) ist hierbei

63 Die Autor/innen betrachten auch die Realisierung des Hochschulwunsches. Hier ist der Anteil derjenigen, die diese Frage bejahten, mit insgesamt $75 \%$ niedriger und variiert zwischen $66 \%$ bei Studierenden des Fachs Medizin und 83\% in den Ingenieurswissenschaften. Zudem zeigt sich, dass Zulassungsbeschränkungen bei $74 \%$ der nicht-realisierten Hochschulwünsche als Ursache genannt wurden (ebd.: 112ff.). 
problematisch, dass die Fachintention ein halbes Jahr nach dem Abitur erfasst wurde, was erneut Umorientierungen und Rationalisierungsprozesse auf Grundlage erfolgloser Bewerbungen beinhalten kann. Außerdem beziehen sich die Ergebnisse damit nur auf Studienberechtigte, die nicht direkt mit dem Studium begonnen haben. ${ }^{64}$ Insgesamt konnten $68 \%$ der Befragten ihre Fachintention umsetzen. Die Variation nach angestrebtem Fach ist dabei beträchtlich: Während Intentionen für MINT Fächer und auch für Lehramtsstudiengänge häufig realisiert wurden (circa 80\%), kann dies deutlich seltener für Medizin $(50 \%)$, Kunst $(39 \%)$ und Psychologie (33\%) beobachtet werden. Hinzu kommt, dass Befragte mit diesbezüglichen Fachplänen drei Jahre später überdurchschnittlich häufig kein Studium aufgenommen haben (Medizin: 27\%, Kunst: 24\%, Psychologie: 20\%, Durchschnitt: 12\%). Wie die Autor/innen feststellen, deuten diese Ergebnisse darauf hin, dass die Umsetzungschancen von Studienfachintentionen mit fachspezifischen Zulassungshürden variieren. Medizin und Psychologie gehören zu den selektivsten zulassungsbeschränkten Fächern, während künstlerische Studiengänge zwar oft formal zulassungsfrei sind, aber in der Regel aufwändige Eignungsfeststellungsverfahren durchgeführt werden (vgl. Abschnitt 2.2.2). Die Autor/innen zeigen weiterhin, dass die Realisierungschancen - wenig überraschend - deutlich mit der Abiturnote zusammenhängen. Sie differenzieren jedoch nicht nach sozialer Herkunft.

Erkenntnisse hierzu liefern Spangenberg et al. (2011: 70ff.). Für die Studienberechtigtenkohorte 2006 zeigen sie zunächst, dass 67\% derjenigen, die ihre Studienintention direkt nach Schulende umsetzen, im zuvor intendierten Fach studieren. Von denjenigen, die nicht sofort studieren, dies aber weiterhin planen, bleiben $56 \%$ bei ihrer ursprünglichen Studienfachintention. In einem zweiten Schritt differenzieren die Autor/innen die Umsetzungschancen der Fachintentionen nach sozialer Herkunft und Studienfeld. ${ }^{65}$ Ausgewählte Ergebnisse sind in Abbildung 4.1 dargestellt. Insgesamt setzen 62\% der Akademikerkinder, aber nur 55\% der Nichtakademikerkinder ihre Studienfachintention um. Der Anteil der „Fachwechsler/innen“ unterscheidet sich kaum, dafür gehen Nichtakademikerkinder häufiger gar nicht ins Studium über. Ähnlich wie bereits bei Heine et al. (2007) variiert die Umsetzungswahrscheinlichkeit deutlich mit der ursprünglichen Fachintention. Akademikerkinder haben dabei in elf von 16 betrachteten Studienfeldern bessere Realisierungschancen als ihre Peers aus Elternhäusern ohne akademische Bildung. Am deutlichsten zeigt

64 Für den Jahrgang 2002 handelt es sich dabei um circa 65\% der Studienberechtigten. Der Anteil direkter Übergänge liegt aufgrund der damals noch nicht abgeschafften Wehrpflicht unter dem heutigen Anteil von etwa 50\%.

65 Dabei werden die Übereinstimmungen/Wechsel zwischen Welle 1 (Intention vor Schulabschluss) und Welle 2 (Studienfach nach Schulabschluss) sowie zwischen Welle 2 (Intention nach Schulabschluss, sofern nicht übergegangen) und Welle 3 (Studium drei Jahre später) zusammengefasst. 
sich dies beim Fach Medizin mit einem Herkunftsunterschied von 31 Prozentpunkten.

Abb. 4.1: Studienfachintention und -realisierung nach Bildungsherkunft

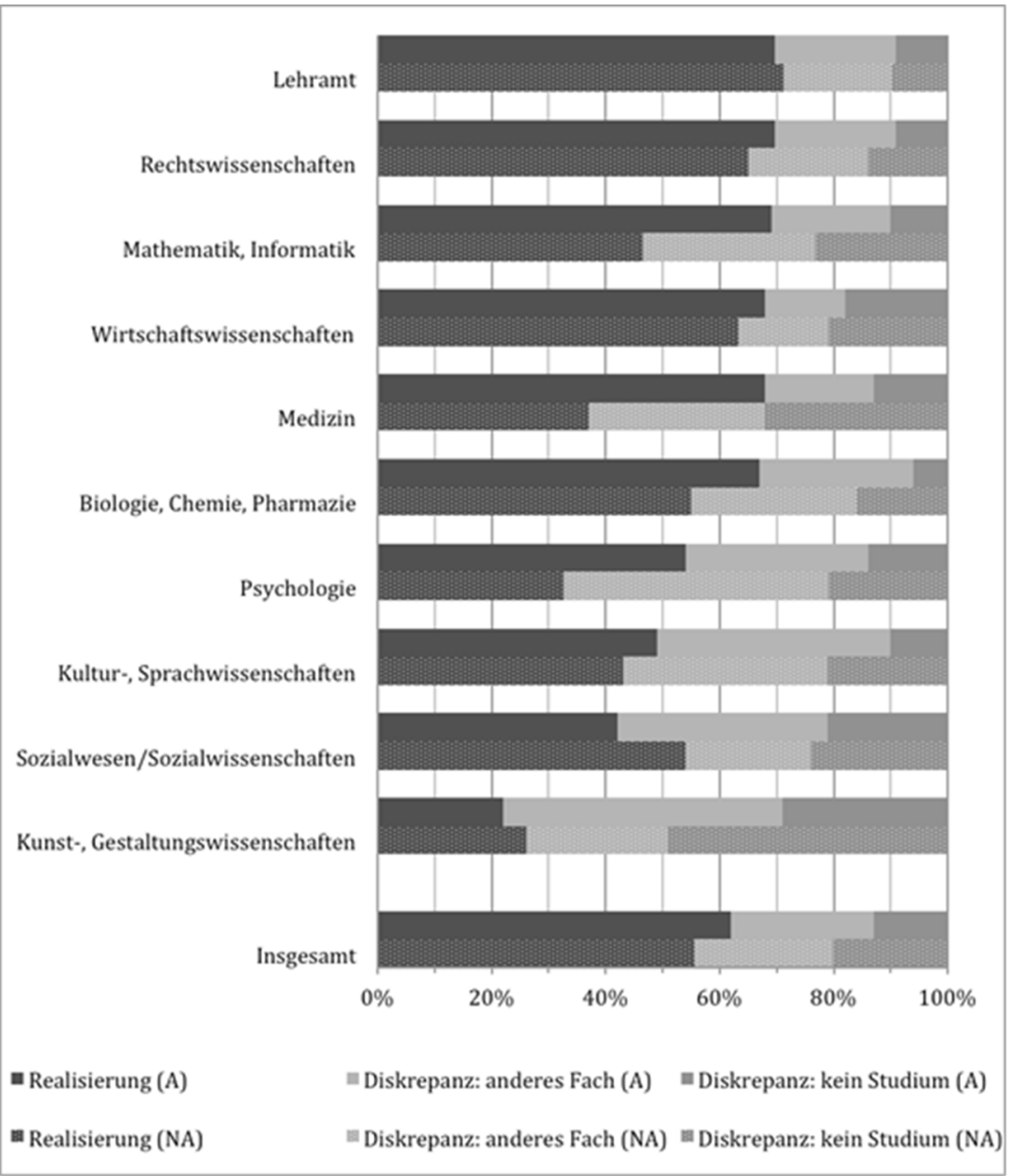

Quelle: Spangenberg et al. (2011: 83), DZHW Studienberechtigtenpanel Jahrgang 2006, Wellen 1-3, eigene Darstellung ausgewählter Ergebnisse

A (ohne Muster, jeweils oberer Balken): Befragte aus Akademikerfamilien (mindestens ein Elternteil mit Hochschulabschluss)

NA (mit Muster, jeweils unterer Balken): Befragte aus Nichtakademikerfamilien 
Insgesamt sind die empirischen Befunde zur Umsetzung von Studienaspirationen bzw. -intentionen aufgrund unterschiedlicher Datengrundlagen, Konzeptualisierungen und Operationalisierungen sehr divers. Einig sind sich alle Studien jedoch darin, dass nicht alle Schüler/innen ihre Aspirationen/Intentionen umsetzen können und dass - sofern untersucht - dies sozial schwächere Schüler/innen häufiger betrifft. Dies zeigt sich vor allem in den USA, wo die Gruppe der Studienaspirant/innen groß und heterogen ist. In Deutschland, wo aufgrund vorhandener Selektionsschwellen im Schulsystem und attraktiver Ausbildungsalternativen die Gruppe der Hochschulzugangsberechtigten mit Studienaspiration bereits viel stärker (positiv) selektiert ist, scheinen Herkunftsunterschiede bei der Umsetzung von Studienintentionen hingegen gering zu sein. Etwas deutlicher scheinen diese bei der Umsetzung von Studienfachintentionen hervorzutreten.

Neben den bereits diskutierten Einschränkungen der existierenden (deutschen) Studien ergeben sich zusammenfassend folgende Forschungslücken: Erstens beschäftigten sich die genannten Studien vornehmlich mit der Frage der Realisierungschancen von Studienintentionen. Die berichteten Umsetzungschancen sind meist entsprechend hoch. Weitestgehend offen bleibt daher bislang, inwiefern diese Ergebnisse auf die Umsetzungschancen von Studienaspirationen übertragbar sind. Im vorangegangenen Abschnitt wurde argumentiert, dass Aspirationen in größerem Maße als Intentionen ein idealistisches Element enthalten (können), das nicht im Zusammenhang mit ihren Umsetzungschancen stehen muss. Die konzeptionelle Differenzierung zwischen Aspirationen und Intentionen beruht genau auf der Idee, dass Intentionen realistischer sind, da sie in Auseinandersetzung mit wahrgenommenen individuellen und institutionellen Barrieren entstehen. Entsprechend sollten ihre Umsetzungschancen auch größer sein als die von Aspirationen, insbesondere dann, wenn externe Zwänge umfassend (und korrekt) reflektiert werden (vgl. Azjen 1991). Die Fragen, inwiefern Studienaspirationen umgesetzt werden können und ob hierbei soziale Herkunftsunterschiede existieren, bleiben also zunächst weitestgehend offen.

Zweitens gibt es nur sehr wenige und rein deskriptive Befunde zur Frage des Zusammenspiels von sozialer Herkunft, spezifischen Fachaspirationen und ihrer Umsetzung. Dabei stellt sich nicht nur die Frage, ob es Diskrepanzen zwischen spezifischen Aspirationen und entsprechenden Übergängen ins Studium gibt, sondern auch, ob nicht gelungene Umsetzungen mit Umorientierungen innerhalb des Hochschulsystems verbunden sind oder ob sie zu einer $U m$ entscheidung und der Wahl alternativer Bildungswege führen (vgl. Spangenberg et al. 2011).

Drittens gibt es zu sozialen Herkunftsunterschieden bezüglich der Umsetzungschancen von Studienaspirationen/-intentionen zwar erste empirische Befunde. Verglichen mit der empirischen Evidenz bezüglich sozialer Ungleichheit bei der Studienaufnahme (oder Studienintention) (vgl. Kapitel 3) und den 
dabei zugrundeliegenden Mechanismen gibt es hier aber noch deutlichen Nachholbedarf. Inwiefern können die theoretischen Mechanismen und empirischen Befunde, die für den Übergang ins Hochschulstudium gezeigt werden, auf die Gruppe von Schüler/innen mit Studienaspiration übertragen werden?

Viertens wurde in Kapitel 3 argumentiert, dass für ein umfassenderes Verständnis sozialer Selektionsprozesse der Übergang ins Studium differenzierter betrachtet werden sollte. Für den deutschen Kontext wurde bisher nicht untersucht, in welchen selbst- und fremdselektiven Phasen der Umsetzung von Studienaspirationen Schüler/innen den Hochschulpfad (zumindest vorläufig) verlassen und in welchen Phasen hierbei soziale Herkunftsunterschiede beobachtet werden können. Dieser letzte Punkt steht im Mittelpunkt der folgenden Ausführungen.

\subsection{Die Phasen der Aspirationsumsetzung}

Die in Kapitel 3 vorgestellten Phasenmodelle stellen eine wichtige Orientierung für die vorliegende Arbeit dar. Folgende Annahmen sind dabei auch hier zentral: (1) Schüler/innen müssen auf dem Weg in die Hochschule verschiedene Phasen durchlaufen bzw. Meilensteine bewältigen, (2) institutionelle Merkmale des Hochschulsystems spielen dabei eine zentrale Rolle, entsprechende Opportunitäten und Barrieren werden - mehr oder weniger vollständig und korrekt - antizipiert und in die Entscheidungsfindung einbezogen, (3) sowohl individuelle als auch institutionelle Entscheidungen - Selbst- und Fremdselektion - spielen eine Rolle und (4) die Phasen sind nicht unabhängig voneinander, sondern beeinflussen sich ,in subtle and complex ways" (Cabrera und La Nasa 2000: 5).

Die Entstehung von Studienaspirationen stellt in den meisten existierenden Modellen die erste Phase dar. Im Gegensatz dazu sind in der vorliegenden Arbeit bereits bestehende Aspirationen der Ausgangspunkt für die Untersuchung von deren Umsetzungschancen (vgl. Hanson 1994, Roderick et al. 2011). Hierfür stehen im Folgenden - in Anlehnung an Roderick et al. (2011) vier Phasen im Mittelpunkt, die in Abbildung 4.2 modellhaft dargestellt sind.

In der ersten Phase betrachte ich die Übersetzung von idealistischen Aspirationen in realistische Intentionen. Hier steht also die Frage im Mittelpunkt, inwiefern Schüler/innen, die sich wünschen zu studieren, ein Studium nach Einbezug wahrgenommener individueller und institutioneller Hürden auch intendieren. Die folgenden Phasen umfassen die Umsetzung von Aspirationen. In der zweiten Phase fokussiere ich dabei auf das konkrete Bewerbungsverhalten als wichtigen ersten Schritt dieser Umsetzung. Darauf folgt in der dritten Phase die Zulassung zum Studium und damit der direkte Einfluss institutioneller Selektionsprozesse. Wenn die vorangegangenen Meilensteine - hier: 
Bewerbung und Zulassung - gemeistert wurden, stellt sich zuletzt in der vierten Phase die Frage, ob Studienaspirationen durch die Studienaufnahme final umgesetzt werden. ${ }^{66}$

Abb. 4.2: Modellhafte Darstellung der Phasen der Aspirationsumsetzung

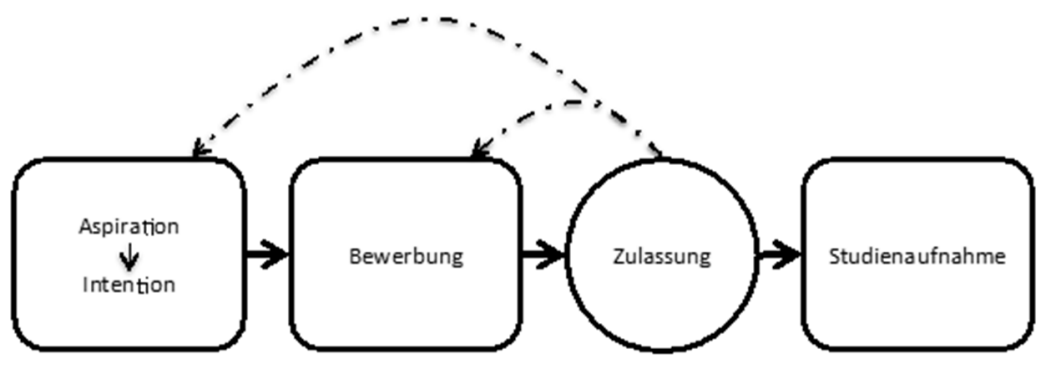

Quelle: Eigene Darstellung

In Abbildung 4.2 beziehen sich rechteckige Felder auf die Phasen der Selbstselektion, während das kreisförmige Feld auf Fremdselektion verweist, die sowohl eine direkte Wirkung entfalten als auch indirekt (durch Antizipation) auf die vorgelagerten Phasen zurückwirken kann (gestrichelte Pfeile). Bereits in der ersten Phase können hiernach Auswahlverfahren und Zulassungschancen als Teil der institutionellen Opportunitätsstruktur antizipiert werden. Und auch das Bewerbungsverhalten in der zweiten Phase ist nicht nur oft Voraussetzung für eine Zulassung zum Studium, sondern selbst durch die zur Anwendung kommenden Auswahlkriterien und antizipierte Zulassungschancen beeinflusst (vgl. DesJardins et al. 2006, Drewes und Michael 2006).

Bevor der Forschungsstand zu den einzelnen Phasen dargestellt wird, soll noch kurz auf den Stellenwert von Intentionen für die folgenden Phasen eingegangen werden. Alternativ könnten Intentionen, wie auch bei Roderick et al. (2011), als notwendiger Schritt für den Eintritt in die folgenden Phasen modelliert werden (Abbildung A4.1) und die Analysen zum Bewerbungsverhalten auf diejenigen Schüler/innen mit Studienintentionen beschränkt werden. In der vorliegenden Arbeit werden Aspirationen und Intentionen zu Beginn des letzten Schuljahres gemessen. Ein solches Vorgehen würde ausschließen, dass sich

66 Nicht in allen Kontexten ist Phase 2 Bedingung für Phase 3 und die Phasen 2 oder 3 Bedingung für Phase 4. Auch in Deutschland ist es möglich, sich nach Erhalt der Hochschulzugangsberechtigung in (wirklich) zulassungsfreie Studiengänge einzuschreiben und somit die Phasen 2 und 3 zu umgehen. Wie in Kapitel 8 noch ausführlicher dargelegt wird, verfolge ich hier ein breites Verständnis von Bewerbung als „studiengerichtetes Verhalten“ (Einschreiben oder Bewerben) und Zulassung als „Zugang gewähren“ (durch Zulassung oder fehlende Zugangshürden). 
Schüler/innen im letzten Schuljahr umentscheiden (etwa auf Grundlage neuer Informationen) (vgl. Spangenberg und Quast 2016). Um ein Ergebnis vorwegzunehmen: Im in dieser Arbeit betrachteten empirischen Fall zeigt sich, dass Intentionen weder hinreichend noch notwendig für die Umsetzung von Studienaspirationen sind (vgl. Abschnitt 8.2.1). Alternativ könnten Intentionen als wichtiger Prädiktor von Studienbewerbungen und Mediator von Herkunftsunterschieden in der folgenden Bewerbungsphase betrachtet werden. Dieser Frage wird sich in Kapitel 8 empirisch angenähert.

Im Folgenden sollen zentrale empirische Ansätze und Befunde zu den vier Phasen und hierbei auftretender sozialer Ungleichheit dargestellt und Forschungslücken - insbesondere für den deutschen Kontext - identifiziert werden. Die im Folgenden diskutierten Studien und Ergebnisse beziehen sich dabei - mit Ausnahme der ersten Phase - aufgrund der diesbezüglich eingeschränkten Befundlage nicht ausschließlich auf die Umsetzung von Studienaspirationen, da sonst wichtige Erkenntnisse zu den einzelnen Phasen keine Berücksichtigung finden würden. Es werden daher zum Beispiel auch zentrale Befunde vorgestellt, die sich generell mit Bewerbungen oder Zulassungen beschäftigen. Nachdem diese diskutiert wurden, sollen in einem letzten Schritt empirische Erkenntnisse zu der Frage aufgezeigt werden, ob Studienberechtigte eher in den Phasen der Selbst- oder Fremdselektion „verloren gehen“ (Hillmert und Jacob 2010: 59), ob also soziale Ungleichheit bei der Umsetzung von Aspirationen eher durch individuelle Entscheidungen oder institutionelle Auswahl zustande kommt.

\subsubsection{Phase 1: die Übersetzung von Studienaspirationen in Studienintentionen}

Wie bereits in Abschnitt 4.1 angedeutet, gibt es einige (wenige) Studien, die sowohl die Diskrepanzen zwischen Aspirationen und Intentionen als auch diesbezügliche Herkunftsunterschiede zeigen. ${ }^{67}$ Eine Diskrepanz besteht in den al-

67 Empirische Forschung zu Aspirationen und Intentionen fand und findet zum Großteil mit Bezug auf das status attainment-Modell statt (siehe Kapitel 5 für Details), in dem Erwartungen und Vorbildfunktionen aus dem sozialem Umfeld und - hierüber vermittelt - die soziale Herkunft sowie Schulleistung von Schüler/innen deren Bildungsaspirationen beeinflussen (vgl. Sewell et al. 2004). Zwischen Aspirationen und Intentionen wird in diesem Forschungszweig häufig nicht differenziert, sodass entsprechend auch die Übersetzung von Aspirationen in Intentionen nicht untersucht wird. Dies ist umso erstaunlicher, bedenkt man, dass Archibald Haller einer der frühen Co-Autoren vieler status attainment-Studien - eine explizite Trennung zwischen beiden Konzepten befürwortete (Haller 1968). Empirisch wird meist auf Intentionen zurückgegriffen: ,,most analyses of status attainment do not 
lermeisten Fällen (und entsprechend der theoretischen Erwartung) zwischen höheren Aspirationen und niedrigeren Intentionen: So berichten verschiedene Autor/innen auf Grundlage unterschiedlicher Daten, für 16\% (Hanson 1994) bis 30\% (Katthab 2015, siehe auch Kirk et al. 2012, Roderick et al. 2011, Vaisey 2010) der befragten Schüler/innen höhere Aspirationen als Intentionen. Vaisey (2010) und Hanson (1994) differenzieren diese Ergebnisse nach sozialer Herkunft und berichten deutliche Unterschiede von 17 bzw. 13 Prozentpunkten. ${ }^{68}$ Zudem herrscht Einigkeit bezüglich des positiven Zusammenhangs zwischen Schulleistung und der Übereinstimmung zwischen hohen Aspirationen und Intentionen (vgl. Katthab 2015, Roderick et al. 2011). Die zitierten Ergebnisse beziehen sich auf anglo-amerikanische Kontexte (USA und Großbritannien). Für Deutschland liegen hierzu bisher keine Ergebnisse vor. ${ }^{69}$

Nur zwei mir bekannte Studien (Hanson 1994, Roderick et al. 2011) untersuchen mithilfe multivariater Modelle Gründe für eine erfolgreiche oder erfolglose Übersetzung von Studienaspirationen in Studienintentionen. In beiden Studien wird das Sample auf high school seniors mit Studienaspiration beschränkt (bei Hanson zusätzlich auf solche mit überdurchschnittlich hoher Testleistung in den Bereichen Lesen und Mathematik) und analysiert, welche Faktoren mit dem Vorhandensein einer ebenso hohen Intention zusammenhängen. Hanson (1994: 172) findet, dass der oben bereits berichtete signifikant positive Zusammenhang zwischen sozialer Herkunft und der Übersetzung von Aspirationen in Intentionen auch nach Hinzufügen einer Vielzahl weiterer Variablen (z.B. Schulleistung, Aspirationen von Eltern und Peers) bestehen bleibt. Roderick et al. (2011: 192ff.) berichten, dass sich neben einer hohen Bildung der Mutter (college Abschluss vs. kein high school-Abschluss) auch elterlicher Druck, Schulleistung, extracurriculare Aktivitäten und - auf Schulebene - ein studienaffines Klima positiv auf die Übersetzungschancen auswirken.

Mit Blick auf die Übersetzung von weitestgehend uneingeschränkten Studienaspirationen in individuelle und institutionelle Barrieren antizipierende Studienintentionen ergeben sich aus diesem Literaturüberblick verschiedene Forschungslücken. Gezeigt werden konnte bisher der Zusammenhang zwischen verschiedenen Individualvariablen sowie - auf institutioneller Ebene -

index motivation as much as they index knowledge of the 'real world"' (Kerckhoff 1976: 371, siehe auch Morgan 2007).

68 Vaisey (2010) zeigt, dass 31\% der Jugendlichen aus armen Familien geringere Intentionen als Aspirationen nennen, während dies nur auf 14\% der Jugendlichen aus finanziell besser gestellten Familien zutrifft. Hanson (1994) berichtet Anteile von 25\% (unteres SES-Quartil) und 12\% (oberes SES-Quartil) (für ähnliche Ergebnisse bezüglich beruflicher Aspirationen und Intentionen siehe Stephenson 1957).

69 Einige deutsche Studien beschäftigen sich mit angrenzenden Themen, fokussieren aber auf frühere Bildungsstufen (vgl. Kleine et al. 2009, Kurz und Paulus 2006, Trebbels 2015). 
einigen Schulvariablen und der Übersetzungswahrscheinlichkeit. Während dabei einzelne Variablen aus der status attainment-Literatur als erklärende Faktoren einbezogen wurden, wurden soziologische RC-Modelle bisher nicht auf diese Fragestellung angewandt, obwohl gerade die Entstehung realistischer Intentionen oft als Rationalisierungsprozess verstanden wird, der der Logik rationaler Entscheidungsmodelle folgt (etwa Becker 2010, Morgan 1998, Stocké 2005b).

Hieran anknüpfend stellt sich die Frage nach dem potentiellen Einfluss institutioneller Faktoren bereits in der ersten Phase. In den in Abschnitt 3.3 vorgestellten college choice-Modellen spielen zwar institutionelle Merkmale eine zentrale Rolle. Jedoch ist eine konzeptionelle Trennung zwischen Aspirationen und Intentionen meist nicht explizit vorgesehen, sodass der institutionellen Ausgestaltung des Hochschulsystems in der ersten Phase noch kaum Relevanz zugesprochen wird (vgl. Hossler et al. 1989: 270, siehe auch Chapman 1986, Jackson 1982). Betrachtet man weitestgehend uneingeschränkte Studienwünsche, mag diese Annahme zutreffen. Doch schließt man mögliche Diskrepanzen zwischen idealistischen Aspirationen und realistischen Intentionen aufgrund der Antizipation institutioneller Hürden und Opportunitäten im Bereich des Hochschulsystems nicht aus, sollten diese auch auf die - entsprechend umfassendere - erste Phase zurückwirken. Forschung hierzu ist mir nicht bekannt.

Letztlich beziehen sich die mir bekannten Ergebnisse - sicherlich zum Teil aufgrund von Datendesideraten (vgl. Abschnitt 7.1.1) - allein auf den anglo-amerikanischen Kontext. Die empirische Untersuchung in Kapitel 7 setzt an diesen Forschungslücken an.

\subsubsection{Phase 2: Bewerbungsentscheidungen und Bewerbungsstrategien}

Als zweite Phase soll hier das Bewerbungsverhalten von studienberechtigten Schüler/innen näher betrachtet werden, denn (sozialgruppenspezifische) Studienchancen ,depend crucially on who applies in the first place“ (Koffman und Tienda 2008: 2). Mittlerweile gibt es eine Vielzahl an Studien, die sich mit dem Thema Studienbewerbung befassen, erneut vor allem, aber nicht ausschließlich in den USA (vgl. An 2010, DesJardins et al. 2006, Griffith und Rothstein 2009, Grodsky und Riegele-Crumb 2010, Hoxby und Avery 2013, Hoxby und Turner 2013, Klasik 2012, Lloyed et al. 2008, Long 2004, Roderick et al. 2011, Turley et al. 2007, Großbritannien: Anders 2012, Boliver 2013, Shiner und Noden 2015, Israel: Ayalon 2007, Kanada: Drewes und Michael 2006, Portugal: Sá und Tavares 2018, Schweden: Hällsten 2010). Quantitative empirische Untersuchungen beruhen hier zum einen auf Individualdaten von Schüler/innen, in denen Bewerbungsentscheidungen zum Teil standardmäßig erfasst werden. Zum anderen kann in einigen Ländern auch auf administrative 
Daten zurückgegriffen werden. In den USA ist zum Beispiel das Versenden der Ergebnisse des Scholastic Assessment Tests (SAT) oder American College Tests (ACT) Voraussetzung für die Bewerbung an vielen Hochschulen, sodass umfangreiche Daten zu diesen Bewerbungsproxies inklusive der Testwerte und im Zuge des Testverfahrens erhobenen sozio-demographischen Angaben der Teilnehmer/innen vorliegen. In einigen anderen Ländern laufen sämtliche Studienbewerbungen über ein zentrales Bewerbungsportal, sodass auch hier umfassende Informationen zu den Bewerbungen und zu Hintergrundvariablen der Bewerber/innen vorliegen. Dies ist mit dem Universities \& Colleges Admissions Service (UCAS) der Fall in Großbritannien, aber zum Beispiel auch in Portugal, Schweden oder Dänemark. Für Deutschland wird das Bewerbungsverhalten von Studienberechtigten in den einschlägigen Befragungen bisher kaum erfasst. ${ }^{70}$ Mit der Stiftung für Hochschulzulassung existiert allerdings auch hier ein zentrales Bewerbungsportal für die bundesweit zulassungsbeschränkten Studiengänge (vgl. Abschnitt 2.2.2) und somit erste Untersuchungen zu Bewerbungen für diese spezifischen Studienfächer (vgl. Braun et al. 2007, Horstschräer 2012).

Im Folgenden fokussiere ich auf drei Forschungsstränge zum Studienbewerbungsverhalten (und innerhalb dieser auf den Einfluss sozialer Herkunft): Erstens werden Studien vorgestellt, die sich mit der Frage befassen, $o b$ überhaupt eine oder mehrere Bewerbungen getätigt werden. Zweitens solche, die sich überwiegend auf Bewerber/innen beschränken und analysieren, wofür diese sich bewerben und welchen Einfluss institutionelle Merkmale hierbei spielen. Drittens liegt der Fokus auf empirischen Ergebnissen zum „Wie“, also zu sozialgruppenspezifischen Bewerbungsmustern und -strategien. ${ }^{71}$

Bewerbungen: empirische Erkenntnisse zum „Ob“

Einige Studien untersuchen auf Grundlage meist repräsentativer Befragungen, ob sich studienberechtigte Schüler/innen überhaupt für ein Studium (im USamerikanischem Kontext häufig für ein four-year college) bewerben (vgl. USA: An 2010, Griffith und Rothstein 2009, Grodsky und Riegele-Crumb 2010, Klasik 2012, Lloyed et al. 2008, Roderick et al. 2011, Turley et al. 2007;

70 Neben den in der vorliegenden Arbeit genutzt Best Up Daten, stellt wiederum das NEPS eine Ausnahme dar. Hier wurden Studienberechtigte der Startkohorte 4 erstmalig nach ihrem Bewerbungsverhalten gefragt (z.B.: ob, wie oft, für welche Fächer, für welche Hochschulen). Diese Daten wurden im Sommer 2017 kurz nach Fertigstellung der vorliegenden Dissertation veröffentlicht. Hierauf basierende Publikationen liegen noch nicht vor.

71 Zudem gibt es Studien, die sich auf Bewerbungen für nur eine oder einige wenige Hochschulen (z.B. in einem Bundesstaat) beschränken (vgl. DesJardins et al. 2006, Koffmann und Tienda 2008, Touthousian 1999, Weiler 1994). Diese werden im Folgenden nicht systematisch berücksichtigt. 
UK: Anders 2012, Khattab 2015). Die Mehrzahl dieser Studien betrachten auch den Zusammenhang zwischen der sozialen Herkunft (in Form von elterlicher Bildung, Klasse oder Einkommen) und Bewerbungsentscheidungen, wenn nicht als zentrale Fragestellung, so zumindest als Kontrollvariable, und berichten einen substantiellen positiven Zusammenhang, der oft auch in multivariaten Modellen signifikant bleibt (vgl. Anders 2012, An 2010, Grodsky und Riegele-Crumb 2010, Klasik 2012, Turley et al. 2007). ${ }^{72}$ Einig sind sich diese Studien zudem darin, dass leistungsbezogene Merkmale generell positiv mit Bewerbungsentscheidungen zusammenhängen. Zudem wirkt der elterliche Einfluss auch in dieser Phase: Interaktion, Unterstützung und Studienerwartungen der Eltern sind positiv mit Bewerbungsentscheidungen assoziiert (vgl. An 2010, Khattab 2015, Lloyed et al. 2008).

Die bisher zitierten Studien untersuchen meist die Bewerbungsentscheidungen aller Studienberechtigten. Wie in Abschnitt 4.2 bereits beschrieben, werden in einigen Studien Bewerbungen auch als abhängige Variable genutzt, um die Umsetzung von Studienaspirationen - oder in den meisten Fällen: Studienintentionen $-\mathrm{zu}$ untersuchen. Mit Fokus auf soziale Herkunftsunterschiede untersuchen etwa Grodsky und Riegele-Crumb (2010) die Bewerbungswahrscheinlichkeit von texanischen high school seniors mit Studienintention. Sie argumentieren, dass eine in den USA mittlerweile fast universelle Studienintention an sich nicht in jedem Fall ausreicht, um diese umzusetzen, sondern dass es zudem darauf ankommt, wie früh sich diese herausgebildet hat und - damit einhergehend - wie selbstverständlich sie ist (siehe auch Alexander und Cook 1979, Bozick et al. 2010). Davon ausgehend analysieren sie, inwiefern ein „college-going habitus“ im Sinne einer früh herausgebildeten Selbstverständlichkeit zu studieren dazu beiträgt, dass sich Schüler/innen in ihrem letzten Schuljahr für four-year colleges bewerben. Empirisch zeigt sich, dass die Bildung der Eltern als Indikator für soziale Herkunft positiv mit dem college-going habitus ihrer Kinder assoziiert ist und dieser wiederum positiv mit Bewerbungsentscheidungen zusammenhängt. Die Bildungsherkunft hat aber auch einen direkten Einfluss auf die Studienbewerbung: Haben die Eltern einen Hochschulabschluss (im Vergleich zu einem high school-Abschluss), erhöht sich die Bewerbungswahrscheinlichkeit auch in den multivariaten Modellen um circa 20 Prozentpunkte. Zudem profitieren nicht nur sozial privilegierte Schüler/innen von einem college-going habitus: „Disadvantaged youth, although less likely to evidence a college-going habitus, benefit from a collegegoing habitus as much as their more advantaged peers." (ebd.: 16).

72 Bemerkenswerte Herkunftsunterschiede findet etwa Anders (2012: 202) für englische Jugendliche. Während sich 77\% der Schüler/innen aus dem Top-Einkommensquintil für ein Studium bewerben, tun dies nur 35\% ihrer Peers aus dem unteren Einkommensquintil. Auch nach Kontrolle verschiedener weiterer sozio-demographischer, Leistungs- und Schulvariablen bleibt ein Unterschied von 9 Prozentpunkten. 
In der einzigen Studie, die Bewerbungsentscheidungen im deutschen Kontext untersucht, und auf Grundlage derselben Daten, die auch in der vorliegenden Arbeit verwendet werden (Best Up), zeigen Ehlert et al. (2017a) für Deutschland, dass sich etwa zwei Drittel der Berliner Schüler/innen mit Studienintention am Ende des vorletzten Schuljahres direkt nach Erhalt der Hochschulzugangsberechtigung für ein Studium bewerben. Zudem besteht eine Herkunftsdifferenz von etwa 10 Prozentpunkten zwischen Schüler/innen aus Doppelakademikerfamilien auf der einen und Schüler/innen aus Ein- bzw. Nichtakademikerfamilien auf der anderen Seite. Als ein wichtiger Grund hierfür werden Informationsasymmetrien bezüglich der Erträge, Kosten und Finanzierungsmöglichkeiten eines Hochschulstudiums identifiziert. ${ }^{73}$

Bewerbungen: empirische Erkenntnisse zum „Wofür“

Für die erste Phase wurde kritisch festgestellt, dass der Einfluss institutioneller Barrieren und Opportunitäten des Hochschulsystems kaum Beachtung findet. Im Gegensatz dazu rücken diese in der zweiten Phase in einer Reihe von Studien in den Vordergrund. Diese fokussieren auf Studienbewerber/innen und untersuchen, welche institutionellen und individuellen Variablen die Bewerbungswahrscheinlichkeit für bestimmte Hochschultypen oder Hochschulen erhöhen bzw. senken (vgl. USA: Hoxby und Avery 2013, Griffith und Rothstein 2009, Kanada: Drewes und Michael 2006, UK: Boliver 2013, Shiner und Noden 2015, Schweden: Hällsten 2010, Deutschland: Horstschräer 2012). Bewerbungsentscheidungen für bestimmte Studienfächer werden hingegen kaum untersucht, sicherlich auch, weil die Entscheidung für bestimmte Hochschulinstitutionen im Vergleich zur fachlichen Ausrichtung in vielen (anglo-amerikanischen) Ländern - zumindest mit Blick auf grundständige Studiengänge - vordergründig ist und die zentrale Stratifizierungsdimension darstellt.

Bezüglich des Prestiges von Hochschulen zeigt sich für Großbritannien, dass die Herkunftsklasse signifikant mit Bewerbungen für prestigereiche Hochschulen assoziiert ist und dass dieser Zusammenhang zum Teil, aber nicht vollständig, durch leistungsbezogene Variablen sowie den Schultyp (öffentlich vs. privat) erklärt werden kann (vgl. Boliver 2013, Shiner und Noden 2015, für die USA siehe Griffith und Rothstein 2009).

73 Wie in Kapitel 6 noch ausführlicher beschrieben wird, wurde im Rahmen des Best Up Projekts eine Informationsintervention mit Schüler/innen aus acht zufällig ausgewählten Schulen durchgeführt. Die oben berichteten Anteile beziehen sich ausschließlich auf die Kontrollgruppe (19 Schulen). Der Informationsworkshop zu Erträgen und Finanzierungsmöglichkeiten eines Studiums erhöhte die Wahrscheinlichkeit einer Studienbewerbung. Dies war vor allem bei Befragten aus Ein- und Nichtakademikerfamilien der Fall. Bei Doppelakademikerkindern konnte kein Einfluss des Informationstreatments festgestellt werden (Ehlert et al. 2017a). 
Weitere Studien untersuchen, welche Hochschulmerkmale die Wahrscheinlichkeit der Bewerbung für bestimmte Hochschulen im Vergleich zu anderen verfügbaren Optionen erhöhen. Hoxby und Avery (2013) differenzieren ihre Ergebnisse zwischen leistungsstarken Schüler/innen (10\% Jahrgangsbeste) aus einkommensstarken und einkommensschwachen Familien und zeigen, dass die Wahrscheinlichkeit, sich an einer bestimmten Hochschule zu bewerben, mit steigender geographischer Distanz für beide Gruppen sinkt, dass finanziell benachteiligte Schüler/innen aber zusätzlich Studienoptionen stark präferieren, die einen Auszug aus dem Elternhaus nicht erforderlich machen. Weiterhin favorisieren diese nicht selektive Hochschulen oder selektive Hochschulen, deren durchschnittlicher SAT-Wert unter ihrem individuellen liegt, sowie Hochschulen mit geringeren Gebühren. Schüler/innen aus finanziell besser ausgestatteten Familien präferieren hingegen insbesondere selektive und gut ausgestattete Hochschulen.

Auch Hällsten (2010) beschäftigt sich mit herkunftsspezifischen Präferenzen für unterschiedliche Hochschul- bzw. Programmmerkmale, allerdings für den schwedischen Kontext, in dem - wie auch in Deutschland - die direkten monetären Kosten ein weitaus kleineres Gewicht haben als in den USA. Seine Ergebnisse deuten darauf hin, dass sich zwar die Stärke, nicht aber die Richtung der Zusammenhänge zwischen institutionellen Faktoren und Bewerbungsentscheidungen zwischen Bewerber/innen höherer und niedrigerer sozialer Herkunft unterscheiden (manual vs. service class): So entscheiden sich Bewerber/innen seltener für entfernte Hochschulen, vor allem wenn sie aus der Arbeiterklasse stammen. Eine größere Selektivität von Studiengängen sowie traditionelle (vs. nicht-traditionelle) Hochschulen ziehen hingegen Bewerber/innen aus der Dienstklasse stärker an. Zudem favorisieren diese Studiengänge, die zum Beruf ihrer Eltern führen. Insgesamt schließt Hällsten, dass die Präferenzen sozial schwächerer Bewerber/innen deren Entscheidungsspielraum stark einschränken. Weiterhin weist er darauf hin, dass sich Präferenzen auch deutlich zwischen Bewerber/innen mit unterschiedlichem Leistungsstand unterscheiden. In einer kanadischen Studie finden auch Drewes und Michael (2006) einen differentiellen Einfluss der Leistungsselektivität von Studiengängen, die positiv auf leistungsstärkere und negativ auf leistungsschwächere Bewerber/innen wirkt, was darauf hindeutet, dass Bewerber/innen ihre Zulassungschancen antizipieren und in ihre Bewerbungsentscheidungen einfließen lassen.

Wie weiter oben bereits erwähnt, sind für den deutschen Kontext Untersuchungen zum Bewerbungsverhalten von Studienberechtigten aufgrund fehlender Daten kaum vorhanden. Eine Ausnahme stellen administrative Daten der $\mathrm{SfH}$ zu Bewerbungen für bundesweit zulassungsbeschränkte Studiengänge dar, die allerdings keine Angaben zur sozialen Herkunft der Bewerber/innen enthalten. Horstschräer (2012) nutzt die Bewerbungsdaten der Jahre 2002 bis 2008 und beschränkt sich dabei auf Top-Abiturient/innen (Durchschnittsnote 
$1,0)$ und deren Bewerbungen über die Abiturbestenquote für das Fach Medizin (vgl. Kapitel 2.2.2). Im Zeitvergleich zeigt sie, dass diese sehr leistungsstarken Studienberechtigten auf die Exzellenzinitiative reagierten und sich nach deren Initiierung häufiger für Hochschulen mit dem Exzellenzlabel bewarben als zuvor. Zudem zeigt sie, dass selbst in dieser positiv selektierten Gruppe die Wahrscheinlichkeit, sich für eine bestimmte Hochschule zu bewerben, deutlich mit steigender geographischer Distanz sinkt.

Bewerbungsmuster und Bewerbungsstrategien: empirische Erkenntnisse zum „Wie“

$\mathrm{Zu}$ Beginn dieses Abschnittes wurde die Bewerbungsphase als zentral für den Übergang ins Studium interpretiert, da Bewerbungen in vielen Fällen notwendige Voraussetzungen für den Zugang zum Hochschulstudium sind. Dabei spielt aber nicht nur das $O b$ und Wofür, sondern insbesondere auch das Wie eine wichtige Rolle. Die Antwort auf diese Frage fällt oft wenig optimistisch aus, und der Bewerbungsprozess wird für viele (oder eher: bestimmte) Schüler/innen als suboptimal beschrieben (vgl. Hoxby und Avery 2013, Hoxby und Turner 2013). Als Gründe hierfür werden vor allem Informationsdefizite genannt: ,Some students may lack access to information; others may be overwhelmed by the process of parsing information on the volume of potential postsecondary options. Either circumstance may drive students to make important choices that are haphazard." (Page und Scott-Clayton 2016: 10, siehe auch Avery et al. 2014). Wie in Kapitel 5 noch ausführlicher argumentiert werden wird, nutzen sozial privilegierte Schüler/innen und ihre Familien die vom System gebotenen Optionen, um ihre Studienchancen zu steigern (vgl. Alon 2009, McDonough 1997), während sozial schwächeren Schüler/innen hierzu oft die nötige Unterstützung und Informationen fehlen. Dies zeigt sich auch in sozialgruppenspezifischen Bewerbungsstrategien: Für die USA wurde gezeigt, dass bei sozial privilegierten Schüler/innen dieses strategische Handeln bereits lange vor der eigentlichen Bewerbungsphase beginnt und sich in der Schulwahl, in der Wahl extracurricularer Aktivitäten und in der Nutzung professioneller und oft kostspieliger Beratungs- und Testvorbereitungsangebote zeigt. Die Folge sind hochgradig rationalisierte Bewerbungsmuster. Schüler/innen aus sozial schwächeren Familien treten hingegen weniger gut informiert und vorbereitet in die Bewerbungsphase ein (vgl. Buchmann et al. 2010, McDonough 1994, 1997; für Großbritannien siehe Reay et al. 2001a).

Hoxby und Avery (2013) zeigen diesen Gegensatz am Beispiel von leistungsstarken Schüler/innen in der oben bereits diskutierten Studie. Schüler/innen aus finanziell gut gestellten Familien befolgen die gängigen Expertenrat- 
schläge für einen optimalen Bewerbungsprozess. ${ }^{74}$ Ihre finanziell schlechter gestellten Peers weichen hingegen häufig davon ab. Nur $8 \%$ bewerben sich „achievement-typical“, also den Ratschlägen entsprechend. 53\% hingegen bewerben sich ,income-typical“, d.h. ausschließlich für nicht selektive oder nur geringfügig selektive Hochschulen, die oft schlecht mit Ressourcen ausgestattet sind, hohe Abbruchquoten verzeichnen und deren Studierende im Durchschnitt weit unter ihrem eigenen Leistungsniveau liegen. Das Bewerbungsmuster der restlichen 39\% finanziell benachteiligten, leistungsstarken Schüler/innen beschreiben Hoxby und Avery (2013: 27) schlicht als „odd“. Als einen Hauptgrund für das unterschiedliche Bewerbungsmuster nennen die Autor/innen regionale und schulische Segregation, die dazu führt, dass finanziell benachteiligte Schüler/innen trotz ihres Leistungspotentials im Durchschnitt weniger Informationen und Unterstützung bekommen. Entsprechend zeigen Hoxby und Turner (2013) in einem Folgeprojekt mithilfe eines Feldexperiments, dass zusätzliche Informationen unter anderem zu einem höheren Anteil leistungstypischen Bewerbungsverhaltens unter finanziell benachteiligten Schüler/innen beitragen.

Die Anzahl an Bewerbungen identifizieren weitere Studien als wichtige Strategie (vgl. Israel: Ayalon 2007, USA: Hurtado et al. 1997, Smith 2014): „By applying to several colleges candidates for higher education widen their opportunities and increase the likelihood of entering a "good" college“ (Ayalon 2007: 888). Übereinstimmend stellen diese Studien fest, dass die Anzahl der Bewerbungen mit dem elterlichen Einkommen oder der elterlichen Bildung steigt. Hurtado et al. (1997) berichten zum Beispiel, dass sich 52\% der Schüler/innen aus finanziell gut ausgestatteten Familien für mindestens zwei und $17 \%$ für mindestens fünf Hochschulen bewerben. Die Werte für Schüler/innen aus finanziell benachteiligten Familien liegen bei 25\% bzw. 3\%. Auch in multivariaten Modellen ist der Einkommenseffekt signifikant. Ayalon (2007) untersucht retrospektiv das Bewerbungsverhalten von israelischen Studienanfänger/-innen und findet, dass sich Akademikerkinder nicht nur häufiger mehr als einmal bewarben als ihre Kommiliton/-innen aus nicht-akademischem Elternhaus, sondern dass sie dies vor allem dann taten, wenn sie lukrative Fächer an Universitäten anstrebten und gleichzeitig eher durchschnittliche Schulnoten vorweisen konnten. Ayalon interpretiert dieses Muster als Strategie privilegierter Klassen, sich eben diese Privilegien auch weiterhin zu sichern (siehe auch McDonough 1994). Diese Strategie scheint aufzugehen, da die Anzahl der Bewerbungen signifikant positiv mit Zulassungschancen (vgl. Roderick et

74 Demnach sollten sich Schüler/innen für einige ,reach colleges“ (durchschnittliche Testwerte der Studierenden liegen über den eigenen), einige ,peer colleges“ (durchschnittliche Testwerte der Studierenden liegen im eigenen Bereich) und einige ,safe colleges“ (durchschnittliche Testwerte der Studierenden liegen unter den eigenen, sodass Zulassungschancen relativ sicher sind) bewerben. 
al. 2011), der Studienaufnahme (vgl. Smith 2014) sowie dem Übergang in prestigereiche Studienoptionen (vgl. Ayalon 2007) zusammenhängt. ${ }^{75}$

Insbesondere im Vergleich zum US-amerikanischen Kontext sind langfristig angelegte Strategien im Kontext des deutschen Zulassungssystems sicherlich nicht in gleichem Maße möglich und nötig. Nichtsdestotrotz wurde in Kapitel 2 gezeigt, dass die Zulassungsregeln komplexer geworden sind und eine Studienaufnahme - je nach eigenem (Leistungs-)Profil, fachlicher und örtlicher Präferenz - nicht als von vorn herein gesichert angesehen werden kann. Auch hier sollten Systemkenntnis und damit verbundene Bewerbungsmuster Zulassungschancen optimieren. Entsprechend wurde strategisches Bewerbungsverhalten aus einer ökonomischen Perspektive auch für deutsche Bewerber/innen der bundesweit zulassungsbeschränkten Studiengänge beobachtet, die ihre Liste an Studiengängen in der Abiturbestenquote trunkieren. So minimieren sie das Risiko, über diese Quote einer weniger präferierten Hochschule zugewiesen zu werden und halten sich gleichzeitig die Zulassungschance an ihrer präferierten Hochschule offen (vgl. Braun et al. 2007). Es ist jedoch aufgrund von Datenrestriktionen unklar, inwiefern diese Strategie vor allem von sozial privilegierten Studienbewerber/innen angewendet wird.

Zusammenfassend konnte gezeigt werden, dass sich Schüler/innen unterschiedlicher sozialer Herkunft darin unterscheiden, ob sie sich überhaupt bewerben, wofür sie sich bewerben und welche Bewerbungsstrategien sie anwenden. Die Ergebnisse beziehen sich mit wenigen Ausnahmen auf den angloamerikanischen Kontext. Für Deutschland existieren zwar erste Studien, die sich mit dem Ob (Ehlert et al. 2017a), dem Wofür (Horstschräer 2012) und dem Wie (Braun et al. 2007) beschäftigen, insgesamt stellen die Bewerbungsphase und insbesondere hierbei auftretende soziale Ungleichheit jedoch im deutschen Kontext ein großes Forschungsdesiderat dar. In Kapitel 8 wird die Frage nach sozialen Herkunftsunterschieden im Bewerbungsverhalten von Studienberechtigten mit Studienaspirationen ausführlich empirisch untersucht.

75 So berichtet Smith (2013), dass eine Erhöhung von einer auf zwei Bewerbungen die Studienwahrscheinlichkeit um 40 Prozentpunkte steigert und eine Erhöhung von zwei auf drei Bewerbungen um 10 Prozentpunkte. Weitere Bewerbungen bringen hingegen keinen substantiellen Zuwachs. Die höhere Studienwahrscheinlichkeit liegt dabei sowohl an einer erhöhten Zulassungswahrscheinlichkeit als auch an einer größeren Auswahl zwischen mehreren Studiengängen, zu denen Bewerber/innen zugelassen wurden, da somit Präferenzen eher realisiert werden können. Neben der Anzahl an Bewerbungen zeigen erste Befunde, dass auch die Breite der Bewerbungen bezüglich der Fächer und der Hochschulen Zulassungschancen erhöht (nicht aber die Chance, für den präferierten Studienplatz zugelassen zu werden) (vgl. Sá und Tavares 2018). 


\subsubsection{Phase 3: Zulassung}

Wie bereits argumentiert, beeinflussen individuelle Entscheidungen „die Gestaltung der tatsächlichen Bildungsverläufe nur zu einem gewissen Grad. Relevante Entscheidungen werden auch von anderen Instanzen getroffen (Lehrer oder Aufnahme- und Bewerbungskommissionen)“ (Hillmert 2005: 177). Der Übergang ins Studium ist damit - mit Ausnahme von zulassungsfreien Hochschulsystemen oder Studiengängen - auch abhängig von institutionellen Selektionsentscheidungen. Im Vergleich zu individuellen Bildungsentscheidungen sind die „komplementären Prozesse“ der Auswahl von Bewerber/innen durch Hochschulen deutlich seltener erforscht (vgl. Sackmann 2015: 31). Diese Feststellung gilt allgemein, vor allem aber auch für den deutschen Kontext.

Gleichwohl befassen sich einige Studien mit dieser Phase, die sich in zwei zentrale Forschungsstränge unterteilen. Der erste besteht aus Studien, die $\mathrm{Zu}-$ lassungschancen meist gegeben einer oder mehrerer Bewerbungen untersuchen. Die bereits mehrfach zitierten Studien von Klasik (2012) und Roderick et al. (2011) gehören hierzu. Letztere berichten, dass $14 \%$ der four-year college Bewerber/innen (insgesamt 59\% der Schüler/innen mit Studienaspiration) an keiner entsprechenden Hochschule zugelassen wurden. Hochgradig prädiktiv für die Zulassung sind dabei die Schulleistung, aber auch strategisches Bewerbungs- und Informationsverhalten (Besuch von Messen, Nutzung von Ratgebern und Mehrfachbewerbung). Die Bildung der Mutter als sozialer Herkunftsindikator hat in dieser Phase keinen Einfluss. Klasik (2012) hingegen findet eine Herkunftsdifferenz (gemessen über das Einkommen der Eltern), die auch in multivariaten Modellen signifikant bleibt. ${ }^{76}$ Auch für Großbritannien zeigt Boliver (2013) eine Herkunftsdifferenz (Eltern in oberer Dienstklasse vs. Arbeiterklasse) von 8 Prozentpunkten bezüglich der Zulassungschance zu einer prestigereichen Russel Group University gegeben einer entsprechenden Bewerbung. Dieser Unterschied verschwindet in multivariaten Modellen nach Kontrolle der Kurswahl sowie Schulleistung. Bezüglich der Zulassungschancen zu weniger prestigereichen neuen Hochschulen ist die Herkunftsdifferenz von vorn herein kleiner (4 Prozentpunkte).

Der zweite Forschungsstrang besteht aus quantitativen und qualitativen Studien, die die Wirkung verschiedener Auswahlkriterien und -verfahren analysieren. Bezüglich standardisierter, meritokratisch orientierter Kriterien wie Testergebnisse oder Schulnoten zeigt sich - wiederum für die USA - dass nicht nur die Schulleistung sozial ungleich verteilt ist, sondern auch die Ergeb-

76 Im Gegensatz zu Roderick et al. (2011) beschränkt Klasik (2012) das Sample allerdings nicht auf diejenigen, die den vorherigen Meilenstein (hier: Bewerbung) gemeistert haben, wodurch die Ergebnisse nur sehr eingeschränkt vergleichbar sind (siehe auch Abschnitt 3.3). 
nisse standardisierter Tests, da diese „shadow education“ fördern, die vorwiegend von sozial privilegierten Schüler/innen genutzt wird, deren Eltern sowohl über die nötigen finanziellen Ressourcen sowie das nötige Systemwissen verfügen (vgl. Alon und Tienda 2007, Ayalon 2009, Buchmann et al. 2010, Grodsky et al. 2008, McDonough 1994, 1997). Standardisierte Verfahren wirken somit vor allem auf indirektem Wege.

Daneben gibt es Befunde, die zeigen, dass auch „,weiche“ Verfahren“" (Helsper und Krüger 2015: 12) wie Auswahlgespräche durch die Prüfung von „,implizite[m] Wissen um milieu- und situationsspezifische Handlungsweisen“ (Rothmüller 2011: 94) zu sozial ungleich verteilten Zulassungschancen führen können. Insbesondere mit Blick auf den Zugang zu künstlerischen Studiengängen wurde dies in qualitativen Studien gezeigt (vgl. Burke und McManus 2011, Rothmüller 2011). In einer quantitativen Studie demonstrieren Zimdars et al. (2009), dass kulturelles Wissen und Lesegewohnheiten mit Zulassungschancen zur University of Oxford, wo Interviews häufig angewandt werden, zusammenhängen und Herkunftsdifferenzen zum Teil mediieren. Auch Thomsen (2018) zeigt für Dänemark, dass vor allem sozial privilegierte Männer bei schlechterem Notendurchschnitt mithilfe qualitativer Verfahren (vor allem Interviews) zugelassen werden, insbesondere für Studiengänge der Professionen Medizin und Architektur. Diese helfen somit sozial privilegierten, leistungsschwachen Studienaspiranten dabei, rein leistungsbezogene Kriterien zu umgehen und somit den sozialen und vermutlich auch professionellen Status ihrer Eltern zu reproduzieren.

Im deutschen ,notendominierte[n] Auswahlsystem“ (Sackmann 2015: 41) spielen, wie bereits in Kapitel 2 gezeigt wurde, sowohl weiche Kriterien als auch Tests eine eher untergeordnete Rolle. Inwiefern Zulassungschancen in diesem Kontext mit der sozialen Herkunft variieren, ist bisher nicht untersucht worden. Zwar werden mögliche Konsequenzen verschiedener Auswahlverfahren aus ungleichheitstheoretischer Perspektive besprochen (vgl. Helsper und Krüger 2015, Täger 2010), die empirische Forschung beschränkt sich aber weitestgehend auf den psychologisch-diagnostischen Bereich, in dem die Vorhersagekraft von Auswahlverfahren für den Studienerfolg im Vordergrund steht (vgl. Hänsgen 2008, Hissbach et al. 2011; Reuter und Spoun 2009; Simmenroth-Nayda et al. 2014; Waydhas et al. 2007). Soziale Ungleichheit spielt allenfalls eine indirekte Rolle, zum Beispiel in einer Evaluation des Tests für medizinische Studiengänge, in der zum einen gezeigt wird, dass die Testergebnisse zu einem gewissen Grad trainiert werden können und dass zum anderen Testteilnehmer/innen, deren Väter mindestens ein Abitur haben, etwas bessere Testergebnisse erzielen als die Vergleichsgruppe (Trost et al. 1998). Da diese jedoch auch bessere Studienleistungen zeigen, wird dies als fair interpretiert. Fairness wird in diesem Forschungsbereich als wichtiges Bewertungskriterium von Auswahlinstrumenten angesehen und ist gleichzeitig - aus soziologischer Perspektive - mit definitorischen Schwierigkeiten behaftet (vgl. Tara- 
zona 2006). Fairness wird zum einen als „Chancengleichheit“ definiert. Gemeint ist damit aus eignungsdiagnostischer Sicht, dass bei ,gleicher Eignung [...] gleiche Chancen auf Zulassung bestehen" (Hänsgen 2008: 149). Zum anderen soll „Fairness durch prädiktive Validität“ (Tarazona 2006: 72) erreicht werden. Entsprechend wird die Auswahl von Bewerber/innen mit den größten Chancen auf Studienerfolg als „fair“ bewertet. Als sozial neutral kann dieses Verständnis von Fairness und Chancengleichheit allerdings nicht bewertet werden, da es Gefahr läuft, „Potential“ mit unterschiedlichen Startbedingungen zu konfundieren (vgl. Grodsky et al. 2008, Tarazona 2006).

\subsubsection{Phase 4: Studienaufnahme}

Wie bereits in Kapitel 3 diskutiert, gibt es sowohl für den deutschen als auch für andere Kontexte zahlreiche Studien, die sich mit der Studienaufnahme „realisierten Übergängen“ - und hierbei auftretender sozialer Ungleichheit beschäftigen. Diese Studien sollen hier nicht (erneut) diskutiert werden. Hingegen soll die Studienaufnahme als letzte Phase im Übergangsprozess betrachtet werden, nachdem die vorangegangenen Hürden - zuletzt die Zulassung - gemeistert wurden. Wie sich zeigt, wird nicht nur die Gruppe der Schüler/innen mit jedem Schritt selektiver, sondern auch die Anzahl entsprechender Studien nimmt ab. Erneut können hier die Arbeiten von Roderick et al. (2011) und Klasik (2012) zitiert werden. Erstere zeigen, dass trotz der zuvor stattfindenden starken Selektionsprozesse nur 80\% der zugelassenen Chicagoer high schoolAbsolvent/innen (insgesamt 51\% des Ausgangssamples) ein Studium an einem four-year college aufnehmen. Differenziert nach sozialer Herkunft berichtet Klasik (2012), dass etwa 84\% der Zugelassenen aus einkommensstarken Familien, aber nur $62 \%$ ihrer finanziell benachteiligten Peers ein Studium aufnehmen. ${ }^{77}$ In den USA kann die Unsicherheit bei Fragen der Studienfinanzierung ein zentraler Grund für diese Lücke zwischen Zulassung und Studienaufnahme sein. Entsprechend zeigen beide Studien, dass eine Bewerbung für finanzielle Unterstützung signifikant positiv mit der Studienaufnahme zusammenhängt.

In Deutschland kann dieser Grund zwar nicht ausgeschlossen werden, ist aber aufgrund der deutlich geringeren Studienkosten unwahrscheinlicher. Abgesehen von der finanziellen Förderung, besteht natürlich die Möglichkeit, dass Bewerber/innen zwar Zusagen erhalten, aber nicht für ihren präferierten Studiengang (im präferierten Fach, am präferierten Ort) und sich daher im letzten Schritt doch gegen ein Studium entscheiden oder die Studienaufnahme auf

77 Dieser deutliche Herkunftsunterschied im letzten Schritt ist bemerkenswert, da zugelassene Schüler/innen aus einkommensschwachen Familien bereits viel stärker selektiert sind (47\% des Ausgangssamples) als Schüler/innen aus einkommensstarken Familien (80\%). 
einen späteren Zeitpunkt mit möglicherweise besseren Zulassungschancen für ihre erste Präferenz verschieben.

\subsubsection{Soziale Ungleichheit durch Selbst- oder Fremdselektion?}

Die vier diskutierten Phasen der Umsetzung von Studienaspirationen unterteilen sich in Selbst- und Fremdselektionsphasen. Während die Übersetzung von Aspirationen in Intentionen, Bewerbungen und die letztliche Studienaufnahme (vorwiegend) ersteren zugewiesen werden können, sind es die Hochschulen, die in Phase 3 (Zulassung) über die Aufnahme oder Ablehnung von Studienaspirant/innen entscheiden. Welche Selektionsart ist relevanter für die Umsetzung von Studienaspirationen und hierbei auftretender sozialer Ungleichheit?

Die in Kapitel 3 vorgestellten Phasenmodelle und die hieran anknüpfenden Studien, die in den vorangegangenen Abschnitten wiederholt diskutiert wurden (insbesondere Klasik 2012 und Roderick et al. 2011), berücksichtigen meist sowohl Phasen der Selbst- als auch der Fremdselektion. Je nachdem, ob die Analyse unkonditional oder konditional erfolgt, ob also alle Studienberechtigten berücksichtigt werden oder nur diejenigen, die die vorangegangenen Phasen erfolgreich gemeistert haben, sind es entweder die späten (selbst- und fremdselektiven) Stufen, die im unkonditionalen Fall nur noch von wenigen erreicht werden und besonders selektiv sind (vgl. Klasik 2012), oder - im Einklang mit dem Selektionsmodell von Mare (1980) - eher die frühen selbstselektiven Phasen, in denen viele (sozial schwächere) Studienaspirant/innen verloren gehen (vgl. Berkner und Chavez 1997, Horn 1997, Roderick et al. 2011).

Explizit mit der Frage nach dem Verhältnis von Selbst- und Fremdselektion beschäftigen sich weitere Studien im US-amerikanischen und britischen Kontext und differenzieren dabei meist zwischen Bewerbungsentscheidungen auf der einen und Zulassungsentscheidungen bzw. Übergängen ${ }^{78}$ auf der anderen Seite (vgl. Anders 2012, Boliver 2013, Brown und Hirschman 2006, Hoxby und Avery 2013, Koffman and Tienda 2008). Dabei zeigen sich Herkunftsunterschiede im Bewerbungsverhalten oft auch nach Kontrolle von leistungsbezogenen Indikatoren. Bei gleichem Bewerbungsverhalten und unter Kontrolle von Schulleistung und weiteren Qualifikationen können hingegen keine her-

78 Auch wenn der letztliche Übergang gegeben einer Zulassung wiederum eine individuelle Entscheidung darstellt, interpretieren einige Autor/innen diesen als Proxy für Zulassungsentscheidungen, da die finale Studienentscheidung in der Regel eine Zulassungsentscheidung voraussetzt (vgl. Anders 2012, Hoxby und Avery 2013). Damit werden die Anteile an Zugelassenen unterschätzt, während soziale Herkunftsunterschiede bezüglich der Zulassung möglicherweise überschätzt werden (sofern auch der letzte Schritt - Studienaufnahme gegeben einer Zulassung - sozialen Selektionsprozessen unterliegt). 
kunftsspezifischen Zulassungschancen nachgewiesen werden (vgl. Anders 2012, Boliver 2013, Hoxby und Avery 2013).

Insgesamt besteht große Einigkeit darin, dass soziale Ungleichheit vor allem auf Selbstselektion zurückgeführt werden kann und dass entsprechende Reformen in den frühen Phasen des Übergangsprozesses ansetzen sollten. So schlussfolgern etwa Berkner und Chavez (1997: iii):

"High school graduates whose parents have low levels of income and education are able to attend four-year colleges at the same rates as students from middleincome families, if they do what four-year colleges expect them to do. That is, if low-income students have an academic record and aptitude test scores which demonstrate even the minimal qualifications for admission to a four-year institution, if they take a college entrance examination, and if they submit an application for admission, the majority of low-income students enroll in postsecondary education."

Es wäre allerdings ein Fehlschluss, Hochschulen als Gatekeeper gänzlich von einem möglichen Beitrag an sozialer Ungleichheit im Übergangsprozess freizusprechen. Zulassungsentscheidungen an sich erscheinen zwar im Lichte dieser Befunde weitestgehend (und im Durchschnitt) neutral. Dies trifft jedoch nicht zwangsläufig auf das zu, was sie voraussetzen. Soziale Ungleichheit durch Fremdselektion scheint daher vor allem auf indirektem Wege zustande zu kommen, zum einen indem verschiedene Auswahlverfahren und -kriterien Adaptionsstrategien eröffnen (vgl. Alon 2009, Buchmann et al. 2010), zum anderen indem sie antizipiert werden und damit auf Prozesse der Selbstselektion zurückwirken können (vgl. DesJardins et al. 2006, Drewes und Michael 2006). Auf diese Mechanismen wird im folgenden Kapitel noch detaillierter eingegangen.

\subsection{Zusammenfassung}

Kapitel 4 diente der konzeptionellen und empirischen Bestandsaufnahme zur Frage sozialer Ungleichheit bei der Umsetzung von Studienaspirationen als wichtige Grundlage für die folgenden theoretischen Überlegungen und empirischen Untersuchungen.

Zusammenfassend wurden Studienaspirationen, in Abgrenzung zu Intentionen, idealtypisch als weitestgehend uneingeschränkte, idealistische Wünsche definiert. Dass ein beachtlicher Anteil an Schüler/innen mit Studienaspirationen diese nicht umsetzt und hierbei soziale Herkunftsunterschiede beobachtet werden können, wurde insbesondere im US-amerikanischen Kontext gezeigt. Im Vergleich zu den hierbei betrachteten high school seniors handelt es sich bei deutschen Studienberechtigten mit Studienaspiration um eine stär- 
ker positiv selektierte Gruppe: Sie haben erstens die früheren Schwellen im Schulsystem überwunden und zweitens die Hochschulzugangsberechtigung nicht mit dem vorrangigen Ziel erworben, ihre Chancen auf eine attraktive Ausbildungsstelle zu verbessern. Entsprechend ist hier die Befundlage weniger eindeutig und erste Ergebnisse verweisen eher auf relativ hohe Umsetzungschancen und geringe Herkunftsunterschiede. Deutlicher ist der (rein deskriptive) Befund einer Diskrepanz zwischen Studienfachaspirationen (bzw. -intentionen) und deren Umsetzung, die überdurchschnittlich häufig bei sozial schwächeren Studienberechtigten beobachtet werden kann. Dies kann durchaus als Anzeichen dafür interpretiert werden, dass fachlich variierende institutionelle Barrieren Umsetzungschancen (sozialgruppenspezifisch) strukturieren. Insgesamt beziehen sich diese ersten Befunde zum deutschen Kontext jedoch vorwiegend auf Studienintentionen als Ausgangspunkt, die durch die Antizipation individueller und institutioneller Hürden bereits eingeschränkt sein können und somit per Definition in stärkerem Zusammenhang mit den entsprechenden Outcomes stehen sollten als weniger eingeschränkte Bildungswünsche.

Inwiefern können Studienberechtigte ihre idealistischen Studienaspirationen umsetzen und wie können etwaige Herkunftsunterschiede erklärt werden? Um sich diesen Fragen anzunähern, wird die Umsetzung von Studienaspirationen modellhaft in vier zentrale Phasen unterteilt: die Übersetzung von Studienaspirationen in Intentionen (Phase 1) sowie die Umsetzung von Studienaspirationen in Form von Bewerbungen (Phase 2), Zulassungen (Phase 3) und der Studienaufnahme (Phase 4). Wie in Kapitel 3 bereits diskutiert, liegt der Fokus der deutschen Forschung auf der Untersuchung von Studienintentionen oder realisierten Übergängen. Der Literaturüberblick zu den zwischenliegenden (bzw. vorgelagerten) Phasen der Aspirationsumsetzung bestätigte, dass die diesbezügliche Befundlage als äußerst dünn bezeichnet werden kann. Lediglich zur Bewerbungsphase existieren einige erste Befunde, die jedoch meist nicht nach sozialer Herkunft differenzieren (für eine Ausnahme siehe Ehlert et al. 2017a).

Für andere Kontexte, erneut vornehmlich, jedoch nicht ausschließlich aus dem anglo-amerikanischen Raum, existieren allerdings zum Teil zahlreiche Befunde, die zeigen, dass in allen vier Phasen Studienberechtigte ,verloren gehen" und dass dies in stärkerem Maße für sozial schwächere Schüler/innen beobachtet werden kann: Sozial privilegierte Schüler/innen übersetzen ihre Studienaspirationen häufiger in Intentionen, bewerben sich häufiger (und anders), bekommen häufiger Zulassungen zum Studium und entscheiden sich letztlich häufiger für eine Studienaufnahme. Zudem zeigt sich, dass institutionelle Merkmale, wie Prestige, Selektivität oder die geographische Distanz, Umsetzungschancen sozialgruppenspezifisch beeinflussen.

Die ,späten“ Phasen, insbesondere die letztliche Studienaufnahme, ist insbesondere dann sehr sozial selektiv, wenn sie unkonditional betrachtet wird, 
das heißt hier: wenn alle Studienberechtigten (mit Studienaspiration) in die Analysen einbezogen werden. Liegen konditionale Modelle zugrunde, wird also die Umsetzung des vorherigen Schrittes als Voraussetzung für die „Teilnahme“ am nächsten Schritt modelliert, nimmt soziale Slektivität jedoch über die Phasen ab. Es sind dann die frühen Phasen, in denen viele Schüler/innen „verloren gehen“. Entsprechend wird eher Selbstselektion als Fremdselektion als ursächlich für soziale Ungleichheit identifiziert. Es handelt sich hierbei jedoch in der Regel um ceteris paribus Befunde, d.h. Fremdselektion scheint eine untergeordnete Rolle zu spielen, sofern Studienberechtigte unterschiedlicher sozialer Herkunft sich bezüglich weiterer relevanter Variablen nicht unterscheiden: wenn sie sich zu gleichen Anteilen, mit dem gleichen Profil und auf die gleiche Art und Weise bewerben. Inwiefern hiervon ausgegangen werden kann, sollte neben der individuellen Ressourcenausstattung auch von den institutionellen Bedingungen abhängen, mit denen sie konfrontiert sind, wenn sie ihre Studienaspirationen (mehr oder weniger erfolgreich) umsetzen. Hieran anknüpfend sollen im folgenden Kapitel theoretische Überlegungen zu möglichen Mechanismen hinter (potentiell) sozial ungleich verteilten Umsetzungschancen von Studienaspirationen angestellt werden. 


\section{Soziale Herkunft, Studienaspirationen und ihre Umsetzung: theoretische Perspektiven}

Nachdem im vorangegangenen Kapitel die Phasen der Umsetzung von Studienaspirationen und diesbezügliche empirische Befunde ausführlich beschrieben wurden, widmet sich Kapitel 5 theoretischen Ansätzen, mit deren Hilfe hierbei auftretende soziale Herkunftsunterschiede erklärt werden können.

Da Studienaspirant/innen unterschiedlicher sozialer Herkunft im Fokus dieser Arbeit stehen, soll im ersten Abschnitt (5.1) ein grundlegendes theoretisches Verständnis davon entwickelt werden, welche Merkmale diese Gruppe auszeichnen. Hierfür wird zunächst den Fragen nachgegangen, in welchem Zusammenhang die soziale Herkunft von Schüler/innen mit deren idealistischen Bildungsaspirationen steht und - hieran anknüpfend - welchen Stellenwert Aspirationen in verschiedenen Ungleichheitstheorien einnehmen. Dabei stehen der mikrotheoretisch fundierte rational choice-Ansatz, Bourdieus Reproduktionstheorie sowie das sozialpsychologisch orientierte status attainmentModell im Fokus. In diesen theoretischen Ansätzen stellen Aspirationen vor allem einen wichtigen Mediator sozialer Ungleichheit im Bildungssystem dar (5.1.1). Die in Abschnitt 4.1 zitierten empirischen Befunde deuten jedoch darauf hin, dass auch unter sozial schwächeren Schüler/innen hohe Bildungsaspirationen verbreitet sein können. Daher soll auf Grundlage der genannten Theorien auch diskutiert werden, unter welchen Bedingungen Studienaspirationen theoretisch auch bei sozial schwächeren Schüler/innen erwartet werden können (5.1.2).

In den hierauf folgenden Abschnitt verlagert sich der Fokus auf die Umsetzung vorhandener Studienaspirationen: Was sind potentielle Gründe für unrealisierte Studienaspirationen, und inwiefern sind selbst innerhalb der hoch selektiven Gruppe von (Fach-)Abiturient/innen mit Studienaspiration noch Herkunftsunterschiede zu erwarten? Welche grundlegenden Mechanismen kommen als mögliche Erklärung in Frage? Für ein umfassenderes Verständnis sozialer Ungleichheit ist dabei nicht nur die Frage wichtig, ob Studienaspirationen überhaupt umgesetzt werden, sondern auch, inwiefern eine Umsetzung mit der Wahl unterschiedlicher, horizontal und vertikal stratifizierter Studienoptionen einhergeht, die im Zuge der Bildungsexpansion zunehmend wichtige Ungleichheitsdimensionen darstellen.

Zunächst wird grundlegend argumentiert, dass sozialgruppenspezifische Realisierungschancen durch das Zusammenspiel von individuellen Ressourcen und institutionellen Opportunitäten und Barrieren entstehen können (5.2). Hieran anknüpfend werden unter Rückgriff auf die bereits in Kapitel 3 eingeführte Unterscheidung zwischen primären und sekundären Effekten (Boudon 1974) mögliche ungleichheitsgenerierende Mechanismen abgeleitet. Das Zusam- 
menspiel individueller Ressourcen und institutioneller Opportunitäten und Barrieren kann sich dabei in der Wechselwirkung zwischen Leistungsunterschieden und institutionellen Auswahlkriterien (5.3) sowie zwischen (mehr oder weniger rational reflektierten) Bildungsentscheidungen und der (hochschulischen) Opportunitätsstruktur (5.4) zeigen.

\subsection{Aspirationen im Spiegel soziologischer Ungleichheitstheorien}

In Kapitel 4 wurden Aspirationen als Bildungswünsche definiert, denen ein idealistisches Element innewohnt und die weniger stark von wahrgenommenen Barrieren beeinflusst sind als Intentionen. Damit stellt sich die Frage, ob auch Aspirationen bereits sozialgruppenspezifisch eingeschränkt sind und damit Bildungsaufstiegen entgegenwirken. Oder ob über das Stratifikationssystem hinaus ähnliche Bildungsziele als erstrebenswert geteilt werden, deren Umsetzungschancen aber nach sozialer Stellung variieren. Sind es also ,limited desires“ oder „limited opportunities“ (Keller und Zavalloni 1964: 58, siehe auch Rodman 1963, Stephenson 1957, Swidler 1986), die zu Bildungsungleichheiten führen? Die Antwort auf diese Frage ist zentral für die vorliegende Arbeit, denn wären Aspirationen universell geteilt, wären sie weder als Explanandum noch als Explanans geeignet, um Bildungsverläufe zu modellieren. Realisierungschancen von Studienaspirationen wären damit nichts weiter als ein Synonym für Übergangschancen ins Hochschulstudium.

Theoretischer Konsens besteht diesbezüglich nicht (vgl. Stephenson 1957): Einige Autor/innen interpretieren Aspirationen als Teil des gesellschaftlichen Wertesystems, das von großen Teilen der Bevölkerung geteilt wird - unabhängig von sozialer Klasse, Ethnie oder Geschlecht (vgl. Merton 1957, Swidler 1986). Dieses Verständnis von Aspirationen als universell geteilt findet vor allem in den USA Anklang, wo der „college-for-all“ Mythos (Rosenbaum 2001) in den letzten Jahrzehnten über alle sozialen Gruppen hinweg zu einem extremen Anstieg von Studienaspirationen und somit zu einer Entkopplung von Aspirationen und sozialer Herkunft sowie deren Umsetzungschancen geführt hat (vgl. Domina et al. 2011: 94, Goyette 2008, Jacob und Wilder 2010). Ähnliche Ergebnisse zitierend schlussfolgert zum Beispiel Ann Swidler (1986: 274), dass Aspirationen aufgrund fehlender Varianz sowohl für die Erklärung sozialer Ungleichheit als auch individuellen Verhaltens weitestgehend ungeeignet sind: „What people want [...] is of little help in explaining their action".

Andererseits wird argumentiert, dass Aspirationen nicht uneingeschränkt sind, sondern als Teil des klassenspezifischen Wertesystems nach sozialer Lage variieren (vgl. Hyman 1953); dies ergibt sich gerade aus ihrer sozial eingebet- 
teten Entstehung, aus der eigenen Position im gesellschaftlichen Gefüge, die (wahrgenommene) Möglichkeiten entweder eröffnet oder einschränkt. ${ }^{79}$ Diese Argumentation erscheint dem stark stratifizierten deutschen Bildungssystem, das als angemessen und als möglich wahrgenommene Bildungsalternativen sozialgruppenspezifisch definiert, zunächst besser zu entsprechen. Verschiedene, häufig genutzte Ungleichheitstheorien wie etwa Pierre Bourdieus Reproduktionstheorie, das sozialpsychologisch orientierte status attainment-Modell oder soziologische Spielarten des RC-Ansatzes argumentieren größtenteils in diese Richtung. Sozialgruppenspezifische Aspirationen können aus dieser Perspektive als zentraler Mediator von Bildungsungleichheit verstanden werden. Die grundlegenden Mechanismen, die einer sozialgruppenspezifischen Einschränkung von Aspirationen zugrunde liegen, sollen im Folgenden kurz vorgestellt werden.

\subsubsection{Der Regelfall: Aspirationen als Mediator von Herkunftsunterschieden}

Nach Pierre Bourdieu werden Aspirationen als ein mehr oder weniger internalisiertes Streben nach Bildung im Zuge der Sozialisation entlang gesellschaftlicher Stratifikationslinien inkorporiert und wirken damit als Teil des klassenspezifischen Habitus (Bourdieu 1973, 1982, Bourdieu und Passeron 1973). Vermittelt über den Habitus sind Praxis und Struktur zirkulär miteinander verbunden. Aus dieser Perspektive werden Aspirationen zu sich selbst erfüllenden Prophezeiungen, wenn „die objektiven Bedingungen [...] die Bestrebungen bestimmen, indem sie den Grad bestimmen, in dem sie befriedigt werden können“ (Bourdieu 1973: 108). Ein Hochschulstudium einschränkende (oder ermöglichende) Strukturen werden demnach im Zuge der Sozialisation internalisiert und in entsprechende Bildungsaspirationen übersetzt, was zur „Selbsteliminierung" der unteren Klassen führt (Bourdieu und Passeron 1971: 175).

Auch im stärker funktionalistisch orientiertem Wisconsin model of status attainment stellen Aspirationen einen zentralen Ankerpunkt dar (vgl. Haller und Portes 1969, Sewell et al. 1969, Sewell und Hauser 1972, Sewell und Shah

79 Eine Integration beider Perspektiven liefert Rodmans (1963) Ansatz vom lowerclass value stretch. Demzufolge hängt die Universalität von Werten (und synonym: Aspirationen) von deren Abstraktionsgrad ab: Je abstrakter, desto eher über Klassengrenzen hinweg geteilt. Die unteren Klassen teilen demnach zwar die generellen, abstrakten Werte mit den oberen Klassen, entwickeln diese aber weiter, dehnen und ergänzen sie, um sie in Einklang mit ihrer nachteiligen Position zu bringen (ebd.: 209f.; siehe auch Mickelsons (1990) Unterscheidung zwischen abstrakten und konkreten Einstellungen). 
1967, für einen umfassenden Überblick siehe Sewell et al. 2004). ${ }^{80}$ Das vor allem in der US-amerikanischen Forschung verbreitete Modell baut auf der Arbeit von Blau und Duncan (1967) zur intergenerationalen Mobilität auf, die zeigen konnten, dass der größte Anteil des Einflusses von sozialer Herkunft und leistungsbezogenen Faktoren auf die spätere berufliche Stellung über die Bildungsbeteiligung mediiert wird. Um die zugrundeliegenden Mechanismen für Unterschiede in der Bildungsbeteiligung besser zu verstehen, integrierten Forscher der University of Wisconsin Madison sozialpsychologische Variablen in das ursprüngliche Blau-Duncan-Modell. Zentral ist hierbei, dass die Erwartungshaltung, Ermutigung und Vorbildfunktion sogenannter signifikanter Anderer - vor allem Eltern, Peers und zu einem geringeren Anteil auch Lehrkräfte - positiv mit den Aspirationen von Schüler/innen zusammenhängen und gleichzeitig mit deren sozialem Hintergrund und Schulleistung variieren. Die eigenen Aspirationen stehen wiederum in starkem Zusammenhang mit der Fortführung der angestrebten Bildungslaufbahn und erklären somit einen Großteil des Zusammenhangs zwischen sozialer Herkunft und schulischer Leistung auf der einen und Bildungserfolg auf der anderen Seite (vgl. Hossler et al. 1999, Jacob und Wilder 2010). Als stärkster Mediator wurden Aspirationen zum „strategic center“ des status attainment-Models (Haller und Portes 1973: 68).

Letztlich nehmen Aspirationen auch in vor allem auf Raymond Boudon (1974) zurückgehenden mikro-theoretischen (rationalen) Entscheidungsmodellen, die bereits in Kapitel 3 als Hauptstrang der deutschen Bildungssoziologie identifiziert wurden (vgl. Solga und Becker 2012), einen zentralen Stellenwert ein. In Anlehnung an die social position theory von Keller und Zavalloni (1964) haben Aspirationen laut Boudon (1974: 23) eine strukturelle Komponente, die durch die Position der sozialen Herkunft bestimmt wird und vorgibt, wie weit junge Menschen ,reisen“ müssen, um ein bestimmtes Bildungsniveau und damit zusammenhängend einen bestimmten sozialen Status erreichen zu können. Dementsprechend können Schüler/innen aus sozial schwächeren Familien relativ gesehen ebenso hohe Aspirationen wie ihre sozial privilegierten Peers haben, auch wenn sie (absolut gesehen) geringere Ziele anstreben (vgl. ebd.: 23, Keller und Zavalloni 1964: 66). Das absolute Aspirationsniveau ist entsprechend sozial ungleich verteilt, da Familien aller sozialer Klassen Statuserhalt anstreben: "[they] seek to ensure, so far as they can, that their children acquire a class position at least as advantageous as that from which they originate or, in other words, they seek to avoid downward social mobility" (Breen und Goldthorpe 1997: 283). Diese grundlegenden Annahmen wurden als Sta-

80 Das Konzept von Aspirationen als ,the cognitive orientational aspect of goal-oriented behavior" (Haller 1968: 484) wurde zwar von (Sozial-)Psychologen bereits früh verwendet (z.B. Lewin 1939), erlangte aber seine breite Popularität durch das status attainment-Modell, das im Zuge der Wisconsin Longitudinal Study formalisiert und empirisch geprüft wurde. 
tus-erhaltmotiv oder relative Risikoaversion (relative risk aversion, $R R A$ ) formalisiert (insbesondere Breen und Goldthorpe 1997). Bildungsaspirationen in Form eines Strebens nach Statuserhalt sind ein wichtiger Treiber sozialgruppenspezifischer Bildungsentscheidungen (sekundärer Herkunftseffekte, vgl. Abschnitt 5.3).

Aspirationen als Teil des klassenspezifischen Habitus, als Resultat der sozialgruppenspezifischen Einflussnahme signifikanter Anderer und als Antrieb zum Erhalt des familiären sozialen Status dienen in den kurz vorgestellten theoretischen Ansätzen als wichtige Erklärung für soziale Ungleichheit im Bildungssystem. Wenn sie ein zentraler Mediator von Bildungsungleichheit sind, wie können dann hohe Aspirationen innerhalb der Gruppe sozial schwächerer Schüler/innen erklärt werden?

\subsubsection{Die Ausnahme? Hohe Aspirationen trotz niedriger sozialer Herkunft}

Keller und Zavalloni (1964) sehen neben der strukturellen Komponente von Aspirationen auch eine persönliche (,needs, capacities and talents“, ebd.: 60), durch die Variationen im Aspirationsniveau innerhalb einer sozialen Herkunftsgruppe zustande kommen können. Die verschiedenen, auf Boudon aufbauenden RC-Ansätze gehen - wie oben beschrieben - zwar aufgrund der treibenden Kraft des Statuserhaltmotivs von sozial ungleich verteilten (absoluten) Bildungsaspirationen als Regelfall aus, doch handelt es sich bei dieser Regelmäßigkeit nicht um ein ,iron law“ (Goldthorpe 1996a: 498). Der Link zwischen sozialer Herkunft und Aspirationen kommt vor allem durch eine Grenzziehung nach unten zustande: Da Statusverlust unter allen Umständen vermieden werden soll, bestehen Aspirationen für höhere Bildungsstufen, solange die Reproduktion des sozialen Status durch die bereits erreichte Bildungsstufe nicht als gesichert gilt. Ein Aspirationsniveau unterhalb von Hochschulbildung ist daher für sozial privilegierte Schüler/innen - im Gegensatz zu sozial benachteiligten Schüler/innen - extrem unwahrscheinlich (für eine Diskussion von Ausnahmen vgl. Jæger und Holm 2012). Weniger unwahrscheinlich ist hingegen, dass sozial benachteiligten Schüler/innen Aufwärtsmobilität anstreben. Eine Bedingung hierfür ist allerdings, dass die Erfolgsaussichten ausreichend hoch sind und somit das Risiko eines Misserfolgs (und damit einhergehender Kosten und Abstiegsrisiken) minimiert wird (vgl. Breen und Goldthorpe 1997: 291f., Gambetta 1987, Goldthorpe 1996a). ${ }^{81}$

81 Wie gravierend ein etwaiger Misserfolg ist, hängt dabei vom Bildungssystem und dem Link zwischen Zertifikaten und Arbeitsmarkterfolg ab. Bildungsjahre ohne Abschluss müssen nicht zwangsläufig verschwendet sein, wenn die Chancen auf Statuserhalt auch ohne Abschluss gesteigert werden (Breen und Goldthorpe 1997: 282). Im deutschen Kontext, in dem formale Bildungszertifikate für den Arbeits- 
Auch im status attainment-Modell besteht zwar vermittelt über die Erwartungshaltung und Vorbildfunktion von Eltern und Peers ein Zusammenhang zwischen sozialer Herkunft und Bildungsaspirationen. Es ist jedoch nicht ausgeschlossen, dass auch sozial schwächere Schüler/innen Freunde und Peers mit eigenen Studienaspirationen haben und Eltern, die ihnen eine hohe Erwartungshaltung entgegen bringen und sie unterstützen (vgl. Sewell und Shah 1968). Dies sollte vor allem dann der Fall sein, wenn Schüler/innen aus sozial schwächeren Familien überdurchschnittlich gute Schulleistung demonstrieren, da diese beeinflusst, wie hoch deren Potential eingeschätzt wird.

Auch der oben mit Bezug auf Bourdieu diskutierte „Selbsteliminierungsmechanismus" ist nicht absolut. Zwar hat sich mit Bezug auf die durch den Habitus vermittelte Zirkularität von Agency und Struktur unter Bourdieus Kritikern eine deterministische Leseart durchgesetzt (vgl. Goldthorpe 1996a, 2007, Sullivan 2002), doch Bourdieus Texte lassen auch eine andere Interpretation zu (z.B. Bourdieu 1990, 1981: 171, vgl. weiterhin Edgerton und Roberts 2014, Lamont und Lareau 1988, Lizardo 2004, Reay 1998, Reay et al. 2001a, van de Werfhorst 2010), nach der der Habitus ein ,dynamisches Konzept“ ist (Reay 1998: 521), „kein Schicksal, dem man sich fatalistisch zu fügen hätte“ (Müller 2014: 34) - er leitet zwar Praxis und setzt Grenzen, determiniert sie aber nicht (vgl. Bourdieu 1992). Damit ist der Habitus durchaus vereinbar mit Abweichung und Innovation:

"If habitus is open, in some degree, to change in the face of new environments and experiences, if it is a generative principle that enables a diverse range of actions, and if an individual's habitus is not fully coterminous with any single group, but rather a unique sedimentation of their situated history, then habitus-mediated individual action can still be novel and innovative, and social origins do not necessarily determine social destinations; social reproduction is not inevitable." (Edgerton und Roberts 2014: 202)

Der Habitus wird hiernach zwar von Angehörigen derselben sozialen Klasse in seinen Grundzügen geteilt, ist aber keineswegs für jeden Einzelnen identisch. Damit erklären Bourdieu und Passeron (1971: 43f.) die „Ausnahmefälle", die trotz der unterprivilegierten sozialen Stellung ihrer Familie ein Hochschulstudium anstreben. Die Ursachen hierfür seien in einem ,außergewöhnlichen Familienmillieu“ zu suchen, das sie ,von einem der mächtigsten Handikaps ihrer sozialen Schicht befreit: dem resigniertem Verzicht auf eine Fortführung ,unmöglicher' Studien“" (ebd.). Dennoch ist habituelle Anpassung

markterfolg eine große Rolle spielen, ist dies jedoch selten der Fall. Hier sollten Studienzeiten ohne Abschluss schlechter verwertbar sein, sodass mit erfolglosem Studium die (Opportunitäts-)Kosten steigen, da auch Schüler/innen aus nicht-akademisch gebildeten Elternhäusern in der Regel einen Ausbildungsabschluss benötigen, um ihren sozialen Status zu sichern (vgl. Bol und van de Werfhorst 2010, Solga und Konietzka 2000). 
und die Herausbildung hoher Bildungsaspirationen meist nicht gleichzusetzen mit einer kompletten Aufhebung internalisierter, klassenspezifischer „DenkWahrnehmungs-, Bewertungs- und Aktionsschemata" (Bourdieu und Passeron 1973: 55). Hier kommt der stabile, dauerhafte Teil des Habitus zum Tragen, der trotz Anpassung weiterhin beeinflussen kann, ob man sich wie ,ein Fisch im Wasser" (Bourdieu und Wacquant 1996:161) fühlt und den spezifischen Anforderungen des Hochschulsystems inklusive dessen Zugangs gerecht wird.

Gerade deutsche Abiturient/innen aus sozial schwächeren Familien haben sich nicht bereits aufgrund der Internalisierung ,objektiver Erfolgswahrscheinlichkeiten“ (Bourdieu 1973: 83) an früheren Selektionsstufen „selbst eliminiert", was auf eine positivere Haltung gegenüber Bildung hindeutet als es auf Grundlage ihrer Position im sozialen Raum allgemein zu erwarten wäre. Dies kann zum einen das Resultat positiver schulischer Erfahrungen sein, wenn ,academic success and the rewards of increased cultural capital positively alter the student's aspirations and orientation toward school (habitus), which in turn feeds back into their practices and performance" (Edgerton und Roberts 2014: 199f.). Zum anderen sind solche Schüler/innen, wie oben beschrieben, besonders anpassungsfähig oder wurden in einem ,,außergewöhnlichen Familienmilieu“" (Bourdieu und Passeron 1971: 43) sozialisiert.

Zusammenfassend wurde aus den diskutierten Ungleichheitstheorien - im Gegensatz zu dem eingangs vorgestellten Verständnis von Aspirationen als universell, über Klassenlinien hinweg verbreitetet - ein deutlicher Zusammenhang zwischen sozialer Herkunft und Aspirationen abgeleitet, der ihren Stellenwert als zentraler Mediator von Bildungsungleichheit hervorhebt. Obwohl die Theorien also von deutlich sozialstrukturell beeinflussten Aspirationen als Regelfall ausgehen, schließen sie nicht aus, dass auch sozial schwächere Schüler/innen Studienaspirationen ausbilden. Dies ist vor allem dann wahrscheinlich, wenn förderliche Faktoren wirken (z.B. gute Schulleistung oder Unterstützung und Zuspruch aus dem sozialen Netzwerk). Bei sozial schwächeren Schüler/innen mit hohen Bildungsaspirationen sollte es sich demnach um eine weiter positiv selektierte Gruppe handeln (wenn auch nicht unbedingt um die von Bourdieu und Passeron (1971: 43) vor fast 50 Jahren identifizierten „Ausnahmefälle“). Inwiefern können also selbst innerhalb der Gruppe von Studienaspirant/innen noch soziale Herkunftsunterschiede bezüglich der Umsetzungschancen von Studienaspirationen angenommen werden? Wie argumentiert wurde, liefern die oben angeführten, verbreiteten Ungleichheitstheorien vor allem Erklärungen für das Zustandekommen sozialgruppenspezifischer Aspirationen. Doch können die hierin angelegten Mechanismen auch für deren Umsetzung relevant sein. Im Folgenden werden diese daher erneut aufgegriffen und ergänzt. 


\subsection{Die Umsetzung von Studienaspirationen: Zusammenspiel von individuellen Ressourcen und institutionellen Strukturen}

Grundsätzlich folge ich der Annahme, dass soziale Ungleichheit im Bildungssystem durch das Zusammenspiel individueller Ressourcen und institutioneller Opportunitäten und Barrieren verstanden werden kann, die die Handlungen von Akteuren - Schüler/innen und deren Familien - sowohl einschränken als auch ermöglichen können. Bevor dieses Zusammenspiel weiter ausbuchstabiert wird, sollen hierfür im Folgenden zunächst zentrale Definitionen geliefert werden.

Aspirationen als „motivational factors“ (Azjen 1991: 181) oder „well-formulated goals“ (Schoon und Lyons-Amos 2016: 13) können als wichtige Komponente der Agency individueller Akteure verstanden werden, die diesen Handlungsimpulse gibt, sie motiviert, entsprechende Entscheidungen zu treffen und Handlungen auszuführen - hier etwa, sich für ein Studium zu bewerben. Daran schließt sich die Frage an, ob Aspirationen für Angehörige unterschiedlicher sozialer Gruppen dieselbe Bedeutung haben, in gleicher Weise motivieren und Handlungsimpulse setzen oder ob nicht nur ihre Entstehung, sondern auch Wirkung sozialgruppenspezifisch eingeschränkt ist. Denn gleichzeitig treffen Akteure auf Strukturen und sind mit unterschiedlichen sozialgruppenspezifischen - Ressourcen ausgestattet, die Handlungen und Handlungsergebnisse sowohl einschränken als auch ermöglichen können (Goldthorpe 1996a, Sewell 1992).

Ressourcen - ebenso wie der ähnlich häufig genutzte Kapitalbegriff werden hier als Mittel verstanden, auf die Akteure (mehr oder weniger bewusst) zurückgreifen können, um ihre Ziele zu verwirklichen. Diese Mittel sind nicht ausschließlich materiell, sondern können auch in sozialen Beziehungen oder den Akteuren selbst liegen. Entsprechend wurden unabhängig von den genutzten Begrifflichkeiten aus unterschiedlichen theoretischen Perspektiven drei Arten von Ressourcen - ökonomische, kulturelle und soziale - identifiziert (vgl. Bourdieu 1983, Coleman 1988, Erikson und Jonsson 1996). Auch wenn diese, wie oben argumentiert, bereits Bildungsaspirationen selbst mitprägen und Ressourcenunterschiede somit in der Gruppe der Studienaspirant/innen kleiner sein sollten, kann auch hier von einem gewissen Maß an Heterogenität ausgegangen werden, da die Ressourcenausstattung auch immer das „Resultat sozialstrukturell unterschiedlicher Lebensgeschichten“ (Solga 2005a: 53) ist, folglich von der eigenen sozialen Herkunft beeinflusst wird.

Während ökonomische Ressourcen in Form monetärer Mittel leicht zu definieren sind, ist die Abgrenzung zwischen kulturellen oder sozialen Ressourcen weniger eindeutig, da deren Ausprägung und Wirkung (etwa in Form spezifischer Informationen, kulturellen Wissens und Fähigkeiten oder internali- 
sierter Werte und Einstellungen) nicht unabhängig von ihrer Entstehung innerhalb sozialer und gleichzeitig mit mehr oder weniger kulturellen Ressourcen ausgestatteter Netzwerke verstanden werden kann..$^{82}$ Unabhängig davon, ob als kulturell oder sozial bezeichnet, sind im Bildungssystem zwei Formen von Ressourcen besonders relevant: zum einen im Netzwerk verbreitete Normen und Werte und zum anderen hier verfügbare Informationen.

Im sozialen Netzwerk verbreitete und durch interne und externe Sanktionen gefestigte Bildungsnormen werden, insbesondere wenn sie homogen sind, adaptiert oder internalisiert. Sozial privilegierte Schüler/innen sind häufig in Netzwerke eingebettet, in denen die Studiennorm tief verankert ist, was dazu führen kann, dass ein Hochschulstudium unhinterfragt verfolgt wird. Im Netzwerk verbreitete und als Teil des Habitus internalisierte Normen sind dabei nicht nur ermöglichend, sondern auch einschränkend (Coleman 198: 105); sie konstituieren „ein System von Grenzen“ (Bourdieu 1992: 33), das „lower and upper boundaries" (Glaesser und Cooper 2014: 477) innerhalb und außerhalb des Hochschulsystems klassenspezifisch definiert.

Mit zunehmender Komplexität des Bildungssystems steigt die Relevanz von im Netzwerk verfügbaren Informationen (vgl. Pfeffer 2008). Quantität und Qualität des Wissens zu verschiedenen Bildungswegen innerhalb und außerhalb des Hochschulsystems sind dabei aufgrund des unterschiedlichen Wissensschatzes und der (Bildungs-)Erfahrungen in der Familie und im sozialen Netzwerk sozial ungleich verteilt (vgl. Bourdieu 1982, Erikson und Jonsson 1996, Grodsky und Jones 2007, Hossler et al. 1999, McDonough 1997, Slack et al. 2012). So können Eltern und ein soziales Netzwerk mit Hochschulerfahrung nicht nur darüber informieren, ,dass man gar nicht besonders clever sein muss, um es an der Universität zu schaffen“ (Müller und Pollak 2008: 310), sondern auch entscheidendes System- und Navigationswissen weitergeben (vgl. Erikson und Jonsson 1996: 22).

Die Strukturen, mit denen Studienaspirant/innen heute konfrontiert sind und die sozial ungleich verteilte Studienchancen mit beeinflussen, ergeben sich unter anderem aus der Allokations- oder Selektionsfunktion des Bildungssystems, durch die die Zuteilung zu gesellschaftlichen Positionen über objekti-

82 Entsprechend werden die verschiedenen Formen kultureller und sozialer Ressourcen je nach theoretischem Ansatz anders zugeordnet. So besteht laut Coleman (1988) Sozialkapital aus in Netzwerken bestehenden Verpflichtungen, Normen und Informationen. Letztere werden sowohl von Erikson und Jonsson (1996) als auch Bourdieu $(1973,1982)$ kulturellen Ressourcen bzw. Kapital zugeordnet. Colemans Konzeption der normativen Komponente sozialen Kapitals befindet sich in argumentativer Nähe zur Erwartungshaltung signifikanter Anderer im status attainment-Modell (in dem allerdings die Begriffe kulturelle oder soziale Ressourcen/Kapital kaum Verwendung finden). Bei Bourdieu (1983) macht dies einen wichtigen Bestandteil des inkorporierten kulturellen Kapitals aus, das während der Sozialisationszeit von den Eltern auf die Kinder übertragen wird. 
vierte und vergleichbare Bildungszertifikate sicherstellt wird (vgl. Keller 2014, Parsons 1972). Allokation findet heute vermehrt durch das Hochschulsystem statt, da die Bildungsexpansion vor allem zum Ausbau des primären und sekundären Bildungsbereichs führte (vgl. Abschnitt 2.2.1). Funktionalistische Ansätze gehen dabei davon aus, dass Zertifikate - und damit einhergehend hierarchisch differenzierte Positionen in einer arbeitsteiligen Gesellschaft - allein auf Grundlage natürlicher Begabung und Anstrengungen vergeben werden. Bildungserfolge und -misserfolge werden so individualisiert - ,winners can self-congratulate and losers have only themselves to blame" (Swartz 2008: 418) - und gesellschaftliche Stratifizierung und soziale Ungleichheit als ,gesellschaftliches Funktionserfordernis“ (Solga 2005b: 26) legitimiert. Aus konflikttheoretischer Perspektive (vgl. Bourdieu 1973, 1982, Collins 1971, 1979, Karabel 1972) sollten statushohe Familien hingegen ,zur Wahrung des relativen Seltenheitsgrades ihrer Abschlüsse und damit einhergehend zur Aufrechterhaltung ihrer Position innerhalb der Struktur der Klassen“ (Bourdieu 1982: 222) bestrebt sein, das Hochschulsystem zumindest in Teilen gegen die wachsende Zahl an Studienaspirant/innen zu schließen. Dafür können neue SchlieBungsmechanismen eingeführt oder alte verstärkt werden. So kann Hochschulbildung zum Beispiel durch Studiengebühren oder Studienzeitverlängerungen verteuert werden. ${ }^{83}$ Zudem kann es zu einer Verknappung des Angebots an Hochschulbildung (bzw. fehlendem Ausbau trotz steigender Nachfrage) (vgl. Bourdieu 1982) und damit einhergehend einer stärkeren Selektion der Studienaspirant/innen kommen (vgl. Alon 2009). Zudem wird die Bildungsexpansion meist von einer Differenzierung innerhalb verschiedener Bildungsebenen begleitet (vgl. Abschnitt 2.2.1), die wiederum mit unterschiedlich prestigereichen beruflichen Positionen verknüpft sind. Solch eine Hierarchisierung ,can be every bit as effective as exclusion in sorting people, and it has the added advantage of giving credibility to the myth of equal opportunity" (Karabel 1972: 42).

Aus diesen (möglichen) Entwicklungen ergibt sich ein institutionelles Geflecht, das - im Zusammenspiel mit individuell verfügbaren Ressourcen - auf direktem und indirektem Wege auf die Umsetzung von Studienaspirationen

83 Wie in Abschnitt 2.2.1 dargelegt, haben sich Studiengebühren über den kleinen Sektor privater Hochschulen hinaus in Deutschland nicht etabliert. Auch bezüglich der Studiendauer zeigt sich im Zuge des Bologna Prozesses zunächst ein gegenteiliger Trend. Diese Entwicklung ist allerdings im Kontext einer zunehmenden vertikalen Stratifizierung des Hochschulstudiums zu sehen. Soziale Ungleichheit beim Zugang zum Masterstudium (Auspurg und Hinz 2011a, Lörz et al. 2015, Neugebauer et al. 2016) bei gleichzeitig fehlendem Rückgang sozialer Ungleichheit beim Übergang in ein Bachelorstudium (Neugebauer 2015), deuten dabei auf Schließungsprozesse hin. Die Verkürzung der Studienzeit betrifft zudem nicht die Professionen Medizin und Jura, die für soziale Schließung besonders prädestiniert sind (Thomsen 2018). 
einwirken und dabei zu sozialer Ungleichheit beitragen kann. Welche Mechanismen sind hierfür grundlegend? Zur Annäherung an diese Frage wird die verbreitete Unterscheidung zwischen primären und sekundären Herkunftseffekten als zentrales theoretisches Ordnungselement herangezogen.

Auch wenn der Ursprung dieser theoretischen Differenzierung weiter zurückliegt (vgl. Jackson 2013), war es Raymond Boudon (1974), der sie durch sein weit rezipiertes Buch „Education, Opportunity and Social Inequality“ in den Kanon der (mikro-theoretisch orientierten) soziologischen Bildungsforschung einführte. Die getroffene Unterscheidung beruht auf der Beobachtung, dass ein Teil der sozialen Ungleichheit im Bildungssystem durch sozial ungleich verteilte (non-)kognitive Fähigkeiten und schulische Leistung (primäre oder auch indirekte Effekte) zustande kommt. Wie im Folgenden argumentiert wird, werden diese (durch sozialgruppenspezifische Ressourcenausstattung zustande kommenden) Unterschiede im Leistungsprofil erst im Zusammenspiel mit entsprechenden Selektionskriterien institutioneller Gatekeeper relevant (5.3). Über die Wirkung primärer Effekte hinaus entsteht soziale Ungleichheit auch durch sozialgruppenspezifische Bildungsentscheidungen (sekundäre oder auch direkte Herkunftseffekte). Verschiedene Bildungsoptionen innerhalb und außerhalb des Hochschulsystems und hiermit in Verbindung stehende institutionelle Opportunitäten und Barrieren werden hierbei im Lichte der eigenen Ressourcenausstattung (mehr oder weniger bewusst) reflektiert und in die Entscheidungsfindung einbezogen (5.4).

\subsection{Primäre Herkunftseffekte und ihre Relevanz in Auswahlverfahren}

Insgesamt können primäre Herkunftseffekte als das Resultat kumulierter Strategien, Ressourcen und Sozialisationsbedingungen verstanden werden, die in Interaktion mit der Institution Schule Bildungserfolg begünstigen oder erschweren (vgl. Erikson und Jonsson 1996: 10ff., Jackson 2013: 12f.). Sie manifestieren sich nicht nur in sozialgruppenspezifischen kognitiven und nonkognitiven Fähigkeiten (vgl. Blossfeld et al. 2015: 147), die sich (zum Teil) in unterschiedliche Schulabschlüsse und zertifizierte Schulleistungen übersetzen, sondern darüber hinaus auch im jeweiligen Qualifikationsprofil, auf dessen Grundlage Studienberechtigte um einen Studienplatz konkurrieren (z.B. durch Schulwahl, Kurswahl, die Teilnahme an verschiedenen extra-curricularen Tätigkeiten oder auch eine während der Sozialisation erworbene kulturelle Passung zwischen Auswählenden und Auszuwählenden).

Zur Erklärung primärer Herkunftseffekte (im Gegensatz zu sekundären) wird häufig mehr oder weniger explizit auf Bourdieus reproduktionstheoretisches Paradigma (insbesondere 1973, 1982, 1983, Bourdieu und Passeron 
1971) zurückgegriffen (vgl. Barone 2006, Becker 2012, Erikson und Jonsson 1996, Maaz 2006, van de Werfhorst und Hofstede 2007). Im Kern geht dieses davon aus, dass kulturelles Kapital - vor allem in seiner inkorporierten Form $^{84}$ - während der Sozialisationszeit zunächst von den Eltern auf ihre Kinder übertragen und in einem zweiten Schritt in Bildungserfolg (Bildungszertifikate) übersetzt wird. Dies gelingt vor allem Kindern aus sozial privilegierten Elternhäusern, da das kulturelle Kapital und damit verbunden auch der Habitus der Mittel- und Oberschicht - „familiarity with the dominant culture“ (Sullivan 2002: 145) - im Einklang mit den Anforderungen und Vermittlungslogiken im Bildungssystem stehen und dieses gleichzeitig kaum dazu beiträgt, ungleiche Startbedingungen auszugleichen (vgl. Bourdieu 1973). Unterschiede in den sozialisations- und daher klassenbedingten Voraussetzungen für Bildungserfolg werden so als natürliche Begabung wahrgenommen und dadurch legitimiert. Durch diesen Deckmantel der Neutralität kann das Bildungssystem ,seine Funktion der Perpetuierung sozialer und kultureller Privilegien wahrnehmen“ (Bourdieu und Passeron 1971: 190).

Für den Übergang in die Hochschule wurde vor allem im deutschen Kontext argumentiert und gezeigt, dass primäre Effekte weniger Relevanz besitzen als sekundäre. So zeigen etwa Schindler und Reimer (2010) für verschiedene Studienberechtigtenkohorten, dass nur etwa 20\% der Herkunftsdifferenz beim Übergang ins Studium auf primäre Effekte zurückgeführt werden können (siehe auch Neugebauer et al. 2013, Schindler und Lörz 2012, Watermann et. al. 2014). Neben der stark segmentierten Struktur postsekundärer Bildung in Deutschland (vgl. Müller und Pollak 2008), die sekundäre Effekte verstärkt, ist ein weiterer Grund für die eingeschränkte Relevanz primärer Effekte in der „großen Selektivität der verbleibenden Population“ zu suchen, die die Hochschulzugangsberechtigung erreicht. „Gerade das Leistungsniveau der Abiturienten scheint sich kaum mehr nach der sozialen Herkunft zu unterscheiden“ (Schindler und Reimer 2010: 647). ${ }^{85}$ Wie in Abschnitt 5.1.2 diskutiert, sollte

84 Bourdieu (1983) unterscheidet drei Arten kulturellen Kapitals: Dieses kann objektiviert sein, d.h. in Form kultureller Objekte wie etwa Büchern, Gemälden oder Instrumenten vorliegen. Es kann weiter in Form von Bildungstiteln institutionalisiert sein und seinem Träger somit institutionelle Anerkennung und Legitimität bringen. Letztlich bezieht sich inkorporiertes kulturelles Kapital auf sämtliche kulturelle Fähigkeiten, Wissen und Werte, die sich Personen insbesondere während ihrer Sozialisation in der Familie und Schule aneignen konnten.

85 Der Befund der relativ geringen Erklärungskraft primärer Herkunftseffekte und somit der großen Erklärungskraft sekundärer Effekte wird zusätzlich dadurch gestützt, dass mögliche ,antizipatorische Bildungsentscheidungen“ dazu führen, dass sekundäre Effekte unterschätzt werden. ,This is because anticipatory decisions can be expected, especially through their positive or negative effects on motivation, to influence students' performance in the examinations they subsequently take, and [...] they will thus contribute, perversely, to primary effects rather than to secondary effects, i.e. effects that derive from choice." (Jackson et al. 2007: 222) 
diese Argumentation in noch stärkerem Maße auf Schüler/innen mit Studienaspirationen zutreffen. Als weiteren Grund für geringe primäre Herkunftseffekte führt Schindler (2012: 21) die geringe (Leistungs-)Selektivität des deutschen Hochschulsystems an:

\begin{abstract}
„Zwar existieren in einigen Studienfächern Zulassungsbeschränkungen, die sich etwa durch einen Numerus Clausus auf Schulleistungskriterien stützen, prinzipiell wird aber mit der Hochschulreife die Berechtigung zum Eintritt in das Hochschulsystem erworben. Daher sind für die Frage, ob überhaupt ein Studium aufgenommen werden kann oder nicht, die konkreten Abiturnoten von nachrangiger Bedeutung.“
\end{abstract}

Wäre der Hochschulzugang nur an das Berechtigungszertifikat geknüpft, würden primäre Herkunftseffekte - sobald die große Hürde des Erhalts des (Fach)Abiturs gemeistert ist - in der Tat keine Rolle spielen. Wie in Abschnitt 2.2.2 beschrieben wird mit der Expansion der Sekundarstufe II die Allokationsfunktion des Bildungssystems jedoch zunehmend von den Schulen auf die Hochschulen verschoben (vgl. Kreckel 2015), sodass diese nicht alle Anwärter/innen aufnehmen (können). Dies stärkt ihre Rolle als Gatekeeper, die durch Zulassungsbeschränkung und Eignungsfeststellungsverfahren darüber entscheiden, wer Zugang zu höherer Bildung und den damit verbundenen Zertifikaten und Arbeitsmarktpositionen erhält (vgl. Blossfeld und Shavit 1993, Keller 2014: 32ff.). Zur Wirkung primärer Herkunftseffekte sollte dies jedoch nur führen, wenn die Kriterien, die zur Auswahl genutzt werden, bestimmte soziale Gruppen eher erfüllen (vgl. hierzu Abschnitt 4.3.3). Warum könnte dies der Fall sein?

Aus schließungstheoretischer Sicht sind Statusgruppen kontinuierlich bemüht, sich Macht und Privilegien gegen steigende Konkurrenz zu sichern. Statusgruppen mit viel kulturellem (und teilweise auch ökonomischem) Kapital dominieren die Hochschulinstitutionen und versuchen ihren Einfluss zu nutzen, um den Zugang von ,,ihresgleichen“ zunächst zu Bildungszertifikaten und daran anknüpfend zu beruflichen Positionen sicherzustellen (vgl. Bourdieu 1982, Collins 1971, Karabel 1984, 2005, Weber 1980). Statusgruppenkämpfe sind dabei auch immer Kämpfe um die Definitionsmacht von Zulassungskriterien: „Professional classes assert their influence on the changing definitions of merit to implement admission procedures that operate de facto to ensure that children from privileged backgrounds gain access to elite education" (Thomsen 2018: 338). Dies funktioniert vor allem im Zusammenspiel mit den institutionellen Entscheidungsträgern, die meist der gleichen Statusgruppe angehören und die Interessen ihrer Organisation durch eben diese Linse betrachten (vgl. Karabel 1984: 5). ${ }^{86}$

86 Karabel (2005) demonstriert zum Beispiel anhand der Zulassungspraxis der „Big Three" (Harvard, Princton, Yale), wie wandelbar und instrumentalisiert die Definition von Meriten sein kann, wenn sie nicht mehr den doppelten Zweck Aus- 
Die Festlegung von Auswahlkriterien ist dabei theoretisch nahezu unbegrenzt und kann neben leistungsbezogenen Merkmalen wie Testergebnissen oder Schulnoten auch finanzielle Ressourcen, Motivation, Charaktereigenschaften, Zeit oder praktische Erfahrung beinhalten und bis zu Erbschaft (legacy status, vgl. Espenshade et al. 2004) oder explizit askriptiven Merkmalen (Geschlecht, Nationalität) reichen (für einen Überblick siehe Sackmann 2015). Verbunden mit der Definition von Kriterien legen Gatekeeper mit der Form des Auswahlprozesses individuelle und institutionelle Freiräume fest. Auswahlprozesse können standardisiert ohne direkten Kontakt erfolgen, wenn Testergebnisse, Schulnoten oder andere (durchaus auch qualitativere) Nachweise verlangt und durch ein Punkteverfahren objektiviert werden. Auswahlprozesse können aber auch interaktiv gestaltet sein, wenn Bewerber/innen auf Gatekeeper treffen und ihre Eignung zum Beispiel im persönlichen Gespräch demonstrieren müssen.

Alle Auswahlkriterien und -verfahren haben das Potential, Ungleichheit zu reproduzieren, ,for a particular definition always advantages some groups and disadvantages others" (Swartz 2008: 415). ${ }^{87}$ Dies trifft aufgrund unterschiedlicher Sozialisationsbedingungen und Aneignungsressourcen faktisch auch dann zu, wenn sie wegen ihrer Nähe zu bürokratischen Verfahren den Anschein von Neutralität erwecken. So kann auch die Festlegung leicht erfassbarer leistungsbezogener Kriterien im engeren Sinne (z.B. die im deutschen Kontext so relevanten Schulnoten) aufgrund sozialgruppenspezifischer Leistungsunterschiede zu sozialer Ungleichheit von Zulassungschancen führen.

„Weiche“ Verfahren und Kriterien wie die Prüfung der „Persönlichkeit“" von Bewerber/innen durch Auswahlgespräche (vgl. Helsper und Krüger 2015: 12) werden in der deutschen Debatte um „Profil und Passung“ (vgl. Heine et al. 2008, Lewin und Lischka 2004) zwar vor allem als erfolgversprechendes Mittel hervorgehoben, um über Anforderungen zu informieren und die Motivation und Eignung von Bewerber/innen für den jeweiligen Studiengang über

schluss und Monopolisierung von Privilegien erfüllt. So wurden in den 1920ern die ursprünglich leistungsbezogenen Kriterien in ,highly subjective qualities such as 'character', 'personality' and 'leadership'” (ebd.: 1) umgewandelt. Dies war der Tatsache geschuldet, dass immer mehr ,ungewollte“ Bewerber/innen die ursprünglichen Anforderungen erfüllten, sodass neue, auf kultureller Passung beruhende Kriterien zur Schließung der amerikanischen Elitebildung genutzt wurden.

87 Eine Ausnahme ist das Los, das alle Bewerber/innen zu „merkmalslosen Wesen“ (Sackmann 2015: 50) macht und damit sozialer Ungleichheit zumindest bei der Auswahl vorbeugt (eine weitere Voraussetzung für Chancengleichheit besteht in der universellen Teilnahme am Losverfahren). Allerdings ist ein Losverfahren mit dem meritokratischen Ideal nicht vereinbar und wird ,durch das erlebte Maß an Willkür manchmal als befremdlich wahrgenommen“ (ebd.). Losverfahren spielen bei der Zulassung zu deutschen Hochschulen eine sehr untergeordnete Rolle und werden in der Regel nur für übriggebliebene Studienplätze angewendet (nach dem regulären und dem Nachrückverfahren). 
deren Schulleistung hinaus zu ermitteln (vgl. Wissenschaftsrat 2004, siehe Abschnitt 2.2.2 für Details). Die Frage, inwiefern dies zur Reproduktion sozialer Ungleichheit beiträgt, wird jedoch eher ausgeblendet. Dabei kann habituelle und kulturelle Passung in persönlichen Gesprächssituationen konstruiert werden (vgl. Bourdieu und Passeron 1971, Burke und McManus 2011, Thomsen 2018, Zimdars et al. 2009). Diese Passung (oder: der passende Habitus) wird im Zuge der Sozialisation in einem hochschulerfahrenen Umfeld erworben und trägt so dazu bei, dass sich Bewerber/innen ohne solche Sozialisationserfahrungen weniger gut bewähren können. Verschärft wird dies durch fehlende Transparenz, wenn trotz komplexer Auswahlverfahren - wie etwa beim Zugang zu künstlerischen Studiengängen - oft wenig konkrete Informationen zu Ablauf und Anforderungen zur Verfügung gestellt werden (vgl. Burke und McManus 2011, Rothmüller 2011, 2012):

„This lack of information in prospectuses and on websites implies that institutions expect applicants to instinctively know what will happen in the admissions process, or that they should be able to find out this information for themselves, when actually there is nowhere for them to look, further exacerbating their confusion and lack of clarity." (Burke und McManus 2011: 19)

Intransparenz durch mangelnde Informationen eröffnet somit denjenigen potentiellen Bewerber/innen einen Vorteil im Bewerbungsprozess, die ,von Haus aus" wissen, welche Anforderungen gestellt werden und wie sie diese bewältigen können oder die über ihr soziales Netzwerk Zugang zu relevanten Informationen haben.

Daneben können manche Auswahlkriterien Adaptionsmöglichkeiten eröffnen. Bezug nehmend auf Parkins (1979) schließungstheoretischen Ansatz zeigt Alon (2009), wie sich sozial privilegierte Familien an neue Schließungspraktiken anpassen, um ihre Privilegien zu sichern. Ein Beispiel hierfür ist die Anwendung vermeintlich meritokratischer Tests zur Selektion. Dies trifft zunächst alle: „All students - affluent, poor, and middle class - face the same academic barriers because exclusionary rules are universal“ (ebd.: 736). Es sind jedoch vor allem sozial privilegierte Familien, die ihre Strategien ändern, um ihre Kinder im Kampf um begehrte Studienplätze bestmöglich zu rüsten. Diese sozial ungleiche Anpassungsfähigkeit führt trotz universeller Kriterien $\mathrm{zu}$ sozialer Ungleichheit: "The adaptation of the privileged, and the failure of the underprivileged to keep pace, creates a remarkable class-based polarization in the level of test scores, which, in turn, intensifies and expedites the formation of inequality" (ebd.: 750).

In den USA, wo standardisierte Tests als zentrales Selektionskriterium genutzt werden, hat sich entsprechend eine ganze Industrie herausgebildet, die Schüler/innen auf verschiedene Weise auf diesen „Meilenstein“ auf dem Weg in ein selektives College vorbereitet (vgl. Buchmann et al. 2010, McDonough 1994). Damit trägt die Wichtigkeit der Optimierung von Testergebnissen zur Entstehung von einem privatwirtschaftlichen Bildungssektor (,,shadow educa- 
tion $^{\text {") }}$ bei, dessen Dienste teuer erkauft werden müssen. Es sind jedoch nicht nur ökonomische Ressourcen, die hierbei Ungleichheit verschärfen, sondern wiederum das sozialgruppenspezifische Wissen darüber, wann und wie bestimmte Schritte eingeleitet werden sollten, um Studienchancen zu erhöhen (vgl. Avery und Kane 2004, McDonough 1997). Selbst im oft als egalitär charakterisierten Schweden führt spezifisches Wissen über den hier verwendeten Test (SweSAT) zu sozial ungleich verteilten Optimierungsstrategien (in Form wiederholter Teilnahme) und damit Zulassungschancen (vgl. Berggren 2007).

Welche Relevanz haben diese theoretischen Überlegungen und empirischen Befunde im deutschen Kontext? Wie in Abschnitt 2.2.3 dargelegt, nutzen viele Hochschulen den vorhandenen gesetzlichen Spielraum, um durch Eignungsfeststellungsverfahren oder im Rahmen der AdH-Quote eigene (meist standardisierbare und objektivierbare) Kriterien zu definieren und Verfahren anzuwenden. Qualitative Zulassungsverfahren wie Auswahlgespräche kommen dabei zwar zum Einsatz (vgl. etwa Bloch et al. 2015), spielen jedoch rein quantitativ eine eher untergeordnete Rolle. Gleiches gilt für standardisierte Tests: Zum einen kommen diese weniger flächendeckend zur Anwendung als etwa in den USA (vgl. Buchmann et al. 2010). Zum anderen gibt es nicht den einen Test, auf den alle testbasierten Zulassungsverfahren zurückgreifen, sodass sich in Deutschland die oben beschriebene Vorbereitungsindustrie bisher nicht herausgebildet hat. Doch auch dies ist wiederum abhängig vom Studienfach. Für die Zulassung zu medizinischen Studiengängen kommen fachbezogene Studierfähigkeitstests mittlerweile fast flächendeckend zum Einsatz (vgl. Abschnitt 2.2.2), sodass kommerzielle Vorbereitungsmöglichkeiten - wenn auch in deutlich geringerem Ausmaß als in den USA - existieren. ${ }^{88}$ Ebenso haben die spezifischen Anforderungen der künstlerischen Auswahlverfahren durch sogenannte „Mappenkurse“ auch in Deutschland ,einen eigenen ,Markt" von Bildungs- und Beratungsangeboten entstehen lassen“ (Hölscher und Zymek 2015: 223).

Auch andere Kriterien, die in den Auswahlverfahren deutscher Hochschulen häufiger zum Einsatz kommen, wie etwa der Nachweis extracurricularer Aktivitäten (ehrenamtliches Engagement, Praktika) oder Fremdsprachenkenntnisse, können mit den nötigen kulturellen, sozialen und ökonomischen Ressourcen ohne große Schwierigkeit nachgewiesen werden und so Adaptionsstrategien fördern. So zeigen etwa Carlson et al. (2017), dass Austauschprogramme überdurchschnittlich häufig von sozial privilegierten Schüler/innen genutzt werden, um „transnationales kulturelles Kapital“ zu erwerben.

88 Bei den Tests handelt es sich mehrheitlich um den bereits in Kapitel 2 vorgestellten TMS und den Hamburger Naturwissenschaftstest (HAM-Nat). Zum Beispiel bietet das Institut für Testforschung und Testtraining Köln TMS-Vorbereitungskurse (5 Tage, $895 €$ ) oder individuelle Einzeltrainings (2 Tage, 1000€) an. (https://tms-medizinertest.de/[letzter Zugriff: 09.08.2021]). 
Dies kann die Zulassungschancen zu bestimmten Studiengängen gerade bei schlechteren Abiturnoten verbessern (vgl. Abschnitt 2.2.2) ${ }^{89}$

Trotz dieser Entwicklungen hin zu komplexeren und diversifizierteren Auswahlverfahren ist das deutsche Zulassungssystem insgesamt klar notendominiert (Sackmann 2015). Wie die gesetzlich festgelegte Zentralität der Abiturdurchschnittsnote als Auswahlkriterium verdeutlicht, wird nach wie vor von einem starken Signalwert schulischer Zertifikate ausgegangen, an dem sich auch institutionelle Gatekeeper orientieren (müssen) (vgl. Watermann et al. 2014). Für den Zugang zu Hochschulbildung sollten sich primäre Herkunftseffekte daher in der Tat vor allem in sozialgruppenspezifischer Schulleistung widerspiegeln, sofern diese selbst innerhalb der Gruppe der Studienaspirant/innen noch nachweisbar sind. Dies könnte sich aufgrund der stark fachlich (und regional) variierenden Selektivität von Studiengängen insbesondere dann zeigen, wenn bestimmte, sehr selektive Fächer angestrebt werden.

Auch im Zusammenspiel mit den im Folgenden im Fokus stehenden sekundären Herkunftseffekten können hierdurch sozialgruppenspezifische Zugangsmuster entstehen, zum Beispiel, wenn sozial schwächere Studienberechtigte hohe Zugangshürden antizipieren und sich infolgedessen gegen ein Studium entscheiden. Andersherum könnten leistungsschwächere, sozial privilegierte Schüler/innen eher dazu bereit sein, ihre fachlichen Präferenzen aufzugeben, um überhaupt studieren zu können (vgl. Glaesser und Cooper 2014). Ebenso könnten vorhandene Ressourcen eine ausreichend lange Wartezeit oder ein Studium an einer entfernten Hochschule erlauben und somit die Realisierungschancen aufgrund einer breiteren Auswahl an passenden Studienoptionen erhöht werden (vgl. Smith 2014).

\subsection{Sekundäre Effekte: Bildungsentscheidungen innerhalb institutioneller Optionen und Barrieren}

Über Leistungsunterschiede und Unterschiede im Qualifikationsprofil hinaus kann soziale Ungleichheit beim Übergang in die Hochschule durch sozialgruppenspezifische Bildungsentscheidungen entstehen, die insbesondere an späten Schwellen im Bildungssystem eine zentrale Rolle spielen (vgl. Neugebauer et al. 2013). Dabei stehen Studienberechtigte nicht nur vor der Entscheidung für

89 Generell kann hier die Kausalitätsfrage nicht eindeutig beantwortet werden: Handelt es sich bei solchen Tätigkeiten tatsächlich um (Adaptions-)Strategien, um Zulassungschancen für spezifische Studienangebote zu erhöhen, oder sind es erst diese Tätigkeiten, die das Interesse für ein bestimmtes Studienangebot wecken? Unabhängig von der Kausalität ist das Resultat - bessere oder schlechtere Zulassungschancen - jedoch das gleiche. 
oder gegen ein Studium, sondern auch für oder gegen bestimmte Studienoptionen.

Wie bereits in Kapitel 3 diskutiert, ist für die theoretische Einbettung sekundärer Effekte der Rückgriff auf RC-Ansätze - trotz ebenfalls verbreiteter Kritik (vgl. Yee 1997) - sowohl international als auch in der deutschen Ungleichheitsforschung ,one of the most significant trends in the study of inequalities in educational attainment in the past decade[s]" (Breen und Jonsson 2005: 227, siehe auch Solga und Becker 2012: 24). Jedoch müssen Bildungsentscheidungen nicht zwangsläufig auf einer bewusst reflektierten und rationalen Entscheidungslogik beruhen, sondern können auch zum Beispiel durch internalisierte Werte unterbewusst geleitet werden (vgl. Jackson 2013: 15, Schindler und Reimer 2010: 628). Beide Entscheidungslogiken führen in ihrer Konsequenz zu sozialgruppenspezifischen Bildungsentscheidungen, die ungleichheitsgenerierenden Mechanismen unterscheiden sich jedoch (zum Teil).

Im Folgenden sollen - unter Rückbezug auf die bereits eingeführten theoretischen Perspektiven - zunächst die verschiedenen Entscheidungslogiken und deren mögliche Integration kurz beschrieben werden. Im Anschluss daran werden mithilfe beider Perspektiven zentrale Mechanismen hinter sozial ungleichen Bildungsentscheidungen von Studienaspirant/innen abgeleitet, die im Zusammenspiel von institutionellen Barrieren und Opportunitäten und individuellen Ressourcen gesehen werden.

\subsubsection{Entscheidungslogiken: unbewusst oder intentional?}

Aus einer Sozialisations- oder ,pushed-from-behind“ Perspektive (Gambetta 1987: 11ff.) wird individuelles Handeln auf normativ-kulturelle Einflüsse des sozialen Umfelds zurückgeführt (vgl. Kroneberg et al. 2009). Diese wirken auf sozial-psychologische Prozesse wie etwa Imitation (von Peers) und Adaption (an Erwartungen des sozialen Umfeldes) (vgl. Haller 1982), auf deren Grundlage Akteure handeln. Das status attainment-Modell argumentiert weitestgehend aus dieser Perspektive. Dabei findet keine bewusst rationale Entscheidung statt. Auch die Rolle institutioneller Barrieren wird weitestgehend ausgeblendet. Hieran ansetzende Kritik übte etwa Kerckhoff (1976: 369), demzufolge Unterschiede im Bildungserwerb nicht allein das Resultat sozialisationsbedingter Einflüsse seien (Sozialisationsmodell), sondern auch durch ,structural limitations and selection criteria" zustandekämen (Allokationsmodell).

Auch habituelles Handeln, wie es Bourdieu (1982, Bourdieu und Passeron 1973) beschreibt, ist zunächst vorbewusst - „,behind their backs“ (Gambetta 1987: 11) - da es durch den internalisierten und im Normalfall unbewusst wirkenden Habitus geleitet wird. Dies ist immer dann der Fall, wenn der familiäre, der individuelle und der institutionelle Habitus konflikt- und störungsfrei ineinander greifen; nachschulische Bildungsentscheidungen haben dann eine ,se- 
amless quality“ (Mc Donough 1997: 154). Strukturelle Barrieren (und Anreize) sind dabei durchaus zentral. Doch wirken diese im Regelfall weniger durch bewusste Antizipation auf Bildungsentscheidungen, als vielmehr dadurch, dass sie als Teil des Habitus internalisiert werden. Dies kann zum „,selbstgewählten Verzicht“ für die einen und zum Studium als Selbstverständlichkeit, der unhinterfragt gefolgt wird, für die anderen führen. Handeln als "unbewusstes Folgen" bedeutet jedoch nicht, dass es abseits von Eigeninteressen und Strategien erfolgen muss. Doch gerade, wenn der Habitus einer Person und das soziale Feld, in der sie sich bewegt, gut zusammen passen, wenn die Person also einen „Sinn für das Spiel“ hat (Bourdieu 1985: 145), wird Nutzenmaximierung - um RC-Vokabular zu bemühen - oft ganz automatisch erreicht: ,[they] need not engage in rational computation in order to reach the goals that best suit their interests" (Bourdieu 1990: 108).

Aus den gerade vorgestellten Perspektiven ist die normative Dimension kultureller und sozialer Ressourcen zentral, um individuelle Entscheidungen $\mathrm{zu}$ verstehen. Anders in RC-Modellen, deren theoretische Grundlagen in mikro-ökonomischen Ansätzen, vor allem der Humankapitaltheorie (Becker 1993) begründet liegen. Das zugrundeliegende Akteursmodell ist das des homo economicus, des individuellen Nutzenmaximierers, der, durch seine eigenen Interessen geleitet, die Kosten und Erträge verschiedener Optionen im Lichte seiner Budgetbeschränkungen abwägt und - rational und konsistent - diejenige mit dem höchsten Nutzen wählt (vgl. Becker 1993, DesJardins und Toutkoushian 2005, Kroneberg und Kalter 2012, Perna 2006). Dieses Verständnis von rationalem Handeln ist sparsam und enthält keine ,black boxes“, wie sie Boudon (2003: 3) etwa in sozialisationsbasierten Erklärungsversuchen sieht. Dennoch erscheint diese ,enge“ oder ,dünne“ RC-Variante (vgl. Elster 1983, Opp 1999) als universelle Handlungstheorie ungeeignet: „The application of RCT has clearly demonstrated the empirical inadequacy of this set of restrictive assumptions” (Kroneberg und Kalter 2012: 81).$^{90}$ In der „,weiten“ Version wird Rationalität jedoch nahezu grenzenlos (vgl. Quackenbush 2004). Verschiedenste Motive können demnach rationales Handeln anleiten, wie etwa "altruism and fairness or, more generally, the desire to act according to one's identity, values, and internalized norms" (Kroneberg und Kalter 2012: 81). ${ }^{91}$

90 Auch in der ökonomischen Literatur wurden an verschiedenen Stellen - auch in den Schriften zur Humankapitaltheorie von Becker selbst - die sehr restriktiven Annahmen der engen RC-Version gelockert, sodass auch risikohafte und unsichere Entscheidungen modelliert werden können (vgl. Becker 1986: 111, DesJardins und Toutkoushian 2005).

91 Hieran geäußerte Kritik bezieht sich darauf, dass neue Erklärungen für beobachtete Phänomene jederzeit ad hoc integriert werden können, sodass der Ansatz tautologisch und kaum mehr falsifizierbar erscheint (für einen Überblick zur Kritik und Entgegnungen siehe Opp 1999). 
Von einer zu starken Ausweitung des Rationalitätsbegriffs nehmen die meisten bildungssoziologischen Anwendungen Abstand (insbesondere Breen und Goldthorpe 1997: 278, siehe auch Becker und Hecken 2009b, Hillmert und Jacob 2003, Schindler und Reimer 2010). So werden „tastes for eduction“ (Holm und Jæger 2008: 202), ,cognitive frames and traditional habits“ (Becker und Hecken 2009b: 244) oder „values, norms or beliefs“ (Breen und Goldthorpe 1997: 278) zwar als Handlungsmotivationen anerkannt und genannt, aber von rationalen Entscheidungen abgegrenzt (vgl. Goldthorpe 1996b). Bildungsentscheidungen werden hingegen als Resultat einer (im engeren Sinne) rationalen Abwägung erwarteter Kosten, Erträge und Erfolgswahrscheinlichkeiten verschiedener (Bildungs-)Optionen modelliert (vgl. Boudon 1974, Breen und Goldthorpe 1997, Erikson und Jonsson 1996, Esser 1999). Die institutionelle Opportunitätsstruktur definiert dabei den Rahmen, innerhalb dessen diese Abwägung stattfindet und Bildungsentscheidungen getroffen werden. Kosten können in Form von sozialen Kosten, Opportunitätskosten und direkten finanziellen Kosten auftreten. Neben erwarteten Einkommenserträgen $^{92}$ ist Statuserhalt - wie in Abschnitt 5.1.1 beschrieben - die zentrale Ertragsdimension in soziologischen RC-Modellen (vgl. insbesondere Breen und Goldthorpe 1997, Esser 1999). Rationale Akteure streben demnach nach mindestens dem Bildungsabschluss, der es ihnen ermöglicht, den sozialen Status ihrer Eltern zu reproduzieren (vgl. Breen und Goldthorpe 1997: 283). Die wahrgenommene Erfolgswahrscheinlichkeit drückt letztlich die Unsicherheit aus, den erwarteten Ertrag auch tatsächlich ernten zu können. Die Erfolgswahrscheinlichkeit als Teil sekundärer Herkunftseffekte hängt zusammen mit den bereits eingeführten primären Effekten und tatsächlich sollte die (sozialgruppenspezifische) Leistung in die wahrgenommene Erfolgswahrscheinlichkeit eingehen. Doch geht Letztere über vorhandene Fähigkeiten und ihre (mehr oder weniger akkurate) Übersetzung in Schulleistung hinaus und enthält auch subjektive Bewertungen der Wahrscheinlichkeit, einen bestimmten Bildungsgang erfolgreich abschließen zu können, in die die wahrgenommenen eigenen Fähigkeiten, die wahrgenommenen Anforderungen eines Bildungsganges sowie die zur Verfügung stehende akademische (und auch finanzielle) Unterstützung einfließen.

92 Vor allem in der Ökonomie werden diese finanziellen Erträge als mit bestimmten Bildungsabschlüssen verbundenes Lebenseinkommen modelliert. Dieses Vorgehen wird sowohl von Soziologen als auch Ökonomen als zu voraussetzungsvoll kritisiert, da die Annahme, dass Schüler/innen ihr Lebenseinkommen in Abhängigkeit verschiedener Bildungsabschlüsse errechnen können, kaum zu halten sei (vgl. DesJardins und Toutkoushian 2005: 218, Erikson und Jonsson 1996: 14). Daher greifen zum Beispiel Erikson und Jonsson (1996) auf die weniger anspruchsvolle Annahme zurück, dass Individuen zumindest in der Lage sind, Einkommensrenditen (sowie andere Ertragsdimensionen) grob zu schätzen und die verschiedenen Alternativen in Beziehung zueinander zu setzen. 
Ein zentraler Unterschied zwischen den vorgestellten Ansätzen besteht in der Frage, welchen Raum sie individueller Agency einräumen, d.h. inwiefern Schüler/innen intentional und rational auf Anreize reagieren können oder durch Strukturen und unbewusst ablaufende kognitive Prozesse in ihrer Wahl und ihren Handlungen eingeschränkt oder gar determiniert sind (vgl. Gambetta 1987).

Eine Integration der verschiedenen Handlungsformen versucht das von Hartmut Esser (2001) entwickelte und vor allem von Clemens Kroneberg (2005) weiterentwickelte Modell der Frameselektion (MFS) ${ }^{93}$, das von einer „variablen Rationalität" ausgeht: Stark vereinfacht kann Handeln danach in zwei Modi erfolgen: dem automatisch-spontanen (as-Modus) oder dem reflexiv-kalkulierenden (rc-Modus) (Kroneberg 2005). Im as-Modus werden stark internalisierte Normen unhinterfragt verfolgt, die im Falle von Bildungsentscheidungen zum Beispiel durch ,homogene Bildungsansprüche der signifikanten Anderen“" (Stocké 2013: 275) zustande kommen. Alternativen werden dann gar nicht erst in Erwägung gezogen (vgl. Kroneberg et al. 2009: 4) und mögliche Restriktionen und Barrieren eher ausgeblendet. Im rc-Modus werden hingegen zur Verfügung stehende Alternativen einer systematischen KostenNutzen-Kalkulation unterworfen. Ob eine Handlung automatisch-spontan oder reflexiv-kalkulierend erfolgt, hängt vor allem davon ab, wie groß die Möglichkeiten und Motivation für eine bewusste Auseinandersetzung sind, wie viel Kosten und Mühen hiermit verbunden sind und wie einfach Akteure auf ein mentales Modell zurückgreifen können, das ihnen das ,,angemessene“ oder „natürliche“ Handeln in der gegebenen Situation vorgibt. Für Bildungsentscheidungen ist laut Kroneberg et al. (2009: 22) die Aktivierung des rc-Modus wahrscheinlich, da es sich um eine folgenreiche und langfristig bekannte Entscheidung handelt und sich die Abwägungskosten in Grenzen halten sollten. Sind jedoch Bildungsnormen oder auch Bildungsaspirationen stark internalisiert, sollten diese eher automatisch-spontan verfolgt werden (vgl. auch Becker 2010: 22; für empirische Evidenz siehe Kroneberg et al. 2009, Stocké 2013).

Das MFS ist insbesondere für Überlegungen zur Umsetzung von Studienaspirationen interessant, da es in ihnen einen wichtigen Handlungsimpuls in Form eines möglichen normativen Skriptes sieht (vgl. Kroneberg et al. 2009: 21), aber gleichzeitig Raum für Variation lässt. Ist dieses Skript leicht verfügbar und stark internalisiert, wird es ohne rationale Abwägungsprozesse automatisch verfolgt, sodass die Umsetzungschancen - zumindest im Sinne individueller Entscheidungen (Selbstselektion) - hoch sein sollten. Die Wahl, vor der Schüler/innen stehen, kann dann eher als habitualisierte „Nicht-Wahl“" cha-

93 Grundlegend geht das MFS davon aus, dass jeder Handlung eine Situationsdeutung vorausgeht (Frameselektion: Welche Art von Situation liegt vor?), ein Handlungsskript aktiviert (Skriptselektion: Welches Verhalten ist in dieser Situation angemessen?) und letztlich eine entsprechende Handlung ausgewählt wird (Handlungsselektion: Wie verhalte ich mich in der Situation?) (Kroneberg 2005: 346). 
rakterisiert werden (vgl. Ball et al. 2002: 57, Grodsky und Riegele-Crumb 2010), weil ,den Akteuren gar nichts anderes in den Sinn kommt [...]. In solchen Fällen bestimmt die idealistische Bildungsaspiration das Handeln“ (Becker 2010: 22). Stehen aber zum Beispiel Studienaspirationen - obwohl geäußert - als normatives Skript nur eingeschränkt zur Verfügung, hängt deren Umsetzungswahrscheinlichkeit (im Sinne individueller Entscheidungen) von weiteren Faktoren ab, die rational gegeneinander abgewogen werden. Für sozial privilegierte Schüler/innen sollte meist Ersteres zutreffen. So schreiben etwa Becker und Hecken (2009) im Lichte ihrer Ergebnisse zu Studienentscheidungen sächsischer Abiturient/innen, dass insbesondere Akademikerkinder hier vermutlich nicht rein rational abwägen:

“... their education decision can - in contrast to the working classes - not be characterized as rationally and carefully considered cost-benefit calculations that also taking [sic] into account alternative educational courses. It has to be assumed that these classes already have cognitive frames and traditional habits with regard to academic education and culture." (Becker und Hecken 2009b: 244) ${ }^{94}$

Dies bedeutet jedoch nicht, dass Entscheidungen abseits jeglicher strategischer Überlegungen getroffen werden. Sozial privilegierte Schüler/innen scheinen zwar aufgrund stark internalisierter Normen spontan, gleichzeitig aber durch die nötige Systemkenntnis und Unterstützung auch strategisch und abwägend zu entscheiden, um ihr hohes Bildungsziel zu erreichen. So berichten Reay et al. (2001a), dass gerade sozial privilegierte Privatschüler/innen ihre (stark internalisierten) Bildungsziele zwar grundsätzlich mit dem as-Modus in Übereinstimmung verfolgen, dies aber besonders gut informiert, reflektiert und strategisch tun (siehe auch McDonough 1994, 1997). Die Umsetzung von Studienaspirationen in sozial privilegierten Familien ist damit weitaus reflektierter als das Ziel an sich, und die Schüler/innen und ihre Eltern können als strategische, „highly organised choosers” (Reay et al. 2001: 8.1) charakterisiert werden. Solch ein Handeln kann mit Max Weber (1980: 12) auch als wertrational beschrieben werden. ${ }^{95}$

Weniger eindeutig verhält es sich bei der insbesondere im deutschen Kontext sehr positiv selektierten Gruppe sozial benachteiligter (Fach-)Abitu-

94 Weitere empirische Evidenz hierzu liefern umfangreiche qualitative Studien zum Studienentscheidungsprozess von US-amerikanischen und englischen high schoolAbsolvent/innen (USA: McDounough 1997, UK: Ball et al. 2002, Reay 1998, Reay et al. 2001a/b). Die Autor/innen berichten, dass gerade sozial privilegierte Schüler/innen vom Studium als ,'automatic', ,taken for granted', always assumed“" (Ball et al. 2002: 57) sprechen und diesem ,vorbestimmten“"Weg unhinterfragt folgen.

95 Wertrationales Handeln ist am ,unbedingten Eigenwert eines Sichverhaltens ausgerichtet" (Weber 1980: 12). Handlungsfolgen sind dabei zwar an Werten orientiert, die Mittel zu deren Erreichen können aber zweckrational (d.h. egoistisch instrumentell zur Erreichung eigener Ziele) gewählt werden. 
rient/innen mit Studienaspiration. Für sie stellt sich die Frage, inwiefern ihre Studienaspirationen tatsächlich weniger stark internalisiert und als Skript verfügbar sind und damit Handlungen weniger spontan leiten und ob ihnen tatsächlich weniger Unterstützung zuteil wird. Es könnte - im Einklang mit der „Mobilitätsthese“ (Di Maggio 1982) und entgegen streng reproduktionstheoretischen Auslegungen - auch davon ausgegangen werden, dass positive Einstellungen zu Bildung, Ermutigungen, Unterstützungen und Studienaspirationen gerade auch in als ,sozial schwächer“ kategorisierten Familien zu positiven Bildungsoutcomes führen (vgl. De Graaf et al. 2000, Grodsky und RiegeleCrumb 2010, Maaz 2006). Stark internalisierte Studienaspirationen und deren Befürwortung und Unterstützung durch das soziale Umfeld könnten demnach unabhängig von der sozialen Herkunft positiv auf die individuelle Studienentscheidung wirken. Im Einklang mit Bourdieu zeigt sich jedoch auch, dass Studienaspirationen sozial schwächerer Schüler/innen im Durchschnitt weniger gefestigt und somit auch weniger handlungsleitend sind, da sie oft widersprüchliche oder entgegengesetzte Signale aus schulischen und familiären Kontexten erhalten (vgl. Bozick et al. 2010). Ist dies der Fall, sollten sie ihre Entscheidung stärker rational abwägen und sich zum Teil trotz ihres Wunsches gegen ein Studium entscheiden.

Insbesondere in rationale Entscheidungen sollte die institutionelle Ausgestaltung des Hochschulsystems mit einfließen, die Opportunitäten und Barrieren zum Beispiel in Form von verfügbaren Optionen, Kosten, Zulassungschancen, Mobilitätszwängen und Informationsanforderungen definiert. Diese werden im Lichte der verfügbaren Ressourcen (mehr oder weniger umfassend und korrekt) antizipiert, fließen in die Abwägung von Kosten, Erträgen und Erfolgswahrscheinlichkeiten ein und tragen so zur Entscheidung für oder gegen ein (bestimmtes) Studium bei. Dies kann ein komplettes Abwenden vom Studium, ein Aufschieben oder eine Anpassung der ursprünglichen Aspirationen innerhalb des Hochschulsystems zur Folge haben. Doch auch bei einem unhinterfragten Folgen der generellen Studienaspiration sollte die institutionelle Ausgestaltung des Hochschulsystems eine Rolle spielen, da sie Optionen vorgibt, die als mehr oder weniger angemessen und realisierbar wahrgenommen werden können. Selbst wenn sich sozial schwächere Studienaspirant/innen in gleichem Maße für ein Studium entscheiden wie ihre sozial privilegierten Peers, können sowohl unhinterfragte Entscheidungen als auch rationale Abwägungen zu sozialer Ungleichheit innerhalb des Hochschulsystems beitragen.

Im Folgenden wird diskutiert, inwiefern die verfügbaren institutionellen Optionen innerhalb und außerhalb des Hochschulsystems und damit verbundene institutionelle Barrieren die (mehr oder weniger bewussten) Studienentscheidungen von Studienaspirant/innen unterschiedlicher sozialer Herkunft strukturieren können. 


\subsubsection{Institutionelle Optionen: Wahlmöglichkeiten innerhalb und außerhalb des Hochschulsystems}

Die institutionelle Differenzierung des Bildungssystems kann sozial ungleiche Übergangsprozesse strukturieren, indem sie unterschiedlich ertragreiche, kostenintensive, risikoreiche und leistungsselektive Bildungsoptionen bietet, die für Studienberechtigte unterschiedlicher sozialer Herkunft unterschiedlich attraktiv oder erreichbar sein können. Bereits in Kapitel 2 wurden empirische Befunde für Deutschland zitiert, die verdeutlichen, dass sowohl die Entscheidung für oder gegen ein Studium als auch für oder gegen bestimmte Studienoptionen sozial selektiv sind. Im Folgenden stehen die Mechanismen im Vordergrund, auf deren Grundlage Herkunftsunterschiede erklärt werden können.

\section{Studium oder Berufsausbildung}

Soziale Ungleichheit bei der generellen Entscheidung für oder gegen ein Studium kann zum einen durch sozialgruppenspezifische Einschätzungen von Kosten, Erfolgswahrscheinlichkeiten und Erträgen entstehen. Aufgrund unterschiedlicher Ressourcenausstattung wiegen Kosten für sozial schwächere Schüler/innen schwerer, und die Erfolgswahrscheinlichkeit wird von ihnen als geringer eingeschätzt. Gleichzeitig steht mit dem Berufsausbildungssystem eine attraktive Alternative zum Studium zur Auswahl, die mit geringeren Kosten und Risiken bei gleichzeitig guten Arbeitsmarktchancen einhergeht. Zwar sollte die Bewertung von mit einem Studium verbundenen Einkommenserträgen nicht mit der sozialen Herkunft variieren (vgl. Davies und Guppy 1997, Erikson und Jonsson 1996, Lörz 2012, Obermeier und Schneider 2015), doch führt das über alle Herkunftsgruppen hinweg geteilte Statuserhaltmotiv zur sozialgruppenspezifischen Bewertung der Ertragskomponente. Demnach weisen sozial privilegierte Schüler/innen höherer Bildung per Definition einen höheren Nutzen zu: ,individuals with different social class backgrounds have different optimal stopping points or thresholds where the costs of continuing education (real costs, earnings foregone, and the risk of failure) outweigh the utility of further education“" (Holm und Jæger 2008: 200). ${ }^{96}$

96 Für deutsche (Fach-)Abiturient/innen berichten verschiedene Autor/innen auf Grundlage des DZHW Studienberechtigtenpanels und der Sächsischen Abiturientenbefragung (vgl. Becker und Hecken 2007, Lörz 2012, Schindler und Reimer 2010), dass gerade das Motiv des Statuserhalts im Vergleich zur Einschätzung von Kosten und Erfolgswahrscheinlichkeit einen geringen Erklärungsbeitrag für sozial ungleiche Studienentscheidungen liefert. Theoretisch und empirisch widersprüchliche Ergebnisse mögen - neben der Möglichkeit, dass RC-Modelle individuelles Handeln generell nur eingeschränkt erklären können (vgl. Kroneberg und Kalter 2012) - an der Herausforderung liegen, das Statuserhaltmotiv sauber direkt zu 
Zum anderen können im Netzwerk verbreitete und als Teil des Habitus internalisierte Normen individuelles Handeln leiten. Dabei kann - wie unter 5.2 beschrieben - von einer sozialgruppenspezifischen Grenzziehung ausgegangen werden (vgl. Bourdieu 1992, Glaesser und Cooper 2014). Gerade für Studienberechtigte aus Akademikerfamilien liegt eine Berufsausbildung oft bereits unterhalb ihrer selbstgesteckten Grenzen, und der Studienweg wird weitestgehend unhinterfragt verfolgt. Andersherum ist bei Studienberechtigten aus sozial schwächeren Familien, insbesondere denjenigen mit Studienaspiration, jedoch kaum wahrscheinlich, dass eine Grenzziehung ,nach oben“ stattfindet und ein Studium an sich komplett außerhalb ihrer Überlegungen liegt. Eine solche Abgrenzung ist eher innerhalb des Hochschulsystems zu erwarten. Der Studienentscheidungsprozess ist für sie jedoch oft konfliktreicher und muss stärker bewusst rationalisiert werden. Für sie ist daher ein rationales Abwägen wahrscheinlicher, das auch zur Entscheidung gegen ein Studium führen kann.

Optionen innerhalb des Hochschulsystems

Die gerade beschriebenen Mechanismen können weitestgehend auf Bildungsentscheidungen innerhalb des Hochschulsystems übertragen werden. Aufgrund der geringeren Ressourcen und des engeren Zeithorizontes von Abiturient/innen aus sozial schwächeren Familien entscheiden diese eher ,kostenorientiert", während ihre sozial privilegierten Peers sich stärker an den Erträgen verschiedener Studienoptionen orientieren können (vgl. Preißer 2003: 220). Während also sozial schwächere Studienberechtigte in weniger kostenintensive, weniger akademisch fordernde und - damit im Zusammenhang stehend - auch weniger prestigereiche Studienoptionen „kanalisiert“ oder ,abgelenkt" werden, ist das Gegenteil für sozial privilegierte Studienaspirant/innen der Fall. Laut der effectively maintained inequality (EMI) These von Lucas (2001: 1652) wird Statuserhalt nicht nur durch ein bestimmtes Bildungsniveau angestrebt, sondern auch durch vorteilhafte Wahlen innerhalb dessen: ,the socioeconomically advantaged seek out whatever qualitative differences there are [...] and use their advantages to secure quantitatively similar but qualitatively better education". Vorangetrieben werden solche Entscheidungen theoretisch durch die Expansion des Bildungssystems und insbesondere auch des Hochschulsystems, durch die ,einfache“ Hochschulzertifikate an Wert verlieren und sozial privilegierte Akteure auf ertragreichere Bildungsformen ausweichen, um Statusreproduktion soweit wie möglich sicherzustellen. ${ }^{97}$

messen, was zu ,suboptimalen“ Operationalisierungen führen kann (Schindler 2014: 62, siehe auch Becker 2012, Watermann et al. 2014).

97 Empirische Studien, die sich mit der Entwicklung sozialer Ungleichheit innerhalb des Hochschulsystems beschäftigen, finden für andere Kontexte tendenziell eine Verstärkung sozialer Ungleichheit in Bezug auf die Wahl prestigereicher Hoch- 
Bourdieu (1982: 238f.) sieht hierfür den Distinktionssinn sozial privilegierter Schüler/innen - ,eine der kostbarsten Mitgiften des ererbten kulturellen Kapitals" - als ursächlich. Diese habituelle Strategie lässt sie besonders exklusive Bildungsformen ganz natürlich identifizieren, ,,weil man eben ein Gespür dafür hat, wann die abgewerteten Schulzweige oder Karrieren beizeiten aufzugeben sind, um sich Zukunftsträchtigerem zuzuwenden, statt sich weiter an schulische Werte zu klammern, die in ihren Profitchancen doch nur einer früheren Marktlage entsprechen. "Sie müssen also nur ihrer Disposition folgen, um Praktiken zu produzieren ,that are ,naturally“ distinguished“ (Bourdieu 1990: 109) und somit soziale Reproduktion voranzutreiben. Aus Sicht von Schüler/innen aus sozial schwächeren Familien stellt sich die Wahl innerhalb des stratifizierten Hochschulsystems hingegen aus reproduktionstheoretischer Sicht als „erzwungene Wahl“ (Bourdieu und Passeron 2007: 18), als weiterer Schritt der Selbsteliminierung dar: Sie wählen Bildungsgänge (oder werden in diese ,abgedrängt"), die ihren Dispositionen eher entsprechen, die weniger kulturelles (akademisches) Kapital erfordern und dafür stärker praktisch orientiert (bodenständiger) sind. Andere Studienoptionen werden hingegen häufig als ,inaccessible“ (Evans 2009: 342) wahrgenommen. Die Wahlmöglichkeiten der „wenigen [Ü]briggebliebenen“ (Bourdieu und Passeron 1971: 25) sind damit stark eingeschränkt.

Innerhalb von Hochschulsystemen unterscheiden sich Hochschulinstitutionen entlang zweier „Stratifikationsachsen“ (Davies und Guppy 1997: 1419): einer vertikalen (institutionelles Prestige und Hochschultypen) und einer horizontalen (Studienfach). Im Folgenden werden zunächst vertikal und im Anschluss daran horizontal differenzierte Studienoptionen und -entscheidungen genauer betrachtet.

Hochschultypen: Universität vs. Fachhochschule

Prestigeunterschiede zwischen Institutionen oder Hochschultypen spielen vor allem in stark vertikal stratifizierten Hochschulsystemen (etwa den USA, UK oder Frankreich) eine ungleichheitsreproduzierende Rolle (vgl. USA: An 2010, Davies und Guppy 1997, Hoxby und Avery 2013, Karabel und Astin 1975, Karen 2002, Turley et al. 2007; UK: Boliver 2013, Shiner und Noden 2015; Frankreich: Duru-Bellat et al. 2008; vergleichend: Jerrim et al. 2015), da hier ein ,unmistakable set of connections between high-prestige institutions and high-prestige careers" besteht (Karen 2002: 192, siehe auch Brewer et al. 1999, Hussain et al. 2008). Im binären deutschen Hochschulsystem sollte hin-

schulen (USA: vgl. Turley et al. 2007, Long 2004) und Studienfächer (Italien: vgl. Triventi et al. 2017). Für Deutschland bestätigt sich dieser Zusammenhang eher nicht: Reimer und Pollak (2010) finden deutliche Herkunftsunterschiede bezüglich des Studienfachs und Hochschultyps, aber keine Verschärfung über die Zeit. 
gegen nach wie vor die Differenzierung zwischen Universitäten und Fachhochschulen relevanter sein, auf der daher im Folgenden der Fokus liegt. Bezüglich der Arbeitsmarkterträge unterscheiden sich Universitäts- und Fachhochschulabsolvent/innen kaum noch voneinander. Dennoch besteht eine klare Statusdifferenz zwischen beiden Hochschultypen, die sich zum Beispiel im Promotionsrecht widerspiegelt (vgl. Abschnitt 2.2.1). Zudem bieten Fachhochschulen einige besonders prestigereiche Fächer nicht an, sodass angestrebter Statuserhalt (oder Distinktion) für sozial privilegierte Studierende durchaus einen Grund für ein Universitätsstudium darstellen kann.

Weiterhin ist das Curriculum an Universitäten oft akademisch anspruchsvoller oder wird auf diese Weise wahrgenommen (vgl. Reimer und Pollak 2010), und die Studiendauer ist (trotz formaler Angleichung seit der Einführung von Bachelorstudiengängen) faktisch oft länger und weniger gut einschätzbar als beim stärker vorstrukturierten Fachhochschulstudium (vgl. Maaz 2006), was die wahrgenommenen Studienkosten erhöht. Weiterhin sind Fachhochschulen stärker praxisorientiert, was auf eine größere kulturelle Nähe zu Schüler/innen aus Nichtakademikerfamilien hindeutet, während sich Akademikerkinder stärker in der akademischen Ausrichtung von Universitäten wiederfinden sollten. Das Streben nach Statuserhalt und Abgrenzungsprozesse „nach unten“ machen somit die Wahl einer Universität für sozial privilegierte Schüler/innen wahrscheinlicher, während die geringeren Kosten, die als höher wahrgenommene Erfolgswahrscheinlichkeiten und größere kulturelle Nähe zu Fachhochschulen (bei gleichzeitig guten Arbeitsmarktchancen) Schüler/innen aus sozial schwächeren Familien vom Universitätsstudium eher ablenken sollte (für empirische Evidenz hierzu siehe Abschnitt 2.2.4).

\section{Studienfach}

Aufgrund der vergleichsweise geringen vertikalen Stratifizierung ist die horizontale Differenzierung zwischen Studienfächern in Deutschland besonders relevant. Studienfächer zeichnen sich durch eine Kombination verschiedener Merkmale aus. Dies trifft zwar auch auf Hochschultypen zu, jedoch variieren viele Merkmale (z.B. Einkommenserträge, Studiendauer, die regionale Abdeckung und Zugangsbarrieren) im deutschen Kontext stärker zwischen Studienfächern als zwischen Hochschultypen (vgl. Triventi 2013b). Dabei ist es kaum möglich, die Fächer insgesamt in eine Rangordnung von besonders attraktiv zu besonders unattraktiv zu bringen, was neben der oft nötigen Aggregation zu Fächergruppen (Fallzahlprobleme) auch die Hypothesenbildung zum Teil erschwert: "there are countervailing forces acting on students (and families) when it comes to the choice of field of study" (Jackson et al. 2008: 383).

Aus RC-Perspektive wird argumentiert, dass der Studienfachwahl als „Spezialfall der Ausbildungsentscheidung“ (Becker et al. 2010) ähnliche Mechanismen der rationalen Wahl zugrunde liegen wie der generellen Entschei- 
dung für oder gegen ein Studium (vgl. auch Reimer und Pollak 2010). Vor allem das Statuserhaltmotiv in Verbindung mit hohen Anforderungen und Kosten (durch die weit überdurchschnittliche Studiendauer bis zum ersten berufsqualifizierenden Abschluss ${ }^{98}$ und kostenintensive Examenskurse, z.B. in Form von Repetitorien) werden dabei als zentraler Grund für die empirisch nachgewiesene starke soziale Selektivität der Fächer Medizin und Jura angesehen (vgl. Becker et al. 2010, Duru-Bellat et al. 2008, Lörz 2012, Triventi 2013, van de Werfhorst et al. 2001, 2002). Wirtschafts- und ingenieurswissenschaftliche Studiengänge, die neben ihrer fachlichen Nähe zu vielen Ausbildungsberufen durch gute Einkommenserträge gekennzeichnet sind, sind hingegen deutlich weniger sozial selektiv (vgl. Becker et al. 2010, Lörz 2012, van de Werfhorst et al. 2001).

Dass Einkommenserträge insbesondere für sozial schwächere Schüler/innen wichtig sind, zeigen auch Davies und Guppy (1997) für die USA. Hier hängt der sozioökonomische Status nach Kontrolle der Testleistung signifikant negativ mit dem Durchschnittseinkommen im gewählten Fach zusammen. Sie interpretieren dies zum einen als Mobilitätsstrategie sozial schwächerer Studienberechtigter, zum anderen als Anzeichen dafür, dass die Wertschätzung nicht-monetärer Erträge vor allem unter sozial privilegierten (und eventuell zusätzlich leistungsschwächeren) Studienberechtigten verbreitet ist. Für Letztere ist soziale Reproduktion damit nicht nur an ein hohes Einkommen oder einen hohen Status gebunden, sondern kann auch über andere Erträge erzielt werden - zum Beispiel einen ,alternativen“ Lebensstil und damit verbundenes kulturelles Prestige (Davies und Guppy 1992: 1429, siehe hierzu auch Bourdieu 1982, van de Werfhorst 2001).

Für die Wahl eines Studienfaches können auch normative Einflüsse angenommen werden, die durch die intergenerationale Transmission von Interessen und Fähigkeiten, die hiermit verbundene fachlich-kulturelle Nähe und vorhandene Rollenvorbilder sozialgruppenspezifisch geprägt wird (vgl. Lörz 2012, van de Werfhorst et al. 2001). ${ }^{99}$ Vor allem in Familien mit einer spezifischen akademischen Tradition erfolgt die Fachwahl oft unhinterfragt (automatischspontan) und wird nur durch bestehende Zulassungsbarrieren eingeschränkt (vgl. Becker et al. 2010, Preißer 2003). Familien ohne akademische Tradition

98 Während die Regelstudienzeit in den meisten Fächer, die zu einem Bachelorabschluss führen, formal bei sechs Semestern liegt, sind für das Erreichen des Staatsexamens in den rechtswissenschaftlichen und medizinischen Fächern neun und zwölf Semester vorgesehen.

99 Entsprechend spielt auch der Beruf der Eltern und hiermit verbundene Informations- und Unterstützungsressourcen sowie Gelegenheitsstrukturen (Netzwerke, „Vererbung“ von Praxen, Kanzleien) eine wichtige Rolle. Für Deutschland zeigt sich empirisch, dass der Beruf der Eltern deutlich mit der Wahl eines ähnlichen Fachs zusammenhängt (vor allem in den Professionen Medizin und Jura) (vgl. Lörz 2012). 
schaffen hingegen einen Erfahrungsraum, der eher praktisch, manuell, technisch oder wirtschaftlich orientiert ist, sodass Interessen eher in diesen Bereichen ausgebildet und verfolgt werden (vgl. Lörz 2012).

Da Entscheidungen für ein bestimmtes Bildungsniveau und verschiedene Alternativen innerhalb dessen nicht zwangsläufig chronologisch, sondern auch simultan ablaufen können, können Kompromisse zwischen einem Studium und zum Beispiel einer bestimmten fachlichen Ausrichtung notwendig sein (vgl. James et al. 1999, Lörz 2012). Inwiefern solche Kompromisse notwendig sind und $\mathrm{zu}$ sozialgruppenspezifischen Bildungsentscheidungen beitragen, sollte mit vorhandenen institutionellen Barrieren (insbesondere fachlich strukturierten Zulassungshürden) variieren. Dieser Gedanke wird im Folgenden nochmals aufgegriffen und eingehender diskutiert.

\subsubsection{Institutionelle Barrieren und deren Antizipation}

Im Folgenden werden einige potentielle institutionelle Hürden nochmals eingehender diskutiert. Dabei müssen die hier als „Hürden“ oder „Barrieren“ bezeichneten Merkmale nicht immer einschränkend sein, sondern können auf bestimmte Gruppen auch anziehend wirken und als Opportunitäten wahrgenommen werden. Die zuvor geschilderten Optionen und die im Folgenden im Fokus stehenden Barrieren sind nicht unabhängig voneinander, da Letztere über die verschiedenen Wahlmöglichkeiten variieren. Entsprechend sind Rückbezüge und argumentative Überschneidungen im Folgenden stellenweise notwendig.

\section{Studienkosten}

Die Beurteilung der mit einem Studium verbundenen Kosten sollte mit der sozialen Herkunft variieren. Zwar sind - ohne Einbezug von Stipendien- und Fördersystemen - die absoluten Kosten für einen bestimmten Bildungsgang in der Regel für alle Schüler/innen oder Studierenden gleich. Doch können sich diese Kosten relativ zur eigenen und familiären Vermögens- und Einkommenssituation beträchtlich unterscheiden (vgl. Becker 2012). Entsprechend nennen im deutschen Kontext (trotz der hier insgesamt geringen direkten Kosten und der Fördermöglichkeiten durch das BAföG, vgl. Abschnitt 2.2.1) Schüler/innen, deren Eltern kein Hochschulstudium absolviert haben, Finanzierungsschwierigkeiten deutlich häufiger als „Problem bei der Wahl des nachschulischen Werdegangs" (33\% vs. 23\% Akademikerkinder) (Schneider und Franke 2014: 34, 37). Multivariat zeigt sich, dass die stärkere Kostensensibilität sozial schwächerer Schüler/innen einen großen, teilweise den größten Teil sekundärer Herkunftseffekte bei der Entscheidung für oder gegen ein Studium ausma- 
chen (vgl. Becker und Hecken 2007, 2009a, Lörz 2012, Schindler und Reimer 2010).

Kosten entstehen in Deutschland, wo Studiengebühren eine untergeordnete Rolle spielen, zum einen durch die Dauer eines Studiums (siehe Abschnitt 2.2.1). Diese beeinflusst neben der Wahrnehmung direkt anfallender Kosten (z.B. Lebensunterhalt während des Studiums, Höhe gegebenenfalls zurückzuzahlender Förderungen) vor allem die wahrgenommenen Opportunitätskosten (verlorenes Einkommen). Da Schüler/innen aus sozial schwächeren Familien „typischerweise [...] einen kürzeren Zeithorizont [haben], innerhalb dessen sich Bildungsinvestitionen auszahlen müssen“ (Müller und Pollak 2008: 310, vgl. auch Hillmert und Jacob 2003), kann dies zu sozial selektiven Bildungsentscheidungen beitragen.

Zudem können Kosten durch die Verknappung des Studienangebots entstehen (bzw. unzureichender Aufstockung bei steigender Nachfrage). Dies kann zu regionaler Varianz in der institutionellen Abdeckung und damit verbunden in den Zulassungsbeschränkungen führen. Dadurch können Mobilitätszwänge entstehen. Dies führt zum einen zu finanziellen Kosten, die sich in erhöhten Lebenshaltungs-, Umzugs- und Reisekosten niederschlagen. Finanzielle Ressourcen helfen dabei, diese Kosten zu kompensieren, sodass eine größere Flexibilität bei der Ortswahl besteht (vgl. Spieß und Wrohlich 2010). Zum anderen können hierdurch soziale oder auch emotionale Kosten durch die Loslösung vom sozialen Netzwerk entstehen (vgl. Helbig et al. 2017). Damit verbunden ist oft der Wunsch nach regelmäßigen Besuchen, was wiederum die Reisekosten und somit den Bedarf an finanziellen Ressourcen erhöht (vgl. Spieß und Wrohlich 2010). Beide Kostenarten sollten in stärkerem Maße mit der Studienentscheidung von sozial schwächeren Studienberechtigten zusammenhängen: Zum einen verfügen diese über weniger ökonomische Ressourcen, zum anderen sind sie oft emotional stärker in ihre lokalen Netzwerke eingebettet (vgl. Evans 2009, Lörz 2008, McDonough 1997, Reay et al. 2001b: 861).

Sowohl für andere Länderkontexte (vgl. Drewes und Michael 2006, Frenette 2006, Hoxby und Avery 2013, Kjellström und Regnér 1999, Turley 2009, Parker et al. 2016) als auch für Deutschland (vgl. Helbig et al. 2017, Lörz 2008, Spieß und Wrohlich 2010) konnte wiederholt gezeigt werden, dass mit steigender geographischer Distanz zum nächsten Hochschulort die Wahrscheinlichkeit der Studienbewerbung oder Studienaufnahme für alle Studienberechtigten sinkt. Gleiches gilt für die Distanz zu einem bestimmten Hochschultyp oder einem bestimmten Fachangebot (vgl. Griffith und Rothstein 2009, Suhonen 2014). Studien, die nach sozialer Herkunft differenzieren, finden mehrheitlich, dass sich Distanz negativer auf sozial schwächere Schüler/innen auswirkt (vgl. Denzler und Wolter 2010, Do 2004, Frenette 2006, Gibbons und Vignoles 2012, Hällsten 2010, Turley 2009). Für Deutschland sind die Ergebnisse hier nicht eindeutig. So berichtet Lörz (2008: 423), dass 
Studienberechtigte aus Akademikerfamilien häufiger ein Studium an einer entfernten Hochschule aufnehmen. Auch Helbig et al. (2017) zeigen, dass geographische Distanz mit der Studienaufnahme (sowie Studienplänen) sozial schwächere Schüler/innen stärker negativ zusammenhängt. Spieß und Wrohlich (2010) finden hierfür jedoch keine empirische Evidenz.

Mobilitätsbereitschaft sollte nicht nur mit der Studienentscheidung an sich zusammenhängen, sondern auch mit der Wahl bestimmter Studienfächer, da deren regionale Abdeckung stark variiert und sich Zulassungschancen entsprechend dem regionalen Angebot-Nachfrage-Verhältnis unterscheiden können. ${ }^{100}$ Gerade mit Blick auf stark nachgefragte Hochschulstandorte kann eine größere regionale Flexibilität daher nicht nur die Studienchancen an sich erhöhen, sondern vor allem auch die Chancen, bestimmte, stark nachgefragte Fächer zu studieren (vgl. Lörz 2012). Für Italien zeigt sich etwa, dass die Einführung einer finanziellen Förderung für leistungsstarke einkommensschwache Schüler/innen zwar nicht deren generelle Studienwahrscheinlichkeit beeinflusste, jedoch positiv mit der Wahrscheinlichkeit zusammenhing, ein Fach zu studieren, das nicht in Wohnortnähe angeboten wurde (vgl. Vergolini und Zanini 2015).

Letztlich können sich wahrgenommene Kosten nicht nur auf das Studium an sich, sondern auch auf dessen Zugang beziehen, etwa in Form von Kosten für die Vorbereitung auf spezifische Auswahlverfahren (z.B. finanzielle Kosten für Materialien und Kurse, Opportunitätskosten für Vorbereitungszeit) oder deren tatsächliche Durchführung (z.B. Reisekosten, Bewerbungs- und Testgebühren).

\section{Zugangshürden}

Zulassungsverfahren sind jedoch nicht allein aufgrund entstehender Kosten relevant, sondern auch indem sie Zugangschancen definieren, deren Antizipation sich auf individuelle Entscheidungsprozesse auswirken kann. Auswahlverfahren üben damit nicht nur im Zusammenspiel mit primären Effekten einen direkten Einfluss auf Zulassungschancen aus, wenn sie auf Kriterien beruhen, die sozial ungleich verteilt sind bzw. Adaptionsstrategien fördern (vgl. Abschnitt 5.3). Sondern sie wirken auch indirekt, wenn sie als abschreckend oder anziehend wahrgenommen werden oder in die Einschätzung der Erfolgswahrscheinlichkeit eingehen, die entsprechenden Zugangshürden meistern zu kön-

100 So wird zum Beispiel Betriebswirtschaftslehre an vielen der insgesamt fast 400 Hochschulen im gesamten Bundesgebiet angeboten, während etwa Veterinärmedizin derzeit nur an fünf Standorten studiert werden kann. Gleichzeitig ist die Wahrscheinlichkeit für Letzteres einen Studienplatz am Heimatort zu bekommen aufgrund der großen Nachfrage gering, selbst wenn das Fach hier angeboten wird. 
nen. ${ }^{101}$ Institutionelle Fremdselektion und individuelle Selbstselektion sind damit nicht unabhängig voneinander (vgl. Brown und Hirschman 2006, DesJardins et al. 2006, Drewes und Michael 2006, Long 2004, Krüger und Helsper 2015, siehe auch Abschnitt 4.4.5).

Für in Konkurrenz zueinander stehende Hochschulen können Auswahlverfahren zum einen als „Attraktionsmerkmal“ dienen und von potentiellen Bewerber/innen als Anzeichen für die Qualität des Studiengangs sowie die Motivation der Studierenden interpretiert werden. Zum anderen sollen sie auch „abschreckend“ wirken und „die Zahl der Bewerber auf eine Gruppe [reduzieren], die meint, die Qualitätsanforderungen erfüllen zu können“ (Bloch et al. 2015: 196, siehe auch Rothmüller 2011, Täger 2009). Dass solch ein Abschreckungseffekt tatsächlich Wirkung zeigt, demonstrieren Smith et al. (2014) für die USA, wo die Einführung von Essays zur Bewerber/innenauswahl an verschiedenen Hochschulen einen Bewerbungsrückgang zur Folge hatte. Weiterhin konnten verschiedene Studien zeigen, dass die Abschaffung von positiver Diskriminierung als Zulassungskriterium in verschiedenen US-Staaten (affirmative action) und die dadurch als geringer wahrgenommenen Zulassungschancen der vormals bevorzugten Gruppe (racial minorities) zu deutlichen Bewerbungsrückgängen führten (vgl. Brown und Hirschman 2006, Harris und Tienda 2010, Long 2004). Auch für Deutschland zeigt Täger (2009) einen Abschreckungseffekt exemplarisch anhand der Einführung eines Eignungsfeststellungsverfahrens (in Form eines vor Ort zu verfassenden neunzigminütigen Aufsatzes zu einem vorgegebenen Thema) für den Soziologiestudiengang an der Ludwig-Maximilians-Universität München, das zu einer Reduktion der Bewerber/innenanzahl von 400 (vor der Einführung) auf 200 führte. ${ }^{102}$

101 In RC-Modellen bezieht sich Erfolg meist auf die eher längerfristige Dimension eines erfolgreichen Bildungsabschlusses, also etwa auf den Erhalt eines Hochschuldiploms. Erfolg kann sich aber auch auf einen erfolgreichen Zugang zu bestimmten Bildungsgängen beziehen. Diese Dimension spielt in den Modellen eine untergeordnete Rolle. Erikson und Jonsson (1996) gehen etwa davon aus, dass formale Zugangsbarrieren die Alternativen, die gegeneinander abgewogen werden, definieren: erfordert zum Beispiel der Zugang zu einem Studiengang eine bestimmte Abschlussnote, erwägen nur diejenigen diesen Studiengang, die mindestens diese Abschlussnote erreicht haben. Oft wird aber, wie auch in Deutschland (siehe Abschnitt 2.2.2), kein fester Schwellenwert festgelegt, sondern die Zugangschancen hängen von der jeweiligen Bewerber/innenlage und einer Kombination verschiedener Zulassungskriterien ab. Daher besteht für viele Ausbildungs- und Studienoptionen von vornherein ein gewisses Maß an Unsicherheit bezüglich der tatsächlichen Zulassungschancen.

102 Die Abbruchquote bis zum vierten Semester liegt in den Kohorten mit Eignungsfeststellungsverfahren deutlich unter den Werten der Vorjahre, was als positives Zeichen dafür gewertet wird, dass sich nur diejenigen mit „ernsthaftem Interesse“ beworben haben (Täger 2009: 92). Allerdings muss hinzugefügt werden, dass die Anzahl der nach vier Semestern nach wie vor Studierenden vor der Einführung des 
Dabei kann von einem differentiellen Einfluss verschiedener Auswahlverfahren nach sozialer Herkunft ausgegangen werden. Komplexe Auswahlverfahren als Qualitätsmarker sollten zwar prinzipiell auf alle Studienberechtigten attraktiv wirken, doch könnten damit verbundene Exklusivitätswahrnehmungen - ähnlich der Argumentation zur sozial selektiven Wahl prestigereicherer Hochschultypen, Institutionen oder Fächer - sozial privilegierte Studienberechtigte stärker anziehen. Für Studienberechtigte aus sozial schwächeren Familien kann hingegen auch ein umgekehrter Zusammenhang abgeleitet werden:

„Sie bewerten Auswahl- und Eignungsfeststellungsverfahren als eine noch höher angelegte Hürde für die Aufnahme und den Eintritt in die Hochschule und zeigen sich dadurch in ihrer Studierneigung von vorneherein [sic] entmutigt und ,abgeschreckt ${ }^{\star}$. Dieses Verhalten könnte zu einer sich verstärkenden Selbstselektion der weniger begünstigten, so genannten hochschulfernen Hochschulzugangsberechtigten führen. Ein zulassungsfreies Studium hingegen signalisiert einen offenen $\mathrm{Zu}$ gang, an dem kein Gatekeeper nach bestimmten fachlichen und persönlichen Kriterien vorab auswählt." (Täger 2010: 123)

Insbesondere interaktive Verfahren, etwa persönliche Auswahlgespräche, können nicht nur - wie in Abschnitt 5.3 beschrieben - aufgrund variierender kultureller Nähe während ihrer Durchführung Zulassungschancen sozialgruppenspezifisch beeinflussen, sondern aufgrund der Begegnung mit dem akademischen Personal bereits im Vorhinein abschreckend wirken, wenn die antizipierte Situation und das hierfür benötigte kulturelle Kapital nicht zur eigenen Erfahrungswelt passt. ${ }^{103}$ Die Antizipation fehlender habitueller Passung ist für stärker standardisierte Verfahren, etwa Tests, kaum zu erwarten, da diese in der Regel nicht mit potentiell abschreckenden direkten Begegnungen verbunden sind. Alternativ könnten komplexere Auswahlverfahren, wie von Hochschulakteuren erhofft, gerade von sozial schwächeren Studienberechtigten auch genutzt werden, um bessere Informationen über die eigene „Studierfähigkeit" und damit über die Wahrscheinlichkeit eines Studienerfolgs zu erhalten und auch ein möglicherweise skeptisches soziales Umfeld hiervon zu überzeugen.

Wie mehrfach betont, ist in Deutschland die Abiturnote zentrales Auswahlkriterium. Die als „NC“ bekannte Auswahlgrenze variiert zwar je nach

Verfahrens deutlich höher war als nach der Einführung. Dies unterstützt Tägers Vermutung, dass nicht nur Nicht-Interessierte vom neuen Verfahren abgeschreckt werden, sondern ebenfalls Abiturient/innen, die ,ein ernsthaftes Interesse am Fach Soziologie hegen“ (ebd.: 86).

103 Persönliche Auswahlgespräche werden oft als sozial exklusive Veranstaltungen wahrgenommen (vgl. Evans 2009: 347), in denen nicht nur Motivation und fachliche Kenntnisse, sondern auch ein angemessener Kommunikationsstil und Auftritt - ,habituelle Passung“ - geprüft wird (vgl. Burke und McManus 2011, Karabel 2005, Rothmüller 2011). 
Bewerber/innenlage, doch sollten die meisten Studienberechtigten aufgrund von privaten, schulischen und medialen Diffusionsprozessen zumindest ein grundlegendes Verständnis davon haben, wie selektiv verschiedene Fächer oder zumindest das angestrebte Fach in etwa sind. Ist dies der Fall, kann die Selektivität von Studienfächern aufgrund der Wirkung primärer Effekte nicht nur direkt auf Studienchancen wirken (vgl. Abschnitt 5.2.1), sondern darüber hinaus kann ihre Antizipation die wahrgenommene Erfolgswahrscheinlichkeit einer Bewerbung beeinflussen.

Inwiefern dies zu einer Umentscheidung, also ein komplettes Abwenden vom Studium, oder einer Umorientierung innerhalb des Hochschulsystems führt, sollte - wie in Abschnitt 5.4.3 bereits angedeutet - von der Kompromissbereitschaft Studienberechtigter bezüglich der fachlichen Ausrichtung auf der einen und dem generellen Bildungsniveau auf der anderen Seite abhängen. Während es für Studienberechtigte aus sozial schwächeren Familien wahrscheinlicher ist, dass sie vom Studium absehen und eine verwandte Berufsausbildung aufnehmen, sollten sozial privilegierte Studienberechtigte eher dazu neigen, von ihrem fachlichen Wunsch abzuweichen und ein anderes Studienfach zu wählen, um Statuserhalt sicherzustellen. Wie argumentiert, liegt für sie eine Berufsausbildung oft bereits unterhalb ihrer selbstgesteckten Grenzen (vgl. Glaesser und Cooper 2014). Alternative und kostenintensive Möglichkeiten bestehen darin, Zugangshürden zu umgehen, etwa durch eine ausreichend lange Wartezeit oder ein Studium im Ausland (vgl. Gerhards und Németh 2015).

Prestige: institutionelle Hürde oder Pull-Faktor?

Hochschulen mit unterschiedlicher Reputation oder Prestige können zum einen als vertikal stratifizierte Wahloption innerhalb des Hochschulsystems besprochen werden (5.4.2). Prestige kann aber auch als institutionelle Hürde oder Pull-Faktor diskutiert werden. Einig ist sich die Forschung aus stärker stratifizierten Hochschulsystemen, dass institutionelles Prestige sozial privilegierte Schüler/innen, auch unter Kontrolle individueller Leistungsfaktoren, deutlich stärker anzieht und entsprechend als Pull-Faktor fungiert (vgl. Jerrim et al. 2015, Karen 2002, Shiner und Noden 2015). Für sozial schwächere Schüler/innen demonstrieren qualitative Studien hingegen, dass ihre oft getroffene Entscheidung gegen prestigereiche Hochschulen vor allem auf Selbstexklusion beruht, die sich gegen eine fremde und kaum zugängliche Elitekultur richtet, gegen einen institutionellen Habitus, der mit dem eigenen nicht vereinbar ist (vgl. Ball et al. 2002, Evans 2009, Reay 1998, Reay et al. 2001a).

Für den deutschen Kontext wurde die Entscheidung für oder gegen unterschiedlich prestigereiche Hochschulen aufgrund der geringen Institutionalisierung diesbezüglicher Unterschiede nicht als zentrale Wahloption innerhalb des Hochschulsystems diskutiert (vgl. Abschnitt 2.2.1). Nichtsdestotrotz werden 
auch hier erste Signale gesendet, insbesondere durch die Exzellenzinitiative, die zu einer „distinktiven Absetzung“ (Krüger et al. 2012: 329) einiger Universitäten beiträgt und deren Attraktivität für Studienberechtigte steigert (vgl. Horstschräer 2012). Dies könnte insbesondere der Fall für Studienberechtigte aus sozial privilegierten Familien sein (Statuserhalt, Distinktionssinn) (für erste vorsichtige Evidenz hierzu siehe Lörz und Quast 2011, Winkler 2014). Andersherum ist ein Abschreckungsmechanismus, der sich auf bestimmte Institutionen bezieht, in Deutschland bislang jedoch sehr unwahrscheinlich, da eine potentiell abschreckende „Elitekultur“ kaum institutionalisiert ist.

\section{Informationen und Transparenz}

Zuletzt soll hier die Rolle von (In-)Transparenz durch fehlende oder vorhandene institutionell bereitgestellte Informationen diskutiert werden. Diese kann auf zwei Arten wirken: zum einen direkt, indem fehlende Informationen selbst eine Hürde darstellen; zum anderen indirekt, indem sie beeinflussen, wie korrekt und vollständig existierende Barrieren überhaupt antizipiert werden können.

Fehlende Transparenz als institutionelle Hürde kann insbesondere im $\mathrm{Zu}-$ sammenspiel mit sozial ungleich verteilten Informationsressourcen wirken. Denn zum einen stellt das engere soziale Netzwerk eine zentrale Informationsquelle dar. Zum anderen variiert dessen Nutzen aufgrund hierin enthaltener Hochschulerfahrung und Systemkenntnis nach sozialer Herkunft (vgl. Slack et al. 2012). So schätzen Akademikerkinder Gespräche mit Eltern und Verwandten deutlich häufiger als nützlich ein als Nichtakademikerkinder (vgl. Heine et al. 2010: 53). Letztere sind also stärker auf externe Quellen angewiesen, da fehlende Informationen zum Hochschulsystem weniger gut über private Kanäle ergänzt werden können (vgl. Slack et al 2012). ${ }^{104}$ Aufgrund fehlender Systemkenntnis im sozialen Netzwerk ist die Informationsbeschaffung zu hochschulbezogenen Themen für sozial schwächere Schüler/innen jedoch kostenintensiver (vgl. Erikson und Jonsson 1996), insbesondere wenn Informationen nicht einfach zugänglich oder undurchsichtig sind. Gleichzeitig sind Informationen zu alternativen Optionen, etwa zur Berufsausbildung, im eigenen sozialen Netzwerk leichter verfügbar. Fehlende Transparenz oder schwer beschaffbare Informationen könnten somit zur Entscheidung gegen ein Studium (oder spezifische Studienoptionen) beitragen.

104 Das Bereitstellen von Informationen bedeutet keine komplette Aufhebung von Informationsasymmetrien, denn institutionelle Informationen werden häufig als weniger glaubwürdig wahrgenommen als private. Zudem ist es unter Umständen nicht ausreichend, Informationen zur Verfügung zu stellen, denn diese müssen auch aktiv gesucht und entschlüsselt werden, was nicht immer geschieht bzw. gelingt (vgl. Morgan 2010, Slack et al. 2012). 
Ein transparentes System mit zugänglichen Informationen ist auch deshalb wichtig, weil sozial schwächere Schüler/innen sich ihres Informationsdefizites oft nicht bewusst sind und daher seltener aktiv nach Informationen suchen (vgl. Cabrera und Nasa 2000, Menon et al. 2007, Slack et al. 2012) und weil sie oft nicht die nötige Kompetenz und Unterstützung haben, um die in Zeiten des Internets existierende Informationsflut richtig filtern und einordnen zu können (vgl. Morgan 2010: 82). Ein Mangel an leicht verfügbaren und zugänglichen Informationen stellt damit nicht nur selbst eine potentielle Hürde dar, sondern beeinflusst auch die Antizipation weiterer institutioneller Barrieren und Opportunitäten. Eine umfassende Antizipation ist für sozial schwächere Schüler/innen unwahrscheinlicher, was sowohl zur Unter- als auch Überschätzung von institutionellen Hürden und damit verbundenen Kosten und Erfolgswahrscheinlichkeiten führen kann. Damit spiegelt die eigene Wahrnehmung tatsächliche Studienchancen nur in Teilen wider (vgl. Azjen 1991).

Dass höhere Transparenz tatsächlich ungleichheitsreduzierend wirken kann, zeigen Long et al. (2010) am Beispiel der Einführung des „Top Ten Percent Law" in Texas, das Schüler/innen im obersten Leistungspercentil ihrer Schule die Zulassung zu den staatlichen flagship Universitäten garantiert. De facto wären auch vor der Reform die besten zehn Prozent zugelassen worden, doch erst die Ablösung durch eine transparente ,de jure policy“ (ebd.: 102) führte zu mehr Bewerbungen und daraufhin auch Zulassungen von Schüler/innen aus strukturell benachteiligten Schulen.

Welche Bedeutung sozial ungleich verteiltes Systemwissen auf der einen und damit Transparenz und bereitgestellte Informationen auf der anderen Seite haben, hängt davon ab, wie komplex die Struktur und Zulassungsregelungen von Hochschulsystemen sind. Zwar kann das deutsche Hochschulsystem im internationalen Vergleich als homogen bezeichnet werden, doch wie in Kapitel 2 gezeigt wurde, führen auch hier Differenzierungsprozesse und die Reform der Hochschulzulassung zu einer zunehmenden institutionellen Komplexität und Vielfalt. Da die Studienzulassung in Deutschland nicht wie etwa in Großbritannien (vgl. Boliver 2013, Shiner und Noden 2015), Schweden (vgl. Hällsten 2010) oder Dänemark (vgl. Thomsen 2018) zentral organisiert ist, müssen auch Informationen dezentral, über verschiedene Quellen zusammengetragen werden (vgl. Abschnitt 2.2.3). Neben den Webseiten der Hochschulen existiert eine kaum überschaubare Anzahl kommerzieller Internetseiten. Dieser ,Informationsreichtum" kann ohne die richtige Filter- und Entschlüsselungskompetenz schnell zur „Informationsüberlastung“ werden (Morgan 2010: 82, siehe auch Hoxby und Turner 2013, Page und Scott-Clayton 2010), die zu Überforderung und Fehleinschätzungen führen kann.

Fehlende Informationen können dabei nicht nur zur Entscheidung gegen ein Studium oder bestimmte Studienoptionen führen, wenn zum Beispiel $\mathrm{Zu}-$ lassungschancen (teils unberechtigterweise) als gering eingeschätzt werden, sondern auch zu dem bereits diskutierten sehr unterschiedlichen Bewerbungs- 
verhalten (vgl. Abschnitt 4.3.3), das für sozial privilegierte Schüler/innen aufgrund ihrer Vielzahl an privaten und schulischen Informations- und Unterstützungsressourcen als zielgerichtet und strategisch, für ihre sozial stärker benachteiligten Peers hingegen häufig als „odd“ (Hoxby und Avery 2013: 27), „random” (Hällsten 2010: 813) oder „directionlessness” (Reay et al. 2001a: 8.1) charakterisiert wurde. Auch das deutsche Zulassungssystem bietet Möglichkeiten Studienchancen zu erhöhen, die über die Optimierung des Leistungs- und Qualifikationsprofils (die hier den weiter gefassten primären Effekten zugeordnet wurden) hinausgehen (vgl. Abschnitt 5.3). Durch das numerus clausus Verfahren sind Zulassungschancen für weniger nachgefragte Städte, Hochschulen und Studienfächer höher, sodass sich regional und inhaltlich breitere Bewerbungsmuster auszahlen können (vgl. Gehlke et al. 2019b). Neben dem Systemwissen spielt hierfür auch die Bereitschaft und Fähigkeit eine Rolle, hiermit verbundene Kompromisse einzugehen und entstehende (monetäre und soziale) Kosten zu tragen.

\subsection{Zusammenfassung und Hypothesen}

Abschließend sollen hier die theoretischen Überlegungen zu sozialer Ungleichheit bei der Umsetzung von Studienaspirationen zusammengefasst und auch im Rückblick auf die in Kapitel 4 vorgestellten Phasen - Hypothesen abgeleitet werden, die in Kapitel 7 und 8 empirisch geprüft werden.

Zunächst wurde argumentiert, dass Aspirationen theoretisch als wichtiger Mediator sozialer Ungleichheit im Bildungssystem angesehen werden können. Doch ist nicht ausgeschlossen, dass auch sozial schwächere Schüler/innen hohe Bildungsaspirationen ausbilden. Ist dies der Fall, handelt es sich um eine weiter positiv selektierte Gruppe. Doch selbst innerhalb der Gruppe der Studienaspirant/innen können soziale Herkunftsunterschiede bei der Umsetzung von Studienaspirationen erwartet werden, wenn förderliche Ressourcen ungleich verteilt sind und - im Zusammenspiel mit institutionellen Barrieren zu sozialgruppenspezifischen Leistungsprofilen und Bildungsentscheidungen führen.

Aspirationen werden als wichtige Motivationsquelle, als Teil der Agency individueller Akteure verstanden. Wie handlungsleitend sie sind, sollte allerdings davon abhängen, wie stark sie im Zuge der Sozialisation internalisiert wurden. Bei sozial privilegierten Schüler/innen kann von einer starken Internalisierung ausgegangen werden, die dazu führt, dass Studienaspirationen unhinterfragt, habitualisiert oder automatisch-spontan verfolgt werden. Bei sozial schwächeren Schüler/innen ist hingegen eine (rationale) Abwägung im Lichte der eigenen Ressourcenausstattung, der verfügbaren institutionellen Optionen und bestehenden Barrieren wahrscheinlicher, die - trotz Studienwunsch - auch 
zu einer Entscheidung gegen ein Studium führen kann. Zusätzlich wurde theoretisch argumentiert, dass sich Studienaspirant/innen unterschiedlicher sozialer Herkunft nicht nur darin unterscheiden, ob sie überhaupt den Hochschulpfad weiter verfolgen, sondern auch bezüglich ihrer Wahl verschiedener Studienoptionen.

Auf Grundlage dieser Überlegungen sollen im Folgenden Erwartungen zu sozialen Herkunftsunterschieden bei der Umsetzung von Studienaspirationen abgeleitet werden. Diese unterteilen sich in vier inhaltliche Blöcke (A: generelle Annahmen zu Herkunftsunterschieden, B: individuelle Einflussfaktoren, C: institutionelle Einflussfaktoren, D: differenzierte Bildungsentscheidungen) und umspannen die vier vorgestellten Phasen: Übersetzung von Aspiration in Intention (I), Bewerbung (B), Zulassung (Z) sowie Studienaufnahme (S). ${ }^{105}$ Insgesamt ist zu beachten, dass sich nicht jede Hypothese in jeder Phase wiederfindet. Dies hat zum einen theoretische Gründe (zum Beispiel beziehen sich Annahmen zu sozialgruppenspezifischen, individuellen Bildungsentscheidungen auf die selbstselektiven Phasen); zum anderen können einige Hypothesen aufgrund von Datenrestriktionen nur in bestimmten Phasen getestet werden (auch wenn sie für weitere Phasen theoretisch ebenso abgeleitet werden könnten). Die Hypothesen sind in Tabelle 5.1 zusammengefasst.

Hypothesenblock A: generelle Annahmen zu Herkunftsunterschieden

Hypothesenblock A enthält zunächst allgemeine Erwartungen zu sozialer Ungleichheit in den vier Phasen der Aspirationsumsetzung: Zunächst erwarte ich, dass sich soziale Ungleichheit vor allem in den frühen selbstselektiven Phasen der Aspirationsumsetzung zeigt: bei der Übersetzung von Studienaspirationen in (rationalisierte, Barrieren antizipierende) Studienintentionen (HA/I) und der Bewerbung für ein Hochschulstudium (HA/B). Bei der Zulassung gegeben einer Bewerbung erwarte ich hingegen zunächst kaum Herkunftsunterschiede (HA/Za). Diese Hypothesen stehen im Einklang mit den in Kapitel 4 präsentierten empirischen Befunden, die vor allem Selbstselektion als ursächlich für das Zustandekommen sozialer Ungleichheit identifizierten. Gerade im deutschen Kontext, in dem auch für Schüler/innen mit Studienaspirationen attraktive berufliche Alternativen zur Auswahl stehen, könnte sich soziale Ungleichheit tatsächlich vor allem in individuellen Entscheidungen widerspiegeln. Es ist jedoch auch ein alternatives Szenario denkbar, in dem sowohl Selbst- als auch Fremdselektionsprozesse zu sozialer Ungleichheit beitragen können. Wenn Aspirationen auch für Schüler/innen aus sozial schwächeren Familien

105 Der Benennung der Hypothesen unterliegt folgende Logik: $\mathrm{H}$ steht allgemein für Hypothese. Der darauf folgende Buchstabe (A-D) bezieht sich auf den Hypothesenblock, und die folgende Zahl nummeriert die Hypothesen innerhalb eines Blockes fortlaufend. Am Ende der Hypothese wird mit den Buchstaben I, B, Z und S auf die jeweilige Phase verwiesen. 
handlungsleitend sind und somit auch entgegen wahrgenommener Hürden zumindest der Versuch gestartet wird, diese umzusetzen, könnten sich auch Herkunftsunterschiede in den Zulassungschancen zeigen $(\mathbf{H A} / \mathbf{Z b})$. Dies sollte dann der Fall sein, wenn sozial schwächere Studienaspirant/innen den Auswahlkriterien weniger gut entsprechen oder sich weniger zielgerichtet bewerben. Ein stringenter empirischer Test dieser letzten Annahmen ist auf Grundlage der verwendeten Daten leider nicht oder nur sehr eingeschränkt möglich (mit Ausnahme primärer Effekte, siehe unten).

Individuelle Entscheidungen für oder gegen ein Studium sollten sich bereits im Bewerbungsverhalten äußern. Sofern sowohl Bewerbungen als auch Zulassungen vorliegen, erwarte ich daher in der vierten Phase keine Herkunftsunterschiede bei der (konditionalen) Studienaufnahme (HA/Sa). Anders sollte es sich verhalten, wenn der Übergang ins Studium - wie häufig geschehen (vgl. Kapitel 3) - unkonditional betrachtet wird, im vorliegenden Fall also alle Studienaspirant/innen eingeschlossen werden. In diesem Fall sollten sich die Herkunftsdifferenzen im Bewerbungsverhalten hier fortführen und mit einer sozial ungleich verteilten Studienaufnahme einhergehen $(\mathbf{H A} / \mathbf{S b})$.

Hypothesenblock B: individuelle Einflussfaktoren und Bildungsentscheidung

Mit Fokus auf die ersten beiden Phasen enthält Hypothesenblock B zunächst Erwartungen zu ungleichheitsgenerierenden Mechanismen auf der Individualebene. ${ }^{106}$ Sofern selbst innerhalb der Gruppe von Schüler/innen mit Studienaspiration noch Unterschiede in den Schulleistungen bestehen, wird zunächst erwartet, dass die Herkunftsdifferenz bei der Übersetzung von Aspirationen in Intentionen und bei Studienbewerbungen zum Teil hierüber erklärt werden kann (HB1/I, HB1/B). Weiter sollten sozialgruppenspezifische Erwartungen von Kosten, Erträgen und Erfolgswahrscheinlichkeit (HB2/I, HB2/B) sowie zur Verfügung stehende soziale und kulturelle Ressourcen in Form normativer Erwartungen und Unterstützungsleistung (HB3/I, HB3/B) Herkunftsdifferenzen weiter erklären. Neben der Mediation sozialer Herkunftsdifferenzen durch die genannten Faktoren könnte - in Einklang mit dem MfS - auch angenommen werden, dass bestimmte förderliche Bedingungen sowie die Einschätzung von Kosten und Nutzen den Einfluss sozialer Herkunft moderieren, indem sie insbesondere die Studienentscheidungen von sozial schwächeren Schüler/innen beeinflussen (vgl. Becker und Hecken 2009b, De Graaf et al. 2000, Maaz 2006). Ein stringenter empirischer Test solcher Moderationshypothesen ist je-

106 Die hier beschriebenen Mechanismen sollten sich - sofern sie für die Bewerbungsphase nachgewiesen werden können, auch im unkonditionalen Übergang ins Studium widerspiegeln. Da dies nicht im Fokus der vorliegenden Arbeit steht, werden diesbezügliche Erwartungen nicht als Hypothesen formuliert und empirisch getestet. 
doch (aufgrund von Fallzahlrestriktionen) in dieser Arbeit empirisch nicht umsetzbar.

An dieser Stelle sei kurz auf die Rolle herkunftsspezifischer Schulleistung eingegangen. In Anlehnung an Boudon (1974) wird diese in empirischen Anwendungen standardmäßig als primärer Effekt diskutiert. Das hier verwendete Phasenmodell erlaubt einen differenzierteren Blick auf Leistungsunterschiede. Primäre Effekte sollten sich entsprechend der Argumentation in Abschnitt 5.3 in der Zulassungsphase zeigen, da Schulleistung als zentrales Kriterium Zulassungschancen mitbestimmt. Sofern die oben formulierte $\mathrm{HA} / \mathrm{Zb}$ zutrifft wird daher angenommen, dass sich Herkunftsunterschiede in den Zulassungschancen durch Unterschiede in den Schulleistungen teils erklären lassen $(\mathbf{H B 1} / \mathbf{Z})$. In den selbstselektiven Phasen kann Schulleistung hingegen primär als Teil der erwarteten Erfolgswahrscheinlichkeit (bezüglich des Zugangs und des Studienerfolgs) interpretiert werden und somit als Teil sekundärer Effekte. Um einen Vergleich der nachfolgenden Modelle und Ergebnisse mit existierender Forschung zu ermöglichen, folge ich hier jedoch empirisch dem verbreiteten Vorgehen, den Einfluss von Leistungsindikatoren (HB1/I und B) zunächst gesondert zu prüfen.

Hypothesenblock C: institutionelle Einflussfaktoren und Bildungsentscheidung

In Hypothesenblock C rückt der Einfluss institutioneller Barrieren und Opportunitäten in den Fokus, die (mehr oder weniger vollständig) antizipiert, im Lichte der eigenen Ressourcenausstattung bewertet und in die Entscheidungsfindung mit einbezogen werden. Zum einen sollten hierbei antizipierte Kosten eine Rolle spielen, die im deutschen Kontext durch etwaige Mobilitätskosten also die zurückzulegende Distanz zwischen dem Wohnort und dem Studienort - zustande kommen können. Empirische Studien konnten wiederholt zeigen, dass Schüler/innen aller Herkunftsgruppen nahe gelegene Studienoptionen präferieren. Jedoch ist zu erwarten, dass eine längere Distanz Schüler/innen aus sozial schwächeren Familien stärker abschreckt, sowohl bei der Übersetzung von Aspirationen in Intentionen (HC1/I), bei Bewerbungsentscheidungen (HC1/B) als auch beim letztlichen Übergang ins Studium (HC1/S).

Daneben sollten auch Zugangshürden antizipiert werden, die in Deutschland stark mit dem Studienfach variieren. Diese können erstens in Form von Zulassungsbeschränkungen (oder Eignungsfeststellungsverfahren) und der Selektivität des angestrebten Studienfaches vorliegen. Während ich nicht davon ausgehe, dass Zugangshürden im angestrebten Studienfach sozial privilegierte Schüler/innen vom Hochschulstudium abbringen, erwarte ich, dass die Übersetzungswahrscheinlichkeit von Aspirationen in Intentionen (HC2/I) sowie die Bewerbungswahrscheinlichkeit (HC2/B) sozial schwächerer Schüler/innen mit steigender Selektivität und Zulassungsbeschränkungen in ihrem 
präferiertem Fach sinkt, also eher zu einer Umentscheidung führt. Wie argumentiert, können Zugangshürden nicht nur zu einer Umentscheidung führen (gegen ein Studium), sondern auch zu einer Umorientierung (Wahl eines anderen Studienfaches). Diese Umorientierung kann auch bei sozial privilegierten Schüler/innen erwartet werden, wenn sie antizipieren, dass ihre Zugangschancen zu ihrem Wunschfach gering sind, aber dennoch beim Studium ,bleiben“" müssen (oder wollen), um Statuserhalt sicherzustellen. Bezüglich der Umorientierung erwarte ich entsprechend keinen differentiellen Einfluss von Zulassungshürden, die daher generell in einem negativen Zusammenhang mit der Übersetzung und Umsetzung der Studienfachaspiration stehen sollten (HC3/I, HC3/B).

Zweitens könnten spezifische „komplexe Auswahlverfahren“ (wie Interviews oder Tests) - und die Antizipation hiermit verbundener Chancen, Kosten oder Interaktionen - mehr oder weniger anziehend oder abschreckend wirken. Zum einen könnte erwartet werden, dass sie als „Attraktivitätssignal“ insbesondere für sozial privilegierte Studienaspirant/innen als Pull-Faktor fungieren, während sie (insbesondere Interviews) ihre sozial schwächeren Peers abschrecken (HC4a/I). Andersherum könnten komplexe Auswahlverfahren auch auf sozial schwächere Studienberechtigte anziehend wirken, etwa wenn sie wie politisch gewollt - als Informationsmöglichkeit interpretiert werden oder als „Pretests“, mit dem die eigene Studierfähigkeit unter Beweis gestellt werden kann. In diesem Fall ist mit einem generell positiven Einfluss zu rechnen (HC4b/I).

Zuletzt sollten auch von den Hochschulen bereitgestellte Informationen und institutionelles Prestige als „Pull-Faktoren“ wirken. Bezüglich der Informationen kann dies insbesondere für sozial schwächere Studienaspirant/innen erwartet werden, da diese Informationsdefizite weniger gut mithilfe privater Kanäle ausgleichen können und somit institutionell bereitgestellte Informationen eher schätzen (sofern sie sich ihres Informationsdefizits bewusst sind) (HC5/I). Mit Blick auf institutionelles Prestige sollte es sich genau andersherum verhalten. Zwar gehe ich im deutschen Kontext nicht davon aus, dass Prestige abschreckend auf sozial schwächere Studienaspirant/innen wirkt (sondern die Reputation der Hochschule generell positive Signale sendet), doch sollte die anziehende Wirkung institutionellen Prestiges stärker auf sozial privilegierte Schüler/innen zutreffen (HC6/I).

Die Hypothesen zu komplexen Auswahlverfahren, Informationen und Reputation können nur für die erste Phase getestet werden (mithilfe eines Faktoriellen Surveys, siehe Abschnitt 7.1.3).

Hypothesenblock D: differenzierte Bildungsentscheidungen

Hypothesenblock D befasst sich schließlich mit den differenzierten Bildungsentscheidungen innerhalb des Hochschulsystems von denjenigen Studienaspi- 
rant/innen, die sich generell für ein Studium „entschieden“ haben (also in Phase 1 eine Intention äußern, sich in Phase 2 bewerben und in Phase 4 ins Studium übergehen). Bestimmte Studienoptionen - im deutschen Kontext insbesondere unterschiedliche Hochschultypen und Fächer - sollten dabei auf Schüler/innen unterschiedlicher sozialer Herkunft unterschiedlich attraktiv wirken. So kann davon ausgegangen werden, dass sich sozial stärkere Studienaspirant/innen häufiger für ein Universitätsstudium und seltener für ein $\mathrm{FH}$ Studium entscheiden als ihre sozial schwächeren Peers (HD1/I, HD1/B, HD1/S).

Insbesondere Studienfächer zeichnen sich durch deutlich variierende Merkmale aus. Hier soll die Ertragsdimension bezüglich des zu erwartenden Prestiges und Einkommens im Vordergrund stehen. Es werden keine Herkunftsunterschiede bezüglich des mit einem Studium verbundenen erwartbaren Einkommensertrages angenommen (HD2/I, HD2/B, HD2/S), da Schüler/innen aus sozial schwächeren Familien einem hohen Einkommen einen ebenso hohen (wenn nicht höheren) Wert beimessen sollten. Mit Blick auf das Berufsprestige kann jedoch (im Einklang mit der EMI These) ein positiver Zusammenhang zwischen der sozialen Herkunft und der Intention und Bewerbung für ein prestigereiches Fach sowie das Studium eines solchen Faches selbst erwartet werden (HD3/I, HD3/B, HD3/S) 


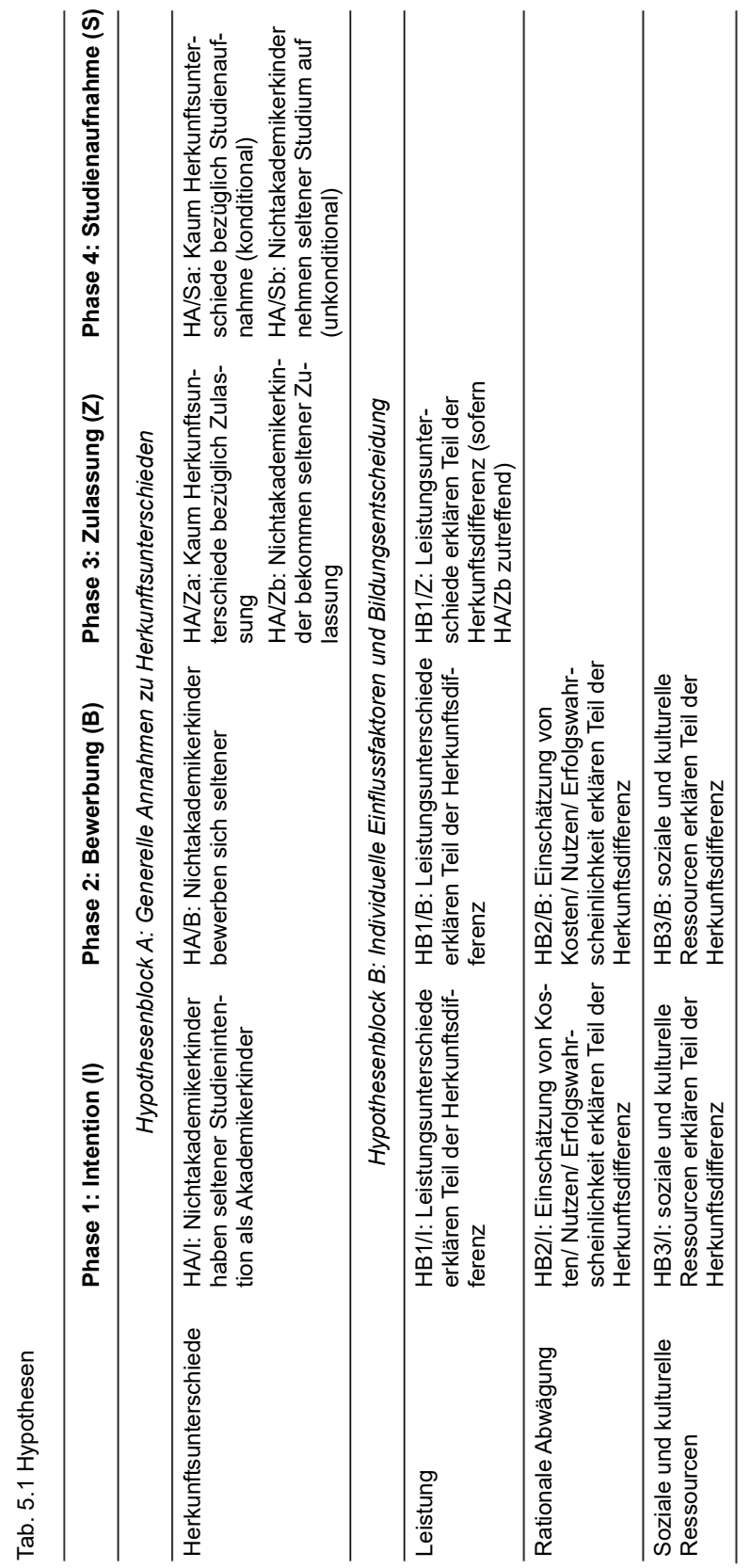




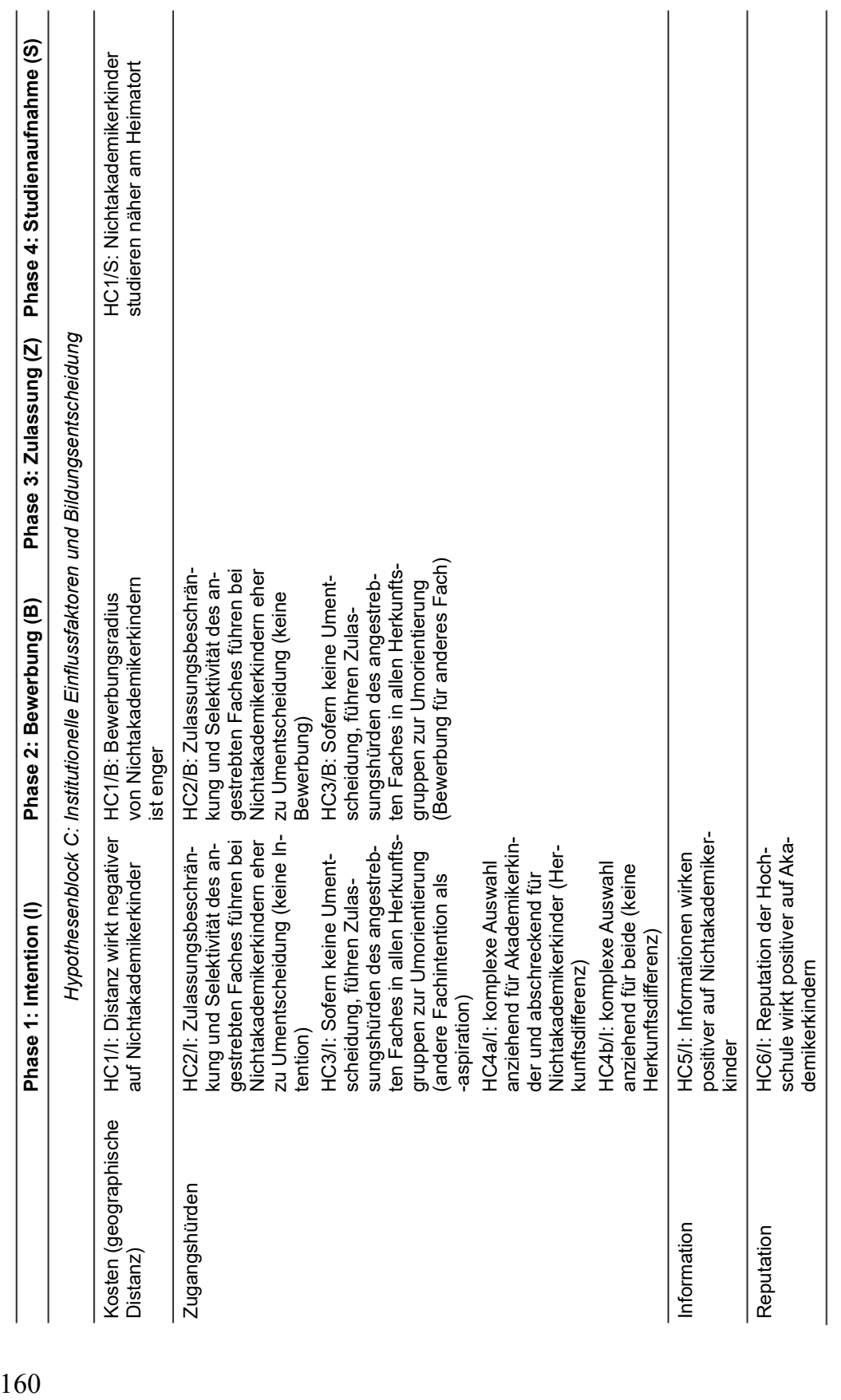




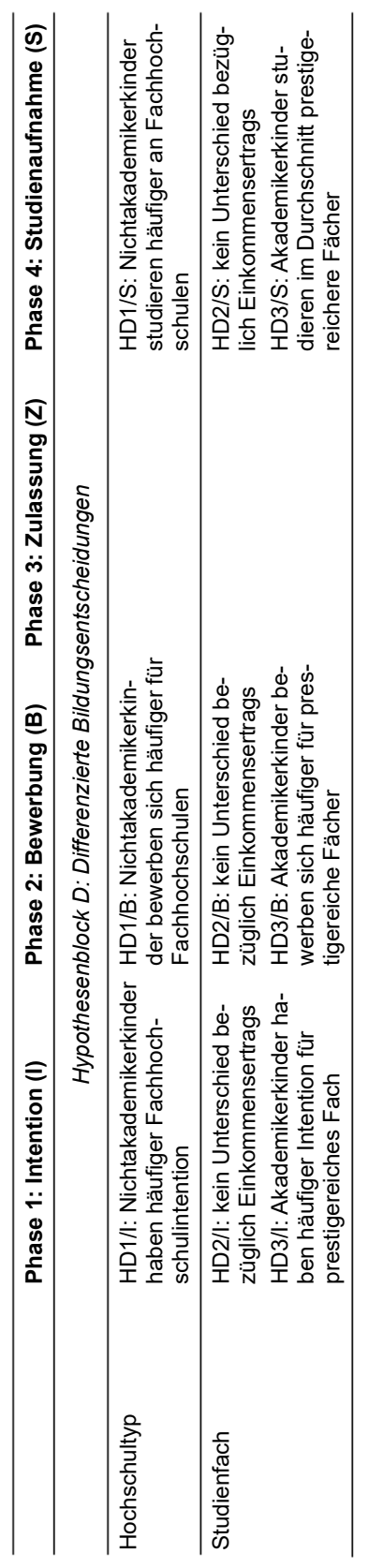




\section{Forschungsdesign}

Bevor die im vorangegangenen Kapitel formulierten theoretischen Annahmen in Kapitel 7 und 8 empirisch geprüft werden, soll im Folgenden das hierfür verwendete Forschungsdesign vorgestellt werden. Zunächst werden die analytischen Schritte kurz dargestellt, die sich aus dem in den Kapiteln 3 und 4 eingeführten und in Kapitel 5 theoretisch angereicherten Phasenmodell ergeben (6.1). Die folgenden Abschnitte geben Auskunft über die verwendeten Daten (6.2), die Operationalisierung zentraler theoretischer Konstrukte (6.3), grundsätzliche Sampleeinschränkungen (6.4) sowie zur Anwendung kommende Auswertungsmethoden und hierbei zu beachtende Besonderheiten (6.5). Kapitel 6 dient der Beschreibung des generellen methodischen Vorgehens. Phasenspezifische Operationalisierungen, Sampleeinschränkungen sowie Methoden (etwa der Faktorielle Survey) werden zu Beginn der jeweiligen empirischen Kapitel genauer erläutert.

\section{1 Übersetzung der Phasen in analytische Schritte}

Ich folge in dieser Arbeit der grundlegenden Annahme, dass soziale Ungleichheit bei der Umsetzung von Studienaspirationen mithilfe einer differenzierten Betrachtung verschiedener Phasen, in denen sowohl individuelle als auch institutionelle Entscheidungsprozesse eine Rolle spielen, umfassender verstanden werden kann. Entsprechend teilt sich auch die empirische Untersuchung in die bereits eingeführten vier Phasen. Mit Phase 1 steht die Übersetzung von Studienaspirationen in Studienintentionen im Vordergrund. Mit den Phasen 2 bis 4 wird schließlich die Umsetzung von Studienaspirationen in Form von Bewerbungen, Zulassungen und der Studienaufnahme genauer betrachtet. Institutionelle Entscheidungen (Fremdselektion) spielen dabei sowohl eine direkte Rolle (in Phase 3) als auch eine indirekte, indem deren Antizipation auf individuelle Entscheidungsprozesse der Phasen 1 und 2 zurückwirkt.

Bereits in Kapitel 4 wurde argumentiert, dass die erste Phase - die Übersetzung von Aspirationen in Intentionen - zwar einen wichtigen ersten Schritt darstellt, jedoch keine notwendige Voraussetzung für die letztliche Umsetzung von Aspirationen ist. Daher wird Phase 1 in Kapitel 7 gesondert analysiert, und die folgenden Analyseschritte werden nicht auf Befragte mit Studienintention beschränkt. Anders verhält es sich mit den folgenden Phasen 2 bis 4, in denen der Eintritt in die jeweils nächste Phase von dem ,erfolgreichen“ Durchlaufen der vorangegangenen Phasen abhängt. Hierfür werden entsprechend konditionale Analysen durchgeführt (vgl. Roderick et al. 2010 für ein ähnliches Vor- 
gehen). Das bedeutet, dass für die Untersuchung von Phase 3 nur Befragte berücksichtigt werden, die sich beworben haben, und für Phase 4 (zunächst) nur solche, die eine Zulassung zum Studium erhielten. Durch dieses Vorgehen reduziert sich die Fallzahl mit jeder Phase, sodass die Schätzungen unsicherer werden. Zudem wird das Analysesample mit jeder Phase zunehmend (positiv) selektiert. Die detaillierten Befunde der jeweils vorangegangenen Phase verhelfen dabei zu einem Überblick über die jeweiligen Selektionsprozesse. Zusätzlich wird in Anhangstabelle A6.1 die Verteilungen zentraler Variablen in den Analysesampeln der jeweiligen Phasen allgemein und getrennt nach sozialer Herkunft dokumentiert.

Die letzte Phase der Studienaufnahme soll jedoch auch unkonditional betrachtet werden, um unabhängig von den zwischenliegenden individuellen und institutionellen Entscheidungsprozessen das Ausmaß sozialer Ungleichheit bei der Umsetzung von Studienaspirationen einschätzen zu können.

\subsection{Daten}

Um die zentrale Fragestellung dieser Arbeit untersuchen zu können, benötige ich Längsschnittdaten, die möglichst detaillierte Informationen zu den verschiedenen Phasen beinhalten. Da andere, für Deutschland repräsentative Datenquellen (etwa die Panelbefragungen vom DZHW oder NEPS) hierzu keine ausführlichen Informationen enthalten, werden Daten des Berliner-Studienberechtigten-Panels (Best Up) herangezogen. Diese ermöglichen erstmals eine differenzierte Untersuchung des Übergangsprozesses, etwa durch einen Faktoriellen Survey, ausführliche Bewerbungs- und Zulassungsmodule sowie die in Abschnitt 2.2.3 bereits vorgestellten Rekrutierungsdaten.

Im Folgenden werden das Best Up-Studiendesign und die hieraus resultierenden individuellen (Abschnitt 6.2.1) und institutionellen (Abschnitt 6.2.2) Daten sowie ihre Möglichkeiten und Grenzen beschrieben. Weitere Details zur Fragestellung und dem Design der Studie können dem Methodenbericht entnommen werden (Ehlert et al. 2017b). Neben den Best Up-Daten wird mit dem Mikrozensus auf eine zweite Datenquelle zurückgegriffen, um das durchschnittliche Einkommen und Prestige von Absolvent/innen verschiedener Studienfächer zu erfassen. Die verwendeten Mikrozensusdaten sowie die hieraus generierten Variablen werden in Abschnitt 6.2.3 dargestellt. 


\subsubsection{Best Up-Individualdaten}

Das Berliner-Studienberechtigten-Panel ${ }^{107}$ beruht auf einem feld-experimentellen Längsschnittdesign. Schüler/innen aus 27 Berliner Schulen mit gymnasialer Oberstufe wurden erstmals am Ende ihres vorletzten Schuljahres vor Erhalt des (Fach-)Abiturs - im Mai/Juni 2013 - befragt und ihre weiteren (Bildungs-)Verläufe in insgesamt fünf Wellen bis Anfang 2016 verfolgt. Auf Grundlage der Best Up-Daten sollen verschiedene Fragestellungen beantwortet werden: Mithilfe von zwei Feldexperimenten wird erstens untersucht, ob der Ausgleich von Informationsasymmetrien und finanziellen Hürden soziale Ungleichheit beim Übergang ins Studium reduzieren kann. ${ }^{108}$ Die Interventionsstudien sind nicht Teil der vorliegenden Arbeit. Nichtsdestotrotz müssen die hierdurch entstandenen Besonderheiten im Design auch für die folgenden Analysen beachtet werden (siehe Abschnitt 6.4). Eine zweite Fragestellung von Best Up - die auch zentraler Bestandteil dieser Arbeit ist - bezieht sich auf den direkten und indirekten Einfluss hochschulischer Auswahlverfahren auf soziale Ungleichheit beim Übergang in die Hochschule.

Die Best Up-Daten beruhen auf einem Sample von 27 Berliner Schulen, an denen die Hochschulzugangsberechtigung erworben werden kann. Insgesamt war dies zum Zeitpunkt der Schulauswahl in Berlin an 137 Schulen möglich, die sich in drei Schultypen unterteilen (Gymnasien, ISS, OSZ, vergleich Abschnitt 2.3). In Berlin wählen Schüler/innen und ihre Eltern das erste Mal nach Klassenstufe 6 zwischen unterschiedlichen sekundären Schultypen. Eine Ausnahme bilden 33 sogenannte grundständige Gymnasien, die leistungs-

107 Best Up ist ein Kooperationsprojekt zwischen der Freien Universität Berlin, dem Deutschen Institut für Wirtschaftsforschung (DIW, Abteilung Bildung und Familie. Beteiligte Forscher/innen: Mathias Hübner, Frauke Peter, C. Katharina Spieß, Johanna Storck, Vaishali Zambre) und dem Wissenschaftszentrum Berlin für Sozialforschung (WZB, Abteilung Ausbildung und Arbeitsmarkt. Beteiligte Forscher/innen: Martin Ehlert, Claudia Finger, Cindy Fitzner, Alessandra Rusconi, Heike Solga). Finanziert wurde das Projekt von der Einstein Stiftung Berlin (Fördernummer: A-2010-25 „Berliner-Studienberechtigten-Panel/Best Up“").

108 Hierfür wurden Schulen zufällig in Kontrollschulen und zwei Gruppen von Treatmentschulen unterteilt. Für das erste Treatment (Informationsintervention) nahmen alle Schüler/innen der ausgewählten Schulen im Anschluss an die erste Befragung an einem Workshop teil, in dem ihnen Informationen zu Finanzierungsmöglichkeiten, Kosten und Erträgen eines Studiums und einer beruflichen Ausbildung präsentiert wurden (für Details und Ergebnisse siehe Ehlert et al. 2017a/b, Finger et al. 2020, Peter et al. 2016a, Peter und Zambre 2017). Als Teil des zweiten Feldexperiments (finanzielle Intervention) wurde allen Schüler/innen der ausgewählten Schulen, die zu Beginn des letzten Schuljahres (Welle 2) eine Ausbildungsintention äußerten, eine finanzielle Unterstützung von monatlich $300 €$ für ihr erstes Studienjahr angeboten, sollten sie doch ein Studium aufnehmen (siehe Ehlert et al. 2017b, Peter et al. 2016b). 
starke Schüler/innen bereits nach Klasse 4 aufnehmen. Da hier Schüler/innen aus sozial privilegierten Familien überrepräsentiert sind (Helbig und Nikolai 2017), wurden grundständige Gymnasien bei der Auswahl der Schulen nicht berücksichtigt. Aus den verbleibenden 104 Schulen (56 Gymnasien, 33 ISS, 15 OSZ) wurde ein stratifiziertes Sample von 27 Schulen gewonnen, das sich gleichmäßig auf die drei Schultypen verteilt.

Schüler/innen aus Familien ohne akademischen Hintergrund sind für die zentralen Fragestellungen des Best Up-Projekts von besonderer Bedeutung. Um ein Sample mit möglichst vielen Schüler/innen aus nichtakademischen Elternhäusern zu gewinnen, lag der Fokus bei der Auswahl der Schulen daher auf Bezirken mit einem überdurchschnittlichen Anteil an Erwachsenen (25 Jahre und älter) mit vergleichsweise niedriger formaler Bildung. ${ }^{109}$

Durch die geographische Einschränkung auf spezielle Bezirke innerhalb Berlins sind keine generalisierenden Aussagen für deutsche oder Berliner Hochschulzugangsberechtigte möglich (zu Besonderheiten des Berliner Schulund Hochschulsystems siehe Abschnitt 2.3). So führt der Ausschluss von „,bildungsbürgerlichen" Bezirken wahrscheinlich zur Unterschätzung von Herkunftsdifferenzen und dem Einfluss der Schulebene, da die Varianz existierender Privilegien, die sich auch in der Wahl der Wohn- und Schulgegend zeigen können, hierdurch eingeschränkt wird. Die Beschränkung der Analysen auf einen bestimmten Kontext birgt aber auch Vorteile, da der Einfluss von divergierenden institutionellen und strukturellen Besonderheiten zwischen und innerhalb der Bundesländer (vgl. Helbig et al. 2015, Helbig und Nikolai 2015) und Bezirke konstant(er) gehalten wird und Herkunftsdifferenzen so eindeutiger der Familie zugeordnet werden können. ${ }^{110}$ Tabelle 6.1 gibt einen Überblick über die fünf Erhebungswellen (W1-W5).

109 „Niedrige formale Bildung“ wurde anhand der International Standard Classification of Education (ISCED) definiert. Hierfür wurden auf Grundlage des Sozialberichts Berlin Brandenburg Bezirke ermittelt, in denen der Anteil der erwachsenen Bevölkerung mit ISCED Level 0 (vorschulische Erziehung) bis 2 (Sekundarstufe I) über dem Berliner Durchschnitt von 16,8\% lag (für nähere Angaben zur stratifizierten Stichprobenziehung siehe Ehlert et al. 2017b). Durch dieses Vorgehen sind Schulen folgender Bezirke im Best Up Sample vertreten: Neukölln (Anteil ISCED 0-2: 30,3\%), Mitte (25,1\%), Spandau (22,9\%), Friedrichshain-Kreuzberg (20,1\%), Tempelhof-Schöneberg (18,9\%), Reinickendorf (17,8\%) (vgl. Amt für Statistik Berlin-Brandenburg 2011: 55).

110 Die Randverteilungen einiger grundsätzlicher Variablen wurden mit einem möglichst vergleichbaren Subsample der deutschlandweit repräsentativen NEPS Startkohorte 4 verglichen (siehe Ehlert et al. 2017b: 25ff.). Leider ist es aus Datenschutzgründen nicht möglich, den Vergleich auf Berliner Befragte zu beschränken. Daher wurden die Best Up-Befragten mit NEPS-Befragten in Großstädten verglichen. Wie bezweckt, ist der Anteil an Befragten aus Nichtakademikerfamilien in den Best Up-Daten höher (62 vs. 44\%). Entsprechend haben die Best Up-Befragten etwas schlechtere Schulnoten, etwas seltener eine Studienintention und seltener 
Tab. 6.1: Best Up-Erhebungsdesign

\begin{tabular}{|c|c|c|c|c|c|}
\hline & $\begin{array}{l}\text { Welle } 1 \\
\text { Mai/Juni } \\
2013\end{array}$ & $\begin{array}{l}\text { Welle } 2 \\
\text { Aug./Sep. } \\
2013\end{array}$ & $\begin{array}{l}\text { Welle } 3 \\
\text { Juli/Okt. } \\
2014\end{array}$ & $\begin{array}{l}\text { Welle } 4 \\
\text { April/Juni } \\
2015\end{array}$ & $\begin{array}{l}\text { Welle } 5 \\
\text { Jan./März } \\
2016\end{array}$ \\
\hline $\mathrm{N}$ & $\begin{array}{l}1578 \\
\text { Response } \\
\text { Rate: } 60 \%\end{array}$ & $\begin{array}{l}1105 \\
\text { Rücklauf: } 70 \%\end{array}$ & $\begin{array}{l}1034 \\
\text { Rücklauf: } 66 \%\end{array}$ & $\begin{array}{l}1005 \\
\text { Rücklauf: } 64 \%\end{array}$ & $\begin{array}{l}972 \\
\text { Rücklauf: } 62 \%\end{array}$ \\
\hline Modus & $\begin{array}{l}\text { Klassenraum- } \\
\text { Befragung } \\
\text { (PAPI), Postali- } \\
\text { sche Eltern- } \\
\text { Befragung }\end{array}$ & $\begin{array}{l}\text { Online- } \\
\text { Befragung }\end{array}$ & $\begin{array}{l}\text { Online- } \\
\text { Befragung }\end{array}$ & $\begin{array}{l}\text { Online- } \\
\text { Befragung }\end{array}$ & $\begin{array}{l}\text { Online- } \\
\text { Befragung }\end{array}$ \\
\hline $\begin{array}{l}\text { Besondere } \\
\text { Inhalte }\end{array}$ & $\begin{array}{l}\text { Kognitions- } \\
\text { fähigkeiten } \\
\text { Informations- } \\
\text { intervention }\end{array}$ & $\begin{array}{l}\text { Faktorieller } \\
\text { Survey } \\
\text { Finanzielle } \\
\text { Intervention }\end{array}$ & $\begin{array}{l}\text { Bewerbungs- } \\
\text { modul WiSe } \\
2014 / 15\end{array}$ & $\begin{array}{l}\text { Zulassungs- } \\
\text { modul WiSe } \\
2014 / 15 \\
\text { Übergang ins } \\
\text { Studium }\end{array}$ & $\begin{array}{l}\text { Bewerbungs-/ } \\
\text { Zulassungs- } \\
\text { modul WiSe } \\
2015 / 16 \\
\text { Übergang ins } \\
\text { Studium }\end{array}$ \\
\hline
\end{tabular}

Quelle: Ehlert et al. 2017b. Eigene Darstellung

Die erste Erhebung fand im Mai/Juni 2013 statt, circa ein Jahr vor dem regulären Erhalt der Hochschulzugangsberechtigung (je nach Schultyp Klassenstufe 11 oder 12, siehe Abschnitt 2.3). Es handelte sich hierbei um eine insgesamt etwa 90minütige Klassenraumbefragung (Paper and Pencil Interview, PAPI). Die Folgebefragungen fanden online statt. ${ }^{111}$ Trotz des Wechsels im Befragungsmodus betrug die Rücklaufquote der zweiten Befragung knapp $70 \%$ und stabilisierte sich in den Folgewellen auf einem hohen Niveau.

Der Befragungszeitraum erstreckte sich über insgesamt fast drei Jahre. Abbildung 6.1 gibt einen Überblick über die zeitliche Anordnung kritischer (hochschulbezogener) Ereignisse, wie etwa dem üblichen Bewerbungsschluss für zulassungsbeschränkte Studiengänge, und das hierin eingebettete Timing der Befragungswellen. Mit der letzten Welle 5 besteht die Möglichkeit, den Übergang ins Studium nach einer einjährigen Auszeit (gap year) zu beobachten. Befragte, die ihr Studium direkt nach dem Abitur aufnahmen, befinden sich entsprechend im dritten Semester. Damit können spätere Entscheidungen für ein Studium (etwa nach einer für Abiturient/innen in der Regel zweijährigen Ausbildung) nicht nachvollzogen werden. Wie in Abschnitt 2.1.2 gezeigt, kann trotz dieser Einschränkung ein Großteil aller Übergänge ins Studium beobachtet werden, da die meisten Abiturient/innen, die schließlich ein Studium

mehrheitlich Freunde mit Studienintention. Zudem sind Befragte mit Migrationshintergrund in den Best Up-Daten deutlich überrepräsentiert (53 vs. 37\%).

111 Mit der Panelpflege, Programmierung und Durchführung der Onlinebefragungen wurde TNS Infratest Sozialforschung (jetzt Kantar Infratest) beauftragt. 
aufnehmen, dies bereits im ersten oder zweiten Jahr nach Erhalt des Abiturs tun (Autorengruppe Bildungsberichterstattung 2020: F2-4web). Hängt die spätere Aufnahme eines Studiums mit der sozialen Herkunft zusammen, sind Aussagen zum Ausmaß sozialer Ungleichheit dennoch eingeschränkt. Empirische Ergebnisse hierzu sind nicht eindeutig (vgl. Quast et al. 2014: 82, Weiss und Steininger 2013: 196).

Abb. 6.1: Zeitstrahl zum Timing wichtiger Ereignisse im Best Up-Studienverlauf

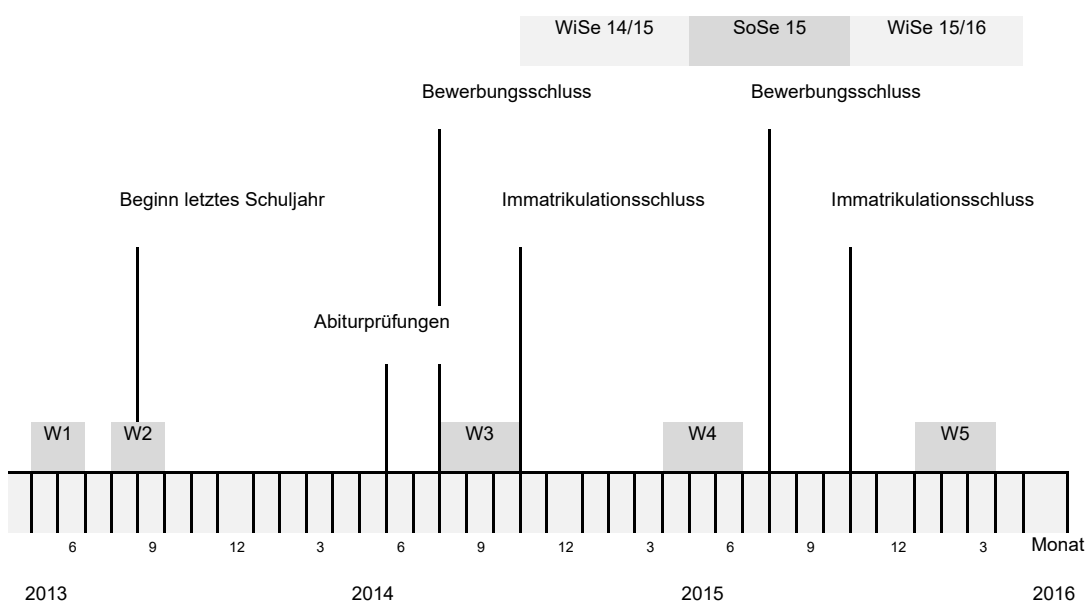

Eigene Darstellung. Das genaue Timing der Ereignisse variiert zum Teil und ist daher hier näherungsweise zu betrachten.

Für die Entwicklung der Fragebögen orientierten wir uns vor allem an bereits erprobten Instrumenten des NEPS (insbesondere Fragebögen der Klassen 11 und 12, Startkohorte 4) und des DZHW (insbesondere Studienberechtigtenpanel). ${ }^{112}$ Wie eingangs bereits beschrieben, enthielten diese Befragungen aber während der Laufzeit des Best Up-Projekts kaum Items zum Bewerbungsverhalten und zu Zulassungen, sodass hierfür neue Module entwickelt und im Vorhinein mit Personen der Zielgruppe getestet wurden. Neben den eigens für die Best Up-Studie entwickelten Bewerbungs- und Zulassungsmodulen wurde zudem für die Untersuchung der ersten Phase - der Übersetzung von idealistischen Studienaspirationen in realistische Studienintentionen - ein Faktorieller Survey (FS) entwickelt (Welle 2), mit dessen Hilfe der Einfluss institutioneller

112 Alle Best Up-Fragebögen stehen online zur Verfügung: http://www.diw.de/six cms/detail.php?id=diw_01.c.561227.de [letzter Zugriff 07.09.2021] 
Merkmale auf die Bewerbungsintention von Schüler/innen quasi-experimentell untersucht werden kann (siehe Abschnitt 7.2.3 für Details).

Da mit den unterschiedlichen Zugangswegen (vgl. Abschnitt 2.2.2) ein deutliches $\mathrm{Ma}$ an Komplexität einhergeht, spiegeln die Bewerbungsmodule dieses zu einem gewissen Grad wider. Die Gratwanderung bei der Entwicklung lag dabei zwischen Komplexitätsreduktion (zur besseren technischen Durchführbarkeit, empirischen Verwertbarkeit und um eine kognitive Überforderung der Befragten zu verhindern) und dem Vermeiden zu starker Vereinfachung. Wie Abbildung 6.1 verdeutlicht, unterscheidet sich das Timing zwischen den Befragungen zu Bewerbungen und Zulassungen direkt nach dem (Fach-)Abitur und ein Jahr später. Um diesem Umstand Rechnung zu tragen, wurden unterschiedliche und gleichzeitig möglichst vergleichbare Module entwickelt, die im Folgenden kurz beschrieben werden:

Welle 3 und 4 (direkte Bewerbung und Zulassung): Die Erhebung der dritten Welle startete kurze Zeit nach Erhalt des (Fach-)Abiturs und direkt nach Ende der üblichen Bewerbungsfrist für zulassungsbeschränkte Studiengänge (15. Juli). Die Feldzeit umspannte außerdem einen großen Teil der Einschreibphase für zulassungsfreie Studiengänge. Da sich der Bewerbungsvorgang zwischen unterschiedlichen Studienarten deutlich unterscheidet, unterteilt sich das W3-Bewerbungsmodul entsprechend in verschiedene „Bewerbungstypen“: 1) Bewerbungen für ein duales Studium, die wiederum entweder an Unternehmen/Behörden/soziale Einrichtungen oder an die Hochschulen selbst gerichtet werden müssen; 2) Bewerbungen für bundesweit zulassungsbeschränkte Studiengänge über die Stiftung für Hochschulzulassung; 3) Bewerbungen für örtlich zulassungsbeschränkte Studiengänge bei den Hochschulen oder bereits erfolgte Einschreibungen für zulassungsfreie Studiengänge und 4) Bewerbungen für Studiengänge im Ausland.

Nacheinander für die verschiedenen Typen erfolgte die Abfrage, ob und (wenn ja) für wie viele Studiengänge sich die Befragten beworben/bereits eingeschrieben haben. Danach konnten die Befragten pro Bewerbungstyp bis zu zehn Studiengänge auflisten (Fach, Hochschule, Hochschulort und gegebenenfalls Land und Unternehmen/Behörde/soziale Einrichtung). Anschließend wurden die (vollständig) angegebenen Studiengänge ${ }^{113}$ eingeblendet und die Befragten gebeten, diese nach ihren Vorlieben zu ordnen. Zu den bis zu fünf erstplatzierten Studiengängen wurden einige typspezifische Nachfragen gestellt.

113 Vollständigkeit war aufgrund der häufig erfolgten automatischen Einblendungen (in derselben Befragung und als Preloads in Folgebefragungen) zentral für eine erfolgreiche Durchführung der Befragungen. Konkret bedeutet dies, dass mindestens Nennungen zum Studienfach und zur Hochschule (bei dualen Studiengängen außerdem zum Unternehmen) vorliegen mussten, um bei Mehrfachbewerbungen eine eindeutige Identifizierung einzelner Studiengänge sicherzustellen. 
Zusagen und Übergänge ins Studium wurden unter Verwendung von Preloads in Welle 4 abgefragt. Zunächst wurden hierfür die Bewerbungen gesichtet und unstimmige Angaben aus der Preloadliste gelöscht. Die verbleibenden, vollständigen Bewerbungsangaben wurden in Welle 4 eingeblendet. Befragte, die im WiSe 2014/15 studierten, wurden zunächst gefragt, ob es sich bei ihrem Studium um einen der eingeblendeten Studiengänge handelte. Sofern dies nicht der Fall war, hatten sie die Möglichkeit, einen neuen Studiengang anzugeben. Unabhängig von ihrem Studienstatus im WiSe 2014/15 wurden außerdem alle Befragten mit W3-Bewerbungen nach Zulassungen gefragt. Hierfür wurde erneut die Preload-Liste verwendet (zusätzlich stand die Kategorie „Zulassung für einen oder mehrere andere Studiengänge“ zur Auswahl). Befragte, die nicht an Welle 3 teilnahmen, bekamen in Welle 4 ein stark verkürztes Bewerbungsmodul (Anzahl Bewerbungen und Zulassungen).

Welle 5 (verzögerte Bewerbung und Zulassung): Die fünfte und letzte Erhebung wurde Anfang 2016 durchgeführt. Hierfür musste das Bewerbungsmodul angepasst und gekürzt werden. ${ }^{114}$ Die Befragten sollten daher zunächst allgemein angeben, ob sie sich für das WiSe 2015/16 beworben hatten, wie oft und ob es sich hierbei um Bewerbungen an deutschen oder ausländischen Hochschulen (oder beidem) handelte. Im Anschluss sollten sie - geordnet nach ihrer Vorliebe - bis zu fünf Studiengänge nennen (Fach, Hochschule, Ort, gegebenenfalls Unternehmen und Land). Die Abfrage nach Zulassungen für die genannten und gegebenenfalls weiteren Studiengänge erfolgte direkt im Anschluss.

Die Bewerbungs- und Zulassungsmodule für die WiSe 2014/15 und 2015/16 unterscheiden sich entsprechend in folgenden Punkten: Erstens wurde zum zweiten Bewerbungszeitpunkt nicht von vorn herein zwischen den verschiedenen Bewerbungstypen unterschieden. Zweitens konnten hier weniger Studiengänge angegeben werden als zum ersten Bewerbungszeitpunkt. Drittens wurden die Studiengänge in Welle 5 erst gerankt, nachdem Informationen über $\mathrm{Zu}$ - oder Absagen bereits vorlagen, sodass die Wahrscheinlichkeit von Rationalisierungsprozessen größer ist (vgl. Sudman et al. 1996). ${ }^{115}$ Letztlich wurden Bewerbungen und Zulassungen für das WiSe 2015/16 direkt nacheinander erfragt, während dies für das WiSe 2014/15 über zwei Wellen verteilt

114 Dies erfolgte aus zwei Gründen: Erstens musste dem Timing der Befragung Rechnung getragen werden, denn die Fragen zu Bewerbungen für das WiSe 2015/16 wurden deutlich später gestellt als die Fragen zu Bewerbungen für das WiSe 2014/15 in Welle 3 (circa ein halbes Jahr vs. direkt nach Bewerbungsschluss). Zweitens waren die vorgesehene Befragungszeit in Welle 5 sowie die Mittel zur Incentivierung der Befragten deutlich geringer.

115 Die Erhebungsdauer von Welle 3 erstreckte sich über drei Monate. Es ist daher auch hier möglich, dass für einige Befragte $\mathrm{Zu}$ - oder Absagen bereits vorlagen, sofern sie den Fragebogen relativ spät ausfüllten (mehr als 75\% der Welle 3 Teilnehmer/innen nahmen allerdings bereits in den ersten vier Wochen am Survey teil). 
erfolgte. Mögliche Auswirkungen für die Vergleichbarkeit der Daten und die Interpretation der Ergebnisse werden an gegebener Stelle in den empirischen Kapiteln diskutiert.

Mithilfe der entwickelten Module können das Bewerbungsverhalten von Schüler/innen sowie Zulassungsentscheidungen von Hochschulen erstmals auch im - wenn auch nicht repräsentativen - deutschen Kontext detailliert dargestellt werden. Zudem können durch die genaue Abfrage der Studiengänge weitere Merkmale wie der Hochschultyp, die Distanz zwischen Hochschulund Heimatort oder erwartbare studienfachspezifische Erträge nicht nur für den im letzten Schritt gewählten Studiengang, sondern für das (weitestgehend) gesamte Bewerbungsspektrum der Befragten abgebildet werden.

\subsubsection{Best Up-Rekrutierungsdaten}

Ein weiteres Novum der Best Up-Daten stellt die umfangreiche institutionelle Recherche dar, mit deren Hilfe dargestellt werden kann, inwiefern Hochschulen durch die Festlegung und Nutzung von Auswahlkriterien und -verfahren von ihrer Rolle als Gatekeeper Gebrauch machen und inwiefern Schüler/innen hierdurch entstehende Zugangsrestriktionen antizipieren und in entsprechende Entscheidungen für oder gegen eine Bewerbung übersetzen. Zentrale Ergebnisse der Rekrutierungsrecherche wurden bereits in Kapitel 2 vorgestellt. Im Folgenden soll das Vorgehen bei der Erstellung der Rekrutierungsdaten beschrieben werden.

Der Rekrutierungsdatensatz enthält valide Angaben zu 466 Studiengängen an deutschen Hochschulen (circa 5\% des gesamtdeutschen Studienangebots). Recherchiert wurden bis auf einige Ausnahmen alle Studiengänge, in die sich die Befragten im WiSe 2014/15 oder 2015/16 immatrikulierten (W4/W5), sowie die zwei höchstgerankten Studiengänge, für die sich die Befragten bewarben (W3/W5). ${ }^{116}$ Die Ausnahmen umfassen Studiengänge an Hochschulen auBerhalb Deutschlands sowie duale Studiengänge, bei denen die Auswahl den Unternehmen, sozialen Einrichtungen oder Behörden obliegt und erfolgreichen Bewerber/innen ein Studienplatz an einer kooperierenden Hochschule meist ohne weitere Auswahl zugeteilt wird. Außerdem wurden Studiengänge einer möglichen zweiten Bewerbungsphase (für das WiSe 2015/16) nicht für Studienabbrecher/innen oder Studiengangswechsler/innen recherchiert, son-

116 Neben dem weiter oben beschriebenen Bewerbungsmodul wurden Welle 3 Teilnehmer/innen ohne Bewerbung gefragt, ob sie planen, sich in einen zulassungsfreien Studiengang für das WiSe 2014/15 einzuschreiben. Weiterhin wurden alle Befragten mit (Fach-)Abitur gefragt, ob sie sich gern für einen bestimmten Studiengang beworben hätten, dies aber aus unterschiedlichen Gründen nicht taten. Auch diese Studiengänge gingen in die Rekrutierungsrecherche ein. 
dern nur für diejenigen Befragten, die nach der ersten Bewerbungsphase (WiSe 2014/15) nicht ins Studium übergingen.

Recherchiert wurden jeweils Angaben für das zum Zeitpunkt der Bewerbung/der Immatrikulation aktuelle Semester sowie für das vorangegangene Wintersemester; Ersteres, um die tatsächlichen Zugangschancen zum Zeitpunkt der Bewerbung abzubilden, Letzteres, um auch deren Antizipation mit einschließen zu können. Konkret bedeutet dies, dass für W3-Bewerbungsangaben und W4-Immatrikulationen Angaben für die WiSe 2013/14 (Antizipation) und 2014/15 (tatsächliche Situation) recherchiert wurden. Für W5-Bewerbungen und Immatrikulationen wurden entsprechend Informationen zu den WiSe 2014/15 und 2015/16 recherchiert. Damit liegt die größte Anzahl an recherchierten Studiengängen für das WiSe 2014/15 vor.

Insgesamt wurden so 582 Studiengänge identifiziert: 492 für das WiSe 2013/14, 580 $0^{117}$ für das WiSe 2014/15 und 215 für das WiSe 2015/16. Allerdings konnten nur 446 der 582 Studiengänge recherchiert werden (77\%). ${ }^{118} 72$ Angaben existierten in der angegebenen Form nicht (z.B. Journalismus an der Technischen Universität Berlin). Diese „Problemfälle“ können zum einen eine spezifische Form der Antwortverweigerung darstellen oder auf Fehler bei der Auswahl der Hochschulen aus der eingeblendeten Liste zurückzuführen sein. Inhaltlich weniger problematisch, aber dennoch nicht recherchierbar sind 54 Studiengänge, bei denen die offene Studiengangsangabe keinem der an der Hochschule angebotenen Studiengänge genau zugeordnet werden konnte (z.B. „Wirtschaft“ an der Humboldt-Universität zu Berlin, an der sowohl Betriebswirtschafts- als auch Volkswirtschaftslehre studiert werden kann). Letztlich gab es zehn Studiengänge mit der Bezeichnung „Lehramt“, aber ohne konkretere Angabe zur Art des Lehramtes oder zum Fach, die allerdings für die Recherche zwingend erforderlich sind.

Nach dem Ausschluss der Problemfälle wurde die Rekrutierungsrecherche für die verbleibenden 446 validen Studiengänge durchgeführt: 391 für das WiSe 2013/14, 444 für das WiSe 2014/15 und 171 für das WiSe 2015/16. Hierfür wurde zunächst auf Grundlage einer Literaturrecherche und öffentlich zugänglicher Dokumente (Hochschulrahmengesetz, Staatsverträge, Hochschulzulassungsgesetze und -verordnungen der Länder, Zulassungssatzungen verschiedener Hochschulen, Informationen der $\mathrm{SfH}$ ) Kategorien identifiziert, die

117 Zwei Studiengänge wurden erstmals zum WiSe 2015/16 angeboten und daher nicht für das vorangegangene Semester recherchiert. Gleiches gilt für vier Studiengänge, die erstmals zum WiSe 2014/15 angeboten wurden.

118 Nicht recherchierbar waren überdurchschnittlich häufig Angaben von Studiengängen, für die sich die Befragten gern beworben hätten, und Angaben zu Einschreibplänen. Es handelt sich dabei also eher um Studiengänge, die für die Befragten möglicherweise nicht genauso konkret waren wie tatsächliche Bewerbungen und Immatrikulationen. 
im Zuge der Recherche ergänzt und angepasst wurden. Tabelle 6.2 gibt einen Überblick über die recherchierten Informationen. ${ }^{119}$

Tab. 6.2: Zentrale Inhalte der Rekrutierungsrecherche

\begin{tabular}{|c|c|}
\hline Basisangaben & $\begin{array}{l}\text { - } \quad \text { Name des Studiengangs (Programmname) } \\
\text { - } \quad \text { Hochschule, Hochschulort } \\
\text { - } \quad \text { Lehrienfächer (bis zu drei) } \\
\text { - } \quad \text { Mono/Kombibachelor }\end{array}$ \\
\hline Quotenregelung & $\begin{array}{l}\text { - Zur Anwendung kommende Quoten (Abiturientenbesten-, Wartezeit-, } \\
\text { AdH-Quote) } \\
\text { - } \quad \text { Höhe der Quoten (\%) }\end{array}$ \\
\hline Wettbewerbssituation & $\begin{array}{l}\text { - Anzahl Studienplätze } \\
\text { - Anzahl Bewerber/innen } \\
\end{array}$ \\
\hline Zulassungsbeschränkung & $\begin{array}{l}\text { - Zulassungsbeschränkung: zulassungsfrei, bundesweit oder örtlich } \\
\text { zulassungsbeschränkt } \\
\text { - Wurden alle Bewerber/innen zugelassen? }\end{array}$ \\
\hline Auswahlgrenzen & $\begin{array}{l}\text { - } \quad \text { Abiturbestenquote: Durchschnittsnote (DN) und nachrangige Kriterien } \\
\text { (in der Regel Wartezeit, WZ) } \\
\text { - Wartezeitquote: WZ und nachrangige Kriterien (in der Regel DN) } \\
\text { - } \quad \text { AdH-Endwert (in Form einer Punktzahl und/oder Schulnote) }\end{array}$ \\
\hline AdH-Verfahren & $\begin{array}{ll} & \text { DN (Ranking im Verfahren*) } \\
- & \text { Schulfächer (Ranking, Fachangabe, Form des Nachweises) } \\
-\quad \text { Berufserfahrung (Ranking, Art, Dauer) } \\
\text { - } \quad \text { Fremdsprachen (Ranking, Art, Form des Nachweises) } \\
\text { - } \quad \text { Studienvorbereitender Kurs (Ranking) } \\
\text { - Weitere (z.B. Tests, Auswahlgespräche) }\end{array}$ \\
\hline $\begin{array}{l}\text { Eignungsfeststellungs- } \\
\text { verfahren }\end{array}$ & $\begin{array}{ll} & \text { Tests } \\
\text { - } & \text { Fremdsprachen (Art, Form des Nachweises) } \\
\text { - } & \text { Berufserfahrung } \\
\text { - } & \text { Einzureichende Materialen (z.B. Mappe, Motivationsschreiben) } \\
\text { - } & \text { Weitere (z.B. Auswahlgespräche, Atteste, Gebühren) }\end{array}$ \\
\hline
\end{tabular}

Quelle: Ehlert et al. 2017b. Eigene Darstellung

*mit dem „Ranking“ innerhalb der AdH-Quote wurde gekennzeichnet, welches relative Gewicht den einzelnen Kriterien bei der Erstellung der Rangliste zugewiesen wird.

119 Die konkreten Kriterien und Verfahren, die zur Auswahl und Eignungsfeststellung herangezogen werden, werden von den Hochschulen in der Regel nur für die aktuelle Bewerbungsphase veröffentlicht. Daher konnten diese für das WiSe 2013/14 nicht gesondert ermittelt werden, da die Recherche erst mit Vorlage der Bewerbungsangaben in Welle 3 (WiSe 2014/15) beginnen konnte. Der Recherche lag daher die Annahme zugrunde, dass die zur Anwendung kommenden Verfahren und Kriterien zur Auswahl und Eignungsfeststellung in der Regel stabil sind. Dies wird durch den Abgleich zwischen den Bewerbungsphasen für die WiSe 2014/15 und 2015/16 bestätigt: Die Eignungsfeststellungsverfahren änderten sich bei keinem, das Auswahlverfahren der Hochschule nur bei sechs der zu beiden Zeitpunkten recherchierten Studiengänge (N:171). Dies gilt nicht für die Auswahlgrenzen, die in der Regel auch für zurückliegende Semester veröffentlicht werden und sich entsprechend des Angebot-Nachfrageverhältnisses verändern können (siehe Abschnitt 2.2.2). 
Die Recherche war vor allem internetbasiert und beruhte auf den Angaben der Hochschulen und der Stiftung für Hochschulzulassung, die Informationen zu den bundesweit zulassungsbeschränkten Studiengängen bereitstellt. Für nicht zentral vergebene Studienplätze veröffentlichen einige Hochschulen - insbesondere Universitäten - Informationen zu Zulassungsmodi, Verfahren und Auswahlgrenzen auf einer zentralen Webseite. Hingegen war das Zusammentragen der Informationen bei kleineren und privaten Hochschulen zum Teil aufwändig. Zudem sind nicht alle Angaben immer frei recherchierbar. Sofern wichtige Angaben nicht recherchiert werden konnten, wurde telefonisch oder via Email Kontakt mit den verantwortlichen Stellen aufgenommen. Doch auch nach diesem Schritt weisen einige Variablen einen hohen Anteil fehlender Werte auf: Auswahlgrenzen Abiturbestenquote (8\%), Auswahlgrenze Wartezeitquote (11\%), AdH-Endwert (28\%), Anzahl Bewerber/innen (37\%) (bei allen weiteren Variablen liegt der Anteil fehlender Werte im unteren einstelligen Bereich).

Die 446 Studiengänge verteilen sich auf insgesamt 68 Hochschulorte, 107 Hochschulen und 125 (unaggregierte) Studienfelder. Wenig überraschend ist die Mehrzahl der recherchierten Studiengänge an Berliner Hochschulen angesiedelt. ${ }^{120}$ Außerdem wurde ein hoher Anteil - jeweils circa die Hälfte - des Studienangebots der drei Berliner Universitäten recherchiert (Freie Universität: 50, Humboldt Universität: 50, Technische Universität: 36).

Tabelle 6.3 gibt einen Überblick über die Verteilung der Studiengänge in den Rekrutierungsdaten. Ein Vergleich mit den im Hochschulkompass gelisteten Studiengängen zeigt, dass Studiengänge an Berliner Hochschulen (im Hochschulkompass: 5\%), an Universitäten (im Hochschulkompass: circa 60\%) und an privaten Hochschulen (im Hochschulkompass: 1\%) ${ }^{121}$ in den Rekrutierungsdaten überrepräsentiert sind. Innerhalb Berlins kommen Studiengänge an Universitäten im Vergleich zu Fachhochschulen deutlich häufiger vor (Rekrutierungsdaten: circa $60 \%$, Hochschulkompass: circa $40 \%) .{ }^{122}$

120 Laut Hochschulkompass umfasste das Studienangebot in Berlin circa 500 Studiengänge (Stand 2017), von denen über 50\% recherchiert wurden. Von dem verbleibenden Studienangebot außerhalb Berlins wurden allerdings nur circa 2-3\% der Studiengänge recherchiert.

121 Der vergleichsweise hohe Anteil an Studiengängen an privaten Hochschulen ist sicherlich durch das hohe Angebot an privaten Hochschulen in Berlin zu erklären: Etwa 35\% der Berliner Studiengänge wurden an privaten Hochschulen angeboten. Dies ist nicht zu verwechseln mit dem Angebot an Studienplätzen, bei dem die staatlichen Hochschulen einen wesentlich höheren Anteil ausmachen sollten.

122 Die unterschiedlichen Kategorisierungen der Studienfächer in den Best Up Daten und im Hochschulkompass lassen diesbezüglich leider keinen Vergleich zu. 
Tab. 6.3: Recherchierte Studiengänge nach Region, Hochschultyp, Trägerschaft, Fächergruppe und Semester

\begin{tabular}{llll}
\hline & WiSe 2013/14 & WiSe 2014/15 & WiSe 2015/16 \\
\hline Total & 391 & 444 & 171 \\
\hline Berlin & $214(55 \%)$ & $236(53 \%)$ & $112(66 \%)$ \\
Außerhalb Berlin & $177(45 \%)$ & $208(47 \%)$ & $59(34 \%)$ \\
\hline Universität & $271(69 \%)$ & $305(69 \%)$ & $124(73 \%)$ \\
Fachhochschule & $112(29 \%)$ & $129(29 \%)$ & $43(25 \%)$ \\
Kunst-/Musikhochschule & $8(2 \%)$ & $10(2 \%)$ & $4(2 \%)$ \\
\hline Staatlich/kirchlich* & $364(93 \%)$ & $415(93 \%)$ & $168(98 \%)$ \\
Privat & $27(7 \%)$ & $29(7 \%)$ & $3(2 \%)$ \\
\hline Geistes-/Sozialwissenschaften/Kunst & $103(26 \%)$ & $126(28 \%)$ & $50(29 \%)$ \\
Sprach-/Literaturwissenschaften & $34(9 \%)$ & $36(8 \%)$ & $9(5 \%)$ \\
Kulturwissenschaften & $28(7 \%)$ & $40(9 \%)$ & $20(12 \%)$ \\
Sozialwissenschaften & $20(5 \%)$ & $26(6 \%)$ & $14(8 \%)$ \\
Kunst & $21(5 \%)$ & $24(5 \%)$ & $7(4 \%)$ \\
\hline Jura/Medizin & $53(14 \%)$ & $53(12 \%)$ & $10(7 \%)$ \\
Rechtswissenschaften & $18(5 \%)$ & $18(4 \%)$ & $6(4 \%)$ \\
Human/Zahnmedizin & $25(6 \%)$ & $25(6 \%)$ & $3(2 \%)$ \\
Gesundheitswissenschaften & $6(2 \%)$ & $6(1 \%)$ & - \\
Veterinärmedizin & $4(1 \%)$ & $4(1 \%)$ & $1(1 \%)$ \\
\hline MINT & $121(31 \%)$ & $132(30 \%)$ & $51(30 \%)$ \\
Naturwissenschaften & $67(17 \%)$ & $72(16 \%)$ & $27(16 \%)$ \\
Ingenieurswissenschaften & $54(14 \%)$ & $60(14 \%)$ & $24(14 \%)$ \\
\hline Wirtschaftswissenschaften & $51(13 \%)$ & $58(13 \%)$ & $21(12 \%)$ \\
\hline Lehramt/Pädagogik & $53(14 \%)$ & $60(13 \%)$ & $31(18 \%)$ \\
Erziehungswissenschaften/Pädagogik & $10(3 \%)$ & $10(2 \%)$ & $7(4 \%)$ \\
Lehramt (Primar-/Sekundarstufe etc.) & $43(11 \%)$ & $50(11 \%)$ & $24(14 \%)$ \\
\hline Sonstige & $10(3 \%)$ & $15(3 \%)$ & $8(5 \%)$ \\
Sport & $4(1 \%)$ & $4(1 \%)$ & - \\
Agrar-/Forst-/Ernährungswissenschaften & $6(2 \%)$ & $11(2 \%)$ & $8(5 \%)$ \\
\hline & & & \\
\hline & & & \\
\hline
\end{tabular}

Quelle: Best Up-Rekrutierungsdaten, eigene Berechnungen

*In allen drei Jahren sind jeweils fünf Studiengänge an Hochschulen in kirchlicher Trägerschaft angesiedelt.

Wie beschrieben, orientierte sich die Auswahl der Studiengänge an den Angaben der Best Up-Befragten und basiert nicht auf einer Stichprobe aller Studiengänge an deutschen Hochschulen. Die recherchierten Studiengänge sind daher nicht für Deutschland oder Berlin repräsentativ. Nichtsdestotrotz ermögli- 
chen es die Rekrutierungsdaten durch die Vielzahl an recherchierten Variablen, Zugangsmodalitäten für die Teilnehmer/innen der Best Up-Studie weitaus differenzierter und detaillierter darzustellen, als dies mit anderen Datenquellen möglich ist.

\subsubsection{Mikrozensus}

Neben den Best Up-Daten dient der Mikrozensus als weitere Quelle zur Generierung der fachspezifischen Einkommens- und Berufsprestigevariablen, die an die Individualdaten gespielt und hier in den verschiedenen Phasen als abhängige Variablen zur Untersuchung horizontaler Ungleichheiten genutzt werden. Der Mikrozensus ist eine seit 1957 jährlich durchgeführte repräsentative Haushaltsbefragung der amtlichen Statistik, bei der 1\% der Bevölkerung in Deutschland unter anderem zu Ausbildung, Beruf und Erwerbstätigkeit befragt wird.

Um das durchschnittlich erzielte Einkommen und Berufsprestige von Absolvent/innen verschiedener Studienfächer zu generieren, wurden sechs Erhebungsjahre (2007 - 2012) gepoolt. ${ }^{123}$ Die genutzten Daten beschränken sich auf erwerbstätige ${ }^{124}$ Mikrozensus-Teilnehmer/innen bis zu einem Alter von 64 Jahren mit (Fach-)Abitur und Hochschulabschluss (Fallzahl insgesamt 228.638, zwischen 33.968 im Jahre 2007 und 42.332 im Jahre 2012).

Zur Generierung der Einkommensvariable wurde zunächst das arithmetische Mittel der berichteten Einkommen für alle Studienfächer gebildet und mithilfe des Verbraucherpreisindexes inflationsbereinigt (Referenzjahr: 2007). ${ }^{125} \mathrm{Da}$ im Mikrozensus das Einkommen kategorial erhoben wird, wurde der mittlere Wert der Kategorien verwendet (z.B. 800 bei der Kategorie „700 bis unter 900 Euro"). Für den oberen Extremwert („18.000 und mehr Euro") wurde auf den SOEP Durchschnittswert für diese Einkommensgruppe zurückgegriffen (21.778 Euro, vgl. Glocker und Storck 2014: 119). Letztlich wurde das Nettomonatseinkommen durch die monatliche Arbeitszeit geteilt, um die finale Variable Nettostundenlohn zu generieren. Um eine möglichst stabile Schätzung zu garantieren, wurden nur Studienfächer mit mindestens $100 \mathrm{Be}-$

123 DOIs: $10.21242 / 12211.2007 .00 .00 .1 .1 .0$ bis $10.21242 / 12211.2012 .00 .00 .1 .1 .0$

124 Die eigene Erwerbstätigkeit muss dabei überwiegend zum Lebensunterhalt beitragen.

125 Bei der Bildung der Einkommensvariable wurde nicht zwischen Männern und Frauen unterschieden. Dieses Vorgehen vernachlässigt zwar Geschlechterungleichheiten beim Einkommen, trägt dafür aber der Geschlechtersegregation Rechnung (also dem variierenden Einkommensniveau von Berufen aufgrund unterschiedlicher Männer- und Frauenanteile, siehe Davies und Guppy 1997). Da der Fokus auf der (finanziellen) Attraktivität eines Faches im Vergleich zu anderen liegt, ist der zuletzt genannte Aspekt zentraler. 
obachtungen berücksichtigt (insgesamt 70). Der so errechnete durchschnittliche Nettostundenlohn liegt zwischen 25,77 Euro für Zahnmedizin und 10,65 Euro für Tourismus (für eine detaillierte Darstellung siehe Anhangstabelle A6.2).

Für die Messung des Berufsprestiges wurde auf die von Wegener (1985) entwickelte Magnitude-Prestigeskala (MPS) zurückgegriffen. ${ }^{126}$ Berufsprestige ist natürlicherweise an die Berufe von Personen gekoppelt. Im vorliegenden Fall interessieren jedoch nicht die einzelnen Berufe, sondern zu welchem Berufsprestige bestimmte Studienfächer im Durchschnitt führen. Daher wurde - wie bereits beim Einkommen - das arithmetische Mittel der Magnitude-Prestigeskala für alle Studienfächer gebildet, unabhängig von dem Beruf der Mikrozensus-Teilnehmer/innen. Auch hier wurden nur Fächer mit mindestens 100 Beobachtungen berücksichtigt. Die Werte der 70 verbleibenden Fächer liegen zwischen 64 (Pflegewissenschaften) und 208 (Zahnmedizin) (siehe Anhangstabelle A6.2).

Das durchschnittliche Einkommen und Prestige, zu dem die 70 Studienfächer führen, hängen zwar zusammen, die Korrelation ist aber mit 0,61 alles andere als perfekt. Zwar führt das Studium der Zahnmedizin sowohl zum höchsten Durchschnittseinkommen als auch -prestige, doch gibt es bei anderen Fächern deutliche Verschiebungen (vgl. Anhangstabelle A6.2).

\subsection{Operationalisierung phasenübergreifender Variablen}

Im Folgenden wird die Operationalisierung der unabhängigen Variablen beschrieben, die in mehreren oder allen Phasen des Übergangsprozesses berücksichtigt werden (für Details zu den genutzten Items und zur Operationalisierung siehe Anhangstabelle A6.3). Die verschiedenen abhängigen Variablen zur Intention, Bewerbung, Zulassung und Studienaufnahme sind per Definition phasenspezifisch. Deren Operationalisierung, hiermit verbundene Besonder-

126 Die MPS beruht auf der Wahrnehmung des gesellschaftlichen Ansehens unterschiedlicher Berufe und damit verbundener sozialer Hierarchien. Ein weit zitierter Vorteil der MPS im Vergleich zu anderen Reputationsskalen (wie etwa der ISEI Skala) ist deren offene Skalierung, die zum einen Deckeneffekte vermeidet und somit auch die Maximaldistanz zwischen den Berufen nicht begrenzt, und zum anderen eine sehr detaillierte Differenzierung der Prestigewerte erlaubt (vgl. Christoph 2005, Frietsch und Wirth 2001, Wegener 1985). Da die MPS auf der ISCO68 Klassifikation beruht, im Mikrozensus Berufe jedoch standardmäßig anhand der KldB kodiert werden, wird auf die von Frietsch und Wirth (2001) entwickelte Routine zur Rekonstruktion der MPS anhand der KldB zurückgegriffen. Die Werte dieser angepassten Skala liegen zwischen 30 (mithelfende Landarbeitskräfte) und 216 (Zahnärzt/innen). 
heiten und Samplerestriktionen werden daher nicht im übergreifenden Methodenteil, sondern in den jeweiligen empirischen Kapiteln vorgestellt.

\section{Soziale Herkunft}

Die zentrale unabhängige Variable ist die soziale Herkunft, konkreter: die Bildungsherkunft der Befragten. Operationalisiert wird diese über den höchsten Bildungsabschluss beider Eltern. Soziale Herkunft ist unbestreitbar ein mehrdimensionales Konstrukt (vgl. Buis 2013, Bukodi und Goldthorpe 2013), das auch durch Klasse, berufliche Stellung und Prestige oder Einkommen geprägt ist. Gerade für Deutschland zeigt sich aber, dass die Bildung der Eltern und die damit verbundene Systemkenntnis, Navigationsfähigkeit und kulturelle Nähe zu höheren Bildungsinstitutionen heute der entscheidendere Prädiktor für Bildungsbeteiligung und -erfolg ist (vgl. Becker 2006: 52, Lörz und Schindler 2011: 467, Schimpl-Neimanns 2000: 646).

Ich nutze die Angaben beider Eltern (joined model) im Gegensatz zu Messungen, die sich nur auf den Vater (conventional model) oder den Elternteil mit dem höchsten Bildungsabschluss (dominance model) beziehen, aus zwei Gründen: Erstens zeigt sich, dass Kinder von Eltern mit unterschiedlichen Merkmalen nicht zwangsläufig stärker von dem Elternteil beeinflusst werden, das den höheren Wert vorweist: ,, children are not unequivocally pulled towards the higher status parent's platform, but range somewhere between them" (Korupp et al. 2002: 37). Zweitens kann durch die Berücksichtigung beider Eltern Bildungserfolg besser vorhergesagt werden, da sich die kulturellen Ressourcen beider Elternteile (wenn auch nicht unbedingt gleichmäßig) kumulieren (vgl. Beller 2009, Buis 2013). Daher operationalisiere ich Bildungsherkunft mithilfe von drei Kategorien: Schüler/innen aus Nichtakademikerfamilien (beide Eltern ohne Hochschulabschluss), aus Einakademikerfamilien (ein Elternteil mit Hochschulabschluss) sowie aus Doppelakademikerfamilien (beide Eltern mit Hochschulabschluss). ${ }^{127}$

\section{Leistungsindikatoren}

Schulleistung ist das zentrale Kriterium für den Zugang zu zulassungsbeschränkten Studiengängen in Deutschland und variiert gleichzeitig mit der sozialen Herkunft. Daher ist es unerlässlich, entsprechende Variablen in die Mo-

127 Fehlende Angaben aus Welle 1 wurden, wenn möglich, auf Grundlage der Folgebefragungen und Elternbefragung ergänzt. Sofern die Angabe nur für einen Elternteil vorlag, wurde diese für die Bildung der Variable verwendet. In den folgenden Tabellen und Abbildungen werden die Abkürzungen Uni0, Uni1, Uni2 genutzt, um die Lesbarkeit zu erhöhen. Dies bezieht sich dennoch nicht allein auf Universitätsabschlüsse, sondern beinhaltet alle Hochschulabschlüsse. 
delle aufzunehmen, um Studienchancen und hierbei auftretende soziale Ungleichheit sauber schätzen zu können. Wie in Abschnitt 5.5 diskutiert, stehen Leistungsunterschiede je nach Phase für primäre Effekte (fremdselektive $\mathrm{Zu}$ lassungsphase) oder sind Teil der wahrgenommeine Erfolgswahrscheinlichkeit (selbstselektive Intentions- und Bewerbungsphase).

Operationalisiert wird Schulleistung erstens mithilfe der erreichten Punktzahl in den Fächern Mathe und Deutsch (0-15). Diese wurden in drei Kategorien unterteilt, die als Dummyvariablen in die Modelle aufgenommen werden: unteres Quartil (schlechte Leistung), zweites und drittes Quartil (mittlere Leistung), oberes Quartil (gute Leistung). Für die Analysen zur ersten Phase werden hierfür die in Welle 2 berichteten Zeugnisnoten des zweiten Kurshalbjahres $(\mathrm{KH})$ genutzt, während in den folgenden Analyseschritten die in Welle 3 berichteten Zeugnisnoten des vierten Kurshalbjahres sowie der Abiturdurchschnitt herangezogen werden.

Für Studienchancen wichtiger noch als die einzelnen Schulnoten ist der Abiturdurchschnitt als gesetzlich festgelegtes primäres Kriterium bei der Studienplatzvergabe. In ihrem Literaturüberblick empfehlen Watermann et al. (2014: 239) daher die Abiturdurchschnittsnote für die Messung primärer Herkunftseffekte, da diese eine hohe Sichtbarkeit und ,praktische Relevanz für den Vergleich von Mitschülern und im Wettbewerb um Studien-, Ausbildungsbzw. Arbeitsplätze" habe (siehe auch Schindler und Reimer 2010: 632). Operationalisiert wird sie hier ebenfalls in Form der drei bereits vorgestellten Kategorien; die Unterteilung erfolgt hierbei allerdings nicht entlang von Punkten, sondern Noten $(1,0-4,0)$. Die obere und untere Kategorie wurde entsprechend umgepolt, sodass das untere Quartil immer für eine eher schlechte Leistung steht.

Zur zusätzlichen Kontrolle werden Testergebnisse zur verbalen und figuralen Kognitionsfähigkeit als metrische Variablen in z-standardisierter Form genutzt. ${ }^{128}$

Rational Choice (RC)-Indikatoren

Wie in Kapitel 5 diskutiert, gehen RC-Ansätze davon aus, dass Schüler/innen (und deren Familien) Kosten, Nutzen und Erfolgswahrscheinlichkeiten verschiedener Bildungsoptionen gegeneinander abwägen und auf dieser Grundlage Bildungsentscheidungen treffen. Mittlerweile liegen zahlreiche empirische Anwendungen dieses Ansatzes vor (vgl. Becker und Hecken 2007, Lörz 2012, Schindler und Reimer 2010), die die RC-Komponenten je nach Verfüg-

$128 \mathrm{Im}$ Anschluss an das Ausfüllen des Fragebogens wurden die Befragten gebeten zwei Testhefte zu bearbeiten - eines zu figuralen und eines zu verbalen fluiden Kognitionsfähigkeiten. Es handelt sich hierbei um den I-S-T 2000-Test (Intelligenzstrukturtest), der auch im SOEP zur Messung kognitiver Kompetenzen von Jugendlichen angewendet wird (vgl. Schupp und Hermann 2009). 
barkeit von Variablen auf unterschiedliche, teils nicht optimale Art messen (vgl. Schindler 2014, Watermann et al. 2014). Auf Grundlage der zur Verfügung stehenden, vor allem vom NEPS entlehnten Variablen (vgl. Stocké et al. 2011) wurde versucht, die RC-Komponenten so direkt wie möglich zu approximieren. Alle genutzten Items wurden in Welle 1 gemessen und jeweils in drei Dummy-Variablen unterteilt (für weitere Details siehe Tabelle A6.3).

- Wahrgenommene Kosten: Wie schwer würde eine Studienfinanzierung fallen?

- Wahrgenommene Erfolgswahrscheinlichkeit: Wie wahrscheinlich ist es, ein Studium erfolgreich abzuschließen?

- Wahrgenommene Erträge: Aussichten auf einen a) gut bezahlten, b) gesellschaftlich angesehenen Job nach Studienabschluss.

Indikatoren für soziale und kulturelle Ressourcen

In Kapitel 5 wurde diskutiert, dass Erwartungen und Aspirationen des sozialen Umfelds sowie hierdurch verfügbare Informations- und Unterstützungsleistungen den Übergang ins Studium erleichtern können. Operationalisiert werden diese theoretischen Konstrukte mithilfe von vier Variablen:

- Wahrgenommene elterliche Aspiration: Welche Art von Ausbildung wünschen sich Eltern für ihre Kinder?

- Studienpläne von Freund/innen: Wie viele Freund/innen planen nach der Schule zu studieren?

- Studienerfahrung in der Verwandtschaft: Wie viele Verwandte studieren/haben studiert?

- Unterstützung bei Studienbewerbung: Wie wahrscheinlich ist eine Bewerbungsunterstützung aus dem sozialen Netzwerk?

Zugangshürden der angestrebten Studiengänge

Weiterhin wurde argumentiert, dass Schüler/innen Zugangshürden antizipieren und möglicherweise einbeziehen, wenn sie ihre Aspiration in Intentionen und spätere Bewerbungen übersetzen. Weitere zentrale unabhängige Variablen beziehen sich daher auf Informationen zur durchschnittlichen Zulassungsbeschränkung und Selektivität der angestrebten Studienfächer (W2). Diese wurden mithilfe der Rekrutierungsvariablen operationalisiert.

Hierfür musste zunächst entschieden werden, für welche Fächer Durchschnittswerte gebildet werden können. Die Studienfächer in den Best Up-Daten wurden in Anlehnung an die Fächersystematik des Statistischen Bundesamtes (2012) als Zwei- und Sechssteller vercodet. Da der Sechssteller sich aufgrund seiner starken Differenziertheit nicht anbietet, um aussagekräftige 
Durchschnittswerte zu bilden, ${ }^{129}$ wurde hierfür zunächst auf den Zweisteller zurückgegriffen. Um weitestgehend aussagekräftige Durchschnittswerte über Studienfächer bilden zu können, wurde eine Mindestzahl von zehn recherchierten Studiengängen pro Fach festgelegt. Auf Grundlage dieser Mindestzahl mussten selbst einige selten vorkommende Zweisteller anderen Kategorien zugeordnet werden. ${ }^{130}$ Häufig vorkommende Sechssteller (Psychologie, BWL) sowie Lehramtsstudiengänge konnten hingegen gesondert ausgewiesen werden. Für die identifizierten Studienfächer (insgesamt 13 im WiSe 2013/14 und 14 im WiSe 2014/15) wurden anschließend auf Grundlage der Rekrutierungsrecherche für die WiSe 2013/14 und 2014/15 jeweils sechs Variablen gebildet, die Zugangshürden (Zulassungsbeschränkung, Eignungsfeststellungsverfahren) sowie die (Leistungs-)Selektivität von Studiengängen approximieren sollen:

\section{Zulassungshürden (pro Fach)}

- Anteil zulassungsbeschränkte Studiengänge in \%

- Anteil Studiengänge mit Eignungsfeststellungsverfahren in \%

- Anteil Studiengänge mit Zulassungsbeschränkung oder Eignungsfeststellung in $\%{ }^{131}$

Selektivität (pro Fach)

- Auswahlgrenze Abiturbestenquote: Durchschnittliche Abiturnote (DN) der in dieser Quote zuletzt zugelassenen Bewerber/innen (zulassungsbeschränkte Studiengänge)

- $\quad$ DN + ,alle zugelassen“ (Übersteigt das Angebot an Studienplätzen die Nachfrage, werden auch in zulassungsbeschränkten Studiengängen alle

129 Viele Sechssteller wurden im Zuge der Rekrutierungsrecherche selten recherchiert, sodass zum Beispiel der „Durchschnitt“ der benötigten Abiturnote sich im Extremfall arbiträr auf einen einzelnen Studiengang bezieht und somit nicht als aussagekräftig angesehen werden kann.

130 Veterinärmedizin etwa wurde Humanmedizin/Gesundheitswissenschaften zugeordnet. Die wenigen sportwissenschaftlichen Studiengänge wurden entweder der Lehramtskategorie, den Ingenieurswissenschaften (Sports Engineering) und die verbleibenden drei dem Fach Kunst zugeordnet, da sich die Zulassungsbeschränkungen hier ähneln. Für das WiSe 2013/14 mussten zudem die Studiengänge der Agrar-, Forst- und Ernährungswissenschaften den Ingenieurs- oder Naturwissenschaften zugeordnet werden. Die Zuordnung erfolgte auf Grundlage zusätzlicher Recherchen, z.B. der Studiengangsordnungen.

131 Die zugrunde liegende Idee hierbei ist, dass nur für zulassungsfreie Studiengänge ohne Eignungsfeststellungsverfahren die Möglichkeit besteht, sich nach Vorlage der Hochschulzugangsberechtigung „einfach einzuschreiben“. 
Bewerber/innen zugelassen. Ist dies der Fall, werden keine Auswahlgrenzen veröffentlicht. Die benötigte Abiturnote wurde in solchen Fällen auf bestanden $(4,0)$ gesetzt.)

- DN + ,alle zugelassen“ + zulassungsfreie Studiengänge (Auch zulassungsfreie Studiengänge erfordern eine Hochschulzugangsberechtigung. Diese erhält man bis zu einer DN von 4,0. In dieser letzten Variable wurde daher die benötigte Abiturnote für alle zulassungsfreien Studiengänge zusätzlich auf 4,0 gesetzt. Diese Variable misst somit Zulassungsbeschränkungen und die Selektivität zulassungsbeschränkter Studiengänge simul$\tan$.)

Für die Untersuchung der ersten Phase (Übersetzung Aspiration in Intention) wird im Folgenden auf die Werte des WiSe 2013/14 zurückgegriffen. Für die Analysen zur Bewerbungsphase wurde nach dem Timing der Bewerbung unterschieden: Für Bewerbungen direkt nach dem Abitur (Sommer 2014) wurden ebenfalls die Werte des WiSe 2013/14 genutzt, für Bewerbungen ein Jahr später hingegen die Werte des WiSe 2014/15.

Tabelle 6.4 gibt einen Überblick über die letztlich verwendeten Studienfächer, die Anzahl der recherchierten Studiengänge sowie die Durchschnittswerte der gebildeten Variablen pro Fach und Semester. Der Anteil zulassungsbeschränkter Studiengänge liegt etwa für das WiSe 2013/14 zwischen 100\% (Erziehungswissenschaften) und 33\% (Kunst). Künstlerische Studiengänge wenden dafür - wie bereits in Abschnitt 2.2.3 angemerkt - am häufigsten Eignungsfeststellungsverfahren an. Tatsächlich „einfach einschreiben“ konnte man sich im WiSe 2013/14 am häufigsten in naturwissenschaftliche Studiengänge (29\%). Entsprechend der oben beschriebenen Logik nimmt die durchschnittliche (Leistungs-)Selektivität der Studienfächer über die Variablen DN (Auswahlgrenze Abiturbestenquote) bis NF (+, ,alle zugelassen“ + zulassungsfreie Studiengänge) ab. Nur im Fall erziehungswissenschaftlicher Studiengänge trifft dies nicht $\mathrm{zu}$, da diese zu 100\% zulassungsbeschränkt sind und offensichtlich nie alle Bewerber/innen zugelassen wurden (Letzteres trifft auf weitere Studienfächer zu, etwa Psychologie oder Sozialwissenschaften). 
Tab. 6.4: Durchschnittliche Zugangshürden pro Studienfächer und Semester

\begin{tabular}{|c|c|c|c|c|c|c|c|}
\hline Studienfach & Anzahl & $\mathrm{Z}(\%)$ & $E(\%)$ & ZE (\%) & DN (Note) & NA (Note) & NF (Note) \\
\hline \multicolumn{8}{|c|}{ WiSe 2013/2014 } \\
\hline Sprach-/Literaturwiss. & 34 & 82 & 47 & 91 & 2,1 & 2,8 & 3,0 \\
\hline Kulturwiss. & 13 & 62 & 31 & 77 & 1,9 & 1,9 & 2,8 \\
\hline Erziehungswiss. & 10 & 100 & 50 & 100 & 2,0 & 2,0 & 2,0 \\
\hline Rechtswiss. & 18 & 89 & 17 & 89 & 1,9 & 2,1 & 2,4 \\
\hline Wirtschaftswiss. & 19 & 68 & 37 & 74 & 2,3 & 2,6 & 3,2 \\
\hline Sozialwiss. & 20 & 95 & 30 & 100 & 2,1 & 2,1 & 2,2 \\
\hline Naturwiss. & 69 & 70 & 9 & 71 & 1,8 & 2,5 & 3,0 \\
\hline Medizin $^{132}$ & 35 & 89 & 9 & 94 & 1,1 & 1,2 & 1,5 \\
\hline Ingenieurwiss. & 59 & 71 & 22 & 78 & 2,2 & 2,5 & 3,2 \\
\hline Kunst & 24 & 33 & 79 & 92 & 2,0 & 2,3 & 3,5 \\
\hline Psychologie & 15 & 87 & 13 & 93 & 1,3 & 1,3 & 1,7 \\
\hline BWL & 32 & 66 & 56 & 91 & 2,0 & 2,2 & 3,2 \\
\hline Lehramt & 43 & 91 & 30 & 100 & 2,0 & 2,6 & 2,7 \\
\hline \multicolumn{8}{|c|}{ WiSe 2014/2015 } \\
\hline Sprach-/Literaturwiss. & 36 & 72 & 44 & 89 & 2,4 & 3,2 & 3,4 \\
\hline Kulturwiss. & 23 & 74 & 26 & 87 & 2,0 & 2,5 & 2,9 \\
\hline Erziehungswiss. & 10 & 100 & 50 & 100 & 2,3 & 2,3 & 2,3 \\
\hline Rechtswiss. & 18 & 89 & 17 & 89 & 1,8 & 2,0 & 2,3 \\
\hline Wirtschaftswiss. & 24 & 58 & 37 & 75 & 2,1 & 2,7 & 3,3 \\
\hline Sozialwiss. & 26 & 92 & 23 & 96 & 2,1 & 2,3 & 2,4 \\
\hline Naturwiss. & 72 & 67 & 7 & 68 & 1,8 & 2,5 & 3,1 \\
\hline Medizin & 35 & 89 & 9 & 94 & 1,1 & 1,1 & 1,4 \\
\hline Ingenieurwiss. & 61 & 66 & 25 & 75 & 2,3 & 2,9 & 3,4 \\
\hline Kunst & 27 & 30 & 81 & 93 & 2,2 & 3,0 & 3,7 \\
\hline Psychologie & 17 & 88 & 18 & 94 & 1,3 & 1,3 & 1,8 \\
\hline BWL & 34 & 65 & 53 & 88 & 1,9 & 2,1 & 3,1 \\
\hline Lehramt & 50 & 80 & 28 & 94 & 2,2 & 2,8 & 3,0 \\
\hline $\begin{array}{l}\text { Agrar-/Forst-/ } \\
\text { Ernährungswiss. }\end{array}$ & 11 & 82 & 27 & 91 & 2,3 & 2,5 & 2,8 \\
\hline
\end{tabular}

Quelle: Best Up-Rekrutierungsdaten, eigene Berechnungen

Anzahl: Anzahl recherchierter Studiengänge pro Fach und Semester; Z: Anteil zulassungsbeschränkte Studiengänge (\%); E: Anteil Eignungsfeststellungsverfahren (\%); ZE: Anteil Zulassungsbeschränkung oder Eignungsfeststellungsverfahren (\%); DN: durchschnittliche Auswahlgrenze Abiturbestenquote (Durchschnittnote); NA: durchschnittliche Auswahlgrenze + alle zugelassen; NF: durchschnittliche Auswahlgrenze + alle zugelassen + zulassungsfreie Studiengänge

Einschränkend muss hier festgehalten werden, dass mit diesem Vorgehen die tatsächlichen Zugangshürden nur approximiert werden können. Hierfür gibt es

132 Die Kategorie „Medizin“ bezieht sich hier nicht ausschließlich auf die bundesweit zulassungsbeschränkten Studiengänge, sondern auch auf gesundheitswissenschaftliche Programme. Dies ist der Grund dafür, dass der Anteil an Zulassungsbeschränkungen hier nicht bei 100\% liegt. 
mindestens drei Gründe: Erstens mussten die Studienfächer, für die Durchschnittswerte gebildet wurden, zum Teil relativ stark aggregiert werden, sodass eine mögliche Varianz innerhalb dieser aggregierten Fächergruppen verborgen bleibt. Zweitens wird mit der Abiturnote, die für die Zulassung innerhalb der Abiturbestenquote benötigt wird, die durchschnittliche Selektivität insgesamt überschätzt, da über die anderen Quoten in der Regel auch leistungsschwächere Bewerber/innen zugelassen werden. Da jedoch in den seltensten Fällen Informationen über die Auswahlgrenzen für alle bereitgestellten Studienplätze zur Verfügung stehen, ist es eben diese Angabe, an der sich potentielle Bewerber/innen orientieren können. Drittens beruht die Rekrutierungsrecherche, wie in Abschnitt 6.2.2 dargelegt, nicht auf einer Vollerhebung oder repräsentativen Stichprobe aller Studiengänge an deutschen Hochschulen. Daher ist es möglich (jedoch aufgrund der Datenlage schwer überprüfbar), dass die gebildeten Variablen nicht die tatsächlichen Durchschnittswerte pro Fach widerspiegeln. ${ }^{133}$ Jedoch kann generell nicht klar gesagt werden, welche Auswahl an Studiengängen hierfür angemessen wäre, um fachspezifische Zugangshürden abzubilden. Beziehen Studienberechtigte tatsächlich alle theoretisch verfügbaren Studienoptionen ein oder beschränken sich viele auf einen (regional) eingeschränkteren Optionsradius? Ist Letzteres der Fall (vgl. Abschnitt 2.3), orientieren sich die betrachteten Berliner Studienberechtigten wahrscheinlich stärker an Zugangshürden zum regionalen Studienangebot, das durch die Rekrutierungsdaten vollständiger (wenn auch nicht perfekt) repräsentiert wird. ${ }^{134}$

Trotz der genannten mehr oder weniger gravierenden Einschränkungen stellen die mithilfe der Best Up-Rekrutierungsdaten gebildeten Variablen die für den deutschen Kontext einzige Möglichkeit dar, fachspezifische Zugangshürden (über die Angabe zu Zulassungsbeschränkungen im Hochschulkompass hinaus) zu erfassen.

133 Ein Vergleich der in Kapitel 2 präsentierten Daten des Hochschulkompass mit den Rekrutierungsdaten legt nahe, dass zulassungsbeschränkte Studiengänge in Letzteren überrepräsentiert sind. Dies ist schon deswegen plausibel, weil neben den tatsächlich erfolgten Immatrikulationen ausschließlich von den Befragten hoch gerankte und somit wahrscheinlich überdurchschnittlich stark nachgefragte Studiengänge recherchiert wurden. Dieser generelle Niveauunterschied wäre jedoch vor allem dann problematisch, wenn auch die Relationen zwischen den Fächern deutlich verzerrt wären. Leider lässt sich dies aufgrund der unterschiedlichen Fächerkodierungen kaum nachprüfen.

134 Ähnlich argumentieren Niu und Tienda (2008) in ihrer Studie zu Bewerbungsentscheidungen US-amerikanischer high school-Absolvent/innen: "Theoretically, students choice set can potentially include any postsecondary institution in the country, but in reality students cannot and do not consider all of the possible alternatives" (ebd.: 417). Um sich den choice sets verschiedener Gruppen empirisch anzunähern, greifen auch sie auf die von den Befragten berichteten Hochschulen zurück. 
Kontrollvariablen

Zusätzlich zu den zentralen erklärenden Variablen wird in den multivariaten Modellen für weitere vier Variablen - Geschlecht, Migrationshintergrund (mindestens ein Elternteil außerhalb Deutschlands geboren), Schultyp (Gymnasium, ISS, OSZ) und Abiturart (Abitur oder Fachabitur, ab Phase 2) - kontrolliert, die zwar nicht inhaltlicher Bestandteil der vorliegenden Untersuchung sind, von denen aber angenommen werden kann, dass sie mit den abhängigen Variablen und teilweise auch mit der Bildungsherkunft der Befragten zusammenhängen.

Zwar studieren mittlerweile etwa genauso viele Frauen wie Männer, doch erlangen Erstere häufiger die Hochschulzugangsberechtigung. (Fach-)Abiturientinnen studieren demnach deutlich seltener als (Fach-)Abiturienten: Für den Abiturjahrgang 2013 betrug etwa der Unterschied zwei Jahre nach dem Abitur circa 9 Prozentpunkte (Autorengruppe Bildungsberichterstattung 2016: F2$7 \mathrm{web}$ ). Zahlreiche Studien demonstrieren außerdem deutliche Geschlechterunterschiede bezüglich der Fachwahl (vgl. Bradley 2000, Lörz et al. 2011b).

Die Eltern von Abiturient/innen mit Migrationshintergrund haben häufig keine Hochschulerfahrung (vgl. Kristen et al. 2008). Gleichzeitig streben ihre Kinder überdurchschnittlich häufig ein Studium an (vgl. Kao und Tienda 1998, Lörz et al. 2011a, siehe auch Abschnitt 6.4) und nehmen dies - zumindest im Kontext des deutschen, stratifizierten Schulsystems - auch überdurchschnittlich häufig auf. Ein Grund hierfür wird in der mangelnden Kenntnis des deutschen Ausbildungssystems gesehen (vgl. Kristen et al. 2008).

Letztlich können der Schultyp und die Art des Abiturs Übergangschancen strukturieren, indem sie spezielle fachliche Orientierung und Lernumwelten bieten oder vorhandene Studienoptionen einschränken (vgl. Abschnitt 2.1). Gleichzeitig sind Schüler/innen aus sozial schwächeren Familien sowohl an traditionellen Gymnasien als auch in der Gruppe der Abiturient/innen (allgemeine Hochschulzugangsberechtigung) unterrepräsentiert (vgl. Maaz 2006, Schindler 2012, Watermann und Maaz 2006).

\subsection{Definition des Ausgangssamples}

Das den folgenden Analysen zugrundeliegende Ausgangssample muss sowohl aus methodischen als auch inhaltlichen Gründen eingeschränkt werden. Wie in Abschnitt 6.2 dargelegt, fanden im Rahmen der Best Up-Studie zwei Interventionen statt. Wie erste Veröffentlichungen hierzu zeigen, beeinflusste insbesondere der Informationsworkshop sowohl die Studienintention als auch spätere Entscheidungen der Schüler/innen (bzw. bestimmter Schüler/innengruppen) (vgl. Ehlert et al. 2017a, Finger et al. 2020, Peter et al. 2016a, Peter und 
Zambre 2017). Da die Wirkung der Informationsintervention nicht Teil der vorliegenden Arbeit ist, werden die betroffenen acht Schulen aus den folgenden Analysen ausgeschlossen, um durch die (erfolgreiche) Intervention hervorgerufene Verzerrungen der Ergebnisse zu vermeiden. Im Vergleich hierzu konnte für die finanzielle Intervention kein Einfluss nachgewiesen werden (vgl. Peter et al. 2016b), sodass diesbezüglich kein Ausschluss stattfindet. ${ }^{135}$ Vom ursprünglichen Sample (1578) verbleiben nach dem Ausschluss von Informationsinterventionsschulen 1086 Befragte und 19 Schulen im Sample. Um ein möglichst einheitliches Ausgangssample für die verschiedenen analytischen Schritte zu erzielen, wurden außerdem nur Teilnehmer/innen der zweiten Welle berücksichtigt (762).

Eine weitere Einschränkung ist inhaltlicher Natur: Um die Fragestellung nach sozialer Ungleichheit bei der Umsetzungswahrscheinlichkeit von Studienaspirationen zu beantworten, werden im Folgenden nur Befragte mit Studienaspiration berücksichtigt (für ein ähnliches Vorgehen vgl. Hanson 1994, Roderick et al. 2011). ${ }^{136}$ Da die Herausbildung von Aspirationen von verschiedenen, insbesondere sozialen und leistungsbezogenen Faktoren (vgl. Abschnitt 5.1) beeinflusst wird, ist das verbleibende Sample (positiv) selektiv. Tabelle 6.5 gibt einen Überblick über die Unterschiede zwischen Schüler/innen mit und ohne Studienaspiration in Welle 2 sowie denjenigen mit fehlender Angabe bei dieser Variable. Da die Frage keine „weiß nicht“ Kategorie beinhaltete,

135 Nach der ersten Online-Befragung (W2) wurden diejenigen Schüler/innen in den neun vorgesehenen Schulen getreatet, die in Welle 2 eine Ausbildungsintention äußerten. Dadurch sind zum einen die Analysen der ersten Phase nicht von der finanziellen Intervention betroffen, da diese auf pre-treatment Messungen beruhen. Zum anderen werden im nächsten Schritt Schüler/innen ohne Studienaspiration in Welle 2 ausgeschlossen und somit auch fast alle getreateten Schüler/innen. Es verbleiben nur diejenigen im Ausgangssample, die zwar eine Studienaspiration, nicht aber eine Studienintention äußern (N: 28). Eine einfache Kontrolle des Treatmentstatus bietet sich nicht an, da dieser mit der Studienintention konfundiert ist. Auch ein Ausschluss der getreateten Schüler/innen ist kaum empfehlenswert, da so ein Teil einer sehr spezifischen Gruppe (Schüler/innen mit Studienaspiration, aber ohne Intention in Interventions-, nicht aber Kontrollschulen) ausgeschlossen würde. Da die Intervention keinerlei Effekte zeigte, sollte die Berücksichtigung der wenigen verbleibenden getreateten Schüler/innen unproblematisch sein.

136 Hierfür wurde auf eine NEPS Frage zurückgegriffen (A49_T_Panel_2012CNEPS, siehe auch Stocké et al. 2011): ,Wenn es allein nach Ihren Wünschen ginge: Welche Art von Ausbildung würden Sie nach Beendigung der Schule am liebsten machen? (Wenn Sie vorhaben, nach der Schule zunächst ein freiwilliges soziales Jahr, ein Praktikum o.Ä. zu machen, geben Sie die Art von Ausbildung an, die Sie danach am liebsten machen würden.) 15 Kategorien standen als Antwortmöglichkeiten zur Auswahl, die grob zwischen Studium, Ausbildung, keine Ausbildung unterschieden. Studium und Ausbildung unterteilten sich weiter in verschiedene Hochschul- und Ausbildungsarten. 
kann bei Letzteren nicht eindeutig festgestellt werden, ob es sich hierbei um eine Antwortverweigerung oder um Unentschlossenheit handelt. Insgesamt äußern etwa $80 \%$ der Befragten eine Studienaspiration. Wenig überraschend sind dabei vor allem Schüler/innen aus Doppelakademikerhaushalten und solche mit überdurchschnittlich guten Deutsch- und Matheleistungen im Analysesample überrepräsentiert. Befragte mit Studienaspiration besuchen zudem etwas häufiger Gymnasien als Integrierte Sekundarschulen oder berufliche Gymnasien. In Übereinstimmung mit bisheriger Forschung sind Schüler/innen mit Migrationshintergrund in der Gruppe der Studienaspirant/innen überrepräsentiert (vgl. DeBord et al. 1977, Hauser und Anderson 1991, Kao und Tienda 1998, Portes und Wilson 1976, für Deutschland: Relikowski et al. 2012, Salikutluk 2016, Trebbels 2015). Bezüglich des Geschlechts besteht kein Unterschied. Jedoch treten die theoretisch erwartbaren Zusammenhänge zwischen ausgewählten RC-/Ressourcenvariablen und der Studienaspiration der Befragten deutlich zu Tage (vgl. insbesondere Abschnitt 5.1).

Tab. 6.5: Vergleich der Befragten mit Studienaspiration (im Analysesample) und der Befragten ohne Studienaspiration (nicht im Analysesample), Welle 2

\begin{tabular}{|c|c|c|c|c|}
\hline & & $\begin{array}{l}\text { Studienaspiration: } \\
\text { k.A. }\end{array}$ & $\begin{array}{l}\text { Studienaspiration: } \\
\text { Nein }\end{array}$ & $\begin{array}{l}\text { Studienaspiration: } \\
\text { Ja }\end{array}$ \\
\hline & Total N & & Zeilenprozente & \\
\hline Total & 762 & 6,6 & 13,9 & 79,5 \\
\hline Soziale Herkunft & 752 & & & \\
\hline Uni0 & 471 & 5,7 & 16,6 & 77,7 \\
\hline Uni1 & 162 & 9,3 & 11,7 & 79 \\
\hline Uni2 & 119 & 5,9 & 5,9 & 88,2 \\
\hline Migrationshintergrund & 753 & & & \\
\hline $\mathrm{Ja}$ & 345 & 7,3 & 10,7 & 82 \\
\hline Nein & 408 & 5,9 & 16,7 & 77,5 \\
\hline Geschlecht & 761 & & & \\
\hline Weiblich & 442 & 6,1 & 14,3 & 79,6 \\
\hline Männlich & 319 & 7,2 & 13,2 & 79,6 \\
\hline Schultyp & 762 & & & \\
\hline Gymnasium & 242 & 3,7 & 13,2 & 83,1 \\
\hline ISS & 276 & 8,7 & 13,8 & 77,5 \\
\hline OSZ & 244 & 7 & 14,8 & 78,3 \\
\hline Note Deutsch (2. KH) & 750 & & & \\
\hline Oberes Quartil (+) & 192 & 4,2 & 4,2 & 91,7 \\
\hline Mitte & 344 & 7,3 & 16,6 & 76,2 \\
\hline Unteres Quartil (-) & 214 & 7,5 & 18,7 & 73,8 \\
\hline Note Mathe (2. KH) & 751 & & & \\
\hline Oberes Quartil (+) & 253 & 4,4 & 8,7 & 87 \\
\hline Mitte & 264 & 6,8 & 15,2 & 78 \\
\hline Unteres Quartil (-) & 234 & 8,6 & 18 & 73,5 \\
\hline
\end{tabular}




\begin{tabular}{|c|c|c|c|c|}
\hline & & $\begin{array}{l}\text { Studienaspiration: } \\
\text { k.A. }\end{array}$ & $\begin{array}{l}\text { Studienaspiration: } \\
\text { Nein }\end{array}$ & $\begin{array}{l}\text { Studienaspiration: } \\
\text { Ja }\end{array}$ \\
\hline & Total N & & Zeilenprozente & \\
\hline $\begin{array}{l}\text { Erfolgswahrschein- } \\
\text { lichkeit Studium }\end{array}$ & 756 & & & \\
\hline $\begin{array}{l}\text { (Sehr) unwahr- } \\
\text { scheinlich }\end{array}$ & 298 & 8,7 & 25,5 & 65,8 \\
\hline Wahrscheinlich & 336 & 4,8 & 6,3 & 89 \\
\hline Sehr wahrscheinlich & 122 & 4,9 & 5,7 & 89,3 \\
\hline $\begin{array}{l}\text { Schwierigkeit } \\
\text { Studienfinanzierung }\end{array}$ & 750 & & & \\
\hline (Sehr) schwer & 294 & 5,8 & 18,7 & 75,5 \\
\hline Weder noch & 244 & 7 & 12,3 & 80,7 \\
\hline (Sehr) leicht & 212 & 7,6 & 9 & 83,5 \\
\hline $\begin{array}{l}\text { Studienaspiration } \\
\text { Eltern }\end{array}$ & 740 & & & \\
\hline $\mathrm{Ja}$ & 568 & 4,2 & 8,6 & 87,2 \\
\hline Nein & 172 & 14 & 30,8 & 55,2 \\
\hline $\begin{array}{l}\text { Freunde mit Studien- } \\
\text { aspiration }\end{array}$ & 756 & & & \\
\hline Bis zu $50 \%$ & 358 & 8,7 & 19,3 & 72,1 \\
\hline Mehr als die Hälfte & 398 & 4,8 & 9,3 & 85,9 \\
\hline $\begin{array}{l}\text { Unterstützung Studien- } \\
\text { bewerbung }\end{array}$ & 745 & & & \\
\hline $\begin{array}{l}\text { Sehr/eher unwahr- } \\
\text { scheinlich }\end{array}$ & 100 & 7 & 24 & 69 \\
\hline Eher wahrscheinlich & 205 & 9,8 & 12,7 & 77,6 \\
\hline Sehr wahrscheinlich & 440 & 4,3 & 12,3 & 83,4 \\
\hline
\end{tabular}

Quelle: Best Up-Individualdaten, eigene Berechnungen

Einige (Kontroll-)Variablen sind wichtiger Bestandteil der Modellschätzungen aller nachfolgenden Analyseschritte, sodass zudem Befragte mit fehlenden Werten auf diesen Variablen aus dem Ausgangssample ausgeschlossen werden. Hierbei handelt es sich zunächst um 13 Befragte (2\%) mit fehlenden Werten auf den zeitkonstanten soziodemographischen Variablen Bildungshintergrund, Migrationshintergrund und Geschlecht. Das grundlegende Ausgangssample umfasst somit 593 Befragte. ${ }^{137}$ Für die einzelnen Analyseschritte sind zudem weitere Einschränkungen notwendig, deren Ursachen und Auswirkungen in den empirischen Kapiteln eingehender beschrieben werden.

137 Zudem sind Leistungsindikatoren zentraler Bestandteil der Modellschätzungen. Hierbei handelt es sich zum Teil um phasenspezifische Variablen (vgl. vorheriger Abschnitt). Da diese damit nicht identisch sind, erfolgt dieser Ausschluss nicht als Teil der Definition des grundlegenden Ausgangssamples, sondern in den jeweiligen empirischen Kapiteln. 


\subsection{Methodisches Vorgehen}

\subsubsection{Hierarchische Datenstruktur}

Wie in Abschnitt 6.2.1 beschrieben, beruhen die Best Up-Daten nicht auf einem zufälligen Individualsample, sondern auf einem stratifizierten Sample von insgesamt 27 Schulen, von denen 19 in die folgenden Analysen eingehen. Damit sind die Daten hierarchisch strukturiert mit Individuen auf Ebene 1 und Schulen auf Ebene 2. Aufgrund möglicher Selektionsprozesse und ähnlicher schulischer Erfahrungen und Einflüsse, etwa durch Lehrer/innen, Peers, Schulausstattung oder Informationsangebote, ist davon auszugehen, dass sich Schüler/innen innerhalb einer Schule ähneln.

Damit ist eine zentrale Annahme klassischer Regressionsmodelle - die Unabhängigkeit von Beobachtungen - verletzt. Dies hat zur Folge, dass Standardfehler verzerrt (in der Regel zu klein) sind, sodass die Wahrscheinlichkeit eines Typ-I-Fehlers unterschätzt wird (vgl. Hox 2010: 4-5, Rabe-Hesketh und Skrondal 2008: 129). Wie stark Beobachtungen voneinander abhängen, kann mithilfe der Intraklassenkorrelation spezifiziert werden, die angibt, welcher Anteil der Varianz der abhängigen Variable durch die höhere Ebene - hier also durch Unterschiede zwischen den Schulen - zustande kommt. Für das Problem abhängiger Beobachtungen haben sich verschiedene Korrekturmöglichkeiten etabliert, zum Beispiel die Berechnung clusterrobuster Standardfehler oder die Spezifizierung von Mehrebenenmodellen.

In der vorliegenden Arbeit werden Modelle genutzt, die zur Familie der Generalized Linear Mixed Models (GLMM) gehören. Diese bestehen aus einem festen (fixed) und einem zufälligen (random) Teil und beruhen auf dem Maximum Likelihood (ML) Schätzverfahren. Je nach Metrik der abhängigen Variable kommen dabei unterschiedliche Linkfunktionen (hier: identity und logit, siehe nächster Abschnitt) zum Einsatz. Die abhängige Variable wird dabei immer auf dem untersten Level gemessen (hier: den Schüler/innen oder den Vignetten, vgl. Abschnitt 7.1.3). GLMM können als random intercept oder random slope/coefficients Modelle spezifiziert werden. Den folgenden Analysen liegen random intercept Modelle zugrunde, in denen die Gesamtvarianz der Konstanten in ebenenspezifische Varianzkomponenten zerlegt wird (vgl. Hox 2010: 57). Dadurch werden Niveauunterschiede in der abhängigen Variable zwischen den Level 2 Beobachtungen (Schulen) modelliert und so die Annahme unabhängiger Beobachtungen gelockert.

Formel (1) veranschaulicht das grundsätzlich zur Anwendung kommende GLMM exemplarisch am Beispiel einer kontinuierlichen abhängigen Variable (vgl. Auspurg und Hinz 2015: 88f., Rabe-Hesketh und Skrondal 2008: 94). Das Outcome $Y_{i j}$ für Schüler/in i in Schule $\mathrm{j}$ wird dabei wie folgt spezifiziert: 


$$
\mathrm{Y}_{i j}=\beta_{0}+\beta_{1} \mathrm{X}_{i j 1}+\ldots+\beta_{p} \mathrm{X}_{i j p}+\gamma_{1} \mathrm{Z}_{j 1}+\ldots+\gamma_{q} \mathrm{Z}_{j q}+\mu_{j}+\varepsilon_{i j}
$$

$\beta_{1}$ bis $\beta_{p}$ stellen die geschätzten Koeffizienten der Level 1 Variablen $X_{1}-X_{p}$ und $\varepsilon_{i j}$ den Level 1 (schüler/innenspezifischen) Fehlerterm dar. Entsprechend der Mehrebenenstruktur der Daten kommen mit $\gamma_{1}$ bis $\gamma_{\mathrm{q}}$ die geschätzten Koeffizienten der Level 2 Variablen $Z_{1}$ bis $Z_{q}$ hinzu. $\beta_{0}$ kann zusammen mit dem zweiten Fehlerterm $\mu_{j}$ als schulspezifische Konstante oder auch random intercept verstanden werden (vgl. Auspurg und Hinz 2015: 89). Die Koeffizienten $\beta_{0}$ bis $\gamma_{\mathrm{q}}$ konstituieren den festen Teil des Modells, die Fehlerterme $\mu_{j}$ und $\varepsilon_{i j}$ (und damit zusammenhängend die Varianzkomponenten) den zufälligen.

Eine hierarchische Datenstruktur führt häufig dazu, dass die Anzahl der Cluster, der Beobachtungen innerhalb der Cluster oder beides relativ klein sind. International vergleichende Studien mit Ländern als Clustervariable haben zwar in der Regel viele Level 1 Beobachtungen, aber nur eine begrenzte Anzahl an Clustern (vgl. Bryan und Jenkins 2016, Elff et al. 2021). Stellen Schulen oder Klassen die höhere und Schüler/innen die untere Ebene dar, wächst die Wahrscheinlichkeit, dass die Fallzahl auf beiden Ebenen relativ klein ist. Dies kann Fehlspezifikationen von Modellparametern zur Folge haben. In diesem Kontext wird vermehrt die Frage diskutiert, wie viele Level 2 und Level 1 Beobachtungen ausreichen, um sowohl Punktschätzer als auch Standardfehler und somit Signifikanzniveaus verlässlich zu schätzen und welche Methoden auch für kleine Fallzahlen, insbesondere der Cluster, hierfür angemessen sind. Die Antwort auf diese Frage fällt sehr uneinheitlich aus. Daumenregeln oder auf Simulationen beruhende Empfehlungen beinhalten zum Beispiel eine Mindestzahl von 30 Clustern und 30 Beobachtungen pro Cluster (vgl. Hox 2010: 235). Ebenso lassen sich Empfehlungen von 50:50 finden (vgl. Moineddin et al. 2007). Die dieser Arbeit zugrundeliegenden Daten verfehlen mit 19 Schulen und durchschnittlich 31 Schüler/innen pro Schule (im größtmöglichen Ausgangssample) diese Richtwerte. Glücklicherweise trifft das Problem nicht auf alle Modellbestandteile in gleichem Maße zu, sodass dessen Schwere insbesondere vom Forschungsinteresse abhängt. Verschiedene auf Monte Carlo Simulationen basierende Studien, in denen die Anzahl der Cluster (vgl. Bryan und Jenkins 2016, Stegmueller 2013) und zusätzlich die Anzahl der Fälle innerhalb der Cluster (vgl. Austin 2010, Maas und Hox 2005, Moineddin et al. 2007) variiert werden, zeigen, dass vor allem die Schätzung von Level 2 Parametern sowie der Varianzkomponenten bei kleinen Fallzahlen nicht verlässlich sind. Für die Koeffizienten der unteren Ebene und deren Standardfehler sind sich die verschiedenen Autor/innen einig, dass diese mithilfe von GLMM auch bei kleinen Fallzahlen (auf beiden Ebenen) ausreichend präzise geschätzt werden können. Dies trifft sowohl auf kontinuierliche als auch dichotome abhängige Variablen zu. Austin (2010) etwa überprüft mit verschiedenen Statistikprogrammen logistische Mixed Modelle mit sehr kleinen Fallzahlen auf beiden Ebenen (Level 2: 5 (5) 20; Level 1: 5 (5) 50). Es zeigt sich, dass mit Stata die Level 1 Koeffizienten sowie deren Standardfehler bereits ab 
Fallzahlen von 10:10 verlässlich geschätzt werden können. Zudem zeigt Austin, dass größere Fallzahlen vor allem dann notwendig sind, wenn das durch die abhängige Variable gemessene Ereignis selten auftritt (low-prevalent outcomes). Wie sich im empirischen Teil zeigen wird, treten die untersuchten Ereignisse in der vorliegenden Studie mit großer Häufigkeit auf.

Insgesamt erscheinen für die Fragestellung der vorliegenden Arbeit GLMM trotz gegebener Fallzahl angemessen, da der Fokus klar auf den fixen Level 1 Parametern liegt.

\subsubsection{Modelle für lineare und binäre abhängige Variablen}

In den folgenden Analysen werden sowohl kontinuierlich als auch binär skalierte abhängige Variablen getestet. GLMM können als Erweiterung oder Verallgemeinerung linearer Mehrebenenmodelle verstanden werden, auf deren Grundlage - je nach verwendeter Link-Funktion - auch nicht-lineare Modelle geschätzt werden können. Grundlegend gehen diese von einer latenten (also unbeobachteten), linearen Variable $y^{*}$ aus, deren Erwartungswert über eine spezifische Transformation (die Link-Funktion) mit den beobachteten Werten der abhängigen Variable verbunden wird (vgl. Hardin und Hilbe 2003: 8f., Long und Freese 2014: 188ff.). Im Fall einer kontinuierlichen abhängigen Variable wird in der Regel die Identität als Link-Funktion herangezogen, d.h. auf eine Transformation der Erwartungswerte verzichtet. Diesem Vorgehen folge ich in den Analysen zum Einkommen und Prestige von Studienfächern sowie der Vignettenstudie. Dabei kommt nicht das oft verwendete Full Maximum Likelihood (FML), sondern das Restricted Maximum Likelihood (RML) Verfahren zum Einsatz, da dieses besonders bei geringer Clusteranzahl weniger verzerrte Varianzkomponenten schätzt (vgl. Elff et al. 2021, Hox 2010: 41). ${ }^{138}$

Die meisten abhängigen Variablen, die in dieser Arbeit analysiert werden sollen, sind jedoch binär kodiert (wie etwa die Entscheidung für oder gegen eine Studienbewerbung), sodass die Verwendung einer logistischen LinkFunktion (Logit-Link) zum Einsatz kommt. Hierfür müssen Annahmen getroffen werden, die zur Folge haben, dass zwar die Signifikanz und Richtung der Koeffizienten, nicht aber ihre Stärke über hierarchisch geschachtelte oder

138 Beim FML werden sowohl die Varianzkomponenten als auch die Regressionskoeffizienten in die Wahrscheinlichkeitsfunktion eingeschlossen; beim RML hingegen zunächst nur die Varianzkomponenten (die Regressionskoeffizienten werden in einem separaten Schritt geschätzt). Das Problem am FML Verfahren ist, dass die Regressionskoeffizienten als fixe Bestandteile im Modell belassen werden, wenn die Varianzkomponenten geschätzt werden, ohne jedoch die für die Berechnung der Regressionskoeffizienten benötigten Freiheitsgrade einzubeziehen (für eine detaillierte Beschreibung siehe Hox 2010: 41). 
gruppenspezifische Modelle verglichen werden können. ${ }^{139}$ Dieses sogenannte Skalierungsproblem existiert sowohl für logistische Ein- als auch Mehrebenenmodelle (vgl. Hox 2010: 134) und soll im Folgenden kurz beschrieben werden.

Für die Schätzung von GLMM mit einer beobachteten binären abhängigen Variable y wird zunächst davon ausgegangen, dass ab einem bestimmten Schwellenwert der latenten linearen Variable $y^{*}$, der meist arbiträr auf 0 festgesetzt wird, die beobachtete Variable y den Wert 1 annimmt. Um die Wahrscheinlichkeit des Auftretens von $\mathrm{y}=1$ berechnen zu können, muss die Varianz des Fehlerterms, oder auch die unbeobachtete Heterogenität, bekannt sein. Da jedoch auch diese für $\mathrm{y}^{*}$ unbeobachtet ist, wird sie als logistisch definiert und auf den Wert $\pi^{2} / 3$ fixiert. In linearen Modellen ergibt sich die konstante Gesamtvarianz von y aus der Summe der Fehlervarianz und der durch die unabhängigen Variablen erklärten Varianz: Steigt Letztere durch das Hinzufügen weiterer Variablen an, sinkt Erstere. Durch die Fixierung der Fehlervarianz in logistischen Regressionsmodellen steigt bei steigender erklärter Varianz auch die Gesamtvarianz von y*. Dies hat zur Folge, dass sich die Koeffizienten verschachtelter Modelle auf unterschiedlich skalierte abhängige Variablen beziehen. Daher können sie ,in ihrer Größe nicht sinnvoll verglichen werden, da auch sie notwendigerweise unterschiedlich skaliert sind" (Best und Wolf 2012: 383). Anders ausgedrückt: Ob unterschiedliche (oder identische) Effektstärken aufgrund von Interkorrelation unabhängiger Variablen oder aufgrund der Anpassung des Skalierungsparameters zustande kommen, kann nicht festgestellt werden.

Das Vorhandensein dieses Problems wird zunehmend auch in der soziologischen Forschung erkannt und Lösungsvorschläge diskutiert (vgl. Auspurg und Hinz 201 1b, Best und Wolf 2012, Mood 2010). Theoretische Herleitungen sowie Simulationsstudien verweisen darauf, dass sich marginale Effekte ( $m a r$ ginal effects) für kontinuierliche Prädiktoren bzw. diskrete Veränderungen (discrete changes) für kategoriale Prädiktoren sowohl für den Vergleich von Effektstärken zwischen geschachtelten Modellen (vgl. Best und Wolf 2012, Mood 2010) als auch zwischen unterschiedlichen Gruppen (vgl. Auspurg und Hinz 2011b, Mood 2010) eignen, da diese ,nur zu einem vernachlässigbar geringen Ausmaß durch unbeobachtete Heterogenität verzerrt sind" (Auspurg und Hinz 2011b: 67). Zusätzlich ist ihre Interpretation als Prozentpunkteveränderung bzw. -unterschiede im Gegensatz zu logistischen Regressionskoeffizienten und auch Odds Ratios intuitiv erfassbar. Die nachfolgenden, auf logis-

139 Hierarchisch strukturiert oder verschachtelt sind Modelle dann, wenn zu den in einem Ausgangsmodell enthaltenen erklärenden Variablen sukzessive weitere Variablen hinzugefügt werden, um zum Beispiel Mediationseffekte zu testen. Gruppenspezifisch sind Modelle dann, wenn die gleichen Zusammenhänge für verschiedene Samples (z.B. getrennt für Akademikerkinder und Nichtakademikerkinder) getestet werden. 
tischen Regressionen beruhenden Punktschätzer werden daher - je nach Metrik der unabhängigen Variablen - als durchschnittliche marginale Effekte (average marginal effects, $A M E$ ) oder durchschnittliche diskrete Veränderungen ((average) discrete changes, $D C$ ) ausgegeben.

Gegeben die beobachteten Ausprägungen aller anderen Prädiktoren gibt der AME den über alle Beobachtungen gemittelten Effekt einer marginalen „,instanteneous or infinitly small“ (Long und Freese 2014: 239) - Veränderung eines Prädiktors x auf die Wahrscheinlichkeit des Auftretens von y=1 an. Damit beziehen sie sich auf die (durchschnittliche) Steigung der Regressionslinie an einem bestimmten Punkt (vgl. Hilbe 2009: 605, Kohler und Kreuter 2012: 344). Ist der Prädiktor kategorial, sollte hingegen nicht der AME, sondern der durchschnittliche DC angegeben werden (vgl. Hilbe 2009: 609). ${ }^{140}$ Erneut gegeben die beobachteten Ausprägungen aller anderen Prädiktoren, gibt dieser die über alle Fälle gemittelte Höhe des Effekts an, die mit einer Veränderung des Prädiktors um eine Einheit einhergeht (also einem Statuswechsel von der Referenz- in die Zielkategorie, z.B. von Nichtakademikerkind auf Akademikerkind).

AME oder DC für Interaktionsterme können nicht gesondert berechnet werden, da der Interaktionseffekt sich nicht unabhängig von dessen Haupteffekten verändern kann (vgl. Williams 2012). Interaktionen werden daher im Folgenden zunächst grafisch dargestellt und deren Signifikanz auf Grundlage logistischer Regressionskoeffizienten getestet. Zudem wird getestet, ob sich die Wahrscheinlichkeit von $\mathrm{y}=1$ zwischen den Herkunftsgruppen an bestimmten Werten der betrachteten unabhängigen Variablen signifikant unterschiedet (vgl. Mitchell 2012).

\subsubsection{Anmerkungen zur Bedeutung von Signifikanz}

Wie in Abschnitt 6.2.1 beschrieben, bestehen die Best Up-Daten nicht aus einer repräsentativen Stichprobe. Die folgenden Ergebnisse beziehen sich somit nicht auf eine wohl definierte Population (deutscher oder Berliner Studienberechtigter), sodass deren Interpretation nicht als generalisierend verstanden werden sollte. Nichtsdestotrotz werden Signifikanztests durchgeführt, um die Präzision der Schätzungen einordnen zu können.

Nicht jeder (statistisch) signifikante Effekt ist auch substantiell und damit soziologisch relevant (vgl. Bernardi et al. 2017). Gleichzeitig bedeutet fehlende Signifikanz nicht, dass kein Zusammenhang zwischen der unabhängigen

140 Oft wird der Begriff AME als Oberbegriff verwendet und schließt somit auch die diskrete Veränderung eines Statuswechsels kategorialer Variablen ein (vgl. Williams 2012). Nutzt man den Stata Befehl margins, wird automatisch der DC ausgewiesen, sobald die empfohlene Faktornotation für kategoriale Variablen verwendet wird (vgl. Long und Freese 2014: 163). 
und der abhängigen Variable besteht, sondern dass die Existenz eines potentiellen (sichtbaren) Zusammenhangs nicht mit der nötigen Präzision geschätzt werden konnte, um statistische Signifikanz zu erreichen (was gerade bei kleinen Fallzahlen - wie auch in dieser Studie - nicht ausgeschlossen werden kann). Daher sollen im Folgenden nicht nur die statistische Signifikanz beobachteter Herkunftsunterschiede (und somit die Präzision der Schätzung) diskutiert werden, sondern auch deren vorhandene oder fehlende Substanz (also die Stärke des Effekts). ${ }^{141}$

141 Wenn ich im Folgenden von Einflüssen, Effekten oder Wirkungen spreche und nicht ausschließlich von Zusammenhängen und Assoziationen, geschieht dies zur leichteren Lesbarkeit. Es sei darauf hingewiesen, dass mit den verwendeten Methoden (mit Ausnahme des Faktoriellen Surveys) Zusammenhänge erfasst, jedoch keine kausalen Aussagen getroffen werden können. 


\section{Phase 1: Die Übersetzung von Studienaspiration in Studienintention}

Das erste empirische Kapitel dieser Arbeit untersucht Phase 1 im „Übergangsprozess", in der Schüler/innen mit Studienaspiration diese in eine ebenso hohe Intention übersetzen oder aufgrund antizipierter individueller und/oder institutioneller Hürden hiervon abweichen. Dies erfolgt in mehreren Schritten: Zunächst wird den Fragen nachgegangen, ob es Herkunftsunterschiede bei der Übersetzung von Studienaspirationen in Studienintentionen gibt und wie diese erklärt werden können. Als mögliche Erklärungen werden dabei Unterschiede in den Bildungsentscheidungen untersucht, die sowohl aufgrund sozialgruppenspezifischer Abwägungen von Kosten, Nutzen und Erfolgswahrscheinlichkeiten (RC) als auch aufgrund sozialgruppenspezifischer Erwartungshaltungen und Unterstützung aus dem sozialen Umfeld zustande kommen können (Abschnitt 7.2.1). Zudem wird getestet, inwiefern antizipierte institutionelle Barrieren und Opportunitäten sozialgruppenspezifisch auf die Übersetzungswahrscheinlichkeit wirken (Abschnitt 7.2.2). Sofern eine Studienintention besteht, soll weiterhin die Frage beantwortet werden, inwiefern sich Schüler/innen unterschiedlicher sozialer Herkunft bezüglich ihrer Intention für verschiedene Hochschultypen und Fächer unterscheiden (Abschnitt 7.3).

Die entsprechenden Hypothesen wurden in Kapitel 5 abgeleitet und sind in Tabelle 7.1 zur Übersicht nochmals zusammengefasst. Im Folgenden werden unterschiedliche Messungen des Konzepts Studienintention sowie verschiedene methodische Ansätze herangezogen, um diese Hypothesen zu testen.

\subsection{Methodisches Vorgehen}

Während allgemeine und phasenübergreifende methodische Erläuterungen bereits in Kapitel 6 beschrieben wurden, soll hier zunächst das spezifische, methodische Vorgehen zur Untersuchung der ersten Phase erläutert werden. In Abschnitt 7.2.1 werden zunächst Vor- und Nachteile verschiedener Operationalisierungsmöglichkeiten der abhängigen Variable „Studienintention“ diskutiert: einer abstrakten, globalen (direkte Abfrage) und einer konkreten, plastischen (Faktorieller Survey (FS) oder Vignettenstudie), die in der vorliegenden Arbeit ergänzend zur Anwendung kommen. In Abschnitt 7.1.2 liegt der Fokus auf den Operationalisierungen und Analyseschritten, die auf der direkten Intentionsabfrage beruhen. In Abschnitt 7.1.3 wird anschließend - nach einer kurzen Beschreibung der grundlegenden Methodik - das Design der Best Up- 
Vignettenstudie vorgestellt. Zuletzt thematisiert Abschnitt 7.1.4 in den verschiedenen Analyseschritten notwendige Sampleeinschränkungen aufgrund fehlender Werte.

Tab. 7.1: Hypothesen Phase 1

\begin{tabular}{|c|c|}
\hline \multicolumn{2}{|c|}{ Hypothesenblock A: Generelle Annahmen zu Herkunftsunterschieden } \\
\hline Herkunftsunterschiede & $\begin{array}{l}\text { HA/l: Nichtakademikerkinder haben seltener Studienintention } \\
\text { als Akademikerkinder. }\end{array}$ \\
\hline \multicolumn{2}{|c|}{ Hypothesenblock B: Individuelle Einflussfaktoren und Bildungsentscheidung } \\
\hline Leistung & HB1/l: Leistungsunterschiede erklären Teil der Herkunftsdifferenz. \\
\hline Rationale Abwägung & $\begin{array}{l}\text { HB2/l: Einschätzung von Kosten/Nutzen/Erfolgswahrscheinlichkeit } \\
\text { erklären Teil der Herkunftsdifferenz. }\end{array}$ \\
\hline $\begin{array}{l}\text { Soziale und kulturelle } \\
\text { Ressourcen }\end{array}$ & $\begin{array}{l}\text { HB3/l: Soziale und kulturelle Ressourcen erklären Teil der } \\
\text { Herkunftsdifferenz. }\end{array}$ \\
\hline \multicolumn{2}{|c|}{ Hypothesenblock C: Institutionelle Einflussfaktoren und Bildungsentscheidung } \\
\hline $\begin{array}{l}\text { Kosten } \\
\text { (geographische Distanz }\end{array}$ & HC1/I: Distanz wirkt negativer auf Nichtakademikerkinder. \\
\hline \multirow[t]{4}{*}{ Zugangshürden } & $\begin{array}{l}\text { HC2/l: Zulassungsbeschränkung und Selektivität des angestrebten } \\
\text { Faches führen bei Nichtakademikerkindern eher zu Umentscheidung } \\
\text { (keine Intention). }\end{array}$ \\
\hline & $\begin{array}{l}\text { HC3/l: Sofern keine Umentscheidung, führen Zulassungshürden des } \\
\text { angestrebten Faches in allen Herkunftsgruppen zur Umorientierung } \\
\text { (andere Fachintention als -aspiration). }\end{array}$ \\
\hline & $\begin{array}{l}\text { HC4a/l: Komplexe Auswahl wirkt anziehend für Akademikerkinder } \\
\text { und abschreckend für Nichtakademikerkinder (Herkunftsdifferenz). }\end{array}$ \\
\hline & $\begin{array}{l}\text { HC4b/l: Komplexe Auswahl anziehend für beide (keine } \\
\text { Herkunftsdifferenz). }\end{array}$ \\
\hline Information & HC5/l: Informationen wirken positiver auf Nichtakademikerkinder. \\
\hline Reputation & HC6/l: Reputation der Hochschule wirkt positiver auf Akademikerkinder. \\
\hline \multicolumn{2}{|c|}{ Hypothesenblock D: Differenzierte Bildungsentscheidungen } \\
\hline Hochschultyp & HD1/l: Nichtakademikerkinder haben häufiger Fachhochschulintention. \\
\hline Studienfach & HD2/l: Kein Unterschied bezüglich des Einkommensertrags. \\
\hline & $\begin{array}{l}\text { HD3/l: Akademikerkinder haben häufiger Intention für prestigereiches } \\
\text { Fach. }\end{array}$ \\
\hline
\end{tabular}

\subsubsection{Die Messung von Studienintentionen}

Wie bereits in Abschnitt 4.1 angedeutet, ist die Differenzierung zwischen Aspirationen und Intentionen zwar theoretisch sinnvoll, aber empirisch herausfordernd. Die am häufigsten verbreitete empirische Umsetzung dieser Differenzierung basiert auf getrennten Surveyabfragen nach Bildungsaspirationen/- 
wünschen (z.B. „How far would you like to go in school?”) und Bildungsintentionen/-erwartungen/-plänen (z.B. "Realistically speaking, how far do you think you will go in school?", siehe Lloyed et al. 2008: 10), die mittlerweile in vielen (englischsprachigen) Surveys integriert ist. ${ }^{142}$ Im deutschen Kontext befragt das Nationale Bildungspanel (NEPS) Schüler/innen der gymnasialen Oberstufe getrennt nach ihren Bildungsaspirationen und -intentionen (vgl. Stocké et al. 2011). ${ }^{143}$ Beide Items wurden mit leichten Anpassungen in der Best Up-Befragung übernommen.

Die Aspirationsfrage: „Wenn es allein nach Ihren Wünschen ginge: Welche Art von Ausbildung würden Sie nach Beendigung der Schule am liebsten machen?" wurde - wie in Abschnitt 6.4 beschrieben - verwendet, um das Ausgangssample zu definieren. Die Intentionsfrage, die die als realistisch wahrgenommene Wahrscheinlichkeit einer Studienaufnahme widerspiegeln soll, lautet wie folgt: „Jetzt denken Sie an alles, was Sie derzeit wissen: Welche Art von Ausbildung werden Sie nach Beendigung der Schule wahrscheinlich machen? (Wenn Sie vorhaben nach der Schule zunächst ein freiwilliges soziales Jahr, ein Praktikum o. Ä. zu machen, geben Sie die Art von Ausbildung an, die Sie danach wahrscheinlich machen werden.) ". ${ }^{144}$ Die Antworten auf diese Frage werden im Folgenden in einem ersten Schritt genutzt, um die Übersetzung von Studienaspirationen - die alle Befragten im Ausgangssample äußerten - in Studienintentionen zu messen.

Diese Operationalisierung konstituiert ein sehr abstraktes, globales Maß, das die Gesamtheit wahrgenommener individueller und institutioneller Barrieren und Opportunitäten widerspiegelt. Die Frage nach den Ursachen für eine mögliche Diskrepanz zwischen Studienaspirationen und -intentionen bleibt zunächst offen. Auch wenn es möglich ist, sich dieser Frage mithilfe multivariater Modelle anzunähern, ist der Kern individuell und institutionell eingeschränkter Intentionen mit solch einem globalen Item nicht direkt erfassbar.

Dieser Einschränkung soll durch eine ergänzende Messung von Intentionen im Rahmen eines quasi-experimentellen Faktoriellen Surveys (oder Vignettenstudie) Rechnung getragen werden. Hierfür wurden den Befragten konkrete und plastische Bewerbungsszenarien für Studiengänge mit variierenden

142 Ähnliche Abfragen finden sich zum Beispiel auch in folgenden Datensätzen: USA: High School and Beyond, High School Longitudinal Study, National Study of Youth and Religion, Beginning Postsecondary Study, UK: Longitudinal Study of Young People in England.

143 Hierfür wurden die von Volker Stocké (2005a, b) vorgeschlagenen, ursprünglich für Eltern von Grundschulkindern entwickelten Operationalisierungen verwendet und entsprechend der Zielgruppen des NEPS angepasst.

144 Wie auch bei der Aspirationsfrage stehen als Antwortkategorien „ein Studium“, „,eine berufliche Ausbildung“ und „,keine Ausbildung“ zur Verfügung, wobei die ersten beiden Kategorien in verschiedene Hochschul- und Ausbildungstypen unterteilt werden. 
Merkmalen vorgelegt. Für jedes dieser Szenarien wurden sie gebeten anzugeben, wie wahrscheinlich es ist, dass sie sich unter den skizzierten Bedingungen für den beschriebenen Studiengang bewerben würden. So kann präzise und isoliert untersucht werden, welche Merkmale die Übersetzung von Aspiration in Intention positiv oder negativ beeinflussen.

Trotz dieses Vorteils sind die Erkenntnisse auf die über die Vignetten variierten Merkmale beschränkt, die - im Gegensatz zur globalen Abfrage - zwar einige, jedoch sicherlich nicht alle individuell variierenden Barrieren umfassen. Eine ergänzende Nutzung des gloablen (jedoch sehr abstrakten und daher unkonkreten) Surveyitems und des konkreten (jedoch auf spezifische Einflussfaktoren beschränkten) FS wird daher hier verfolgt.

\subsubsection{Analyseschritte und Operationalisierungen}

Die Analysen zu Phase 1 unterteilen sich in drei Schritte. Im ersten Schritt werden zunächst die Hypothesen der Blöcke A und B getestet. Hierbei wird untersucht, ob sich Schüler/innen unterschiedlicher sozialer Herkunft bei der Übersetzung ihrer Studienaspirationen in Studienintentionen unterscheiden (HA/I). Und - sofern dies der Fall ist - ob dieser Unterschied durch Leistungsindikatoren (HB1/I), ${ }^{145}$ die Abwägung der drei RC-Komponenten (Kosten/Nutzen/Erfolgswahrscheinlichkeit) (HB2/I) und soziale und kulturelle Ressourcen erklärt werden (HB3/I) kann.

Als abhängige Variable dient hierfür das oben diskutierte direkte Intentionsitem, dessen Antwortmöglichkeiten zu zwei Kategorien zusammengefasst wurden: Studienintention vs. keine Studienintention (umfasst die Kategorien „Ausbildung“ und „keine Ausbildung“). In mehreren Schritten werden dem Basismodell (soziale Herkunft + Kontrollvariablen) die in drei Blöcke unterteilten erklärenden Variablen hinzugefügt. Den geschachtelten Modellen kann so zum einen entnommen werden, ob Leistungs- RC- und Ressourcenindikatoren mit der Herausbildung einer Studienintention zusammenhängen, und zum anderen, inwiefern diese Indikatoren zur Reduktion sozialer Herkunftsunterschiede führen (vgl. Abschnitt 6.5.2).

Im zweiten Schritt werden die Hypothesen zum (sozialgruppenspezifischen) Zusammenhang zwischen institutionellen Merkmalen und der Studienintention getestet (Block C). Für die Frage, ob der Zusammenhang zwischen Studienintention und sozialer Herkunft mit antizipierten Zulassungshürden va-

145 Wie in Abschnitt 5.5 erläutert, können aufgrund der empirischen Trennung selbstund fremdselektiver Phasen Leistungsunterschiede hier als Teil der wahrgenommenen Erfolgswahrscheinlichkeit interpretiert werden. Um einen Vergleich mit existierender Forschung zu ermöglichen, werden zunächst Schulleistunen als Mediator aufgenommen, bevor das Modell mit den weiteren Variablen angereichert wird. 
riiert (HC2/I), wird die Studienintention in Abhängigkeit der sozialen Herkunft und den in Kapitel 6 beschriebenen Variablen zur durchschnittlichen Beschränkung und Selektivität des angestrebten Studienfaches sowie deren Interaktion modelliert und grafisch dargestellt. Der gleichen Logik folgt der Test der Hypothese HC3/I. Hier wird nicht mehr die „Umentscheidung“ vom Studium zu einem anderen nachschulischen (Bildungs-)Weg untersucht, sondern eine potentielle „Umorientierung“ innerhalb von als unterschiedlich selektiv wahrgenommenen Fächern. Die Analysen sind somit konditional und beziehen sich nur auf Befragte mit Studienintention, da nur für diese auch eine Studienfachintention vorliegt. Die zweite abhängige Variable „Übereinstimmung Studienfach" nimmt den Wert 1 an, wenn sich das Studienfach zwischen der Aspirations- und Intentionsfrage nicht unterscheidet. ${ }^{146}$

Der (sozialgruppenspezifische) Einfluss weiterer institutioneller Merkmale wird mithilfe des FS untersucht. Hier spielen erneut potentielle Zugangshürden in Form komplexer Auswahlverfahren wie Auswahlgespräche oder Tests eine Rolle (HC4a/I und HC4b/I) sowie geographische Distanz (HC1/I), verfügbare Informationen (HC5/I) und die Reputation der Hochschule (HC6/I). Genauere Informationen zum FS sind in Abschnitt 7.1.3 zusammengetragen.

Im dritten Schritt stehen sozialgruppenspezifische Hochschultyp- (HD1/I) und Fachintentionen (HD2/I und HD3/I) und somit die Frage nach sozialer Ungleichheit innerhalb des vertikal und horizontal differenzierten Hochschulsystems im Mittelpunkt. Auch diese Analysen werden (wie der Test von HC3/I) nur für Befragte mit Studienintention durchgeführt. Für die abhängige Variable Hochschultypintention wurden die zur Auswahl stehenden Hochschultypen in zwei Kategorien zusammengefasst (0: Universität, 1: Fachhochschule inklusive duale Hochschule). Die Fachintention wird nicht wie häufig geschehen kategorial erfasst, sondern über die bereits vorgestellten metrischen Mikrozensusvariablen zum durchschnittlichen Einkommen und Berufsprestige (vgl. Abschnitt 6.2.3), zu dem die intendierten Studienfächer führen. Bei den

146 Wie bereits in Kapitel 6 erwähnt, wurden die Studienfächer in Anlehnung an die Fächersystematik des Statistischen Bundesamtes (2012) vercodet und liegen in den Best Up-Daten als Zweisteller (13 Kategorien) und Sechssteller (circa 80 Kategorien) vor. Der Sechsteller diskriminiert zum Beispiel zwischen Sozialpädagogik und Sozialwesen oder Biochemie und Chemie, während der Zweisteller zwischen den Kategorien Sprach-/Literaturwissenschaften, Kulturwissenschaften, Erziehungswissenschaften/Pädagogik, Sport, Rechtswissenschaften, Wirtschaftswissenschaften, Sozialwissenschaften, Mathematik/Naturwissenschaften, Humanmedizin/Gesundheitswissenschaften, Veterinärmedizin, Agrar-/Forst-/Ernährungswissenschaften, Ingenieurwissenschaften und Kunst unterscheidet. Für die Operationalisierung der Übereinstimmung zwischen Fachaspiration und -intention wird auf den Zweisteller zurückgegriffen, da hier vor allem substantielle Wechsel von Interesse sind. 
Analyseschritten zur Überprüfung des Hypothesenblocks D (sowie auch bei der Hypothese HC3/I) handelt es sich um konditionale Analysen, die nur für Befragte mit Studienintention durchgeführt werden.

\subsubsection{Faktorieller Survey zur Modellierung institutioneller Einflussfaktoren}

Wie angekündigt, soll die direkte Intentionsfrage durch einen Faktoriellen Survey ergänzt werden. Der Fokus liegt dabei auf vom Hochschulsystem ausgehenden, institutionellen Opportunitäten und Barrieren und deren (sozialgruppenspezifischen) Einfluss auf die Übersetzung von Studienaspirationen in Studienintentionen. Im Folgenden soll zunächst die Methode, ihre Vor- und Nachteile in gebotener Kürze beschrieben werden. Im Anschluss daran werden die Best Up-Vignetten vorgestellt.

Faktorielle Surveys: Anmerkungen zur Methodik

Die Methode des FS wurde von Rossi insbesondere seit den 1970ern in die soziologische Forschung eingeführt (vgl. Rossi 1979, Rossi und Nock 1982, für einen Überblick über Entstehung und Anwendungsbereiche siehe Auspurg und Hinz 2015, Wallander 2009). Während zunächst die Untersuchung von Einstellungen, Normen und der Bewertung sozialer Situationen im Mittelpunkt stand (vgl. Alves und Rossi 1978, Denk et al. 1997, Jasso und Opp 1997, Garrett 1982), werden zunehmend auch Entscheidungs- und Handlungsintentionen erforscht, z.B. in Studien zu Anstellungs- und Weiterbildungsentscheidungen von Arbeitgeber/innen (vgl. Daniel et al. 2019, Di Stasio 2014, Di Stasio und Van de Werfhorst 2016, Karpinska et al. 2015), zu Spendenintentionen (vgl. Kinsbergen und Tolsa 2013) oder zur Umzugsbereitschaft (vgl. Abraham et al. 2010). Zudem gibt es erste Untersuchung von Bildungsintentionen auf Grundlage von FS (vgl. Daniel und Watermann 2018, für die Anwendung eines methodisch verwandten Conjoint Experiments siehe Soutar und Turner 2002). ${ }^{147}$

In FS werden den Befragten nicht einzelne Merkmale zur Bewertung vorgelegt, sondern sogenannte Vignetten. Hierbei handelt es sich um hypothetische Situationsbeschreibungen, in denen die Ausprägungen ausgewählter, theoretisch relevanter Merkmale (Dimensionen) experimentell variiert werden. Die Gesamtheit aller möglichen Merkmalskombinationen wird als Vignettenuniversum (full factorial) bezeichnet. Um die Effizienz des Designs zu maximieren wird in der Regel (und speziell bei einer großen Anzahl an Merkmals-

147 Eine Studie auf Grundlage der Best Up-Vignetten, die große Überschneidungen mit der in diesem Kapitel vorgestellten Untersuchung ausweist, liegt zudem seit 2016 als Veröffentlichung vor (Finger 2016). 
kombinationen ${ }^{148}$ ) nicht das gesamte Vignettenuniversum verwendet, sondern ein Sample an Vignetten (fractional factorial). Anschließend wird den Befragten eine Auswahl dieser Vignetten - oft bereits in feste Blöcke oder Decks unterteilt - zufällig zur Bewertung vorgelegt (vgl. Auspurg und Hinz 2015 für zentrale Definitionen und Vorgehensweisen).

In der Literatur werden FS mit verschiedenen Vorteilen in Verbindung gebracht. So folgen sie der Logik experimenteller Designs und können gleichzeitig in größer angelegte Surveys integriert werden, sodass zum einen kausale Aussagen getroffen werden können und zum anderen eine große Fallzahl zur Verfügung steht, die nicht nur aufgrund der Anzahl an Befragten erzielt wird, sondern auch weil jede/r Befragte in der Regel mehrere Vignetten bewertet (vgl. Mutz 2011). So können in der Realität selten vorkommende Phänomene und der (potentielle) Einfluss selten vorkommender Merkmale getestet werden (vgl. Nisic und Auspurg 2009). Im vorliegenden Fall trifft dies zum Beispiel auf den Einfluss verschiedener Auswahlverfahren wie Auswahlgespräche oder Tests zu. Neben bisher generell fehlenden Daten stellt auch deren aktuell (noch?) eingeschränkte Abdeckung die quantitative Forschung vor Herausforderungen.

Durch die experimentelle Variation der Merkmalsausprägungen lässt sich der Einfluss einzelner Dimensionen isolieren, die ansonsten oft konfundiert sind: ,the multicollinearity of the ,real" world is avoided“" (Rossi 1979: 179). Durch die randomisierte Verteilung der Vignetten auf Befragte sind zudem die Befragtenmerkmale nicht mit den Ausprägungen der Vignettendimensionen korreliert, sodass ein potentieller Selektionsbias ausgeschaltet wird und die geschätzten Effekte als Nettoeffekte der Dimensionen interpretiert werden können (vgl. Nisic und Auspurg 2009: 220).

Ein weiterer Vorteil von Vignetten im Vergleich zu direkten Abfragen ist, dass sie das institutionelle Setting, mit dem die Befragten konfrontiert sind, konkret beschreiben und nicht nur abstrakt (und unstandardisiert) zusammenfassen und so ,reale“ Entscheidungssituationen approximieren. Zudem hat der Forscher/die Forscherin ein hohes Maß an Kontrolle über die verwendeten Stimuli, was die Gefahr minimiert, dass Befragte weitere, inter-individuell variierende und dem Forscher/der Forscherin unbekannte Informationen hinzufügen (vgl. Alexander und Becker 1978: 93f.). Bei der direkten Intentionsfrage „Jetzt denken Sie an alles, was Sie derzeit wissen..." - ist dies sicherlich der Fall. Die Standardisierung der Stimuli hat in der vorliegenden Arbeit einen weiteren Vorteil: Hierdurch werden potentielle Informationsasymmetrien zwi-

148 Das Produkt aller Merkmalsausprägungen (Kartesisches Produkt) kann schnell im vier- und auch fünfstelligen Bereich liegen, wenn einzelne Dimensionen viele Ausprägungen aufweisen (z.B. Einkommen oder Alter), und dabei die Anzahl an Befragten überschreiten. Gleichzeitig sollte jede Vignette von mehreren Befragten bewertet werden, um eine Konfundierung von Befragten- und Vignettenmerkmalen zu vermeiden (Auspurg und Hinz 2015: 61). 
schen Befragten (unterschiedlicher sozialer Herkunft) ausgeglichen und deren Präferenzen für spezifische institutionelle Charakteristika besser approximiert. Sozialgruppenspezifische Informationen sind damit nicht mit sozialgruppenspezifischen Präferenzen konfundiert.

Der eigentliche Vorteil der Konstruktion realistischer Szenarien kann sich zum Nachteil verkehren, wenn durch die Kreuzung aller Merkmalsausprägungen auch unrealistische Kombinationen konstruiert werden, die Befragungsabbrüche oder inkonsistente Antworten zur Folge haben können (vgl. Auspurg et al. 2009a, Wallander 2009). Um die Gefahr der Messung methodischer Artefakte zu minimieren, wird daher oft empfohlen, Vignetten mit unlogischen Merkmalskombinationen auszuschließen, auch wenn dadurch die Ziehung einer orthogonalen Stichprobe, in der die Vignettendimensionen möglichst unkorreliert sind, erschwert wird (vgl. Auspurg und Hinz 2015: 57).

Ist das experimentelle Design wohl durchdacht, kann von einer hohen internen Validität ausgegangen werden. Bezweifelt wird jedoch häufig deren externe Validität, da hier lediglich Entscheidungsintentionen und nicht tatsächliche Entscheidungen gemessen werden (vgl. Collet und Childs 2011). Für die Fragestellung dieses Kapitels kann dieser Kritikpunkt entkräftet werden, da das genuine Interesse hier in der Untersuchung von Studienintentionen liegt. Ermutigend sind zudem Evaluationsstudien, die eine relativ hohe Übereinstimmung zwischen den Ergebnissen von Vignettenstudien und Befragungs- bzw. Beobachtungsdaten zeigen (vgl. Hainmüller et al. 2015, Nisic und Auspurg 2009, Petzold und Wolbring 2018).

Best Up-Vignetten: Operationalisierung, experimentelles Design und Analysemethoden

Die Konzeption von FS umfasst zwei zentrale Schritte: Zum einen müssen die Dimensionen, deren Einfluss getestet werden soll, operationalisiert und $\mathrm{zu}$ Vignetten zusammengeführt werden. Zum anderen ist die Wahl des experimentellen Designs zentral, um sicherzustellen, dass der Einfluss der Dimensionen isoliert erfasst werden kann. Im Folgenden wird das entsprechende Vorgehen bei der Konstruktion der Best Up-Vignettenstudie vorgestellt.

Die Best Up-Vignetten beschreiben Bewerbungsszenarien für hypothetische Studiengänge an deutschen Hochschulen. Hierfür wurden anhand theoretischer Überlegungen und vorhandener Forschungsergebnisse sieben Dimensionen mit zwei bis vier Ausprägungen ausgearbeitet und zu Vignettentexten zusammengefasst. Diese wurden mit 23 Schüler/innen der Zielgruppe getestet, um sicherzustellen, dass die Vignetten für die Zielgruppe aussagekräftig sind 
und keine zentralen Dimensionen vergessen wurden. Eine Beispielvignette ist in Abbildung 7.1 dargestellt. ${ }^{149}$

Abb. 7.1: Beispielvignette

Im Bewerbungsverfahren für einen Studienplatz in Ihrem Lieblingsfach zählt zum einen Ihre Abiturnote. Zusätzlich müssen Sie an einem persönlichen Auswahlgespräch teilnehmen.

Auf der Website der Hochschule finden Sie umfassende Informationen zum Bewerbungsprozess, die Ihnen eine gute Vorstellung darüber vermitteln, was Sie erwartet. Außerdem haben Sie Bekannte, die sich mit Bewerbungsverfahren im Hochschulbereich sehr gut auskennen und Ihnen hilfreiche Tipps geben können.

Die Hochschule hat weder einen sehr guten noch einen schlechten Ruf. Sie liegt in einer etwas weiter entfernten Großstadt, wie zum Beispiel Hamburg oder Leipzig. Diese ist aber von Berlin aus bezüglich Fahrzeit und -kosten gut erreichbar, sodass Sie am Wochenende nach Berlin pendeln könnten.

Würden Sie sich für diesen Studienplatz bewerben?

Bitte kreuzen Sie ein Kästchen auf der Skala an, wobei der Wert 0 bedeutet: „Nein, auf keinen Fall“ und der Wert 10: ,Ja, aufjeden Fall. Mit den Werten dazwischen können Sie Ihre Einschätzung abstufen.

Nein/auf keinen Fall

$\mathrm{Ja} /$ auf jeden Fall
0
1
2
3
5
6
$7 \quad 8$
$9 \quad 10$
k.A.

149 Um die Befragten auf das neue Fragenformat einzustimmen, wurde vor Beginn des Vignettenmoduls folgender Text eingeblendet: „Im Folgenden interessiert uns, unter welchen Bedingungen Sie sich nach Ihrer Schulzeit für einen bestimmten Studienplatz bewerben würden. Stellen Sie sich daher bitte vor, dass Sie sich nach dem Abitur für ein Studium bewerben möchten. Wenn Sie eigentlich andere Pläne haben, wie zum Beispiel eine berufliche Ausbildung aufnehmen, versetzen Sie sich einfach in die Rolle eines Studienbewerbers/einer Studienbewerberin. Im Folgenden haben wir verschiedene Situationen zusammengestellt. Bitte lesen Sie diese Situationen gründlich durch. Möglicherweise sind einige der Situationen für Sie nicht $100 \%$ realistisch. Uns interessiert dennoch Ihre Einschätzung: Kreuzen Sie daher bitte für jeden der vorgestellten Studienplätze an, ob Sie sich bewerben würden." 
Aus dem Produkt der Dimensionsausprägungen $(3 \times 2 \times 4 \times 2 \times 2 \times 2 \times 2)$ ergibt sich ein vergleichsweise kleines Vignettenuniversum von 384 Vignetten. Unplausible Kombinationen wurden bereits bei der Konzeption der Vignetten vermieden, sodass ein Ausschluss einzelner Vignetten nicht nötig ist. Neben den inhaltlichen Überlegungen folgt dieses Design den methodischen Empfehlungen von circa sieben Dimensionen mit möglichst wenigen Ausprägungen. ${ }^{150}$

Tabelle 7.2 gibt einen Überblick über die Dimensionen, deren Ausprägungen und Verteilung im Analysesample. In den folgenden Analysen sind vor allem die ersten vier Dimensionen relevant, die sich auf Merkmale der Hochschule bzw. des Studiengangs beziehen und sich in den Hypothesen widerspiegeln: die Entfernung zum Hochschulort, der Ruf der Hochschule, die zur Anwendung kommenden Auswahlverfahren sowie zur Verfügung stehende Informationen zum Bewerbungsprozess.

Während in den frühen Vignettenstudien ein zufälliges Samplingdesign zur Ziehung der benötigten Vignetten aus dem Vignettenuniversum verbreitet war, wird vermehrt die Verwendung D-effizienter Designs empfohlen, die Orthogonalität (minimale Korrelationen zwischen den Dimensionen generell und innerhalb eines Decks) und Balanciertheit (Ausprägungen sind zu gleichen Anteilen repräsentiert) maximieren. Dieses Vorgehen stellt sicher, dass die einzelnen Parameter präzise geschätzt werden können (vgl. Auspurg und Hinz 2015: 22ff.). Aus dem 384 Vignetten umfassenden Universum wurde ein Deffizientes Sample von 298 Vignetten gezogen und auf 76 Decks à fünf Vignetten verteilt (D-Effizienz: 99,93). ${ }^{151}$ Jeder/jedem Befragten wurde eines der

150 Eine zu hohe Anzahl an Dimensionen kann zu kognitiver Überforderung führen, während eine zu kleine Anzahl oft mit einer geringen Standardisierung und somit hoher unbeobachteter Heterogenität einhergeht, wenn Befragte sich die nötigen Informationen für die Bewertung der Szenarien selbst hinzudenken (vgl. Auspurg et al. 2009b). Auspurg und Hinz (2015: 18ff.) empfehlen in ihrem umfassenden Methodenband zu FS etwa sieben Dimensionen. Ein effizientes Design wird durch eine geringe Anzahl an Ausprägungen und somit ein kleines Vignettenuniversum erleichtert.

151 Das effizienteste Design, das von dem Softwarepaket SAS identifiziert wurde, enthält 298 Vignetten, von denen einige dupliziert und im Anschluss daran auf 76 Decks (380/5) verteilt wurden. Es wurde ein Resolution V Design gewählt, in dem die Orthogonalität zwischen allen Dimensionen und deren Zweifachinteraktionen maximiert wird (vgl. Auspurg und Hinz 2015: 26f.). Der D-Effizienzwert ist mit über 99 hoch (als ausreichend werden Werte von 90 auf einer Skala von 0 bis 100 interpretiert) (vgl. ebd: 29). Insbesondere für die Verteilung der Vignetten auf Decks wurde ein D-effizientes Design präferiert, um hohe Korrelationen oder komplette Konfundierung der Dimensionen innerhalb der Decks zu vermeiden, die mit einer zufälligen Aufteilung wahrscheinlich gewesen wären (vgl. ebd.: 39). Die Anzahl der Vignetten pro Person wurde mit fünf gering gehalten, zum einen um die zur Verfügung stehende Bearbeitungszeit nicht zu überschreiten, zum anderen um das Risiko von Ermüdungs- und Lerneffekte zu reduzieren. 
76 Decks zufällig zugeteilt. Dabei wurde die Reihenfolge der Vignetten innerhalb der Decks zufällig variiert, um Reihenfolgeeffekte zu vermeiden (vgl. ebd.: 72). ${ }^{152}$

Tab. 7.2: Operationalisierung und Verteilung der Vignettendimensionen

\begin{tabular}{|c|c|c|c|}
\hline \multicolumn{2}{|c|}{ Dimension } & \multirow{2}{*}{$\begin{array}{l}\text { Ausprägungen } \\
\text { gering/identisch (tägliches Pendeln möglich) } \\
\text { mittel (Pendeln am Wochenende möglich) } \\
\text { hoch (sporadische Besuche möglich) } \\
\text { (zur Illustration wurden jeweils zwei Hochschul- } \\
\text { standorte als Beispiele genannt) }\end{array}$} & \multirow{2}{*}{$\begin{array}{l}\text { Prozent (N) } \\
\text { N Vignetten: } \mathbf{2 8 3 2} \\
\text { N Befragte: } \mathbf{5 7 8} \\
33,4(946) \\
33,1(937) \\
33,5(949)\end{array}$} \\
\hline 1) & $\begin{array}{l}\text { Entfernung zwi- } \\
\text { schen Heimat- } \\
\text { und Hochschulort }\end{array}$ & & \\
\hline 2) & Ruf der Hochschule & $\begin{array}{l}\text { sehr gut } \\
\text { weder sehr gut noch schlecht }\end{array}$ & $\begin{array}{l}50,3(1424) \\
49,7(1408)\end{array}$ \\
\hline 3) & Auswahlverfahren & $\begin{array}{l}\text { Nur Abiturnote } \\
+ \text { Motivationsschreiben } \\
+ \text { Auswahlgespräch } \\
+ \text { Test }\end{array}$ & $\begin{array}{l}25,4(721) \\
25,2(713) \\
24,8(702) \\
24,6(696)\end{array}$ \\
\hline 4) & $\begin{array}{l}\text { Information zum } \\
\text { Bewerbungsprozess }\end{array}$ & $\begin{array}{l}\text { kaum Hinweise auf Hochschulwebsite } \\
\text { umfassende Informationen auf Hochschulwebsite }\end{array}$ & $\begin{array}{l}49,5(1401) \\
50,5(1431)\end{array}$ \\
\hline 5) & $\begin{array}{l}\text { Interesse am } \\
\text { Studienfach }\end{array}$ & $\begin{array}{l}\text { mittel (nicht Lieblingsfach, aber dennoch } \\
\text { interessant) } \\
\text { hoch (Lieblingsfach) }\end{array}$ & $\begin{array}{l}50,3(1423) \\
49,7(1409)\end{array}$ \\
\hline 6) & $\begin{array}{l}\text { Unterstützung beim } \\
\text { Bewerbungsprozess } \\
\text { („Netzwerk”) }\end{array}$ & $\begin{array}{l}\text { keine Bekannte für hilfreiche Tipps } \\
\text { Bekannte }\end{array}$ & $\begin{array}{l}50,2(1421) \\
49,8(1411)\end{array}$ \\
\hline 7) & $\begin{array}{l}\text { Größe der } \\
\text { Hochschulstadt }\end{array}$ & $\begin{array}{l}\text { mittelgroße Stadt } \\
\text { Großstadt }\end{array}$ & $\begin{array}{l}49,4(1399) \\
50,6(1433)\end{array}$ \\
\hline
\end{tabular}

Quelle: Best Up-Individualdaten (Welle 2), eigene Berechnungen

Die Tabellen A7.1 und A7.2 im Anhang bestätigen, dass die Korrelationen zwischen den Vignettendimensionen (Orthogonalität) und zwischen zentralen Merkmalen der Befragten und den Dimensionen (randomisierte Zuweisung)

152 Obwohl das Analysesample im Vergleich zum kompletten Best Up-Sample deutlich kleiner ist, wurde jedes Deck ausreichend häufig bearbeitet (von mindestens 5 Personen) (vgl. Auspurg und Hinz 2015: 58).

153 Hierbei wurde auf eine Passung zwischen Dimension 1 und 7 geachtet. Zum Beispiel wurde für die Kombination: sehr weit entfernt und Großstadt die Beispiele Köln und München gewählt, während für eine mittelgroße und nahegelegene Stadt die Beispiele Potsdam und Franfurt/Oder angezeigt wurden. 
minimal sind. Die Balanciertheit der Ausprägungen kann in Tabelle 7.1 nachvollzogen werden.

Wie Abbildung 7.1 verdeutlicht, wurden die Befragten gebeten, für jedes der fünf Szenarien auf einer 11 Punkte Skala anzugeben, ob sie sich unter den beschriebenen Umständen für den beschriebenen Studiengang bewerben würden. Die Antworten auf diese Frage konstituieren die abhängige Variable „Bewerbungsintention". ${ }^{154}$ Methodische Vergleiche zeigen, dass sich die Ergebnisse zwischen linearen oder ordinalen logistischen Modellen bei ähnlichen Vignettenskalen kaum unterscheiden (vgl. Auspurg und Hinz 2015: 88), sodass hier auf die intuitiveren linearen Modelle zurückgegriffen wird.

Im Gegensatz zu allen anderen Analyseschritten, in denen der Mehrebenenstruktur der Daten durch Berücksichtigung von zwei Ebenen (Schüler/innen und Schulen) Rechnung getragen wird, kommt hier mit den Vignetten eine dritte Ebene hinzu. In den Analysen wird entsprechend die fehlende Unabhängigkeit der Fehlerterme auf Schüler/innen und Schulebene einbezogen (Ebene 1: Vignetten, Ebene 2: Schüler/innen, Ebene 3: Schulen).

\subsubsection{Analysesample und Umgang mit fehlenden Werten}

In Kapitel 6 wurde das grundlegende Ausgangssample der vorliegenden Arbeit definiert: Es besteht aus insgesamt 593 Best Up-Teilnehmer/innen, die nicht die acht zufällig ausgewählten Informationsinterventions-Schulen besuchten, die mindestens an Welle 1 und 2 teilnahmen und die in Welle 2 - zu Beginn des letzten Schuljahres - eine Studienaspiration äußerten. In allen Analyseschritten zu Phase 1 wird das Sample - wie bereits in Abschnitt 6.4 angekündigt - zudem auf Befragte beschränkt, die valide Werte auf den Leistungsindikatoren haben, da diese in allen Modellen berücksichtig werden. Der hieraus resultierende Ausschluss betrifft nur 8 Befragte (1\%). Zudem können $23 \mathrm{Be}-$ fragte (4\%) nicht berücksichtigt werden, die fehlende Angaben auf der abhängigen Variable „Studienintention“" haben. Da keine ,weiß nicht" Kategorie oder Ähnliches zur Verfügung stand, kann nicht mit Sicherheit gesagt werden, ob es sich hierbei um Antwortverweigerung oder einen Ausdruck von Unent-

154 Einige Befragte beantworteten das Vignettenmodul so schnell, dass response sets wahrscheinlich sind. Als Sensitivitätscheck wurden daher 31 Befragte ausgeschlossen, die das Vignettenmodul in weniger als 30 Sekunden bearbeiteten. Die Ergebnisse unterscheiden sich kaum, sodass im Folgenden auf das größere Sample zurückgegriffen wird. Insgesamt entspricht die durchschnittliche Bearbeitungszeit (mean: 3,1 min, median: 2,4 min) den von Sauer et al. (2011: 96) berichteten Bearbeitungszeiten für die ersten fünf Vignetten ähnlicher Komplexität. 
schlossenheit/Unsicherheit handelt. Das Ausgangssample für die Analysen der direkten Intentionsfrage basiert somit auf 562 Fällen. ${ }^{155}$

In den verschiedenen vorgestellten Analyseschritten fallen zudem weitere Befragte aus verschiedenen Gründen aus. Der am stärksten ins Gewicht fallende Ausfallgrund kommt durch fehlende Werte auf den Fachaspirations- und Fachintentionsvariablen zustande. Zum Beispiel ist zwar für alle Befragten im Ausgangssample bekannt, dass sie eine Studienaspiration haben, jedoch liegt für 62 von ihnen (11\%) keine Angabe zur Fachaspiration vor. Erneut ist bei diesen Variablen unklar, inwiefern dies ein Ausdruck von Antwortverweigerung oder Unsicherheit ist. Dass Letzteres zumindest teilweise der Fall ist, wird dadurch verdeutlicht, dass Befragte, die sich über ihre Interessen und Fähigkeiten unklar sind, seltener eine Fachaspiration nennen (vgl. auch Barone et al. 2016, Schneider und Franke 2014). Die folgenden Analysen beruhen auf vollständigen Fällen (complete cases). Auch wenn ich nicht davon ausgehe, dass die Ausfälle weder mit den abhängigen noch mit den unabhängigen Variablen zusammenhängen (MCAR, missing completely at random), wird von einer Imputation fehlender Werte abgesehen (vgl. hierzu etwa Allison 2001). Erstens ist dies technisch aufgrund der Vielzahl an Kategorien der Fachaspirationsund Fachintentionsvariable in Kombination mit der Gesamtzahl an Fällen kaum möglich. Zweitens gehe ich aufgrund der zumindest teilweise inhaltlichen Bedeutung der fehlenden Angabe bei diesen Variablen nicht davon aus, dass Befragte mit Angabe diejenigen ohne Angaben hier gut repräsentieren. Drittens sind durch fehlende Werte auf der Fachaspirations/intentionsvariable auch abhängige Variablen betroffen (etwa wenn untersucht wird, zu welchem durchschnittlichen Einkommen die intendierten Studienfächer führen oder ob eine Übereinstimmung von Studienfachaspiration und -intention besteht), von deren Imputation häufig abgeraten wird (vgl. von Hippel 2007).

Die Einschränkung auf vollständige Fälle wird für die einzelnen Analyseschritte separat vorgenommen: Um die statistische Power nicht von vorn herein zu stark einzuschränken, arbeite ich nicht mit einem einheitlich großen (sprich: dem kleinsten) Sample. Da Personen mit geäußerter Fachaspiration sich von solchen ohne unterscheiden (vgl. Tabelle A7.3), wäre die Aussagekraft aller Ergebnisse somit von vorn herein auf Befragte beschränkt, die nicht nur den Wunsch haben zu studieren, sondern diesen Wunsch auch genauer benennen können. Die Interpretation der Ergebnisse über die verschiedenen Analyseschritte hinweg kann und wird daher nur sehr eingeschränkt erfolgen.

Um möglichst transparent zu sein und der Leserin/dem Leser die Beurteilung der Ergebnisse zu erleichtern, wird für jeden Analyseschritt in Anhangsta-

155 Bei den Auswertungen des FS haben nur 7 Befragte keine der fünf Vignetten bewertet und somit keine Angabe auf der abhängigen Variable. Auf Personenebene besteht das Analysesample somit aus 578 Befragten. Auf Vignettenebene liegen valide Angaben zu 2832 Vignetten vor; 58 Vignetten (2\%) wurden nicht bewertet und können somit in den Analysen nicht berücksichtigt werden. 
belle A7.3 dokumentiert, wie groß das Analysesample im Vergleich zum Ausgangssample ist, welches die Ausfallgründe sind und mit welchen Variablen der Ein- oder Ausschluss aus dem Analysesample signifikant zusammenhängt.

\subsection{Ergebnisse I: Herkunftsunterschiede bei der Übersetzung und deren Erklärungen}

\subsubsection{Leistung, rationale Abwägung und soziale/kulturelle Ressourcen}

Zunächst soll gezeigt werden, ob selbst innerhalb der positiv selektierten Gruppen der Studienaspirant/innen sozial schwächere Schüler/innen (,Nichtakademikerkinder") ihre Studienaspiration tatsächlich seltener in eine Studienintention übersetzen als ihre Peers aus akademisch gebildeten Elternhäusern („Einakademikerkinder“ und „Doppelakademikerkinder"). Die Beantwortung dieser Frage stellt den zentralen Ausgangspunkt für die folgenden Analysen dar, in denen es um mögliche Erklärungen für diese potentiellen Herkunftsunterschiede geht.

Zunächst zeigt sich deskriptiv, dass der Anteil derjenigen Befragten, die ihre Aspiration in eine genauso hohe Intention übersetzen, mit insgesamt $90 \%$ sehr hoch ist. Gleichzeitig zeigt sich wie erwartet, dass es hierbei Unterschiede nach sozialer Herkunft gibt: Während 88\% der Nichtakademikerkinder eine Studienintention äußern, tun dies 97\% der Schüler/innen mit zwei akademisch gebildeten Eltern. Schüler/innen aus einem Einakademikerhaushalt ordnen sich mit $92 \%$ in der Mitte ein (vgl. Tabelle 7.3).

Können die gezeigten Herkunftsunterschiede in dieser ,vorweggenommenen Bildungsentscheidung" (Kurz und Paulus 2008: 5490) durch sozialgruppenspezifische Schulleistung, Einschätzungen von Kosten, Nutzen und Erfolgswahrscheinlichkeiten eines Studiums und die Verfügbarkeit sozialer und kultureller Ressourcen erklärt werden? Tabelle 7.3 gibt hierzu einen deskriptiven Überblick. In der zweiten Spalte ist zunächst die Verteilung der Variablen im Analysesample dargestellt. Es folgen die Zusammenhänge zwischen den erklärenden Variablen und der Bildungsherkunft (mittlere Spalten, Spaltenprozente) und der abhängigen Variable Studienintention (letzte Spalte, Zeilenprozente). ${ }^{156}$ Zum Beispiel befinden sich 42,9\% der Doppelakademikerkinder im

156 In Tabelle 7.3 ist das Sample bereits auf Schüler/innen beschränkt, die keine fehlenden Werte auf den erklärenden Variablen haben (N: 518). Die Herkunftsdifferenz bezüglich der abhängigen Variable ist im größeren Ausgangssample (N: 562) praktisch identisch. Zudem zeigt sich, dass der Ausfall aus dem Analysesample nicht mit den Ausprägungen der abhängigen Variable zusammenhängt (Studienin- 
oberen Leistungsquartil bezüglich ihrer Deutschnote, während dies nur für 26,3\% der Nichtakademikerkinder der Fall ist. Es bestehen also trotz vorgelagerter Selektionsprozesse deutliche Leistungsunterschiede zwischen den Herkunftsgruppen. Gleichzeitig haben 95,5\% der sehr guten Deutschschüler/innen eine Studienintention, jedoch „nur“ $86,7 \%$ der schlechteren.

Insgesamt zeigt sich, dass die aufgeführten unabhängigen Variablen mit der abhängigen Variable Studienintention zwar mehr oder weniger stark, jedoch ausnahmslos in die erwartete Richtung zusammenhängen. Mit Blick auf die RC-Indikatoren zeigen sich ebenfalls deutliche Herkunftsunterschiede: Akademikerkinder schätzen ihre Erfolgswahrscheinlichkeit höher und die finanzielle Belastung durch ein Studium geringer ein. Weniger eindeutig ist das Muster bezüglich der erwarteten Aussichten auf einen angesehenen Job. So bringen Doppelakademikerkinder einen angesehenen Job etwas seltener mit einem Studium in Verbindung als die beiden Vergleichsgruppen. ${ }^{157}$ Neben der Ertragseinschätzung verschiedener Bildungswege sollte aus RC-Perspektive auch relevant sein, als wie wichtig diese Erträge eingeschätzt werden. Hier zeigt sich, dass Nichtakademikerkinder sowohl ein hohes Einkommen als auch einen angesehenen Job seltener als unwichtig für ihre Berufswahl bewerten als Akademikerkinder (Einkommen: 11\% (Uni0) - 13\% (Uni1) - 18\% (Uni2), Ansehen: $28 \%$ - 33\% - 46\%). Mit der Studienintention hängen beide Variablen nicht zusammen (Ergebnisse nicht ausgewiesen). Dies spiegelt die zuvor zitierten Befunde wider (Abschnitt 5.4.2), dass Schüler/innen aus sozial schwächeren Familien Einkommenserträgen einen ebenso großen oder größeren Stellenwert zuweisen als ihre sozial privilegierten Peers.

Deutliche (und theoriekonforme) Herkunftsunterschiede zeigen sich auch hinsichtlich der Indikatoren für soziale und kulturelle Ressourcen. Besonders augenscheinlich ist dies mit Blick auf die Studienerfahrung in der Verwandtschaft, die verbreitete Studiennormen, Informations- und Unterstützungsmöglichkeiten im weiteren sozialen Netzwerk widerspiegeln. Mit der Studienintention scheint diese Variable hingegen nicht stark assoziiert zu sein. Der ge-

tention Ausfall: 88,6\%, Studienintention kein Ausfall: 90,3\%, n.s.). Ist dies der Fall, bietet listwise deletion valide Schätzungen von Zusammenhängen in multiplen Regressionsmodellen (vgl. Allison 2014a). Anfälliger für eine Verletzung der MCAR-Annahme sind hingegen Anteilswerte, sodass bei der Interpretation der deskriptiven Statistiken größere Zurückhaltung geboten ist (ebd.).

157 Der Wert von sozialem Status, der sich stärker noch in der zweiten Dimension (angesehener Job) widerspiegelt, sollte jedoch laut RC-Ansätzen absolut gesehen sozial ungleich verteilt sein, sodass die Ergebnisse zunächst erstaunen. Dies könnte neben sozial erwünschtem Antwortverhalten (,Status ist mir nicht wichtig“) ein Anzeichen dafür sein, dass sozial privilegierte Schüler/innen den Nutzen eines Studiums stärker aus anderen, möglicherweise eher kulturellen Ertragsdimensionen ziehen (vgl. Davies und Guppy 1997, van de Werfhorst 2001). Zudem bedeutet die Verteilung dieser Variablen nicht, dass soziale Reproduktion über Einkommen und berufliches Ansehen nicht dennoch stattfinden wird. 
ringste Herkunftsunterschied (5 Prozentpunkte) zeigt sich bei den wahrgenommenen Studienaspirationen der Eltern, die in allen Herkunftsgruppen bei über $80 \%$ liegt. ${ }^{158}$

Tab. 7.3: Verteilung der erklärenden Variablen nach sozialer Herkunft (Spaltenprozente) und Studienintention (Zeilenprozente)

\begin{tabular}{|c|c|c|c|c|c|}
\hline & $\begin{array}{l}\text { Total } \\
\text { N: } \mathbf{5 1 8}\end{array}$ & $\begin{array}{l}\text { Uni0 } \\
\mathrm{N}: 316\end{array}$ & $\begin{array}{l}\text { Uni1 } \\
\mathrm{N}: 111\end{array}$ & $\begin{array}{l}\text { Uni2 } \\
\mathrm{N}: 91\end{array}$ & $\begin{array}{l}\text { Intention } \\
\mathrm{N}: 468\end{array}$ \\
\hline \multicolumn{6}{|c|}{ Soziale Herkunft } \\
\hline Uni0 & 61 & - & - & - & 88 \\
\hline Uni1 & 21,4 & - & - & - & 91,9 \\
\hline Uni2 & 17,6 & - & - & - & 96,7 \\
\hline \multicolumn{6}{|c|}{ Leistungsindikatoren } \\
\hline \multicolumn{6}{|l|}{ Note Deutsch (2.KH) } \\
\hline Oberes Quartil (+) & 30,3 & 26,3 & 31,5 & 42,9 & 95,5 \\
\hline Mitte & 43,6 & 43,3 & 41,5 & 47,2 & 88,9 \\
\hline Unteres Quartil (-) & 26,1 & 30,4 & 27 & 9,9 & 86,7 \\
\hline \multicolumn{6}{|l|}{ Note Mathe $(2 . \mathrm{KH})$} \\
\hline Oberes Quartil (+) & 37,8 & 37,3 & 34,2 & 43,9 & 92,9 \\
\hline Mitte & 34 & 32,9 & 37,9 & 33 & 89,8 \\
\hline Unteres Quartil (-) & 28,2 & 29,8 & 27,9 & 23,1 & 87,7 \\
\hline \multicolumn{6}{|c|}{ Rational Choice } \\
\hline \multicolumn{6}{|c|}{ Wahrgenommene Erfolgswahrscheinlichkeit } \\
\hline (Sehr) unwahrscheinlich & 30,9 & 34,8 & 27 & 22 & 79,4 \\
\hline Wahrscheinlich & 50,4 & 50 & 55,9 & 45 & 93,5 \\
\hline Sehr wahrscheinlich & 18,7 & 15,2 & 17,1 & 33 & 100 \\
\hline \multicolumn{6}{|c|}{ Kostenerwartung: Wie schwer fällt Studienfinanzierung } \\
\hline (Sehr) schwer & 37,9 & 46,2 & 28,8 & 19,8 & 85,7 \\
\hline Weder noch & 32,6 & 33,5 & 30,6 & 31,9 & 94,7 \\
\hline (Sehr) leicht & 29,5 & 20,3 & 40,6 & 48,3 & 91,5 \\
\hline \multicolumn{6}{|c|}{ Ertragserwartung: Aussicht auf hohes Einkommen durch Studium } \\
\hline Schlecht/mittel & 17,6 & 16,5 & 20,7 & 17,6 & 82,4 \\
\hline Gut & 58,9 & 61,4 & 58,6 & 50,5 & 90,8 \\
\hline Sehr gut & 23,5 & 22,1 & 20,7 & 31,9 & 95,1 \\
\hline
\end{tabular}

158 Ein Grund hierfür liegt im hohen Anteil an Schüler/innen mit Migrationshintergrund, deren Eltern häufig selbst nicht studiert haben, sich aber gleichzeitig ein Studium für ihre Kinder wünschen (vgl. Becker 2010). 


\begin{tabular}{|c|c|c|c|c|c|}
\hline & $\begin{array}{l}\text { Total } \\
\text { N: } 518\end{array}$ & $\begin{array}{l}\text { Uni0 } \\
\mathrm{N}: 316\end{array}$ & $\begin{array}{l}\text { Uni1 } \\
\mathrm{N}: 111\end{array}$ & $\begin{array}{l}\text { Uni2 } \\
\mathrm{N}: 91\end{array}$ & $\begin{array}{l}\text { Intention } \\
\mathrm{N}: 468\end{array}$ \\
\hline \multicolumn{6}{|c|}{ Ertragserwartung: Aussicht auf angesehenen Job durch Studium } \\
\hline Schlecht/mittel & 17,6 & 14,6 & 20,7 & 24,2 & 87,9 \\
\hline Gut & 55 & 57,9 & 49,6 & 51,6 & 89,8 \\
\hline Sehr gut & 27,4 & 27,5 & 29,7 & 24,2 & 93 \\
\hline \multicolumn{6}{|c|}{ Soziale und kulturelle Ressourcen } \\
\hline \multicolumn{6}{|c|}{ Wahrgenommene Studienaspiration Eltern } \\
\hline $\mathrm{Ja}$ & 83 & 81,7 & 83,8 & 86,8 & 92,1 \\
\hline Nein & 17 & 18,3 & 16,2 & 13,2 & 81,8 \\
\hline \multicolumn{6}{|l|}{ Freunde mit Studienaspiration } \\
\hline Bis zu $50 \%$ & 42,5 & 48,7 & 34,2 & 30,8 & 85,9 \\
\hline Mehr als die Hälfte & 57,5 & 51,3 & 65,8 & 69,2 & 93,6 \\
\hline \multicolumn{6}{|c|}{ Studienerfahrung in Verwandtschaft } \\
\hline (Fast) keine & 32,2 & 45,3 & 18,9 & 3,3 & 88,6 \\
\hline Bis zu $50 \%$ & 42,1 & 46,2 & 41,4 & 28,6 & 90,4 \\
\hline Mehr als die Hälfte & 25,7 & 8,5 & 39,7 & 68,1 & 92,5 \\
\hline \multicolumn{6}{|c|}{ Unterstützung bei Studienbewerbung } \\
\hline Sehr/eher unwahrscheinlich & 11,2 & 13,3 & 6,3 & 9,9 & 86,2 \\
\hline Eher wahrscheinlich & 26,6 & 28,2 & 29,7 & 17,6 & 89,9 \\
\hline Sehr wahrscheinlich & 62,2 & 58,5 & 64 & 72,5 & 91,3 \\
\hline
\end{tabular}

Quelle: Best Up-Individualdaten, eigene Berechnungen

Die (weitestgehend) erwarteten Zusammenhänge zwischen den erklärenden Variablen und sozialer Herkunft auf der einen und der abhängigen Variable Studienintention auf der anderen Seite sprechen zunächst für eine mögliche Mediation der Herkunftsdifferenz. Im Folgenden wird dies mithilfe multivariater Modelle geprüft. Hierfür werden dem Basismodell (Bildungsherkunft und Kontrollvariablen, M1) sukzessive die drei Indikatorenblöcke hinzugefügt: Modell 2 enthält zusätzlich die Leistungsvariablen, denen in den Modellen 3 und 4 weitere RC-Indikatoren bzw. die Indikatoren für soziale und kulturelle Ressourcen hinzugefügt werden. Im letzten Modell (M5) sind schließlich alle Variablen enthalten. Abbildung 7.2 zeigt den geschätzten Prozentpunkteunterschied (Discrete Changes) in der Intentionswahrscheinlichkeit zwischen Nichtakademikerkindern (Referenzkategorie) und den beiden anderen Herkunftsgruppen über die verschiedenen Modellspezifikationen hinweg. In Anhangstabelle A7.4 finden sich vollständige Informationen zu allen Modellen. 
Abb. 7.2: Intentionswahrscheinlichkeit: Veränderung sozialer Herkunftsunterschiede über verschiedene Modellspezifikationen (90\%-Cl)

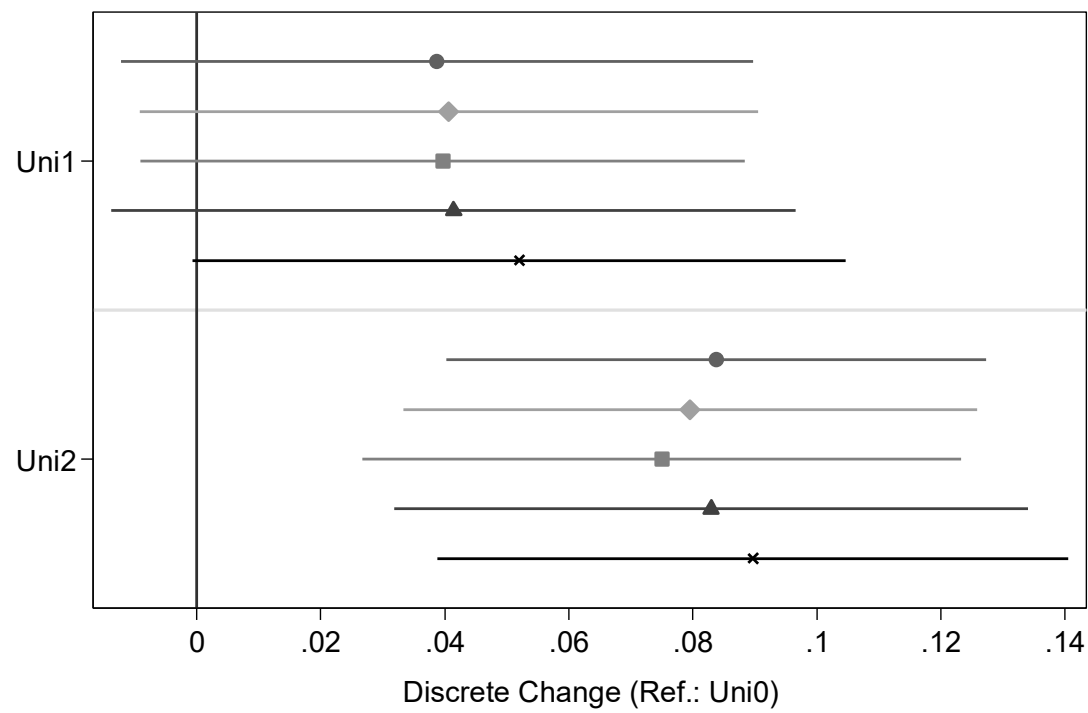

- M1 $\bullet \mathrm{M} 2-\mathrm{M} 3 \triangle \mathrm{M} 4 \times \mathrm{M} 5$

M1: Basismodell: soziale Herkunft + zentrale Kontrollvariablen

M2: + Leistungsindikatoren (HB1/I)

M3: + Leistungsindikatoren + RC (HB2/l)

M4: + Leistungsindikatoren + soziale und kulturelle Ressourcen (HB3/I)

M5: vollständiges Modell: alle Kovariate

Quelle: Best Up-Individualdaten, eigene Berechnungen, ausführliche Darstellung der Modelle: Tabelle A7.4

Logistische random-intercept Modelle, ausgewiesen: DC mit 90\%-Cl (alle weiteren Variablen gehen wie beobachtet in die Berechnung ein)

Das Hinzufügen der erklärenden Variablen zum Basismodell scheint insgesamt die Herkunftsdifferenz von circa 8 Prozentpunkten zwischen Nichtakademikerkindern und Doppelakademikerkindern nur geringfügig zu ändern (vgl. Abbildung 7.2). Während die Herkunftsdifferenz bei Berücksichtigung der Leistungs- und RC-Indikatoren zunächst leicht sinkt (von 8,4 Prozentpunkten auf 8 und 7,5), steigt sie nach Einschluss der Ressourcenindikatoren und insbesondere im vollen Modell wieder an (auf 8,3 und 9 Prozentpunkte). Weitere, hier nicht präsentierte Analysen zeigen, dass insbesondere die Variablen „Studienerfahrung in Verwandtschaft“ sowie „Unterstützung bei Studienbewerbung" als Suppressorvariablen fungieren und so für die geringe Erklärungskraft der Ressourcenvariablen verantwortlich sind. Werden diese nicht 
berücksichtigt, sinkt die Herkunftsdifferenz zwischen Befragten mit zwei und ohne akademisch gebildete Eltern in Modell 4 auf 6,8 Prozentpunkte (von 8,3). Es scheint hier also vor allem die normative Dimension sozialer und kultureller Ressourcen in Form von Erwartungshaltung und Rollenvorbildern im engeren sozialen Netzwerk zu sein, die Herkunftsdifferenzen zumindest tendenziell vermitteln.

Für einen stringenten Test der Mediationshypothesen sollten die Koeffizienten nicht nur ,,per Augenschein“ (Auspurg und Hinz 2011b: 67) über verschiedene Modelle verglichen werden, sondern der Unterschied auch auf Signifikanz getestet werden (vgl. auch Gelman und Stern 2006). Im vorliegenden Fall verweist jedoch bereits der Augenschein aufgrund der extremen Überlappung der Konfidenzintervalle darauf, dass die Unterschiede zwischen den Punktschätzern nicht nur gering, sondern sehr wahrscheinlich auch nicht signifikant sind. ${ }^{159}$

Zuletzt soll in Tabelle 7.4 das vollständige Modell kurz besprochen werden, aus dem hervorgeht, ob die erklärenden Variablen mit der Übersetzungswahrscheinlichkeit von Aspirationen in Intentionen zusammenhängen. Neben der Bildungsherkunft, der Erwartung eines hohen Einkommens sowie der wahrgenommenen Aspiration der Eltern ist es vor allem die wahrgenommene Erfolgswahrscheinlichkeit, die positiv mit der Studienintention assoziiert ist. Zudem hängt die mittlere Kostenkategorie (im Vergleich zu sehr hohen wahrgenommenen Kosten) positiv mit der Studienintention zusammen. Gleiches gilt erstaunlicherweise nicht für als gering eingeschätzte Kosten. Die Schulleistung hat hingegen keinen deutlichen (und sicheren) Einfluss.

159 Bestätigt wird dies mithilfe des in Stata implementierten Tests für "seemingly unrelated estimates“" (suest), der auch für Vergleiche zwischen Modellen, die auf denselben Daten beruhen, geeignet ist. Da dieser nicht mit den hier verwendeten Modellen kompatibel ist, wurden lineare Wahrscheinlichkeitsmodelle mit cluster-robusten Standardfehlern spezifiziert. (Da die Intraklassenkorrelation (Rho, vgl. Tabelle A7.4) extrem klein ist, sollte ein Wechsel von GLMM auf Modelle mit cluster-robusten Standardfehlern hier trotz der geringen Anzahl an Clustern kein Problem darstellen.) Der Test bestätigt den Eindruck, dass sich die Herkunftseffekte nicht zwischen den verschiedenen Modellspezifikationen unterscheiden. 
Tab. 7.4: Übersetzungswahrscheinlichkeit von Studienaspirationen in -intentionen: multivariate Ergebnisse

\begin{tabular}{ll}
\hline & M5 \\
\hline Soziale Herkunft Ref.: Uni0 & \\
Uni1 & $0,052(0,032)$ \\
Uni2 & $0,090^{* \star *}(0,031)$ \\
\hline
\end{tabular}

Leistungsindikatoren

Note Deutsch 2. KH Ref.: unteres Quartil

Mitte

$-0,004(0,035)$

Oberes Quartil

$0,053(0,035)$

Note Mathe 2. KH Ref.: unteres Quartil

Mitte

$0,001(0,033)$

Oberes Quartil

$0,016(0,035)$

Grundfähigkeiten (z-standardisiert)

Figural

$-0,035^{\star \star}(0,015)$

Verbal

$0,003(0,015)$

Rational Choice

Erfolgswahrscheinlichkeit* Ref.: (sehr) unwahrscheinlich - teils/teils

(Sehr) wahrscheinlich

$0,114^{* * *}(0,033)$

Kostenerwartung: Wie schwer fällt Studienfinanzierung Ref.: (sehr) schwer

Weder noch

$0,063^{* *}(0,029)$

(Sehr) leicht

$-0,001(0,037)$

Ertragserwartung: hohes Einkommen durch Studium Ref.: schlecht/mittel

Gut

Sehr gut

$0,056(0,040)$

Ertragserwartung: angesehener Job durch Studium Ref.: schlecht/mittel

$0,087^{*}(0,044)$

Gut

$-0,003(0,033)$

Sehr gut

$0,008(0,039)$

Soziale und kulturelle Ressourcen

Freunde mit Studienaspiration: mehr als die Hälfte

$0,042(0,027)$

Wahrgenommene Studienaspiration Eltern

$0,072^{*}(0,037)$

Studienerfahrung in Verwandtschaft Ref.: (fast) keine

Bis zu $50 \%$

$-0,014(0,026)$

Mehr als $50 \%$

$-0,065(0,048)$

Unterstützung bei Studienbewerbung Ref.: eher/sehr unwahrscheinlich

Eher wahrscheinlich

$0,023(0,040)$

Sehr wahrscheinlich

$0,004(0,038)$

Pseudo-R² (Maddala)

0,09

Rho

0,02

N Befragte/Schulen

$518 / 19$

Quelle: Best Up-Individualdaten, eigene Berechnungen logistisches random-intercept Modell, AME/DC (Standardfehler), *** $p<0,01 ;{ }^{* *} p<0,05 ;{ }^{*} p<0,1$ zusätzlich enthalten: Kontrollvariablen (Migrationshintergrund, Geschlecht und Schultyp)

*Für die multivariaten Modelle wurden die Kategorien „teils/teils“ und "sehr wahrscheinlich“ der Variable Erfolgswahrscheinlichkeit zusammengefasst, da "sehr wahrscheinlich" das Outcome perfekt vorhersagt (siehe Tabelle 7.3). 
Insgesamt haben die Ergebnisse verdeutlicht, dass zwar die meisten Studienaspirant/innen auch Studienintentionen äußern, hierbei jedoch gleichzeitig Herkunftsunterschiede - insbesondere zwischen Nichtakademikerkindern und Doppelakademikerkindern - bestehen (HA/I).

Das Hinzufügen der Leistungsindikatoren reduziert den Herkunftseffekt kaum. Im Falle der RC-Indikatoren (und auch der Aspirationen von Eltern und Freunden) reduziert dieser sich zwar geringfügig, doch kann ein tatsächlicher Unterschied zwischen den Modellspezifikationen nicht präzise geschätzt und somit nicht sicher festgestellt werden. Insgesamt finden die Hypothesen des Blocks B, die von einer Mediation des Herkunftseffektes durch Schulleistung (HB1/I), RC-Indikatoren (HB2/I) und soziales und kulturelles Kapital (HB3/I) ausgingen, in dieser ersten Phase also kaum Unterstützung. Selbst bei gleicher Leistung, gleicher Einschätzung von Kosten, Nutzen und Erfolgswahrscheinlichkeit eines Studiums und gleicher Ausstattung an kulturellen und sozialen Ressourcen sind hier also Herkunftsunterschiede bezüglich der Übersetzung von Studienaspirationen in Studienintentionen zu verzeichnen. Erklärungen für diese sehr robusten Herkunftsunterschiede müssen also an anderer Stelle gesucht werden.

Wie bereits in Kapitel 5 angedeutet, wäre theoretisch auch denkbar, dass die erklärenden Variablen den Einfluss sozialer Herkunft moderieren. Aufgrund der sehr schiefen Verteilung der abhängigen Variable und der teilweise sehr geringen Zellbesetzung ist die Berechnung kategorialer Interaktionen nicht möglich oder sinnvoll, da die Schätzungen zum Teil auf Fallzahlen im unteren einstelligen Bereich beruhen würden.

\subsubsection{Institutionelle Opportunitäten und Barrieren}

Im Folgenden wird getestet, ob institutionelle Hürden (und deren Wahrnehmung) mit der Übersetzungswahrscheinlichkeit von Studienaspirationen in -intentionen zusammenhängen. Hier steht zunächst die durchschnittliche $\mathrm{Zu}$ gangsbeschränkung und Selektivität des angestrebten Studienfaches im Mittelpunkt und die Frage, ob diese sozialgruppenspezifisch auf eine mögliche „Umentscheidung“ (d.h. auf die Intentionswahrscheinlichkeit) oder eine „Umortientierung“" (d.h. auf die Übereinstimmung zwischen angestrebtem und intendiertem Fach) wirkt. Hier ist zu beachten, dass nur über Befragte Aussagen getroffen werden können, für die eine Angabe zu ihrer Fachaspiration vorliegt, die ihre Wünsche also bereits konkretisiert haben.

Im Anschluss daran wird mithilfe des FS der sozialgruppenspezifische Einfluss weiterer institutionelle Hürden und Opportunitäten (geographische Distanz, komplexe Auswahlverfahren, Informationen und Reputation) untersucht. 


\section{„Umentscheidung“: Zugangshürden und Intentionswahrscheinlichkeit}

Wie mehrfach verdeutlicht wurde, variieren Zugangshürden im deutschen Kontext stark über verschiedene Studienfächer. In Abschnitt 5.4.3 wurde die Annahme formuliert, dass Schüler/innen diese antizipieren und einbeziehen, wenn sie ihre idealistische Aspiration in eine realistische Intention übersetzen und dass sozial schwächere Schüler/innen hiervon stärker abgeschreckt werden könnten (HC2/I).

Tabelle 7.5 liefert deskriptive Informationen zu den in Abschnitt 6.3 vorgestellten Variablen zur durchschnittlichen Zugangsbeschränkung und Selektivität der Studienfächer, die die Befragten anstreben (für detaillierte Informationen differenziert nach sozialer Herkunft und den Ausprägungen der abhängigen Variable siehe Anhangstabelle A7.5). Zum Beispiel streben die Befragten im Durchschnitt Fächer an, in denen $76,1 \%$ der angebotenen Studiengänge (in den Rekrutierungsdaten) zulassungsbeschränkt sind und in denen im Vorjahr im Durchschnitt eine Abiturnote von 1,9 benötigt wurde, um über die Abiturbestenquote zugelassen zu werden.

Tab. 7.5: Durchschnittliche Zugangshürden des angestrebten Studienfaches: Deskriptive Information

\begin{tabular}{llll}
\hline & Mean & SD & Min-Max \\
\hline Zulassungsbeschränkung (\%) & 76,1 & 15,8 & $33,3-100$ \\
Eignungsfeststellung (\%) & 28,4 & 20,9 & $7,5-79,2$ \\
Zulassungsbeschränkung oder Eignungsfeststellung (\%) & 86,3 & 10,6 & $70,1-100$ \\
DN (durchschnittliche Auswahlgrenze) & 1,9 & 0,4 & $1,2-2,3$ \\
DN inkl. „alle zugelassen“ & 2,2 & 0,5 & $1,2-2,8$ \\
DN inkl. „alle zugelassen“ + zulassungsfrei & 2,7 & 0,6 & $1,5-3,6$ \\
\hline
\end{tabular}

Quelle: Best Up-Individualdaten + Rekrutierungsdaten, eigene Berechnungen, N: 500

Bivariat zeigen sich zunächst kaum Unterschiede bezüglich der Durchschnittwerte dieser Variablen, weder zwischen Schüler/innen mit und ohne Intention noch zwischen den Herkunftsgruppen (vgl. Tabelle A7.5). Letzteres bedeutet, dass Schüler/innen unterschiedlicher sozialer Herkunft im Durchschnitt sehr ähnlich selektive und beschränkte Fächer anstreben. ${ }^{160}$ Multivariat bestätigt sich, dass keine der Zugangshürden mit der Intentionswahrscheinlichkeit zu-

160 Der einzig nennenswerte Herkunftsunterschied kann bezüglich des Anteils an Studiengängen mit Eignungsfeststellungsverfahren im angestrebten Fach beobachtet werden: Befragte aus nichtakademisch gebildeten Elternhäusern scheinen im Durchschnitt eher Studiengänge mit einem etwas höheren Anteil an Eignungsfeststellungsverfahren studieren zu wollen (30\%) als Befragte aus einem Doppelakademikerhaushalt (25\%, Uni1: 28\%). 
sammenhängt. Zudem zeigt sich erneut eine über die Modelle stabile Herkunftsdifferenz (Tabelle 7.6). ${ }^{161}$

Trotz fehlenden generellen Zusammenhangs können Zugangshürden wie in Hypothese HC2/I angenommen - mit der sozialen Herkunft interagieren, d.h. als mehr oder weniger stark einschränkend wahrgenommen werden. Dass dies in der Tendenz tatsächlich der Fall ist, zeigt Abbildung 7.3. Die Plots basieren auf den in Tabelle 7.6 präsentierten Modellen, denen zusätzlich ein Interaktionsterm zwischen der Bildungsherkunft und der jeweiligen Zugangshürde hinzugefügt wurde. Dargestellt sind die vorhergesagten Wahrscheinlichkeiten der Übersetzung einer Studienaspiration in eine Studienintention für die drei Herkunftsgruppen an unterschiedlichen Werten der Zugangshürden. Oben links zeigt sich, dass die Intentionswahrscheinlichkeit für Akademikerkinder kaum mit der durchschnittlichen Zulassungsbeschränkung ihres angestrebten Faches variiert, während sie für Nichtakademikerkinder sinkt, wenn die durchschnittliche Zulassungsbeschränkung steigt. Gleiches gilt nicht für den Anteil an Eignungsfeststellungsverfahren: Dieser scheint in der Tendenz minimal positiv (jedoch nicht signifikant ${ }^{162}$ ) mit der Intentionswahrscheinlichkeit aller Herkunftsgruppen zusammenzuhängen, geringfügig stärker jedoch für Akademikerkinder. Zusammengenommen spiegelt sich dies im deutlich differentiellen Zusammenhang zwischen der ,durchschnittlichen Zulassungsbeschränkung oder Eignungsfeststellungsverfahren" und der Intentionswahrscheinlichkeit im oberen rechten Teil von Abbildung 7.3 wider.

$161 \mathrm{Im}$ Gegensatz zu den Analysen in Abschnitt 7.2.1 können hier Befragte ohne genannte Fachaspiration nicht berücksichtigt werden (vgl. Tab. A7.3 für hierdurch entstehende Selektivitäten). Dieser angepasste Samplezuschnitt (und nicht das Hinzufügen der Zugangshürden-Variablen) ist der Grund für den leichten Anstieg (und die Signifikanz) des Unterschieds zwischen Einakademikerkindern und Nichtakademikerkindern.

162 Getestet wurde hierfür nicht nur der Gesamteinfluss der Zugangshürden-Variablen (Tabelle 7.6), sondern auch, ob die Steigungen der einzelnen Herkunftsgruppen in Abbildung 7.3 sich signifikant von Null unterscheiden (vgl. Mitchell 2012). 
Tab. 7.6: Intentionswahrscheinlichkeit: soziale Herkunft und Zugangsbarrieren des angestrebten Studienfaches

\begin{tabular}{|c|c|c|c|c|c|c|}
\hline & M1 & M2 & M3 & M4 & M5 & M6 \\
\hline \multicolumn{7}{|c|}{ Soziale Herkunft (Ref. Unio) } \\
\hline Uni1 & $\begin{array}{l}0,067^{* *} \\
(0,028)\end{array}$ & $\begin{array}{l}0,068^{* *} \\
(0,028)\end{array}$ & $\begin{array}{l}0,066^{* *} \\
(0,028)\end{array}$ & $\begin{array}{l}0,067^{* *} \\
(0,028)\end{array}$ & $\begin{array}{l}0,067^{* \star} \\
(0,028)\end{array}$ & $\begin{array}{l}0,067^{\star \star} \\
(0,028)\end{array}$ \\
\hline Uni2 & $\begin{array}{l}0,082^{\star \star *} \\
(0,029)\end{array}$ & $\begin{array}{l}0,083^{* * *} \\
(0,029)\end{array}$ & $\begin{array}{l}0,081^{* * *} \\
(0,029)\end{array}$ & $\begin{array}{l}0,082^{* * *} \\
(0,029)\end{array}$ & $\begin{array}{l}0,083^{\star \star *} \\
(0,029)\end{array}$ & $\begin{array}{l}0,082^{\star \star *} \\
(0,029)\end{array}$ \\
\hline \multicolumn{7}{|c|}{ Durchschnittliche Zugangsbeschränkung/Selektivität } \\
\hline $\begin{array}{l}\text { Zulassungs- } \\
\text { beschränkung }\end{array}$ & $\begin{array}{l}-0,0006 \\
(0,0008)\end{array}$ & & & & & \\
\hline Eignungsfeststellung & & $\begin{array}{l}0,0005 \\
(0,0006)\end{array}$ & & & & \\
\hline $\begin{array}{l}\text { Zulassungs- } \\
\text { beschränkung oder } \\
\text { Eignungsfeststellung }\end{array}$ & & & $\begin{array}{l}-0,0007 \\
(0,001)\end{array}$ & & & \\
\hline $\mathrm{DN}$ & & & & $\begin{array}{l}0,023 \\
(0,035)\end{array}$ & & \\
\hline $\begin{array}{l}\text { DN inkl. „alle } \\
\text { zugelassen“ }\end{array}$ & & & & & $\begin{array}{l}0,024 \\
(0,025)\end{array}$ & \\
\hline $\begin{array}{l}\text { DN inkl. „alle } \\
\text { zugelassen“ }+ \\
\text { zulassungsfrei }\end{array}$ & & & & & & $\begin{array}{l}0,02 \\
(0,02)\end{array}$ \\
\hline Rho & 0,10 & 0,10 & 0,10 & 0,10 & 0,10 & 0,10 \\
\hline $\mathrm{BIC}$ & 379,68 & 379,79 & 380,05 & 379,93 & 379,48 & 379,34 \\
\hline AIC & 316,46 & 316,57 & 316,83 & 316,71 & 316,27 & 316,12 \\
\hline $\mathrm{R}^{2}$ (Maddala) & 0,035 & 0,034 & 0,034 & 0,034 & 0,035 & 0,035 \\
\hline N Befragte/Schulen & $500 / 19$ & $500 / 19$ & $500 / 19$ & $500 / 19$ & $500 / 19$ & $500 / 19$ \\
\hline
\end{tabular}

Quelle: Best Up-Individualdaten + Rekrutierungsdaten, eigene Berechnungen logistisches random-intercept Modell, AME/DC (Standardfehler), ${ }^{* *} p<0,01 ;{ }^{* *} p<0,05 ;{ }^{*} p<0,1$ In allen Modellen enthalten: Leistungsindikatoren und Kontrollvariablen (Migrationshintergrund, Geschlecht und Schultyp)

Ein ähnliches Bild vermitteln die Variablen zur Selektivität des angestrebten Studienfaches. Hier ist zu beachten, dass die x-Achse entgegengesetzt zu der oberen Reihe verläuft. Links sind die sehr selektiven Studiengänge abgetragen, für die im Vorjahr im Durchschnitt ein einser Abitur nötig war, um über die Abiturbestenquote zugelassen zu werden. Zur Erinnerung: Im mittleren und rechten Teil der Abbildung wurde zulassungsbeschränkten Studiengängen, für die alle Bewerber/innen zugelassen wurden, und zusätzlich zulassungsfreien Studiengängen der Wert 4,0 (Abitur bestanden) zugewiesen, sodass die Variablen höhere Werte annehmen und der Range größer wird. Während am sehr selektiven Ende ein deutlicher Herkunftsunterschied von fast 20 Prozentpunkten geschätzt wird, ist dieser am anderen Ende der Selektivitätsachse weitestgehend verschwunden. 
Die Konfidenzintervalle in Abbildung 7.3 geben zwar einen Eindruck von der Präzision der Schätzung an den verschiedenen Werten der ZugangshürdenVariablen, jedoch kann auf Grundlage sichtbarer Überschneidungen nicht abgeleitet werden, dass der Unterschied zwischen den Herkunftsgruppen nicht signifikant ist. Dies wurde separat getestet, erstens durch Betrachtung der Interaktionsterme in den logistischen random intercept Modellen, auf denen die Plots basieren. Diese erreichen meist keines der standardmäßig ausgewiesenen Signifikanzniveaus (nur der Einfluss der Variable „Zulassungsbeschränkung oder Eignungsfeststellung" variiert signifikant zwischen Uni0 und Uni2, p: 0,081 ; nicht ausgewiesen).

Abb. 7.3: Vorhergesagte Intentionswahrscheinlichkeit (90\%-Cl) nach Zugangshürden des angestrebten Faches und sozialer Herkunft
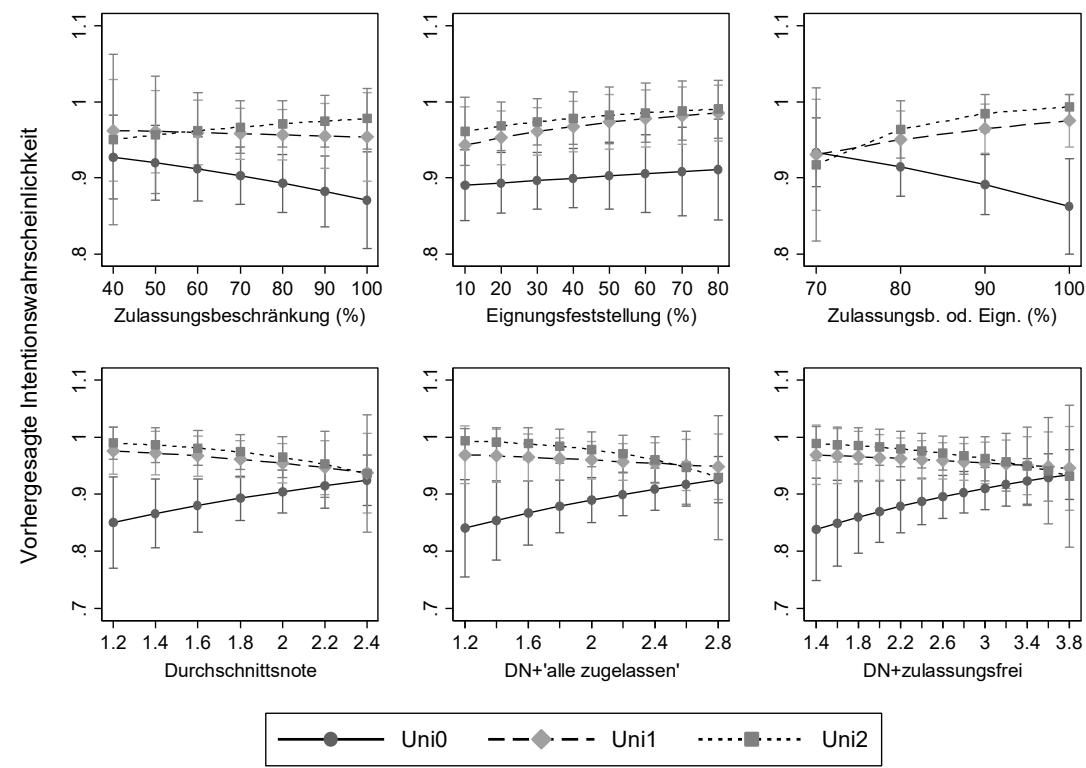

Quelle: Best Up-Individualdaten + Rekrutierungsdaten, eigene Berechnungen Logistische random-intercept Modelle (Leistungsindikatoren und Kontrollvariablen jeweils bei ihrem Mittelwert).

An bestimmten Werten der Variablen zur Messung der Zulassungsbeschränkung/Selektivität des angestrebten Faches kann sich die Intentionswahrscheinlichkeit von Befragten unterschiedlicher sozialer Herkunft dennoch signifikant unterscheiden. Daher wird zweitens der „Kontrast“ in der Intentionswahrscheinlichkeit zwischen Befragten aus Doppel-/Einakademikerhaushalten und 
Befragten aus Nichtakademikerhaushalten mithilfe eines Wald-Tests auf Signifikanz geprüft und in Abbildung 7.4 visualisiert (vgl. Mitchell 2012). Schneidet das Konfidenzintervall die Null-Linie, ist der Unterschied nicht auf dem 10\%-Niveau signifikant. Zum Beispiel erreichen die Unterschiede von etwa 6 bis etwa 11 Prozentpunkten zwischen den Kategorien Uni0 und Uni2 ab einer durchschnittlichen Zulassungsbeschränkung von etwa 70\% statistische Signifikanz.

Insgesamt zeigt sich, dass die Unterschiede zwischen den Herkunftsgruppen im oberen und oft auch mittleren Beschränkungs-/Selektivitätsbereich signifikant und durchaus substantiell sind, mit sinkender Selektivität jedoch sowohl an Substanz als auch Signifikanz einbüßen. Wie sich bereits angedeutet hat, fällt nur der Anteil an Studiengängen mit Eignungsfeststellungsverfahren aus diesem Muster. Insgesamt deuten die Ergebnisse in Richtung von HC2/I, die von einem differentiellen Einfluss antizipierter Zulassungshürden ausgeht: Während sie deutliche Hürden für Nichtakademikerkinder darzustellen scheinen, scheinen Akademikerkinder zumindest in dieser ersten Phase kaum von Zugangsbarrieren beeinflusst zu sein.

Abb. 7.4: Herkunftsunterschiede in der vorhergesagten Intentionswahrscheinlichkeit an unterschiedlichen Werten der Zugangshürden des angestrebten Faches $(90 \%-\mathrm{Cl})$
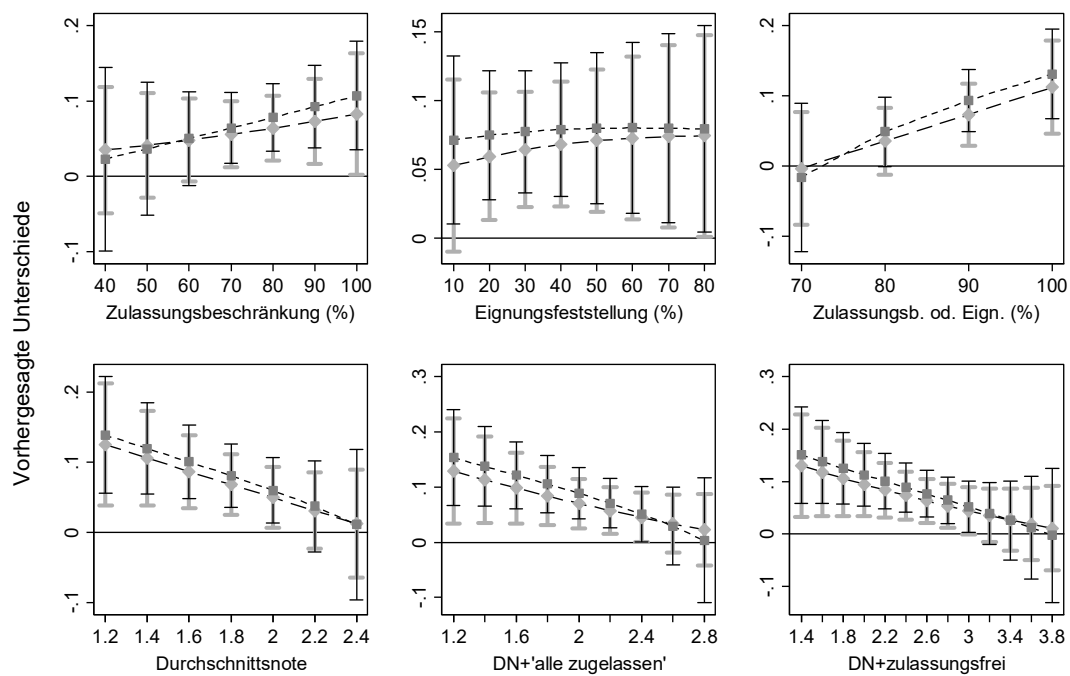

$$
\text { - - - Uni1 vs. Uni0 -..-. Uni2 vs. Uni0 }
$$

Quelle: Best Up-Individualdaten + Rekrutierungsdaten, eigene Berechnungen Logistische random-intercept Modelle (Leistungsindikatoren und Kontrollvariablen jeweils bei ihrem Mittelwert). Graues Konfidenzintervall: Uni1 vs. Uni0, schwarzes Konfidenzintervall: Uni2 vs. Uni0 
„Umorientierung“: Zugangshürden und die Wahrscheinlichkeit übereinstimmender Studienfachaspirationen und -intention

Neben dieser „Umentscheidung“ zwischen Studienaspiration und Studienintention, die für Nichtakademikerkinder stärker mit den wahrgenommenen $\mathrm{Zu}-$ gangshürden zu ihrem angestrebten Fach zusammenzuhängen scheint als für Akademikerkinder, wurde zudem angenommen, dass diese Zugangshürden auch zu einer „Umorientierung“ innerhalb tertiärer Bildung führen können (HC3/I). Die folgenden Analysen sind damit konditional und beschränken sich auf Befragte, die ihre Studienaspiration zunächst generell in eine Studienintention übersetzten.

Deskriptiv zeigt sich, dass die Übersetzung von Fachwünschen in entsprechende Fachintentionen zwar etwas geringer als die zuvor berichtete Übersetzung einer generellen Studienaspiration in eine Studienintention (90\%) ist, doch ist auch die fachliche Übereinstimmung mit insgesamt $79 \%$ hoch.

Auch hier zeigt sich eine Herkunftsdifferenz zwischen den beiden extremen Herkunftsgruppen (Uni0: 78\% vs. Uni2: 84\%), die allerdings etwas geringer ausfällt und statistisch nicht sigifikant ist (p: 0,266). Schüler/innen aus Einakademikerhaushalten liegen in diesem Fall nicht in der Mitte, sondern mit 77\% sehr nah an der Gruppe der Nichtakademikerkinder. Weiterhin deutet sich deskriptiv bereits an, dass die durchschnittliche Zulassungsbeschränkung sowie Selektivität leicht negativ mit der Übersetzungswahrscheinlichkeit von Fachaspirationen in Fachintentionen zusammenhängt (vgl. Anhangstabelle A7.5). ${ }^{163}$

Diese deskriptiven Befunde spiegeln sich auch in den multivariaten Modellen wieder (Tabelle 7.7). Zum einen zeigen sich weder substantielle noch signifikante Herkunftsunterschiede. Zum anderen zeigt sich die „Umorientierungswirkung" (der meisten) Zugangshürden, auch nach Kontrolle der Leistungsindikatoren und weiterer Variablen. So verringert sich die Wahrscheinlichkeit einer fachlichen Übereinstimmung um 0,3 Prozentpunkte, wenn der Anteil zulassungsbeschränkter Studiengänge im angestrebten Fach um 1\% steigt. ${ }^{164}$ Sinkt die durchschnittliche Selektivität des angestrebten Faches um

163 Zum Beispiel streben Befragte mit Übereinstimmung im Durchschnitt Fächer an, die insgesamt weniger selektiv sind (DN: 2,8), als Befragte ohne Übereinstimmung (DN: 2,4). Aus dieser Logik heraus fällt der Anteil an Eignungsfeststellungsverfahren im angestrebten Fach, der positiv mit der Übereinstimmung zwischen fachlicher Aspiration und Intention assoziiert zu sein scheint (vgl. Tabelle A7.5).

164 Geht man von einem linearen Zusammenhang aus, sind dies fast 10 Prozentpunkte bei einem Anstieg der durchschnittlichen Zulassungsbeschränkung um 30\%. Neben dem linearen Zusammenhang wurden auch andere Spezifikationen getestet, die allerdings die Modellgüte nicht verbesserten. 
einen Notenpunkt (etwa von 1 auf 2), dann steigt die Wahrscheinlichkeit einer Übereinstimmung um 26 Prozentpunkte. ${ }^{165}$

Tab. 7.7: Wahrscheinlichkeit der Übereinstimmung zwischen Studienfachaspiration und -intention: soziale Herkunft und Zugangsbarrieren des angestrebten Studienfaches

\begin{tabular}{lllllll}
\hline & M1 & M2 & M3 & M4 & M5 & M6 \\
\hline \multicolumn{7}{c}{ Soziale Herkunft (Ref. Uni0) } \\
\hline Uni1 & $-0,031$ & $-0,024$ & $-0,033$ & $-0,019$ & $-0,013$ & $-0,018$ \\
& $(0,053)$ & $(0,053)$ & $(0,054)$ & $(0,052)$ & $(0,051)$ & $(0,052)$ \\
Uni2 & 0.0304 & 0,042 & 0,028 & 0,056 & 0,057 & 0,049 \\
& $(0.0536)$ & $(0,052)$ & $(0,054)$ & $(0,05)$ & $(0,05)$ & $(0,05)$ \\
\hline
\end{tabular}

Durchschnittliche Zugangsbeschränkung/Selektivität

\begin{tabular}{|c|c|c|c|c|c|c|}
\hline Zulassungsbeschränkung & $\begin{array}{l}-0,003^{*} \\
(0,001)\end{array}$ & & & & & \\
\hline Eignungsfeststellung & & $\begin{array}{l}0,003^{* * *} \\
(0,001)\end{array}$ & & & & \\
\hline $\begin{array}{l}\text { Zulassungsbeschränkung } \\
\text { oder Eignungsfeststellung }\end{array}$ & & & $\begin{array}{l}-0,003 \\
(0,002)\end{array}$ & & & \\
\hline DN & & & & $\begin{array}{l}0,260^{* * *} \\
(0,05)\end{array}$ & & \\
\hline DN, inkl. „alle zugelassen“ & & & & & $\begin{array}{l}0,193^{\star * *} \\
(0,037)\end{array}$ & \\
\hline $\begin{array}{l}\text { DN, inkl. „alle zugelassen“ + } \\
\text { zulassungsfrei }\end{array}$ & & & & & & $\begin{array}{l}0,143^{\star \star \star} \\
(0,03)\end{array}$ \\
\hline Rho & 0 & 0 & 0 & 0 & 0 & 0 \\
\hline $\mathrm{BIC}$ & 483,8 & 477,29 & 485 & 462,82 & 461,18 & 464,89 \\
\hline AIC & 423,66 & 417,16 & 424,86 & 402,68 & 401,05 & 404,75 \\
\hline $\mathrm{R}^{2}$ (Maddala) & 0,05 & 0,06 & 0,05 & 0,09 & 0,09 & 0,09 \\
\hline N Befragte/Schulen & $407 / 19$ & $407 / 19$ & $407 / 19$ & $407 / 19$ & $407 / 19$ & $407 / 19$ \\
\hline
\end{tabular}

Quelle: Best Up-Individualdaten + Rekrutierungsdaten, eigene Berechnungen logistisches random-intercept Modell, AME/DC (Standardfehler), ${ }^{* * *} p<0,01 ;{ }^{* *} p<0,05 ;{ }^{*} p<0,1$ In alle Modellen enthalten: Leistungsindikatoren und Kontrollvariablen (Migrationshintergrund, Geschlecht und Schultyp)

Auch hier zeigt sich der zunächst eher unintuitive Zusammenhang zwischen Eignungsfeststellungsverfahren und der abhängigen Variable: Die Wahrscheinlichkeit einer Übereinstimmung zwischen Fachaspiration und -intention

165 Neben den untransformierten Werten wurden die Berechnungen auch mit z-standardisierten Variablen für die Zugangshürden vorgenommen. Entsprechend zeigt sich, dass die Abnahme der Effektstärke über die Selektivitätsvariablen der unterschiedlichen Skalierung geschuldet ist. Tatsächlich unterscheiden sich die Z-standardisierten Koeffizienten nur sehr geringfügig. Zudem wird ersichtlich, dass die Selektivitätsvariablen mit der abhängigen Variable etwas stärker positiv zusammenhängen: So liegt der standardisierte Koeffizient für Zulassungsbeschränkungen bei 0,04, für Eignungsfeststellungsverfahren bei -0,07 und für die Auswahlgrenzen $(\mathrm{DN})$ bei 0,09 . 
steigt mit steigendem Anteil an Studiengängen mit Eignungsfeststellungsverfahren im angestrebten Fach. Mögliche Gründe hierfür werden am Ende dieses Abschnittes diskutiert.

Im vorangegangenen Abschnitt wurde angenommen und gezeigt, dass $\mathrm{Zu}-$ gangshürden bei sozial privilegierten Schüler/innen, deren Statuserhalt stärker an ein Hochschulstudium geknüpft ist, nicht zu einer Umentscheidung (hier: keine Studienintention) führen. Zugangshürden im angestrebten Fach könnten aber durchaus auch in dieser Gruppe zu einer „realistischeren“ Wahl und somit zu einer Diskrepanz zwischen Fachaspiration und Fachintention führen. Eine Interaktion wurde daher in diesem Schritt nicht angenommen (HC3/I).

Abb. 7.5: Vorhergesagte Wahrscheinlichkeit der Übereinstimmung zwischen Studienfachaspiration und -intention $(90 \%-\mathrm{Cl})$ nach Zugangshürden des angestrebten Faches und sozialer Herkunft
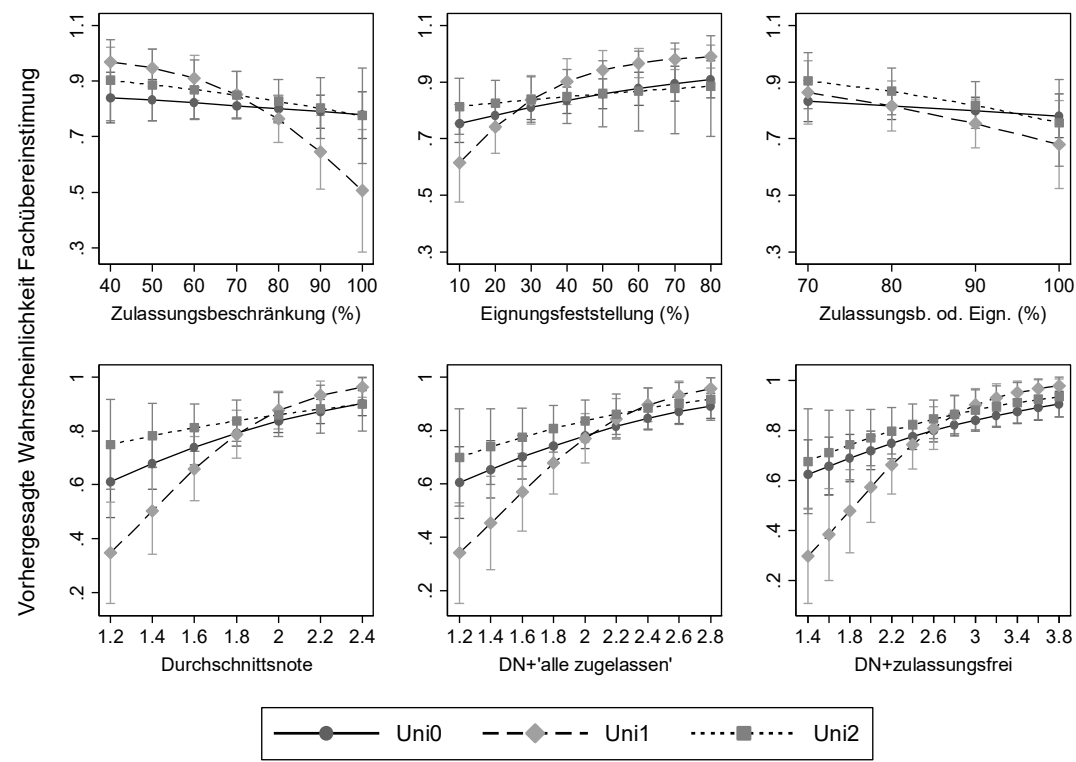

Quelle: Best Up-Individualdaten + Rekrutierungsdaten, eigene Berechnungen Logistische random-intercept Modelle (Leistungsindikatoren und Kontrollvariablen jeweils bei ihrem Mittelwert).

Dies bestätigt sich weitestgehend in Abbildung 7.5. Die Zusammenhänge zwischen Zugangshürden und der Wahrscheinlichkeit einer Übereinstimmung von Fachaspiration und Fachintention gehen für alle Herkunftsgruppen in die gleiche Richtung. Hier zeigt sich, dass es gerade Einakademikerkinder sind, die tendenziell sensibler auf Hürden reagieren und sich insbesondere dann umorientieren, wenn sie sehr selektive Fächer anstreben: Für sie sind die 
Steigungen in den meisten Fällen am stärksten, während sie für Befragte aus Doppel- und Nichtakademikerhaushalten weitestgehend parallel verlaufen. ${ }^{166}$

Generell stellt sich die bereits in Kapitel 5 diskutierte Frage, wie vollständig und korrekt Schüler/innen vorhandene (Zugangs-)Barrieren antizipieren (können). Die präsentierten Ergebnisse zeigen, dass Zugangshürden zumindest in Teilen Studien(fach)intentionen beeinflussen und deuten damit durchaus darauf hin, dass generell Informationen zu Zulassungsbeschränkungen und Selektivität vorhanden sind und zumindest grob eingeschätzt werden können. Bezüglich zur Anwendung kommender Eignungsfeststellungsverfahren ist dies weniger eindeutig. Wie in Abschnitt 2.2.3 beschrieben, sind Informationen hierzu kaum verfügbar, sodass eine einigermaßen korrekte Antizipation (über alle Herkunftsgruppen hinweg) unwahrscheinlich erscheint. Eine Ausnahme stellen sicherlich Fächer wie Sport oder Kunst dar, bei denen eine über den Erhalt der Hochschulzugangsberechtigung hinausgehende Eigungsfeststellung eine lange Tradition hat. Eine alternative Interpretation des fehlenden bzw. leicht positiven Zusammenhangs zwischen dem Anteil an Eignungsfeststellungsverfahren im angestrebten Studienfach und den abhängigen Variablen könnte daher gerade auch in ihrem großen Bekanntheitsgrad für einige Fächer liegen. Streben Schüler/innen etwa ein Kunst- oder Sportstudium an, könnten Eignungsfeststellungsverfahren weniger als Hürde als vielmehr als bekannte Notwendigkeit interpretiert werden, mit der man sich länger auseinandersetzt und auf die man sich entsprechend (mit dem nötigen wahrgenommenen „Talent") vorbereitet hat. Getestet werden können diese Vermutungen sowie die Frage nach hierbei existierenden Herkunftsunterschieden aufgrund zu kleiner Fallzahlen leider nicht.

Wenn Zugangshürden antizipiert werden und tatsächlich ein wichtiger Grund für die (fachspezifische) Diskrepanz zwischen Aspirationen und Intentionen sind, dann sollte sich dies in einer im Vergleich zur Fachaspiration weniger selektiven Fachintention widerspiegeln. Zusätzliche Analysen zeigen, dass dies mit Blick auf die Zulassungsbeschränkungen bei etwa der Hälfte der „Fachwechsel“ der Fall ist (mit Blick auf Eignungsfeststellungsverfahren sogar nur bei etwa 40\%). Bezüglich der benötigten Durchschnittsnote liegt der Anteil von Umorientierungen in Richtung weniger selektiv bei etwas mehr als $70 \%$. Eine Differenzierung nach sozialer Herkunft ist auch hier aufgrund $\mathrm{zu}$ kleiner Fallzahlen nicht möglich. Diese mit der nötigen Vorsicht zu interpretierenden Befunde weisen darauf hin, dass Zulassungsbeschränkungen an sich eine weniger große Hürde darstellen als die hiermit verbundene (Leistungs-)Selektivität, die für Schüler/innen ohne ausreichende Schulleis-

166 Wie zuvor in Abbildung 7.4 wurde geprüft, ob die „Kontraste“ - in diesem Fall zwischen Uni1 und den beiden Vergleichsgruppen - signifikant sind. Dies ist an den sehr selektiven und beschränkten „Rändern“ der Fall. Da diese Ergebnisse nicht zum Test von Hypothese HC3/I beitragen, werden sie hier nicht weiter besprochen, können aber im Anhang nachvollzogen werden (Abbildung A7.1). 
tung relevant sein kann. Weiterhin könnten diese Befunde sowohl ein Anzeichen dafür sein, dass die Antizipationsfähigkeit von Schüler/innen an ihre Grenzen stößt, als auch dafür, dass neben den Zugangshürden weitere mit Studienfächern verbundene Barrieren eine Rolle spielen.

Bewerbungsintention: Ergebnisse des Faktoriellen Surveys

Weitere potentielle institutionelle Barrieren (und Opportunitäten) und deren sozialgruppenspezifischer Einfluss werden im Folgenden mithilfe des Faktoriellen Surveys ${ }^{167}$ untersucht: die geographische Distanz und damit verbundene Studienkosten ( $\mathrm{HCl} / \mathrm{I})$, von den Hochschulen zur Verfügung gestellte Informationen (HC5/I), die Reputation der Hochschule, die im deutschen Kontext als Pull-Faktor interpretiert wurde (HC6/I), sowie die Anwendung komplexer Auswahlverfahren, die den Zugangshürden zugeordet werden können $(\mathrm{HC} 4 \mathrm{a} / \mathrm{I}$ und $\mathrm{HC} 4 \mathrm{~b} / \mathrm{I})$. Wichtig zu beachten ist hier, dass in den folgenden Analysen wieder alle Befragte mit Studienaspiration berücksichtigt werden.

Die gerade diskutierte Frage, wie vollständig und korrekt institutionelle Faktoren überhaupt antizipiert werden können, stellt sich bei der Untersuchung des FS - wie in Abschnitt 7.1.3 beschrieben - nicht, da mit den Vignetten die entsprechenden Informationen standardisiert vorgegeben und damit fehlende Informationen geliefert sowie mögliche Informationsasymetrien ausgeglichen werden. Die Ergebnisse spiegeln damit wider, mit welcher Wahrscheinlichkeit sich Befragte für einen Studiengang mit spezifischen Merkmalen ,entscheiden" würden, sofern grundlegende Informationen zu diesen Merkmalen vorliegen. So können tatsächliche Präferenzen sauberer abgebildet und untersucht werden, wie anziehend oder abschreckend bestimmte Faktoren wirken, wenn alle über die gleichen grundsätzlichen Informationen verfügen.

Ein weiterer - ebenfalls bereits angedeuteter - Vorteil des FS ist, dass der potentielle Einfluss von in der „Realität“ (noch) nicht häufig vorkommenden und (noch) nicht stark institutionalisierten Faktoren überprüft werden kann. Im vorliegenden Fall betrifft dies zum einen die Reputation von Hochschulen. Trotz der Exzellenzinitiative und einigen Hochschulrankings existieren in Deutschland keine klaren und einer breiten Öffentlichkeit bekannten Distinktionsmarker (vgl. Abschnitt 2.2.1). Zudem gehören Informationen hierzu in Deutschland kaum - im Gegensatz zu den USA („Ivy League“), zu Großbritannien („Oxbridge“ oder „Russel Group“) oder Frankreich („Grandes Écoles“) - zum „,common knowledge“, sodass hier durchaus von Informationsasymetrien ausgegangen werden kann. Zum anderen ist es bislang

167 Die folgenden Analysen wurden in Anlehnung an einen bereits in Research in Social Stratification and Mobiliry erschienenen Artikel durchgeführt. Geringfügige Unterschiede in den Ergebnissen sind vor allem auf einen anderen Samplezuschnitt zurückzuführen. 
schwierig den (sozialgruppenspezifischen) Einfluss komplexer Auswahlverfahren mithilfe quantitativer Methoden zu erfassen, da aufgrund ihrer bislang eingeschränkten Abdeckung und lückenhafter Daten die Grundlage hierfür fehlt.

Wie in Abschnitt 7.1.3 beschrieben, wurde die abhängige Variable „Bewerbungsintention“ auf einer 11-Punkteskala erhoben. Der Wert 5 stellt entsprechend die mittlere Kategorie dar. Abbildung 7.6 zeigt die Verteilung nach sozialer Herkunft. Doppelakademikerkinder sind wie erwartet stärker im positiven Bereich vertreten, während sich die Antworten der anderen beiden Gruppen in der Tendenz stärker im mittleren Bereich konzentrieren. Ein eindeutiges Muster zeigt sich insgesamt jedoch nicht, und die Mittelwerte sind fast identisch (Uni0: 6,0, Uni1: 6,1, Uni2: 6,3).

Abb. 7.6: Bewerbungsintention (Vignettenurteil), nach sozialer Herkunft
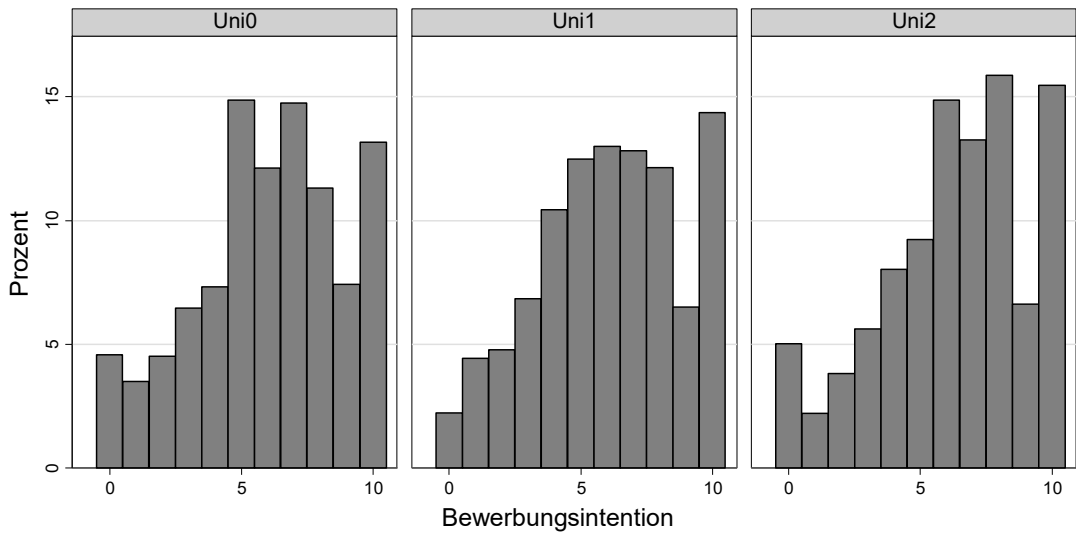

Quelle: Best Up-Individualdaten (Welle 2), eigene Berechnungen

Der Einfluss der institutionellen Faktoren für das gesamte Sample und getrennt nach sozialer Herkunft kann Tabelle 7.8 entnommen werden. Zunächst bestätigt sich in Modell 1 der bereits deskriptiv angedeutete Befund, dass die soziale Herkunft nicht signifikant mit dem Vignettenurteil assoziiert ist. Akademikerkinder scheinen also hier nicht die Tendenz zu haben, unabhängig vom präsentierten Szenario einen höheren Wert zu vergeben.

Bezüglich der Distanz zeigt sich im Einklang mit existierenden Befunden (vgl. Abschnitt 5.4.3), dass diese insgesamt und über alle Herkunftsgruppen hinweg einen deutlichen negativen Einfluss auf die Bewerbungsintention hat. So senkt eine große Entfernung zwischen der Heimatstadt und der Hochschule, an der der beschriebene Studiengang angesiedelt ist, im Vergleich zu einer kurzen Entfernung (gleicher Ort oder in unmittelbarer Nähe) die Wahrscheinlich- 
keit einer Bewerbungsintention um etwa 2 Punkte auf der 11-Punkte Skala. Während sich diesbezüglich keine Herkunftsunterschiede zeigen, wirkt eine mittlere Distanz anscheinend etwas negativer auf Nichtakademikerkinder als auf Akademikerkinder (insbesondere Doppelakademikerkinder).

Bezüglich der Auswahlverfahren zeigt sich zunächst, dass Motivationsschreiben insgesamt keinen nennenswerten Einfluss auf die Bewerbungsintention ausüben. Hingegen haben Auswahlgespräche und Tests einen leicht positiven Einfluss, was hier eher für die Annahme spricht, dass sie weniger als Barriere denn als „Attraktionsmerkmal“ (vgl. Bloch et al. 2015) wahrgenommen werden. Insbesondere für die Bewerbungsintention von Einakademikerkindern scheinen Auswahlgespräche einen leicht positiven Einfluss zu haben. Anders verhält es sich bei Tests, die insbesondere für Nichtakademikerkinder einen tendenziell positiven Einfluss haben.

Wie erwartet fungiert die Reputation von Hochschulen im deutschen Kontext eher als Pull-Faktor und wirkt positiv auf alle Herkunftsgruppen. Zwar steigt die Effektstärke leicht mit der sozialen Herkunft, jedoch sind die Unterschiede nicht sehr groß.

Letztlich spielen von den Hochschulen bereitgestellte Informationen keine Rolle für Doppelakademikerkinder, während sie die Bewerbungsintention der anderen beiden Gruppen leicht erhöhen. Mit Blick auf vorhandene Informationen zeigt sich ein weiteres bemerkenswertes Ergebnis. Private Informationen in Form von Hilfestellungen durch Bekannte mit Hochschul- und Bewerbungserfahrung (,Netzwerk") haben auf die Bewerbungsintention von Akademikerkindern, insbesondere von Doppelakademikerkindern einen deutlich stärkeren positiven Einfluss als auf Nichtakademikerkinder. Dies weist darauf hin, dass Schüler/innen aus sozial privilegierteren Familien wissen, wie wichtig die „richtigen“ Informationen und wie hilfreich daher ihre privaten Informationskanäle sein können, um den Bewerbungsprozess zu erleichtern (vgl. Slack et al. 2012). Sozial schwächere Schüler/innen hingegen scheinen zu antizipieren, dass die Informationen, die sie von „Bekannten“ erhalten, ihnen im Hochschulkontext nur bedingt weiterhelfen können (vgl. Heine et al. 2010). ${ }^{168}$

Insgesamt zeigt sich also, dass geographische Distanz deutlich negativ mit der Bewerbungsintention der Befragten zusammenhängt, während diese insbe-

168 Dies könnte ein Grund für die geringe Erklärungskraft der Variable „Unterstützung bei der Studienbewerbung" in den weiter oben präsentierten Modellen sein (Tab. 7.4), da diese lediglich misst, ob die Befragten überhaupt denken, Unterstützung aus dem sozialen Netzwerk zu bekommen. Alternativ wäre eine Kreuzung mit der Variable, ob die Unterstützung von hochschulerfahrenen Personen ausgeht, denkbar. Da diese Variable sehr stark mit der Bildungsherkunft der Befragten korreliert, wurde hiervon jedoch abgesehen. 
sondere durch die Reputation von Hochschulen und in der Tendenz auch durch Auswahlverfahren und Informationen erhöht wird. ${ }^{169}$

Tab. 7.8: Bewerbungsintention (FS): institutionelle Barrieren und Opportunitäten nach sozialer Herkunft

\begin{tabular}{|c|c|c|c|c|}
\hline & $\begin{array}{l}\text { M1: } \\
\text { Gesamt }\end{array}$ & $\begin{array}{l}\text { M2: } \\
\text { Uni0 }\end{array}$ & $\begin{array}{l}\text { M3: } \\
\text { Uni1 }\end{array}$ & $\begin{array}{l}\text { M4: } \\
\text { Uni2 }\end{array}$ \\
\hline \multicolumn{5}{|l|}{ Soziale Herkunft Ref.: Uni0 } \\
\hline Uni1 & $0,043(0,199)$ & & & \\
\hline Uni2 & $0,260(0,220)$ & & & \\
\hline \multicolumn{5}{|l|}{ Distanz Ref.: Kurz } \\
\hline Mittel & $\begin{array}{l}-1,000^{\star \star \star} \\
(0,094)\end{array}$ & $\begin{array}{l}-1,121^{\star \star \star} \\
(0,123)\end{array}$ & $\begin{array}{l}-0,858^{\star \star \star} \\
(0,197)\end{array}$ & $\begin{array}{l}-0,726^{\star \star *} \\
(0,215)\end{array}$ \\
\hline Lang & $\begin{array}{l}-1,988^{\star \star \star} \\
(0,094)\end{array}$ & $\begin{array}{l}-1,991^{\text {}} \\
(0,122)\end{array}$ & $\begin{array}{l}-1,941^{\star \star \star} \\
(0,197)\end{array}$ & $\begin{array}{l}-2,025^{\text {}} \\
(0,215)\end{array}$ \\
\hline Reputation: gut & $\begin{array}{l}0,849^{\star \star \star} \\
(0,077)\end{array}$ & $\begin{array}{l}0,799^{\star \star \star} \\
(0,100)\end{array}$ & $\begin{array}{l}0,871^{\star \star *} \\
(0,162)\end{array}$ & $\begin{array}{l}0,966^{\star \star \star} \\
(0,175)\end{array}$ \\
\hline \multicolumn{5}{|l|}{ Auswahlverfahren Ref.: nur DN } \\
\hline $\mathrm{DN}+$ Motivationsschreiben & $\begin{array}{l}0,130 \\
(0,108)\end{array}$ & $\begin{array}{l}0,080 \\
(0,141)\end{array}$ & $\begin{array}{l}0,142 \\
(0,228)\end{array}$ & $\begin{array}{l}0,287 \\
(0,245)\end{array}$ \\
\hline $\mathrm{DN}+$ Auswahlgespräch & $\begin{array}{l}0,298^{\star \star \star} \\
(0,108)\end{array}$ & $\begin{array}{l}0,189 \\
(0,141)\end{array}$ & $\begin{array}{l}0,486^{\star \star} \\
(0,228)\end{array}$ & $\begin{array}{l}0,397 \\
(0,249)\end{array}$ \\
\hline DN+ Test & $\begin{array}{l}0,271^{\star \star} \\
(0,108)\end{array}$ & $\begin{array}{l}0,341^{\star *} \\
(0,142)\end{array}$ & $\begin{array}{l}0,255 \\
(0,227)\end{array}$ & $\begin{array}{l}0,035 \\
(0,247)\end{array}$ \\
\hline Information: umfassend & $\begin{array}{l}0,269^{\star \star *} \\
(0,077)\end{array}$ & $\begin{array}{l}0,299^{\star * *} \\
(0,100)\end{array}$ & $\begin{array}{l}0,337^{\star *} \\
(0,163)\end{array}$ & $\begin{array}{l}0,176 \\
(0,175) \\
\end{array}$ \\
\hline Interesse: hoch & $\begin{array}{l}0,758^{\star \star \star} \\
(0,076)\end{array}$ & $\begin{array}{l}0,797^{\star \star \star} \\
(0,100)\end{array}$ & $\begin{array}{l}0,635^{\star \star \star} \\
(0,161)\end{array}$ & $\begin{array}{l}0,722^{\star \star *} \\
(0,175)\end{array}$ \\
\hline Netzwerk: vorhanden & $\begin{array}{l}0,231^{* * *} \\
(0,077)\end{array}$ & $\begin{array}{l}0,100 \\
(0,100)\end{array}$ & $\begin{array}{l}0,285^{*} \\
(0,163)\end{array}$ & $\begin{array}{l}0,635^{\star \star \star} \\
(0,176)\end{array}$ \\
\hline Größe der Stadt: groß & $\begin{array}{l}0,389^{\star \star \star *} \\
(0,077)\end{array}$ & $\begin{array}{l}0,331^{\text {} * *} \\
(0,100)\end{array}$ & $\begin{array}{l}0,495^{\star \star *} \\
(0,162)\end{array}$ & $\begin{array}{l}0,458^{\star \star \star} \\
(0,175)\end{array}$ \\
\hline Konstante & $\begin{array}{l}5,551^{\star \star \star} \\
(0,292)\end{array}$ & $\begin{array}{l}5,638^{\star \star \star} \\
(0,372)\end{array}$ & $\begin{array}{l}5,747^{\star \star \star} \\
(0,594)\end{array}$ & $\begin{array}{l}5,185^{\star \star \star} \\
(0,817)\end{array}$ \\
\hline Rho Befragte & 0,39 & 0,37 & 0,43 & 0,47 \\
\hline Rho Schulen & 0 & 0 & 0 & 0,01 \\
\hline $\mathrm{R}^{2}$ (Maddala) & 0,22 & 0,22 & 0,26 & 0,28 \\
\hline N Vignetten & 2.832 & 1.749 & 585 & 498 \\
\hline N Befragte/Schulen & $578 / 19$ & $357 / 19$ & $121 / 19$ & $100 / 18$ \\
\hline
\end{tabular}

Quelle: Best Up-Individualdaten, eigene Berechnungen

lineare random-intercept Modelle, Standardfehler in Klammern, ${ }^{* * *} p<0,01 ;{ }^{* *} p<0,05 ;{ }^{*} p<0,1$

Alle Modelle enthalten Position der Vignetten innerhalb des Decks, Dauer des Vignettenmoduls, Leistungsindikatoren und Kontrollvariablen (Migrationshintergrund, Geschlecht und Schultyp).

$169 \mathrm{Da}$ die Vignettendimensionen unterschiedlich viele Ausprägungen haben, ist es nicht möglich von der Effektstärke auf ihre relative Erklärungskraft zu schließen. Daher wurden semi-partielle Korrelationen berechnet, die den Anteil der erklärten Varianz durch die einzelnen Dimensionen wiedergeben (vgl. Auspurg und Hinz 2015: 99). Auch hier zeigt sich, dass die Distanz die größte partielle Korrelation aufweist $(-0,29)$, gefolgt von der Reputation $(0,15)$ und den Auswahlverfahren und Informationen, die in etwa dieselben Werte annehmen $(0,04)$. 
Die Herkunftsunterschiede bezüglich der Effektstärken sind insgesamt nicht sehr groß. Entsprechend fallen Ratio-Likelihood Chi-Quadrat Tests (Ergebnisse nicht ausgewiesen), mit deren Hilfe ein Modell ohne Interaktion gegen Modelle mit jeweils einer Interaktion zwischen der Bildungsherkunft und einer Vignettendimension getestet wurden, nicht signifikant aus (eine Ausnahme stellt hier die Interaktion zwischen privaten Informationen und der Bildungsherkunft dar). Dies sagt jedoch noch nichts darüber aus, ob sich einzelne Merkmalsausprägungen signifikant zwischen den Herkunftsgruppen unterscheiden. Entsprechend zeigt sich, dass sich bei einer mittleren Distanz der Unterschied in der Bewerbungsintention signifikant zwischen Doppelakademikerkindern und Nichtakademikerkindern unterscheidet (Differenz: 0,53, p: 0,043). Dies unterstützt Hypothese $\mathrm{HCl} / \mathrm{I}$ zumindest in Teilen, die davon ausgeht, dass eine weitere Distanz negativer mit der Bewerbungsintention von Nichtakademikerkindern zusammenhängt. Alle weiteren hier interessierenden Koeffizienten unterscheiden sich nicht signifikant (und substantiell) nach sozialer Herkunft. Ein differentieller Einfluss der Reputation (positiver für Akademikerkinder, HC6/I), Auswahlverfahren (generell positiv für Akademikerkinder, negativ für Nichtakademikerkinder, HC4a/I) und Informationen (positiver für Nichtakademikerkinder HC5/I) kann daher auf Grundlage der Ergebnisse nicht bestätigt werden. Zwar zeichnen sich zum Beispiel bezüglich des Einflusses von institutionell bereitgestellten Informationen leichte Herkunftsunterschiede ab, doch sind diese zu klein und unsicher, um die Hypothesen aufrechterhalten zu können. Jedoch verweisen die Ergebnisse vorsichtig in Richtung HC4b/I, die davon ausgeht, dass Auswahlverfahren auf alle Herkunftsgruppen positiv wirken. Dies gilt jedoch generell nicht für Motivationsschreiben, und auch Tests und Auswahlgespräche gehen nicht für alle Herkunftsgruppen mit einer signifikanten Erhöhung ihrer Bewerbungswahrscheinlichkeit einher. Zudem muss der Einfluss von komplexen Auswahlverfahren (wie auch von Informationen) hier als eher gering bewertet werden.

\subsection{Ergebnisse II: vertikal und horizontal differenzierte Intentionen}

Bisher wurde gezeigt, dass sich Schüler/innen unterschiedlicher sozialer Herkunft darin unterscheiden, mit welcher Wahrscheinlichkeit sie ihre Studienaspiration in eine Studienintention übersetzen. Zuletzt liegt der Fokus auf der Frage, inwiefern Schüler/innen unterschiedlicher sozialer Herkunft intendieren, unterschiedliche vertikal (Hochschultyp) und horizontal (Studienfach) differenzierte Studienoptionen aufzunehmen.

Bivariate Zusammenhänge hierzu können Tabelle 7.9 entnommen werden. Alle Herkunftsgruppen planen häufiger an einer Universität als an einer Fach- 
hochschule zu studieren. Dieser Anteil ist bei Doppelakademikerkindern mit 82,5\% jedoch mit Abstand am höchsten. Zudem planen Letztere, Fächer zu studieren, die im Durchschnitt mit einem um circa 10 Punkte höheren Magnitude-Prestige-Wert assoziiert sind und zu einem um $1 €$ höheren Nettostundenlohn führen (bei Vollzeiterwerbstätigkeit also im Durchschnitt zu einem circa $2000 €$ höheres Nettojahresgehalt). ${ }^{170}$

Tab. 7.9: Hochschultyp- und Fachintention: deskriptive Information nach sozialer Herkunft

\begin{tabular}{lllll}
\hline & Uni0 & Uni1 & Uni2 & Total \\
\hline $\begin{array}{l}\text { Hochschultyp (N: 507), Prozent (N) } \\
\text { Universität (vs. FH) }\end{array}$ & $68,9(208)$ & $73,2(79)$ & $82,5(80)$ & $72,4(367)$ \\
\hline $\begin{array}{l}\text { Durchschnittliches Einkommen/ } \\
\text { Berufsprestige (N: 407), Mean (SD) }\end{array}$ & & & & \\
\hline Stundenlohn & $16,8(2,3)$ & $16,9(2,4)$ & $17,8(2,3)$ & $17,0(2,3)$ \\
Range & $12,1-23,2$ & $12,1-23,2$ & $12,3-23,2$ & $12,1-23,2$ \\
\hline Berufsprestige & $97,8(23,7)$ & $95,0(22,5)$ & $106,4(26,6)$ & $98,8(24,3)$ \\
Range & $64,9-191,8$ & $67,0-183,4$ & $76,8-183,4$ & $64,9-191,8$ \\
\hline
\end{tabular}

Quelle: Best Up-Individualdaten, Mikrozensus 2007-2012 DOIs: 10.21242/12211.2007.00.00.1.1.0 bis 10.21242/12211.2012.00.00.1.1., eigene Berechnungen

Die Ergebnisse bestätigen sich in den multivariaten Modellen (Tabelle 7.10). Hier zeigt sich erneut, dass es vor allem Schüler/innen mit zwei akademisch gebildeten Eltern sind, die sich signifikant von Nichtakademikerkindern (und zum Teil auch Einakademikerkindern, M3) unterscheiden. Sie intendieren häufiger ein Studium an einer Universität (HD1/I) und in einem prestigereicheren Fach (HD3/I) als Nichtakademikerkinder. Zudem führen ihre Fachintentionen zu einem im Durchschnitt höheren Einkommen, was gegen Hypothese HD2/I spricht, die davon ausgeht, dass diesbezüglich keine Herkunftsunterschiede bestehen (etwa wenn sozial schwächere Studierende häufiger Intentionen für ingenieurs- oder wirtschaftswissenschaftliche Studienfächer äußern, die häufig $\mathrm{zu}$ einem höheren Einkommen als Berufsprestige führen). Der in Abschnitt 7.2.1 beschriebene Befund, dass Nichtakademikerkinder ein hohes Einkommen (und Prestige) sogar etwas wichtiger einschätzen als Akademikerkinder spiegelt sich in diesen Ergebnissen nicht wider.

170 Um die Herkunftsunterschiede zwischen den beiden Fachvariablen vergleichen zu können, wurden diese z-standardisiert. Hier zeigt sich, dass die Bildungsherkunft etwas stärker mit dem Einkommen assoziiert ist: Der Status Uni2 im Vergleich zu Uni0 erhöht den standardisierten Einkommenswert um etwa eine halbe Standardabweichung, den Prestigewert jedoch nur um circa ein Drittel einer Standardabweichung. 
Tab. 7.10: Soziale Herkunft und Hochschultyp-/Fachintention: multivariate Ergebnisse

\begin{tabular}{llll}
\hline & $\begin{array}{l}\text { M1: } \\
\text { FH-Intention }\end{array}$ & $\begin{array}{l}\text { M2: Fachintention } \\
\text { Einkommen }\end{array}$ & $\begin{array}{l}\text { M3: Fachintention } \\
\text { Prestige }\end{array}$ \\
\hline $\begin{array}{l}\text { Soziale Herkunft Ref. Uni0 } \\
\text { Uni1 }\end{array}$ & $-0,022(0,050)$ & $0,201(0,290)$ & $-3,167(3,074)$ \\
Uni2 & $-0,090^{*}(0,051)$ & $1,097^{* * *}(0,310)$ & $8,24^{* *}(3,290)$ \\
\hline Konstante & & $16,89^{\star * *}(0,39)$ & $93,22^{\star * *}(3,819)$ \\
\hline Rho & 0,02 & 0,03 & 0 \\
Pseudo-R ${ }^{2}$ (Maddala) & 0,06 & 0,10 & 0,07 \\
N Befragte/Schulen & $507 / 19$ & $407 / 19$ & $407 / 19$ \\
\hline
\end{tabular}

Quelle: Best Up-Individualdaten, Mikrozensus 2007-2012, DOls: 10.21242/12211.2007.00.00.1.1.0 bis 10.21242/12211.2012.00.00.1.1., eigene Berechnungen

M1: logistisches random-intercept Modell, DC (Standardfehler), M2, M3: lineare random-intercept Modelle, Koeffizienten (Standardfehler), ${ }^{* * *} p<0,01 ;{ }^{* *} p<0,05 ;{ }^{*} p<0,1$

Alle Modelle enthalten Leistungsindikatoren und Kontrollvariablen (Migrationshintergrund, Geschlecht und Schultyp).

Exkurs: Intentionsunterschiede $=$ Aspirationsunterschiede?

An diese Ergebnisse anknüpfend stellt sich die Frage, ob die gerade berichteten Herkunftsunterschiede sich bereits in den spezifischen Aspirationen von Schüler/innen widerspiegeln. Sind es also bereits die Wünsche und Präferenzen - „limited desires“ (Keller und Zavalloni 1964: 58) -, die dazu führen, dass Nichtakademikerkinder (und auch Einakdemikerkinder) Intentionen für weniger prestigereiche Studienoptionen haben? Oder unterscheiden sich die Intentionen von Schüler/innen unterschiedlicher sozialer Herkunft, obwohl sich ihre Fach- und Hochschultypwünsche sehr ähneln? Letzteres wäre ein weiteres Indiz für eine unterschiedliche Bewertung wahrgenommener Hürden.

$\mathrm{Zu}$ diesen Fragen soll hier lediglich ein erster, deskriptiver Eindruck gegeben werden. Tabelle 7.11 zeigt dazu getrennt nach sozialer Herkunft den Anteil an Befragten, die sich wünschen, an einer Universität zu studieren (Aspiration) und denjenigen, die dies auch planen. Zudem werden die Einkommens- und Prestigemittelwerte des angestrebten und intendierten Faches gegenübergestellt. Bezüglich des Hochschultyps kann eine etwas stärkere Diskrepanz bei Nichtakademikerkindern beobachtet werden: $74 \%$ wünschen sich ein Universitätsstudium und 69\% intendieren ebenfalls ein solches. Doppelakademikerkinder haben hier identische Werte. Auch bezüglich des Einkommenswertes unterscheidet sich der durchschnittliche Aspirationswert bei Letzteren nicht vom durchschnittlichen Intentionwert. Ein- und Nichtakademikerkinder liegen hier bereits bezüglich ihrer Aspiration leicht unter dem Wert der Doppelakademikerkinder und korrigieren sich zudem im Durchschnitt etwas „,nach unten“, was zu dem oben berichteten Unterschied 
führt. Ein sehr ähnliches Muster zeigt sich bezüglich des durchschnittlichen Berufspretiges, zu dem die Fachaspiration und -intention führt. Insgesamt bestehen also bereits Unterschiede in den Aspirationen, doch werden diese mit Blick auf die Intentionen größer.

Tab. 7.11: Unterschiede zwischen Hochschultyp- und Fachaspirationen und -intentionen

\begin{tabular}{llll}
\hline & $\begin{array}{l}\text { Hochschultyp Uni: } \\
\text { Aspiration - Intention (\%) }\end{array}$ & $\begin{array}{l}\text { Einkommen: Aspiration - } \\
\text { Intention (Mean, SD) }\end{array}$ & $\begin{array}{l}\text { Prestige: Aspiration - } \\
\text { Intention (Mean, SD) }\end{array}$ \\
\hline Uni0 & $73,8-68,9$ & $17,1(2,6)-16,8(2,3)$ & $102,4(29,7)-97,8(23,7)$ \\
Uni1 & $75,9-73,2$ & $17,5(2,6)-16,9(2,4)$ & $104,8(30,4)-95,0(22,5)$ \\
Uni2 & $82,5-82,5$ & $17,8(2,7)-17,8(2,3)$ & $108,2(21,6)-106,4(26,6)$ \\
Total & $75,9-72,4$ & $17,3(2,6)-17,0(2,3)$ & $104,0(30,2)-98,8(24,3)$ \\
\hline
\end{tabular}

Quelle Best Up-Individualdaten, Mikrozensus 2007-2012, DOIs: 10.21242/12211.2007.00.00.1.1.0 bis 10.21242/12211.2012.00.00.1.1., eigene Berechnungen

N Hochschultyp:507, N Fach: 407

\subsection{Zusammenfassung}

Abschließend sollen die Ergebnisse zur ersten Phase zusammengefasst und deren Bezug zum bereits existierenden Befunden und den theoretischen Annahmen stärker hergestellt werden. Eine kurze Zusammenfassung der Ergebnisse mit Blick auf die aufgestellten Hypothesen kann Tabelle 7.12 entnommen werden.

Mit Phase 1 wurden soziale Herkunftsunterschiede bei der Übersetzung von weitestgehend uneingeschränkten Studienaspirationen in individuelle und institutionelle Barrieren antizipierende Studienintentionen untersucht. Studienintentionen wurden dabei zum einen mithilfe einer direkten Abfrage operationalisiert, die sich eher global auf wahrgenommene Hürden bezieht. Ergänzt wurde diese zum anderen mithilfe eines Faktoriellen Surveys, durch den der konkrete Einfluss bestimmter institutioneller Merkmale isoliert erfasst werden kann.

Wie ausschließlich Aspirationen idealistische Wünsche und Intentionen realistische Einschätzungen abbilden, hängt auch vom Messzeitpunkt ab (vgl. Alexander und Cook 1979, Goyette 2008, Trebbels 2015). In der vorliegenden Studie wurden Aspirationen und Intentionen zu Beginn des letzten Schuljahres erfasst, also bereits relativ spät in der Schullaufbahn, sodass Studienwünsche wahrscheinlich nicht komplett losgelöst von realistischen Einschätzungen geäußert werden und Studienintentionen andererseits bereits relativ realistisch sein sollten. Entsprechend zeigte sich, dass die Übersetzung von Aspirationen 
in -intentionen mit insgesamt etwa $90 \%$ hoch ist. Wie erwartet zeigen sich aber auch Herkunftsunterschiede von fast 10 Prozentpunkten, die sich insbesondere im Vergleich zwischen Doppelakademikerkindern und Nichtakademikerkindern zeigen. Einakademikerkinder nehmen hier die Mittelposition ein. Für den deutschen Kontext sind dies die ersten mir bekannten Befunde dieser Art. Sie spiegeln grundsätzlich die in Abschnitt 4.3.1 zitierten internationalen Befunde wider (vgl. Hanson 1994, Vaisey 2010). Jedoch sind sowohl die Diskrepanz zwischen Aspiration und Intention als auch diesbezügliche Herkunftsdifferenzen insgesamt kleiner, was erneut auf die Positivselektion von Studienaspirant/innen im deutschen Kontext verweist.

Die Herkunftsunterschiede sind auch nach Hinzufügen weiterer Variablen robust und können entsprechend kaum durch die deskriptiv durchaus sichtbaren sozialgruppenspezifischen Leistungsunterschiede, Einschätzungen von Kosten, Nutzen und Erfolgswahrscheinlichkeit sowie kulturelle und soziale Ressourcen erklärt werden, sodass die Hypothesen des Blocks B nicht von den Ergebnissen gestützt werden.

Theoretisch wird die Entstehung von Intentionen oft als Rationalisierungsprozess verstanden, der der Logik rationaler Entscheidungsmodelle folgt (vgl. Becker 2010, Morgan 1998, Stocké 2005b). Zwar zeigen einige der RC-Indikatoren in die erwartete Richtung. Doch finden die in Abschnitt 5.4 zitierten Befunde, nach denen insbesondere die größere Kostensensibilität und als unsicherer eingeschätzte Erfolgswahrscheinlichkeit unter sozial schwächeren Schüler/innen sozialgruppenspezifische Bildungsentscheidungen erklären können (vgl. Becker und Hecken 2007, 2009a, Lörz 2012), für die Gruppe der Studienaspirant/innen und die Herausbildung einer Studienintention kaum Bestätigung.

Obwohl theoretisch davon ausgegangen wird, dass die Antizipation institutioneller Barrieren und Opportunitäten in die Übersetzung von Aspirationen in Intentionen eingeht, gibt es hierzu bisher kaum empirische Evidenz. Mithilfe des Faktoriellen Surveys sowie auf Grundlage von aus den Rekrutierungsdaten generierten Variablen zu Zugangshürden wurde dies untersucht. Es zeigt sich, dass die durchschnittlichen Zugangsbarrieren im angestrebten Fach die Intentionswahrscheinlichkeit von Akademikerkindern kaum beeinflussen, diese bei Nichtakademikerkindern aber deutlich senken. Erstere „bleiben“ also beim Studium, etwa weil sie ein solches benötigen, um Statuserhalt sicherzustellen, weil sie auf mehr Ressourcen zurückgreifen können, um ihren Studienwunsch auch gegen existierende Hürden umsetzen zu können oder weil sie ihre Aspiration unabhängig von potentiellen Hürden unbedingt und unhinterfragt weiterverfolgen. Für sozial schwächere Schüler/innen scheint hingegen eine als gering wahrgenommene Zulassungschance tatsächlich eine Hürde darzustellen. Dass Aakdemikerkinder dabei keineswegs unrealistisch zu sein scheinen, verdeutlicht der Befund, dass sie (ebenso wie Nichtakademikerkinder) sich innerhalb des Hochschulsystems umorientieren, d.h. auf ein anderes Fach aus- 
weichen, wenn das Fach, das sie anstreben, sehr selektiv ist. Wie in Abschnitt 7.2.2 diskutiert, deuten diese Ergebnisse darauf hin, dass Zugangshürden (scheinbar mit Ausnahme von Eignungsfeststellungsverfahren, zu denen Informationen kaum verbreitet sind) antizipiert werden und in die Entscheidungsfindung (sozialgruppenspezifisch) einfließen.

Der Einfluss weiterer institutioneller Hürden und auch Pull-Faktoren wurde mithilfe des FS getestet. Zunächst wurde hier eine weitere potentielle Zugangshürde - oder auch „Attraktionsmerkmal“ - in Form von über den Abiturdurchschnitt hinausgehenden „komplexen Auswahlverfahren“" (Motivationsschreiben, Auswahlgespräche und Tests) geprüft. Es zeigt sich, dass diese (mit Ausnahme von Motivationsschreiben) einen leicht positiven Einfluss auf die Bewerbungsintention von Studienaspirant/innen haben, hierbei jedoch keine Herkunftsunterschiede verzeichnet werden können. Stärker positiv wirkt die Reputation der Hochschule, die jedoch nicht, wie angenommen, einen stärkeren Pull-Faktor für Akademikerkinder darstellt. Dieses Ergebnis weist darauf hin, dass institutionelles Prestige in seiner heutigen Form im deutschen Kontext bisher kaum zu sozialer Ungleichheit beiträgt. Dies liegt jedoch nicht daran, dass es nicht attraktiv auf sozial privilegierte Schüler/innen wirkt, sondern dass es auch für sozial schwächere Schüler/innen einen Pull-Faktor darstellt und sie nicht - wie in anderen Kontexten mit einer stärkeren „Elitekultur“ gezeigt (vgl. Ball et all. 2002, Evans 2009, Reay et al. 2001a/b) - abschreckt. Die Distanz zwischen dem Heimat- und dem Hochschulort hat hingegen einen deutlich negativen Effekt für alle Herkunftsgruppen, was den bekannten Befund einer geringen Mobilitätsbereitschaft bestätigt (vgl. Helbig et al. 2017, KMK 2014, Spieß und Wrohlich 2010). Doch deutet sich an, dass eine mittlere Distanz die Bewerbungsintention von Nichtakademikerkindern im Vergleich zu Doppelakademikerkindern deutlicher senkt. Eine mögliche Interpretation hierfür ist, dass ein mit dem Umzug in eine weit entfernte Stadt einhergehendes komplettes Loslösen vom sozialen Netzwerk für Schüler/innen aller Herkunftsgruppen im Mittel unattraktiv zu sein scheint. Eine mittlere Distanz, die zumindest ein wöchentliches Pendeln theoretisch erlaubt, scheint hingegen weniger abschreckend auf diejenigen zu wirken, die sich die hierdurch entstehenden Kosten eher leisten können. Von Hochschulen bereitgestellte Informationen haben zwar einen leicht positiven Einfluss auf die Bewerbungsintention von Nichtakademikerkindern und auch Einakdemikerkindern im Gegensatz zu Doppelakademikerkindern, doch sind die Unterschiede nicht sehr deutlich und auch nicht signifikant. Dies ist zunächst erstaunlich, da sozial schwächere Schüler/innen fehlende Informationen weniger gut durch private Kanäle ausgleichen können. Dies könnte als Hinweis dafür interpretiert werden, dass sie sich eines potentiellen Informationsdefizits nicht bewusst sind und daher bereitgestellten Informationen keine sehr große Wertschätzung entgegenbringen (vgl. Morgan 2010). Mit Blick auf die Hypothesen des Blocks C fallen die Ergebnisse damit ambivalent aus (siehe Tabelle 7.12). 
Tab. 7.12: Ergebnisübersicht Phase 1

\begin{tabular}{|c|c|c|}
\hline Hypothesen & Ergebnis & \\
\hline $\mathrm{HA} / \mathrm{I}$ & $\begin{array}{l}\text { Uni0 übersetzen ihre Studienaspiration seltener in eine } \\
\text { Studienintention als Uni2 (keine Unterschiede zu Uni1). }\end{array}$ & + \\
\hline HB1/I & $\begin{array}{l}\text { Keine Reduktion der Herkunftsunterschiede durch } \\
\text { Leistungsindikatoren. }\end{array}$ & - \\
\hline HB2/l & Keine Reduktion der Herkunftsunterschiede durch RC-Indikatoren. & - \\
\hline $\mathrm{HB} 3 / \mathrm{l}$ & $\begin{array}{l}\text { Keine Reduktion der Herkunftsunterschiede durch soziale und } \\
\text { kulturelle Ressourcen. }\end{array}$ & - \\
\hline $\mathrm{HC} 1 / \mathrm{l}$ & $\begin{array}{l}\text { Große Distanz negativ für alle Herkunftsgruppen. Mittlere Distanz } \\
\text { negativer für Uni0 vs. Uni2. }\end{array}$ & $(+)$ \\
\hline $\mathrm{HC} 2 / \mathrm{I}$ & $\begin{array}{l}\text { Zulassungsbeschränkung und Selektivität des angestrebten Faches } \\
\text { haben kaum Einfluss auf Intentionswahrscheinlichkeit von Uni1/2, } \\
\text { wirken jedoch negativ auf Uni0 („Umentscheidung“). }\end{array}$ & + \\
\hline $\mathrm{HC} 3 / \mathrm{l}$ & $\begin{array}{l}\text { Zulassungsbeschränkung und Selektivität des angestrebten Faches } \\
\text { führen in allen Herkunftsgruppen zu „Umorientierung“ (Diskrepanz } \\
\text { zwischen Fachaspiration und Fachintention). }\end{array}$ & + \\
\hline $\mathrm{HC} 4 \mathrm{a} / \mathrm{l}$ & Keine nennenswerten Herkunftsunterschiede bezüglich des Einflus- & - \\
\hline $\mathrm{HC} 4 \mathrm{~b} / \mathrm{l}$ & $\begin{array}{l}\text { ses „komplexer Auswahlverfahren“. Tests und Auswahlgespräche } \\
\text { haben in der Tendenz einen leicht positiven Einfluss. }\end{array}$ & $(+)$ \\
\hline $\mathrm{HC} 5 / \mathrm{l}$ & $\begin{array}{l}\text { Informationen haben zwar für Uni0 und Uni1 leicht positiven Einfluss, } \\
\text { Unterschiede zu Uni2 sind aber nicht groß und nicht signifikant. }\end{array}$ & $(-)$ \\
\hline HC6/l & $\begin{array}{l}\text { Reputation hat in allen Herkunftsgruppen positiven Einfluss, kaum } \\
\text { Herkunftsunterschiede. }\end{array}$ & - \\
\hline HD1/l & $\begin{array}{l}\text { Uni0 haben häufiger Fachhochschulintention als Uni2 (kaum } \\
\text { Unterschiede zu Uni1). }\end{array}$ & + \\
\hline $\mathrm{HD} 2 / \mathrm{l}$ & $\begin{array}{l}\text { Uni2 haben Intention für Fächer, die im Durchschnitt zu einem höhe- } \\
\text { ren Einkommen führen als UniO und Uni1. }\end{array}$ & - \\
\hline HD3/l & $\begin{array}{l}\text { Uni2 haben Intention für Fächer, die im Durchschnitt zu einem höhe- } \\
\text { ren Berufsprestige führen als Uni0 und Uni1. }\end{array}$ & + \\
\hline
\end{tabular}

Zuletzt wurde der Frage nachgegangen, ob sich bei Schüler/innen mit Studienintention Herkunftsunterschiede innerhalb des Hochschulsystems bezüglich des Hochschultyps und des Studienfaches zeigen. Im Einklang mit existierenden Forschungsergebnissen (vgl. Lörz 2012, Reimer und Pollak 2010) scheint sich - zumindest beim Vergleich zwischen Doppel- und Nichtakademikerkindern - zu bestätigen, dass sozial stärkere Schüler/innen prestigereichere Studienoptionen „wählen“ (Universitäten und Studienfächer, die im Durchschnitt $\mathrm{zu}$ einem höheren Berufsprestige führen). Basierend auf dem Befund, dass Nichtakademikerkinder einem hohen Einkommen einen ebenso hohen oder gar höheren Wert zuweisen (vgl. Lörz 2012), wurden bezüglich dieser Dimension von Studienfächern keine Herkunftsunterschiede erwartet. Dies bestätigt sich beim Vergleich von Ein- und Nichtakademikerkindern. Für Doppelakademikerkinder zeigt sich jedoch auch hier der höchste Wert. 
Wie argumentiert, stellt die Übersetzung von Studienaspirationen in -intentionen zwar einen wichtigen ersten Schritt dar, doch können hieraus noch keine Aussagen zu den letztlichen Umsetzungschancen getroffen werden. Daher ist im nächsten Kapitel zu prüfen, inwiefern sich die hier gezeigten Befunde auch im Bewerbungsverhalten und letztlichen Übergang ins Studium widerspiegeln. 


\section{Phasen 2-4: Die Umsetzung von Studienaspirationen}

Nachdem im vorangegangenen Kapitel soziale Herkunftsunterschiede bei der Übersetzung von Studienaspirationen in -intentionen untersucht wurden, steht im zweiten empirischen Kapitel dieser Arbeit die Umsetzung von Studienaspirationen im Vordergrund, die die Phasen 2 (Bewerbung), 3 (Zulassung) und 4 (Studienaufnahme) umfasst. Abschnitt 8.2 fokussiert dabei - weitestgehend parallel zu den Analysen in Kapitel 7 - auf soziale Ungleichheit im Bewerbungsverhalten: Unterscheiden sich Studienberechtigte unterschiedlicher sozialer Herkunft darin, ob und wofür sie sich bewerben? Weiterhin soll hier eine erste deskriptive Exploration möglicher Bewerbungsstrategien durchgeführt werden. Im Anschluss daran liegt in Abschnitt 8.3 der Fokus auf der Fremdselektion und der Frage, ob die soziale Herkunft von Bewerber/innen mit deren Zulassungswahrscheinlichkeit zusammenhängt. Zuletzt soll in Abschnitt 8.4 die Studienaufnahme und damit die letzte Phase im Übergangsprozess betrachtet werden. Zunächst wird hier untersucht, ob Studienberechtigte mit einer oder mehreren Zulassungen ein Studium aufnehmen (konditionale Analyse). Der Übergang soll aber auch unkonditional, d.h. für alle Befragten mit Hochschulzugangsberechtigung und Studienaspiration betrachtet werden, um das Ausmaß sozialer Ungleichheit bei der Umsetzung von Studienaspirationen abbilden zu können.

Die Hypothesen zu den Phasen 2 bis 4 wurden in Kapitel 5 abgeleitet und sind zum Überblick in Tabelle 8.1 zusammengefasst. Bevor diese im Folgenden getestet werden, sollen einige methodische Besonderheiten der folgenden Untersuchung in Abschnitt 8.1 beschrieben werden.

\subsection{Methodisches Vorgehen}

Zunächst ist es an dieser Stelle erforderlich, die Definition des Ausgangssample erneut zu besprechen, da neben den grundsätzlichen, in Abschnitt 6.4 beschriebenen, Samplerestriktionen weitere inhaltliche und methodische Restriktionen getroffen werden müssen (8.1.1). Im Anschluss daran werden die abhängigen Variablen der verschiedenen Phasen und Analyseschritte (8.1.2) sowie die zur Anwendung kommende Analysestrategie vorgestellt (8.1.3), bevor zum Schluss erneut auf den Umgang mit fehlenden Werten eingegangen wird (8.1.4). 
Tab. 8.1: Hypothesen Phase 2-4

\begin{tabular}{|c|c|c|c|}
\hline & 2: Bewerbung (B) & 3: Zulassung (Z) & 4: Studienaufnahme (S) \\
\hline \multicolumn{4}{|c|}{ Hypothesenblock A: Generelle Annahmen zu Herkunftsunterschieden } \\
\hline \multirow[t]{2}{*}{$\begin{array}{l}\text { Herkunfts- } \\
\text { unterschiede }\end{array}$} & \multirow[t]{2}{*}{$\begin{array}{l}\text { HA/B: Nichtakademikerkin- } \\
\text { der bewerben sich seltener. }\end{array}$} & $\begin{array}{l}\text { HA/Za: Kaum Unterschiede } \\
\text { bezüglich Zulassung. }\end{array}$ & $\begin{array}{l}\text { HA/Sa: Kaum Unterschiede } \\
\text { bezüglich Studienaufnahme }\end{array}$ \\
\hline & & $\begin{array}{l}\mathrm{HA} / \mathrm{Zb} \text { : Nichtakademikerkin- } \\
\text { der bekommen seltener Zu- } \\
\text { lassung. }\end{array}$ & $\begin{array}{l}\text { (konditional) } \\
\text { HA/Sb: Nichtakademiker- } \\
\text { kinder nehmen seltener } \\
\text { Studium auf (unkonditional). }\end{array}$ \\
\hline \multicolumn{4}{|c|}{ Hypothesenblock B: Individuelle Einflussfaktoren und Bildungsentscheidung } \\
\hline Leistung & $\begin{array}{l}\text { HB1/B: Leistungsunter- } \\
\text { schiede erklären Teil der } \\
\text { Herkunftsdifferenz. }\end{array}$ & $\begin{array}{l}\text { HB1/Z: Leistungsunter- } \\
\text { schiede erklären Teil der } \\
\text { Herkunftsdifferenz. }\end{array}$ & \\
\hline $\begin{array}{l}\text { Rationale } \\
\text { Abwägung }\end{array}$ & $\begin{array}{l}\text { HB2/B: Einschätzung von } \\
\text { Kosten/Nutzen/Erfolgswahr- } \\
\text { scheinlichkeit erklären Teil } \\
\text { der Herkunftsdifferenz. }\end{array}$ & & \\
\hline $\begin{array}{l}\text { Soziale und } \\
\text { kulturelle } \\
\text { Ressourcen }\end{array}$ & $\begin{array}{l}\text { HB3/B: soziale und kultu- } \\
\text { relle Ressourcen erklären } \\
\text { Teil der Herkunftsdifferenz. }\end{array}$ & & \\
\hline
\end{tabular}

Hypothesenblock C: Institutionelle Einflussfaktoren und Bildungsentscheidung

\begin{tabular}{|c|c|c|}
\hline $\begin{array}{l}\text { Kosten } \\
\text { (geographische } \\
\text { Distanz) }\end{array}$ & $\begin{array}{l}\text { HC1/B: Bewerbungsradius } \\
\text { von Nichtakademikerkindern } \\
\text { ist enger. }\end{array}$ & $\begin{array}{l}\text { HC1/S: Nichtakademiker- } \\
\text { kinder studieren näher am } \\
\text { Heimatort. }\end{array}$ \\
\hline \multirow[t]{2}{*}{ Zugangshürden } & $\begin{array}{l}\text { HC2/B: Zulassungsbe- } \\
\text { schränkung und Selektivität } \\
\text { des angestrebten Faches } \\
\text { führen bei Nichtakademiker- } \\
\text { kindern eher zu Ument- } \\
\text { scheidung (keine Bewer- } \\
\text { bung). }\end{array}$ & \\
\hline & $\begin{array}{l}\text { HC3/B: Sofern keine Ument- } \\
\text { scheidung, führen Zulas- } \\
\text { sungshürden des ange- } \\
\text { strebten Faches in allen } \\
\text { Herkunftsgruppen zur Um- } \\
\text { orientierung (Bewerbung für } \\
\text { anderes Fach). }\end{array}$ & \\
\hline \multicolumn{3}{|c|}{ Hypothesenblock D: Differenzierte Bildungsentscheidungen } \\
\hline Hochschultyp & $\begin{array}{l}\text { HD1/B: Nichtakademiker- } \\
\text { kinder bewerben sich häufi- } \\
\text { ger für Fachhochschulen. }\end{array}$ & $\begin{array}{l}\text { HD1/S: Nichtakademiker- } \\
\text { kinder studieren häufiger } \\
\text { an Fachhochschulen. }\end{array}$ \\
\hline \multirow[t]{2}{*}{ Studienfach } & $\begin{array}{l}\text { HD2/B: kein Unterschied } \\
\text { bezüglich des Einkommens- } \\
\text { ertrags. }\end{array}$ & $\begin{array}{l}\text { HD2/S: kein Unterschied } \\
\text { bezüglich des Einkommens- } \\
\text { ertrags. }\end{array}$ \\
\hline & $\begin{array}{l}\text { HD3/B: Akademikerkinder } \\
\text { bewerben sich häufiger für } \\
\text { prestigereiche Fächer. }\end{array}$ & $\begin{array}{l}\text { HD3/S: Akademikerkinder } \\
\text { studieren prestigereichere } \\
\text { Fächer. }\end{array}$ \\
\hline
\end{tabular}




\subsubsection{Definition des Ausgangssamples}

Im Vergleich zum Sample für die Analysen zur Übersetzung von Studienaspirationen in -intentionen, müssen für die Analysen zu den verbleibenden Phasen aus inhaltlichen und methodischen Gründen weitere Befragte ausgeschlossen werden. Im Folgenden werden zunächst das Zustandekommen und die Zusammensetzung des neuen Ausgangssamples beschrieben. Der Ausschluss von Fällen erfolgte hier in drei Schritten: 1) Ausschluss von Befragten ohne (Fach-) Abitur 2014, 2) Ausschluss von Befragten, die ihr Studium im Sommersemester aufnahmen und 3) Ausschluss von Befragten mit fehlenden Werten auf den abhängigen und zentralen unabhängigen Variablen. Durch dieses Vorgehen wird das Ausgangssample für die Analysen der verschiedenen Umsetzungsphasen so weit wie möglich harmonisiert.

Die Analysen zur Bewerbung, Zulassung und Studienaufnahme werden nur für Befragte durchgeführt, von denen bekannt ist, dass sie die Hochschulzugangsberechtigung regulär 2014 erworben haben. ${ }^{171}$ Der Nachweis der Hochschulzugangsberechtigung stellt in Deutschland die wesentliche formale Voraussetzung für die Studienaufnahme dar (vgl. Abschnitt 2.1.1) und ist somit zentrales Definitionskriterium des risk sets für Studienbewerbungen und die spätere Studienaufnahme. Durch dieses Vorgehen sind Schüler/innen ohne Migrationshintergrund, aus Doppelakademikerhaushalten und mit sehr guter Schulleistung im verbleibenden Sample überrepräsentiert (für Details siehe Anhangstabelle A8.1). Insgesamt verbleiben damit 506 Personen (von ursprünglich 593) im Sample.

Der Übergang ins Studium kann bis zu einem Jahr nach dem Abitur abgebildet werden (vgl. Abb. 6.1 in Abschnitt 6.2.1). Die für diese Arbeit zentralen Angaben zu Bewerbungen, Zulassungen und Auswahlverfahren der Hochschulen wurden allerdings nur für die beiden WiSe (2014/15 und 2015/16) er-

171 Dieser Ausschlussgrund basiert damit sowohl auf Panelausfällen (N: 27), Befragten ohne (Fach-)Abitur (N: 28) und Befragten, die das (Fach-)Abitur nicht 2014 absolvierten, sondern ein Jahr später nachholten (N: 32). Diese kleine Gruppe unterscheidet sich nicht nur deutlich von ihren Peers mit regulärem Schulabschluss (zum Beispiel bezüglich der Schulleistung, des Bildungs- und Migrationshintergrunds, vgl. Tabelle A8.1), sondern betritt zudem erst zum WiSe 2015/16 (und nicht wie alle anderen 2014/15) das risk set. Damit variieren zum einen die Zulassungsbedingungen in der ersten Bewerbungsphase zwischen regulären und verzögerten (Fach-)Abiturient/innen; zum anderen liegen den Angaben die in Kapitel 6 beschriebenen unterschiedlichen Bewerbungsmodule zugrunde. Zwar wurde in Abschnitt 6.2.1 gezeigt, dass die W3- und W5-Bewerbungsmodule möglichst vergleichbar konzipiert wurden. Mögliche Unterschiede im Antwortverhalten können jedoch nicht ausgeschlossen werden. Um eine Verzerrung der Ergebnisse durch diese potentiellen Störfaktoren auszuschließen, werden diese Personen in den Analysen nicht berücksichtigt. 
hoben, nicht aber für das dazwischen liegende SoSe. Für 30 Befragte $(5,8 \%)$ im verbleibenden Sample ist jedoch bekannt, dass sie ihr Studium im SoSe 2015 begannen. Aufgrund fehlender Angaben zu zentralen Variablen zum Bewerbungsverhalten und Zulassungen, müssen diese Befragten von vornherein aus den folgenden Analysen ausgeschlossen werden. Ein potentielles Problem dieses Ausschlusses besteht darin, dass so Personen ausgeschlossen werden, die sich für das SoSe erfolgreich beworben haben, während möglicherweise Personen mit erfolgloser Bewerbung im Sample verbleiben, da diese Angabe nicht vorliegt. Befragte mit Studienstart im SoSe (N: 30) unterscheiden sich nur geringfügig von den verbleibenden Fällen (N: 476) (vgl. Anhangstabelle A8.2).

Neben diesen inhaltlichen Ausschlüssen werden ähnlich wie in den Analysen zu Phase 1 zusätzlich Befragte ausgeschlossen, für die die abhängigen Variablen Bewerbung, Zulassung und Studienaufnahme (meist aufgrund von Ausfällen) sowie die Variablen zur Erfassung der Schulleistung (Abiturdurchschnitt, Note Mathe und Deutsch im 4. Kurshalbjahr) nicht gebildet werden können (N: 57, 12\%). ${ }^{172}$ Das verbleibende Ausgangssample zur Untersuchung der Phasen 2-4 besteht damit aus 419 Fällen.

\subsubsection{Analyseschritte und Operationalisierungen}

Im Folgenden werden die abhängigen Variablen zur Untersuchung der einzelnen Phasen sowie die verschiedenen Analyseschritte vorgestellt.

Bewerbungen

Die Analyseschritte zur Bewerbungsphase erfolgen weitestgehend parallel zur ersten Phase. Allerdings ergeben sich aufgrund unterschiedlicher Befragungslogiken auch einige zentrale Unterschiede: Erstens liegt hier kein Faktorieller Survey zum Einfluss institutioneller Merkmale vor, sodass der Einfluss von spezifischen Auswahlverfahren, institutioneller Reputation und Informationen

172 Von den insgesamt 57 Befragten haben nur 10 (2,3\% des verbleibenden Ausgangssamples) fehlende Werte auf den Prädiktoren. Dieser Ausfallgrund ist nicht mit den abhängigen Variablen der Phasen 2-4 assoziiert (vgl. Allison 2014a). Die verbleibenden 47 Ausschlüsse resultieren aus fehlenden Werten auf den abhängigen Variablen, die vorwiegend durch Panelmortalität zustande kommen (38). Insgesamt werden Befragte mit folgenden Merkmalen signifikant häufiger ausgeschlossen: Uni0 vs. Unil (b:0,07, p: 0,052), Befragte, die die Wahrscheinlichkeit, ein Studium zu schaffen, als gering einschätzen (vs. sehr wahrscheinlich, b:-0,12, p: 0,004 ) und Befragte mit weniger als 50\% Freund/innen mit Studienplänen (b: 0,06, p: 0,46$)$. 
nicht getestet werden kann und der Einfluss der Distanz auf andere Weise indirekt geprüft wird.

In der vorliegenden Arbeit folge ich einem breiten Begriffsverständnis von Bewerbungen, das sich auf ein aktives studiengerichtetes Verhalten als notwendige Voraussetzung für die Studienaufnahme bezieht. Im deutschen Kontext kann dies, wie in Kapitel 2 beschrieben, zwei Formen annehmen, die beide eine aktive Handlung von Seiten der Studienberechtigten erfordern: Entweder müssen sich Studienberechtigte für zulassungsbeschränkte Studiengänge oder Studiengänge mit Eignungsfeststellungsverfahren bei den Hochschulen (und im Fall der meisten dualen Studiengänge: bei Unternehmen, Behörden oder sozialen Einrichtungen) bewerben oder sie müssen sich fristgerecht in zulassungsfreie Studiengänge einschreiben. Auf Grundlage der Best Up-Daten ist es nicht in allen Fällen möglich, sauber zwischen beiden Formen zu unterscheiden. Zudem dürfte es auch für Studienberechtigte mitunter schwierig sein, diese auseinanderzuhalten, da auch die Hochschulen die Begriffe nicht einheitlich verwenden (vgl. Abschnitt 6.2.1). Im Folgenden werde ich zur besseren Lesbarkeit weiterhin den Begriff Bewerbung nutzen und subsumiere darunter beide Formen ,studiengerichteten Verhaltens“.

Bewerbungen können aufgrund der oben beschriebenen Einschränkungen bezüglich des SoSe 2015 im vorliegenden Fall zu zwei Zeitpunkten erfolgen: direkt nach dem (Fach-)Abitur (WiSe 2014/15) oder ein Jahr später (WiSe 2015/16). Für die Messung von Bewerbungen direkt nach dem (Fach-)Abitur wurde das in Abschnitt 6.2.1 vorgestellte W3-Bewerbungsmodul genutzt. Befragte, die hier angaben, sich für einen Studienplatz beworben oder in einen Studiengang eingeschrieben zu haben, wurden unabhängig vom Bewerbungstyp (dual vs. nicht-dual, über die SfH, deutsche oder ausländische Hochschulen) als Bewerber/innen klassifiziert. Datenlücken, die durch fehlende Angaben im Bewerbungsmodul und durch temporäre Ausfälle entstanden, wurden durch Welle 4 Angaben möglichst ergänzt. Für die Identifizierung verzögerter Bewerbungen wurde parallel hierzu das Bewerbungsmodul aus Welle 5 genutzt.

Die Analysen zu Bewerbungen erfolgen in drei Schritten. Im ersten Schritt werden die Hypothesen der Blöcke A und B getestet. Hierbei wird untersucht, ob sich die Wahrscheinlichkeit einer Studienbewerbung zwischen Schüler/innen unterschiedlicher sozialer Herkunft unterscheidet (HA/B). Und - sofern dies der Fall ist - ob diese Herkunftsunterschiede durch Leistungsindikatoren (HB1/B), die Abwägung der drei RC-Komponenten (Kosten/Nutzen/Erfolgswahrscheinlichkeit) (HB2/B) und soziale und kulturelle Ressourcen erklärt werden (HB3/B) können. Wie bereits in Kapitel 7 werden dem Basismodell (soziale Herkunft + Kontrollvariablen) in mehreren Schritten die in drei Blöcke unterteilten erklärenden Variablen hinzugefügt und mithilfe der KHB-Methode getestet, ob die Unterschiede der Herkunftseffekte zwischen den verschiedenen Modellspezifikationen signifikant sind. 
Im zweiten Schritt werden die Hypothesen zum (sozialgruppenspezifischen) Zusammenhang zwischen antizipierten Zugangshürden im angestrebten Fach und der Bewerbungswahrscheinlichkeit getestet (Block C). Für die Frage, ob der Zusammenhang zwischen Studienbewerbung und sozialer Herkunft mit antizipierten Zulassungshürden variiert (HC2/B), wird die Bewerbungswahrscheinlichkeit in Abhängigkeit der sozialen Herkunft und den bereits bekannten Variablen zur durchschnittlichen Beschränkung und Selektivität des angestrebten Studienfaches sowie deren Interaktion modelliert. Der gleichen Logik folgt der Test der Hypothese HC3/B, mit der eine potentielle „Umorientierung“ innerhalb von als unterschiedlich selektiv wahrgenommenen Fächern untersucht wird. Die Analysen sind damit konditional und beziehen sich nur auf Bewerber/innen. Ähnlich wie in den Analysen zu Phase 1 wurde eine dichotome abhängige Variable gebildet, die den Wert 1 annimmt, wenn keine Diskrepanz zwischen der Studienfachaspiration (Zweisteller) und mindestens einer der getätigten Bewerbungen besteht. ${ }^{173}$

Inwiefern sich geographische Distanz und somit wahrgenommene Kosten als weitere institutionelle Hürde auf die Bewerbungswahrscheinlichkeit von Studienberechtigten unterschiedlicher sozialer Herkunft auswirken, kann für Phase 2 nicht direkt getestet werden. Für Bewerber/innen kann jedoch untersucht werden, inwiefern sich unterschiedliche Präferenzen für mehr oder weniger entfernte Hochschulen in ihrem Bewerbungsverhalten widerspiegeln (HC1/B). Dies erfolgt gemeinsam mit den Analysen zu Bewerbungen für verschiedene Hochschultypen (HD1/B) und Studienfächer (HD2/B und HD3/B) im dritten Schritt. Da Studienberechtigte sich oft für mehrere Studiengänge bewerben, wurden hierfür jeweils verschiedene abhängige Variablen gebildet:

Hochschultyp: a) erstgerankter (also präferierter) Studiengang an Universität oder Fachhochschule, b) ausschließlich Bewerbungen an Universitäten, c) ausschließlich Bewerbungen an Fachhochschulen.

Fach: a) Einkommen/Berufsprestige des erstgerankten Studienganges, b) Maximalwert aller Bewerbungen, c) Minimalwert aller Bewerbungen.

173 Bei der Frage nach der Übereinstimmung/Diskrepanz zwischen Fachaspirationen zu Beginn des letzten Schuljahres und Bewerbungen circa ein oder zwei Jahre später können Umorientierungen auf verschiedene Weise auftreten. Zum einen kann es sich bei fehlender Übereinstimmung - wie schon in Phase 1 angenommen - um eine tatsächliche durch (antizipierte) Barrieren verursachte Diskrepanz handeln. Zum anderen sind Umorientierungsprozesse auf Grundlage neuer Informationen im letzten Schuljahr und darüber hinaus auch bezüglich der Aspirationen möglich, sodass eine beobachtete Diskrepanz den angepassten Wünschen der Befragten entsprechen kann. Der Vergleich zwischen der fachlichen Übereinstimmung bei der erstgerankten Bewerbung und allen getätigten Bewerbungen ermöglicht eine Annäherung an diese Frage. 
Ort: a) erstgerankter Studiengang in Berlin bzw. in Berlin/Brandenburg (Pendelnähe), b) ausschließlich Bewerbungen in Berlin bzw. in Berlin/Brandenburg, c) Maximaldistanz aller Bewerbungen. ${ }^{174}$

Beim dritten Analyseschritt (sowie auch bei der Hypothese HC3/B) handelt es sich um konditionale Analysen, die nur für Befragte mit direkter oder verzögerter Studienbewerbung (N: 359) durchgeführt werden. Für Befragte mit Bewerbungen zu beiden Zeitpunkten wird die erste Bewerbungsphase berücksichtigt und für deren Timing kontrolliert (WiSe 2014/15 oder WiSe 2015/16).

Bewerbungsmuster und -strategien

Neben den bereits aus Kapitel 7 weitestgehend bekannten Analyseschritten kommt für Phase 2 zudem eine erste Beschreibung (sozialgruppenspezifischer) Bewerbungsstrategien hinzu, deren Operationalisierung im Folgenden vorgestellt wird.

In Abschnitt 4.3.2 wurden empirische Studien vorgestellt, die zum Beispiel die Anzahl (vgl. Ayalon 2007, Hurtado et al. 1997, Roderick et al. 2011, Smith 2014) und Breite (vgl. Sá und Tavares 2018) von Bewerbungen als wichtige Bewerbungsstrategien identifizieren. Bei Präferenzen für selektive Studienprogramme mit geringen bzw. unsicheren Zulassungschancen wurde zudem das Versenden von back-up Bewerbungen für weniger selektive Studienoptionen (peer/safe colleges) als wichtige Strategie identifiziert (vgl. Hoxby und Avery 2013). Für den deutschen Kontext stellt die Untersuchung von Studienbewerbungsmustern und -strategien bislang Neuland dar. Mithilfe der Best Up-Bewerbungsmodule in Verbindung mit den Rekrutierungsdaten ist es erstmals möglich, Bewerbungsmuster und -strategien auch für deutsche Studienberechtigte zu approximieren.

Nicht alle Bewerbungsmuster müssen mit einer intendierten Strategie verbunden sein; in welchen Fällen dies zutrifft, ist schwer festzustellen. Unabhängig davon, ob bewusst intendiert oder nicht, können bestimmte Muster in ihrer Wirkung mehr oder weniger strategisch sein. Welche Muster strategisch sind, hängt jedoch vom angestrebten Ziel ab. Bestehen zum Beispiel starke Präferenzen für ein bestimmtes Studienfach, kann eine andere Strategie sinnvoll sein als bei sehr starken örtlichen Präferenzen. Eine solche Differenzierung ist hier nicht möglich. Es soll daher neben der Beschreibung von Bewerbungsmustern

174 Für die Maximaldistanz wurde die Entfernung in Kilometern zwischen Berlin (Zentrum) und der jeweiligen Hochschulstadt (Zentrum) mit Hilfe des Onlinetools „https://www.luftlinie.org/“ berechnet. Es wurde sowohl die Luftlinie als auch die PKW-Distanz berechnet. Da sich die Ergebnisse nicht unterscheiden, wird im Folgenden nur die Luftlinie genutzt. Für Auslandsbewerbungen wurde der Wert auf $700 \mathrm{~km}$ festgesetzt. 
versucht werden, allgemeine Strategien zu operationalisieren, die Zulassungschancen generell erhöhen.

In Anlehnung an vorhandene empirische Studien zum Thema, wurden verschiedene Variablenblöcke operationalisiert: erstens die Anzahl und Breite der Bewerbungen, zweitens die zur Anwendung kommenden Zulassungsbeschränkungen und Auswahlverfahren und drittens die Zulassungschancen, die sich aus dem Verhältnis der eigenen Abiturleistung und Wartezeit sowie den jeweiligen Auswahlgrenzen der gewählten Studiengänge ergeben. Für den ersten Variablenblock zur Anzahl und Breite wurden ausschließlich die Best UpIndividualdaten genutzt (Bewerbungsmodule aus Welle 3 für direkte Bewerbungen und Welle 5 für verzögerte Bewerbungen). Für den zweiten und dritten Variablenblock wurden zudem die Rekrutierungsdaten herangezogen. Die Rekrutierungsrecherche wurde - wie in Abschnitt 6.2.2 beschrieben - für die jeweils erst- und zweitgerankte Bewerbung durchgeführt, sodass sich die Variablen auf diese beiden Bewerbungen pro Befragte/n beziehen. Zudem wurde keine Rekrutierungsrecherche für duale Studiengänge (Zugangsweg: Unternehmen) sowie Studiengänge an ausländischen Hochschulen durchgeführt, sodass Befragte deren erst- und zweitgerankte Bewerbungen in diese Kategorie fallen, hier nicht berücksichtig werden können (direkte Bewerbung: $23 \mathrm{Be}-$ fragte, verzögerte: 4 Befragte). Die einzelnen Variablen und deren Operationalisierung sind in Tabelle 8.2 zusammengefasst.

Betrachtet wird auch hier die jeweils erste Bewerbungsphase, die entweder im WiSe 2014/15 oder 2015/16 stattfand. Ein Vergleich mit einer möglichen zweiten Bewerbungsphase ist aufgrund zu geringer Fallzahlen nicht möglich. ${ }^{175} \mathrm{Da}$ es sich bei der Untersuchung von Studienbewerbungsmustern und -strategien - wie oben bereits angedeutet - für den deutschen Kontext um bisher unerforschtes Gebiet handelt, haben die präsentierten Ergebnisse einen vornehmlich explorativen Charakter und dienen der erstmaligen, systemati-

175 Best Up befasst sich zentral mit nachschulischen Bildungsentscheidungen (Studium versus Ausbildung), nicht jedoch mit Studienabbrüchen und -wechseln. In Welle 5 wurde daher das in Abschnitt 6.2.1 beschriebene, ausführliche Bewerbungsmodul nur Befragten vorgelegt, die zuvor nicht bereits ein Studium aufgenommen hatten. Ebenso wurde die Rekrutierungsrecherche für verzögerte Bewerbungen nur durchgeführt, sofern keine Studienaufnahme zum WiSe 2014/15 oder zum SoSe 2015 erfolgte. Ausführliche Informationen zur zweiten Bewerbungsphase liegen damit nur vor, wenn die erste nicht in einem Studium mündete (je nach Variable für circa 20 bis 30 Befragte). Alternativ könnte zwischen direkten und verzögerten Bewerbungsstrategien unterschieden werden. Verzögerte Bewerbungen wären mit circa 90 Befragten zwar besser besetzt als die zweite Bewerbungsphase. Für eine Differenzierung nach sozialer Herkunft bietet sich aber auch dies nicht an. Zudem wären in diesem Fall diejenigen Befragten zusammengefasst, die sich zum WiSe 2015/16 erstmalig und wiederholt bewarben (sofern kein Übergang ins Studium nach einer direkter Bewerbung erfolgte). 
schen Beschreibung von Bewerbungsmustern/-strategien (für das gesamte Analysesample und getrennt nach Herkunftsgruppen).

Tab. 8.2: Operationalisierung Bewerbungsmuster und -strategien

\begin{tabular}{|c|c|}
\hline Variable & Operationalisierung \\
\hline \multicolumn{2}{|r|}{ Anzahl/Breite der Bewerbungen } \\
\hline Anzahl & $\begin{array}{l}\text { Anzahl der Bewerbungen: metrisch und kategorial mit } 4 \text { Kategorien }(1,2-3 \text {, } \\
4-5,>5)\end{array}$ \\
\hline $\begin{array}{l}\text { Studienart (dual } \\
\text { über Unternehmen) }\end{array}$ & $\begin{array}{l}\text { Bewerbung duales/nicht-duales Studium (Zugangsweg: Unternehmen) } \\
3 \text { Kategorien: } \\
\text { - Bewerbungen ausschließlich für duale Studiengänge } \\
\text { - Bewerbungen sowohl duale als auch nicht-duale Studiengänge } \\
\text { - Bewerbungen ausschließlich für nicht-duale Studiengänge }\end{array}$ \\
\hline Breite Hochschultyp & Bewerbungen an einem/mehr als einem Hochschultyp \\
\hline Breite Fächer & Bewerbungen nur für ein Fach/für mehrere Fächer (Zweisteller) \\
\hline Breite Standorte & Bewerbungen nur an einem/an mehr als einem Ort \\
\hline \multicolumn{2}{|r|}{ Zulassungsbeschränkungen und Auswahlverfahren } \\
\hline $\begin{array}{l}\text { Zulassungs- } \\
\text { beschränkung }\end{array}$ & $\begin{array}{l}\text { Art der Zulassungsbeschränkung zum Bewerbungszeitpunkt (direkt: WiSe } \\
\text { 2014/15, verzögert: WiSe 2015/16), jeweils für erst- und zweitgerankten } \\
\text { Studiengang, } 3 \text { Kategorien: } \\
\text { - zulassungsfrei } \\
\text { - örtlich zulassungsbeschränkt } \\
\text { - } \quad \text { bundesweit zulassungsbeschränkt } \\
\text { Kommen Zulassungsbeschränkungen und/oder Eignungsfeststellungs- } \\
\text { verfahren zum Einsatz, jeweils für erst- und zweitgerankten Studiengang, } \\
3 \text { dichotome Variablen: } \\
\text { - Zulassungsbeschränkung } \\
\text { - Eignungsfeststellungsverfahren } \\
\text { - Zulassungsbeschränkung oder Eignungsfeststellungsverfahren }\end{array}$ \\
\hline $\begin{array}{l}\text { Komplexe } \\
\text { Auswahlverfahren }\end{array}$ & $\begin{array}{l}\text { Kommen Verfahren zum Einsatz, die über das Zusenden von Nachweisen } \\
\text { (z.B. in Form des Abiturzeugnisses oder Praktikumsnachweises) hinausge- } \\
\text { hen? Tests, Auswahlgespräche sowie Motivationsschreiben/Arbeitsproben } \\
\text { wurden aufgrund der geringen Fallzahl zu einer Variable zusammengefasst. } \\
\text { Dichotome Variable, jeweils für erst- und zweitgerankten Studiengang }\end{array}$ \\
\hline \multicolumn{2}{|r|}{ Zulassungschancen } \\
\hline $\begin{array}{l}\text { Zulassungschancen, } \\
\text { gegeben der } \\
\text { individuellen DN } \\
\text { und WZ }\end{array}$ & $\begin{array}{l}\text { Schritt 1: Bildung der Differenz zwischen der in der Abiturientenbestenquote } \\
\text { erforderlichen Durchschnittsnote (DN) im Vorjahr und der eigenen DN. } \\
\text { Zulassungsfreien Studiengängen oder solchen, bei denen im Vorjahr alle } \\
\text { Bewerber/innen zugelassen wurden, wurde der Wert } 4,0 \text { zugewiesen als } \\
\text { Mindestnote für den Erhalt der Hochschulzugangsberechtigung. }\end{array}$ \\
\hline & $\begin{array}{l}\text { Empirisch vorkommender Range: } 2,9 \text { Notenpunkte bessere - } \\
2,4 \text { Notenpunkte schlechtere individuelle DN. }\end{array}$ \\
\hline
\end{tabular}




\begin{tabular}{|c|c|}
\hline \multirow[t]{4}{*}{ Variable } & Operationalisierung \\
\hline & $\begin{array}{l}\text { Schritt 2: Identifizierung von Fällen, für die die Auswahlgrenze des Vorjahres } \\
\text { in der Wartezeitquote für eine Zulassung ausreicht (nur verzögerte } \\
\text { Bewerbungen) }{ }^{176}\end{array}$ \\
\hline & $\begin{array}{l}\text { Schritt 3: Bildung einer dichotomen Variable für erst- und zweitgerankten } \\
\text { Studiengang (oder einem von beiden): } \\
\text { - weitestgehend realistische Zulassungschancen: Eigene DN ist weniger } \\
\text { als } 0,3 \text { Notenpunkte schlechter als im Vorjahr zuletzt zugelassene DN } \\
\text { (Abiturbestenquote) und/oder Auswahlgrenze in WZ-Quote betrug bis zu } \\
2 \text { Semester (verzögerte Bewerbungen) }\end{array}$ \\
\hline & $\begin{array}{l}\text { - unsichere Zulassungschancen: Eigene DN ist mindestens } 0,3 \\
\text { Notenpunkte schlechter als im Vorjahr zuletzt zugelassene DN und } \\
\text { Auswahlgrenze in WZ-Quote betrug mehr als } 2 \text { Semester (verzögerte } \\
\text { Bewerbungen) }\end{array}$ \\
\hline \multirow[t]{5}{*}{$\begin{array}{l}\text { Ausgleichs- } \\
\text { möglichkeit } \\
\text { individuelle DN/WZ } \\
\text { durch AdH-Kriterien }\end{array}$} & $\begin{array}{l}\text { Kommen im Auswahlverfahren des erst- und zweitgerankten Studiengangs } \\
\text { (oder einem von beiden) weitere AdH-Kriterien zur Anwendung, mit denen } \\
\text { eine potentiell zu schlechte DN (im oben definierten Sinne) und - im Falle } \\
\text { verzögerter Bewerbungen - eine zu kurze WZ ausgeglichen werden } \\
\text { können? Im Falle von direkten Bewerbungen wurde Berufserfahrung als } \\
\text { Kriterium ausgeschlossen, da die geforderte Dauer in der Regel nicht von } \\
\text { „frischen“ Abiturient/innen erreicht werden kann. }\end{array}$ \\
\hline & 3 Kategorien: \\
\hline & \\
\hline & $\begin{array}{l}\text { - DN/WZ wahrscheinlich nicht ausreichend + weitere Auswahlkriterien zum } \\
\text { Ausgleich }\end{array}$ \\
\hline & - DN/WZ wahrscheinlich ausreichend \\
\hline \multirow[t]{2}{*}{$\begin{array}{l}\text { Ausgleichs- } \\
\text { möglichkeit } \\
\text { individuelle DN/WZ } \\
\text { durch zusätzliche } \\
\text { Bewerbungen }\end{array}$} & $\begin{array}{l}\text { Gibt es neben der ersten und gegebenenfalls zweiten Bewerbung weitere } \\
\text { Bewerbungen, durch die Zulassungschancen erhöht werden können (sofern } \\
\text { DN und WZ für erst- oder zweitgerankten Studiengang wahrscheinlich nicht } \\
\text { ausreichen)? } 3 \text { Kategorien: }\end{array}$ \\
\hline & $\begin{array}{l}\text { - DN/WZ wahrscheinlich nicht ausreichend + keine weiteren Bewerbungen } \\
\text { - } \mathrm{DN} / \mathrm{WZ} \text { wahrscheinlich nicht ausreichend + weitere Bewerbungen } \\
\text { - } \mathrm{DN} / \mathrm{WZ} \text { wahrscheinlich ausreichend }\end{array}$ \\
\hline
\end{tabular}

\section{Zulassung}

In Phase 3 steht die Frage im Mittelpunkt, ob die Zulassungschancen zum Studium mit der sozialen Herkunft der Studienbewerber/innen zusammen hängt (HA/Za oder HA/Zb) und - sofern dies der Fall ist - ob dies durch Leistungsunterschiede (hier: primäre Effekte) erklärt werden kann (HB1/Z).

176 Theoretisch ist dieses Vorgehen auch für direkte Bewerbungen möglich, da die Auswahlgrenze in der Wartezeitquote auch 0 betragen kann. Dies kommt nur in sehr wenigen Fällen vor (hier bei einem erstgeranktem und zwei zweitgerankten Studiengängen). Die betreffenden Befragten haben bereits aufgrund des Verhältnisses ihrer eigenen und der im Vorjahr erforderlichen Abiturnote eine ,realistische Bewerbung“. 
In Phase 2 wurde die Variable Bewerbung breit definiert als aktives studiengerichtetes Verhalten, das sowohl Bewerbungen für zulassungsbeschränkte Studiengänge als auch die Immatrikulation in zulassungsfreie Studiengänge beinhaltet. Der zentralen abhängigen Variable in Phase 3 unterliegt ein ähnliches Verständnis. Als ,zugelassen zum Studium“ werden alle Befragten definiert, die entweder angaben, nach einer Bewerbung für einen zulassungsbeschränkten Studiengang zugelassen worden zu sein oder die sich in einen zulassungsfreien Studiengang immatrikulierten (Welle 4 und 5). Im Fall zulassungsfreier Studiengänge wird die Immatrikulation also sowohl mit dem Ereignis Bewerbung als auch Zulassung gleichgesetzt. ${ }^{177}$

Bei den Analysen zu Zulassungschancen handelt es sich um konditionale Analysen, in denen nur Befragte berücksichtigt werden, die sich für ein Studium beworben haben. Die Studienbewerbung ist also zentrales Kriterium für die Definition des risk sets.

\section{Studienaufnahme}

Mit der vierten und letzten Phase wird schließlich die Studienaufnahme untersucht und somit die finale Realisierung der Studienaspiration.

Auch hier wird im ersten Schritt der Frage nachgegangen, ob die Studienwahrscheinlichkeit und somit die Umsetzungswahrscheinlichkeit von Studienaspirationen mit der sozialen Herkunft der Studienberechtigten variiert. Die abhängige Variable ist dichotom und nimmt den Wert 1 an, wenn Befragte zum WiSe 2014/15 oder 2015/16 ein Studium aufnahmen. Die Analysen sind zweigeteilt: zunächst wird die Studienaufnahme wie bereits die Zulassungswahrscheinlichkeit in der vorangegangenen Phase konditional modelliert, um Hypothese HA/Sa zu testen. Die Analysen beziehen sich damit nur auf Befragte mit einer oder mehreren Zulassungen zum Studium. Zuletzt wird die Studienaufnahme - wie in vielen Studien üblich (vgl. Abschnitt 3.2) - aber auch unkonditional, d.h. im vorliegenden Fall für das gesamte Ausgangssample der Phasen 2-4, unabhängig von getätigten Bewerbungen und erhaltenen Zusagen untersucht. Hiermit soll das letztliche Ausmaß sozialer Ungleichheit bei der Umsetzung von Studienaspirationen erfasst werden (HA/Sb).

Um sich erneut der Frage sozialer Ungleichheiten innerhalb des Hochschulsystems zu nähern, stehen im zweiten Schritt - parallel zu den Phasen 1 und 2 - die Fragen im Mittelpunkt, an welchen Hochschultypen (HD1/S) und

177 Neben dem „Ob“ wäre zudem die Frage relevant, wofür Befragte zugelassen wurden, um Aussagen darüber treffen zu können, vor welchen mehr oder weniger attraktiven Auswahloptionen sie nach der Selektion durch die Hochschulen standen. Die Variable Zulassung ist jedoch nicht für alle Studiengänge rekonstruierbar, für die sich die Befragten bewarben, sodass zum Beispiel keine umfassenden Aussagen darüber getroffen werden können, für welches Fächerspektrum, an welchen Orten oder für welche Hochschultypen Zulassungen vorliegen. 
welche Studienfächer (HD2/S und HD3/S) Schüler/innen unterschiedlicher sozialer Herkunft studieren. Außerdem wird untersucht, ob sie regional mobil sind (Studium in Berlin, Berlin/Brandenburg, Distanz in km zwischen Berlin und Hochschulort) (HB1/S). Bei Schritt 2 handelt es sich erneut um konditionale Analysen, die nur für diejenigen durchgeführt werden, die überhaupt studieren (N: 293). Dabei wird der erste Übergang ins Studium (und kein Studiengangswechsel) berücksichtigt und für das Timing des Übergangs (WiSe 2014/15 oder 2015/16) kontrolliert.

\subsubsection{Auswertungsstrategie}

Auch in den Analysen zur zweiten, dritten und vierten Phase kommen die in Abschnitt 6.5.1 eingeführten Generalized Linear Mixed Modelle zum Einsatz. Anders als bei den Untersuchungen zur ersten Phase ist es hier allerdings notwendig, der zeitlichen Dimension der Ereignisse und hierdurch auftretenden Zensierungen Rechnung zu tragen. Dies erfolgt durch die Anwendung ereignisanalytischer Verfahren, die für die Beantwortung der Frage, $o b$ ein Ereignis - hier eine Studienbewerbung, Zulassung oder Studienaufnahme - eintritt, geeigneter sind als statistische Standardprozeduren (vgl. Allison 1982, Singer und Willet 2003). Um die Bewerbungs-, Zulassungs- und Studienwahrscheinlichkeit bis ein Jahr nach Erhalt des (Fach-)Abiturs zu untersuchen, kommt mit der Berechnung von Hazardratenmodellen für diskrete Zeit ein solches Verfahren zum Einsatz (vgl. Allison 1982, 2014b, Singer und Willet 2003). ${ }^{178}$ Dieses modelliert, wie groß die konditionale Wahrscheinlichkeit ist, dass das jeweilige Ereignis eintritt, sofern es nicht bereits eingetreten ist. ${ }^{179}$

Um Hazardratenmodelle für diskrete Zeit berechnen zu können, muss das Datenformat zunächst so angepasst werden, dass die Analyseeinheit sich nicht auf einzelne Personen bezieht, sondern auf „Personen-Jahre“ (Allison 2014b: 10f.). ${ }^{180}$ Jede Person wird damit solange berücksichtigt, bis das Ereignis eintritt

178 In ereignisanalytische Verfahren kann die Zeit entweder kontinuierlich oder diskret einbezogen werden. Im vorliegenden Fall ist die Berücksichtigung diskreter Zeitintervalle sinnvoll, nicht nur aufgrund der diskreten Erhebungszeitpunkte, sondern vor allem auch, weil die betrachteten Ereignisse inhärent diskret sind, d.h. nur zu bestimmten Zeitpunkten auftreten können (vgl. Singer und Willet 2003: 313).

179 Ereignisanalytische Verfahren eignen sich insbesondere zur Beantwortung von „Ob“ und „Wann“ Fragen (vgl. Singer und Willet 2003). Für die Analyseschritte zum „Wofür“ (Hochschultyp, Studienfach, Hochschulstandort) und zur Diskrepanz zwischen Fachaspirationen und deren Umsetzung ist die Anwendung solcher Verfahren nicht erforderlich und die Daten werden in ihrem ursprünglichem Format belassen. Um für mögliche Timingeffekte zu kontrollieren, wird jedoch für den Zeitpunkt - direkt oder verzögert - der jeweiligen Beobachtung kontrolliert.

180 Umgesetzt wurde die Anpassung des Datenformats in Stata mithilfe des von Singer und Willet bereitgestellten ,prsnperd“-Kommandos. 
oder die Person das risk set verlässt, obwohl das Ereignis noch nicht beobachtet wurde. Letzteres wird als Rechtszensierung bezeichnet, die von statistischen Standardprozeduren nicht angemessen einbezogen wird (vgl. Allison 1982: 629) und zu Verzerrungen der Schätzer führen kann. Ereignisanalytische Methoden beziehen hingegen die Zensierung von Fällen explizit mit ein: „The beauty of survival analysis is that, under the assumption of independent [or non-informative, $\mathrm{CF}$ ] censoring, we can use the risk set to estimate what would have happened to the entire remaining population were there no censoring" (Singer und Willet 2003: 336).

Zensierung kann prinzipiell in zwei Formen auftreten (vgl. Allison 2014: 15ff.): Sie kann erstens festgelegt (fixed) sein, wenn sie designbedingt ist und für alle Befragten zum gleichen Zeitpunkt auftritt. Sie kann weiterhin - in Allisons Terminologie - zufällig (random) sein, wenn Personen ohne Ereignis das risk set frühzeitig verlassen (z.B. durch Panelmortalität oder anderweitige Selektion aus dem risk set). Zufällig bedeutet hier nicht zwangsläufig ,unrelated to anything else" (Allison 2014: 15). Dennoch liegt Hazardratenmodellen die Annahme zugrunde, dass jedwede zufällige Zensierung nicht-informativ ist, d.h., dass sie nicht mit der Wahrscheinlichkeit des Auftretens des Ereignisses zum Zeitpunkt der Zensierung zusammenhängt. Ein formaler Test dieser Annahme ist nicht möglich, da die Ereigniswahrscheinlichkeit per Definition nicht beobachtet werden kann (was dazu führt, dass das Problem häufig ignoriert wird) (vgl. Allison 2014b: 16, Singer und Willet 2003: 318f.).

Allison (2014b) schlägt einen Sensitivitätstest vor, dem ich folgen werde: Hiernach wird das tatsächliche Modell mit zwei extremen Szenarien verglichen. Im ersten Szenario wird ein hoher Hazard für alle zensierten Fälle angenommen und das Ereignis zum Zeitpunkt der Zensierung auf 1 gesetzt. Im zweiten Szenario wird ein konstant niedriger Hazard angenommen und das Ereignis in allen folgenden Perioden (bis zur designbedingten Zensierung) auf 0 gesetzt. Ein Vergleich der Modelle ermöglicht, sich dem Ausmaß des Problems nicht-informativer Zensierung anzunähern.

Neben nicht-informativer Zensierung ist eine weitere Modellannahme, dass der Einfluss der erklärenden Variablen über die Zeit konstant ist (Proportionalitätsannahme). Zur Prüfung dieser Annahme kann das Modell mit zeitkonstanten Effekten mit einer flexibleren Spezifikation, die Interaktionen zwischen der sozialen Herkunft (sowie weiteren unabhängigen Variablen) und den Periodendummies enthält, anhand eines Ratio-Likelihood Chi-Quadrat Tests verglichen werden. ${ }^{181}$ Die Verletzung der Proportionalitätsannahme kann insgesamt als weniger problematisch angesehen werden als etwa informative Zensierung (vgl. Allison 2014b: 43), insbesondere wenn ein ereignisdatenanalyti-

181 Der Ratio-Likelihood Chi-Quadrat Test testet die Null-Hypothese, dass alle zusätzlich zu einem Modell hinzugefügten Parameter gemeinsam 0 sind. Ist der ChiQuadrat Wert nicht signifikant, kann die Null-Hypothese und somit die Proportionalitätsannahme aufrechterhalten werden. 
sches Vorgehen vor allem dafür genutzt wird, um das Auftreten von Ereignissen trotz vorhandener Zensierungen sauber zu schätzen, das genuine Forschungsinteresse (wie im vorliegenden Fall) jedoch nicht der Einfluss der Zeit ist. Die geschätzten Effekte können dann als Durchschnittseffekte der unabhängigen Variablen im betrachteten Zeitraum interpretiert werden (vgl. ebd.).

Abschließend soll hier ein Überblick über die Konstruktion der Perioden gegeben werden, da diese sich zwischen den verschiedenen Analyseschritten unterscheidet. Eine zusammenfassende Darstellung kann Tabelle 8.3 entnommen werden. In den Analysen zur Bewerbungswahrscheinlichkeit und zur unkonditionalen Studienwahrscheinlichkeit folgen die Perioden der historischen Zeit (direkt nach dem (Fach-)Abitur, WiSe 2014/15, oder ein Jahr später, WiSe 2015/16). Für die Analysen zur Zulassungswahrscheinlichkeit werden die Perioden hingegen entsprechend der Bewerbungsphasen, also dem Eintritt in das risk set, konstruiert. Zeitpunkt 1 bezieht sich damit auf Zulassungen nach der ersten Bewerbungsphase, die sowohl direkt nach dem (Fach-)Abitur als auch ein Jahr verzögert stattfinden kann. Zeitpunkt 2 geht für diejenigen in die Analysen ein, für die in der ersten Bewerbungsphase das Ereignis Zulassung nicht beobachtet werden konnte und für die eine zweite Bewerbungsphase vorliegt. Die Zensierungsgründe vervielfältigen sich damit (vgl. Tabelle 8.3). Einer ähnlichen Logik folgen die Analysen zur konditionalen Studienwahrscheinlichkeit. Hier wurden die Perioden entsprechend der ersten und gegebenenfalls zweiten Zulassungsphase konstruiert. Allerdings zeigt sich empirisch, dass eine zweite Zulassungsphase nur für 15 Personen in die Analysen eingehen würde, die nach der ersten Zulassungsphase (hier im WiSe 2014/15) nicht ins Studium übergingen und die sich ein Jahr später erneut bewarben und eine $\mathrm{Zu}$ sage erhielten. Alle diese 15 Personen gehen zu Zeitpunkt 2 ins Studium über, sodass ein ereignisanalytisches Verfahren hier nicht sinnvoll erscheint. Das alternative analytische Vorgehen wird in Abschnitt 8.4 vorgestellt.

Abbildung A8.1 im Anhang gibt, getrennt nach sozialer Herkunft, einen zusammenfassenden Überblick über die Verläufe von Phase 2 bis 4. Ersichtlich werden hier die Größe der jeweiligen risk sets sowie die Anteile an Befragten, für die das jeweilige Ereignis (in der ersten oder zweiten Periode) beobachtet werden kann. Zudem können der Anteil an zensierten Fällen sowie die Zensierungsgründe nachvollzogen werden. 
Tab. 8.3: Definition der risk sets, Perioden und hieraus resultierende Zensierungsgründe

\begin{tabular}{|c|c|c|c|c|}
\hline Analyseschritt & Risk Set & T1 & T2 & (potentielle) Zensierungsgründe \\
\hline \multirow[t]{2}{*}{ Bewerbung } & \multirow[t]{2}{*}{$\begin{array}{l}\text { Studien- } \\
\text { berechtigte }^{*}\end{array}$} & \multirow[t]{2}{*}{ WiSe 2014/15 } & \multirow[t]{2}{*}{ WiSe 2015/16 } & $\begin{array}{l}\text { Keine Bewerbung } \mathrm{t} 1+ \\
\text { Ausfall t2 }\end{array}$ \\
\hline & & & & Keine Bewerbung $\mathrm{t} 1$ und $\mathrm{t} 2$ \\
\hline \multirow[t]{4}{*}{ Zulassung } & \multirow[t]{4}{*}{$\begin{array}{l}\text { Bewerber/ } \\
\text { innen }\end{array}$} & \multirow{4}{*}{$\begin{array}{l}\text { 1. Bewer- } \\
\text { bungsphase: } \\
\text { WiSe 2014/15 } \\
\text { oder 2015/16 }\end{array}$} & \multirow{4}{*}{$\begin{array}{l}\text { 2. Bewer- } \\
\text { bungsphase: } \\
\text { WiSe 2015/16 }\end{array}$} & $\begin{array}{l}\text { Keine Zulassung nach } \\
\text { 1. Bewerbung } 2014+\text { Ausfall t2 }\end{array}$ \\
\hline & & & & $\begin{array}{l}\text { Keine Zulassung nach } \\
\text { 1. Bewerbung } 2014+ \\
\text { keine erneute Bewerbung }\end{array}$ \\
\hline & & & & $\begin{array}{l}\text { Keine Zulassung nach } \\
\text { 1. Bewerbung } 2015\end{array}$ \\
\hline & & & & $\begin{array}{l}\text { Keine Zulassung nach 1. und } 2 . \\
\text { Bewerbung }\end{array}$ \\
\hline \multirow{4}{*}{$\begin{array}{l}\text { Studien- } \\
\text { aufnahme } \\
\text { (konditional) }\end{array}$} & \multirow{4}{*}{ Zugelassene } & \multirow{4}{*}{$\begin{array}{l}\text { 1. Zulassungs- } \\
\text { phase: WiSe } \\
2014 / 15 \text { oder } \\
2015 / 16\end{array}$} & \multirow{4}{*}{$\begin{array}{l}\text { 2. Zulassungs- } \\
\text { phase: WiSe } \\
2015 / 16\end{array}$} & $\begin{array}{l}\text { Kein Studium nach } 1 \\
\text { Zulassung } 2014+\text { Ausfall t2 }\end{array}$ \\
\hline & & & & $\begin{array}{l}\text { Kein Studium nach } 1 . \\
\text { Zulassung } 2014+\text { keine erneute } \\
\text { Zulassung (od. Bewerbung) }\end{array}$ \\
\hline & & & & $\begin{array}{l}\text { Kein Studium nach } \\
\text { 1. Zulassung } 2015\end{array}$ \\
\hline & & & & $\begin{array}{l}\text { Kein Studium nach } 1 . \text { und } 2 . \\
\text { Zulassung }\end{array}$ \\
\hline $\begin{array}{l}\text { Studien- } \\
\text { aufnahme } \\
\text { (unkond.) }\end{array}$ & $\begin{array}{l}\text { Studien- } \\
\text { berechtigte* }^{*}\end{array}$ & WiSe 2014/15 & WiSe 2015/16 & $\begin{array}{l}\text { Kein Studium } \mathrm{t} 1 \text { + Ausfall } \mathrm{t} 2 \\
\text { Kein Studium } \mathrm{t} 1 \text { und } \mathrm{t} 2\end{array}$ \\
\hline
\end{tabular}

${ }^{*}$ Für die genaue Definition des Analysesamples siehe Abschnitt 8.1.1.

\subsubsection{Umgang mit fehlenden Werten}

Während die Analysen zu Phase 3 über die generellen Sampleeinschränkungen hinaus nicht von weiteren Einschränkungen betroffen sind, sind die einzelnen Analyseschritte der (selbstselektiven) Phasen 2 und 4 in gleichem Maße von Einschränkungen durch fehlende Werte (insbesondere bezüglich der Fachaspiration sowie den abhängigen Variablen zum Hochschultyp und Fach) betroffen wie die der ersten Phase. Das Vorgehen gleicht damit dem in Abschnitt 7.1.4 beschriebenen.

Für die Variablen zu den Bewerbungsstrategien erscheint eine ergänzende Erläuterung angebracht, da sich hier die Fallzahl leider deutlich reduziert. Zwar ist für 416 Befragte bekannt, dass sie sich bewarben, doch kann nur für 317 rekonstruiert werden, wie oft und wie breit sie dies taten. Dies liegt zum einen daran, dass die Bewerbungsmodule nicht ausreichend ausgefüllt wurden. Zum anderen konnten zwar Bewerbungen temporärer Welle 3 Ausfälle mithilfe der Welle 4 Angaben ergänzt werden, doch ist dies für differenziertere 
Bewerbungsstrategien nicht möglich, da in Welle 4 nur sehr eingeschränkte Informationen hierzu erfasst wurden. Neben diesen Einschränkungen durch die Individualdaten kommen für die Variablenblöcke zu Zulassungsbeschränkungen und Zulassungschancen weitere fehlende Werte durch die Rekrutierungsdaten hinzu, da die Recherche nicht für alle angegebenen Studiengänge durchgeführt werden konnte (etwa im Fall dualer Studiengänge oder „Problemfällen“, siehe Abschnitt 6.2.2) oder einzelne Angaben, etwa zu den Auswahlgrenzen, nicht recherchiert werden konnten. ${ }^{182}$

Wie auch für die Analysen zu Phase 1 sind für alle Analyseschritte die genauen Fallzahlen, Ausfallgründe und hierdurch entstehende Selektivitäten in Anhangstabelle A8.3 dokumentiert.

\subsection{Ergebnisse Phase 2: Herkunftsunterschiede im Bewerbungsverhalten}

In den Analysen zur ersten Phase zeigte sich, dass die meisten Schüler/innen ihre Studienaspiration in eine Studienintention übersetzen. Es konnten aber auch Herkunftsunterschiede beobachtet werden. Ob sich dies im Bewerbungsverhalten widerspiegelt ist grafisch in Abbildung 8.1 in Form der „Überlebenswahrscheinlichkeit" von Studienberechtigten unterschiedlicher sozialer Herkunft dargestellt. Bei der Überlebenswahrscheinlichkeit handelt es sich um einen ereignisdatenanalytischen Begriff, der die Wahrscheinlichkeit angibt, zu den betrachteten Zeitpunkten im risk set zu verbleiben, oder anders ausgedrückt: das Ereignis - in diesem Fall eine Studienbewerbung - noch nicht erlebt zu haben. Das deskriptive Maß „Überlebenswahrscheinlichkeit“ kann in Form von Prozentwerten interpretiert werden.

182 Für Befragte mit unzureichenden Angaben im Bewerbungsmodul ist zumindest zum Teil zu vermuten, dass sie vom Bewerbungsprozess eher überfordert waren bzw. diesen weniger gut durchdacht angingen. Diese Vermutung wird dadurch gestützt, dass Befragte mit schlechter Schulleistung, geringerer wahrgenommener Erfolgswahrscheinlichkeit sowie Bewerbungsunterstützung aus dem sozialen Netzwerk häufiger ausfallen (vgl. Tabelle A8.3). Die im Analysesample verbleibenden Befragten sind damit deutlich positiv selektiert, und die Variablen bilden damit das Bewerbungsverhalten von informierten und organisierten Bewerber/innen vermutlich besser ab als von eher unvorbereiteten, uninformierten Bewerber/innen. Zudem sind Doppelakademikerkinder überrepräsentiert, sodass Herkunftsunterschiede potentiell unterschätzt werden. 
Abb. 8.1: Studienbewerbung nach sozialer Herkunft (Überlebenswahrscheinlichkeit)

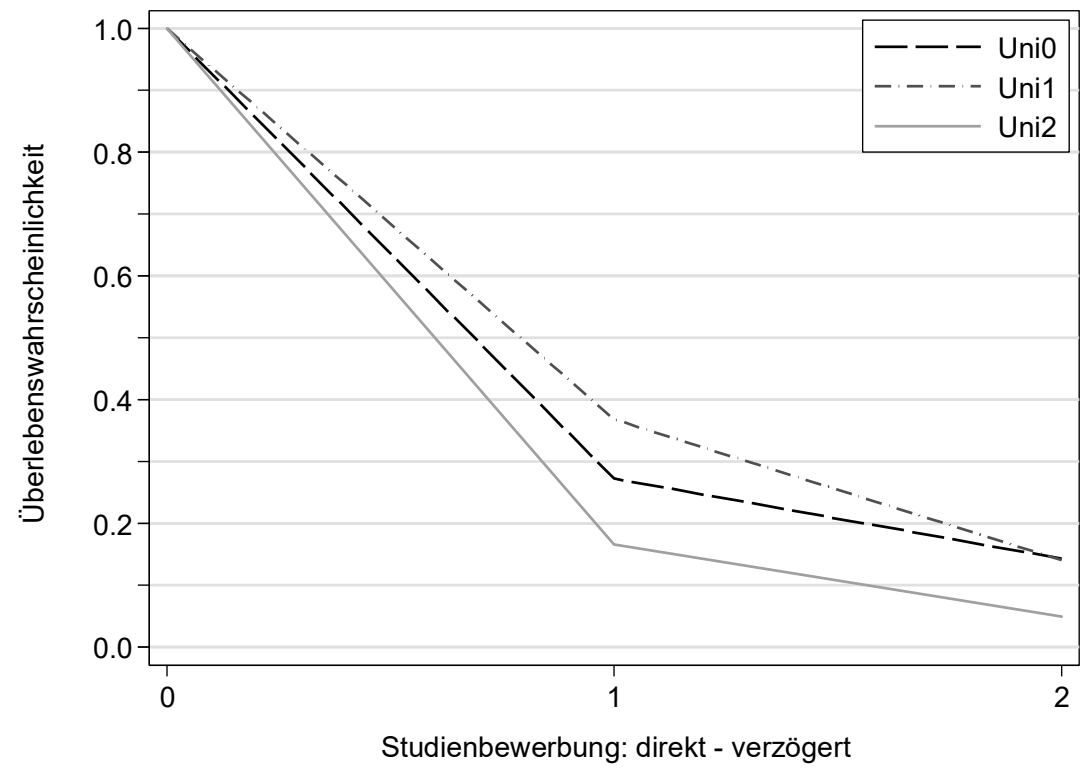

N Uni0: 246 (zensiert: 40); Uni1: 95 (zensiert: 14); Uni2: 78 (zensiert: 6), vgl. Abb. A8.1 Quelle: Best Up-Individualdaten, eigene Berechnungen

Ersichtlich wird, dass Studienberechtigte mit zwei hochschulerfahrenen Eltern sich insgesamt am häufigsten bewerben: direkt nach dem Abitur bereits $83 \%$ (t1). Entsprechend liegt die Überlebenswahrscheinlichkeit nur bei $17 \%$ und sinkt zum WiSe 2015/16 (t2) weiter auf 5\%. Einakademikerkinder nehmen hier allerdings nicht wie in der ersten Phase die Mittelposition ein. Sie bewerben sich seltener direkt nach dem Abitur als ihre Peers aus Nichtakademikerhaushalten (Uni1: 63\%; Uni0: 73\%), ziehen dann aber zum zweiten Bewerbungszeitpunkt mit ihnen gleich (Anteil ohne Bewerbungen zu t2 oder Überlebenswahrscheinlichkeit in beiden Gruppen 14\%).

Wie kommt dieser Unterschied zustande? Wie schon im vorangegangenen Kapitel sollen hier zunächst geprüft werden, inwiefern Herkunftsunterschiede durch Unterschiede in den Schulleistungen, Abwägungen von Kosten, Nutzen und Erfolgswahrscheinlichkeiten sowie durch sozial ungleich verteilte kulturelle und soziale Ressourcen erklärt werden können. 


\subsubsection{Leistung, rationale Abwägung und soziale/kulturelle Ressourcen}

Im Folgenden steht die Überprüfung der Hypothesen des Blocks B im Fokus. Das Vorgehen gleicht dabei grundsätzlich dem in Kapitel 7 (Abschnitt 7.2.1). Ein zentraler Unterschied bezieht sich auf die Referenzkategorie bei der Schätzung sozialer Herkunftsunterschiede: Da sich bereits deskriptiv zeigt, dass sich bezüglich der Bewerbungswahrscheinlichkeit vor allem Doppelakademikerkinder von den beiden anderen Gruppen unterscheiden, dienen diese im Folgenden als Referenzkategorie.

Trotz der Unterschiede zwischen den Analysesamplen für Phase 1 und Phase 2 (vgl. Abschnitt 8.1.1) gleichen sich die deskriptiven Zusammenhänge zwischen der sozialen Herkunft und den erklärenden Variablen weitestgehend und werden daher hier nicht gesondert diskutiert (können aber Anhangstabelle A8.4 entnommen werden). Eine Auffälligkeit sei hier jedoch erwähnt, die sich im vorangegangenen Kapitel nicht zeigte: Studienberechtigte mit einem akademisch gebildeten Elternteil scheinen bezüglich ihrer Schulleistung im Vergleich zu den anderen beiden Gruppen schlechter abzuschneiden: Zum Beispiel befinden sich 35\% im unteren Quartil bei der Mathenotenverteilung, während dies nur auf 23\% der Nichtakademikerkinder und 20\% der Doppelakademikerkinder zutrifft. Ein ähnliches Bild zeigt sich bei weiteren Leistungsindikatoren. ${ }^{183}$ Dies spiegelt sich allerdings kaum in ihrer wahrgenommenen Erfolgswahrscheinlichkeit wider.

Die multivariaten Analysen folgen der gleichen Logik wie die parallelen Analysen zur Intentionsphase in Abschnitt 7.2.1. Zunächst wird das Basismodell (Bildungsherkunft und Kontrollvariablen, M1) sukzessive erweitert (um Leistungsindikatoren (M2), zusätzlich RC-Indikatoren (M3), Ressourcenindikatoren (M4) und letztlich alle Kovariate (M5)) und die Veränderung der Herkunftseffekte über die verschiedenen Modellspezifikationen hinweg nachvollzogen (Abbildung 8.2). Im Anschluss daran wird das vollständige Hazardra-

$183 \mathrm{Zu}$ beachten ist hierbei, dass es sich nicht um dieselben Variablen handelt wie zur Untersuchung der Phase 1, für die die Noten des zweiten (und nicht vierten) Kurshalbjahres verwendet wurden. In dem hier genutzten Analysesample schneiden Einakademikerkinder aber auch bezüglich der Noten im zweiten Kurshalbjahr im Vergleich zu den beiden anderen Herkunftsgruppen etwas schlechter ab als in den Analysen in Kapitel 7. Dies ist ein Indiz dafür, dass leistungsschwächere Doppelund Nichtakademikerkinder überproportional häufig aus dem hier zugrundeliegenden Analysesample ausscheiden (aus diversen Gründen, etwa weil sie häufiger im SoSe mit dem Studium beginnen, häufiger ausfallen oder seltener die Studienberechtigung regulär erhalten, vgl. Abschnitt 8.1.1). Interessanterweise sind die Variablen zur Schulleistung die einzigen, für die dieses Phänomen beobachtet werden kann. Bezüglich aller anderen Variablen unterscheidet sich die Verteilung nach sozialer Herkunft nur geringfügig. 
tenmodell (M5), das in Tabelle 8.4 dargestellt ist, kurz besprochen. Im Anhang können detaillierte Informationen zu allen fünf Modellen nachvollzogen werden (Tabelle A8.5). ${ }^{184}$

Abbildung 8.2 zeigt zunächst, dass die deskriptiv beobachteten Unterschiede zwischen Doppelakademikerkindern und den beiden anderen Herkunftsgruppen über alle Modelle hinweg deutlich zutage treten und statistische Signifikanz erreichen. Dennoch sind auch Veränderungen über die Modelle hinweg sichtbar, die nicht nur in Form einer Reduktion, sondern auch einer Vergrößerung des Herkunftseffektes zutage treten. Das Hinzufügen der erklärenden Variablen zum Basismodell scheint sich allerdings unterschiedlich auf die Bewerbungswahrscheinlichkeit von Nichtakademikerkindern (vs. Uni2) und Einakademikerkindern (vs. Uni2) auszuwirken.

Die geringere Bewerbungswahrscheinlichkeit von Nichtakademikerkindern im Vergleich zu Doppelakademikerkindern scheint nicht mithilfe der Leistungsindikatoren erklärt werden zu können. Im Gegensatz hierzu scheinen die RC-Indikatoren zumindest eine leicht mediierende Wirkung zu haben (die Herkunftsdifferenz reduziert sich in M3 im Vergleich zu M2 um 2,1 Prozentpunkte von 13,4 auf 11,3). Beim Hinzufügen der Ressourcenindikatoren in M4 zeigt sich das bereits aus Kapitel 7 bekannte Bild eines in diesem Fall sogar

184 Wie in Abschnitt 8.1.3 angekündigt, wurden zuvor zwei Annahmen getestet, die Hazardratenmodellen zugrunde liegen. Dass insbesondere die Überlebenswahrscheinlichkeit von Studienberechtigten aus Einakademikerfamilien nicht parallel zu der der anderen beiden Herkunftsgruppen verläuft (Abb. 8.1), könnte ein Indiz für die Verletzung der Proportionalitätsannahme sein. Dies wurde mithilfe eines Ratio-Likelihood Chi-Quadrat Tests geprüft (für Details siehe Abschnitt 8.1.3), der zeigt, dass dies hier in der Tat der Fall ist. Da Herkunftsunterschiede im Timing der Bewerbung nicht im Zentrum dieser Arbeit stehen, wird jedoch auf das Hinzufügen von Interaktionstermen zwischen den Periodendummies und der Bildungsherkunft verzichtet; die Effekte können dennoch als Durchschnittseffekte der sozialen Herkunft im betrachteten Zeitraum interpretiert werden. Folgenreicher wäre die Verletzung der Annahme nicht-informativer Zensierung (vgl. Allison 2014b), nach der Zensierungsgründe nicht mit der Wahrscheinlichkeit des Auftretens des Ereignisses zusammenhängen sollten. Zur Annäherung an die Frage, ob die (nicht designbedingten) Zensierungen informativ sind, wurde, wie zuvor beschrieben, das auf Grundlage der tatsächlichen Daten berechnete Hazardratenmodell (inklusive aller zentralen unabhängigen und Kontrollvariablen) mit high-hazard und low-hazard Szenarien verglichen (vgl. Allison 2014b). Die Ergebnisse sind in Anhangstabelle A8.6 dargestellt und zeigen, dass sich die Modelle grundsätzlich sehr ähneln, sodass die Interpretation der Ergebnisse durch informative Zensierung nicht deutlich gestört sein sollte. (Der Vergleich erfolgt anhand von M2, das neben der sozialen Herkunft die Leistungsindikatoren sowie die Kontrollvariablen enthält und somit die Variablen, die in allen Analyseschritten berücksichtigt werden). 
recht deutlichen Anstiegs der Herkunftsdifferenz (um 3,9 auf 17,3 Prozentpunkte).

Beim Vergleich von Einakademikerkindern mit der Referenzkategorie sind es hingegen eher die - oben bereits erwähnten - Leistungsunterschiede, die einen Teil des Unterschieds in der Bewerbungswahrscheinlichkeit zu vermitteln scheinen (dieser sinkt von 16,7 Prozentpunkten in M1 auf 12,1 Prozentpunkte in M2). RC-Indikatoren spielen beim Vergleich innerhalb der Gruppe von Akademikerkindern keine vermittelnde Rolle. Doch steigt auch hier die Herkunftsdifferenz durch Hinzufügen der Ressourcen-Variablen leicht an (auf 14 Prozentpunkte in M4).

Erneut sind es die Variablen "Studienerfahrung in Verwandtschaft" sowie „Unterstützung bei Studienbewerbung“, die als Suppressor-Variablen fungieren. Werden diese aus dem finalen Modell entfernt, dann reduziert sich die Differenz zwischen Doppel- und Nichtakademikerkindern auf 10 und zwischen Doppel- und Einakademikerkindern auf 11,4 Prozentpunkte.

Auf Grundlage der Punktschätzer und Konfidenzintervalle in Abbildung 8.2 wird auch hier - ähnlich wie in Phase 1 - bereits größtenteils ersichtlich, dass die Unterschiede statistisch nicht signifikant sind. ${ }^{185}$

Tabelle 8.4 zeigt, dass über die soziale Herkunft hinaus nur wenige der Indikatoren signifikant mit der Bewerbungswahrscheinlichkeit zusammenhängen: Insbesondere ein (wahrgenommener) Studienwunsch der Eltern sowie eine sehr hohe wahrgenommene Erfolgswahrscheinlichkeit erhöhen die Bewerbungswahrscheinlichkeit. Zudem zeigt sich ein positiver Zusammenhang zwischen der Erwartung eines hohen gesellschaftlichen Ansehens durch ein Studium und der Bewerbungswahrscheinlichkeit (im Gegensatz zu Phase 1, wo eher die Einkommenserwartung mit der Intentionswahrscheinlichkeit zusammenhing). Die Kosteneinschätzung scheint in der Bewerbungsphase eine untergeordnete Rolle zu spielen. Und auch die Schulnoten sind nicht sehr deutlich mit der Bewerbungswahrscheinlichkeit assoziiert: Die Effektstärken liegen zwischen 4 und 11 Prozentpunkten, und die Konfidenzintervalle sind in den meisten Fällen zu groß, um das Vorhandensein eines Einflusses präzise vorhersagen zu können.

185 Mit einer Ausnahme kann in der Tat keiner der Unterschiede zwischen den Herkunftskoeffizienten präzise genug geschätzt werden, um statistische Signifikanz zu erreichen (auch dann nicht, wenn die beiden Suppressor-Variablen aus dem Endmodell entfernt werden). Die Ausnahme bezieht sich auf den Unterschied im Uni1Koeffizienten zwischen M1 und M2. Doch da gerade die Leistungsindikatoren im Gruppenvergleich - wie oben beschrieben - problematisch zu sein scheinen, sollte dieses Ergebnis hier nicht überbewertet werden. Da in allen weiteren Analyseschritten für die Schulleistung kontrolliert wird, sollten diesbezügliche Selektivitäten die Ergebnisinterpretation nicht deutlich beeinträchtigen. 
Abb. 8.2: Bewerbungswahrscheinlichkeit: Veränderung sozialer Herkunftsunterschiede über verschiedene Modellspezifikationen (Hazardratenmodelle)

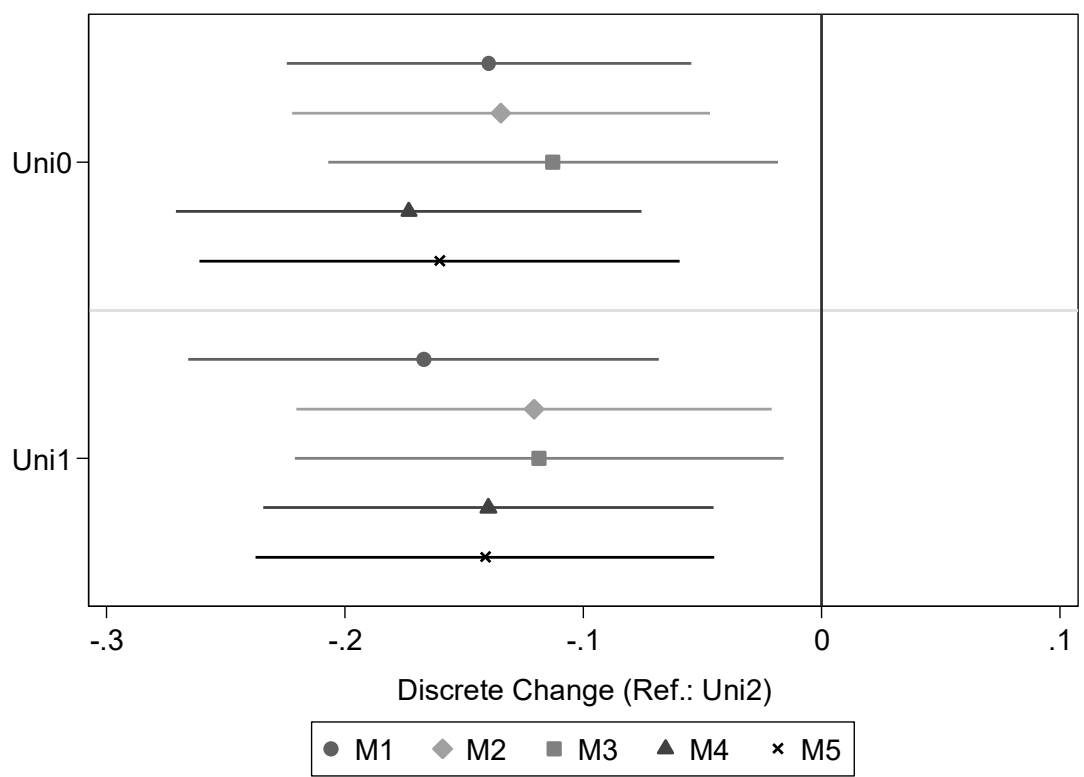

M1: Basismodell: soziale Herkunft + zentrale Kontrollvariablen

M2: + Leistungsindikatoren (HB1/B)

M3: + Leistungsindikatoren + RC (HB2/B)

M4: + Leistungsindikatoren + soziale und kulturelle Ressourcen (HB3/B)

M5: vollständiges Modell: alle Kovariate

Quelle: Best Up-Individualdaten, eigene Berechnungen, ausführliche Darstellung der Modelle: Tabelle A8.5

Logistische random-intercept Modelle, ausgewiesen: DC mit 90\%-Cl (alle weiteren Variablen gehen wie beobachtet in die Berechnung ein) 
Tab. 8.4: Bewerbungswahrscheinlichkeit in Abhängigkeit der sozialen Herkunft, Leistung, RC und Ressourcen (Hazardratenmodell)

\begin{tabular}{|c|c|}
\hline & M5 \\
\hline \multicolumn{2}{|l|}{ Soziale Herkunft Ref.: Uni2 } \\
\hline Uni0 & $-0,160^{* * *}(0,061)$ \\
\hline Uni1 & $-0,141^{\star \star}(0,059)$ \\
\hline \multicolumn{2}{|l|}{ Leistungsindikatoren } \\
\hline \multicolumn{2}{|l|}{ Abiturdurchschnitt Ref.: unteres Quartil } \\
\hline Mitte & $0,107^{\star}(0,058)$ \\
\hline Oberes Quartil & $0,080(0,083)$ \\
\hline \multicolumn{2}{|l|}{ Note Deutsch 4. KH Ref.: unteres Quartil } \\
\hline Mitte & $0,052(0,052)$ \\
\hline Oberes Quartil & $0,107(0,066)$ \\
\hline \multicolumn{2}{|l|}{ Note Mathe 4. KH Ref.: unteres Quartil } \\
\hline Mitte & $0,041(0,052)$ \\
\hline Oberes Quartil & $0,071(0,071)$ \\
\hline \multicolumn{2}{|l|}{ Grundfähigkeiten (z-standardisiert) } \\
\hline Figural & $0,007(0,023)$ \\
\hline Verbal & $-0,012(0,026)$ \\
\hline \multicolumn{2}{|l|}{ Rational Choice } \\
\hline \multicolumn{2}{|c|}{ Wahrgenommene Erfolgswahrscheinlichkeit Ref.: (sehr) unwahrscheinlich - teils/teils } \\
\hline Wahrscheinlich & $-0,022(0,051)$ \\
\hline Sehr wahrscheinlich & $0,112^{*}(0,063)$ \\
\hline \multicolumn{2}{|c|}{ Kostenerwartung: Wie schwer fällt Studienfinanzierung Ref.: (sehr) schwer } \\
\hline Weder noch & $0,062(0,055)$ \\
\hline (Sehr) leicht & $0,040(0,050)$ \\
\hline \multicolumn{2}{|c|}{ Ertragserwartung: Aussicht auf hohes Einkommen durch Studium Ref.: schlecht/mittel } \\
\hline Gut & $-0,057(0,054)$ \\
\hline Sehr gut & $-0,058(0,070)$ \\
\hline \multicolumn{2}{|c|}{ Ertragserwartung: Aussicht auf angesehenen Job durch Studium Ref.: schlecht/mittel } \\
\hline Gut & $0,062(0,057)$ \\
\hline Sehr gut & $0,110 *(0,063)$ \\
\hline \multicolumn{2}{|l|}{ Soziale und kulturelle Ressourcen } \\
\hline Freunde mit Studienaspiration: mehr als die Hälfte & $0,033(0,042)$ \\
\hline Wahrgenommene Studienaspiration Eltern & $0,166^{\star \star *}(0,047)$ \\
\hline \multicolumn{2}{|l|}{ Studienerfahrung in Verwandtschaft Ref.: (fast) keine } \\
\hline Bis zu $50 \%$ & $0,040(0,047)$ \\
\hline Mehr als $50 \%$ & $-0,087(0,066)$ \\
\hline \multicolumn{2}{|c|}{ Unterstützung bei Studienbewerbung Ref.: eher/sehr unwahrscheinlich } \\
\hline Eher wahrscheinlich & $0,021(0,068)$ \\
\hline Sehr wahrscheinlich & $-0,052(0,063)$ \\
\hline T1: WiSe 2014/15 & $0,002(0,135)$ \\
\hline T2: WiSe 2015/16 & $-0,074(0,139)$ \\
\hline Pseudo- $R^{2}$ (Maddala) & 0,18 \\
\hline Rho & 0,01 \\
\hline N Perioden/Befragte/Schulen & $481 / 387 / 19$ \\
\hline
\end{tabular}

Quelle: Best Up-Individualdaten, eigene Berechnungen

logistisches random-intercept Modell, AME/DC (Standardfehler), *** $p<0,01 ;{ }^{* *} p<0,05 ;{ }^{*} p<0,1$ zusätzlich enthalten: Kontrollvariablen (Migrationshintergrund, Geschlecht, Schultyp und Art der Hochschulzugangsberechtigung) 
Zusammenfassend haben die Ergebnisse verdeutlicht, dass auch in der zweiten Phase deutliche und robuste Herkunftsunterschiede in der Bewerbungswahrscheinlichkeit bis ein Jahr nach dem (Fach-)Abitur bestehen (HA/B). Diese zeigen sich hier aber nicht nur zwischen Doppelakademikerkindern und Nichtakademikerkinder, sondern ebenfalls zwischen Doppel- und Einakademikerkindern.

Leistungsindikatoren mediieren zwar einen Teil der Herkunftsdifferenz zwischen Einakademikerkindern und Doppelakademikerkindern, tragen aber nicht zur Erklärung des Unterschieds zu Nichtakademikerkindern bei (gegen HB1/B). Auch die eingeführten RC- und Ressourcen-Indikatoren können Herkunftsunterschiede in der Bewerbungswahrscheinlichkeit kaum erklären (gegen HB2/B und HB3/B). Insgesamt finden die Mediationshypothesen des Blocks B also auch in Phase 2 kaum Unterstützung. Selbst bei gleicher Leistung, gleicher Einschätzung von Kosten, Nutzen und Erfolgswahrscheinlichkeit eines Studiums und gleicher Ausstattung an kulturellen und sozialen Ressourcen sind Herkunftsunterschiede bezüglich der Bewerbungswahrscheinlichkeit zu verzeichnen.

Exkurs: Die Rolle von Intentionen in der Bewerbungsphase

Wie in Abschnitt 4.3 angekündigt, soll an dieser Stelle kurz auf die Rolle von Intentionen für Bewerbungsentscheidungen eingegangen werden. Zunächst soll die Frage geklärt werden, ob Intentionen ein notwendiger Schritt für die folgenden Umsetzungsphasen sind. Auf Grundlage von Tabelle A8.7 im Anhang kann dies klar verneint werden, denn fast $63 \%$ derjenigen ohne Studienintention zu Beginn des letzten Schuljahres bewerben sich dennoch für einen Studienplatz. Dies kann insbesondere für Nichtakademikerkinder beobachtet werden (73\%). Eine mögliche Erklärung liegt im Messzeitpunkt von Intentionen begründet: zu Beginn des letzten Schuljahres. Während des letzten Schuljahres kann es auf Grundlage neuer Informationen zu weiteren Anpassungen kommmen, die letztlich doch in einer Studienbewerbung münden (vgl. Spangenberg und Quast 2016). Ausgeschlossen werden kann zudem nicht, dass unrealisierte Ausbildungsaspirationen zu einer Umentscheidung geführt haben. $\mathrm{Zu}$ beachten ist, dass die Anzahl an Schüler/innen im Bewerbungssample ohne Intention sehr gering ist, insbesondere unter Akademikerkindern, sodass von einer weiteren Interpretation von Herkunftsunterschieden hier abgesehen wird.

Zudem zeigt sich, dass eine Studienintention zu Beginn des letzten Schuljahres auch keine hinreichende Bedingung für eine Bewerbung bis 1,5 Jahre nach Erhalt der Hochschulzugangsberechtigung ist, denn insgesamt 11\% der Schüler/innen mit Intention bewerben sich nicht. Hier deutet sich an, dass insbesondere Doppelakademikerkinder zu Beginn des letzten Schuljahres bereits gefestigtere Intentionen zu haben scheinen, die sie häufig in Form einer Bewerbung verfolgen $(95 \%)$. 
Zudem wurde in Abschnitt 4.3 vorgeschlagen, Intentionen als Prädiktor für folgende Bewerbungsentscheidungen und als Mediator von Herkunftsdifferenzen zu modellieren. Wie sich schon bivariat andeutet, zeigt sich, dass Intentionen die Bewerbungswahrscheinlichkeit um mehr als 20 Prozentpunkte steigern und damit der stärkste Prädiktor im Modell sind. Sie mediieren Herkunftsunterschiede hier aber kaum (vgl. Tabelle A8.8).

\subsubsection{Zugangshürden}

Im zweiten Schritt wird geprüft, inwiefern (antizipierte) Zugangsbarrieren im angestrebten Fach die Bewerbungswahrscheinlickeit senken und ob dies insbesondere der Fall für sozial schwächere Studienberechtigte ist (HC2/B). Neben dieser „Umentscheidung“ wird ebenfalls für Phase 2 untersucht, inwiefern Zugangsbarrieren (sozialgruppenspezifisch) zu einer „Umorientierung“ innerhalb des Hochschulsystems, also zu einer Bewerbung für andere Studienfächer führen ( $\mathrm{HC} 3 / \mathrm{B})$.

„Umentscheidung“: Zugangshürden und Bewerbungswahrscheinlichkeit

Allgemeine deskriptive Informationen zu den Zugangshürden im angestrebten Studienfach wurden bereits in Kapitel 7 sowie insbesondere Tabelle A7.5 gegeben. Letztere enthält auch die entsprechenden Informationen für die Analysesample, auf denen die folgende Untersuchung beruht. Kurz zusammengefasst unterscheiden sich auch hier Studienberechtigte unterschiedlicher sozialer Herkunft im Durchschnitt kaum darin, wie selektiv und zulassungsbeschränkt ihr angestrebtes Studienfach ist.

Die in Tabelle 8.5 berichteten multivariaten Hazardratenmodelle bestätigen die im vorangegangenen Abschnitt berichteten Herkunftsunterschiede in der Bewerbungswahrscheinlichkeit. ${ }^{186}$ Der Anteil an Studiengängen mit $\mathrm{Zu}$ lassungsbeschränkung und Eignungsfeststellungsverfahren im angestrebten Fach hängt nicht mit der Bewerbungswahrscheinlichkeit zusammen. Anders verhält es sich mit den Selektivitätsvariablen: sinkt die durchschnittliche Selektivität des angestrebten Fachs (,DN“) um einen Notenpunkt (etwa von 1 auf 2), erhöht sich die Bewerbungswahrscheinlichkeit um knapp 19 Prozentpunkte. ${ }^{187}$ Hier zeichnet sich ein Unterschied zu Phase $1 \mathrm{ab}$, in der keine der

186 Die Tatsache, dass die Herkunftsdifferenz etwas ansteigt, ist nicht dem Hinzufügen der Zugangshürden-Variablen geschuldet, sondern der für diesen Analyseschritt notwendigen Sampleeinschränkung. Wie in Abschnitt 8.1.4 beschrieben, können hier Befragte nicht berücksichtigt werden, die keine konkrete Fachaspiration nannten. Vgl. Tabelle A8.3 für hierdurch entstehende Selektivitäten.

187 Neben den untransformierten Werten wurden wie schon in Kapitel 7 die Berechnungen auch mit z-standardisierten Variablen für die Zugangshürden vorgenom- 
Zugangshürden signifikant mit der Intentionswahrscheinlichkeit zusammenhing.

Tab. 8.5: Bewerbungswahrscheinlichkeit: soziale Herkunft und Zugangsbarrieren des angestrebten Studienfaches (Hazardratenmodelle)

\begin{tabular}{|c|c|c|c|c|c|c|}
\hline & M1 & M2 & M3 & M4 & M5 & M6 \\
\hline \multicolumn{7}{|c|}{ Soziale Herkunft (Ref. Uni2) } \\
\hline Uni0 & $\begin{array}{l}-0,189^{\star * *} \\
(0,052)\end{array}$ & $\begin{array}{l}-0,192^{\star \star *} \\
(0,052)\end{array}$ & $\begin{array}{l}-0,186^{\star * *} \\
(0,052)\end{array}$ & $\begin{array}{l}-0,191^{\star * *} \\
(0,051)\end{array}$ & $\begin{array}{l}-0,192^{\star * *} \\
(0,051)\end{array}$ & $\begin{array}{l}-0,192^{\star \star \star} \\
(0,051)\end{array}$ \\
\hline Uni1 & $\begin{array}{l}-0,154^{\text {***}} \\
(0,06)\end{array}$ & $\begin{array}{l}-0,153^{\star \star *} \\
(0,059)\end{array}$ & $\begin{array}{l}-0,154^{\star *} \\
(0,06)\end{array}$ & $\begin{array}{l}-0,156^{* * *} \\
(0,058)\end{array}$ & $\begin{array}{l}-0,157^{\star * \star} \\
(0,058)\end{array}$ & $\begin{array}{l}-0,158^{\star \star *} \\
(0,059)\end{array}$ \\
\hline
\end{tabular}

Durchschnittliche Zugangsbeschränkung/Selektivität

\begin{tabular}{|c|c|c|c|c|c|c|}
\hline $\begin{array}{l}\text { Zulassungs- } \\
\text { beschränkung }\end{array}$ & $\begin{array}{l}-0,0006 \\
(0,001)\end{array}$ & & & & & \\
\hline Eignungsfeststellung & & $\begin{array}{l}0,0010 \\
(0,0009)\end{array}$ & & & & \\
\hline $\begin{array}{l}\text { Zulassungs- } \\
\text { beschränkung oder } \\
\text { Eignungsfeststellung }\end{array}$ & & & $\begin{array}{l}-0,002 \\
(0,002)\end{array}$ & & & \\
\hline $\mathrm{DN}$ & & & & $\begin{array}{l}0,187^{* * *} \\
(0,054)\end{array}$ & & \\
\hline $\begin{array}{l}\text { DN inkl. „alle } \\
\text { zugelassen“ }\end{array}$ & & & & & $\begin{array}{l}0,126^{\star \star *} \\
(0,037)\end{array}$ & \\
\hline $\begin{array}{l}\text { DN inkl. „alle } \\
\text { zugelassen“+ } \\
\text { zulassungsfrei }\end{array}$ & & & & & & $\begin{array}{l}0,073^{* *} \\
(0,03)\end{array}$ \\
\hline T1: WiSe 2014/15 & $\begin{array}{l}0,269^{\star *} \\
(0,121)\end{array}$ & $\begin{array}{l}0,20^{* *} \\
(0,095)\end{array}$ & $\begin{array}{l}0,389 * * \\
(0,196)\end{array}$ & $\begin{array}{l}-0,135 \\
(0,139)\end{array}$ & $\begin{array}{l}-0,064 \\
(0,126)\end{array}$ & $\begin{array}{l}0,017 \\
(0,126)\end{array}$ \\
\hline T2: WiSe 2015/16 & $\begin{array}{l}0,159 \\
(0,127)\end{array}$ & $\begin{array}{l}0,090 \\
(0,106)\end{array}$ & $\begin{array}{l}0,281 \\
(0,202)\end{array}$ & $\begin{array}{l}-0,242^{*} \\
(0,144)\end{array}$ & $\begin{array}{l}-0,182 \\
(0,133)\end{array}$ & $\begin{array}{l}-0,093 \\
(0,133)\end{array}$ \\
\hline Rho & 0 & 0 & 0 & 0 & 0 & 0 \\
\hline $\mathrm{BIC}$ & 580,81 & 579,90 & 580,20 & 569,70 & 570,277 & 575,34 \\
\hline $\mathrm{AIC}$ & 502,90 & 501,99 & 502,30 & 491,79 & 492,37 & 497,44 \\
\hline $\mathrm{R}^{2}$ (Maddala) & 0,20 & 0,20 & 0,20 & 0,21 & 0,21 & 0,21 \\
\hline $\begin{array}{l}\text { N Perioden/Befragte/ } \\
\text { Schulen }\end{array}$ & $\begin{array}{l}446 / \\
367 / 19\end{array}$ & $\begin{array}{l}446 / \\
367 / 19\end{array}$ & $\begin{array}{l}446 / \\
367 / 19\end{array}$ & $\begin{array}{l}446 / \\
367 / 19\end{array}$ & $\begin{array}{l}446 / \\
367 / 19\end{array}$ & $\begin{array}{l}446 / \\
367 / 19\end{array}$ \\
\hline
\end{tabular}

Quelle: Best Up-Individualdaten + Rekrutierungsdaten, eigene Berechnungen logistische random-intercept Modelle, AME/DC (Standardfehler), ${ }^{* *} p<0,01 ;{ }^{* *} p<0,05 ;{ }^{*} p<0,1$ In allen Modellen enthalten: Leistungsindikatoren und Kontrollvariablen (Migrationshintergrund, Geschlecht, Schultyp, Timing der Bewerbung)

men. Auch hier zeigt sich, dass die Abnahme der Effektstärke über die Selektivitätsvariablen der unterschiedlichen Skalierung geschuldet ist. Tatsächlich unterscheiden sich die z-standardisierten Koeffizienten weniger stark (DN: 0,067, DN+alle: 0,062, DN+frei: 0,047). 
Variiert der Zusammenhang zwischen antizipierten Zugangshürden und der Bewerbungswahrscheinlichkeit mit der sozialen Herkunft (HC2/B)? Zur Beantwortung dieser Frage wird die Bewerbungswahrscheinlichkeit in Abbildung 8.3 für die drei Herkunftsgruppen an unterschiedlichen Werten der $\mathrm{Zu}-$ gangshürden-Variablen in Form der bereits aus Kapitel 7 bekannten Grafiken (etwa Abb. 7.3) visualisiert. Die Plots basieren auf den in Tabelle 8.5 präsentierten Modellen, denen zusätzlich ein Interaktionsterm zwischen der Bildungsherkunft und der jeweiligen Zugangshürde hinzugefügt wurde. Deutlich sichtbar sind auch hier die Unterschiede in der Bewerbungswahrscheinlichkeit zwischen Doppelakademikerkindern, die in jedem Fall die höchste Bewerbungswahrscheinlichkeit haben, und den anderen beiden Herkunftsgruppen. Interaktionen können jedoch nicht eindeutig nachvollzogen werden; entsprechend sind die Interaktionsterme nicht signifikant (nicht ausgewiesen). In der Tendenz scheinen durchschnittliche Zulassungsbeschränkungen im angestrebten Fach (oberes Panel) am schwächsten mit der Bewerbungswahrscheinlichkeit von Nichtakademikerkindern zusammenzuhängen und die durchschnittliche Selektivität (unteres Panel) am schwächsten mit der Bewerbungswahrscheinlichkeit von Einakademikerkindern. ${ }^{188}$

Zusammenfassend wurde gezeigt, dass die durchschnittliche Zulassungsbeschränkung im angestrebten Fach nicht im Zusammenhang mit der Bewerbungswahrscheinlichkeit steht. Dies ist für alle drei Herkunftsgruppen der Fall. Jedoch sinkt die Bewerbungswahrscheinlichkeit mit steigender durchschnittlicher Selektivität des angestrebten Studienfaches. Für Einakademikerkinder scheint dies jedoch im Vergleich zu Doppel- und Nichtakademikerkindern eine weniger gravierende Hürde darzustellen.

188 Entsprechend zeigt sich, dass die AME der Selektivitätsvariablen im unteren Panel für Nichtakademikerkinder mindestens auf dem 95\%-Niveau signifikant sind. Dies ist nicht der Fall für Einakademikerkinder, bei denen die geringere Steigung grafisch bereits sichtbar ist. Für Doppelakademikerkinder ist nur der AME der mittleren Variable („DN+alle zugelassen“) signifikant verschieden von Null (p:0,09). Ein Grund hierfür ist sicherlich die deutlich kleinere Fallzahl im Vergleich zu Nichtakademikerkindern. Im oberen Panel unterscheidet sich keine der insbesondere für Akademikerkinder sichtbaren leichten Zu- bzw. Abnahmen der Bewerbungswahrscheinlichkeit signifikant von Null. Ebenfalls getestet wurde, an welchen Werten der Zugangshürden-Variablen sich die Bewerbungswahrscheinlichkeit zwischen Nichtakademikerkindern und Akademikerkindern signifikant unterscheidet, da dies nicht anhand der Überlappungen der Konfidenzintervalle in Abbildung 8.3 abgelesen werden kann. Die Ergebnisse hierzu sind im Anhang in Abbildung A8.2 grafisch dargestellt. Wenig überraschend unterscheidet sich die Bewerbungswahrscheinlichkeit von Nichtakademikerkindern an keinem Wert der Zugangshürden signifikant von der von Einakademikerkindern (die Konfidenzintervalle schneiden an allen Werten die Null-Linie). Die Unterschiede zwischen Nichtakademikerkindern und Doppelakademikerkindern sind hingegen - ebenfalls wenig überraschend - häufig signifikant. 
Zusammengenommen sprechen die Ergebnisse gegen eine stärker abschreckende Wirkung von Zugangsbarrieren für Nichtakademikerkinder im Vergleich zu Akademikerkindern bei ihrer Entscheidung für oder gegen eine Studienbewerbung und somit gegen Hypothese HC2/B.

Abb. 8.3: Vorhergesagte Bewerbungswahrscheinlichkeit $(90 \%-\mathrm{Cl})$ nach Zugangshürden des angestrebten Faches und sozialer Herkunft (Hazardratenmodelle)
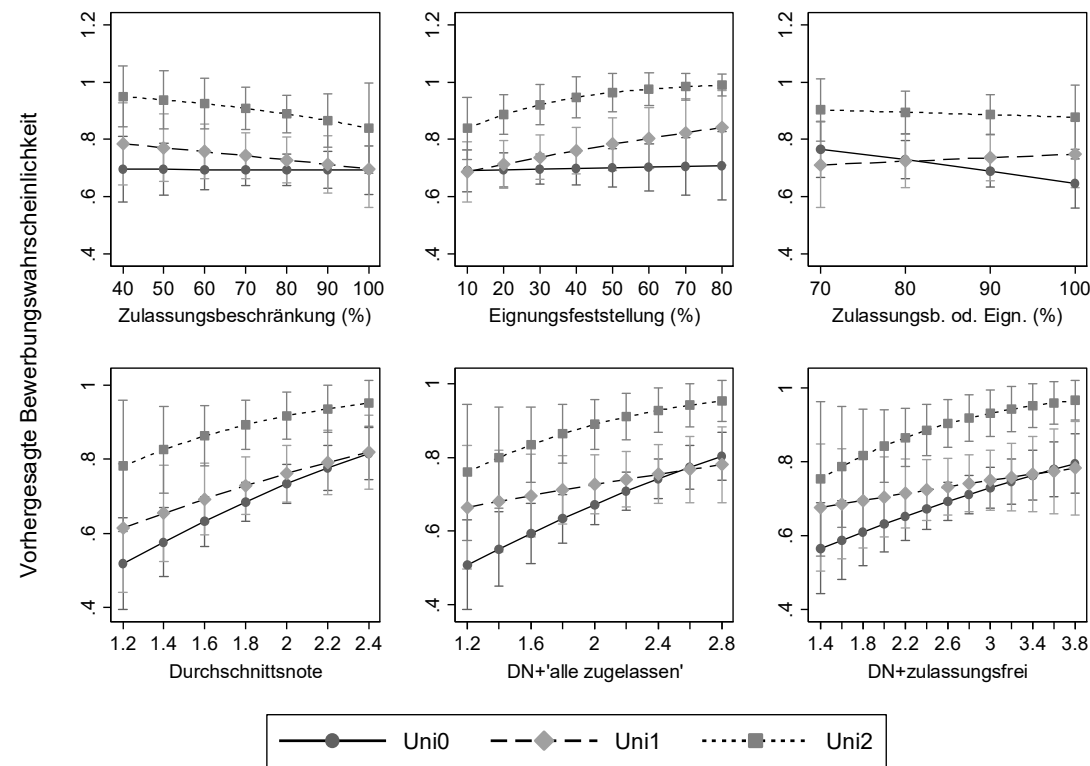

Quelle: Best Up-Individualdaten + Rekrutierungsdaten, eigene Berechnungen

Logistische random-intercept Modelle (Leistungsindikatoren und Kontrollvariablen jeweils bei ihrem Mittelwert).

„Umorientierung“: Zugangshürden und die Wahrscheinlichkeit übereinstimmender Studienfachaspirationen und Bewerbungen

Im nächsten Schritt wird der Frage nachgegangen, ob antizipierte Zugangshürden im angestrebten Fach nicht nur (teilweise) zur Entscheidung gegen eine Studienbewerbung beitragen, sondern ob sie auch mit einer (sozialgruppenspezifischen) Umorientierung zusammenhängen, sodass sich Studienberechtigte seltener für ihr ursprüngliches Wunschfach bewerben (HC3/B). Die Analysen beschränken sich damit auf Befragte, die sich für ein Studium bewarben (und für die Informationen zur Fachaspiration und zum Fach der Bewerbungen vorliegen, vgl. Tabelle A8.3). 
Deskriptiv zeigt sich zunächst, dass sich insgesamt $62 \%$ der Befragten für ihr (Welle 2-)Wunschfach bewarben. Damit ist die fachliche Übereinstimmung insgesamt geringer als die Übereinstimmung zwischen Fachaspiration und Fachintention in der ersten Phase (79\%, siehe Abschnitt 7.2.2). Auch hier zeigen sich leichte (nicht signifikante) Herkunftsunterschiede: Während für 67\% der Doppelakademikerkinder ein „Fachmatch“ beobachtet werden kann, ist dies nur für 60\% der Nichtakademikerkinder der Fall (Unil: 62\%).

Die multivariaten Ergebnisse zur Übereinstimmung zwischen der Fachaspiration und entsprechenden Bewerbungen können Tabelle 8.6 entnommen werden. Der Unterschied zwischen Nichtakademikerkindern und Doppelakademikerkindern von etwa 5 bis 6 Prozentpunkten ist noch sichtbar, verfehlt aber in allen Modellen deutlich das 10\%-Signifikanzniveau. Weiterhin hängt keine der Zugangshürden mit der abhängigen Variable zusammen.

Tab. 8.6: Wahrscheinlichkeit der Übereinstimmung zwischen Studienfachaspiration und -bewerbung: soziale Herkunft und Zugangsbarrieren des angestrebten Studienfaches

\begin{tabular}{|c|c|c|c|c|c|c|}
\hline & M1 & M2 & M3 & M4 & M5 & M6 \\
\hline \multicolumn{7}{|c|}{ Soziale Herkunft (Ref. Uni2) } \\
\hline Uni0 & $\begin{array}{l}-0,054 \\
(0,078)\end{array}$ & $\begin{array}{l}-0,052 \\
(0,078)\end{array}$ & $\begin{array}{l}-0,057 \\
(0,078)\end{array}$ & $\begin{array}{l}-0,059 \\
(0,078)\end{array}$ & $\begin{array}{l}-0,062 \\
(0,078)\end{array}$ & $\begin{array}{l}-0,059 \\
(0,078)\end{array}$ \\
\hline Uni1 & $\begin{array}{l}-0,003 \\
(0,089)\end{array}$ & $\begin{array}{l}-0,006 \\
(0,089)\end{array}$ & $\begin{array}{l}-0,005 \\
(0,089)\end{array}$ & $\begin{array}{l}-0,002 \\
(0,089)\end{array}$ & $\begin{array}{l}-0,001 \\
(0,088)\end{array}$ & $\begin{array}{l}-0,001 \\
(0,089)\end{array}$ \\
\hline \multicolumn{7}{|c|}{ Durchschnittliche Zulassungsbeschränkung/Selektivität } \\
\hline $\begin{array}{l}\text { Zulassungs- } \\
\text { beschränkung }\end{array}$ & $\begin{array}{l}0,002 \\
(0,002)\end{array}$ & & & & & \\
\hline Eignungsfeststellung & & $\begin{array}{l}-0,002 \\
(0,001)\end{array}$ & & & & \\
\hline $\begin{array}{l}\text { Zulassungs- } \\
\text { beschränkung oder } \\
\text { Eignungsfeststellung }\end{array}$ & & & $\begin{array}{l}-0,003 \\
(0,003)\end{array}$ & & & \\
\hline DN & & & & $\begin{array}{l}0,017 \\
(0,082)\end{array}$ & & \\
\hline $\begin{array}{l}\text { DN inkl. „alle } \\
\text { zugelassen“ }\end{array}$ & & & & & $\begin{array}{l}0,040 \\
(0,056) \\
\end{array}$ & \\
\hline $\begin{array}{l}\text { DN inkl. „alle } \\
\text { zugelassen“ + } \\
\text { zulassungsfrei }\end{array}$ & & & & & & $\begin{array}{l}0,001 \\
(0,046)\end{array}$ \\
\hline Rho & 0 & 0 & 0 & 0 & 0 & 0 \\
\hline $\mathrm{BIC}$ & 442,12 & 442,09 & 442,40 & 443,21 & 442,76 & 443,26 \\
\hline AIC & 377,02 & 376,99 & 377,30 & 378,11 & 377,65 & 378,15 \\
\hline $\mathrm{R}^{2}$ (Maddala) & 0,07 & 0,08 & 0,07 & 0,07 & 0,07 & 0,07 \\
\hline N Befragte/Schulen & $275 / 19$ & $275 / 19$ & $275 / 19$ & $275 / 19$ & $275 / 19$ & $275 / 19$ \\
\hline
\end{tabular}

Quelle: Best Up-Individualdaten + Rekrutierungsdaten, eigene Berechnungen logistische random-intercept Modelle, AME/DC (Standardfehler), ${ }^{* * *} p<0,01 ;{ }^{* *} p<0,05 ;{ }^{*} p<0,1$ In allen Modellen enthalten: Leistungsindikatoren und Kontrollvariablen (Migrationshintergrund, Geschlecht, Schultyp, Timing der Bewerbung) 
Einen möglicherweise differentiellen Einfluss der Zugangshürden visualisiert Abbildung 8.4. ${ }^{189}$ Der Anteil an zulassungsbeschränkten Studiengängen ist in keiner der Herkunftsgruppen mit der abhängigen Variable assoziiert (keine der Steigungen unterscheidet sich signifikant von Null). Bezüglich der anderen Variablen zeigen sich hingegen durchaus Unterschiede; jedoch sind diese weniger durch Unterschiede zwischen Nichtakademikerkindern auf der einen und Akademikerkindern auf der anderen Seite getrieben. So scheint sich insbesondere der Anteil an Studiengängen mit Eignungsfeststellungsverfahren im angestrebten Fach positiv auf die Übereinstimmung zwischen Fachaspiration und Bewerbung von Einakademikerkindern auszuwirken, während das Gegenteil der Fall für die beiden anderen Gruppen zu sein scheint. ${ }^{190}$ Ein anderes Bild zeigt sich bezüglich der durchschnittlichen Selektivität des angestrebten Fachs (untere Teil von Abbildung 8.4). Diese ist nicht mit der „Übereinstimmungswahrscheinlichkeit" von Nichtakademikerkindern assoziiert. Für Einakademikerkinder scheint sie tendenziell mit einer fachlichen Umorientierung zusammenzuhängen, während das Gegenteil für Doppelakademikerkinder beobachtet werden kann. ${ }^{191}$

Insgesamt sind die Ergebnisse hier weniger eindeutig als in Phase 1: Höhere durchschnittliche Zugangshürden im angestrebten Fach gehen nicht konsistent mit einer höheren „Umorientierungswahrscheinlichkeit" (also einer niedrigeren Wahrscheinlichkeit einer fachlichen Übereinstimmung zwischen Aspiration und Bewerbung) einher. Dies spricht gegen Hypothese HC3/B. Eindeutige und konsistente Interaktionen mit der sozialen Herkunft können ebenfalls nicht beobachtet werden.

189 Auch in diesem Fall wurde getestet, an welchen Werten der Zugangshürden-Variablen sich die Herkunftsunterschiede signifikant von Null unterscheiden. Dies ist nur am oberen Ende des Anteiles an Eignungsfeststellungsverfahren der Fall. Dies kann im Anhang in Abbildung A8.3 nachvollzogen werden.

190 Die Interaktionsterme zwischen der Variable „Eignungsfeststellungsverfahren“ und Uni1 vs. Uni0/2 sind signifikant (p: 0,019 bzw. 0,049). Zudem ist die Steigung der Regressionsgeraden für Uni1 (p: 0,081) und Uni0 (p: 0,067) signifikant von Null verschieden (die fehlende Signifikanz bei Uni2 trotz ähnlicher Effektstärke kann durch die kleineren Fallzahlen zustande kommen).

191 Der Interaktionsterm zwischen der Variable „Durchschnittnote“ und Uni1 vs. Uni2 ist signifikant (p: 0,066). Knapp signifikant verschieden von Null (p: 0,094) ist jedoch nur der Slope für Einakademikerkinder. In der Tendenz deuten sich diese Ergebnisse auch für die anderen beiden Selektivitätsvariablen an, jedoch ist die Schätzung hier ungenauer. 
Abb. 8.4: Vorhergesagte Wahrscheinlichkeit der Übereinstimmung zwischen Studienfachaspiration und -bewerbung nach Zugangshürden des angestrebten Faches und sozialer Herkunft
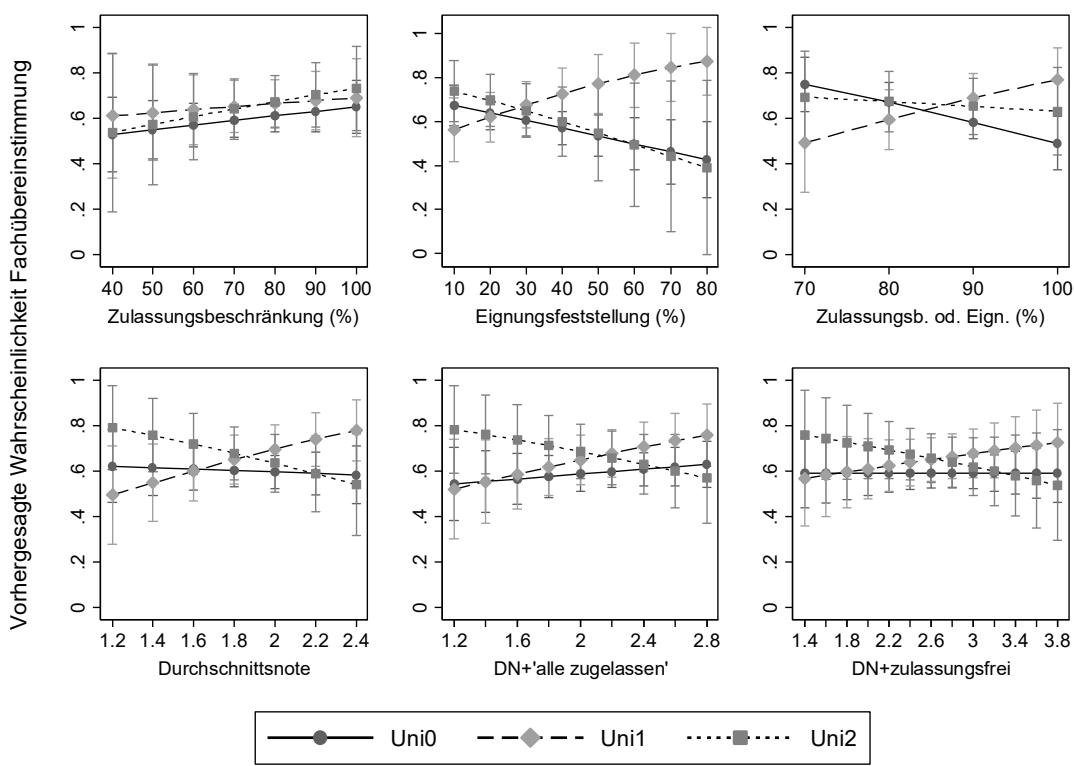

Quelle: Best Up-Individualdaten + Rekrutierungsdaten, eigene Berechnungen

Logistische random-intercept Modelle (Leistungsindikatoren und Kontrollvariablen jeweils bei ihrem Mittelwert).

Exkurs: Was bedeutet „Umorientierung“?

Im Gegensatz zu den Untersuchungen der ersten Phase, für die idealistische Fachaspirationen und realistische Fachintentionen zum selben Zeitpunkt erhoben wurden, liegen bei den gerade präsentierten Analysen die Äußerung von Fachwünschen und deren Umsetzungsversuch in Form einer Bewerbung circa ein bis zwei Jahre auseinander. Daher stellt sich die Frage, wie gefestigt geäuBerte Fachwünsche zu Beginn des letzten Schuljahres bereits sind. Und damit einhergehend: inwiefern die für etwa $40 \%$ der Befragten beobachtete Diskrepanz tatsächlich dadurch zustande kommt, dass sie ihre (stabile) Fachaspiration aufgrund antizipierter Barrieren nicht versuchen umzusetzen. Oder ob ihr geäußerter Wunsch zu Beginn des letzten Schuljahres noch vage ist und erst später konkretisiert und gefestigt wird?

Für Deutschland zeigen Schneider und Franke (2014: 25) auf Grundlage des DZHW Studienberechtigtenpanels, dass insgesamt etwa 40\% der Befragten erst im letzten Schuljahr mit der Informationssuche über Studienalternati- 
ven beginnen. Es ist daher durchaus plausibel anzunehmen, dass die Fachaspirationen, die Schüler/innen zu Beginn des letzten Schuljahres äußern, im letzten Schuljahr und darüber hinaus im Lichte neu gewonnener Informationen konkretisiert, angepasst oder verändert werden. Die Argumentation muss sich hierbei auf eine Gradwanderung begeben, insbesondere wenn die Unterscheidung zwischen idealistischen Aspirationen und realistischen Intentionen aufrechterhalten werden soll. Führen neu hinzugewonnene Informationen über vorhandene Hürden und Erfolgswahrscheinlichkeiten zu der diskutierten Übersetzung von Aspirationen in Intentionen? Bleibt also der ursprüngliche Wunsch bestehen, wird aber als unrealistisch verworfen und daher nicht weiterverfolgt (und äußert sich in der beobachteten Diskrepanz zwischen Aspiration und Bewerbung)? Oder führen neue Informationen dazu, dass der Wunsch, ein bestimmtes Fach zu studieren und damit einen mehr oder weniger stark definierten Berufsweg einzuschlagen, verändert wird (z.B. wenn sich herausstellt, dass das damit verbundene Berufsbild doch nicht $\mathrm{zu}$ den eigenen ebenfalls veränderlichen - Arbeits- und Lebensvorstellungen passt)? Letztlich ist hier von einer starken inter-individuellen Variation auszugehen, und die Frage, ob es sich bei der beobachteten Diskrepanz um stabile unrealisierte oder veränderte realisierte Aspirationen handelt, kann - zumindest mit den vorliegenden Daten - nicht eindeutig beantwortet werden.

Einige Tendenzen lassen sich aus den Best Up-Daten jedoch ableiten. Dies soll im Folgenden kurz rein deskriptiv auf Grundlage von zwei Variablen geschehen. Erstens wurden die Befragten in der zweiten Welle im Anschluss an die Benennung ihres gewünschten Studienfaches gefragt, ob auch andere Fächer in Frage kommen. Ganze 82\% (N gesamt: 263 der oben betrachteten 275 Befragten) bejahten dies. ${ }^{192}$ Herkunftsunterschiede zeigen sich hier nicht. Dieser hohe Anteil lässt vermuten, dass sich bei vielen Schüler/innen zu Beginn des letzten Schuljahres noch keine festen Fachaspirationen herausgebildet haben.

Zweitens sollten die Befragten sowohl für das WiSe 2014/15 als auch 2015/16 ihre Bewerbungen nach ihrer Vorliebe ranken. Neben der bereits bekannten „Fachmatch“-Variable, die den Wert 1 annimmt, wenn das Wunschfach mit mindestens einer Bewerbung übereinstimmt, wurde zusätzlich eine Variable gebildet, die die Übereinstimmung zwischen Aspiration und erstgerankter Bewerbung abbildet. In Abbildung 8.5 sind die Anteile fachlicher Übereinstimmungen für beide Variablen abgetragen. Der Anteil eines „Fachmatches" ist bei der erstgerankten Bewerbung kleiner als bei Berücksichtigung aller Bewerbungen. Das bedeutet, dass sich einige Befragte zwar für ihr zu Beginn des letzten Schuljahres angegebenes Wunschfach bewerben, aber den entsprechenden Studiengang nicht als ihre präferierte Bewerbung kategorisie-

192 Sehr ähnliche Ergebnisse berichten Barone et al. (2017) für Italien: 80\% der Schüler/innen haben zu Beginn des letzten Schuljahres noch keine eindeutige Fachpräferenz und nennen mehrere Alternativen. 
ren. Dieses Muster zeigt sich tendenziell stärker bei Studienberechtigten aus Nichtakademikerfamilien. Für diese insgesamt 14\% könnte eine Neuorientierung bereits vor der Bewerbung stattgefunden haben. ${ }^{193}$ Für diejenigen, die bei keiner Bewerbung eine Übereinstimmung erzielen, kann allerdings keine Aussage darüber getroffen werden, ob dies der Fall ist, weil sie ihre Aspirationen geändert haben oder weil antizipierte Barrieren sie abschreckten.

Abb. 8.5: Übereinstimmung Fachaspiration und Bewerbung nach sozialer Herkunft (in \%)

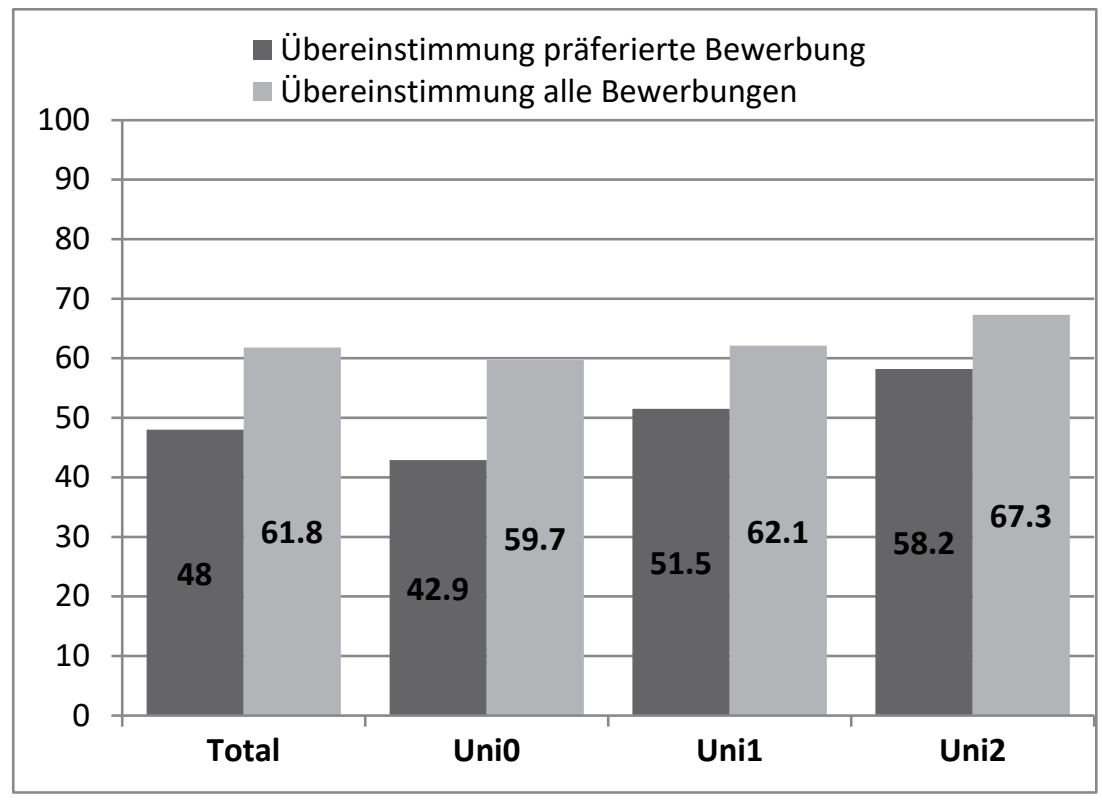

Quelle: Best Up-Individualdaten, eigene Berechnung

Insgesamt lässt sich auf Grundlage dieser Deskriptionen in der Tendenz ableiten, dass nicht für alle Befragten eine fehlende Übereinstimmung zwischen Fachaspiration und Bewerbung mit unverwirklichten Wünschen einhergeht. Es sei daher nochmals darauf verwiesen, dass die präsentierten Ergebnisse sich

193 Ein Studiengang vereinigt immer mehrere Merkmale, wie Fach, Hochschultyp oder Ort. Daher ist es natürlich möglich, dass je nach Priorität trade-offs zwischen den Merkmalen stattfinden und so zum Beispiel zwar der präferierte Ort, nicht aber das präferierte Fach auf Platz 1 gerankt wird. Dieses Problem lässt sich allerdings in den Daten nur sehr begrenzt nachvollziehen und muss daher hier vernachlässigt werden. 
auf die Diskrepanz zwischen Aspirationen zu Beginn des letzten Schuljahres und Bewerbungen bis zu zwei Jahre später beziehen. Dies könnte auch eine Erklärung dafür sein, dass die durchschnittlichen Zugangshürden im (ursprünglich) angestrebten Fach mit fachspezifischen Bewerbungen deutlich schwächer zusammenhängen als mit Fachintentionen, die in Phase 1 zeitgleich zu den (damaligen) Fachaspirationen erhoben wurden.

\subsubsection{Differenzierte Bewerbungen: Hochschultypen/Fächer/ Orte}

Zuletzt steht die Frage im Fokus, an welchen Orten, für welche Hochschultypen und welche Fächer sich Studienberechtigte bewerben. Wie bereits erwähnt, gehört die Analyse des Ortes bzw. der Entfernung thematisch zu Hypothesenblock C. Hier kann nicht - wie auf Grundlage des Faktoriellen Surveys - direkt erfasst werden, welchen Einfluss geographische Distanz und damit Studienkosten auf Bewerbungsentscheidungen haben. Jedoch kann über den Bewerbungsradius indirekt approximiert werden, inwiefern örtliche Präferenzen sozialgruppenspezifisch variieren $(\mathrm{HC} 1 / \mathrm{B})$.

Mit den Analysen zu Bewerbungen für unterschiedliche Hochschultypen und unterschiedlich ertragreiche Studienfächer werden erneut vertikal und horizontal differenzierte Studienentscheidungen untersucht und die Hypothesen des Blocks D getestet.

Ort und geographische Distanz

Für die Untersuchung der regionalen Reichweite von Bewerbungen wurden mithilfe der Bewerbungsmodule verschiedene abhängige Variablen gebildet, die in Abschnitt 8.1.2 vorgestellt wurden. Diese beziehen sich zum einen auf den Ort (Berlin bzw. Berlin/Brandenburg vs. außerhalb) und zum anderen auf die Distanz in Kilometern. Zudem wird zwischen der präferierten (erstgerankten) Bewerbung und allen berichteten Bewerbungen unterschieden. Erstere bildet eher die Präferenzen für bestimmte Orte $a b$, während Letztere die Mobilitätsbereitschaft approximiert.

Bivariate Zusammenhänge zwischen diesen abhängigen Variablen und der sozialen Herkunft sind in Abbildung 8.6 und Tabelle 8.7 zusammengefasst. Zunächst zeigt sich die aus der Literatur bekannte (vgl. Abschnitt 5.4.3) starke Präferenz für ein Studium am Heimatort: Für circa 73\% der Befragten ist ihr präferierter Studiengang in Berlin angesiedelt. Dies ist am häufigsten bei Nichtakademikerkindern (78\%) und am seltensten bei Einakademikerkindern $(63 \%)$ der Fall. Nimmt man Bewerbungen in Brandenburg und somit in Pendelnähe hinzu, erhöht sich der Anteil auf insgesamt 83\%. Nur 11\% der Nicht- 
akademikerkinder haben eine Präferenz für einen weiter entfernten Hochschulort.

Betrachtet man alle Bewerbungen, wird durchaus eine größere Mobilitätsbereitschaft sichtbar. Jedoch bewarben sich immer noch fast die Hälfte der Befragten ausschließlich in Berlin und fast $70 \%$ ausschließlich in Berlin und Brandenburg. Insbesondere bezüglich der zuletzt genannten Variable sind deutliche Herkunftsunterschiede zu beobachten (Uni0 vs. Uni1: 18 Prozentpunkte, Uni0 vs. Uni2: 16 Prozentpunkte).

Abb. 8.6: Bewerbungen in Berlin und Berlin/Brandenburg nach sozialer Herkunft (in \%)

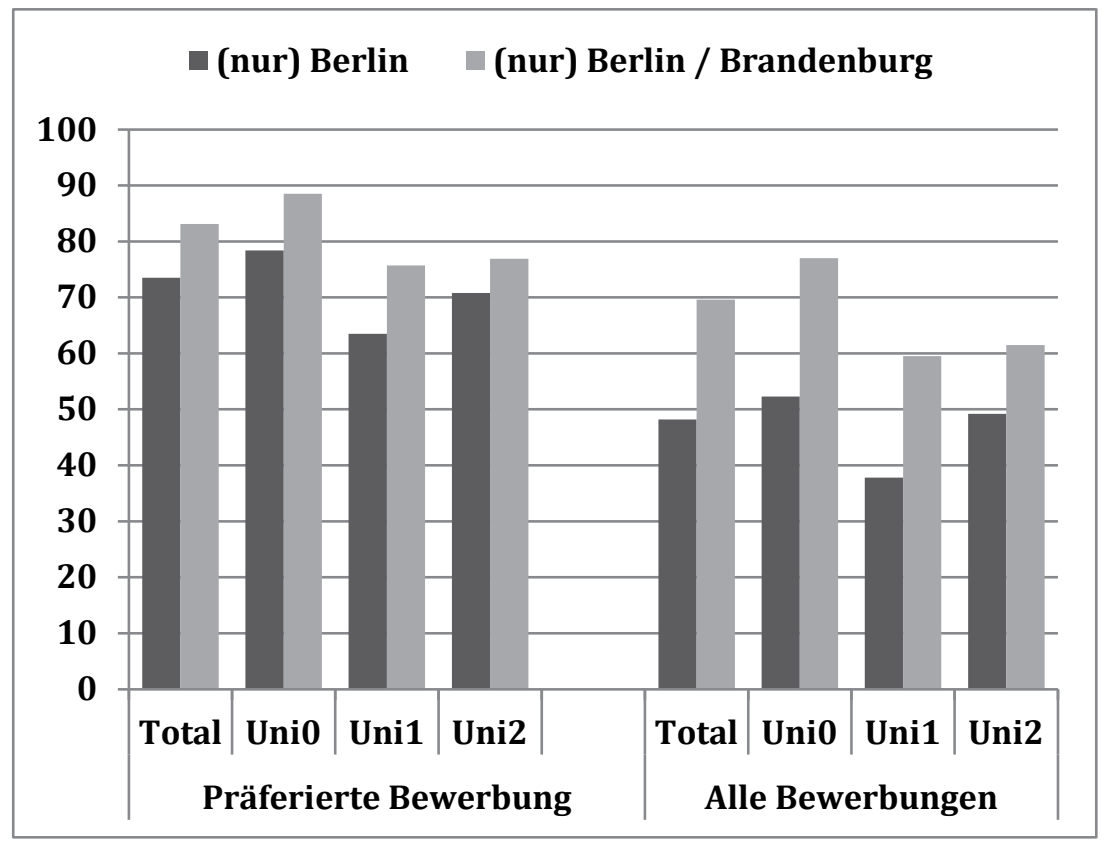

Quelle: Best Up-Individualdaten, eigene Berechnung N Total: 313, Uni0: 174, Uni1: 74, Uni2: 65

Diese Befunde spiegeln sich auch in den durchschnittlichen Distanzwerten wider. Nichtakademikerkinder haben hier bei allen Variablen im Durchschnitt den geringsten Wert. Doppelakademikerkinder scheinen bezüglich der Maximaldistanz am wenigsten eingeschränkt zu sein (im Durchschnitt $185 \mathrm{~km}$ ), während sich die geringere Gebundenheit an Berlin in der im Durchschnitt höheren Minimaldistanz von Einakademikerkindern widerspiegelt $(43,5 \mathrm{~km})$. 
Tab. 8.7: Entfernung der Bewerbungen nach sozialer Herkunft (in km)

\begin{tabular}{lllll}
\hline & $\begin{array}{l}\text { Uni0 } \\
\text { (N: 174) }\end{array}$ & $\begin{array}{l}\text { Uni1 } \\
\text { (N: 74) }\end{array}$ & $\begin{array}{l}\text { Uni2 } \\
\mathbf{( N :} \mathbf{6 5})\end{array}$ & $\begin{array}{l}\text { Total } \\
\text { (N: 313) }\end{array}$ \\
\hline $\begin{array}{llll}\text { Präferierte Bewerbung } \\
\text { Entfernung (Mean/SD) }\end{array}$ & $\begin{array}{l}\text { (15,8 } \\
\text { Range }\end{array}$ & $\begin{array}{l}87,5 \\
(169,7)\end{array}$ & $\begin{array}{l}81,7 \\
(179,5)\end{array}$ & $\begin{array}{l}57,6 \\
(141,7)\end{array}$ \\
\hline Alle Bewerbungen: Maximalwert & $0-512$ & $0-700$ & $0-700$ & $0-700$ \\
Entfernung (Mean/SD) & 101,8 & 169,6 & 184,7 & 135,1 \\
& $(172,7)$ & $(213,8)$ & $(254,1)$ & $(204,7)$ \\
Range & $0-700$ & $0-700$ & $0-700$ & $0-700$ \\
\hline Alle Bewerbungen: Minimalwert & & & & \\
Entfernung (Mean/SD) & 14,4 & 43,5 & 29,9 & 24,5 \\
& $(61,8)$ & $(118,1)$ & $(124,7)$ & $(93,4)$ \\
Range & $0-512$ & $0-541$ & $0-700$ & $0-700$ \\
\hline
\end{tabular}

Quelle: Best Up-Individualdaten, eigene Berechnung

In Tabelle 8.8 wird ersichtlich, dass sich die deskriptiven Befunde auch in den multivariaten Modellen bestätigen. So ist etwa die Hochschule der erstgerankten Bewerbung von Einakademikerkindern um 12 Prozentpunkte seltener in Berlin angesiedelt (MO1) und im Durchschnitt $49 \mathrm{~km}$ weiter entfernt (MD1) als diejenige von Nichtakademikerkindern. Sehr deutlich tritt auch die stärkere Präferenz von Nichtakademikerkindern für Hochschulen in Pendelnähe zu Tage: Die Wahrscheinlichkeit, dass sie sich ausschließlich in Berlin oder Brandenburg bewerben, ist um 21 Prozentpunkte höher als bei Einakademikerkindern. Die Unterschiede zwischen Bewerber/innen ohne und mit zwei akademisch gebildeten Eltern ist insgesamt etwas schwächer (jedoch meist dennoch deutlich) und erreicht nicht in allen Modellen statistische Signifikanz.

Insgesamt verweisen die Ergebnisse auf eine herkunftsspezifische Präferenz für mehr oder weniger weit entfernte Hochschulorte, stärker jedoch noch auf Herkunftsunterschiede in der Mobilitätsbereitschaft, die sich zum Beispiel auch in der Maximaldistanz aller Bewerbungen zeigt, die für Akademikerkinder deutlich höher ist. Die Ergebnisse sprechen damit für Hypothese HC1/B.

Im Vergleich zu den Ergebnissen des Faktoriellen Surveys in Phase 1, die zeigten, dass eine sehr große geographische Distanz die Bewerbungsintention aller Herkunftsgruppen in gleichem Maße senkte, deutet sich hier an, dass ein höherer Anteil sozial stärkerer Schüler/innen durchaus dazu bereit zu sein scheint, auch weitere Entfernungen auf sich zu nehmen, um ihre Studienaspirationen umzusetzen. 
Tab. 8.8: Soziale Herkunft und regionale Reichweite der Bewerbungen: multivariate Ergebnisse

\begin{tabular}{lllll}
\hline & Uni1 vs. Uni0 & Uni2 vs. Uni0 & Konstante & $\begin{array}{l}\text { Rho/Pseudo-R } \\
\text { (Maddala) }\end{array}$ \\
\hline MO1: präferierte Bewerbung & $-0,125^{* *}$ & $-0,084$ & & $0 / 0,04$ \\
Berlin & $(0,066)$ & $(0,068)$ & & \\
MO2: präferierte Bewerbung & $-0,117^{* *}$ & $-0,109^{*}$ & & $0 / 0,05$ \\
Berlin/Brand. & $(0,057)$ & $(0,060)$ & & $0 / 0,07$ \\
MO3: alle Bewerbungen & $-0,157^{* *}$ & $-0,055$ & & \\
Berlin & $(0,069)$ & $(0,075)$ & & $0 / 0,07$ \\
MO4: alle Bewerbungen & $-0,213^{\star * *}$ & $-0,178^{* *}$ & & \\
Berlin/Brandenburg & $(0,067)$ & $(0,072)$ & & $0 / 0,06$ \\
\hline MD1: Distanz präferierte & $49,317^{* *}$ & $38,553^{*}$ & 8,179 & \\
Bewerbung & $(20,542)$ & $(21,757)$ & $(33,158)$ & \\
MD2: Maximaldistanz & $95,335^{* * *}$ & $95,291^{* * *}$ & $-49,195$ & $0 / 0,11$ \\
MD3: Minimaldistanz & $(28,797)$ & $(30,50)$ & $(46,482)$ & \\
& $23,353^{*}$ & 14,811 & 20,326 & $0 / 0,06$ \\
\hline
\end{tabular}

Quelle: Best Up-Individualdaten, eigene Berechnungen

N Personen/Schulen: $313 / 19 ;{ }^{* * *} p<0,01 ;{ }^{* *} p<0,05 ;{ }^{*} p<0,1$

MD1-MD3: lineare random intercept Modelle, Regressionskoeffizienten (Standardfehler)

MO1-MO4: logistische random intercept Modelle, DC (Standardfehler)

In allen Modellen enthalten: Leistungsindikatoren und Kontrollvariablen (Migrationshintergrund, Geschlecht, Schultyp, Timing der Bewerbung)

\section{Hochschultyp}

Auch für den Hochschultyp wurden sowohl Variablen für die präferierte als auch für alle Bewerbungen gebildet, die in Abbildung 8.7 zunächst deskriptiv und in Tabelle 8.9 multivariat dargestellt werden. Wie schon bezüglich der Hochschultypintention in Phase 1 richtet sich ein Großteil der erstgerankten Bewerbungen an Universitäten, insbesondere unter (Doppel-)Akademikerkindern. Mit Blick auf alle Bewerbungen wird ersichtlich, dass insgesamt etwa $30 \%$ der Befragten sich für beide Hochschultypen bewerben. Am seltensten ist dies der Fall bei Doppelakademikerkindern, die sich mit Abstand am häufigsten nur für Universitäten bewerben. 


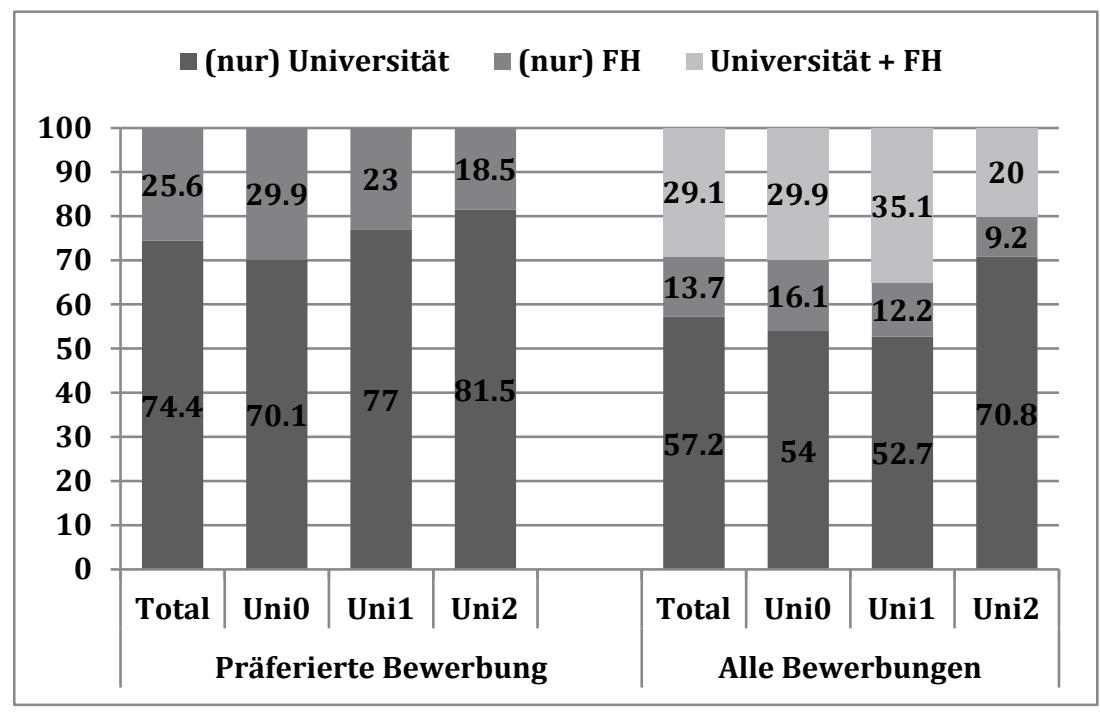

Quelle: Best Up-Individualdaten, eigene Berechnung N Total: 313, Uni0: 174, Uni1: 74, Uni2: 65

Nach Kontrolle der Schulleistung und der bekannten Kontrollvariablen ist bezüglich der präferierten Bewerbung nur der Unterschied zwischen Nichtakademikerkindern und Einakademikerkindern, die ihre erstgerankte Bewerbung um fast 10 Prozentpunkte seltener an eine FH schicken, knapp signifikant. Der Unterschied zwischen Nichtakademikerkindern und Doppelakademikerkindern wird hingegen leicht reduziert und kann nicht mehr sicher geschätzt werden. Letzteres gilt auch für Modell 2 und die Variable, ob Bewerbungen ausschließlich an Universitäten gerichtet werden (der Unterschied von 11 Prozentpunkten zwischen Doppel- und Nichtakademikerkindern verfehlt hier knapp das 10\% Signifikanzniveau, p: 0,120). Nichtakademikerkinder senden jedoch signifikant häufiger ausschließlich Bewerbungen an Fachhochschulen.

Insgesamt zeigt sich also, dass Akademikerkinder tendenziell eine stärkere Präferenz für Universitäten haben (HD1/B). Jedoch sind die Ergebnisse weniger eindeutig als im vorangegangenen Schritt zum Hochschulort. In der Tendenz setzen sich hier die Ergebnisse aus Phase 1 fort. Jedoch tritt der Unterschied zwischen Nichtakademikerkindern und Einakademikerkindern, der sich in Phase 1 kaum zeigt, hier deutlicher zutage. 
Tab. 8.9: Soziale Herkunft und Hochschultypbewerbungen: multivariate Ergebnisse

\begin{tabular}{llll}
\hline & $\begin{array}{l}\text { M1: erstgerankte } \\
\text { Bewerbung FH }\end{array}$ & M2: nur Uni & M3: nur FH \\
\hline Soziale Herkunft (Ref. Uni0) & & & \\
Uni1 & $-0,099^{*}$ & $-0,004$ & $-0,079^{*}$ \\
& $(0,056)$ & $(0,071)$ & $(0,043)$ \\
Uni2 & $-0,076$ & 0,114 & $-0,083^{*}$ \\
& $(0,065)$ & $(0,074)$ & $(0,048)$ \\
\hline Rho & 0 & 0 & 0 \\
Pseudo-R & 0,08 & 0,06 & 0,07 \\
N Personen/Schulen & $313 / 19$ & $313 / 19$ & $313 / 19$ \\
\hline
\end{tabular}

Quelle: Best Up-Individualdaten, eigene Berechnungen

Logistische random intercept Modelle, DC (Standardfehler); ${ }^{* *} p<0,01 ;{ }^{* *} p<0,05 ;{ }^{*} p<0,1$

Alle Modelle enthalten Leistungsindikatoren und Kontrollvariablen (Migrationshintergrund, Geschlecht, Schultyp und Timing der Bewerbung).

Studienfach: Einkommen und Berufsprestige

Anders verhält sich dies bei den Analysen zu den Studienfachbewerbungen. Hier spiegelt sich der bereits aus Phase 1 bekannte Befund wider, dass Nichtakademikerkinder und Einakademikerkinder sich kaum unterscheiden, während sich Doppelakademikerkinder für ertragreichere Fächer bewerben, sowohl bezüglich des durchschnittlich erwartbaren Einkommens als auch des Berufsprestiges (vgl. Tabelle 8.10). Die stärkere Präferenz von Doppelakademikerkindern für ertragreichere Fächer spiegelt sich auch darin wider, dass sich ihre durchschnittlichen Werte im Gegensatz zu den anderen beiden Gruppen kaum zwischen der erstgerankten Bewerbung und dem Maximalwert aller Bewerbungen unterscheiden.

Die deskriptiven Unterschiede können nur zum Teil nach Berücksichtigung weiterer Kovariaten aufrechterhalten werden (Tabelle 8.11). Insbesondere die Einkommensunterschiede bei der erstgerankten Bewerbung zwischen Nichtakademikerkindern und Doppelakademikerkindern treten mit über einem Euro Nettostundenlohn deutlich zutage. Die Unterschiede beim Maximaleinkommen sind jedoch deutlich geringer und nicht signifikant. Beim Minimaleinkommen (also der unteren ,Schmerzgrenze") unterscheiden sich mit 70 Cent nur die Akademikerkinder untereinander. Bezüglich des Berufsprestiges zeigt sich nur zwischen Nichtakademikerkindern und Doppelakademikerkindern bei der erstgerankten Bewerbung ein nennenswerter und signifikanter Unterschied.

Insgesamt haben Doppelakademikerkinder also sowohl eine stärkere Präferenz (erstgerankte Bewerbung) für Fächer, die im Durchschnitt zu einem höheren Einkommen führen, als auch solchen, die mit einem höheren Berufspres- 
tige verbunden sind. Jedoch holen die anderen beiden Herkunftsgruppen auf, wenn alle Bewerbungen berücksichtigt werden.

Tab. 8.10: Studienfachbewerbungen: durchschnittliches Einkommen und Berufsprestige nach sozialer Herkunft

\begin{tabular}{|c|c|c|c|c|}
\hline & $\begin{array}{l}\text { Uni0 } \\
\text { (N: 174) }\end{array}$ & $\begin{array}{l}\text { Uni1 } \\
\text { (N: 74) }\end{array}$ & $\begin{array}{l}\text { Uni2 } \\
\text { (N: 65) }\end{array}$ & $\begin{array}{l}\text { Total } \\
\text { (N: } 313)\end{array}$ \\
\hline \multicolumn{5}{|l|}{$\begin{array}{l}\text { Studienfach Stundenlohn } \\
\text { (in Euro) }\end{array}$} \\
\hline \multicolumn{5}{|l|}{ Präferierte Bewerbung } \\
\hline Stundenlohn (Mean/SD) & $16,8(2,3)$ & $16,8(2,5)$ & $18,0(2,5)$ & $17,0(2,4)$ \\
\hline Range & $12,3-25,8$ & $12,9-25,8$ & $12,9-25,8$ & $12,3-25,8$ \\
\hline \multicolumn{5}{|c|}{ Alle Bewerbungen: Maximalwert } \\
\hline Stundenlohn (Mean/SD) & $17,8(2,5)$ & $17,7(2,9)$ & $18,3(2,5)$ & $17,9(2,6)$ \\
\hline Range & $12,3-25,8$ & $12,9-25,8$ & $12,9-25,8$ & $12,3-25,8$ \\
\hline \multicolumn{5}{|c|}{ Alle Bewerbungen: Minimalwert } \\
\hline Stundenlohn (Mean/SD) & $16,2(2,3)$ & $15,8(2,1)$ & $17,0(2,3)$ & $16,3(2,3)$ \\
\hline Range & $10,6-23,2$ & $12,1-23,2$ & $12,4-23,2$ & $10,6-23,2$ \\
\hline \multicolumn{5}{|l|}{ Studienfach Prestige (MPS) } \\
\hline \multicolumn{5}{|l|}{ Präferierte Bewerbung } \\
\hline Prestige (Mean/SD) & $103,6(27,2)$ & $104,8(28,4)$ & $115,1(35,3)$ & $106,3(29,8)$ \\
\hline Range & $64,9-207,9$ & $67,0-207,9$ & $64,9-207,9$ & $64,9-207,9$ \\
\hline \multicolumn{5}{|c|}{ Alle Bewerbungen: Maximalwert } \\
\hline Prestige (Mean/SD) & $111,5(31,6)$ & $114,2(33,1)$ & $118,6(35,5)$ & $113,6(32,8)$ \\
\hline Range & $64,9-207,9$ & $67,0-207,9$ & $64,9-207,9$ & $64,9-207,9$ \\
\hline \multicolumn{5}{|c|}{ Alle Bewerbungen: Minimalwert } \\
\hline Prestige (Mean/SD) & $95,8(25,4)$ & $93,8(19,9)$ & $103,2(28,5)$ & $96,9(25,1)$ \\
\hline Range & $64,9-191,8$ & $67,0-183,4$ & $64,9-191,8$ & $64,9-191,8$ \\
\hline
\end{tabular}

Quelle: Best Up-Individualdaten, Mikrozensus 2007-2012 DOIs: 10.21242/12211.2007.00.00.1.1.0 bis 10.21242/12211.2012.00.00.1.1., eigene Berechnungen

Damit fällt der Rückbezug zu den Hypothesen ambivalent aus. Hypothese HD2/B geht davon aus, dass es auch in der Bewerbungsphase keine Herkunftsunterschiede bezüglich des Einkommensertrages gibt. Dies bestätigt sich nur, wenn alle Bewerbungen einbezogen werden, nicht aber wenn die präferierte Bewerbung betrachtet wird. Andersherum wird mit Hypothese HD3/B angenommen, dass sich Herkunftsunterschiede bezüglich des erwartbaren Berufsprestiges der Fächer zeigen, für die sich die Studienaspirant/innen bewerben. Dies bestätigt sich wiederum in der Tendenz, wenn die erstgerankte Bewerbung betrachtet wird, nicht aber, wenn alle Bewerbungen einbezogen werden. 
Tab. 8.11: Soziale Herkunft und Studienfachbewerbung: multivariate Ergebnisse

\begin{tabular}{lllllll}
\hline & $\begin{array}{l}\text { ME1: } \\
\text { Einkommen } \\
\text { erstgerankt }\end{array}$ & $\begin{array}{l}\text { ME2: } \\
\text { Maximal- } \\
\text { einkommen }\end{array}$ & $\begin{array}{l}\text { ME3: } \\
\text { Minimal- } \\
\text { einkommen }\end{array}$ & $\begin{array}{l}\text { MP1: } \\
\text { Prestige } \\
\text { erstgerankt }\end{array}$ & $\begin{array}{l}\text { MP2: } \\
\text { Maximal- } \\
\text { prestige }\end{array}$ & $\begin{array}{l}\text { MP3: } \\
\text { Minimal- } \\
\text { prestige }\end{array}$ \\
\hline \multicolumn{7}{c}{ Soziale Herkunft (Ref. Uni2) } \\
\hline Uni0 & $-1,125^{* * *}$ & $-0,575$ & $-0,535$ & $-8,881^{* *}$ & $-5,365$ & $-3,173$ \\
& $(0,337)$ & $(0,363)$ & $(0,331)$ & $(4,292)$ & $(4,662)$ & $(3,661)$ \\
Uni1 & $-0,724^{*}$ & $-0,175$ & $-0,732^{*}$ & $-6,292$ & $-0,652$ & $-6,696$ \\
& $(0,389)$ & $(0,418)$ & $(0,382)$ & $(4,928)$ & $(5,342)$ & $(4,201)$ \\
\hline Konstante & $16,62^{* * *}$ & $16,69^{* * *}$ & $16,94^{* * *}$ & $95,46^{* * *}$ & $93,09^{* * *}$ & $97,76^{* * *}$ \\
& $(0,531)$ & $(0,574)$ & $(0,540)$ & $(7,170)$ & $(7,988)$ & $(6,157)$ \\
\hline & & & & & & \\
Rho & 0 & 0 & 0,02 & 0,03 & 0,05 & 0,04 \\
$\begin{array}{l}\text { Pseudo-R } \\
\text { (Maddala) }\end{array}$ & 0,24 & 0,24 & 0,15 & 0,17 & 0,19 & 0,13 \\
$\begin{array}{l}\text { N Perso- } \\
\text { nen/Schulen }\end{array}$ & $313 / 19$ & $313 / 19$ & $313 / 19$ & $313 / 19$ & $313 / 19$ & $313 / 19$ \\
\hline
\end{tabular}

Quelle: Best Up-Individualdaten, Mikrozensus 2007-2012, DOls: 10.21242/12211.2007.00.00.1.1.0 bis 10.21242/12211.2012.00.00.1.1., eigene Berechnungen.

Lineare random-intercept Modelle, Koeffizienten (Standardfehler), ${ }^{* *} p<0,01 ;{ }^{* *} p<0,05 ;{ }^{*} p<0,1$

Alle Modelle enthalten Leistungsindikatoren und Kontrollvariablen (Migrationshintergrund, Geschlecht, Schultyp und Timing der Bewerbung).

Da sich das theoretische Argument eher auf Präferenzen bezieht (sozial privilegierte Studienberechtigte weisen prestigereichen Fächern einen größeren Wert zu, um Statuserhalt trotz steigender Studierendenzahlen sicherzustellen, während ein hohes Einkommen von allen Herkunftsgruppen im Durchschnitt als ähnlich erstrebenswert angesehen werden sollte), neige ich hier dazu, die Ergebnisse eher als Unterstützung für $\mathrm{HD} 3 / \mathrm{B}$ als $\mathrm{HD} 2 / \mathrm{B}$ zu interpretieren.

Exkurs: Unterschiede in den Bewerbungen $=$ Unterschiede in den Aspirationen?

Wie bereits in Kapitel 7 soll hier deskriptiv gezeigt werden, inwiefern sich die fach- und hochschultypbezogenen Unterschiede in den (erstgerankten) Bewerbungen bereits in entsprechenden Aspirationen wiederfinden.

Tabelle 8.12 zeigt zum einen, dass sich der Anteil an Studienaspirant/ innen, die sich wünschten, an einer Universität zu studieren, kaum von dem Anteil mit (erstgerankter) Bewerbung an einer Universität unterscheidet. Hier spiegeln sich die Herkunftsunterschiede also tatsächlich bereits in den Aspirationen wider. Weiterhin zeigt sich, dass unter den Bewerber/innen im Durchschnitt kaum Herkunftsunterschiede bezüglich des erwartbaren Einkommensund Prestigeertrages ihrer Fachaspiration bestehen. Die oben beschriebenen 
Befunde, dass Doppelakademikerkinder sich im Durchschnitt für ertragreichere Studienfächer bewerben, scheint hier also nicht in unterschiedlichen Aspirationen begründet zu liegen. Interessanterweiser sind es hier jedoch nicht die Ein- und Nichtakademikerkinder, die sich „,nach unten“ korrigieren, sondern die Doppelakademikerkinder, die im letzten Schuljahr Präferenzen für ertragreichere Fächer zu entwickeln scheinen (oder dies als Notwendigkeit wahrnehmen).

Tab. 8.12: Unterschiede zwischen Hochschultyp- und Fachaspirationen und entsprechender erstgerankter Bewerbung

\begin{tabular}{llll}
\hline & $\begin{array}{l}\text { Hochschultyp Uni: Aspira- Einkommen: Aspiration - } \\
\text { tion - Bewerbung (\%) }\end{array}$ & $\begin{array}{l}\text { Prestige: Aspiration - } \\
\text { Bewerbung (Mean, SD) }\end{array}$ & Bewbung (Mean, SD) \\
\hline Uni0 & $74,1-70,1$ & $17,1(2,7)-16,8(2,3)$ & $104,7(31,3)-103,6(28,3)$ \\
Uni1 & $78,4-77,0$ & $17,2(2,9)-16,9(2,6)$ & $105,4(31,7)-105,3(29,3)$ \\
Uni2 & $84,6-81,5$ & $17,3(2,4)-18,0(2,5)$ & $104,0(27,9)-114,3(37,0)$ \\
Total & $77,3-74,4$ & $17,2(2,7)-17,1(2,4)$ & $104,7(30,7)-106,2(30,6)$ \\
\hline
\end{tabular}

Quelle Best Up-Individualdaten, Mikrozensus 2007-2012, DOIs: 10.21242/12211.2007.00.00.1.1.0 bis 10.21242/12211.2012.00.00.1.1., eigene Berechnungen

Es werden nur diejenigen Befragten berücksichtigt, für die sowohl Angaben zur Aspiration als auch Bewerbung vorliegen. N Hochschultyp: 313, N Fach: 275

\subsubsection{Bewerbungsstrategien}

Nachdem Ergebnisse dazu präsentiert wurden, ob und wofür sich Studienberechtigte bewerben, steht im Folgenden das „Wie“ im Vordergrund. Hierfür wurden auf Grundlage der Bewerbungsmodule und Rekrutierungsdaten drei Variablenblöcke zur Messung von Bewerbungsmustern und -strategien operationalisiert: erstens die Anzahl und Breite der Bewerbungen, zweitens die zur Anwendung kommenden Zulassungsbeschränkungen und Auswahlverfahren und drittens die Zulassungschancen (siehe Abschnitt 8.1.2). Insbesondere die Untersuchung zur Breite steht in inhaltlicher Nähe zum vorangegangenen Abschnitt, in dem untersucht wurde, an welchen Orten, für welche Hochschultypen und Fächer sich die Befragten bewarben.

Wie in Abschnitt 8.1.2 bereits erwähnt, handelt es sich bei den folgenden Untersuchungen um erste explorative Deskriptionen zu möglichen Bewerbungsstrategien. Da die Bewerbungsstrategien nicht für alle Befragten gebildet werden konnten und die Analysesample mit Blick auf verschiedene Variablen selektiv sind (vgl. Abschnitt 8.1.4 und Tabelle A8.3), sollten die Ergebnisse mit Vorsicht bewertet werden. Dies betrifft insbesondere die Variablenblöcke zwei und drei, für die neben den Individualdaten auch die Rekrutierungsdaten genutzt wurden. 
Die Verteilung der zentralen Variablen insgesamt und nach sozialer Herkunft werden im Folgenden grafisch dargestellt. Detaillierte Informationen zu den genauen Werten und einigen zusätzlichen Variablen finden sich in Anhangstabelle A8.9.

Abbildung 8.8 zeigt die durchschnittliche Anzahl (kategorisiert) und Breite der Bewerbungen. Mit Blick auf die Anzahl wird deutlich, dass fast ein Drittel und damit der (relativ) größte Anteil der Bewerber/innen sich nur für einen Studiengang bewirbt (31\%). Ein Viertel bewirbt sich häufiger als fünf Mal. Doppelakademikerkinder tendieren dabei stärker zu nur einer Bewerbung (38\%) und seltener zu einer hohen Anzahl (>5: 20\%).

Die größte Breite kann insgesamt bezüglich des Fächerspektrums beobachtet werden. Fast die Hälfte aller Befragten bewerben sich für mehr als ein Fach - oder genauer: für mehr als eine Fächergruppe (Zweisteller). Für mehr als einen Hochschultyp bewerben sich hingegen - in weitestgehender Übereinstimmung mit den Befunden aus Abschnitt 8.2.3 - nur 26\% der Befragten. An mehr als einem Ort bewerben sich 43\%. Andersherum bedeutet dies, dass sich deutlich mehr als die Hälfte der Befragten nur an einem Ort bewarben, in der Regel in Berlin (vgl. Abschnitt 8.2.3). Differenziert nach sozialer Herkunft zeigt sich kein einheitliches Bild. Für Einakademikerkinder bestätigt sich der im vorherigen Abschnitt bereits gezeigte Befunde einer relativ großen örtlichen $(51 \%)$ und hochschultypbezogenen (32\%) Breite. Nichtakademikerkinder scheinen hingegen eher mit Bezug auf das Fach als auf den Ort kompromissbereit zu sein: 53\% bewerben sich für mehrere Fächer, jedoch nur 38\% für mehrere Orte. Doppelakademikerkinder sind insbesondere fachlich als auch bezüglich des Hochschultyps stärker festgelegt als die beiden anderen Gruppen. ${ }^{194}$

Generell kann davon ausgegangen werden, dass die Anzahl und Breite von Bewerbungen die Zulassungschancen erhöhen. Ob solch eine „Strategie“ notwendig ist, hängt aber auch von der Selektivität der Studiengänge in Kombination mit dem eigenen Qualifikationsprofil (insbesondere der Leistung) ab (vgl. Abschnitt 5.3). Dies wird im Folgenden betrachtet.

194 Die im Durchschnitt insgesamt geringere Breite der Bewerbungen von Doppelakademikerkindern hängt natürlich auch mit der geringeren Anzahl ihrer Bewerbungen zusammen. Die Variablen zur Breite nehmen bei nur einer Bewerbung automatisch den Wert 0 an, sodass Doppelakademikerkinder hier schon deswegen geringere „Chancen“ auf eine breite Bewerbung haben. 
Abb. 8.8: Anzahl und Breite der Bewerbungen nach sozialer Herkunft (1. Bew.phase, in \%)

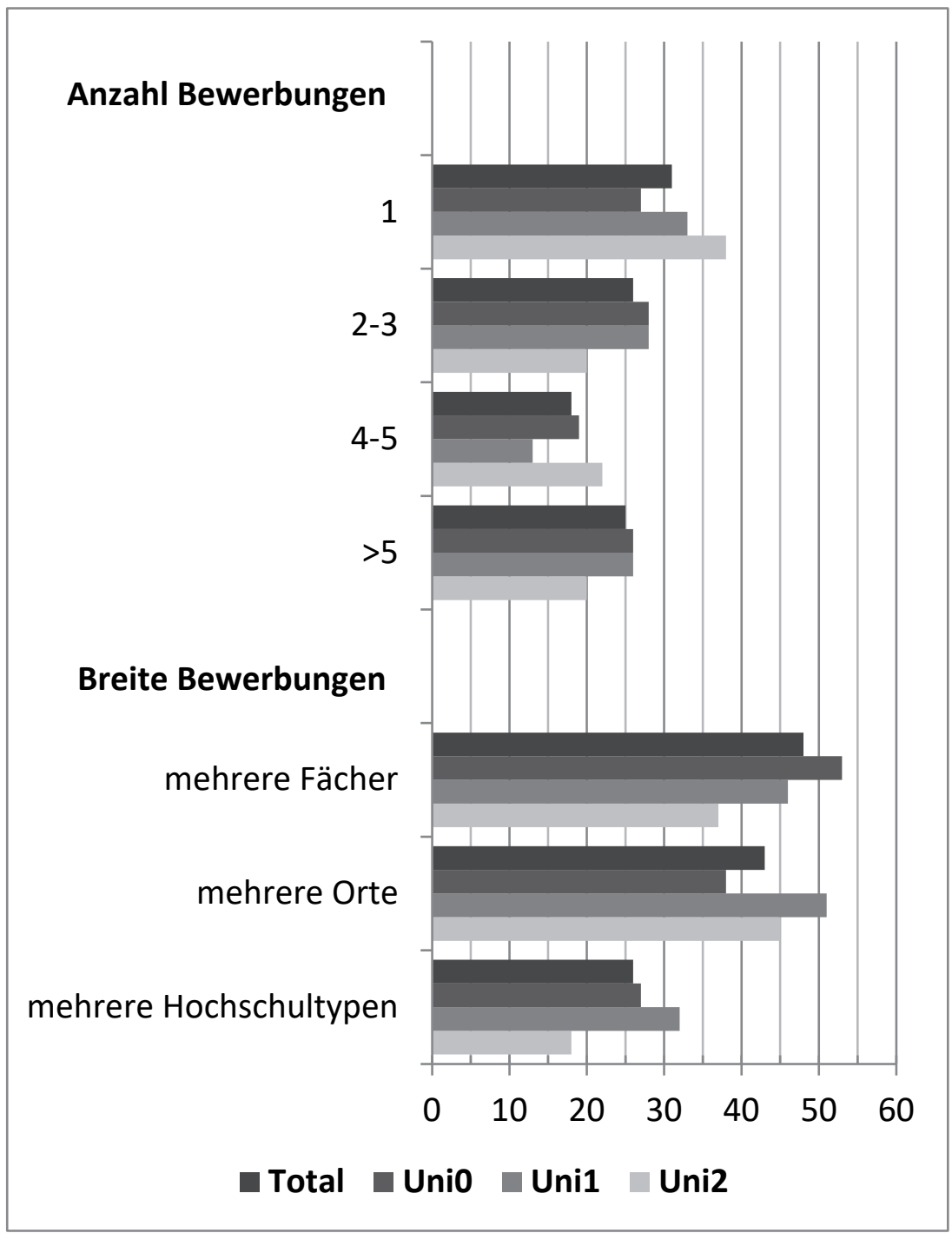

Quelle: Best Up-Individualdaten, eigene Berechnungen N: Total: 317; Uni0: 180; Uni1: 72; Uni2: 65 
Die ersten drei Variablen in Abbildung 8.9 zeigen zunächst allgemeine Informationen zu den Zulassungsmodi des präferierten Studiengangs. Die jeweiligen Angaben liegen ebenfalls für die zweite Präferenz, wenn vorhanden, vor und können Tabelle A8.9 entnommen werden. Bezüglich der Zulassungsbeschränkungen zeigt sich, dass $10 \%$ der Bewerber/innen ihre erstgerankte Bewerbung an bundesweit zulassungsbeschränkte Studiengänge richteten und nur 13\% an zulassungsfreie. Letzteres relativiert die in Abschnitt 2.2.3 dargestellten Statistiken, dass etwa 50\% der Studiengänge (auch in Berlin) zulassungsfrei sind (Stand zum WiSe 2014/15); offensichtlich werden diese nicht von Bewerber/innen präferiert. Doppelakademikerkinder sind dabei sowohl bei den prestigereichen und sehr selektiven bundesweit zulassungsbeschränkten als auch bei zulassungsfreien Studiengängen etwas überrepräsentiert (18\% und $19 \%$ ).

Auf Studiengangsebene wurde in Kapitel 2 gezeigt, dass über $80 \%$ der recherchierten Studiengänge entweder auf Grundlage von Zulassungsbeschränkungen oder Eignungsfeststellungsverfahren auswählen. Auf Befragtenebene richten nur insgesamt $8 \%$ der Bewerber/innen ihre erstgerankte Bewerbung an Studiengänge, für die keine über die Hochschulzugangsberechtigung hinausgehende Einschränkung vorgenommen wird. Dabei zeigen sich kaum Herkunftsunterschiede.

Inwiefern die vorgenommene Auswahl von Bewerber/innen auf ,komplexen "Verfahren beruht (z.B. Tests, Auswahlgespräche, Arbeitsproben), wird in der nächsten Variable dargestellt. Insgesamt ist dies in $18 \%$ der präferierten Bewerbungen der Fall. Es deutet sich ein geringfügig höherer Anteil bei Doppelakademikerkindern an, jedoch ist dieser mit nur 3-4 Prozentpunkten nicht substantiell. Insgesamt scheinen solche Verfahren keine der Herkunftsgruppen besonders abzuschrecken (oder anzuziehen) - ein Befund, der sich bereits in den Analysen des Faktoriellen Surveys zeigte (Abschnitt 7.2.2).

Die letzten beiden Variablen berichten Zulassungschancen und potentielle Wege, diese zu erhöhen. „Nicht erforderlich“ bedeutet jeweils, dass die eigene Abiturnote (bzw. Wartezeit) im Vorjahr für eine Zulassung über die jeweilige Quote ausgereicht hätte. ${ }^{195}$ Dies ist der Fall für insgesamt 65\% der erstgerankten Bewerbungen, für Nichtakademikerkinder mit 69\% etwas häufiger (Uni1:

195 Für diese Variablen wurde sowohl die erst- als auch zweitgerankte Bewerbung genutzt (sofern vorhanden). „Nicht erforderlich“ bedeutet entsprechend, dass entweder für die erste oder die zweite (oder beide) einigermaßen sichere Zulassungschancen bestehen. Wird nur die erste Präferenz betrachtet, reduziert sich der Wert entsprechend auf insgesamt 50\% (vgl. Tabelle A8.9). Die Label wurden für die Abbildungen vereinfacht. In Tabelle A8.9 (und Tabelle 8.13 unten) lauten sie wie folgt: nicht möglich $=\mathrm{DN}+\mathrm{WZ}$ nicht ausreichend + keine weiteren Kriterien $/ \mathrm{Be}-$ werbungen; möglich $=\mathrm{DN}+\mathrm{WZ}$ nicht ausreichend + aber weitere Kriterien $/$ Bewerbungen; nicht erforderlich $=\mathrm{DN}+\mathrm{WZ}$ ausreichend 
58\%, Uni2: 62\%), die sich damit hinsichtlich ihrer Abiturnote und der Selektivität des Studienganges am ,realistischsten“ bewerben.

Abb. 8.9: Zulassungsverfahren und -chancen nach sozialer Herkunft (1. Bew.phase, in \%)

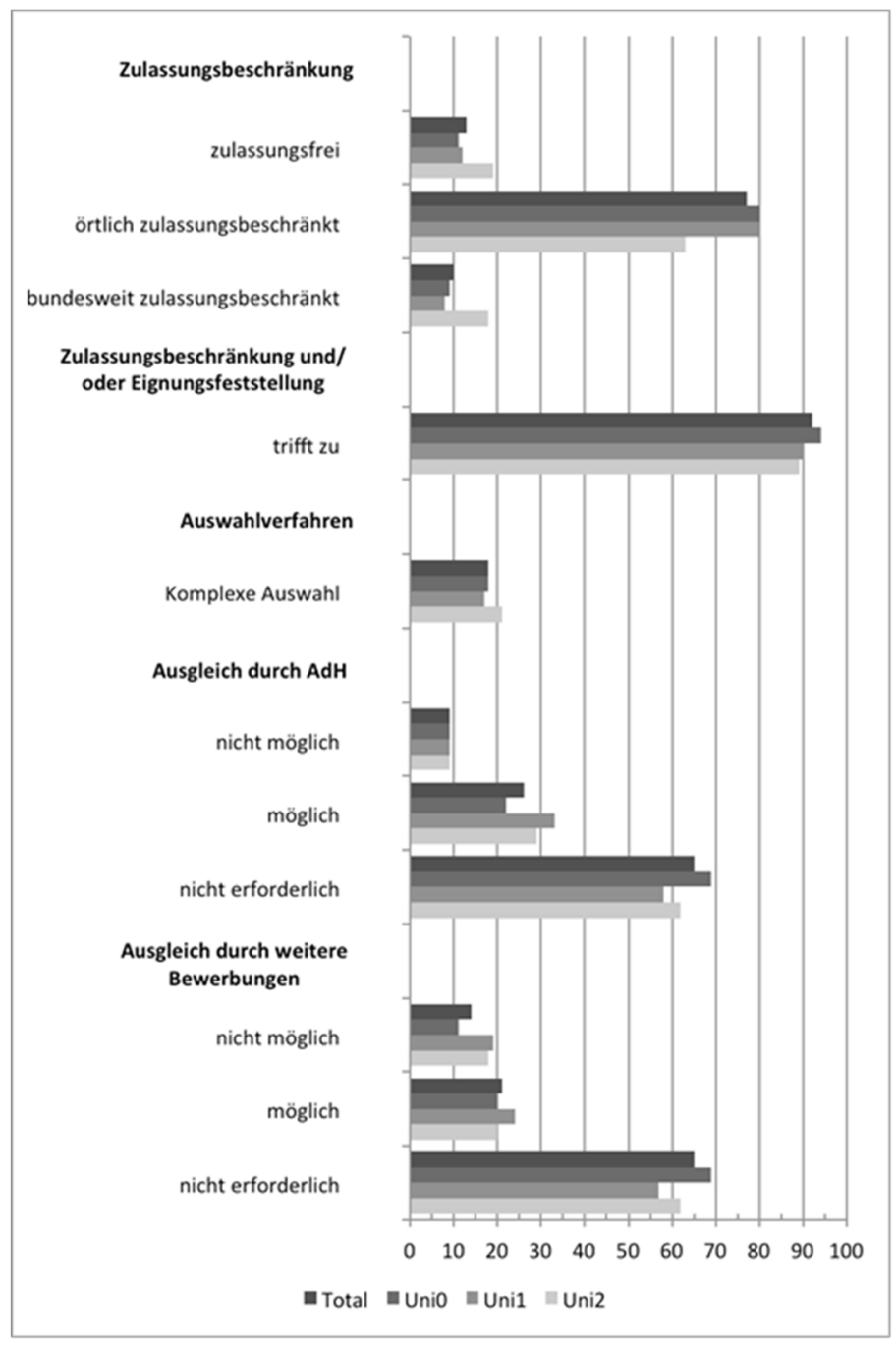

Quelle: Best Up-Individualdaten + Rekrutierungsdaten, eigene Berechnungen

N Zulassungsbeschränkung/Auswahlverfahren: Total: 266; Uni0: 149; Uni1: 60; Uni2: 57

N Ausgleich: Total: 240; Uni0: 130; Uni1: 54; Uni2: 56 
Sind Zulassungschancen unsicher, kann dies entweder durch weitere zur Anwendung kommende Kriterien (in der Regel in der „AdH“-Quote) oder durch zusätzliche Bewerbungen (potentiell) ausgeglichen werden. ${ }^{196}$ Keine Ausgleichsmöglichkeiten oder Ausgleichsversuche durch weitere Kriterien oder Bewerbungen bei unsicheren Zulassungschancen für den präferierten (und gegebenenfalls zweitgerankten) Studiengang kann für 9 bzw. $14 \%$ der Befragten beobachtet werden. Beim fehlenden Ausgleich durch zusätzliche Bewerbungen sind Akademikerkinder leicht überrepräsentiert (Uni1: 19\%, Uni2: 18\% vs. Uni0 11\%).

Zusammenfassend konnte gezeigt werden, dass sich ein hoher Anteil an Studienberechtigten weder sehr häufig noch sehr breit für ein Studium bewirbt: Ein Drittel bewirbt sich nur für einen Studiengang und - hiermit im Zusammenhang stehend - mehr als die Hälfte nur für ein Fach und an einem Ort. Nichtakademikerkinder scheinen dabei eher kompromissbereit bezüglich des Studienfachs zu sein, während Akademikerkinder bei der Ortswahl häufiger flexibel sind.

Ein relativ hoher Anteil (fast zwei Drittel) der Bewerber/innen hat zudem - basierend auf den Auswahlgrenzen im Vorjahr - relativ gute Zulassungschancen oder zumindest potentielle Ausgleichsmöglichkeiten. Insgesamt und gerade auch mit Blick auf den zweiten und dritten Variablenblock deutet sich hier nicht an, dass es Herkunftsunterschiede darin gibt, wie „strategisch“ (im Sinne von Zulassungschancen generell zu erhöhen) sich Studienberechtigte bewerben.

Sind also gegeben einer Bewerbung keine Herkunftsunterschiede in den Zulassungschancen zu erwarten? Diese Frage steht im Fokus der dritten Phase, die im Folgenden untersucht wird.

\subsection{Ergebnisse Phase 3: Herkunftsunterschiede bei der Zulassung}

In Kapitel 2 wurden Ergebnisse aus der Best Up-Rekrutierungsrecherche zusammengefasst und gezeigt, dass in einem Großteil der Studiengänge (85\%), für die sich die Befragten bewarben, die Zulassung entweder über Zulassungsbeschränkungen oder über die Anwendung von Eignungsfeststellungsverfahren eingeschränkt ist, sich Studienberechtigte also nicht einfach einschreiben können. Hochschulen scheinen von ihrer Rolle als Gatekeeper also durchaus

196 Inwiefern ein Ausgleich oder eine Erhöhung der Zulassungschancen hierdurch tatsächlich wahrscheinlich ist, hängt natürlich davon ab, ob das eigene Profil den jeweiligen Auswahlkriterien entspricht bzw. ob die zusätzlichen Bewerbungen realistischer/weniger selektiv sind. 
Gebrauch zu machen. Sie wenden dabei jedoch vor allem leicht erfassbare, standardisierte Kriterien an - allen voran und im Einklang mit gesetzlichen Vorgaben die (sozial ungleich verteilte) Abiturnote.

Mit der Übersetzung von Studienaspirationen in Studienintentionen und dem ersten Umsetzungsschritt in Form von Studienbewerbungen wurden bisher zwei selbstselektive Phasen ausführlich diskutiert. Die indirekte Wirkung der beschriebenen Gatekeepingprozesse stellte dabei einen wichtigen Bestandteil der Untersuchungen dar. Im Folgenden steht mit der fremdselektiven Phase 3 zur Studienzulassung die direkte Wirkung von Gatekeepingprozessen im Vordergrund. Mit den Best Up-Daten kann diese zwar (quantitativ) im Gegensatz zu anderen Datenquellen weitaus ausführlicher (bzw. überhaupt) untersucht werden, doch ist eine ähnlich detaillierte Untersuchung, wie sie zu Phase 1 und 2 vorgelegt wurde, nicht möglich.

Wie bereits in Abschnitt 8.1.3 beschrieben, kommen auch für die Zulassungsphase ereignisdatenanalytische Methoden zum Einsatz. Das Ereignis $\mathrm{Zu}-$ lassung kann zu zwei Zeitpunkten beobachtet werden: Zeitpunkt 1 bezieht sich auf Zulassungen nach der ersten Bewerbungsphase, die direkt nach dem Abitur (N: 304) oder ein Jahr verzögert (N: 55) stattfinden kann. Zeitpunkt 2 geht für 33 Befragte in die Analysen ein, die sich nach einer erfolglosen Bewerbung direkt nach dem Abitur im Folgejahr erneut bewerben.

Abbildung 8.10 zeigt die „Überlebenswahrscheinlichkeit“ für die drei Herkunftsgruppen, also die Wahrscheinlichkeit nach einer Bewerbung keine $\mathrm{Zu}$ lassung zu erhalten. Nach der ersten Bewerbungsphase erhielten insgesamt $21 \%$ der Bewerber/innen keine Zulassung. Während Doppelakademikerkinder sich am häufigsten direkt nach dem Abitur bewarben, sind es Nichtakademikerkinder, die etwas häufiger als Akademikerkinder nach ihrer ersten Bewerbungsphase eine Zulassung erhalten (82\%, Uni1: 75\%, Uni2: 76\%). ${ }^{197}$ Nach der zweiten Bewerbungsphase bekommen fast alle, die sich noch im risk set befinden, eine Zulassung: die Überlebenswahrscheinlichkeit sinkt für Nichtund Einakademikerkinder auf 6\% und für Doppelakademikerkinder sogar auf 0 (siehe auch Abb. A8.1 im Anhang).

Tabelle 8.13 zeigt die Zulassungswahrscheinlichkeit von Akademikerkindern im Vergleich zu Nichtakademikerkindern (diskrete Hazardratenmodelle) unter Einschluss der Kontrollvariablen (M1) sowie Leistungsindikatoren (M2). Wie sich schon deskriptiv angedeutet hat, unterscheidet sich die Zulassungswahrscheinlichkeit nicht zwischen Bewerber/innen unterschiedlicher sozialer Herkunft.

197 Die Ergebnisse aus Phase 2 und 3 können hier nicht direkt in Beziehung zueinander gesetzt werden, da sich die Perioden jeweils auf andere tatsächliche Zeitpunkte beziehen können. Wenn nur direkte Bewerbungen betrachtet werden, sind die $\mathrm{Zu}$ lassungschancen aber sehr ähnlich zu den hier berichteten: (Uni0: 83\%, Uni1/2: $75 \%$ ). 
Abb. 8.10: Studienzulassung nach sozialer Herkunft (Überlebenswahrscheinlichkeit)

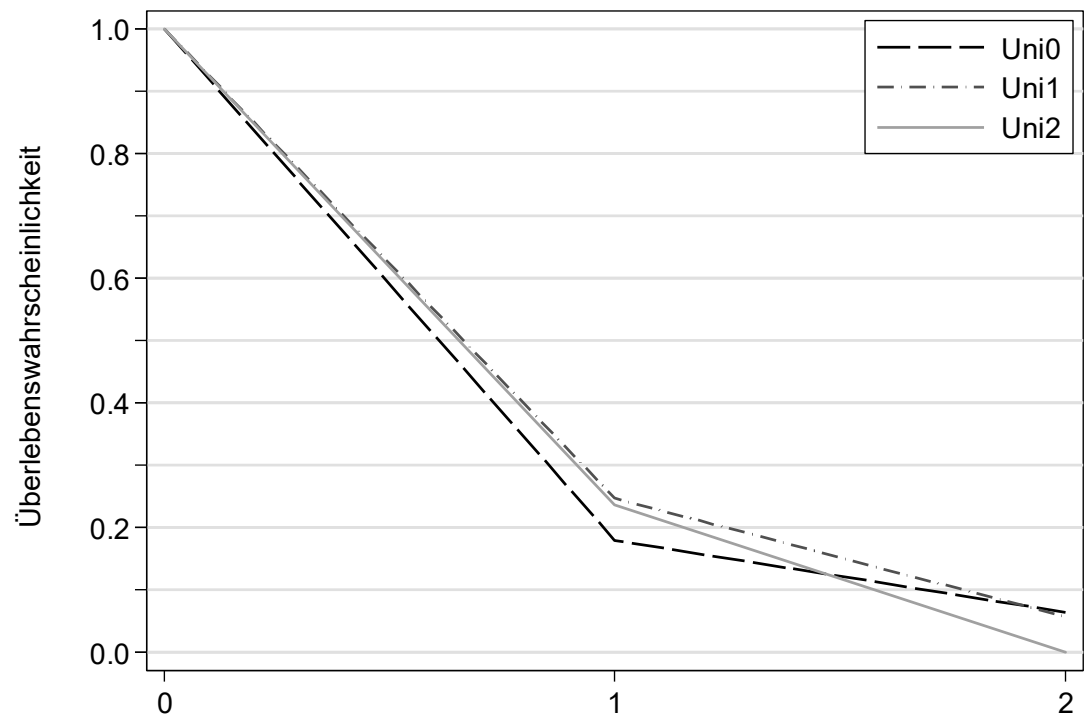

Studienbewerbung: 1.Phase - 2.Phase

N Uni0: 206 (zensiert: 28), Uni1: 81 (zensiert: 10), Uni2: 72 (zensiert: 11), vgl. Abb. A8.1

Quelle: Best Up-Individualdaten, eigene Berechnungen

Modell 2 kann entnommen werden, dass die Zulassungswahrscheinlichkeit signifikant positiv mit dem Abiturdurchschnitt sowie der Mathenote zusammenhängt. Gleichzeitig sind die Schulleistungen auch in diesem fortgeschrittenen Sample noch deutlich sozial ungleich verteilt (vgl. Tabelle A6.1). ${ }^{198}$ Dass sich dennoch keine Herkunftsunterschiede in der Zulassungswahrscheinlichkeit zeigen, kann an der Selektivität der Studiengänge liegen, für die sich Studienberechtigte bewerben. Wie sich im vorangegangenen Abschnitt zeigte, ist mit Blick auf die eigene und erforderliche Schulleistung ein hoher Anteil der

198 Zum Beispiel befinden sich bezüglich der Abiturnote 46\% der Doppelakademikerkinder im obersten Leistungsquartil: (Uni0: 24\%, Uni1: 25\%), jedoch nur 15\% im unteren (Uni0: 18, Uni1: 32). Damit bestätigt sich der bereits für die Bewerbungsphase festgestellte Befund, dass Einakademikerkinder hinsichtlich ihrer Schulleistung eine eher negativ selektierte Gruppe sind. Gleichzeitig hat sich in Abschnitt 8.2 gezeigt, dass sie sich häufiger als die anderen beiden Herkunftsgruppen verzögert bewerben. Dies könnte ein Hinweis darauf sein, dass sie ein Jahr Auszeit häufiger nutzen, um Wartezeit zu sammeln oder ihr Qualifikationsprofil zu verbessern und somit ihre schlechtere Schulleistung ausgleichen. 
getätigten Bewerbungen (in allen Herkunftsgruppen) weitestgehend realistisch.

Tab. 8.13: Zulassungswahrscheinlichkeit und soziale Herkunft (Hazardratenmodell)

\begin{tabular}{|c|c|c|}
\hline & M1 & M2 \\
\hline \multicolumn{3}{|c|}{ Soziale Herkunft Ref.: Uni0 } \\
\hline Uni1 & $-0,024(0,051)$ & $0,020(0,044)$ \\
\hline Uni2 & $-0,003(0,051)$ & $-0,007(0,052)$ \\
\hline
\end{tabular}

Leistungsindikatoren

Abiturdurchschnitt Ref.: unteres Quartil

Mitte

$0,164^{* *}(0,068)$

Oberes Quartil

$0,195^{\star *}(0,087)$

Note Deutsch 4. KH Ref.: unteres Quartil

Mitte

$0,002(0,044)$

Oberes Quartil

$-0,068(0,063)$

Note Mathe 4. KH Ref.: unteres Quartil

Mitte

$0,115^{*}(0,06)$

Oberes Quartil

$0,218^{\star * *}(0,069)$

Grundfähigkeiten (z-standardisiert)

Figural

$-0,033(0,021)$

Verbal

$0,016(0,024)$

T1: erste Bewerbungsphase

$0,179^{* * *}(0,058) \quad-0,066(0,082)$

T2: zweite Bewerbungsphase

$0,181^{\text {** }}(0,084) \quad-0,0001(0,093)$

BIC

449,01

387,88

AIC

409,30

459,36

Pseudo- $R^{2}$

0,14

0,14

Rho

0,07

0,15

N Perioden/Befragte/Schulen

$392 / 359 / 19$

$392 / 359 / 19$

Quelle: Best Up-Individualdaten, eigene Berechnungen

Logistische random-intercept Modelle, AME/DC (Standardfehler), *** $p<0,01 ;{ }^{* *} p<0,05 ;{ }^{*} p<0,1$

In allen Modellen enthalten: Kontrollvariablen (Migrationshintergrund, Geschlecht, Schultyp, Timing der ersten Bewerbungsphase)

Da die Studienbewerbung das zentrale Definitionskriterium des risk sets darstellt, kommt neben den bekannten Zensierungsgründen (Studienende, Antwortverweigerung oder Ausfall) ein weiterer hinzu, wenn sich Befragte nach einer erfolglosen ersten (direkten) Bewerbungsphase verzögert nicht erneut bewerben (N: 22). Gerade dieser Zensierungsgrund ist möglicherweise mit der Zulassungswahrscheinlichkeit assoziiert (und damit informativ), wenn Be- 
fragte mit geringen Zulassungschancen diese nach einer ersten erfolglosen Bewerbungsphase realisieren und sich daher gegen eine erneute Bewerbung entscheiden oder eine solche weiter hinauszögern, um Zulassungschancen über die Wartezeitquote zu optimieren (für ein ähnliches Beispiel siehe Allison 2014b). Tatsächlich scheint dies vor allem für Doppelakademikerkinder und zu einem geringeren Anteil auch für Nichtakademikerkinder der Fall zu sein, deren „Überlebenswahrscheinlichkeit“ (oder: Anteil ohne Zulassung) in Periode 2 deutlich steigt, sofern Befragte ohne verzögerte Bewerbung nicht aus dem risk set entfernt werden (Uni2: von 0 auf 13\%, Uni0: von 6 auf 12\%, Uni1: von 6 auf 8\%). Während dies für sozial schwächere Studienberechtigte ein Abwenden vom Studium zur Folge haben könnte, könnte es für ihre sozial privilegierten Peers, die einen längeren Zeithorizont haben, lediglich mit einem Aufschub verbunden sein. ${ }^{199}$

Insgesamt scheint soziale Ungleichheit bei der Umsetzung von Studienaspirationen nicht durch die direkte Selektionsleistung deutscher Hochschulakteure verschärft zu werden: Sofern Studienbewerbungen getätigt werden, können keine sozialgruppenspezifischen Zulassungschancen festgestellt werden $(\mathrm{HA} / \mathrm{Za}$, gegen $\mathrm{HA} / \mathrm{Zb})$, die somit auch nicht durch sozialgruppenspezifische Schulleistung (primäre Effekte) erklärt werden können (gegen HB1/Z). Immerhin bekommen 20\% aller Bewerber/innen - trotz vorgelagerter Selektionsprozesse - in der ersten Bewerbungsphase keine Zulassung zum Studium. Bewerben diese sich erneut, verbleibt nur noch ein sehr geringer Anteil ohne Zulassung. $\mathrm{Zu}$ bedenken ist dabei, dass sich nicht alle Studienberechtigten nach einer erfolglosen Bewerbungsphase (sofort) wieder bewerben. Weiter zu untersuchen wäre, inwiefern hierbei soziale Selektionsprozesse stattfinden, wie sie etwa Weiss und Steininger (2013) bei späteren Übergängen beobachten. An dieser Stelle stößt die empirische Analyse in diesem Kapitel jedoch an

199 Multivariat wurde sich der Frage nach nicht-informativer Zensierung erneut durch den Vergleich des auf den tatsächlichen Werten beruhenden Modells mit einem high-hazard und einem low-hazard Modell angenähert. Als drittes Vergleichsmodell wurde, wie oben beschrieben, nur für zensierte Befragte mit direkter, aber ohne verzögerte Bewerbung ein geringer Hazard angenommen. Wie Tabelle A8.10 zeigt, sind die (fehlenden) Herkunftsunterschiede über die verschiedenen Modellspezifikationen robust, sodass die Ergebnisinterpretation nicht durch vermeintliche nicht-informative Zensierung verfälscht ist. Ebenfalls getestet wurde die Proportionalitätsannahme. Hierfür mussten die Kategorien Uni1 und Uni2 gepoolt werden, da alle Doppelakademikerkinder, die in der zweiten Phase noch at risk sind, eine Zulassung bekommen, und die Interaktionsterme daher nicht vollständig geschätzt werden können. Der Ratio-Likelihood Chi-Quadrat Tests der M2 (Tab. 8.11) gegen ein Modell mit zusätzlichen Interaktionen testet, ist nicht signifikant (p: 0,237). Gleiches gilt, wenn Doppelakademikerkinder nicht berücksichtigt werden (p: 0,61). In diesem Fall kann die Proportionalitätsannahme also aufrechterhalten werden. 
ihre Grenzen (sowohl bezüglich der Fallzahlen als auch der Laufzeit der Studie).

Exkurs: Bewerbungsstrategien und Zulassungswahrscheinlichkeit

In Abschnitt 8.2.4 wurde der Versuch unternommen, Bewerbungsstrategien für den deutschen Kontext zu operationalisieren, mit deren Hilfe Zulassungschancen potentiell erhöht werden können. Ein kleiner Exkurs dazu, inwiefern diese Zulassungschancen tatsächlich erhöhen, erscheint hier angebracht. Im Folgenden soll für einige ausgewählte Variablen kurz gezeigt werden, ob dies tatsächlich der Fall ist.

Hierfür werden die bereits bekannten Hazardratenmodelle berichtet, die neben den Periodendummies zusätzlich zunächst jeweils eine Bewerbungsvariable enthalten. Diese wurden zeitveränderlich modelliert, d.h. je nach Bewerbungszeitpunkt wurde der entsprechende Wert für die direkte oder verzögerte Variable zugespielt. Erneut ist zu beachten, dass die Analysen nur für $240 \mathrm{Be}-$ fragte durchgeführt werden konnten, für die die Bewerbungsstrategievariablen vorliegen. 200

In Tabelle 8.14 sind die Ergebnisse dargestellt. Jede Zeile bezieht sich dabei auf ein neues Modell. Bezüglich der Anzahl zeigt sich, dass das Versenden von zwei bis drei im Vergleich zu nur einer Bewerbung die Zulassungschancen nicht signifikant erhöht. Bewirbt man sich hingegen vier oder fünf Mal, steigen die Chancen um 15 Prozentpunkte. Weitere Bewerbungen bringen jedoch darüber hinaus keinen zusätzlichen Anstieg.

Auch die Breite der Bewerbungen wirkt sich signifikant positiv auf die Zulassungschancen aus. Insbesondere Bewerbungen für mehrere Fächer (die sich im vorangegangenen Abschnitt am häufigsten bei Nichtakademikerkindern beobachten ließen) erhöhen die Zulassungschancen um 18 Prozentpunkte. Berechnet man den Einfluss aller drei Breite-Variablen simultan (nicht dargestellt), bestätigt sich, dass die fachliche Breite mit Abstand den stärksten Einfluss ausübt.

Weiterhin zeigt sich wenig überraschend, dass es die Zulassungschancen sehr stark erhöht, wenn der eigene Abiturdurchschnitt oder die bereits gesammelte Wartezeit im Vorjahr für eine Zulassung über die jeweilige Quote ausgereicht hätte (circa 50 Prozentpunkte). Als überraschend könnte man eher einstufen, dass der Zusammenhang nicht noch deutlicher zutage tritt. Dies ist ein klares Anzeichen dafür, dass die Vorjahreswerte die Zulassungschancen des aktuellen Jahres tatsächlich nur in Teilen widerspiegeln. Während bei „nicht

200 Der erste Block zu Anzahl und Breite der Bewerbungen könnte theoretisch für 317 Befragte untersucht werden. Um hier konsistent zu sein, werden die Ergebnisse nur anhand des kleineren Samples, für das alle Variablen zur Verfügung stehen, berichtet. Insgesamt unterscheiden sich die Ergebnisse nicht substantiell voneinander. 
ausreichender" Durchschnittsnote oder Wartezeit zusätzliche Bewerbungen die Zulassungswahrscheinlichkeit um 31 Prozentpunkte erhöhen, ist dies bei zur Anwendung kommenden zusätzlichen Kriterien für die Bewerber/innenauswahl nicht bzw. weniger eindeutig der Fall: Immerhin beträgt der DC noch 12 Prozentpunkte, jedoch ist dessen Schätzung bedeutend unsicherer. Zum einen könnte dies ein Hinweis dafür sein, dass aufgrund der Zentralität der Abiturnote weitere Kriterien tatsächlich nur eine geringe Rolle spielen. Andererseits setzt deren strategische Nutzung Informationen dazu voraus, dass solche Verfahren zum Einsatz kommen und worum es sich dabei handelt.

Tab. 8.14: Zulassungswahrscheinlichkeit und Bewerbungsstrategien (Hazardratenmodell)

\begin{tabular}{|c|c|c|}
\hline Bewerbungsstrategie & DC (Standardfehler) & BIC/AIC/Pseudo-R ${ }^{2}$ \\
\hline \multicolumn{3}{|c|}{ Anzahl und Breite der Bewerbungen } \\
\hline \multicolumn{3}{|l|}{ Anzahl, Ref.: 1} \\
\hline $\begin{array}{l}2-3 \\
4-5\end{array}$ & $\begin{array}{l}0,066 \\
(0,063)\end{array}$ & $322,98 / 248,53 / 0,17$ \\
\hline \multirow[t]{2}{*}{$>5$} & $\begin{array}{l}0,151^{\star *} \\
(0,061)\end{array}$ & \\
\hline & $\begin{array}{l}0,126^{*} \\
(0,067)\end{array}$ & \\
\hline Mehrere Orte & $\begin{array}{l}0,102^{\star *} \\
(0,046)\end{array}$ & $313,90 / 246,55 / 0,16$ \\
\hline Mehrere Fächer & $\begin{array}{l}0,180^{\star * *} \\
(0,050)\end{array}$ & $301,11 / 233,75 / 0,16$ \\
\hline Mehrere Hochschultypen & $\begin{array}{l}0,116^{\star *} \\
(0,045)\end{array}$ & $312,74 / 245,38 / 0,16$ \\
\hline \multicolumn{3}{|c|}{ Zulassungschancen } \\
\hline $\begin{array}{l}\text { Ausgleich AdH, Ref. DN+WZ nicht } \\
\text { ausreichend, keine weiteren Kriterien }\end{array}$ & & $260,73 / 189,83 / 0,15$ \\
\hline $\begin{array}{l}\mathrm{DN}+\mathrm{WZ} \text { nicht ausreichend, weitere } \\
\text { Kriterien }\end{array}$ & $\begin{array}{l}0,120 \\
(0,134)\end{array}$ & \\
\hline$D N+W Z$ ausreichend & $\begin{array}{l}0,463^{\star \star *} \\
(0,130)\end{array}$ & \\
\hline $\begin{array}{l}\text { Ausgleich Bewerbung, Ref. DN+WZ nicht } \\
\text { ausreichend, keine weiteren } \\
\text { Bewerbungen }\end{array}$ & & $254,74 / 183,84 / 0,14$ \\
\hline $\begin{array}{l}\text { DN+WZ nicht ausreichend, weitere } \\
\text { Bewerbungen }\end{array}$ & \multicolumn{2}{|l|}{$\begin{array}{l}0,310^{\star \star *} \\
(0,116)\end{array}$} \\
\hline DN+WZ ausreichend & $\begin{array}{l}0,559^{* * *} \\
(0,110)\end{array}$ & \\
\hline
\end{tabular}

Quelle: Best Up-Individualdaten + Rekrutierungsdaten, eigene Berechnungen N (Perioden/Personen/Schulen): 256/240/19

logistische random-intercept Modelle, DC (Standardfehler), ${ }^{* * *} p<0,01 ;{ }^{* *} p<0,05 ;{ }^{*} p<0,1$

In allen Modellen enthalten: soziale Herkunft, Leistungsindikatoren, Kontrollvariablen, Periodendummies 


\subsection{Ergebnisse Phase 4: Herkunftsunterschiede bei der Studienaufnahme}

Bisher hat sich gezeigt, dass sich soziale Herkunftsunterschiede bei der Umsetzung von Studienaspirationen zwar bei der Bewerbung, nicht aber bei der Zulassung zum Studium zeigen. Zuletzt steht der finale Schritt - die Studienaufnahme - im Zentrum der Untersuchung (8.4.1). Diese wird wie im vorangegangenen Schritt zunächst konditional betrachtet, also nur für diejenigen Befragten, die sich sowohl für ein Studium beworben haben als auch eine Zusage erhielten (N: 310) (HA/Sa). Im zweiten Schritt sollen Herkunftsunterschiede bei der Studienaufnahme aber auch unkonditional betrachtet werden, also unabhängig von Bewerbung und Zulassung, um dem letztlichen Ausmaß sozialer Ungleichheit bei der Umsetzung von Studienaspirationen näher zu kommen (HA/Sb). Zuletzt wird für diejenigen Studienberechtigten, die ins Studium übergehen, erneut untersucht, ob es Herkunftsunterschiede bezüglich der Fächer (HD2/S, HD3S), Hochschultypen (HD1/S) und Orte (HC1/S) gibt (8.4.2).

\subsubsection{Studienaufnahme: konditional vs. unkonditional}

Konditionale Studienaufnahme

Generell bietet sich auch für die Analyse zur Studienaufnahme ein ereignisdatenanalytisches Verfahren an, da diese zu zwei Zeitpunkten erfolgen kann und durch das längsschnittliche Design Zensierungen auftreten. Wie bereits in Abschnitt 8.1.3 erwähnt, ist dies jedoch für die Untersuchung des Übergangs der zugelassenen Bewerber/innen nicht sinnvoll, da es nur für 15 Befragte eine zweite Periode gibt (diejenigen, die sich direkt bewarben, eine Zulassung erhielten, nicht mit dem Studium begannen und sich danach erneut bewarben und eine Zulassung erhielten). In dieser zweiten Periode erleben alle $15 \mathrm{Be}-$ fragten, die im risk set verbleiben, das Ereignis Studienaufnahme, sodass hier das Ereignis also perfekt vorhergesagt wird.

Zwei alternative Vorgehensweisen zur Analyse der konditionalen Studienaufnahme werden daher verfolgt: Zum einen wird die Studienaufnahme nach der ersten Zulassung betrachtet (Studienaufnahmen in Periode 2 bekommen damit den Wert 0 zugewiesen). Zum anderen wird die Studienaufnahme bis ein Jahr nach dem Abitur untersucht (Studienaufnahmen in Periode 2 bekommen damit den Wert 1).

Tabelle 8.15 zeigt die bivariaten Zusammenhänge zwischen den beiden abhängigen Variablen und der sozialen Herkunft. Insgesamt ist der Anteil derjenigen, die nach einer Zulassung mit dem Studium beginnen, mit knapp 90\% und knapp 95\% wie erwartet sehr hoch. In der Tat zeigen sich hier Herkunfts- 
unterschiede zwischen den sozialen Herkunftsgruppen - diese verlaufen aber eher ,zugunsten“ von Nichtakademikerkindern, die um etwa 7 Prozentpunkte häufiger ein Studium aufnehmen als Doppelakademikerkinder. Nach Kontrolle weiterer Variablen sind diese nach wie vor sichtbar und im Falle der zweiten abhängigen Variable ist der Unterschied zwischen Doppelakademikerkindern und Nichtakademikerkindern leicht signifikant (Tabelle 8.16).

Tab. 8.15: Konditionale Studienaufnahme nach sozialer Herkunft (Prozent/Fallzahl)

\begin{tabular}{lllll}
\hline & Uni0 & Uni1 & Uni2 & Total \\
\hline $\begin{array}{l}\text { Studienaufnahme nach } \\
\text { erster Zulassung }\end{array}$ & $92,7(165)$ & $85,9(61)$ & $85,3(52)$ & $89,7(278)$ \\
\hline $\begin{array}{l}\text { Studienaufnahme bis } \\
\text { 1 Jahr nach dem Abitur }\end{array}$ & $96,6(172)$ & $93,0(66)$ & $90,2(55)$ & $94,5(293)$ \\
\hline
\end{tabular}

Quelle: Best Up-Individualdaten, eigene Berechnungen

Tab. 8.16: Konditionale Studienwahrscheinlichkeit: multivariate Ergebnisse

\begin{tabular}{|c|c|c|}
\hline & $\begin{array}{l}\text { M1 } \\
\text { nach erster Zulassung }\end{array}$ & $\begin{array}{l}\text { M2 } \\
\text { bis ein Jahr nach dem Abitur }\end{array}$ \\
\hline \multicolumn{3}{|c|}{ Soziale Herkunft Ref.: Uni0 } \\
\hline Uni1 & $-0,068(0,048)$ & $-0,021(0,031)$ \\
\hline Uni2 & $-0,059(0,049)$ & $-0,076^{*}(0,045)$ \\
\hline \multicolumn{3}{|c|}{ Leistungsindikatoren } \\
\hline \multicolumn{3}{|c|}{ Abiturdurchschnitt Ref.: unteres Quartil } \\
\hline Mitte & $0,047(0,064)$ & $0,035(0,043)$ \\
\hline Oberes Quartil & $0,032(0,080)$ & $0,005(0,064)$ \\
\hline \multicolumn{3}{|c|}{ Note Deutsch 4. KH Ref.: unteres Quartil } \\
\hline Mitte & $-0,018(0,041)$ & $-0,003(0,039)$ \\
\hline Oberes Quartil & $-0,061(0,056)$ & $0,046(0,039)$ \\
\hline \multicolumn{3}{|c|}{ Note Mathe 4. KH Ref.: unteres Quartil } \\
\hline Mitte & $-0,010(0,050)$ & $0,034(0,036)$ \\
\hline Oberes Quartil & $0,006(0,060)$ & $0,0002(0,055)$ \\
\hline \multicolumn{3}{|c|}{ Grundfähigkeiten (z-standardisiert) } \\
\hline Figural & $0,003(0,020)$ & $0,013(0,014)$ \\
\hline Verbal & $-0,003(0,022)$ & $0,001(0,016)$ \\
\hline Pseudo- $R^{2}$ & 0,03 & 0,05 \\
\hline Rho & 0 & 0 \\
\hline N Befragte/Schulen & $310 / 19$ & $310 / 19$ \\
\hline
\end{tabular}

Quelle: Best Up-Individualdaten, eigene Berechnungen logistische random-intercept Modelle, AME/DC (Standardfehler), *** $p<0,01 ;{ }^{* *} p<0,05 ;{ }^{*} p<0,1$ Zusätzlich enthalten: Kontrollvariablen (Migrationshintergrund, Geschlecht, Schultyp und Timing der Zulassung). 
Mit Hypothese HA/Sa wurde angenommen, dass sich bezüglich des konditionalen Übergangs ins Studium kaum Herkunftsunterschiede zeigen. Auf Grundlage der Ergebnisse kann diese in der Tendenz nicht bestätigt werden. Warum nehmen Doppelakademikerkinder trotz Zulassung etwas seltener ein Studium auf als Nichtakademikerkinder? Möglicherweise sind sie wählerischer. Verschiedene Befunde, zum Beispiel ihre geringe fachliche Breite bei den Bewerbungen (Abschnitt 2.2.4), weisen darauf hin, dass sie stärkere fachliche Präferenzen haben und diese auch dann umsetzen möchten, wenn sie mit höheren Zulassungshürden konfrontiert sind. Entsprechend zeigt sich, dass diejenigen Doppelakademikerkinder, die ins Studium übergehen, mit 76\% deutlich häufiger angeben, ihren favorisierten Studiengang zu studieren als Einakademikerkinder (68\%) und insbesondere Nichtakademikerkinder (59\%).

\section{Unkonditionale Studienaufnahme}

Welche Befunde zeigen sich, wenn man den unkonditionalen Übergang ins Studium betrachtet, also alle Studienaspirant/innen unabhängig von den vorangegangenen Phasen berücksichtigt?

Im Falle der unkonditionalen Analyse der Studienwahrscheinlichkeit ist ein ereignisdatenanalytisches Vorgehen problemlos möglich. Hier folgen die Perioden - wie schon in der Bewerbungsphase - der historischen Zeit und beziehen sich auf die WiSe 2014/15 (t1) und 2015/16 (t2).

In Abbildung 8.11 ist die Überlebenswahrscheinlichkeit getrennt nach sozialer Herkunft dargestellt. Wenig überraschend zeigt sich das bereits aus der Bewerbungsphase bekannte Bild, dass Einakademikerkinder deutlich seltener direkt ins Studium übergehen (40\%) als die beiden anderen Gruppen (jeweils $55 \%$ ), dies aber zum zweiten Zeitpunkt fast vollständig ausgeglichen wird. Ein Jahr nach Erhalt des (Fach-)Abiturs haben knapp 70\% der Studienberechtigten ihre Studienaspiration umgesetzt, Herkunftsunterschiede zeigen sich kaum (Überlebenswahrscheinlichkeit, also Anteil ohne Studienaufnahme: Uni0: 28\%, Uni1: 30\%, Uni2: 26\%). Auch multivariat bestätigt sich (Tabelle 8.17), dass insgesamt kaum Herkunftsunterschiede bezüglich der finalen Umsetzung von Studienaspirationen beobachtet werden können (gegen $\mathrm{HA} / \mathrm{Sb}$ ). ${ }^{201}$

201 Auch für diesen Analyseschritt wurden die Proportionalitätsannahme sowie die Annahme nicht-informativer Zensierung getestet. Wie bereits in den vorangegangenen Analyseschritten zeigt sich auch hier, dass die Ergebnisse robust sind, wenn den (zufällig) zensierten Fällen ein hoher oder ein niedriger Hazard zugewiesen wird (vgl. Anhangstabelle A8.11). Mit Blick auf die Proportionalitätsannahme deutet sich eine Verletzung bereits in Abbildung 8.11 an. Dies bestätigt der durchgeführte Ratio-Likelihood Chi-Quadrat Tests, auf dessen Grundlage die Null-Hypothese, dass die dem Modell hinzugefügten Interaktionsterme gemeinsam Null sind, abgelehnt wird (p: 0,05). Wie bereits in den Analysen zur Bewerbungsphase 
Abb. 8.11: Unkonditionale Studienaufnahme nach sozialer Herkunft (Überlebenswahrscheinlichkeit)

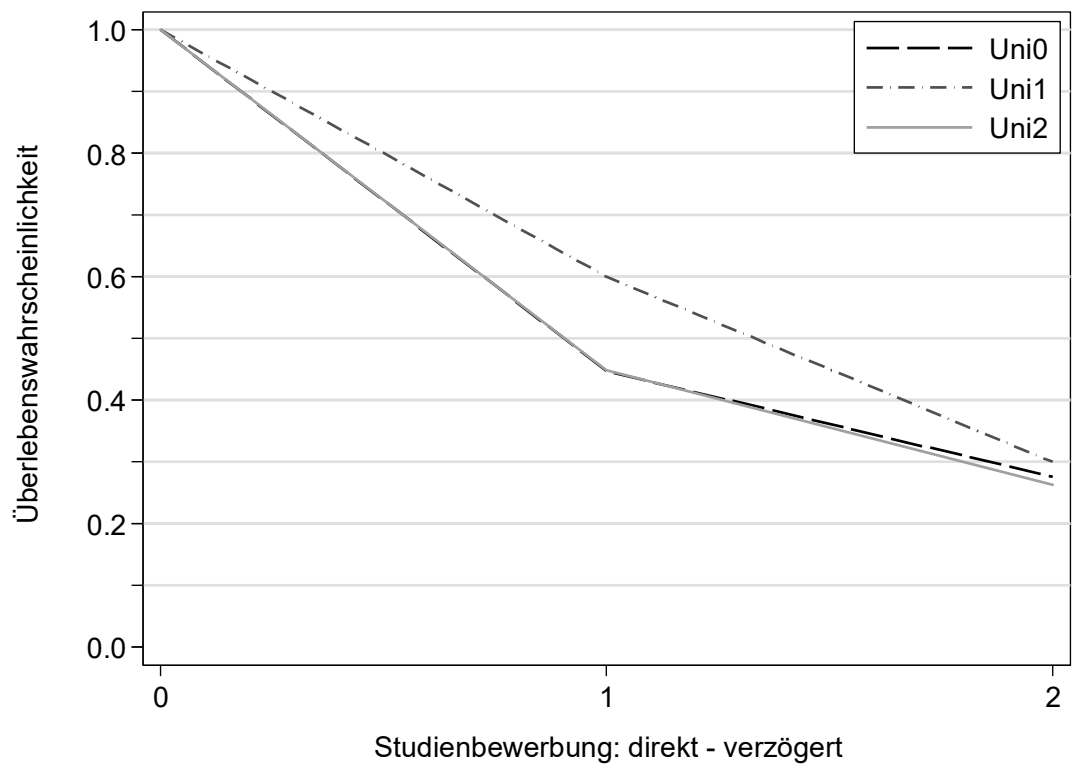

N Uni0: 246 (zensiert nach t1: 16; t2: 58)

N Uni1: 95 (zensiert nach t1: 1 ; t2: 28)

N Uni2: 78 (zensiert nach t1: 6; t2: 17)

Quelle: Best Up-Individualdaten, eigene Berechnungen

Weitere deskriptive Befunde zeigen, dass von denjenigen, die bis ein Jahr nach dem (Fach-)Abitur kein Studium aufgenommen haben, insgesamt 66\% planen, dies noch zu tun. Hierbei sind Nichtakademikerkinder mit $61 \%$ unterrepräsentiert (Uni1: 72\%, Uni2: 74\%).

sei daher hier darauf verwiesen, dass die Herkunftseffekte als Durchschnitteffekte im Beobachtungszeitraum zu interpretieren sind. 
Tab. 8.17: Unkonditionale Studienwahrscheinlichkeit: multivariate Ergebnisse (Hazardratenmodell)

\begin{tabular}{ll}
\hline & DC/AME (Standardfehler) \\
\hline Soziale Herkunft Ref.: Uni0 & $0,005(0,049)$ \\
Uni1 & $0,028(0,055)$ \\
Uni2 & \\
\hline Leistungsindikatoren & \\
Abiturdurchschnitt Ref.: unteres Quartil & $0,142^{* *}(0,057)$ \\
Mitte & $0,128(0,082)$ \\
Oberes Quartil & \\
Note Deutsch 4. KH Ref.: unteres Quartil & $0,056(0,052)$ \\
Mitte & $0,043(0,065)$ \\
Oberes Quartil & \\
Note Mathe 4. KH Ref.: unteres Quartil & $0,121^{* *}(0,051)$ \\
Mitte & $0,219^{* * *}(0,069)$ \\
oberes Quartil & \\
Grundfähigkeiten (z-standardisiert) & $-0,013(0,022)$ \\
Figural & $-0,002(0,025)$ \\
Verbal & $-0,276^{* * *}(0,080)$ \\
\hline T1: WiSe 2014/15 & $-0,282^{* * *}(0,082)$ \\
T2: WiSe 2015/16 & 0,10 \\
\hline Pseudo-R ${ }^{2}$ (Maddala) & 0,03 \\
Rho & $598 / 419 / 19$ \\
N Perioden/Personen/Schulen &
\end{tabular}

Quelle: Best Up-Individualdaten, eigene Berechnungen

Logistische random-intercept Modelle, AME/DC (Standardfehler), ${ }^{* * *} p<0,01 ;{ }^{* *} p<0,05 ;{ }^{*} p<0,1$

Zusätzlich enthalten: Kontrollvariablen (Migrationshintergrund, Geschlecht, Schultyp und Art der Hochschulzugangsberechtigung)

\subsubsection{Differenzierte Übergänge: Hochschulorte, Hochschultypen und Studienfächer}

Insgesamt konnten also in Phase 4 keine Herkunftsunterschiede bezüglich der Umsetzungswahrscheinlichkeit von Studienaspirationen beobachtet werden. Im letzten Schritt wird für diejenigen Befragten, die ein Studium aufnahmen, untersucht, inwiefern sich Herkunftsunterschiede bezüglich des Ortes $(\mathrm{HC} 1 / \mathrm{S})$, des Hochschultyps (HD1/S) und des Faches zeigen (HD2/S und HD3/S). Mit der Untersuchung des Hochschultyps und des Studienfaches soll geprüft werden, inwiefern sich die in Phase 1 und 2 gezeigte soziale Ungleich- 
heit innerhalb des Hochschulsystems auch bezüglich der letztlichen Übergänge fortsetzt.

Tabell 8.18 gibt hierzu zunächst einen deskriptiven Überblick. Die bereits in Phase 1 und 2 beobachteten starken Präferenzen für nahegelegene Studienoptionen spiegeln sich deutlich im letztlichen Studienort wider: Insgesamt studieren nur etwa 15\% nicht in Berlin oder Brandenburg. Die bereits aus Phase 2 bekannten Herkunftsunterschiede können auch hier beobachtet werden, sind insgesamt aber weniger deutlich ausgeprägt. Dies liegt auch daran, dass Akademikerkinder zwar einen größeren Bewerbungsradius hatten und somit eine größere prinzipielle Mobilitätsbereitschaft zeigten ( $40 \%$ bewarben sich auch außerhalb von Berlin/Brandenburg), sich aber letztlich häufig für eine nahegelegene Hochschule entschieden. Weiterhin bestätigt sich der Befund, dass Einakademikerkinder im Durchschnitt die weitesten Distanzen zurücklegen, um zu studieren.

Tab. 8.18: Studienort, Hochschultyp und Studienfach nach sozialer Herkunft

\begin{tabular}{|c|c|c|c|c|}
\hline & $\begin{array}{l}\text { Uni0 } \\
\text { (N: 164) }\end{array}$ & $\begin{array}{l}\text { Uni1 } \\
\text { (N: 63) }\end{array}$ & $\begin{array}{l}\text { Uni2 } \\
\text { (N: } 54)\end{array}$ & $\begin{array}{l}\text { Total } \\
\text { (N: 281) }\end{array}$ \\
\hline \multicolumn{5}{|l|}{ Hochschulort $(\% / \mathrm{N})$} \\
\hline Berlin & $69,5(114)$ & $63,5(40)$ & $64,8(35)$ & $67,3(189)$ \\
\hline Berlin/Brandenburg & $89,0(146)$ & $76,2(48)$ & $83,3(45)$ & $85,1(239)$ \\
\hline \multicolumn{5}{|l|}{ Durchschnittliche Entfernung in $\mathrm{km}$} \\
\hline Entfernung (Mean/SD) & $49,1(123,5)$ & $101,4(179,1)$ & $70,5(172,4)$ & $65(148,5)$ \\
\hline Range & $0-700$ & $0-639$ & $0-700$ & $0-700$ \\
\hline \multicolumn{5}{|l|}{ Hochschultyp (\%/N) } \\
\hline Universität (vs. FH) & $65,9(108)$ & $63,5(40)$ & $74,1(40)$ & $66,9(188)$ \\
\hline \multicolumn{5}{|l|}{$\begin{array}{l}\text { Studienfach: durchschnittliches } \\
\text { Einkommen/Berufsprestige }\end{array}$} \\
\hline Stundenlohn in Euro (Mean/SD) & $16,9(1,8)$ & $16,6(1,8)$ & $17,2(2,2)$ & $16,9(1,9)$ \\
\hline Range & $12,3-22,3$ & $12,9-19,2$ & $12,1-23,2$ & $12,1-23,2$ \\
\hline Berufsprestige (Mean/SD) & $100,1(19,8)$ & $95,0(16,0)$ & $101,1(25,5)$ & $99,1(20,3)$ \\
\hline Range & $64,8-191,8$ & $67,0-136,3$ & $64,9-191,8$ & $64,9-191,8$ \\
\hline
\end{tabular}

Quelle: Best Up-Individualdaten, eigene Berechnungen

Bezüglich des Hochschultyps zeigt sich zunächst das bereits bekannte Muster, dass Doppelakademikerkinder an Universitäten überrepräsentiert sind. Es zeigt sich aber auch, dass die allgemein stärkere Präferenz für Universitäten (insgesamt sendeten $75 \%$ ihre erstgerankte Bewerbung an diesen Hochschultyp, vgl. Abb. 8.8) nicht in allen Fällen umgesetzt werden konnte. Insbesondere Einaka- 
demikerkinder studieren häufiger an Fachhochschulen als mit Blick auf ihre Bewerbungen zu erwarten gewesen wäre.

Hinsichtlich des Einkommens und Berufsprestiges, zu dem das letztliche Studienfach führt, zeigen sich im Durchschnitt kaum Unterschiede zwischen den Herkunftsgruppen.

Auch nach Kontrolle der Schulleistung spiegelt sich in den multivariaten Modellen in Tabelle 8.19 die größere regionale Flexibilität (oder: Mobilitätszwang?) von Einakademikerkindern wider, die signifikant häufiger an entfernteren Hochschulen studieren als Nichtakademikerkinder (M2 und M3). Die Unterschiede zwischen Doppel- und Nichtakademikerkindern sind hingegen klein und nicht signifikant. Hypothese $\mathrm{HC} 1 / \mathrm{S}$, mit der eine geringere regionale Mobilität von Nichtakademikerkindern angenommen wurde, wird damit nur zum Teil von den Befunden gestützt.

Tab. 8.19: Soziale Herkunft, Studienort, Hochschultyp, Studienfach: multivariate Ergebnisse

\begin{tabular}{|c|c|c|c|c|c|c|}
\hline & $\begin{array}{l}\text { M1 } \\
\text { Ort: Berlin }\end{array}$ & $\begin{array}{l}\text { M2 } \\
\text { Ort: Berlin/ } \\
\text { Brandenburg }\end{array}$ & $\begin{array}{l}\text { M3 } \\
\text { Distanz }\end{array}$ & $\begin{array}{l}\text { M4 } \\
\text { Hochschul- } \\
\text { typ: FH }\end{array}$ & $\begin{array}{l}\text { M5 } \\
\text { Fach: } \\
\text { Einkommen }\end{array}$ & $\begin{array}{l}\text { M6 } \\
\text { Fach: } \\
\text { Prestige }\end{array}$ \\
\hline \multicolumn{7}{|c|}{ Soziale Herkunft Ref. Uni0 } \\
\hline Uni1 & $\begin{array}{l}-0,040 \\
(0,072)\end{array}$ & $\begin{array}{l}-0,128^{* *} \\
(0,063)\end{array}$ & $\begin{array}{l}55,457^{\star *} \\
(23,337)\end{array}$ & $\begin{array}{l}-0,031 \\
(0,069)\end{array}$ & $\begin{array}{l}-0,136 \\
(0,275)\end{array}$ & $\begin{array}{l}-5,839^{*} \\
(3,095)\end{array}$ \\
\hline Uni2 & $\begin{array}{l}-0,046 \\
(0,077)\end{array}$ & $\begin{array}{l}-0,028 \\
(0,054)\end{array}$ & $\begin{array}{l}12,115 \\
(24,565)\end{array}$ & $\begin{array}{l}-0,077 \\
(0,072)\end{array}$ & $\begin{array}{l}0,041 \\
(0,291)\end{array}$ & $\begin{array}{l}-1,389 \\
(3,258)\end{array}$ \\
\hline Konstante & & & $\begin{array}{l}46,014 \\
(38,415)\end{array}$ & & $\begin{array}{l}16,581^{\text {***}} \\
(0,498)\end{array}$ & $\begin{array}{l}97,348^{\star \star *} \\
(5,094)\end{array}$ \\
\hline Rho & 0 & 0 & 0 & 0 & 0,06 & 0 \\
\hline $\begin{array}{l}\text { Pseudo-R² } \\
\text { (Maddala) }\end{array}$ & 0,05 & 0,05 & 0,06 & 0,09 & 0,17 & 0,12 \\
\hline $\begin{array}{l}\mathrm{N} \\
\text { Befragte/ } \\
\text { Schulen }\end{array}$ & $281 / 19$ & $281 / 19$ & $281 / 19$ & $281 / 19$ & $281 / 19$ & $281 / 19$ \\
\hline
\end{tabular}

Quelle: Best Up-Individualdaten, eigene Berechnungen

*** $p<0,01 ;{ }^{* *} p<0,05 ;{ }^{*} p<0,1$

M3/5/6: lineare random intercept Modelle, Regressionskoeffizienten (Standardfehler)

M1/2/4: logistische random intercept Modelle, DC (Standardfehler)

Alle Modelle enthalten Leistungsindikatoren und Kontrollvariablen (Migrationshintergrund, Geschlecht, Schultyp und Timing der Studienaufnahme)

Bezüglich des Hochschultyps ist der Unterschied von etwa 8 Prozentpunkten zwischen Doppel- und Nichtakademikerkindern zwar noch sichtbar, aber sehr unsicher geschätzt (gegen HD1/S). Im Gegensatz zu den präferierten Bewerbungen in Phase 2 zeigen sich keine erwartbaren Einkommensunterschiede des Studienfaches zwischen Doppel- und Nichtakademikerkindern (HD2/S). Gleiches gilt für das Berufsprestige, zu dem ein Studienfach im Durchschnitt führt (gegen HD3/S). Der bereits deskriptiv geringere Prestigewert für Einakademi- 
kerkinder kann auch multivariat beobachtet werden; der Unterschied ist insgesamt jedoch kaum substantiell.

Exkurs: Unterschiede im Hochschultyp und Studienfach = Aspirationsunterschiede?

Insgesamt sind die Unterschiede zwischen den Herkunftsgruppen - insbesondere zwischen Nichtakademikerkindern und Doppelakademikerkindern - bezüglich ihres Studienfaches und dem Hochschultyp, an dem sie studieren, geringer, als rückblickend auf Phase 1 und 2 zu erwarten gewesen wäre.

Auch für die Gruppe der Studierenden soll der bereits bekannte Exkurs unternommen werden, um sich der Frage anzunähern, inwiefern sich dies bereits in ihren Aspirationen widerspiegelt. Mit Blick auf den Hochschultyp zeigt sich in Tabelle 8.20, dass in allen Herkunftsgruppen ein größerer Anteil der Studierenden lieber an einer Universität studiert hätte, als dies letztlich der Fall ist. Bezüglich des mit einem Studienfach verbundenen Einkommens zeigen sich nur geringe Herkunftsunterschiede, sowohl bei der Aspiration als auch beim Studienfach. Ein sehr interessanter Unterschied, auch rückblickend auf die parallelen deskriptiven Befunde aus Phase 1 und 2, zeigt sich jedoch beim Berufsprestige. Einakademikerkinder und Nichtakademikerkinder hatten in Phase 2 fast identische Werte bei ihren Aspirationen und der erstgerankten Bewerbung. Die Aspirationswerte sind nach wie vor die gleichen, doch studieren sie im Durchschnitt Fächer, die zu einem niedrigeren Berufsprestige führen (insbesondere Einakademikerkinder). Bei Doppelakademikerkindern sind die Werte zwischen Aspiration und Studienfach fast identisch. Jedoch auf einem deutlich geringeren Niveau als zu erwarten gewesen wäre: Ihre präferierte Bewerbung richtete sich im Durchschnitt an Fächer mit einem Prestigewert von 114,3 (vgl. Tabelle 8.12). Dies ist ein weiterer Hinweis darauf, dass sie wählerischer sind. Doppelakademikerkinder mit Aspirationen (und Bewerbungen) für prestigereiche Fächer, wie etwa Medizin, scheinen sich bei fehlenden (passenden) Zusagen eher gegen ein Studium oder wahrscheinlicher: für ein Verschieben des Studiums zu entscheiden, um ihre fachlichen Präferenzen umsetzen zu können. Hinweise hierfür zeigten sich bereits in dem Befund, dass sie trotz Zusage etwas seltener ins Studium übergehen (Abschnitt 8.3). 
Tab. 8.20: Unterschiede zwischen Hochschultyp-/Fachaspirationen und dem entsprechenden Studium

\begin{tabular}{llll}
\hline & $\begin{array}{l}\text { Hochschultyp Uni: } \\
\text { Aspiration - Studium (\%) }\end{array}$ & $\begin{array}{l}\text { Einkommen: Aspiration - } \\
\text { Studium (Mean, SD) }\end{array}$ & $\begin{array}{l}\text { Prestige: Aspiration - } \\
\text { Studium (Mean, SD) }\end{array}$ \\
\hline Uni0 & $75,6-65,9$ & $16,9(2,6)-17,0(1,8)$ & $103,1(28,4)-99,9(20,3)$ \\
Uni1 & $76,2-63,5$ & $17,4(2,8)-16,6(1,8)$ & $105,7(32,1)-94,6(15,8)$ \\
Uni2 & $87,0-74,1$ & $17,2(2,7)-17,4(2,1)$ & $99,6(21,6)-101,4(27,4)$ \\
Total & $77,9-66,9$ & $17,0(1,9)-16,9(1,9)$ & $103,1(28,4)-99,0(20,9)$ \\
\hline
\end{tabular}

Quelle Best Up-Individualdaten, Mikrozensus 2007-2012, DOIs: 10.21242/12211.2007.00.00.1.1.0 bis 10.21242/12211.2012.00.00.1.1., eigene Berechnungen

Es werden nur diejenigen Befragten berücksichtigt, für die sowohl Angaben zur Aspiration als auch zum Studium vorliegen. N Hochschultyp: 281, N Fach: 246

\subsection{Zusammenfassung}

Zuletzt sollen die Ergebnisse zu sozialer Ungleichheit bei der Umsetzung von Studienaspirationen hier zusammengefasst und diskutiert werden. Die zentralen Befunde und deren Implikation mit Blick auf die formulierten Hypothesen sind in Tabelle 8.21 zusammengetragen.

In der zweiten Phase zeigte sich, dass bis ein Jahr nach dem (Fach-)Abitur ein hoher Anteil von insgesamt fast $90 \%$ der Studienberechtigten versuchen, ihre Studienaspirationen in Form von Bewerbungen umzusetzen. Doppelakademikerkinder sind unter den Bewerber/innen klar überrepräsentiert. Einakademikerkinder nehmen bezüglich ihrer Bewerbungswahrscheinlichkeit nicht die mittlere Position ein, sondern bewerben sich ungefähr ähnlich häufig wie Nichtakademikerkinder. Dabei zeigt sich zudem ein Timingeffekt: Sie bewerben sich seltener direkt nach dem Abitur, holen dies aber ein Jahr später wieder auf.

Die Herkunftsunterschiede zwischen Doppelakademikerkindern auf der einen und Nicht- sowie Einakademikerkindern auf der anderen Seite sind erneut sehr robust und können kaum durch sozialgruppenspezifische Schulleistung und Indikatoren der rationalen Wahl oder soziale und kulturelle Ressourcen erklärt werden. Generell deuten einige Variablen in die vermutete Richtung: So erhöhen die eingeschätzte Erfolgswahrscheinlichkeit, die Erwartung eines angesehenen Jobs durch ein Studium sowie der Studienwunsch der Eltern die Bewerbungswahrscheinlichkeit. Alle weiteren Indikatoren spielen eine eher untergeordnete Rolle für die Bewerbungswahrscheinlichkeit von Studienaspirant/innen. So scheinen etwa antizipierte Kosten in diesem Fall keinen Einfluss zu üben. Dies könnte im vorliegenden Fall auch daran liegen, dass die Berliner Hochschullandschaft viele Studienoptionen in unmittelbarer örtlicher 
Nähe bietet und sich anfallende Mobilitätskosten - bei erfolgreicher Bewerbung - daher in Grenzen halten.

Der Einfluss antizipierte Zugangshürden zeigt sich nicht mit Blick auf die generellen Zulassungsbeschränkungen: Ob ein Fach im Durchschnitt häufiger zulassungsbeschränkt ist oder Eignungsfeststellungsverfahren anwendet, scheint die Bewerbungswahrscheinlichkeit kaum zu beeinflussen. Hingegen ist es die durchschnittliche Selektivität von Studienfächern, die deutlich mit der Bewerbungswahrscheinlichkeit assoziiert ist und damit offenbar eine Hürde im Umsetzungsprozess darstellt. Dies ist jedoch in allen Herkunftsgruppen (weniger stark bei Einakademikerkindern) der Fall. Zu einer Umorientierung innerhalb des Hochschulsystems, d.h. zu einer Diskrepanz zwischen der Fachaspiration zu Beginn des letzten Schuljahres und einer entsprechenden Bewerbung - wie sie in Phase 1 beobachtet werden konnte - tragen Zugangshürden jedoch kaum bei. Generell scheint sich ein beachtlicher Anteil von fast $40 \%$ der Studienaspirant/innen innerhalb des letzten Schuljahres weitestgehend unabhängig von antizipierten Barrieren fachlich neu zu orientieren. Insgesamt zeigt sich jedoch in der Tendenz auch, dass die fachlichen Präferenzen von Akademikerkindern, insbesondere Doppelakademikerkindern, bereits gefestigter zu sein scheinen als die von Nichtakademikerkindern: ihre erstgerankte Bewerbung richtet sich häufiger an Studiengänge in Fächern, die sie bereits zu Beginn des letzten Schuljahres studieren wollten. Eine alternative Interpretation dieses (deskriptiven) Befundes ist, dass Nichtakademikerkinder ihre Präferenzen eher am Hochschulort festmachen und somit fachliche Vorlieben hinten anstellen.

Dies spiegelt sich auch in den Ergebnissen zum Hochschulort und der durchschnittlichen Distanz wider. Generell zeichnet sich im Einklang mit existierenden Befunden (vgl. Abschnitt 5.4.3) ab, dass die Nähe zum Heimatort ein zentrales Wahlmotiv (in allen Herkunftsgruppen) zu sein scheint. Dabei zeigt sich, dass sich Studienaspirant/innen unterschiedlicher sozialer Herkunft bezüglich ihrer Präferenzen (der erstgerankten Bewerbung) ähnlicher sind als bezüglich ihrer Mobilitätsbereitschaft, die hier über den Bewerbungsradius erfasst wurde. Diese scheint bei Akademikerkindern, hier insbesondere bei Einakademikerkindern, größer zu sein.

Die bereits aus Phase 1 bekannten Befunde einer sozialgruppenspezifischen Wahl von Optionen innerhalb des Hochschulsystems sind auch in der Bewerbungsphase sichtbar. Erwähnenswert ist dabei, dass sich Unterschiede beim erwartbaren Einkommen und Berufsprestige, zu dem Studienfächer im Durchschnitt führen, nur bei der präferierten Bewerbung zeigen, nicht aber, wenn alle Bewerbungen einbezogen werden. Dies stützt die Annahmen der EMI-These (Lucas 2001), mit der angenommen wurde, dass sozial privilegierte Schüler/innen versuchen, soziale Reproduktion nicht nur durch ein höheres Bildungsniveau, sondern auch durch ertragreiche Optionen innerhalb einer Bildungsstufe zu erreichen. 
Mit Phase 3 wurde schließlich untersucht, inwiefern die individuelle Entscheidung von Studienaspirant/innen durch fremdselektive Prozesse eingeschränkt wird. Zunächst konnte konstatiert werden, dass der überwiegende Anteil an Bewerber/innen - insgesamt etwa $80 \%$ - bereits nach der ersten Bewerbungsphase eine Zulassung zum Studium erhielt. Andersherum bedeutet dies aber auch, dass $20 \%$ der bereits hochgradig selektiven Gruppe der Studienbewerber/innen keine Zusage für einen Studienplatz erhielt (und scheinbar nicht dazu bereit ist, sich in eine der wirklich zulassungsfreien Optionen einzuschreiben). Herkunftsunterschiede zeigen sich dabei kaum, und wenn, dann tendenziell eher in Richtung größere Zulassungschancen für Nichtakademikerkinder. Diese Tendenz könnte darin begründet liegen, dass sie sich im Durchschnitt fachlich etwas breiter bewerben, was wiederum - entsprechend der starken fachlichen Variation in den Zugangsbarrieren - die Zulassungschancen insgesamt erhöht.

Dabei zeichnet sich ab, dass sich einige erfolglose Bewerber/innen ein Jahr später nicht erneut bewerben. Für die einen könnte dies ein Abwenden vom Studium, für die anderen eher einen Aufschub bedeuten, durch den die ursprünglichen Präferenzen eher verwirklicht werden können. Die Fallzahlen, auf denen diese differenzierten Annahmen beruhen, sind insgesamt sehr klein und die gelieferte Interpretationen daher nur als Hinweise und Tendenzen zu bewerten.

Betrachtet man in der letzten Phase den Übergang ins Studium unkonditional, d.h. für die gesamte Gruppe der Studienaspirant/innen unabhängig von Bewerbungen und Zulassungen, zeigen sich kaum Herkunftsdifferenzen. Studienberechtigte unterschiedlicher sozialer Herkunft scheinen also ihre Studienaspirationen bis ein Jahr nach dem Abitur in gleichem Maße umsetzen zu können. Es handelt sich hierbei um einen Anteil von insgesamt etwa 70\%. Im Umkehrschluss bedeutet dies, dass etwa 30\% der Studienaspirant/innen ihren Wunsch bis ein Jahr nach Erhalt der Hochschulzugangsberechtigung nicht verwirklichen konnten. Der Befund geringer Herkunftsdifferenzen bei der Studienaufnahme trotz deutlich unterschiedlicher Bewerbungswahrscheinlichkeit (bei ähnlichen Zulassungschancen) ist auch darauf zurückzuführen, dass unter den zugelassenen Studienbewerber/innen, Nichtakademikerkinder ein Studium etwas häufiger aufnehmen, während insbesondere einige Doppelakademikerkinder möglicherweise (vorerst) auf ein Studium verzichten, sofern sich dieses nicht mit ihren Präferenzen deckt.

Mit Blick auf den letztlichen Studienort zeigt sich auch in der vierten Phase die starke regionale Verbundenheit. Insgesamt studieren nur 15\% der betrachteten Berliner Studienaspirant/innen außerhalb Berlins oder Brandenburgs. Die Herkunftsunterschiede sind dabei deutlich geringer als in Phase 2. Dies verweist erneut darauf, dass sich die Präferenzen für nahegelegenen Studienoptionen zwischen Studienberechtigten unterschiedlicher sozialer Herkunft kaum unterscheiden - ein Befund, der sich auch in den Ergebnissen des 
Faktoriellen Surveys in Phase 1 wiederfindet und der die Befunde anderer Studien stützt, die für Deutschland keinen oder einen eher geringen sozialgruppenspezifischen Einfluss der Distanz auf die Studienaufnahme feststellen konnten (Helbig et al. 2017, Spieß und Wrohlich 2010). In diesem Kapitel konnte aber anhand des Bewerbungsverhaltens ergänzend gezeigt werden, dass sozial schwächere Studienaspirant/innen generell weniger mobilitätsbereit sind, was sich in ihrem insgesamt engeren Bewerbungsradius widerspiegelt. Sie sind also stärker regional eingeschränkt und müssen damit möglicherweise bezüglich anderer Punkte größere Abstriche machen. Der alleinige Fokus auf bereits realisierte Übergänge würde diesen Befund verbergen.

Ähnliches lässt sich bezüglich des letztlichen Übergangs in unterschiedlich prestigereiche Studienoptionen feststellen: Hier sind die Unterschiede zwischen den Herkunftsgruppen im Vergleich zur präferierten Bewerbung zum Teil deutlich reduziert.

Insgesamt geben die Befunde an verschiedenen Stellen Hinweise darauf, dass sozial privilegierte Studienberechtigte (hier: Doppelakademikerkinder) bezüglich ihrer Studienwahl fachlich stärker festgelegt und wählerischer sind: ${ }^{202} \mathrm{Ihr}$ zu Beginn des letzten Schuljahres geäußertes Wunschfach entspricht häufiger ihrer präferierten Bewerbung. Sie bewerben sich seltener für mehrere Fächer. Sie gehen trotz Zusage etwas seltener ins Studium über. Wenn sie übergehen, dann geben sie häufiger an, dass der Studiengang, den sie studieren, ihren Präferenzen entspricht. Zudem deuten die Ergebnisse darauf hin, dass diejenigen Doppelakademikerinder mit Aspirationen für sehr prestigereiche Studienoptionen, die oft auch überdurchschnittlich selektiv sind, bis ein Jahr nach dem Abitur seltener ins Studium übergehen. $\mathrm{Zu}$ vermuten ist, dass sie ihren längeren Zeithorizont nutzen, um ihre Zulassungschancen in ihrem präferierten Fach zu erhöhen. Soziale Ungleichheit bei der Umsetzung von Studienaspirationen könnte damit eher verspätet auftreten (vgl. Weiss und Steiniger 2013). Diese würde sich dann aber nicht allein in sozialgruppenspezifischen Übergangsraten zeigen, sondern auch in unterschiedlichen Chancen, die eigenen (fachlichen) Präferenzen umzusetzen. Doppelakademikerkinder könnten auf diese Weise einen besseren „Match“ oder eine bessere „Passung“ erzielen, somit möglicherweise zufriedener mit dem Studium sein und dieses seltener abbrechen.

Insgesamt haben die Befunde verdeutlicht, dass der alleinige Blick auf die finalen unkonditionalen Modelle wichtige Erkenntnisse zu den zwischenliegenden Selektionsprozessen verbergen würde. Dies betrifft sowohl die Befunde zur „Studienentscheidung“ an sich als auch die „Wahl“ unterschiedlicher Studienoptionen.

202 Da dieser Aspekt nicht im Fokus der Arbeit steht, sind die zusätzlichen Analysen meist explorativ und deskriptiv, sodass die Befunde als erste Hinweise gedeutet werden sollten. Da diese Hinweise jedoch an verschiedenen Stellen zu finden sind, addieren sie sich zu dem beschriebenen Muster auf. 
Tab. 8.21: Ergebnisübersicht der Phasen 2-4

\begin{tabular}{|c|c|c|}
\hline Hypothesen & Ergebnis & \\
\hline \multicolumn{3}{|c|}{ Phase 2: Bewerbung } \\
\hline $\mathrm{HA} / \mathrm{B}$ & Uni0 (und Uni1) bewerben sich seltener für ein Studium als Uni2 & + \\
\hline HB1/B & Keine Reduktion der Herkunftsunterschiede durch Leistungsindikatoren & - \\
\hline $\mathrm{HB} 2 / \mathrm{B}$ & Keine Reduktion der Herkunftsunterschiede durch RC-Indikatoren & - \\
\hline HB3/B & $\begin{array}{l}\text { Keine Reduktion der Herkunftsunterschiede durch soziale und kulturelle } \\
\text { Ressourcen }\end{array}$ & - \\
\hline $\mathrm{HC} 1 / \mathrm{B}$ & $\begin{array}{l}\text { Uni0 bewerben sich häufiger (ausschließlich) in Berlin oder } \\
\text { Berlin/Brandenburg und haben einen geringeren Bewerbungsradius als } \\
\text { Uni1/2 (insbesondere Uni1) }\end{array}$ & + \\
\hline $\mathrm{HC} 2 / \mathrm{B}$ & $\begin{array}{l}\text { Durchschittliche Selektivität (nicht jedoch Zulassungsbeschränkung) } \\
\text { des angestrebten Faches senkt Bewerbungswahrscheinlichkeit; keine } \\
\text { Interaktion mit der sozialen Herkunft }\end{array}$ & - \\
\hline $\mathrm{HC} 3 / \mathrm{B}$ & $\begin{array}{l}\text { Keine konsistenten Zusammenhänge zwischen den Zugangshürden im } \\
\text { angestrebten Fach und Übereinstimmung zwischen Fachaspiration und } \\
\text { Bewerbung }\end{array}$ & - \\
\hline HD1/B & $\begin{array}{l}\text { Tendenziell bewerben sich Uni0 seltener an Universitäten und häufiger } \\
\text { ausschließlich an FHs als Uni } 1 / 2\end{array}$ & + \\
\hline \multirow[t]{2}{*}{$\mathrm{HD} 2 / \mathrm{B}$} & $\begin{array}{l}\text { Präferierte Bewerbung: Fach führt im Durchschnitt zu höheren } \\
\text { Einkommen bei Uni2 als bei Uni0 (und Uni1) }\end{array}$ & $(-)$ \\
\hline & Alle Bewerbungen: keine Herkunftsunterschiede & \\
\hline \multirow[t]{2}{*}{ HD3/B } & $\begin{array}{l}\text { Präferierte Bewerbung: Fach führt im Durchschnitt zu höherem } \\
\text { Berufsprestige bei Uni2 als bei Uni0 }\end{array}$ & $(+)$ \\
\hline & Alle Bewerbungen: keine Herkunftsunterschiede & \\
\hline
\end{tabular}

Phase 3: Zulassung

\begin{tabular}{lll}
\hline HA/Za & Keine Herkunftsunterschiede in der Zulassungswahrscheinlichkeit & + \\
HA/Zb & $\begin{array}{l}\text { Da keine Herkunftsunterschiede, auch keine Reduktion durch } \\
\text { Leistungsindikatoren (primäre Effekte) }\end{array}$ & - \\
\hline HB1/Z & & - \\
\hline
\end{tabular}

Phase 4: Studienaufnahme

$\mathrm{HA} / \mathrm{Sa} \quad$ Uni0 beginnen nach einer Zulassung etwas häufiger mit dem Studium als Uni2

$\mathrm{HA} / \mathrm{Sb} \quad$ Keine Herkunftsunterschiede in der (unkonditionalen) Studienaufnahme

HC1/S Uni0 studieren häufiger nah am Heimatort als Uni1. Keine Unterschiede zu Uni2

HD1/S Tendenziell studieren Uni2 häufiger an Universitäten als Uni0 (aber unsichere Schätzung)

HD2/S Keine Herkunftsunterschiede bezüglich des Einkommens, zu dem das Studienfach im Durchschnitt führt.

HD3/S Keine (hypothesenkonformen) Herkunftsunterschiede bezüglich des Berufsprestiges, zu dem das Studienfach im Durchschnitt führt. 


\section{Zusammenfassung und Diskussion}

Ausgangspunkt dieser Arbeit ist das für Deutschland immer wieder konstatierte doppelköpfige Problem geringer Studierendenquoten bei gleichzeitig deutlich ausgeprägter sozialer Ungleichheit beim Übergang ins Studium. Gerade in modernen „Bildungs-“ oder „Wissensgesellschaften“ (Mayer 2004, OECD 2008), in denen hohe Bildung und ein hohes Bildungsniveau zentral für individuelles und gesellschaftliches Vorankommen ist, und berufliche Positionen stark an Bildungszertifikate geknüpft sind, werden so Lebenschancen sozial ungleich verteilt.

Für den deutschen Kontext wurden in bildungssoziologischen Beiträgen zwei zentrale Gründe für die ausgeprägten sozialen Herkunftsunterschiede beim Übergang ins Hochschulstudium identifiziert (vgl. Mayer et al. 2007, Müller und Pollak 2008, Powell und Solga 2011): Erstens führt das stark stratifizierte deutsche Schulsystem mit seinen frühen und folgenreichen Entscheidungsschwellen dazu, dass viele Schüler/innen aus sozial schwächeren Familien bereits früh den Hochschulpfad verlassen und die formale Hochschulzugangsberechtigung somit nicht erhalten. Hiermit verbunden bietet zweitens das deutsche Berufsausbildungssystem eine attraktive Alternative zum Hochschulstudium an und trägt so dazu bei, dass einige Schüler/innen - wiederum insbesondere aus sozial schwächeren Familien - die Hochschulzugangsberechtigung nicht mit dem vorrangigen Ziel eines Hochschulstudiums erlangen. Gut erforscht ist damit soziale Ungleichheit an den Hauptgelenkstellen im Bildungssystem. Kaum Erkenntnisse liegen jedoch zu denjenigen Schüler/innen vor, die die Schule mit Hochschulzugangsberechtigung und Studienwunsch verlassen. Setzen selbst in dieser positiv selektierten Gruppe nicht alle ihre Studienaspiration um und sind selbst hier noch soziale Herkunftsunterschiede zu beobachten? Diese Fragen erweitern den Blick für weitere, insbesondere vom Hochschulsystem ausgehende Barrieren, die im Zusammenspiel mit individuellen Ressourcen den Übergangsprozess beeinflussen können. Hier setzt die vorliegende Arbeit an.

Studienberechtige mit Studienaspiration haben wesentliche Hürden auf dem akademischen Pfad bereits genommen und haben nur noch ,einen kurzen Weg“ vor sich. Was auf diesem kurzen Weg passiert, ist für den deutschen Kontext bisher weitestgehend unbekannt. Daher ergänze ich konzeptionell und empirisch mit dieser Arbeit die (deutsche) Forschung zu sozialer Ungleichheit im Hochschulsystem durch eine detailliertere Betrachtung des Übergangsprozesses ins Studium. Die mittlerweile durchaus zahlreichen Studien zum deutschen Kontext untersuchen entweder Studienintentionen oder bereits realisierte Übergänge und können damit nur Teilaspekte des Übergangs abbilden: Während Intentionen vor allem die individuellen Entscheidungen von Studien- 
berechtigten widerspiegeln, die zwar häufig, aber nicht in jedem Fall umgesetzt werden (können) (vgl. Helbig et al. 2015, Jacob et al. 2013, Weiss und Steiniger 2013), birgt die Untersuchung realisierter Übergänge das Problem, dass individuelle und institutionelle Entscheidungsprozesse (Selbstselektion und Fremdselektion) konfundiert sind (Boliver 2013). Die Untersuchung verschiedener Phasen des Übergangsprozesses - die Übersetzung von Studienaspirationen in -intentionen, Bewerbungen, Zulassungen und die finale Studienaufnahme - ermöglicht es erstmalig für den deutschen Kontext zwischen diesen Selektionsarten auch empirisch zu unterscheiden.

Auf Grundlage verfügbarer Datensätze war eine solch detaillierte Betrachtung bisher kaum möglich. Die im Rahmen des Best Up-Projekts erhobenen Längsschnittdaten von Berliner Studienberechtigten füllen diese Datenlücke. Durch detaillierte Abfragen zu Bewerbungen und Zulassungen und die Verbindung dieser Individualdaten mit den gesammelten institutionellen Daten zu Auswahlverfahren an deutschen Hochschulen ist es erstmals möglich, sowohl die indirekte als auch direkte Rolle von Fremdselektionsprozessen empirisch zu prüfen. Zudem wird mit einem Faktoriellen Survey ein quasi-experimentelles Verfahren angewandt, um den (sozialgruppenspezifischen) Einfluss institutioneller Faktoren zu untersuchen. Da hier bestimmte Bewerbungssituationen vorgegeben werden, ist es möglich, den potentiellen Einfluss von Merkmalen, die zwar in der „Realität“ (noch) nicht häufig vorkommen oder (noch) nicht stark institutionalisiert sind (etwa institutionelles Prestige oder komplexe Auswahlverfahren), die aber seit längerem Teil der bildungspolitischen Debatte sind, zu untersuchen. Trotz der Fülle der Best Up-Daten muss einschränkend betont werden, dass diese nicht repräsentativ für deutsche oder Berliner Studienberechtigte sind und dass die Fallzahlen zum Teil an die Grenzen des analytisch Machbaren stoßen. Daher ist der eigentliche Beitrag der Arbeit theoretischer und konzeptionell empirischer Natur; die Auswertungen und Ergebnisse haben - aus genannten Gründen - eher explorativen Charakter.

Im Folgenden werden die theoretischen Argumentationen und zentralen empirischen Befunde dieser Arbeit zusammengefasst, Grenzen aufgezeigt, weiterer Forschungsbedarf identifiziert und zuletzt mögliche politische und gesellschaftliche Implikationen diskutiert.

\section{Theoretische Argumentationslinie}

„Bildungsaspiration“ ist ein schillernder Begriff, auf den in vielen bildungssoziologischen Beiträgen zum Teil auf sehr unterschiedliche Art zurückgegriffen wird (vgl. Haller 1968, Morgan 2007, Stocké et al. 2011). Bildungsaspirationen als zentraler Ausgangspunkt dieser Arbeit werden - in Abgrenzung zu dem ebenfalls häufig genutzten Konzept der Intentionen - als Bildungswünsche definiert, denen ein idealistisches Element innewohnt und die weniger stark von wahrgenommenen individuellen und institutionellen Barrieren beeinflusst 
sind. Welchen Stellenwert nehmen diese in der soziologischen Ungleichheitstheorie ein? Sowohl aus Pierre Bourdieus Reproduktionstheorie (z.B. 1982, 1973, Bourdieu und Passeron 1971), dem sozialpsychologischen status attainment-Modell (z.B. Sewell et al. 1969, Sewell und Hauser 1972, Sewell und Shah 1967) als auch soziologischen RC-Ansätzen (z.B. Boudon 1974, Breen und Goldthorpe 1997, Erikson und Jonsson 1996) kann zunächst die Rolle von Aspirationen als wichtiger Mediator sozialer Ungleichheit im Bildungssystem abgeleitet werden. Obwohl diese Ansätze von deutlich sozialstrukturell beeinflussten Aspirationen als Regelfall ausgehen, schließen sie nicht aus, dass auch sozial schwächere Schüler/innen Studienaspirationen ausbilden. Dies ist vor allem dann wahrscheinlich, wenn förderliche Faktoren wirken (z.B. gute Schulleistung oder Unterstützung und Zuspruch aus dem sozialen Netzwerk). Bei sozial schwächeren Schüler/innen mit hohen Bildungsaspirationen sollte es sich demnach um eine weiter positiv selektierte Gruppe handeln.

Weiter wurde argumentiert, dass selbst innerhalb der Gruppe von Studienaspirant/innen noch soziale Herkunftsunterschiede bezüglich der Umsetzungschancen von Studienaspirationen bestehen können.

Aspirationen werden als wichtige Motivationsquelle, als Teil der Agency individueller Akteure verstanden (vgl. Azjen 1991, Schoon und Lyons-Amos 2016). Doch gleichzeitig treffen diese Akteure auf Strukturen und sind mit unterschiedlichen - sozialgruppenspezifischen - Ressourcen ausgestattet, die Handlungen und Handlungsergebnisse sowohl einschränken als auch ermöglichen können (Goldthorpe 1996a, Sewell 1992).

Um sozialgruppenspezifische Umsetzungschancen von Studienaspirationen theoretisch einzubetten, wurde auf die in der Bildungssoziologie etablierte Unterscheidung zwischen primären und sekundären Herkunftseffekten zurückgegriffen (vgl. Boudon 1974). Primäre Effekte (also Unterschiede in den kognitiven und non-kognitiven Fähigkeiten, Schulleistungen und - weiter gefasst - dem Qualifikationsprofil) - so wurde argumentiert - werden insbesondere im Zusammenspiel mit Auswahlkriterien und -verfahren relevant für den Übergang ins Studium, wenn bestimmte soziale Gruppen diese eher nachweisen oder sich aneignen können (sei es durch eine größere kulturelle Nähe zum Schulsystem, Nachhilfe, Sprachreisen, extracurriculare Aktivitäten oder gezielte Testvorbereitung) (vgl. Alon 2009, Bourdieu und Passeron 1971, Buchman et al. 2010, Thomsen 2018). Aus konflikttheoretischer Perspektive kann dabei argumentiert werden, dass Gatekeepingprozesse von Seiten der Hochschulen - selbst wenn sie auf meritokratischen Kriterien basieren und somit als neutral und legitim wahrgenommen werden - zur Schließung der Hochschulen gegenüber bestimmten Statusgruppen beitragen können (vgl. Karabel 1984, 2005).

Vermeintliche Zugangshürden sowie die weitere hochschulische Opportunitätsstruktur können zudem auf Bildungsentscheidungen zurückwirken und sich somit in sekundären Effekten manifestieren. Optionen und Hürden, so die 
Argumentation, werden antizipiert und im Lichte der eigenen Ressourcen (und Präferenzen) bewertet. Dies kann aufgrund der unterschiedlichen Ausstattung an ökonomischen, vor allem aber sozialen und kulturellen Ressourcen (etwa in Form von Systemkenntnis, Unterstützung und Erwartungen aus dem sozialen Umfeld) zu sozialgruppenspezifischen Entscheidungen für oder gegen ein Studium beitragen (vgl. Erikson und Jonsson 1996). Fragen, die sich dabei stellen, sind, wie korrekt und vollständig institutionelle Barrieren und Opportunitäten - in Anbetracht vorhandener privater und institutionell zur Verfügung gestellter Informationen - überhaupt antizipiert werden können, und welche Rolle die Antizipation von Hürden für Schüler/innen mit Studienaspiration spielt, deren Aspiration stark internalisiert und damit handlungsleitend ist (vgl. Esser 2001, Kroneberg 2005, Kroneberg et al. 2009).

Aus ungleichheitstheoretischer Sicht ist allerdings nicht nur die Entscheidung für oder gegen ein Studium relevant, sondern auch soziale Selektionsprozesse innerhalb des Hochschulsystems (vgl. Bourdieu 1982, Karabel und Astin 1975, Lucas 2001). Die verschiedenen vertikal und horizontal differenzierten Studienoptionen - im deutschen Kontext vor allem der Hochschultyp sowie das Studienfach - zeichnen sich durch verschiedene Merkmale aus, die auf Studienberechtigte unterschiedlicher sozialer Herkunft mehr oder weniger abschreckend oder anziehend wirken können. Sind diese Studienoptionen mit unterschiedlichen Erträgen verbunden, wie im deutschen Fall vor allem mit Blick auf das Studienfach beobachtet werden kann, setzt sich soziale Reproduktion potentiell selbst dann fort, wenn der letzte Schritt im Übergangsprozess genommen ist.

\section{Fremdselektion im deutschen Kontext}

Bevor die Ergebnisse zu sozialer Ungleichheit in den selbstselektiven und fremdselektiven Phasen der Aspirationsumsetzung zusammenfassend beschrieben werden, soll hier kurz erörtert werden, inwiefern im deutschen Kontext überhaupt davon ausgegangen werden kann, dass Gatekeepingprozesse eine Rolle spielen.

In vielen Studien zu Deutschland wird mehr oder weniger explizit angenommen, dass das deutsche Zulassungssystem keine große Hürde für den Übergang ins Studium an sich darstellt, da auch leistungsschwächere Studienberechtigte ausreichend Wahlmöglichkeiten zwischen zulassungsfreien Studiengängen haben (vgl. Gehlke et al. 2019b, Schindler 2012). Jedoch wurde die Rolle der Hochschulen als Gatekeeper in den letzten Jahrzehnten zunehmend institutionalisiert, etwa durch die Novelle des Hochschulrahmengesetzes im Jahre 2004, mit der die Autonomie der Hochschulen bei der Bewerber/innenauswahl gesteigert wurde. Über formale Zulassungsbeschränkungen hinaus liegen aber kaum aktuelle Informationen vor. 
Im Zuge des Best Up-Projekts wurden neben den Individualdaten auch institutionelle Daten zu Zulassungsbeschränkungen und Auswahlkriterien an deutschen Hochschulen erhoben. Da sich die Auswahl der circa 450 Studiengänge nach den Angaben der Befragten richtete, bilden die Rekrutierungsdaten die Berliner Hochschullandschaft besser ab als die bundesweite, sind aber weder für Berlin noch für Deutschland repräsentativ. Sie stellen jedoch die einzige neuere Datenquelle dar, mit deren Hilfe nicht nur aktuelle Zugangsbeschränkungen, sondern darüber hinaus auch die (Leistungs-)Selektivität von Studiengängen sowie konkrete Auswahlkriterien und -verfahren erfasst werden können. Die Auswertung der Daten zeigt, dass im WiSe 2014/15 ein geringer Anteil der recherchierten Studiengängen (circa 15\%) im engeren Sinne zulassungsfrei war, da neben der kapazitätsrechtlichen Zulassungsbeschränkung auch Eignungsfeststellungsverfahren eine zunehmend wichtige Rolle bei der Bewerber/innenauswahl zu spielen scheinen (vgl. auch Winter et al. 2012). Zur Anwendung kommen dabei allerdings neben der Abiturnote, deren zentraler Stellenwert rechtlich verankert ist, vorwiegend bereits vor der Bewerbung erlangte leicht nachzuprüfende und standardisiert erfassbare Kriterien (z.B. Berufserfahrung oder Sprachkenntnisse). Weitere Verfahren - wie Tests oder Auswahlgespräche - werden zwar auch genutzt, jedoch nur in der Minderheit der Fälle.

Insgesamt kann basierend auf diesen Befunden festgehalten werden, dass Fremdselektionsprozesse auch im deutschen Kontext durchaus relevant sein können. Abhängig sein sollte dies aber insbesondere auch von den fachlichen Präferenzen, da Zugangshürden stark mit dem Studienfach variieren (vgl. Gehlke et al. 2019a).

Soziale Ungleichheit bei der Umsetzung von Studienaspirationen: Selbstselektion oder Fremdselektion?

Im Folgenden werden die Ergebnisse zur zentralen Fragestellung dieser Arbeit zusammengefasst: Können selbst innerhalb der Gruppe von Studienberechtigten mit Studienaspiration soziale Herkunftsunterschiede beim Übergang ins Studium beobachtet werden? Und sind hierfür sowohl individuelle als auch institutionelle Entscheidungsprozesse relevant?

Betrachtet man das letztliche Ergebnis - den Übergang ins Studium - kann zunächst konstatiert werden, dass bis ein Jahr nach dem Abitur bei weitem nicht alle (genauer gesagt etwa 70\%) der betrachteten Berliner Studienberechtigten ihre Studienaspiration umsetzen. Ein „Verlust“ kann dabei in allen Phasen festgestellt werden: Etwa 90\% der Schüler/innen mit Studienaspiration haben zu Beginn des letzten Schuljahres auch eine Studienintention. Von den im nächsten Schritt untersuchten Studienberechtigten bewerben sich über $80 \%$ bis ein Jahr nach dem Abitur. Über 80\% der Bewerber/innen werden zugelassen und über 90\% der Zugelassenen nehmen ein Studium auf. 
Betrachtet man den Übergang ins Studium (bis ein Jahr nach Erhalt der Hochschulzugangsberechtigung) unkonditional, kann festgehalten werden, dass hierbei zunächst keine Herkunftsunterschiede verzeichnet werden können. Kommt soziale Ungleichheit also weder durch Selbstselektion noch durch Fremdselektion zustande? Die detaillierte Betrachtung der verschiedenen Phasen kann hierzu Aufschluss geben: Es zeigt sich, dass es vor allem die frühen selbstselektiven Phasen sind, in denen soziale Herkunftsunterschiede verzeichnet werden können. So übersetzen sozial schwächere Schüler/innen ihre Studienaspiration seltener in eine Studienintention und bewerben sich nach Erhalt der Hochschulzugangsberechtigung seltener für ein Studium. Diese Herkunftsunterschiede zeigen sich vor allem (oder ausschließlich) beim Vergleich der beiden betrachteten Extremgruppen: Schüler/innen mit zwei vs. keinem akademisch gebildeten Elternteil.

Haben sie sich beworben, bestehen allerdings keine Unterschiede bezüglich der Zulassungschancen - direkte Fremdselektion scheint also (im Durchschnitt) eine untergeordnete Rolle zu spielen. Fokussiert man in der letzten Phase nur auf Studienaspirant/innen mit einer Zulassung zum Studium, zeigt sich zudem, dass Nichtakademikerkinder sogar etwas häufiger ins Studium übergehen.

Damit reihen sich die Ergebnisse dieser Arbeit zum deutschen Kontext in den internationalen (vor allem anglo-amerikanischen) Forschungsstand ein, nach dem es vor allem Selbstselektionsprozesse sind, die zu sozialer Ungleichheit im Hochschulsystem beitragen (vgl. Anders 2012, Boliver 2013, Hoxby und Avery 2013).

\section{Individuelle Entscheidungsfaktoren}

Für die Übersetzung von Studienaspirationen in Studienintentionen sowie Studienbewerbungen wurde untersucht, inwiefern die hierbei auftretenden Herkunftsunterschiede durch Unterschiede in den Schulleistungen, den wahrgenommenen Kosten, Nutzen und der Erfolgswahrscheinlichkeit sowie in den sozialen und kulturellen Ressourcen begründet liegen. Zwar zeigen einige der Indikatoren in die angenommene Richtung (vor allem die wahrgenommene Erfolgswahrscheinlichkeit sowie der Studienwunsch der Eltern haben in beiden Phasen einen positiven Einfluss), doch können sie - trotz der beobachtbaren ungleichen Verteilung über die Herkunftsgruppen - Unterschiede in der Intentions- und Bewerbungswahrscheinlichkeit nicht erklären: Selbst bei gleicher Leistung, gleicher Einschätzung von Kosten, Nutzen und Erfolgswahrscheinlichkeit eines Studiums und gleicher Ausstattung an kulturellen und sozialen Ressourcen sind hier also Herkunftsunterschiede bezüglich der Übersetzung von Studienaspirationen in Studienintentionen und Studienbewerbungen zu verzeichnen. 
Für die Gruppe der Studienaspirant/innen scheinen also die etablierten Mechanismen, für die in anderen auf alle Studienberechtigten bezogenen Studien zumindest teilweise Unterstützung gefunden werden konnte (vgl. Becker und Hecken 2007, Lörz 2012, Maaz 2006, Schindler und Reimer 2010), keine große Erklärungskraft zu besitzen. Dies kann empirische Gründe haben: Wie mehrfach diskutiert sollten die Analysen eher explorativ interpretiert werden und die Ergebnisse - aufgrund fehlender Repräsentativität und kleiner Fallzahlen - nicht überbewertet werden. Die theoretischen Konstrukte sind in dieser bereits stark vorselektierten Gruppe sehr schief verteilt (etwa schätzen nur wenige ihre Erfolgswahrscheinlichkeit als gering ein). Gepaart mit geringen Fallzahlen, insbesondere in der Gruppe der Akademikerkinder, basieren die Analysen zum Teil auf sehr gering bersetzten Zellen. Natürlich kann auch in der vorliegenden Arbeit das Problem unzureichender Operationalisierung der theoretischen Konstrukte (vgl. Becker 2012, Schindler 2014, Watermann et al. 2014) nicht ausgeschlossen werden. Die Ergebnisse können aber durchaus auch theoretisch erklärt werden: Wie in Abschnitt 5.1 diskutiert handelt es sich bei Studienaspirant/innen, insbesondere bei denjenige aus sozial schwächeren Familien, um eine stark positiv selektierte Gruppe. Möglicherweise sind Herkunftsunterschiede bezüglich des Internalisierungsgrades an Aspirationen klein, sodass diese für alle Herkunftsgruppen ähnlich handlungsleitend sind. Im hier betrachteten Sample an Berliner Schüler/innen aus sozial benachteiligten Bezirken kann dies auch an der Gruppe der Akademikerkinder liegen, die möglicherweise weniger stark internalisierte Aspirationen haben als Akademikerkinder aus stärker bildungsbürgerlichen Bezirken.

\section{Institutionelle Barrieren und Opportunitäten}

Weiterhin wurde untersucht, inwiefern die Antizipation institutioneller Barrieren und Opportunitäten die Umsetzung von Studienaspirationen mit beeinflusst. Zum einen wurde argumentiert, dass Studienkosten hierbei eine (sozialgruppenspezifische) Rolle spielen können. Da direkte Kosten in Deutschland, etwa in Form von Studiengebühren, eine untergeordnete Rolle spielen, könnten es vor allem Mobilitätszwänge sein, die sowohl finanzielle also auch soziale Kosten verursachen. Dass diese Annahme ihre Berechtigung besitzt, verdeutlicht die insgesamt sehr geringe regionale Mobilität deutscher (und insbesondere auch Berliner) Studienberechtigter (vgl. Helbig et al. 2017, KMK 2014, Spieß und Wrohlich 2010).

Die Ergebnisse dieser Arbeit bestätigen diesen Befund. In der ersten Phase wurde der Einfluss der Distanz mithilfe des Faktoriellen Surveys erfasst. Während eine große Distanz die Bewerbungsintention von Studienaspirant/innen aller Herkunftsgruppen deutlich senkt, deutet sich an, dass eine mittlere Distanz die Bewerbungsintention von Nichtakademikerkindern im Vergleich zu Doppelakademikerkindern deutlicher reduziert. Ein mit dem Umzug in eine 
weit entfernte Stadt einhergehendes komplettes Loslösen vom sozialen Netzwerk ist für Schüler/innen aller Herkunftsgruppen im Mittel ähnlich unattraktiv. Eine mittlere Distanz, die zumindest ein wöchentliches Pendeln theoretisch erlaubt, scheint hingegen weniger abschreckend auf diejenigen zu wirken, die sich die hierdurch entstehenden Kosten eher leisten können.

In der Bewerbungsphase wurde der Einfluss der Distanz indirekt erfasst, über die Entfernung der Hochschulorte, für die sich Studienaspirant/innen beworben haben. Auch hier zeigt sich die starke regionale Verbundenheit darin, dass ein Großteil der Bewerbungen an Berliner Hochschulen gesendet wurde. Am deutlichsten trifft dies auf Studienaspirant/innen aus Nichtakademikerfamilien zu. Zudem zeigt sich, dass sich Studienaspirant/innen unterschiedlicher sozialer Herkunft bezüglich ihrer Präferenzen (der erstgerankten Bewerbung) ähnlicher sind als bezüglich ihrer Mobilitätsbereitschaft, die hier über den Bewerbungsradius erfasst wurde. Diese scheint bei Akademikerkindern (hier insbesondere bei Einakademikerkindern) größer zu sein (etwa 40\% bewarben sich auch außerhalb von Berlin/Brandenburg; der Wert für Nichtakademikerkinder liegt bei etwas mehr als 20\%). Nichtakademikerkinder sind also insgesamt stärker regional eingeschränkt und müssen damit möglicherweise bezüglich anderer Präferenzen größere Abstriche machen. Der alleinige Fokus auf bereits realisierte Übergänge würde diesen Befund verbergen.

Als zweite institutionelle Hürde wurden Zugangsbarrieren zum Studium betrachtet. Ein zentrales Argument dieser Arbeit ist, dass Fremdselektionsprozesse nicht allein auf direktem Wege Wirkung entfalten können, indem Bewerber/innen abgewiesen werden oder ihnen Zugang gewährt wird, sondern dass von Gatekeepern gesetzte Kriterien auch indirekt wirken, indem sie antizipiert und so in die individuelle Entscheidung einbezogen werden. Die Verbindung der Individualdaten mit den institutionellen Rekrutierungsdaten bot hier eine bisher einzigartige Möglichkeit, die potentiell ungleichheitsgenerierende Wirkung von Zugangshürden zu testen. Da diese in Deutschland stark mit dem Studienfach variieren, wurden aus den Rekrutierungsdaten für verschiedene Fächer Durchschnittswerte zu Zulassungsbeschränkungen, Eignungsfeststellungverfahren und der (Leistungs-)Selektivität generiert.

Ein sozialgruppenspezifischer Einfluss kann vor allem in der ersten Phase beobachtet werden. Hier zeigt sich, dass die durchschnittlichen Zugangsbarrieren im angestrebten Fach die Intentionswahrscheinlichkeit von Akademikerkindern kaum beeinflussen, diese bei Nichtakademikerkindern aber deutlich senken. Dass Akademikerkinder jedoch keineswegs unrealistisch zu sein scheinen, verdeutlicht der Befund, dass sie (ebenso wie Nichtakademikerkinder) sich innerhalb des Hochschulsystems umorientieren, d.h. auf ein anderes Fach ausweichen, wenn das Fach, das sie anstreben, sehr selektiv ist.

Weniger eindeutig sind die Befunde in der Bewerbungsphase. Der Einfluss von Zugangshürden in dem Fach, das zu Beginn des letzten Schuljahres als Wunschfach genannt wurde, zeigt sich hier insbesondere mit Blick auf die 
durchschnittliche Selektivität von Studienfächern, die deutlich mit der Bewerbungswahrscheinlichkeit assoziiert ist und damit offenbar eine Hürde im Umsetzungsprozess darstellt. Dies ist jedoch tendenziell in allen Herkunftsgruppen der Fall. $\mathrm{Zu}$ einer Umorientierung innerhalb des Hochschulsystems, d.h. zu einer Diskrepanz zwischen der Fachaspiration zu Beginn des letzten Schuljahres und einer entsprechenden Bewerbung, tragen Zugangshürden jedoch kaum bei. Ein Grund für die insgesamt ambivalenteren Befunde in der zweiten Phase kann in Umorientierungsprozessen im Laufe des letzten Schuljahrs gesucht werden. Auswertungen des DZHW Studienberechtigtenpanels zeigen, dass mehr als die Hälfte der befragten Schüler/innen erst im Laufe des letzten Schuljahres mit der Informationssuche zu Studien- und Ausbildungsalternativen beginnen (Heine et al. 2010). Somit ist der Befund dieser Arbeit, dass sich ein beachtlicher Anteil von fast $40 \%$ der Studienaspirant/innen innerhalb des letzten Schuljahres fachlich neu zu orientieren scheint, wenig erstaunlich. Insgesamt zeigt sich jedoch in der Tendenz auch, dass die fachlichen Präferenzen von Akademikerkindern, insbesondere Doppelakademikerkindern, bereits gefestigter zu sein scheinen als die von Nichtakademikerkindern. Erstere richten ihre präferierte Bewerbung häufiger an Studiengänge in Fächern, die sie bereits zu Beginn des letzten Schuljahres studieren wollten.

Eine (quantitative) Untersuchung des Einflusses weiterer Zugangsbarrieren stößt im deutschen Kontext schnell an ihre Grenzen. Zwar kommen komplexere Auswahlverfahren wie Auswahlgespräche und Tests durchaus zum Einsatz, doch sind sie nicht weit verbreitet, sodass ihr spezifischer Einfluss mithilfe der Best Up-Rekrutierungsdaten nicht erfasst werden kann. Mithilfe des Faktoriellen Surveys kann jedoch zumindest für die erste Phase der potentielle Einfluss von Merkmalen getestet werden, die in der „Realität" nicht häufig vorkommen (vgl. Nisic und Auspurg 2009). Es zeigt sich, dass „komplexe“ Auswahlverfahren (Motivationsschreiben, Auswahlgespräche und Tests) im Vergleich etwa zur Distanz einen insgesamt eher geringen Einfluss ausüben. In der Tendenz scheinen sie allerdings eher als „Attraktionsmerkmal“ denn als Abschreckung zu fungieren, weitestgehend unabhängig von der sozialen Herkunft.

Ähnliches kann für die institutionelle Reputation berichtet werden. Auch hier ist eine empirische Untersuchung aufgrund der noch geringen Institutionalisierung von Reputationsunterschieden in Deutschland bisher nur eingeschränkt möglich. Für die vorliegende Studie käme erschwerend hinzu, dass einige Berliner Hochschulen als exzellent gelabelt wurden und somit gerade für Berliner Studienberechtigte hier verschiedene institutionelle Merkmale konfundiert sind. Zu vermuten wäre, dass der Standort Berlin hier ausschlaggebender als der Exzellenzstatus ist. Auch in diesem Fall konnte mithilfe des Faktoriellen Surveys geprüft werden, ob die Reputation von Hochschulen, wie angenommen, tatsächlich positiver auf die Bewerbungsintention von sozial Privilegierten wirkt, die über solche Distinktionsmarker möglicherweise ver- 
suchen, den Erhalt ihres sozialen Status sicherzustellen. Es zeigt sich jedoch, dass die Reputation der Hochschule alle Herkunftsgruppen in etwa gleichem Maße anzieht und auf sozial schwächere Schüler/innen nicht - wie in anderen Kontexten mit einer stärkeren „Elitekultur“ gezeigt (vgl. Ball et all. 2002, Evans 2009, Reay et al. 2001a/b) - abschreckend wirkt.

Letztlich wurde ebenfalls mithilfe des Faktoriellen Surveys der Einfluss von institutionell bereitgestellten Informationen auf die Bewerbungswahrscheinlichkeit getestet. Diese haben nur einen geringen positiven Einfluss auf die Bewerbungsintention von Nichtakademikerkindern, obwohl diese fehlende Informationen weniger gut durch private Kanäle ausgleichen können. Dies legt die Interpretation nahe, dass sie sich eines potentiellen Informationsdefizits nicht bewusst sind und daher bereitgestellten Informationen keine sehr große Wertschätzung entgegenbringen (vgl. Morgan 2010). Im Gegensatz dazu zeigt sich, dass private Informationen in Form von Hilfestellungen durch Bekannte mit Hochschul- und Bewerbungserfahrung die Bewerbungsintention von Akademikerkindern, insbesondere von Doppelakademikerkindern deutlich stärker positiv beeinflussen. Dies weist darauf hin, dass Schüler/innen aus sozial privilegierteren Familien wissen, wie wichtig die ,richtigen“ Informationen, die in ihrem privaten Netzwerk zur Verfügung stehen, sein können, um den Bewerbungsprozess zu erleichtern (vgl. Slack et al. 2012). Sozial schwächere Schüler/innen hingegen scheinen zu antizipieren, dass die Informationen, die sie von „Bekannten“ erhalten, ihnen im Hochschulkontext nur bedingt weiterhelfen können (vgl. Heine et al. 2010). In Kombination mit möglicherweise wenig genutzten institutionellen Informationen könnte dies letztlich einen doppelten Nachteil darstellen.

\section{Horizontal und vertikal differenzierte Studienoptionen}

Zuletzt wurde der Frage nachgegangen, ob sich Studienaspirant/innen unterschiedlicher sozialer Herkunft darin unterscheiden, welche Optionen sie innerhalb des Hochschulsystems, „wählen“. Als vertikale Dimension wurde für das grundsätzlich binäre deutsche Hochschulsystem der Hochschultyp - Universität vs. Fachhochschule - untersucht. Aus ungleichheitstheoretischer Sicht relevanter noch ist jedoch die horizontale Dimension der Studienfächer, da diese spätere Arbeitsmarktchancen stärker strukturieren und somit in ihrer Konsequenz zu vertikalen Reproduktionsprozessen beitragen. Studienfächer wurden dabei nicht wie in anderen Studien (z.B. Becker et al. 2010, Lörz 2012, Reimer und Pollak 2010) kategorial unterteilt, sondern anhand ihres Ertrages bemessen. Der Untersuchung lag dabei die Annahme zugrunde, dass sich zwar soziale Herkunftsunterschiede bezüglich des Berufsprestiges zeigen, zu dem Studienfächer führen, nicht aber mit Blick auf den erwartbaren Einkommensertrag, da ein hohes Einkommen auch (oder gerade auch) von sozial schwächeren Schüler/innen als wichtig eingeschätzt wird (z.B. Lörz 2012, Obermeier und 
Schneider 2015). Dafür wurden auf Grundlage des Mikrozensus Einkommensund Berufsprestigewerte errechnet, die Absolvent/innen verschiedener Studienfächer im Durchschnitt erzielen. Auch wenn es unwahrscheinlich ist, dass Schüler/innen hierzu umfassend informiert sind, gehe ich mit Erikson und Jonsson (1996) davon aus, dass grundlegende Ideen zu möglichen Erträgen vorhanden sind und Studienfächer so zumindest in eine Rangordnung gebracht werden können.

Bezüglich des Hochschultyps zeigt sich prinzipiell sowohl mit Blick auf Intentionen als auch Bewerbungen der erwartete und bereits zuvor gezeigte (vgl. Blossfeld et al. 2015, Reimer und Pollak 2010) Zusammenhang: Nichtakademikerkinder intendieren häufiger ein $\mathrm{FH}$-Studium und bewerben sich entsprechend auch häufiger für ein solches. Generell zeigt sich dieser Befund auch noch beim Übergang ins Studium, kann aber aufgrund der abnehmenden Fallzahlen nicht mehr präzise geschätzt werden.

Mit Blick auf die „Wahl“ der Studienfächer konnte für Phase 1 und 2 gezeigt werden, dass sich Doppelakademikerkinder hier bezüglich des Berufsprestiges und stärker noch bezüglich des Einkommens im Vergleich zu den anderen beiden Herkunftsgruppen ,absetzen“. In der Bewerbungsphase zeigt sich dieser Unterschied jedoch nur bei Betrachtung der präferierten Bewerbung, nicht aber, wenn alle Bewerbungen einbezogen werden. Es deutet sich also an, dass sozial privilegierte Schüler/innen versuchen, soziale Reproduktion nicht nur durch ein höheres Bildungsniveau, sondern auch durch ertragreiche Optionen innerhalb einer Bildungsstufe zu erreichen. Beim letztlichen Übergang in unterschiedliche Studienfächer bis ein Jahr nach Erhalt der Hochschulzugangsberechtigung sind die Unterschiede zwischen den Herkunftsgruppen im Vergleich zur präferierten Bewerbung jedoch deutlich reduziert. Der Einschub „bis ein Jahr nach Erhalt“" ist dabei durchaus bedeutsam. Denn dieser Befund schließt nicht aus, dass sich größere Herkunftsunterschiede im weiteren Verlauf zeigen werden (vgl. Weiss und Steiniger 2013).

Denn insbesondere Doppelakademikerkinder mit Aspirationen (und Bewerbungen) für prestigereiche Fächer, wie etwa Medizin, scheinen sich bei fehlenden (passenden) Zusagen eher gegen ein Studium oder wahrscheinlicher: für ein Verschieben des Studiums zu entscheiden. Insgesamt finden sich an verschiedenen Stellen der Untersuchung Hinweise dafür, dass sozial privilegierte Studienaspirant/innen stärkere fachliche Präferenzen haben und diese auch gegen Hürden versuchen umzusetzen, auch wenn dies mit einer Verzögerung des Übergangs ins Studium verbunden ist.

Soziale Ungleichheit bei der Umsetzung von Studienaspirationen könnte damit eher verspätet auftreten (vgl. Weiss und Steiniger 2013). Dies würde sich dann aber nicht allein in sozialgruppenspezifischen Übergangsraten zeigen, sondern auch in unterschiedlichen Chancen, die eigenen (fachlichen) Präferenzen umzusetzen. Doppelakademikerkinder könnten auf diese Weise einen 
besseren „Match“ oder eine bessere „Passung“ erzielen, somit möglicherweise zufriedener mit dem Studium sein und dieses seltener abbrechen.

Für sozial schwächere Schüler/innen deutet sich hingegen an, dass sie fachlich flexibler sind. Zum einen zeigt sich dies in den explorativen Befunden zu den „Bewerbungsstrategien“, bei denen sich für Nichtakademikerkinder eine größere fachliche, jedoch geringere regionale Breite feststellen ließ. Sie scheinen also örtliche Präferenzen inhaltlichen Vorlieben vorzuziehen. Inwiefern dies zum Studienerfolg beiträgt, ist fraglich.

Insgesamt haben die Befunde verdeutlicht, dass der alleinige Blick auf die finalen unkonditionalen Modelle wichtige Erkenntnisse zu den zwischenliegenden Selektionsprozessen verbergen würde. Dies betrifft sowohl die Befunde zur „Studienentscheidung“ an sich als auch zur „Wahl“ unterschiedlicher Studienoptionen. Gezeigt hat sich, dass realisierte Übergange Präferenzen nur zum Teil widerspiegeln und somit die voraussetzungsvolle Annahme, dass allein vom Outcome (dem Übergang ins Studium) auf individuelle Präferenzen und Entscheidungen geschlossen werden kann, nicht in allen Fällen gehalten werden kann (vgl. Sen 1986).

Grenzen der empirischen Untersuchung und weiterer Forschungsbedarf

Mit dieser Arbeit wird eine detaillierte und differenzierte Betrachtung des Übergangsprozesses in die Hochschule vorgelegt und so Erkenntnisse zu Selektionsprozessen geliefert, die bei alleiniger Betrachtung von Studienintentionen oder realisierten Übergängen verborgen bleiben. Der Fokus auf Studienberechtige mit Studienaspiration, die nicht bereits im Vorfeld durch frühere Bildungsentscheidungen oder berufsbildende Alternativen vom Hochschulstudium ,abgelenkt“" wurden (vgl. Shavit und Müller 2000), eröffnete den Blick auf weitere institutionelle Barrieren, die den Übergang ins Hochschulstudium beeinflussen können.

Grenzen der empirischen Analysen sind zum einen in den genutzten Daten zu suchen. Zwar war es auf Grundlage der Best Up-Daten erstmals möglich, den Übergang ins Studium auf solch detaillierte Weise zu betrachten, doch ist die Aussagekraft der Ergebnisse durch den spezifischen Kontext, auf den sich die Daten beziehen, eingeschränkt.

Im Berliner Kontext treffen unterschiedliche Faktoren aufeinander: Zwar ist sowohl die interne als auch externe Nachfrage nach Hochschulbildung in der Hauptstadt hoch, doch bietet diese gleichzeitig ein großes und vielfältiges Angebot an Studienoptionen. Zwar führt diese Vielfalt zu einer hohen, nur schwer überschaubaren Komplexität, doch sind viele Hochschulen und somit auch Informationsmöglichkeiten für Berliner Schüler/innen einfach erreichbar. $\mathrm{Zu}$ prüfen wäre, inwiefern die auf Berliner Studienberechtigte bezogenen Befunde auch auf andere Regionen Deutschlands zutreffen. Zum Beispiel wäre $\mathrm{zu}$ fragen, inwiefern und mit welchen Konsequenzen sich regionale (Im)Mo- 
bilität im Zusammenspiel mit Zugangshürden auf die (sozialgruppenspezifische) Fachwahl auswirkt und somit der politisch angestrebten „Passung“ zwischen Studienbewerber/innen und Studiengängen entgegenwirkt (für diesbezügliche Befunde aus der Schweiz vgl. Denzler und Wolter 2010).

Die regionale Einschränkung der Best Up-Daten hat noch eine zweite Dimension, da sich die Schulstichprobe auf sozial benachteiligte Bezirke innerhalb Berlins beschränkte. Zum einen bietet dies den Vorteil, dass der Einfluss schulischer Faktoren - die nicht im Fokus dieser Arbeit standen - so konstant(er) gehalten wird. Zum anderen führt der Ausschluss von ,bildungsbürgerlichen“ Bezirken wahrscheinlich zur Unterschätzung von Herkunftsdifferenzen, da die Varianz existierender Privilegien, die sich auch in der Wahl der Wohn- und Schulgegend zeigen können, hierdurch eingeschränkt wird. Die präsentierten Befunde zu sozialen Herkunftsunterschieden können somit als konservativ interpretiert werden.

Da ein vorrangiges Ziel des Best Up-Projektes darin bestand, die Rolle von Informationsasymmetrien für soziale Ungleichheit beim Übergang ins Studium mithilfe eines Feldexperiments aufzudecken, konnten für die Analysen in dieser Arbeit lediglich die Angaben von Befragten aus den 19 Kontrollschulen genutzt werden. Damit war auch die Fallzahl insbesondere in den späteren, konditionalen Analyseschritten deutlich beschränkt, sodass weiterführende Analysen und auch die statistische Power zur präzisen Schätzung von Effekten teilweise an ihre Grenzen stießen. Erstrebenswert wären differenzierte Angaben zum Übergangsprozess auf Grundlage größerer und möglichst repräsentativer Stichproben. Im NEPS wurden Studienberechtigte der Startkohorte 4 erstmalig auch nach ihrem Bewerbungsverhalten gefragt, sodass künftig zumindest grundsätzliche Aussagen zu diesem wichtigen Schritt im Übergangsprozess für den gesamtdeutschen Kontext getroffen werden können.

Mithilfe der Best Up-Rekrutierungsdaten konnte gezeigt werden, dass der Zugang zum Hochschulstudium beschränkter zu sein scheint, als weithin bekannt ist. So existieren kaum Informationen dazu, dass Eignungsfeststellungsverfahren über bestimmte Studienfächer etwa im künstlerischen Bereich hinaus durchaus verbreitet zu sein scheinen. Damit wird eine Offenheit des deutschen Hochschulsystems suggeriert, die in dieser Form nicht zutrifft. Die Best Up-Rekrutierungsdaten stellen einen ersten Schritt zum besseren Verständnis aktueller Zugangsbeschränkungen an deutschen Hochschulen dar. Doch umfassen sie nur circa 5\% des gesamtdeutschen Studienangebots, das mittlerweile mehr als 10.000 Studiengänge umfasst. Die letzte mir bekannte Bestandsaufnahme zur Anwendung von Auswahl- und Eignungsfeststellungsverfahren wurde kurz nach der 7. Novelle des HRG, mit der den Hochschulen größere Autonomie bei der Bewerber/innenauswahl verliehen wurde, vor über zehn Jahren von Heine et al. (2006) vorgelegt. Eine aktuellere Bestandsaufnahme, die zu einem besseren Verständnis der Rolle der Hochschulen beim Übergang ins Studium beitragen kann, ist damit längst überfällig. 
Zwar konnten mit dieser Arbeit mithilfe des Faktoriellen Surveys und der Verbindung der Rekrutierungs- mit den Individualdaten erste Analysen zum Einfluss von Zugangsbeschränkungen und der Selektivität von Studienfächern auf individuelle Entscheidungsprozesse vorgelegt werden. Doch musste die Frage zum direkten Einfluss bestimmter Auswahlverfahren offen bleiben. Denn selbst wenn keine sozialen Herkunftsunterschiede in den Zulassungschancen beobachtet werden konnten, bedeutet dies nicht, dass bestimmte Verfahren nicht ungleichheitssteigernd (oder -reduzierend) wirken können. Qualitative Fallstudien könnten den Forschungsstand hier bereichern (wie etwa Burke und McManus (2011) für Großbritannien oder Rothmüller (2011) für Österreich). Zudem liegt weiteres Potential in den Daten der Stiftung für Hochschulzulassung, mit denen eine Vollerhebung aller Bewerbungen für bundesweit zulassungsbeschränkte und damit sehr selektive Studiengänge vorliegt.

Mögliche Erweiterungen der Analysen bestehen darin, den Blick für weitere Gruppenvergleiche zu öffnen. Durch den Fokus dieser Arbeit auf die soziale Herkunft von Schüler/innen wurde in den Analysen für weitere soziodemographische Merkmale kontrolliert. Besonders relevant erscheint hier aufgrund der geschlechtstypischen Fach- und Berufswahl ein weiterer Vergleich zwischen jungen Männern und Frauen. Aufgrund geschlechtsspezifischer Studienfachpräferenzen in Kombination mit fachspezifischen Zugangsbarrieren, die in bestimmten frauendominierten Fächern besonders hoch sind, liegt die Vermutung nahe, dass Schülerinnen im Durchschnitt mit größeren Hürden konfrontiert sind als Schüler. Inwiefern dies ein Grund für die geringeren Übergangsquoten von Abiturientinnen ist, ist bisher eine offene Frage.

\section{Implikationen}

Was bedeuten die Befunde dieser Arbeit für die Problematik sozialer Ungleichheit im Hochschulsystem und hieran anknüpfend für die Reproduktion gesellschaftlicher Privilegien?

Zunächst ist grundsätzlich festzustellen, dass selbst innerhalb der hoch selektiven Gruppe von deutschen Studienberechtigten mit Studienaspiration bis ein Jahr nach dem Abitur nicht alle ihren Studienwunsch umsetzen. Im Falle der betrachteten Berliner Schüler/innen handelt es sich hierbei um ganze 30\%. Dabei gehen Studienaspirant/innen in allen untersuchten Phasen „verloren“: nicht alle Studienaspirant/innen bewerben sich, nicht alle Bewerber/innen werden zugelassen und nicht alle Zugelassenen nehmen schließlich ein Studium auf. Der „kurze Weg“ in die Hochschule scheint also nicht für alle einfach zu bewältigen zu sein. Insgesamt konnten beim letztlichen Übergang ins Studium zwar keine soziale Herkunftsdifferenzen aufgezeigt werden, doch deuten die Befunde im Einklang mit existierender Forschung (vgl. Spangenberg et. al 2011, Weiss und Steininger 2013) darauf hin, dass Herkunftsunterschiede bei einer längeren Betrachtung stärker zutage treten. 
Nicht gezeigt werden konnte, dass die Zulassungswahrscheinlichkeit zum Studium mit der sozialen Herkunft variiert. Dies sollte Hochschulen als Gatekeeper jedoch nicht gänzlich von der Verantwortung für einen sozial inklusiven Hochschulzugang freisprechen. Denn es gibt durchaus Anzeichen dafür, dass Zugangshürden antizipiert werden und so in die Entscheidung z.B. gegen eine Studienbewerbung einfließen können. Auch wenn das Zulassungssystem in seiner jetzigen Form sozial schwächere Schüler/innen beim Übergang per se nicht zu benachteiligen scheint, kann es dennoch dazu beitragen, dass die Realisierung spezifischer Präferenzen von der Wartezeit abhängt, die Studienberechtigte akzeptieren wollen oder können. In einem System, in dem ein längerer Zeithorizont honoriert wird, kann dies aus einer längerfristigeren Perspektive, als in dieser Arbeit berücksichtigt werden konnte, zu sozialer Ungleichheit beitragen. Gerade mit der Wartezeit-Quote wird das eigentliche Ziel verfolgt, allen Studienberechtigten eine realistische Chance auf einen Studienplatz im Fach ihrer Wahl zu ermöglichen. „Alle“ bezieht sich in der Argumentation aber vor allem auf das gesamte Leistungs- und weniger auf das gesamte Herkunftsspektrum. Zur Folge könnte dies haben, dass insbesondere leistungsschwächere, sozial privilegierte Studienberechtigte, die es sich leisten können, zu warten, ihr präferiertes Studium aufnehmen können (vgl. Hillmert und Jacob 2003). Die abnehmende Relevanz der Wartezeit, die sich durch die Änderung des Zulassungsverfahrens der bundesweit zulassungsbeschränkten Studiengänge ergibt, ist aus dieser Perspektive zu begrüßen.

Eine wichtige Rolle spielt auch die Mobilitätsbereitschaft von Studienberechtigten. Zwar besteht in allen Herkunftsgruppen die Präferenz für ein Studium in Heimatnähe, doch scheint eine geringe Mobilitätsbereitschaft unter sozial schwächeren Schüler/innen verbreiteter zu sein. Befürworter hochschuleigener Auswahlverfahren sehen in diesen vor allen das Potential, die „Passung“ zwischen Studierenden und Studiengang zum beiderseitigen Nutzen zu verbessern. Eine zentrale Voraussetzung hierfür ist, dass dies auch ein wesentliches Ziel der Studienberechtigten ist. Gerade bei sozial schwächeren Schüler/innen ist dies möglicherweise nicht der Fall, zumindest dann nicht, wenn es regionale Mobilität voraussetzt.

Bisher folgte die Argumentation der Annahme, dass Studienberechtigte bestimmte, fachliche Präferenzen haben, diese aber mehr oder weniger gut umsetzen können. Dabei weisen verschiedene Befunde darauf hin, dass viele Schüler/innen sich trotz generellen Studienwunsches erst spät mit konkreteren Fragen des Studiums beschäftigen. Ad hoc-Entscheidungen, die sich eher an leicht realisierbaren Optionen als an informierten Überlegungen orientieren, tragen in vielen Fällen sicherlich nicht zu hoher Studienzufriedenheit und damit einhergehend zu einer hohen Erfolgswahrscheinlichkeit bei. Insbesondere Schüler/innen aus hochschulfernen Familien können diesbezüglich nicht auf (hochschulbezogene) Erfahrungen aus ihrem familiären Netzwerk aufbauen und könnten von einem besseren und früheren Informationsprozess profitieren. 
Gerade in einem Hochschulsystem, in dem sowohl die Studienoptionen als auch der Zugang zunehmend komplex werden, scheint dies zentral für einen informierten (und erfolgreichen) Übergangsprozess.

In anderen stark stratifizierten Hochschulsystemen sind die Selektivität von Hochschulen und deren Reputation eng miteinander verbunden. Hier konnte nachgewiesen werden, dass harte Auswahlverfahren beim Zugang zu selektiven und oft als ,elitär" wahrgenommenen Hochschulen eine stark abschreckende Wirkung auf bestimmte Bewerber/innengruppen haben (vgl. Reay 1998, Evans 2009). In Deutschland sind trotz Exzellenzinitiative und der beschriebenen Autonomiesteigerung der Hochschulen weder Reputationsunterschiede noch komplexere Auswahlverfahren bisher stark institutionalisiert.

Die präsentierten Befunde verweisen darauf, dass unter diesen Bedingungen weder Auswahlverfahren noch institutionelles Prestige eine abschreckende Wirkung für sozial schwächere Schüler/innen haben. Dies sollte von politischen und Hochschulakteuren als Chance gewertet und dafür genutzt werden, weitere Bestrebungen in Richtung vertikaler Stratifizierung nicht nur aus der Perspektive internationaler Wettbewerbsfähigkeit zu diskutieren und voranzutreiben, sondern dabei auch Wege zu einem über die gesamte Bandbreite hinweg inklusiven Hochschulsystem mitzudenken. In den bisherigen Debatten treten solche Überlegungen klar hinter rein wirtschaftlichen zurück.

In dieser Arbeit standen Studienberechtigte mit Studienaspiration im Fokus, die nur noch einen „kurzen“ (wenn auch nicht unbedingt einfachen) Weg vor sich haben. Zuletzt soll der Blick von diesem kurzen Weg zum langen Pfad gelenkt werden. Denn es sollte nicht vergessen werden, dass die Weichen für ein Studium im deutschen Bildungssystem bereits früh gestellt werden und dass es diese frühen und folgenreichen Entscheidungen sind, die viele vom Hochschulpfad ablenken und damit zu ausgeprägter sozialer Ungleichheit beitragen. Auch wenn hierdurch die hochschulpolitischen Akteure nicht aus der Verantwortung genommen werden sollen, ist ein alleiniger Ansatz an dieser späten Schwelle keinesfalls ausreichend, um ein sozial inklusives Bildungssystem zu realisieren, in dem unabhängig von der sozialen Herkunft Wünsche verfolgt werden können. 


\section{Literaturverzeichnis}

Abraham, M., Auspurg, K. \& Hinz, T. (2010). Migration decisions within dualearner partnerships: A test of bargaining theory. Journal of Marriage and $\mathrm{Fa}$ mily, 72(4), 876-892.

Alexander, C.S. \& Becker, H.J. (1978). The use of vignettes in survey research. Public Opinion Quarterly, 42(1), 93-104.

Alexander, K.L. \& Cook, M.A. (1979). The motivational relevance of educational plans: questioning the conventional wisdom. Social Psychology Quarterly, 42(3), 202-213.

Allison, P.D. (1982). Discrete-time methods for the analysis of event histories. Sociological Methodology, 13, 61-98.

Allison, P.D. (2014a). Listwise deletion: it's not evil. In: https://statisticalhorizons.com/listwise-deletion-its-not-evil [letzter Zugriff: 09.08.2021].

Allison, P.D. (2014b). Event history analysis. Second edition. Thousand Oaks, CA: Sage.

Allmendinger, J. (1989). Educational systems and labor market outcomes. European Sociological Review, 5(3), 231-250.

Allmendinger, J. (2013). Bildungsgesellschaft. Über den Zusammenhang von Bildung und gesellschaftlicher Teilhabe in der heutigen Gesellschaft. Dossier Zukunft Bildung. Bonn: Bundeszentrale für politische Bildung.

Alon, S. (2009). The evolution of class inequality in higher education: Competition, exclusion, and adaptation. American Sociological Review, 74(5), 731755.

Alon, S. \& Tienda, M. (2007). Diversity, opportunity, and the shifting meritocracy in higher education. American Sociological Review, 72(4), 487-511.

Alves, W.M. \& Rossi, P.H. (1978). Who should get what? Fairness judgments of the distribution of earnings. American Journal of Sociology, 84(3), 541-564.

Amt für Statistik Berlin-Brandenburg (2011). Regionaler Sozialbericht Berlin und Brandenburg 2011. Potsdam: Amt für Statistik Berlin-Brandenburg.

Amt für Statistik Berlin-Brandenburg (2016). Studierende an Hochschulen in Berlin. Wintersemester 2015/2016. Teil 2: Ausführliche Ergebnisse. Potsdam: Amt für Statistik Berlin-Brandenburg.

An, B.P. (2010). The relations between race, family characteristics, and where students apply to college. Social Science Research, 39(2), 310-323.

Anders, J. (2012). The link between household income, university applications and university attendance. Fiscal Studies, 33(2), 185-210.

Arum, R., Shavit, Y. \& Gamoran, A. (2007). More inclusion than diversion. Expansion, differentiation, and market structure in higher education. In: Y. Shavit, R. Arum \& A. Gamoran (Hrsg.), Stratification in higher education. A comparative study. Stanford, CA: Stanford University Press, 1-35. 
Auspurg, K. \& Hinz, T. (2011a). Master für Alle? Der Einfluss sozialer Herkunft auf den Studienverlauf und das Übertrittsverhalten von Bachelorstudierenden. Soziale Welt, 62(1), 75-99.

Auspurg, K. \& Hinz, T. (2011b). Gruppenvergleiche bei Regressionen mit binären abhängigen Variablen - Probleme und Fehleinschätzungen am Beispiel von Bildungschancen im Kohortenverlauf. Zeitschrift für Soziologie, 40(1), 62-73.

Auspurg, K. \& Hinz, T. (2015). Factorial survey experiments. Thousand Oaks, CA: Sage.

Auspurg, K., Hinz, T. \& Liebig, S. (2009a). Komplexität von Vignetten, Lerneffekte und Plausibilität im Faktoriellen Survey. Methoden, Daten, Analysen, 3(1), 59-96.

Auspurg, K., Hinz, T., Liebig, S. \& Sauer, C. (2009b). Auf das Design kommt es an. Experimentelle Befunde zu komplexen Settings in Faktoriellen Surveys. SoFid Methoden und Instrumente der Sozialwissenschaften, 2, 23-39.

Austin, P.C. (2010). Estimating multilevel logistic regression models when the number of clusters is low: a comparison of different statistical software procedures. International Journal of Biostatistics, 6(1), Artikel 16.

Autorengruppe Bildungsberichterstattung (2012). Bildung in Deutschland 2012. Ein indikatorengestützter Bericht mit einer Analyse zur kulturellen Bildung im Lebenslauf. Bielefeld: W. Bertelsmann Verlag.

Autorengruppe Bildungsberichterstattung (2016). Bildung in Deutschland 2016. Ein indikatorengestützter Bericht mit einer Analyse zu Bildung und Migration. Bielefeld: W. Bertelsmann Verlag.

Autorengruppe Bildungsberichterstattung (2020). Bildung in Deutschland 2020. Ein indikatorengestützter Bericht mit einer Analyse zu Bildung in einer digitalisierten Welt. Bielefeld: wbv.

Avery, C., Howell, J.S. \& Page L.C. (2014). A review of the role of college applications on students' postsecondary outcomes. Research Brief, October 2014. New York: The College Board.

Avery, C. \& Kane, T. (2004). Student perceptions of college opportunities: The Boston COACH program. In: C. Hoxby (Hrsg.), College choices: The economics of where to go, when to go, and how to pay for it. Chicago: University of Chicago Press, 355-391.

Ayalon, H. (2007). College application behavior: who is strategic? Does it help? Higher Education, 54(6), 885-905.

Ayalon, H. \& Shavit, Y. (2004). Educational reforms and inequalities in Israel: The MMI hypothesis revisited. Sociology of Education, 77(2), 103-120.

Ayhan, H.Ö. \& Işikal, S. (2004). Memory recall errors in retrospective surveys: A reverse record check study. Quality \& Quantity, 38(5), 475-493.

Azjen, I. (1991). The theory of planned behavior. Organizational Behavior and Human Decision Processes, 50(2), 179-211.

Baethge, M. (2006). Das deutsche Bildungs-Schisma: Welche Probleme ein vorindustrielles Bildungssystem in einer nachindustriellen Gesellschaft hat. SOFIMitteilungen, Nr. 34. Göttingen: SOFI, 13-27. 
Ball, S.J., Davies, J., David, M. \& Reay, D. (2002). ,Classification“ and ,Judgement': Social class and the ,cognitive structures' of choice of higher education. British Journal of Sociology of Education, 23(1), 51-71.

Banscherus, U. (2010). Hochschulzulassung und Kapazitätsplanung in Westeuropa. Eine Bestandsaufnahme. Die Hochschule, 2/2010, 40-56.

Banscherus, U., Engel, O., Spexard, A. \& Wolter, A. (2015). Differenzierung als Thema von Hochschulpolitik und Hochschulforschung: Ein hochaktueller „Klassiker“. In: U. Banscherus, O. Engel, A. Mindt, A. Spexard \& A. Wolter (Hrsg.), Differenzierung im Hochschulsystem. Nationale und internationale Entwicklungen und Herausforderungen. Münster: Waxmann, 11-40.

Barlösius, E. (2008). „Leuchttürme der Wissenschaft“. Ein metaphorischer Vorgriff auf eine neuorientierte Wissenschaftspolitik. Leviathan, 36(1), 149-169.

Barone, C. (2006). Cultural capital, ambition and the explanation of inequalities in learning outcomes: a comparative analysis. Sociology, 40(6), 1039-1058.

Barone, C., Schizzerotto, A., Abbiati, G. \& Argentin, G. (2017). Information barriers, social inequality, and plans for higher education: Evidence from a field experiment. European Sociological Review, 33(1), 84-96.

Baumert, J., Klieme, E., Neubrand, M., Prenzel, M., Schiefele, U., Schneider, W., Stanat, P., Tillmann, K-J. \& Weiß, M. (Hrsg.) (2001). PISA 2000: Basiskompetenzen von Schülerinnen und Schülern im internationalen Vergleich. Opladen: Leske + Budrich.

Becker, B. (2010). Bildungsaspirationen von Migranten Determinanten und Umsetzung in Bildungsergebnisse. Arbeitspapiere - Working Papers, Nr. 137. Mannheim: Mannheimer Zentrum für Europäische Sozialforschung.

Becker, G.S. (1986). The economic approach to human behavior. In: J. Elster (Hrsg.), Rational Choice. New York: New York University Press, 108-122.

Becker, G.S. (1993). Human capital: A theoretical and empirical analysis with special reference to education (3rd edition). Chicago: University of Chicago Press.

Becker, R. (2000). Klassenlage und Bildungsentscheidungen. Eine empirische Anwendung der Wert-Erwartungstheorie. Kölner Zeitschrift für Soziologie und Sozialpsychologie, 52 (3), 450-474.

Becker, R. (2006). Dauerhafte Bildungsungleichheiten als unerwartete Folge der Bildungsexpansion? In: A. Hadjar \& R. Becker (Hrsg.), Die Bildungsexpansion. Erwartete und unerwartete Folgen. Wiesbaden: VS Verlag für Sozialwissenschaften, 27-62.

Becker, R. (2009). Wie können „bildungsferne“ Gruppen für ein Hochschulstudium gewonnen werden? Eine empirische Simulation mit Implikationen für die Steuerung des Bildungswesens. Kölner Zeitschrift für Soziologie und Sozialpsychologie, 61(4), 563-593.

Becker, R. (2012). Bildungsungleichheit im Lichte aktueller Theorieanwendung in der soziologischen Bildungsforschung. In: M. Bergman, S. Hupka-Brunner, T. Meyer \& R. Samuel (Hrsg.), Bildung - Arbeit - Erwachsenwerden. Ein interdisziplinärer Blick auf die Transition im Jugend- und jungen Erwachsenenalter. Wiesbaden: VS Verlag für Sozialwissenschaften, 43-75. 
Becker, R., Haunberger, S. \& Schubert, F. (2010). Studienfachwahl als Spezialfall der Ausbildungsentscheidung und Berufswahl. Zeitschrift für Arbeitsmarktforschung, 42(4), 292-310.

Becker, R. \& Hecken, A.E. (2007). Studium oder Berufsausbildung? Eine empirische Überprüfung der Modelle zur Erklärung von Bildungsentscheidungen von Esser sowie von Breen und Goldthorpe. Zeitschrift für Soziologie, 36(2), 100-117.

Becker, R. \& Hecken, A.E. (2008). Warum werden Arbeiterkinder vom Studium an Universitäten abgelenkt? Eine empirische Überprüfung der „Ablenkungsthese" von Müller und Pollak (2007) und ihrer Erweiterung durch Hillmert und Jacob (2003). Kölner Zeitschrift für Soziologie und Sozialpsychologie, 60(1), 3-29.

Becker, R. \& Hecken, A.E. (2009a). Higher education or vocational training? An empirical test of the rational action model of educational choices suggested by Breen and Goldthorpe and Esser. Acta Sociologica, 52(1), 25-45.

Becker, R. \& Hecken, A.E. (2009b). Why are working-class children diverted from universities? European Sociological Review, 25(2), 233-250.

Beller, E. (2009). Bringing intergenerational social mobility research into the twenty-first century: why mothers matter. American Sociological Review, 74(4), 507-528.

Berggren, C. (2007). Broadening recruitment to higher education through the admission system: gender and class perspectives. Studies in Higher Education, 32(1), 97-116.

Berkner, L. \& Chavez, L. (1997). Access to postsecondary education for the 1992 high school graduates. Washington: US Department of Education, Office of Educational Research and Improvement, National Center for Education Statistics.

Bernardi, F., Chakhaia, L. \& Leopold, L. (2017). 'Sing me a song with social significance': The (mis)use of statistical significance testing in European sociological research. European Sociological Review, 33(1), 1-15.

Best, H. \& Wolf, C. (2012). Modellvergleich und Ergebnisinterpretation in Logitund Probit-Regressionen. Kölner Zeitschrift für Soziologie und Sozialpsychologie, 64(2), 377-395.

Blau, P.M. \& Duncan, O.D. (1967). The American Occupational Structure. New York: Wiley.

Bloch, R., Gut, M., Klebig, K. \& Mitterle, A. (2015). Die Auswahl der Besten? Auswahlverfahren an sich stratifizierenden Einrichtungen und Programmen im Hochschulbereich. In: W. Helsper, W. \& H.-H. Krüger (Hrsg.), Auswahl der Bildungsklientel. Zur Herstellung von Selektivität in "exklusiven" Bildungsinstitutionen. Wiesbaden: Springer, 185-209.

Bloch, R., Kreckel, R., Mitterle, A. \& Stock, M. (2014). Stratifikation im Bereich der Hochschulbildung in Deutschland. Zeitschrift für Erziehungswissenschaften, 17(3), 243-261. 
Blossfeld, P.N., Blossfeld, G.J. \& Blossfeld, H.-P. (2015). Educational expansion and inequalities in educational opportunity: Long-term changes for East and West Germany. European Sociological Review, 31(2), 144-160.

Blossfeld, H.-P. \& Shavit, Y. (1993). Dauerhafte Ungleichheiten. Zur Veränderung des Einflusses der sozialen Herkunft auf die Bildungschancen in dreizehn industrialisierten Ländern. Zeitschrift für Pädagogik, 39(1), 25-52.

Bode, M. (2013). Hochschulzulassungsrecht im Spannungsfeld von gesamtstaatlicher Planung und lokaler Gerechtigkeit. Wissenschaftsrecht, 46(4), 348-385.

Bode, M. (2015). §32 Auswahlverfahren. Hochschulrecht in Bund und Ländern, Loseblattsammlung, Stand: September 2015.

Bol, T. \& van de Werfhorst, H.G. (2011). Signals and closure by degrees: The education effect across 15 European countries. Research in Social Stratification and Mobility, 29(1), 119-132.

Boliver, V. (2013). How fair is access to more prestigious UK universities? The British Journal of Sociology, 64(2), 344-364.

Boudon, R. (1974). Education, opportunity, and social inequality. New York: Wiley.

Boudon, R. (2003). Beyond Rational Choice Theory. Annual Review of Sociology, 29(1), 1-21.

Bourdieu, P. (1973). Kulturelle Reproduktion und soziale Reproduktion. In: P. Bourdieu (Hrsg.), Grundlagen einer Theorie der symbolischen Gewalt. Frankfurt am Main: Suhrkamp Verlag, 88-137.

Bourdieu, P. (1982). Die feinen Unterschiede. Kritik der gesellschaftlichen Urteilskraft. Frankfurt am Main: Suhrkamp.

Bourdieu, P. (1983). Ökonomisches Kapital, kulturelles Kapital, soziales Kapital. In: R. Kreckel (Hrsg..), Soziale Ungleichheiten (Soziale Welt, Sonderband 2). Göttingen: Schwartz, 183-198.

Bourdieu, P. (1985). Praktische Vernunft. Zur Theorie des Handelns. Frankfurt am Main: Suhrkamp.

Bourdieu, P. (1990). In Other Words. Stanford, CA: Stanford University Press.

Bourdieu, P. (1992). Die verborgenen Mechanismen der Macht. Hamburg: VSA Verlag.

Bourdieu, P. \& Passeron, J.-C. (1971). Die Illusion der Chancengleichheit. Untersuchungen zur Soziologie des Bildungswesens am Beispiel Frankreichs. Stuttgart: Ernst Klett Verlag.

Bourdieu, P. \& Passeron, J.-C. (1973). Grundlagen einer Theorie der symbolischen Gewalt. Frankfurt am Main: Suhrkamp Verlag.

Bourdieu, P. \& Passeron, J.-C. (2007). Die Erben: Studenten, Bildung und Kultur. Konstanz: UVK.

Bourdieu, P. \& Wacquant, L.J.D. (1996). Reflexive Anthropologie. Frankfurt am Main: Suhrkamp.

Bozick, R., Alexander, K., Entwisle, D., Dauber, S. \& Kerr, K. (2010). Framing the future: revisiting the place of educational expectations in status attainment. Social Forces, 88(5), 2027-2052. 
Bradley, K. (2000). The incorporation of women into higher education: paradoxical outcomes? Sociology of Education, 73(1), 1-18.

Braun, S., Dwenger, N. \& Kübler, D. (2007). Telling the truth may not pay off: an empirical study of centralized university admission in Germany. IZA Discussion Paper, No. 3261. Bonn: Forschungsinstitut zur Zukunft der Arbeit.

Breen, R. \& Goldthorpe, J.H. (1997). Explaining educational differentials. Towards a formal rational action theory. Rationality and Society, 9(3), 275-305.

Breen, R. \& Jonsson, J.O. (2000). A multinomial transition model for analyzing educational careers. American Sociological Review, 65(5), 754-72.

Breen, R. \& Jonsson, J.O. (2005). Inequality of opportunity in comparative perspective: Recent research on educational attainment and social mobility. Annual Review of Sociology, 31, 223-243.

Breen, R., Luijkx, R., Müller, W \& Pollak, R. (2009). Nonpersistent inquality in educational attainment: Evidence from eight European countries. American Journal of Sociology, 114(5), 1475-1521.

Brewer, D.J., Eide, E.R. \& Ehrendberg, R.G. (1999). Does it pay to attend an elite private college? Cross-cohort evidence on the effects of college type on earnings. The Journal of Human Resources, 34(1), 104-123.

Brint, S. \& Karabel, J. (1989). The diverted dream. Community Colleges and the promise of educational opportunity in America, 1900-1985. New York, Oxford: Oxford University Press.

Brown, S.K. \& Hirschman, C. (2006). The end of affirmative action in Washington State and its impact on the transition from high school to college. Sociology of Education, 79(2), 106-130.

Bryan, M.L. \& Jenkins, S.P. (2016). Multilevel modelling of country effects: A cautionary tale. European Sociological Review, 32(1), 3-22.

Buchholz, S. \& Schier, A. (2015). New game, new chance? Social inequalities and upgrading secondary school qualifications in West Germany. European Sociological Review, 31(5), 603-615.

Buchmann, C., Condron, D.J. \& Roscigno, V.J. (2010). Shadow education, American style: test preparation, the SAT and college enrollment. Social Forces, 89(2), 435-461.

Buchmann, C. \& Park, H. (2009). Stratification and the formation of expectations in highly differentiated educational systems. Research in Social Stratification and Mobility, 27(4), 245-267.

Buis, M.L. (2013). The composition of family background: the influence of the economic and cultural resources of both parents on the offsping's educational attainment in the Netherlands between 1939 and 1991. European Sociological Review, 29(3), 593-602.

Bukodi, E. \& Goldthorpe, J.H. (2013). Decomposing 'social origins': the effects of parents' class, status, and education on the educational attainment of their children. European Sociological Review, 29(5), 1024-1039.

Burke, P.J. \& McManus, J. (2011). Art for a few: exclusions and misrecognitions in higher education admission practices. Discourse: Studies in the Cultural Politics of Education, 32(5), 699-712. 
Cabrera, A.F. \& La Nasa, S.M. (2000). Understanding the college-choice process. New Directions for Institutional Research, 107, 5-22.

Cabrera, A. \& La Nasa, S. (2001). On the path to college: Three critical tasks facing America's disadvantaged. Research in Higher Education, 42(2), 119-149.

Carlson, S., Gerhards, G. \& Hans, S. (2017). Educating children in times of globalisation: class-specific child-rearing practices and the acquisition of transnational cultural capital. Sociology, 51(4), 749-765.

Chapman, R.G. (1986). Toward a theory of college selection: A model of college search and choice behavior. Advances in Consumer Research, 13, 246-250.

Christoph, B. (2005). Zur Messung des Berufsprestiges: Aktualisierung der Magnitude-Prestigeskala auf die Berufsklassifikation ISCO88. ZUMA-Nachrichten, 29(57), 79-127.

Coleman, J.S. (1988). Social capital and the creation of human capital, American Journal of Sociology, 94, 95-120.

Collett, J.L. \& Childs, E. (2011). Minding the gap: Meaning, affect, and the potential shortcomings of vignettes. Social Science Research, 40(2), 513-522.

Collins, R. (1971). Functional and conflict theories of educational stratification. American Sociological Review, 36(6), 1002-1019.

Collins, R. (1979). The credential society: An historical sociology of education and stratification. New York: Academic Press.

Dahrendorf, R. (1965). Bildung ist Bürgerrecht. Plädoyer für eine aktive Bildungspolitik. Hamburg: Christian Wegner Verlag.

Daniel, A., Neugebauer, M. \& Watermann, R. (2019). Studienabbruch und Einstellungschancen auf dem Ausbildungsmarkt: Ein faktorieller Survey mit Arbeitgeber/innen. Zeitschrift für Erziehungswissenschaft, 22(5), 1147-1174.

Daniel, A. \& Watermann, R. (2018). The Role of Perceived Benefits, Costs, and Probability of Success in Students' Plans for Higher Education. A Quasi-experimental Test of Rational Choice Theory. European Sociological Review, 34(5), 539-553.

Darraz, E.F., Lenhardt, G., Reiz, R.D. \& Stock, M. (2009). Private Hochschulen in Chile, Deutschland, Rumänien und den USA. HoF-Arbeitsberichte, 3/2009. Wittenberg: Institut für Hochschulforschung (HoF).

Davies, S. \& Guppy, N. (1997). Fields of study, college selectivity, and student inequalities in higher education. Social Forces, 75(4), 1417-1438.

DeBord, L.W., Griffin., L. \& Clark, M. (1977). Race and sex influences in the schooling process of rural and small town youth. Sociology of Education, 50(2), 85-102.

De Graaf, N.-D., de Graaf, P.M. \& Kraaykamp, G. (2000). Parental cultural capital and educational attainment in the Netherlands. Sociology of Education, 73(2), 92-111.

Denk, C.E., Benson, J.M., Fletcher, J.C. \& Reigel, T.M. (1997). How do Americans want to die? A factorial vignette survey of public attitudes about end-oflife medical decision-making. Social Science Research, 26(1), 95-120. 
Denzler, S. \& Wolter, S.C. (2010). Der Einfluss des lokalen Hochschulangebots auf die Studienwahl. Zeitschrift für Erziehungswissenschaften, 13(4), 683706.

Deppe, U., Helsper, W., Kreckel, R., Krüger, H.-H. \& Stock, M. (2015). Germany's hesitant approach to elite education. Stratification processes in German secondary and higher education. In: S.J. Ball, A. van Zanten \& B. DarchyKoechlin (Hrsg.), Elites, privilege and excellence: The national and global redefinition of advantage. World Yearbook of Education 2015. London, New York: Routledge, 82-94.

DesJardins, S.L., Ahlburg, D.A. \& McCall, B.P. (2006). An integrated model of application, admission, enrollment and financial aid. The Journal of Higher Education, 77(3), 381-429.

DesJardins, S.L. \& Toutkoushian, R.K. (2005). Are students really rational? The development of rational thought and its application to student choice. In: J.C. Smart (Hrsg.), Higher education: Handbook of theory and research (Vol. 20). Dordrecht: Kluwer Academic Publishers, 191-240.

DFG [Deutsche Forschungsgemeinschaft] (2019). Entscheidungen in der Exzellenzstrategie: Exzellenzkommission wählt zehn Exzellenzuniversitäten und einen Exzellenzverbund aus. Pressemitteilung, 34.

DiMaggio, P. (1982). Cultural capital and school success: The impact of status culture participation on the grades of U.S. high school students. American Sociological Review, 47(2), 189-201.

Di Stasio, V. (2014). Education as a signal of trainability: results from a vignette study with Italian employers. European Sociological Review, 30(6), 796-809.

Di Stasio, V. \& van de Werfhorst, H.G. (2016). Why education matters to employers in different institutional contexts - A vignette study in England and the Netherlands. Social Forces, 95(1), 77-106.

Do, C. (2004). The effects of local colleges on the quality of college attended. Economics of Education Review, 23(3), 249-257.

Domina, T., Conley, A.M. \& Farkas, G. (2011). The link between educational expectations and effort in the college-for-all era. Sociology of Education, 84(2), 93-112.

Drewes, T. \& Michael, C. (2006). How do students choose a university?: an analysis of applications to universities in Ontario, Canada. Research in Higher Education, 47(7), 781-800.

Duru-Bellat, M., Kieffer, A. \& Reimer, D. (2008). Patterns of social inequality in access to higher education in France and Germany. International Journal of Comparative Sociology, 49(4-5), 347-368.

Edding, F. (1963). Ökonomie des Bildungswesens: Lehren und Lernen als Haushalt und als Investition. Freiburg im Breisgau: Rombach.

Edgerton, J.D. \& Roberts, L.W. (2014). Cultural capital or habitus? Bourdieu and beyond in the explanation of enduring educational inequality. Theory and Research in Education, 12(2), 193-220. 
Ehlert, M., Finger, C., Rusconi, A. \& Solga, H. (2017a). Applying to college. Do information deficits lower the likelihood of college-eligible students from lessprivileged families to pursue their college intentions? - Evidence from a field experiment. Social Science Research, 67, 193-212.

Ehlert, M., Peter, F., Finger, C., Rusconi, A., Solga, H., Spieß, C.K. \& Zambre, V. (2017b). The Berliner-Studienberechtigten-Panel (Best Up). Methodological and Data Report. Data Documentation, No. 90. Berlin: DIW.

Elff, M., Heisig, J.P. Schaeffer, M. \& Shikano, S. (2021). Multilevel Analysis with Few Clusters. Improving Likelihood-Based Methods to Provide Unbiased Estimates and Accurate Inference. British Journal of Political Science, 51(1), 412-426.

Elster, J. (1983). Sour grapes. Studies in the subversion of rationality. Cambridge: Cambridge University Press.

Erikson, R. \& Jonsson, J.O. (1996). Introduction: Explaining class inequality in education: the Swedish test case. In R. Erikson \& J.O. Jonsson (Hrsg.), Can education be equalized?: The Swedish case in comparative perspective. Boulder, CO: Westview Press, 1-64.

Espenshade, T.J., Chung, C.Y. \& Walling, J.L. (2004). Admission preferences for minority students, athletes, and legacies at elite universities. Social Science Quarterly, 85(5), 1422-1446.

Esser, H. (1999). Soziologie. Spezielle Grundlagen. Band 1: Situationslogik und Handeln. Frankfurt am Main: Campus.

Esser, H. (2001). Soziologie. Spezielle Grundlagen. Band 6: Sinn und Kultur. Frankfurt am Main: Campus.

Europäische Kommission (2010). Europe 2020. A strategy for smart, sustainable and inclusive growth. In: http://ec.europa.eu/eu2020/pdf/COMPLET\%20EN \%20BARROSO \%20\%20\%20007\%20-\%20Europe\%202020\%20-\%20EN\%2 0version.pdf [letzter Zugriff: 09.08.2021].

Europäische Kommission (2016). Nationale Studiengebühren und Fördersysteme im europäischen Hochschulwesen 2016/2017. Eurydice - Fakten und Zahlen. Luxemburg: Amt für Veröffentlichungen der Europäischen Union.

Evans, S. (2009). In a different place: working-class girls and higher education. Sociology, 43(2), 340-355.

Finger, C. (2016). Institutional constraints and the translation from college aspirations into intentions. Evidence from a factorial survey. Research in Social Stratification and Mobility, 46(B), 112-128.

Finger, C. (2021). Auswahlverfahren an deutschen Hochschulen. Version 1.0.0. Wissenschaftszentrum Berlin für Sozialforschung. Dataset. https://doi.org/ $10.7802 / 2292$

Finger, C., Fitzner, C. \& Heinmüller, J. (2018). Von wegen „einfach Einschreiben“. Wie Hochschulen Ihre Studierenden auswählen. WZBrief Bildung, Berlin: WZB. 
Finger, C., Solga, H., Ehlert, M. \& Rusconi, A. (2020). Gender differences in the choice of field of study and the relevance of income information. Insights from a field experiment. Research in Social Stratification and Mobility, 65, Article: 100457.

Franke, B. \& Schneider, H. (2015). Informationsverhalten bei der Studien- und Berufsausbildungswahl. Studienberechtigte 2012 ein halbes Jahr vor und ein halbes Jahr nach Schulabschluss. Forum Hochschule, 1/2015. Hannover: DZHW.

Frankenberg, P. (2008). Profil und Passung - Hochschulpolitische Thesen zur Studierendenauswahl. In: C. Heine, H.-J. Didi, K. Haase \& H. Schneider (Hrsg.), Profil und Passung. Studierendenauswahl in einem differenzierten Hochschulsystem. HIS: Forum Hochschule, 14/2008. Hannover: HIS, 9-15.

Frenette, M. (2006). Too far to go on? Distance to school and university participation. Education Economics, 14(1), 31-58.

Frietsch, R. \& Wirth, H. (2001). Die Übertragung der Magnitude-Prestigeskala von Wegener auf die Klassifizierung der Berufe. ZUMA-Nachrichten, 25(48), 139163.

Gambetta, D. (1987). Were they pushed or did they jump? Individual decision mechanisms in education. Cambridge: Cambridge University Press.

Garret, K. (1982). Child abuse: Problems of definition. In: P.H. Rossi \& S.L. Nock (Hrsg.), Measuring social judgments. The factorial survey approach. Beverly Hills, CA: Sage, 177-204.

Gehlke, A., Hachmeister, C.-D. \& Hüning, L (2019a). Der CHE Numerus ClaususCheck 2019/20. Eine Analyse des Anteils von NC-Studiengängen in den einzelnen Bundesländern. Arbeitspapier, Nr. 233. Gütersloh: CHE gemeinnütziges Centrum für Hochschulentwicklung.

Gehlke, A., Hachmeister, C.-D. \& Hüning, L. (2019b). Im Blickpunkt: Der Numerus Clausus (NC) im Wintersemester 2019/20. Was man über den Numerus Clausus (NC) wissen muss und wo es die meisten frei zugänglichen Studiengänge gibt. Gütersloh: CHE gemeinnütziges Centrum für Hochschulentwicklung.

Gelman, A. \& Stern, H. (2006). The difference between "significant" and "not significant" is not itself statistically significant. The American Statistician, 60(4), 328-331.

Gentsch, S. \& Gold, A. (2008). Studierendenauswahl durch Interviews - Ergebnisse einer Pilotstudie. In: H. Schuler \& B. Hell (Hrsg.), Studierendenauswahl und Studienentscheidung. Göttingen: Hogrefe, 156-167.

Gerhards, J. \& Németh, B. (2015). Ökonomisches Kapital der Eltern und Medizinstudium im Ausland. Wie Europäisierungs- und Globalisierungsprozesse die Reproduktion sozialer Ungleichheiten verändern. Berliner Journal für Soziologie, 25(3), 283-301.

Gibbons, S. \& Vignoles, A. (2012). Geography, choice and participation in higher education in England. Regional Science and Urban Economics, 42(1), 98-113. 
Glaesser, J. \& Cooper, B. (2014). Using rational action theory and Bourdieu's habitus theory together to account for educational decision-making in England and Germany. Sociology, 48(3), 463-481.

Glocker, D. \& Storck, J. (2014). Risks and returns to educational fields. Economics of Education Review, 42, 109-129.

Goldthorpe, J.H. (1996a). Class analysis and the reorientation of class theory: The case of persisting differentials in educational attainment. The British Journal of Sociology, 47(3), 481-505.

Goldthorpe, J.H. (1996b). The quantitative analysis of large-scale data-sets and rational action theory: for a sociological alliance. European Sociological Review, 12(2), 109-126.

Goldthorpe, J.H. (2007). "Cultural capital": some critical observations. Sociologica, 1(2), 1-23.

Goyette, K. (2008). College for some to college for all: Social background, occupational expectations, and educational expectations over time. Social Science Research, 37(2), 461-84.

Graf, L. (2013). The hybridization of vocational training and higher education in Austria, Germany, and Switzerland. Opladen, Berlin, Toronto: Budrich UniPress.

Griffith, A. \& Rothstein, D. (2009). Can't get there from here: The decision to apply to a selective college. Economics of Education Review, 28(5), 620-628.

Grodsky, E. \& Jones, M.T. (2007). Real and imagined barriers to college entry: perceptions of cost. Social Science Research, 36(2), 745-766.

Grodsky, E. \& Riegle-Crumb, C. (2010). Those who choose and those who don't: social background and college orientation. The Annals of the American Academy of Political and Social Science, 627(1), 14-35.

Grodsky, E., Warren, J.R. \& Felts, E. (2008). Testing and social stratification in American education. Annual Review of Sociology, 34, 385-404.

Guggenberger, H. (1991). Hochschulzugang und Studienwahl. Empirische und theoretische Ergebnisse von Hochschulforschung. Klagenfurt: Kärntner Druck- und Verlagsgesellschaft.

Haase, K. (2008). Studierendenauswahl im internationalen Vergleich. In: H. Schuler \& B. Hell (Hrsg.), Studierendenauswahl und Studienentscheidung. Göttingen: Hogrefe, 21-31.

Hachmeister, C.-D. \& Grevers, J. (2019). Im Blickpunkt: Die Vielfalt der Studiengänge 2019. Entwicklung des Studienangebotes in Deutschland zwischen 2014 und 2019. Gütersloh: CHE gemeinnütziges Centrum für Hochschulentwicklung.

Hachmeister, C.-D., Hüsch \& M., Thiemann, J (2020). Hochschulzugang in Deutschland. Eignungsprüfungen als Ersatz für das Abitur? Stand 2020. Gütersloh: CHE gemeinnütziges Centrum für Hochschulentwicklung.

Hachmeister, C.-D., Röwert, R., de Vries, L. \& Gvozdenko, V. (2016). Der CHE Numerus Clausus-Check 2016/17. Eine Analyse des Anteils von NC-Studiengängen in den einzelnen Bundesländern. Arbeitspapier, Nr. 192. Gütersloh: CHE gemeinnütziges Centrum für Hochschulentwicklung. 
Hainmueller, J., Hangartner, D. \& Yamamoto, T. (2015). Validating vignette and conjoint survey experiments against real-world behavior. Proceedings of the National Academy of Sciences, 112(8), 2395-2400.

Haller, A.O. (1968). On the concept of aspiration. Rural Sociology, 33(4), 484487.

Haller, A.O. (1982). Reflections on the social psychology of status attainment. In: R.M. Hauser, D. Mechanic, A.O. Haller \& T.S. Hauser (Hrsg.), Social structure and behavior: Essays in honor of William Hamilton Sewell. New York: Academic Press, 3-28.

Haller, A. O. \& Portes, A. (1973). Status attainment processes. Sociology of Education, 46(1), 51-91.

Hällsten, M. (2010). The structure of educational decision making and consequences for inequality: a Swedish test case. American Journal of Sociology, $116(3), 806-854$.

Hänsgen, K.-D. (2008). Anforderungen an Zulassungstests dargestellt am Beispiel des Eignungstests für das Medizinstudium in der Schweiz. In: H. Schuler \& B. Hell (Hrsg.), Studierendenauswahl und Studienentscheidung. Göttingen: Hogrefe Verlag, 147-155.

Hanson, K.H. \& Litten L.H. (1982). Mapping the road to academe: a review of research on women, men, and the college-selection process. In: P.J. Perun (Hrsg.), The undergraduate woman: issues in educational equity. Lexington, MA: D.C. Health, 73-97.

Hanson, S.L. (1994). Lost talent: Unrealized educational aspirations and expectations among US youths. Sociology of Education, 67(3), 159-183.

Hardin, J.W. \& Hilbe, J.M. (2003). Generalized estimating equations. Boca Raton, FL: CRC Press.

Harris, A.L. \& Tienda, M. (2010). Minority higher education pipeline: consequences of changes in college admissions policy in Texas. Annals of the American Academy of Political and Social Science, 627(1), 60-81.

Hauck-Scholz, P. (2010). Hochschulzulassung. Ein verfassungsrechtlicher und hochschulpolitischer Beitrag zur aktuellen Diskussion. Die Hochschule, 2/2010, 86-104.

Haug, V. (2006). „Wer die Wahl hat, hat die Qual?“ - Hochschulwahlrecht im Vergleich. Wissenschaftsrecht, 39(2), 96-113.

Hauser, R.M. \& Anderson, D.K. (1991). Post-high school plans and aspirations of black and white high school seniors: 1976-86. Sociology of Education, 64(4), 263-277.

Heine, C., Briedis, K., Didi, H., Haase, K. \& Trost, G. (2006). Auswahl- und Eignungsfeststellungsverfahren beim Hochschulzugang in Deutschland und ausgewählten Ländern. Eine Bestandsaufnahme. HIS Kurzinformation, A3/2006. Hannover: HIS.

Heine, C., Didi, H.-J., Haase, K. \& Schneider, H. (Hrsg.) (2008). Profil und Passung. Studierendenauswahl in einem differenzierten Hochschulsystem. HIS: Forum Hochschule, 14/2008. Hannover: HIS. 
Heine, C., Spangenberg, H. \& Lörz, M. (2007). Nachschulische Werdegänge studienberechtigter Schulabgänger/innen. Zweite Befragung der Studienberechtigten 20023 1/2 Jahre nach Schulabgang im Zeitvergleich. HIS:Forum Hochschule, 11/2007. Hannover: HIS.

Heine, C., Willich, J. \& Schneider, H. (2010). Informationsverhalten und Entscheidungsfindung bei der Studien- und Berufswahl. Studienberechtigte 2008 ein halbes Jahr vor dem Erwerb der Hochschulreife. HIS: Forum Hochschule, 1/2010. Hannover: HIS.

Helbig, M., Jähnen, S. \& Marczuk, A. (2015). Bundesländerunterschiede bei der Studienaufnahme. WZB Discussion Paper, P 2015-001. Berlin: Wissenschaftszentrum Berlin für Sozialforschung.

Helbig, M., Jähnen, S. \& Marczuk, A. (2017). Eine Frage des Wohnorts. Zur Bedeutung der räumlichen Nähe von Hochschulen für die Studienentscheidung in Deutschland. Zeitschrift für Soziologie, 46(1), 55-70.

Helbig, M. \& Nikolai, R. (2015). Die Unvergleichbaren. Der Wandel der Schulsysteme in den deutschen Bundesländern seit 1949. Bad Heilbrunn: Julius Klinkhardt.

Helbig, M. \& Nikolai, R. (2017). Alter Wolf im neuen Schafspelz? Die Persistenz sozialer Ungleichheiten im Berliner Schulsystem. WZB Discussion Paper, $\mathrm{P}$ 2017-001. Berlin: Wissenschaftszentrum Berlin für Sozialforschung.

Hell, B., Trapmann, S. \& Schuler, H. (2008). Synopse der Hohenheimer Metaanalysen zur Prognostizierbarkeit des Studienerfolgs und Implikationen für die Auswahl- und Beratungspraxis. In: H. Schuler \& B. Herrl (Hrsg.), Studierendenauswahl und Studienentscheidung. Göttingen: Hogrefe Verlag, 43-56.

Helsper, W. \& Krüger, H.-H. (2015). Auswahlverfahren in Bildungsinstitutionen eine Einleitung. In: W. Helsper \& H.-H. Krüger (Hrsg.), Auswahl der Bildungsklientel. Zur Herstellung von Selektivität in ,exklusiven “ Bildungsinstitutionen. Wiesbaden: Springer, 9-27.

Hilbe, J.M. (2009). Logistic Regression Models. Boca Raton, FL: CRC Press.

Hillmert, S. (2005). Bildungsentscheidungen und Unsicherheit: soziologische Aspekte eines vielschichtigen Zusammenhangs. Zeitschrift für Erziehungswissenschaft, 8(2), 173-186.

Hillmert, S. \& Jacob, M. (2003). Social inequality in higher education: Is vocational training a pathway leading to or away from university? European Sociological Review, 19(3), 319-334.

Hillmert, S. \& Jacob, M. (2010). Selections and social selectivity on the academic track: A life-course analysis of educational attainment in Germany. Research in Social Stratification and Mobility, 28(1), 59-76.

Holm, A. \& Jæger, M.M. (2008). Does relative risk aversion explain educational inequality? A dynamic choice approach. Research in Social Stratification and Mobility, 26(3), 199-219. 
Hölscher, S. \& Zymek, B. (2015). Notwendig unbestimmt? Verfahren und Strukturen der Aufnahme und Auswahlprozesse an Kunsthochschulen. In: W. Helsper, W. \& H.-H. Krüger (Hrsg.), Auswahl der Bildungsklientel. Zur Herstellung von Selektivität in ,exklusiven“ Bildungsinstitutionen. Wiesbaden: Springer, 211-238.

Horn, L. (1997). Confronting the odds: Students at risk and the pipeline to higher education. Washington: US Department of Education, Office of Educational Research and Improvement, National Center for Education Statistics.

Horstschräer, J. (2012). University rankings in action? The importance of rankings and an excellence competition for university choice of high ability students. Economics of Education Review, 31(6), 1162-1176.

Hossler, D., Braxton, J. \& Coopersmith, G. (1989). Understanding student choice. In: J.C. Smart (Hrsg.), Higher education: Handbook of theory and research $(I V)$. New York: Agathon Press, 231-288.

Hossler, D. \& Gallagher, K.S. (1987). Studying student college choice. College and University, 62(3), 207-221.

Hossler, D., Schmit, J. \& Vesper, N. (1999). Going to college. How social, economic and educational factors influence decisions students make. Baltimore, London: The John Hopkins University Press.

Hox, J.J. (2010). Multilevel Analysis: Techniques and Applications. 2. Auflage. London: Routledge.

Hoxby, C. \& Avery, C. (2013). The missing "one-offs": the hidden supply of highachieving, low-income students. Brookings Paper on Economic Activity, Spring 2013. Washington: Brookings Institution Press, 1-65.

Hoxby, C. \& Turner, S. (2013). Expanding college opportunities for high-achieving, low- income students. SIEPR Discussion Paper, No. 12-014. Stanford, CA: Stanford Institute for Economic Policy Research.

HRK [Hochschulrektorenkonferenz] (2004). Zur Neuregelung des Hochschulzulassungsrechts. Entschließung des 98. Senats vom 10. Februar 2004. In: https://www.hrk.de/positionen/position/beschluss/detail/zur-neuregelungdes-hochschulzulassungsrechts/[letzter Zugriff: 09.08.2021].

Hüber, F. \& Kübler, D. (2011). Hochschulzulassung in Deutschland: Wem hilft die Reform durch das „Dialogorientierte Serviceverfahren“? Perspektiven der Wissenschaftspolitik, 12(4), 430-444.

Hurtado, S., Kurotsuchi Inkelas, K., Briggs, C. \& Rhee, B.-S. (1997). Differences in college access and choice among racial/ethnic groups: identifying continuing barriers. Research in Higher Education, 38(1), 43-75.

Hussain, I., McNally, S. \& Telhaj, S. (2009). University quality and graduate wages in the UK. London: Centre for the Economics of Education.

Hyman, H.H. (1953). The value systems of different classes: A social psychological contribution to the analysis of stratification. In: R. Bendix \& S.M. Lipset (Hrsg.), Class, status and power. Glencoe: Free Press, 426-442.

Jackson, G.A. (1982). Public efficiency and private choice in higher education. Educational Evaluation and Policy Analysis, 4(2), 237-247. 
Jackson, M. (2013). Introduction. How is inequality of educational opportunity generated? The case for primary and secondary effects. In: M. Jackson (Hrsg.), Determined to succeed? Performance versus choice in educational attainment. Stanford, CA: Stanford University Press, 1-33.

Jackson, M., Erikson, R., Goldthorpe, J.H. \& Yaish, M. (2007). Primary and secondary effects in class differentials in educational attainment: the transition to A-level courses in England and Wales. Acta Sociologica, 50(3), 211-229.

Jackson, M. \& Jonsson, J.O. (2013). Why does inequality of educational opportunity vary across countries? Primary and secondary effects in comparative context. In: M. Jackson (Hrsg.), Determined to succeed? Performance versus choice in educational attainment. Stanford, CA: Stanford University Press, 306-338.

Jackson, M., Luijkx, R., Pollak, R., Vallet, L.A. \& van de Werfhorst, H.G. (2008). Educational fields of study and the intergenerational mobility process in comparative perspective. International Journal of Comparative Sociology, 49(45), 369-388.

Jacob, M., Steininger, H.-M. \& Weiss, F. (2013). Bleibt's dabei? Soziale Ungleichheiten in der Studienabsicht und ihrer Realisierung nach einer beruflichen Ausbildung. In: J. Asdonk, S.U. Kuhnen \& P. Bornkessel (Hrsg.), Von der Schule zur Hochschule: Analysen, Konzeptionen und Gestaltungsperspektiven des Übergangs. Münster: Waxmann, 307-322.

Jacob, M. \& Solga, H. (2015). The German vocational education and training system in transformation: Changes in the participation of low- and high-achieving youth over time. European Sociological Review, 31(2), 161-171.

Jacob, B. \& Wilder, T. (2010). Educational expectations and attainment. NBER Working Paper, No. 15683. Cambridge, MA: National Bureau of Economic Research.

Jæger, M.M. \& Holm, A. (2012). Conformists or rebels? Relative risk aversion, educational decisions, and social class reproduction. Rationality and Society, 24(2), 221-253.

James, R. (2001). Participation disadvantage in Australian higher education: An analysis of some effects of geographical location and socioeconomic status. Higher Education, 42(4), 455-472.

James, R., Baldwin, G. \& McInnies, C. (1999). Which university? The factors influencing the choices of prospective undergraduates. Melbourne: Centre for the Study of Higher Education, University of Melbourne.

Jasso, G. \& Opp, K.-D. (1997). Probing the character of norms: A factorial survey analysis of the norms of political action. American Sociological Review, 62(6), 947-964.

Jencks, C., Crouse, J. \& Mueser, P. (1983). The Wisconsin model of status attainment: A national replication with improved measures of ability and aspiration. Sociology of Education, 56(1), 3-19.

Jerrim, J., Chmielewski, A.K. \& Parker, P. (2015). Socioeconomic inequality in access to high-status colleges: A cross-country comparison. Research in Social Stratification and Mobility, 42, 20-32. 
Kamm, R. \& Köller, M. (2010). Hochschulsteuerung im deutschen Bildungsföderalismus. Swiss Political Science Review, 16(4), 649-686.

Kao, G. \& Tienda, M. (1998). Educational aspirations of minority youth. American Journal of Education, 106(3), 349-384.

Karabel, J. (1972). Open admission. Toward meritocracy or democracy? Change, $4(4), 38-43$.

Karabel, J. (1984). Status group struggle, organizational interests, and the limits of institutional autonomy: The transformation of Harvard, Yale, and Princeton, 1918-1940. Theory and Society, 13(1), 1-40.

Karabel, J. (2005). The chosen: the hidden history of admission and exclusion at Harvard, Yale, and Princeton. Boston: Houghton Mifflin.

Karabel, J. \& Astin, A.W. (1975). Social class, academic ability, and college "quality". Social Forces, 53(3), 381-398.

Karen, D. (2002). Changes in access to higher education in the United States: 19801992. Sociology of Education, 75(3), 191-210.

Karpinska, K., Henkens, K., Schippers, J. \& Wang, M. (2015). Training opportunities for older workers in the Netherlands: a vignette study. Research in Social Stratification and Mobility, 41, 105-144.

Keller, A. (2009). Gefahr in Verzug. Das Zulassungschaos an den Hochschulen ist nicht nur bildungspolitisch verfehlt, sondern auch grundrechtwidrig. In: RosaLuxemburg-Stiftung (Hrsg.), Menschenrecht auf Bildung - Zugänge, Übergänge, Abschlüsse. Berlin: Rosa-Luxemburg-Stiftung, 25-31.

Keller, A. (2016). Promotionsrecht für Fachhochschulen und Promotionszugang von Fachhochschulabsolventen und Fachhochschulabsolventinnen in der Bundesrepublik Deutschland. Stand: 1. Juli 2016. In: https://www.htw-berlin.de/fileadmin/HTW/Zentral/HSL_Promotion/Synopse_Promotionsrecht_Fachhochschulen.pdf [letzter Zugriff: 30.03.2017].

Keller, F. (2014). Strukturelle Faktoren des Bildungserfolgs. Wie das Bildungssystem den Übertritt in das Berufsleben bestimmt. Wiesbaden: Springer VS.

Keller, S. \& Zavalloni, M. (1964). Ambition and social class: a respecification. Social Forces, 43(1), 58-70.

Kellermann, P. (1984). Zur Entwicklung des Hochschulzugangs in Österreich Ergebnisse von Sekundäranalysen und Primärerhebungen. In: P. Kellermann (Hrsg.), Studienaufnahme und Studienzulassung. Aspekte des Wandels im Zugang zu den Hochschulen. Klagenfurt: Kärtner Druck- und Verlagsgesellschaft, 243-302.

Kerckhoff, A.C. (1976). The status attainment process: socialization or allocation? Social Forces, 55(2), 368-381.

Kerckhoff, A.C. (1977). The realism of educational ambitions in England and the United States. American Sociological Review, 42(4), 563-571.

Khattab, N. (2015). Students' aspirations, expectations and school achievement: what really matters? British Educational Research Journal, 41(5), 731-748.

Kinsbergen, S. \& Tolsma, J. (2013). Explaining monetary donations to international development organisations: A factorial survey approach. Social Science Research, 42(6), 1571-1586. 
Kirk, C.M., Lewis, R.K., Scott, A., Wren, D., Nilsen, C. \& Colvin, D.Q. (2012). Exploring the educational aspirations-expectations gap in eighth grade students: implications for educational interventions and school reform. Educational Studies, 38(5), 507-519.

Kjellström, C. \& Regnér, H. (1999). The effects of geographical distance on the decision to enrol in university education. Scandinavian Journal of Educational Research, 43(4), 335-348.

Klasik, D. (2012). The college application gauntlet: a systematic analysis of the steps to four-year college enrollment. Research in Higher Education, 53(5), 506-549.

Kleine, L., Paulus, W. \& Blossfeld, H.-P. (2009). Die Formation elterlicher Bildungsentscheidungen beim Übergang von der Grundschule in die Sekundarstufe I. In: J. Baumert, K. Maaz \& U. Trautwein (Hrsg.), Bildungsentscheidungen. Zeitschrift für Erziehungswissenschaft, Sonderheft 12/2009. Wiesbaden: VS Verlag für Sozialwissenschaften, 103-125.

KMK [Kultusministerkonferenz] (2014). Die Mobilität der Studienanfänger und Studierenden in Deutschland von 1980 bis 2012. Statistische Veröffentlichungen der KMK, Dokumentation Nr. 203. Bonn: KMK.

Koffman, D. \& Tienda, M. (2008). Missing in application: The Texas top 10\% law and campus socioeconomic diversity. In: http://theop.princeton.edu/reports/wp/ApplicantSocialClass.pdf [letzter Zugriff: 14.09.2017].

Kohler, U. \& Kreuter, F. (2012). Datenanalyse mit Stata: Allgemeine Konzepte der Datenanalyse und ihre praktische Anwendung. 4. Auflage. München, Wien: Oldenbourg.

Konegen-Grenier, C. (2018). Wer bekommt einen Studienplatz? Die Regelung des Hochschulzugangs im Umbruch. IW-Report, No. 22/2018, Institut der deutschen Wirtschaft: Köln.

Korupp, S.E., Ganzeboom, H.B.G. \& van der Lippe, T. (2002). Do mothers matter? A comparison of models of the influence of mothers' and fathers' educational and occupational status on children's educational attainment. Quality \& Quantity, 36(1), 17-42.

Kreckel, R. (2014). Akademisierungswahn?: Anmerkungen zur Aktualität einer immer wiederkehrenden Debatte aus der Sicht der Hochschulforschung. Die Hochschule, 22(1), 161-175.

Kreckel, R. (2015). Struktur der Studierendenauswahl im expandierenden Hochschulsystem der Bundesrepublik Deutschland. In: W. Helsper \& H.-H. Krüger (Hrsg.), Auswahl der Bildungsklientel. Zur Herstellung von Selektivität in ,exklusiven "Bildungsinstitutionen. Wiesbaden: VS Verlag für Sozialwissenschaften, 405-420.

Kristen, C., Reimer, D. \& Kogan, I. (2008). Higher education entry of Turkish immigrant youth in Germany. International Journal of Comparative Socio$\log y, 49(2-3), 127-151$.

Kroneberg, C. (2005). Die Definition der Situation und die variable Rationalität der Akteure. Ein allgemeines Modell des Handelns. Zeitschrift für Soziologie, 34(5), 344-363. 
Kroneberg, C. \& Kalter, F. (2012). Rational choice theory and empirical research: Methodological and theoretical contributions in Europe. Annual Review of Sociology, 38, 73-92.

Kroneberg, C., Stocké, V. \& Yaish, M. (2009). Norms or rationality? The rescue of jews, electoral participation, and educational decisions. Sonderforschungsbereich 504 Working Paper Series, Nr. 06-09. Mannheim: Universität Mannheim.

Kroth, A. (2015). The effects of the introduction of tuition on college enrollment in Germany: results from a natural experiment with special reference to students from low parental education backgrounds. Dissertation, University of Michigan.

Krüger, H.-H., Helsper, W., Sackmann, R., Breidenstein, G., Bröckling, U., Kreckel, R., Mierendorff, J. \& Stock, M. (2012). Mechanismen der Elitebildung im deutschen Bildungssystem. Ausgangslage, Theoriediskurse, Forschungsstand. Zeitschrift für Erziehungswissenschaften, 15(2), 327-343.

Kurz, K. \& Paulus, W. (2008). Übergänge im Grundschulalter: die Formation elterlicher Bildungsaspirationen. In: K.-S. Rehberg (Hrsg.), Die Natur der Gesellschaft: Verhandlungen des 33. Kongresses der Deutschen Gesellschaft für Soziologie in Kassel 2006. Frankfurt am Main: Campus, 5489-5503.

Lamont, M. \& Lareau, A. (1988). Cultural capital: allusions, gaps and glissandos in recent theoretical developments. Sociological Theory, 6(2), 153-168.

Lewin, D. (2004). Qualität aufgrund hochschuleigener Auswahlverfahren? Das Hochschulwesen, 1/2004, 15-17.

Lewin, D. \& Lischka, I. (2004). Passfähigkeit - ein neuer Ansatz für den Hochschulzugang? Die Hochschule, 2/2004, 81-95.

Lewin, K. (1939). Field theory and experiment in social psychology: concepts and methods. American Journal of Sociology, 44(6), 868-896.

Lischka, I. (2001). Hochschulzugang - Schnittstelle zwischen Hochschule, Gesellschaft, Individualebene. In: I. Lischka \& A. Wolter (Hrsg.), Hochschulzugang im Wandel? Entwicklungen, Reformperspektiven und Alternativen. Weinheim: Deutscher Studienverlag, 27-40.

Lizardo, O. (2004). The cognitive origins of Bourdieu's habitus. Journal for the Theory of Social Behaviour, 34(4), 375-401.

Lloyd, K.M., Leicht, K.T. \& Sullivan, T.A. (2008). Minority college aspirations, expectations and applications under the Texas Top 10\% Law. Social Forces, 86(3), 1105-1137.

Long, J.S. \& Freese, J. (2014). Regression models for categorical dependent variables using Stata. 3. Auflage. College Station, Texas: Stata Press.

Long, M.C. (2004), College applications and the effect of affirmative action. Journal of Econometrics, 121(1), 319-342.

Long, M.C., Saenz, V. \& Tienda, M. (2010). Policy transparency and college enrollment: Did the Texas Top Ten Law broaden access to public flagships? The Annuals of the American Academy, 627(1), 82-105. 
Lörz, M. (2008). Räumliche Mobilität beim Übergang ins Studium und im Studienverlauf: Herkunftsspezifische Unterschiede in der Wahl und Nachhaltigkeit des Studienortes. Bildung und Erziehung, 61(4), 413-436.

Lörz, M. (2012). Mechanismen sozialer Ungleichheit beim Übergang ins Studium. In: R. Becker \& H. Solga (Hrsg.), Soziologische Bildungsforschung. Kölner Zeitschrift für Soziologie und Sozialpsychologie, Sonderheft 52. Wiesbaden: VS Verlag für Sozialwissenschaften, 302-324.

Lörz, M., Netz, N. \& Quast, H. (2016). Why do students from underprivileged families less often intend to study abroad? Higher Education, 72(2), 153-174.

Lörz, M. \& Quast, H. (2011). Soziale Ungleichheit bei der Wahl der Hochschule. HIS Magazin, 4/2011. Hannover: HIS, 2-4.

Lörz, M., Quast, H. \& Roloff, J. (2015). Konsequenzen der Bologna-Reform: Warum bestehen auch am Übergang vom Bachelor- ins Masterstudium soziale Ungleichheiten? Zeitschrift für Soziologie, 44(2), 137-155.

Lörz, M., Quast, H. \& Woish, A. (2011a). Bildungsintentionen und Entscheidungsprozesse Studienberechtigte 2010 ein halbes Jahr vor Schulabgang. HIS: Forum Hochschule, 14/2011. Hannover: HIS.

Lörz, M. \& Schindler, S. (2011). Bildungsexpansion und soziale Ungleichheit: Zunahme, Abnahme oder Persistenz ungleicher Chancenverhältnisse - eine Frage der Perspektive? Zeitschrift für Soziologie, 40(6), 458-477.

Lörz, M., Schindler, S. \& Walter, J.G. (2011b). Gender inequalities in higher education: extent, development and mechanisms of gender differences in enrolment and field of study choice. Irish Educational Studies, 30(2), 179-198.

Lucas, S. (2001). Effectively maintained inequality: education transitions, track mobility, and social background effects. American Journal of Sociology, 106(6), 1642-1690.

Maas, C.J.M. \& Hox, J.J. (2005). Sufficient sample sizes for multilevel modelling. Methodology, 1(3), 86-92.

Maaz, K. (2006). Soziale Herkunft und Hochschulzugang. Effekte institutioneller Öffnung im Bildungssystem. Wiesbaden: VS Verlag für Sozialwissenschaften.

Mare, R.D. (1980). Social background and school continuation decisions. Journal of the American Statistical Association, 75(370), 295-305.

Mayer, K.U. (2004). Die Bildungsgesellschaft. In: A. Pongs (Hrsg.), In welcher Gesellschaft leben wir eigentlich? München: Dilemma Verlag, 193-218.

Mayer, K.U., Müller, W. \& Pollak, R. (2007). Germany: Institutional change and inequalities of access in higher education. In: Y. Shavit, R. Arum \& A. Gamoran (Hrsg.), Stratification in higher education. A comparative study. Stanford, CA: Stanford University Press, 240-265.

McCarron, G.P. \& Inkelas, K.K. (2006). The gap between educational aspirations and attainment for first generation college students and the role of parental involvement. Journal of College Student Development, 47(5), 534-549.

McDonough, P.M. (1994). Buying and selling higher education: the social construction of the college applicant. The Journal of Higher Education, 65(4), 427-446. 
McDonough, P.M. (1997). Choosing Colleges: How Social Class and Schools Structure Opportunity. Albany: State University of New York Press.

Menon, M.E., Saiti, A. \& Socratous, M. (2007). Rationality, information search and choice in higher education: evidence from Greece. Higher Education, 54(5), 705-721.

Merton, R. (1957). Social Theory and Social Structure. New York: Free Press.

Mickelson, R.A. (1990). The attitude-achievement paradox among black adolescents. Sociology of Education, 63(1), 44-61.

Middendorff, E., Apolinarski, B., Becker, K., Bornkessel, P., Brandt, T., Heißenberg, S. \& Poskowsky, J.. (2017). Die wirtschaftliche und soziale Lage der Studierenden in Deutschland 2016. Berlin: BMBF.

Mitchell, M.N. (2012). Interpreting and visualizing regression models using Stata. College Station, Texas: Stata Press.

Moineddin, R., Matheson, F.I. \& Glazier, R.H. (2007). A simulation study of sample size in multilevel regression models. BMC Medical Research Methodology, 7(1), Artikel 34.

Mood, C. (2010). Logistic regression: why we cannot do what we think we can do, and what we can do about it. European Sociological Review, 26(1), 67-82.

Morgan, J.H.M. (2010). The role of financial information in college decision making. Dissertation, Boston College.

Morgan, S.L. (1998). Adolescent educational expectations. Rationalized, fantasized, or both? Rationality and Society, 10(2), 131-162.

Morgan, S.L. (2007). Expectations and aspirations. In G. Ritzer (Hrsg.), The Blackwell Encyclopedia of Sociology. Oxford: Blackwell Publishing, 1528-1531.

Müller, H.-P. (2014). Pierre Bourdieu. Eine systematische Einführung. Berlin: Shurkamp Verlag.

Müller, W., Brauns, H. \& Steinmann, S. (2002). Expansion und Erträge tertiärer Bildung in Deutschland, Frankreich und im Vereinigten Königreich. Berliner Journal für Soziologie, 12(1), 37-62.

Müller, W. \& Haun, D. (1994). Bildungsungleichheit im sozialen Wandel. Kölner Zeitschrift für Soziologie und Sozialpsychologie, 46(1), 1-42.

Müller, W. \& Karle, W. (1993). Social selection in educational systems in Europe. European Sociological Review, 9(1), 1-23.

Müller, W. \& Pollak, R. (2008). Weshalb gibt es so wenige Arbeiterkinder in Deutschlands Universitäten? In: R. Becker \& W. Lauterbach (Hrsg.), Bildung als Privileg. Wiesbaden: VS Verlag für Sozialwissenschaften, 307-346.

Mutz, D.C. (2011). Population-based survey experiments. Princeton: Princeton University Press.

Nash, R. (2002). The educated habitus, progress at school, and real knowledge. Interchange, 33(1), 27-48.

Neugebauer, M. (2015). The introduction of bachelor degrees and the underrepresentation of students from low social origin in higher education in Germany: A pseudo-panel approach. European Sociological Review, 31(5), 591602 . 
Neugebauer, M., Neumeyer, S. \& Alesi, B. (2016). More diversion than inclusion? Social stratification in the Bologna system. Research in Social Stratification and Mobility, 45, 51-62.

Neugebauer, M., Reimer, D., Schindler, S. \& Stocké, V. (2013). Primary and secondary effects at the transitions to secondary school and tertiary education in Germany. In: M. Jackson (Hrsg.), Determined to succeed? Performance versus choice in educational attainment. Stanford, CA: Stanford University Press, 56-88.

Neugebauer, M. \& Schindler, S. (2012). Early transitions and tertiary enrolment: The cumulative impact of primary and secondary effects on entering university in Germany. Acta Sociologica, 55(1), 19-36.

Nickolaus, R. \& Abele, S. (2009). Chancen und Grenzen eines differenzierten Ansatzes zur Hochschulbewerberauswahl. Das Hochschulwesen, 3/2009, 81-88.

Nida-Rümelin, J. (2013). „Wir sollten den Akademisierungswahn stoppen “. In: http://www.faz.net/aktuell/politik/portraets-personalien/im-gespraech-juliannida-ruemelin-wir-sollten-den-akademisierungswahn-stoppen12554497.html [letzter Zugriff 09.08.2021].

Nisic, N. \& Auspurg K. (2009). Faktorieller Survey und klassische Bevölkerungsumfrage im Vergleich - Validität, Grenzen und Möglichkeiten beider Ansätze. In: P. Kriwy \& C. Groß (Hrsg.), Klein aber fein! Quantitative empirische Sozialforschung mit kleinen Fallzahlen. Wiesbaden: VS Verlag für Sozialwissenschaften, 211-246.

Niu, S.X. \& Tienda, M. (2008). Choosing colleges: Identifying and modeling choice sets. Social Science Research, 37(2), 416-433.

Obermeier, V. \& Schneider, T. (2015). Educational choice and risk preferences: How important is relative vs. individual risk preference? Journal of Educational Research Online, 7(2), 99-128.

OECD [Organisation für wirtschaftliche Zusammenarbeit und Entwicklung] (2008). Tertiary Education for the Knowledge Society: Volume 1 and Volume 2. Paris: OECD Publishing.

OECD [Organisation für wirtschaftliche Zusammenarbeit und Entwicklung] (2016). Education at a Glance 2016: OECD Indicators. Paris: OECD Publishing.

Opp, K.D. (1999). Contending conceptions of the theory of rational action. Journal of Theoretical Politics, 11(2), 171-202.

Page, L.C. \& Scott-Clayton, J. (2016). Improving college access in the United States: barriers and policy responses. Economics of Education Review, 51, 4-22.

Parker, P.D., Jerrim, J., Anders, J. \& Astell-Burt, T. (2016). Does living closer to a university increase educational attainment? A longitudinal study of aspirations, university entry, and elite university enrolment of Australian youth. Journal of Youth and Adolescence, 45(6), 1156-1175.

Parkin, F. (1979). Marxism and Class Theory: A Bourgeois Critique. London: Tavistok.

Parsons, T. (1972). Das System moderner Gesellschaften. München: Juventa. 
Paulsen, M. (1990). College choice: Understanding student enrollment behavior. Washington DC: ERIC Clearinghouse on Higher Education.

Perna, L.W. (2006). Studying college access and choice: a proposed conceptual model. In: J.C. Smart (Hrsg.), Higher education: Handbook of theory and research (Vol. 21). Dordrecht: Springer, 99-157.

Peter, F., Rusconi, A., Solga, H. \& Spieß, C.K. (2016a). Informationen zum Studium verringern soziale Unterschiede bei der Studienabsicht von AbiturientInnen. DIW Wochenbericht, 26, 555-565.

Peter, F., Rusconi, A., Solga, H. \& Spieß, C.K. (2016b). Anschubfinanzierung reicht nicht: Neue Studie untersucht, was zur Entscheidung fürs Studium beiträgt. WZB-Mitteilungen, 15, 32-35.

Peter, F. \& Zambre, V. (2017). Intended college enrollment and educational inequality: Do students lack information? Economics of Education Review, 60, 125-141.

Petzold, K. \& Wolbring, T. (2018). What Can We Learn from Factorial Surveys about Human Behavior? A Validation Study Comparing Field and Survey Experiments on Discrimination. Methodology, 15(1), 19-30.

Pfeffer, F.T. (2008). Persistent inequality in educational attainment and its institutional context. European Sociological Review, 24(5), 543-565.

Picht, G. (1964). Die deutsche Bildungskatastrophe: Analyse und Dokumentation. Olten, Freiburg im Breisgau: Walter.

Portes, A. \& Wilson, K. (1976). Black-white differences in educational attainment. American

Sociological Review, 41(3), 414-431.

Powell, J.J.W. \& Solga, H. (2011). Why are higher education participation rates in Germany so low? Institutional barriers to higher education expansion. Journal of Education and Work, 24(1-2), 49-68.

Preißer, R. (1997). Zur Reproduktion sozialer Ungleichheit durch Bildungsentscheidungen: ein Beitrag zum Verhältnis von Sozialstruktur und individuellem Handeln. Dissertation, Freie Universität Berlin.

Preuß, T. (2017). Fünf Jahre DoSV im Produktivbetrieb: Zahlen, Daten, Fakten. Dortmund: Stiftung für Hochschulzulassung.

Pritchard, R. (2006). Trends in the restructuring of German universities. Comparative Education Review, 50(1), 90-112.

Protsch, P. \& Solga, H. (2016). The social stratification of the German VET system. Journal of Education and Work, 29(6), 637-661.

Quackenbush, S. (2004). The rationality of rational choice theory. International Interactions, 30(2), 87-107.

Quast, H., Scheller, P. \& Lörz, M. (2014). Bildungsentscheidungen im nachschulischen Verlauf. Dritte Befragung der Studienberechtigten 2008 viereinhalb Jahre nach Schulabschluss. Forum Hochschule, 9/2014. Hannover: DZHW.

Rabe-Hesketh, S. \& Skrondal, A. (2008). Multilevel and longitudinal modeling $u$ sing Stata. 2. Auflage. College Station, Texas: Stata Press. 
Reay, D. (1998). 'Always knowing' and 'never being sure': familial and institutional habituses and higher education choice. Journal of Education Policy, 13(4), 519-529.

Reay, D., David, M. \& Ball, S. (2001a). Making a difference?: Institutional habituses and higher education choice. In: http://www.socresonline.org.uk/5/4/reay.html [letzter Zugriff: 14.09.2017].

Reay, D., Davies, J., David, M. \& Ball, S.J. (2001b). Choices of degree or degrees of choice? Class, 'race' and the higher education choice process. Sociology, 35(4), 855-874.

Reimer, D. \& Pollak, R. (2010). Educational expansion and its consequences for vertical and horizontal inequalities in access to higher education in West Germany. European Sociological Review, 26(4), 415-430.

Relikowski, I., Yilmaz, E. \& Blossfeld, H.-P. (2012). Wie lassen sich die hohen Bildungsaspirationen von Migranten erklären? Eine Mixed-Methods-Studie zur Rolle von strukturellen Aufstiegschancen und individueller Bildungserfahrung. In: R. Becker \& H. Solga (Hrsg.), Soziologische Bildungsforschung. Kölner Zeitschrift für Soziologie und Sozialpsychologie, Sonderheft 52. Wiesbaden: VS Verlag für Sozialwissenschaften, 111-136.

Reuther, H. \& Spoun, S. (2009). Information und Auswahl von Studierenden als zentraler Faktor für Studienqualität - erste Erfahrungen der Leuphana Universität Lüneburg. Das Hochschulwesen, 3/2009, 89-97.

Roderick, M., Coca, V. \& Nagaoka, J. (2011). Potholes on the road to college: High school effects in shaping urban students' participation in college application, four-year college enrollment, and college match. Sociology of Education, 84(3), 178-211.

Rodman, H. (1963). The lower-class value stretch. Social Forces, 42(2), 205-215.

Rosenbaum, J. (2001). Beyond college for all: career paths for the forgotten half. New York: Russell Sage Foundation.

Rossi, P.H. (1979). Vignette analysis: Uncovering the normative structure of complex judgments. In: R.K. Merton, J.S. Coleman \& P.H. Rossi (Hrsg.), Qualitative and quantitative social research: Papers in honor of Paul. F Lazarsfeld. New York: Free Press, 176-186.

Rossi, P.H. \& Nock, S.L. (Hrsg.) (1982). Measuring social judgments: The factorial survey approach. Thousand Oaks, CA: Sage Publications.

Rothmüller, B. (2011). Chancen verteilen. Ansprüche und Praxis universitärer Zulassungsverfahren. Wien: Löcker.

Sá, C. \& Tavares, O. (2018). How student choice consistency affects the success of applications in Portuguese higher education. Studies in Higher Education, 43(12), 2148-2160.

Sackmann, R. (2015). Die Bedeutung institutioneller Auswahlprozesse für die Erzeugung von Bildungsungleichheit. In: W. Helsper, W. \& H.-H. Krüger (Hrsg.), Auswahl der Bildungsklientel. Zur Herstellung von Selektivität in "exklusiven" Bildungsinstitutionen. Wiesbaden: Springer, 31-67. 
Salikutluk, Z. (2016). Why do immigrant students aim high? Explaining the aspiration-achievement paradox of immigrants in Germany. European Sociological Review, 32(5), 581-592.

Sauer, C., Auspurg, K., Hinz, T. \& Liebig, S. (2011). The application of factorial surveys in general populations samples: the effects of respondent age and education on response times and response consistency. Survey Research Methods, 5(3), 89-102.

Scheller, P., Isleib, S. \& Sommer, D. (2013). Studienanfängerinnen und Studienanfänger im Wintersemester 2011/12. Tabellenband. HIS: Forum Hochschule, 6/2013. Hannover: HIS.

Schimpl-Neimanns, B. (2000). Soziale Herkunft und Bildungsbeteiligung. Empirische Analysen zur Herkunftsspezifischen Bildungsungleichheiten zwischen 1950 und 1989. Kölner Zeitschrift für Soziologie und Sozialpsychologie, 52(4), 636-669.

Schindler, S. (2012). Aufstiegsangst. Eine Studie zur sozialen Ungleichheit beim Hochschulzugang im historischen Zeitverlauf. Düsseldorf: Vodafone Stiftung.

Schindler, S. (2014). Wege zur Studienberechtigung - Wege ins Studium? Eine Analyse sozialer Inklusions- und Ablenkungsprozesse. Wiesbaden: Springer VS.

Schindler, S. \& Lörz, M. (2012). Mechanisms of social inequality development: primary and secondary effects in the transition to tertiary education between 1976 and 2005. European Sociological Review, 28(5), 647-660.

Schindler, S. \& Reimer, D. (2010). Primäre und sekundäre Effekte der sozialen Herkunft beim Übergang in die Hochschulbildung. Kölner Zeitschrift für Soziologie und Sozialpsychologie, 62(4), 623-653.

Schindler, S. \& Reimer, D. (2011). Differentiation and social selectivity in German higher education. Higher Education, 61(3), 261-275.

Schnabel, K. \& Gruehn, S. (2000). Studienfachwünsche und Berufsorientierungen in der gymnasialen Oberstufe. In: J. Baumert, W. Bos \& R.H. Lehmann (Hrsg.), TIMSS/III: Dritte Internationale Mathematik- und Naturwissenschaftsstudie - Mathematische und naturwissenschaftliche Bildung am Ende der Schullaufbahn. Bd. 2: Mathematische und physikalische Kompetenzen am Ende der gymnasialen Oberstufe. Opladen: Leske und Budrich, 405-443.

Schneider, H. \& Franke, B. (2014). Bildungsentscheidungen von Studienberechtigten. Studienberechtigte 2012 ein halbes Jahr vor und ein halbes Jahr nach Schulabschluss. Forum Hochschule, 6/2014. Hannover: DZHW.

Schneider, T. (2008). Social inequality in educational participation in the German school system in a longitudinal perspective: Pathways into and out of the most prestigious school track. European Sociological Review, 24(4), 511-526.

Schofer, E. \& Meyer J.W. (2005). The worldwide expansion of higher education in the twentieth century. American Sociological Review, 70(6), 898-920.

Schoon, I. \& Lyons-Amos, M. (2016). Diverse pathways in becoming an adult: the role of structure, agency and context. Research in Social Stratification and Mobility, 46, 11-20. 
Schröder, M. (2015). Studienwahl unter den Folgen einer radikalen Differenzierung. Bad Heilbrunn: Klinkhardt Verlag.

Schuler, H. \& Hell, B. (2008). Studierendenauswahl und Studienentscheidung aus eignungsdiagnostischer Sicht. In: H. Schuler \& B. Hell (Hrsg.), Studierendenauswahl und Studienentscheidung. Göttingen: Hogrefe, 11-17.

Schupp, J. \& Hermann, S. (2009). Kognitionspotenziale Jugendlicher: Ergänzung zum Jugendfragebogen der Längsschnittstudie Sozio-Oekonomisches Panel (SOEP); Methodenbericht und Variablendokumentation. Data Documentation, No. 43. Berlin: DIW.

Selbmann, F. (2012). „Verwirrende Vielfalt“ oder wie das Recht auf die freie Wahl eines Studienplatzes ausgehebelt wird. Neue Zeitschrift für Verwaltungsrecht, 31(21), 1373-1375.

Sen, A. (1986). Behavior and the concept of preference. In: J. Elster (Hrsg.), Rational choice. Oxford: Basil Blackwell, 60-81.

SenBJF [Berliner Senatsverwaltung für Bildung, Jugend und Familie] (2014). Berliner Bildungs-ABC. Von vorschulischer bis beruflicher Bildung: Ein Überblick mit Hinweisen für Neu-Berliner. Berlin: SenBJF.

Sewell, W.H. Jr. (1992). A theory of structure: Duality, agency, and transformation. American Journal of Sociology, 98(1), 1-29.

Sewell, W.H., Haller, A.O. \& Portes, A. (1969). The educational and early occupational attainment process. American Sociological Review, 34(1), 82-92.

Sewell, W.H. \& Hauser, R.M. (1972). Causes and consequences of higher education: Models of the status attainment process. American Journal of Agricultural Economics, 54(6), 851-861.

Sewell, W.H., Hauser, R.M., Springer, K.W. \& Hauser, T.S. (2004). As we age: A review of the Wisconsin Longitudinal Study, 1957-2001. Research in Social Stratification and Mobility, 20, 3-111.

Sewell, W. H. \& Shah, V.P. (1967). Socioeconomic status, intelligence, and the attainment of higher education. Sociology of Education, 40(1), 1-23.

Sewell, W.H. \& Shah, V.P. (1968). Social class, parental encouragement, and educational aspirations. American Journal of Sociology, 73(5), 559-572.

Shavit, Y. und Müller, W. (2000). Vocational secondary education: Where diversion and where safety net? European Societies, 2(1), 29-50.

Shiner, M. \& Noden, P. (2015). 'Why are you applying there?': 'race', class and the construction of higher education 'choice' in the United Kingdom. British Journal of Sociology of Education, 36(8), 1170-1191.

Simmenroth-Nayda, A., Meskauskas, E., Burckhardt, G. \& Görlich, Y. (2014). Das neue Göttinger Auswahlverfahren für Medizin - welche Bewerber können profitieren? Zeitschrift für Evidenz, Fortbildung und Qualität im Gesundheitswesen, 108(10), 609-617.

Singer, J.D. \& Willet, J.B. (2003). Applied longitudinal data analysis. Modeling change and event occurrence. Oxford: Oxford University Press.

Slack, K., Mangan, J., Hughes, A. \& Davies, P. (2014). 'Hot', 'cold' and 'warm' information and higher education decision-making. British Journal of Sociology of Education, 35(2), 204-223. 
Smith, J. (2014). The effect of college applications on enrollment. The B.E. Journal of Economic Analysis \& Policy, 14(1), 151-188.

Smith, J., Hurwitz, M. \& Howell, J. (2015). Screening mechanisms and student responses in the college market. Economics of Education Review, 44, 17-28.

Solga, H. (2005a). Ohne Abschluss in die Bildungsgesellschaft. Die Erwerbschancen gering qualifizierter Personen aus soziologischer und ökonomischer Perspektive. Opladen: Verlag Barbara Budrich.

Solga, H. (2005b). Meritokratie - die moderne Legitimation ungleicher Bildungschancen In: P.A. Berger \& H. Kahlert (Hrsg.), Institutionalisierte Ungleichheiten? Stabilität und Wandel von Bildungschancen. Weinheim: Juventa, 1938.

Solga, H. \& Becker, R. (2012). Soziologische Bildungsforschung - eine kritische Bestandsaufnahme. In: R. Becker \& H. Solga (Hrsg.), Soziologische Bildungsforschung. Kölner Zeitschrift für Soziologie und Sozialpsychologie, Sonderheft 52. Wiesbaden: VS Verlag für Sozialwissenschaften, 7-43.

Solga, H. \& Konietzka, D. (2000). Das Berufsprinzip des deutschen Arbeitsmarktes: Ein geschlechtsneutraler Allokationsmechanismus? Schweizerische Zeitschrift für Soziologie, 26(1), 111-147.

Soutar, G.N. \& Turner, J.P. (2002). Students' preferences for university: a conjoint analysis. International Journal of Educational Management, 16(1), 40-45.

Spangenberg, H., Beuße, M. \& Heine, C. (2011). Nachschulische Werdegänge des Studienberechtigtenjahrgangs 2006. Dritte Befragung der studienberechtigten Schulabgänger/innen $2006-31 \frac{1}{2}$ Jahre nach Schulabschluss im Zeitvergleich. HIS:Forum Hochschule, 18/2011. Hannover: HIS.

Spangenberg, H., Mühleck, K. \& Schramm, M. (2012). Erträge akademischer und nicht-akademischer Bildung. Ein Vergleich von studienberechtigten mit Berufsausbildungs- und Hochschulabschluss zwanzig Jahre nach Erlangung der Hochschulreife. HIS: Forum Hochschule, 11/2012. Hannover: HIS.

Spangenberg, H. \& Quast, H. (2016). Bildungsentscheidungen und Umorientierungen im nachschulischen Verlauf. Dritte Befragung der Studienberechtigten 2010 viereinhalb Jahre nach Schulabschluss. Forum Hochschule, 5/2016. Hannover: DZHW.

Spieß, C.K. \& Wrohlich, K. (2010). Does distance determine who attends a university in Germany? Economics of Education Review, 29(3), 470-479.

Statistisches Bundesamt (Destatis) (2012). Bildung und Kultur. Studierende an Hochschulen - Fächersystematik. Fachserie 11, Reihe 4.1. Wiesbaden: Statistisches Bundesamt.

Statistisches Bundesamt (Destatis) (2016a). Hochschulen auf einen Blick. Wiesbaden: Statistisches Bundesamt.

Stegmueller, D. (2013). How many countries do you need for multilevel modeling? A comparison of Bayesian and frequentist approaches. American Journal of Political Science, 57(3), 748-761.

Stephenson, R.M. (1957). Mobility orientation and stratification of 1,000 ninth graders. American Sociological Review, 22(2), 204-212. 
Stocké, V. (2005a). Idealistische Bildungsaspirationen. In: A. Glöckner-Rist (Hrsg.), ZUMA-Informationssystem. Elektronisches Handbuch sozialwissenschaftlicher Erhebungsinstrumente. Version 9.00, Zentrum für Umfragen, Methoden und Analysen, Mannheim.

Stocké, V. (2005b). Realistische Bildungsaspirationen. In: A. Glöckner-Rist (Hrsg.), ZUMA-Informationssystem. Elektronisches Handbuch sozialwissenschaftlicher Erhebungsinstrumente. Version 9.00, Zentrum für Umfragen, Methoden und Analysen, Mannheim.

Stocké, V. (2013). Bildungsaspirationen, soziale Netzwerke und Rationalität. In: R. Becker \& A. Schulze (Hrsg.), Bildungskontexte. Strukturelle Voraussetzungen und Ursachen ungleicher Bildungschancen. Wiesbaden: Springer Fachmedien, 269-298.

Stocké, V., Blossfeld, H.-P., Hoenig, K. \& Sixt, M. (2011). Social inequality and educational decisions in the life course. In: H.-P. Blossfeld, H.-G. Roßbach \& J. von Maurice (Hrsg.), Education as a Lifelong Process. The German National Educational Panel Study. Zeitschrift für Erziehungswissenschaft, Sonderheft 14/2011. Wiesbaden: VS Verlag für Sozialwissenschaften, 103-119.

Sudman, S., Bradburn, N.M. \& Schwarz, N. (1996). Thinking about answers: The application of cognitive processes to survey methodology. San Francisco, CA: Jossey-Bass.

Suhonen, T. (2014). Field-of-study choice in higher education: does distance matter? Spatial Economic Analysis, 9(4), 355-375.

Sullivan, A. (2002). Bourdieu and education: How useful is Bourdieu's theory for researchers? The Netherlands' Journal of Social Sciences, 38(2), 144-166.

Swartz, D.L. (2008). Social closure in American elite higher education. Theory and Society, 37(4), 409-419.

Swidler, A. (1986). Culture in action: Symbols and strategies. American Sociological Review, 51(2), 273-286.

Täger, M. (2009). Die neue Zulassungspraxis an deutschen Hochschulen: Die Bedeutung des Eignungsfeststellungsverfahrens für das Soziologiestudium. In: M. Bülow-Schramm (Hrsg.), Hochschulzugang und Übergänge in der Hochschule: Selektionsprozesse und Ungleichheiten. Bern: Peter Lang, 81-96.

Täger, M. (2010). Der Hochschulzugang: Eine bildungs- und organisationssoziologische Untersuchung der Reform der Hochschulzulassung durch Auswahlund Eignungsfeststellungsverfahren. Dissertation, LMU München: Sozialwissenschaftliche Fakultät.

Tarazona, M. (2006). Berechtigte Hoffnung auf bessere Studierende durch hochschuleigene Studierendenauswahl? Eine Analyse der Erfahrungen mit Auswahlverfahren in der Hochschulzulassung. Beiträge zur Hochschulforschung, 28(2), 68-89.

Teichler, U. (1984). Hochschulzugang und Hochschulzulassung im internationalem Vergleich. In: P. Kellermann (Hrsg.), Studienaufnahme und Studienzulassung. Aspekte des Wandels im Zugang zu den Hochschulen. Klagenfurt: Kärtner Druck- und Verlagsgesellschaft, 9-24. 
Teichler, U. (2005). Hochschulstrukturen im Umbruch - eine Bilanz der Reformdynamik seit vier Jahrzehnten. Frankfurt am Main: Campus.

Thomsen, J.-P. (2018). Test-based admission to selective universities: a lever for first-generation students or a safety net for the professional classes? Sociology, 52(2), 333-350.

Trautwein, U., Maaz, K., Lüdtke, O., Nagy, G., Husemann, N., Watermann, R. \& Köller, O. (2006). Studieren an der Berufsakademie oder an der Universität, Fachhochschule oder Pädagogischen Hochschule?: Ein Vergleich des Leistungsstands, familiären Hintergrunds, beruflicher Interessen und der Studienwahlmotive von (künftigen) Studierenden aus Baden-Württemberg. Zeitschrift für Erziehungswissenschaften, 9(3), 393-412.

Trebbels, M. (2015). The transition at the end of compulsory full-time education. Educational and future career aspirations of native and migrant students. Wiesbaden: Springer Fachmedien.

Triventi, M. (2013). The role of higher education stratification in the reproduction of social inequality in the labor market. Research in Social Stratification and Mobility, 32, 45-63.

Triventi, M., Vergolini, L. \& Zanini, N. (2017). Do individuals with high social background graduate from more rewarding fields of study? Changing patterns before and after the 'Bologna Process'. Research in Social Stratification and Mobility, 51, 28-40.

Trost, G., Blum, F., Fay, E., Klieme, E., Maichle, U., Mayer, M. \& Nauels, H.U. (1998). Evaluation des Tests für Medizinische Studiengänge (TMS): Synopse der Ergebnisse. Bonn: Institut für Test- und Begabungsforschung.

Turley, R.N. (2009). College proximity: mapping access to opportunity. Sociology of Education, 82(2), 126-146.

Turley, R.N., Santos, M. \& Ceja, C. (2007). Social origin and college opportunity expectations across cohorts. Social Science Research, 36(3), 1200-1218.

Turner, R.H. (1960). Sponsored and contest mobility and the school system. American Sociological Review, 25(6), 855-867.

Vaisey, S. (2010). What people want: Rethinking poverty, culture, and educational attainment. The Annuals of the American Academy, 629(1), 75-101.

van de Werfhorst, H.G. (2010). Cultural capital: strengths, weaknesses and two advancements. British Journal of Sociology of Education, 31(2), 157-169.

van de Werfhorst, H.G., De Graaf, N.D. \& Kraaykamp, G. (2001). Intergenerational resemblance in field of study in the Netherlands. European Sociological Review, 17(3), 275-293.

van de Werfhorst, H.G. \& Hofstede, S. (2007). Cultural capital or relative risk aversion? Two mechanisms for educational inequality compared. British Journal of Sociology, 58(3), 391-415.

van de Werfhorst, H.G., Sullivan, A. \& Cheung, S.Y. (2003). Social class, ability and choice of subject in secondary and tertiary education in Britain. British Educational Research Journal, 29(1), 41-62. 
Vergolini, L. \& Zanini, N. (2015). Away, but not too far from home. The effects of financial aid on university enrolment decisions. Economics of Education Review, 49, 91-109.

von Hippel, P.T. (2007). Regression with missing Ys: an improved strategy for analyzing multiply imputed data. Sociological Methodology, 37(1), 83-177.

Wagner, A. (2018). Fördert die Exzellenzinitiative soziale Ungleichheit bei der Hochschulwahl? Untersuchung sozialer Folgen einer Prestigedifferenzierung zwischen deutschen Universitäten. Zeitschrift für empirische Hochschulforschung, 2018(2), 133-155.

Wallander, L. (2009). 25 years of factorial surveys in sociology: A review. Social Science Research, 38(3), 505-520.

Watermann, R., Daniel, A. \& Maaz, K. (2014). Primäre und sekundäre Disparitäten des Hochschulzugangs: Erklärungsmodelle, Datengrundlagen und Entwicklungen. Zeitschrift für Erziehungswissenschaft, 17(2), 233-261.

Watermann, R. \& Maaz, K. (2006). Effekte der Öffnung von Wegen zur Hochschulreife auf die Studienintention am Ende der gymnasialen Oberstufe. Zeitschrift für Erziehungswissenschaft, 9(2), 219-239.

Watermann, R. \& Maaz, K. (2010). Soziale Herkunft und Hochschulzugang: Eine Überprüfung der Theorie des geplanten Verhaltens. In W. Bos, E. Klieme \& O. Köller (Hrsg.), Schulische Lerngelegenheiten und Kompetenzentwicklung: Festschrift für Jürgen Baumert. Münster: Waxmann, 311-329.

Waydhas, C., Heue, M. \& Nast-Kolb, D. (2007). Strukturierte Interviews zur Studienplatzvergabe in der Medizin: Erste Erfahrungen. GMS Zeitschrift für Medizinische Ausbildung, 24(4), Doc186.

Weber, J.D. (2010). Kapazitätsrecht. Quantität und Gleichheit versus Qualität und Wettbewerb. Die Hochschule, 2/2010, 73-85.

Weber, M. (1980). Wirtschaft und Gesellschaft: Grundriß der verstehenden Soziologie. Tübingen: Mohr.

Wegener, B. (1985). Gibt es Sozialprestige? Zeitschrift für Soziologie, 14(3), 209235.

Weiss, F., Schindler, S. \& Gerth, M. (2015). Hochschulrankings als Kriterium für neue soziale Ungleichheit im tertiären Bildungssystem? Zeitschrift für Soziologie, 44(5), 366-382.

Weiss, F., Steininger, H.-M. (2013). Educational family background and the realisation of educational career intentions: participation of German upper secondary graduates in higher education over time. Higher Education, 66(2), 189-202.

Williams, R. (2012). Using the margins command to estimate and interpret adjusted predictions and marginal effects. The Stata Journal, 12(2), 308-331.

Williams, T.H. (1972). Educational aspirations: longitudinal evidence on their development in Canadian youth. Sociology of Eduction, 45(2), 107-133.

Winkler, O. (2014). Exzellente Wahl. Soziale Selektivität und Handlungsorientierung bei der Wahl der Spitzenbildung im Hochschulbereich. Zeitschrift für Soziologie der Erziehung und Sozialisation, 24(3), 280-296. 
Winkler, O. (2019). Vertikale Differenzierung und geografische Studierendenmobilität. In: W. Helsper, H.-H. Krüger \& J. Lüdemann (Hrsg.), Neue Stratifizierungen im Bildungssystem? Ergebnisse der DFG- Forschergruppe „, Mechanismen der Elitebildung im deutschen Bildungssystem ". Beiheft der Zeitschrift für Pädagogik, 232-252.

Winter, M., Rathmann, A., Trümpler, D. \& Falkenhagen, T. (2012). Entwicklungen im deutschen Studiensystem. Analysen zu Studienangebot, Studienplatzvergabe, Studienbewerbung und Studienkapazität. HoF-Arbeitspapiere, 7/2012. Wittenberg: Institut für Hochschulforschung (HoF).

Wissenschaftsrat (2004). Empfehlungen zur Reform des Hochschulzugangs. Berlin.

Wissenschaftsrat (2010). Empfehlungen zur Differenzierung der Hochschulen. Lübeck.

Wolter, A. (2001). Neuordnung des Hochschulzugangs durch hochschuleigene Auswahlverfahren. In: I. Lischka \& A. Wolter (Hrsg.), Hochschulzugang im Wandel? Entwicklungen, Reformperspektiven und Alternativen. Weinheim, Basel: Beltz Verlag, 269-299.

Wolter, A. (2008). Hochschulzulassung im differenzierten Hochschulsystem Von der Studienberechtigung zur individuellen Kompetenzmessung. In: C. Heine, H.-J. Didi, K. Haase \& H. Schneider (Hrsg.), Profil und Passung. Studierendenauswahl in einem differenzierten Hochschulsystem. HIS: Forum Hochschule, 14/2008. Hannover: HIS, 16-34.

Wolter, A. (2014). Eigendynamik und Irreversibilität der Hochschulexpansion: Die Entwicklung der Beteiligung an Hochschulbildung in Deutschland. In: U. Banscherus, M. Bülow-Schramm, K. Himpele, S. Staack \& S. Winter (Hrsg.), Übergänge im Spannungsfeld von Expansion und Exklusion. Bielefeld: Bertelsmann, 19-38.

Yee, A.S. (1997). Thick rationality and the missing "brute fact": the limits of rationalist incorporations of norms and ideas. The Journal of Politics, 59(4), 10011039.

Zimdars, A., Sullivan, A. \& Heath, A. (2009). Elite higher education admission in the arts and sciences: is cultural capital the key? Sociology, 43(4), 648-666.

Zimmerhofer, A. \& Trost, G. (2008). Auswahl- und Feststellungsverfahren in Deutschland - Vergangenheit, Gegenwart und Zukunft. In: H. Schuler \& B. Herrl (Hrsg.), Studierendenauswahl und Studienentscheidung. Göttingen: Hogrefe Verlag, 32-42.

Zimmermann, K. (2010). Kapazitätsrechtliche Grundlagen und alternative Regelungsmodelle im hochschulpolitischen Diskurs. Die Hochschule, 2/2010, 920. 


\section{Anhang}

Der Anhang steht online zum kostenfreien Download bereit und ist über die folgende DOI erreichbar: 10.3224/96665040A 


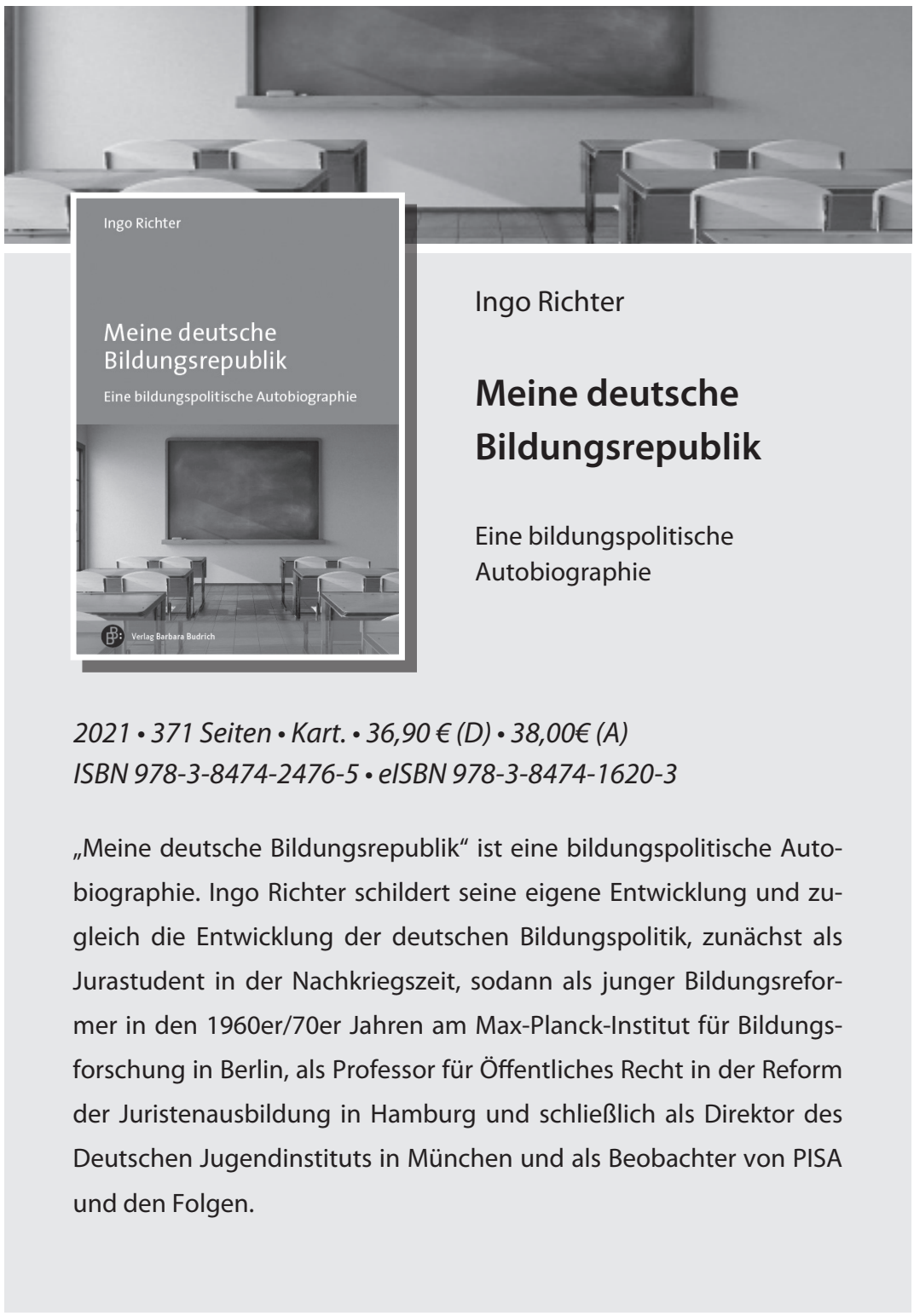

www.shop.budrich.de 


\section{Claudia Finger Soziale Herkunft und die Umsetzung von Studienaspirationen}

Individuelle und institutionelle Einflüsse in Phasen der Selbst- und Fremdselektion

Ausgangspunkt des Buches ist das für Deutschland konstatierte Problem geringer Studierendenquoten bei gleichzeitig ausgeprägter sozialer Ungleichheit im Studium. Das Buch untersucht dabei individuelle und institutionelle Hürden bei der Umsetzung von Studienaspirationen und fragt, ob es selbst auf diesem „kurzen Stück“ ins Studium soziale Herkunftsunterschiede gibt. Im Mittelpunkt der Untersuchung stehen theoretisch wie quantitativ empirisch verschiedene Phasen der Aspirationsumsetzung - Intention, Bewerbung, Zulassung, Studienaufnahme - sowie die direkte und indirekte Rolle von Gatekeepingprozessen an deutschen Hochschulen.

Die Autorin:

Dr. Claudia Finger, wissenschaftliche Mitarbeiterin, Abteilung Ausbildung und Arbeitsmarkt, Wissenschaftszentrum Berlin für Sozialforschung

ISBN 978-3-96665-040-3

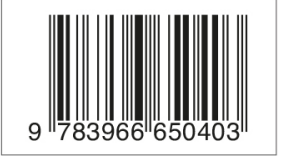

